Portland State University

PDXScholar

Summer 8-1-2013

\title{
"Who Would Have Thought, With a Diagnosis Like \\ This, I Would be Happy?": Portraits of Perceived Strengths and Resources in Early-Stage Dementia
}

Jutta Elisabeth Ataie

Portland State University

Follow this and additional works at: https://pdxscholar.library.pdx.edu/open_access_etds

Part of the Clinical and Medical Social Work Commons, Nervous System Diseases Commons, and the Social Work Commons

Let us know how access to this document benefits you.

\section{Recommended Citation}

Ataie, Jutta Elisabeth, "'Who Would Have Thought, With a Diagnosis Like This, I Would be Happy?":

Portraits of Perceived Strengths and Resources in Early-Stage Dementia" (2013). Dissertations and Theses. Paper 1107.

https://doi.org/10.15760/etd.1107

This Dissertation is brought to you for free and open access. It has been accepted for inclusion in Dissertations and Theses by an authorized administrator of PDXScholar. Please contact us if we can make this document more accessible: pdxscholar@pdx.edu. 
"Who Would Have Thought, With a Diagnosis Like This, I Would be Happy?": Portraits of Perceived Strengths and Resources in Early-Stage Dementia

by

Jutta Elisabeth Ataie

A dissertation submitted in partial fulfillment of the requirements for the degree of

Doctor of Philosophy

in

Social Work and Social Research

Dissertation Committee:

Vicki Cotrell, Chair

Maria Talbott

Rebecca Logsdon

David Morgan

Paula Carder

Portland State University

2013 
(C) 2013 Jutta Elisabeth Ataie 


\begin{abstract}
This study used photovoice methodology to explore how people with early-stage dementia use their perceived strength and resources to cope with the illness. Purposive sampling was used to recruit participants. Thirteen women and seven men age 57 to 90 (mean 73.4) with MMSE scores ranging from 20 to 28 (mean 25.6) participated in the project. Participants were provided with a disposable camera and invited to take photographs relevant to their well-being. The photographs provided the means for participants to take the lead in dialogue sessions about their coping response.

Grounded theory analysis revealed that initially, early-stage dementia precipitated a disruption in well-being. For the participants in this study, learning to live with the illness was an ongoing, continually shifting process of adjustment, where the participants moved from a familiar sense of well-being to an alternative sense of well-being. In this process the participants used a variety of strengths and resources ranging from those that supported them in reconstructing identity, regaining control, and rebuilding relationships to those that assisted them in reestablishing a healthy lifestyle and restoring meaning and purpose. Striving toward well-being while acknowledging the inconceivable notion of losing one's cognitive abilities was the central strength that the participants brought to this process. It captured the paradox the participants faced in living with early-stage dementia, which was to relegate the illness to the background of their lives while managing its symptoms in the foreground.
\end{abstract}




\section{Acknowledgements}

There are many people who have contributed to this dissertation. First and foremost, I would like to express my admiration, appreciation, and respect for the 20 amazing people with early-stage dementia who so generously shared their lives, fears, and hopes with me. I am humbled by their willingness to talk so openly with me. Secondly, I would like to express my appreciation to the members of my dissertation committee. I owe a special thanks to Vicki Cotrell, my dissertation chair, who provided invaluable and tireless guidance from inception through completion of this study. She had a great impact on my scholarly development and I am grateful for her leadership, insights, encouragement, and confidence in me. Gratitude beyond words goes to David Morgan. I feel deeply honored that he was willing to be part of my dissertation committee. He is a mentor in every sense of the word, who challenged and transformed the way I think and conduct research. No words can convey the gratitude I feel for Rebecca Logsdon and her enthusiasm and commitment to me, my research, and people with dementia. Her expertise, guidance, and belief in me helped me grow as a researcher. Meeting with her always left me passionate about the possibilities for creative scholarship. I would also like to thank Maria Talbott for her encouragement throughout my doctoral studies. Her enthusiasm was a source of inspiration and nurtured my interest in gerontology. My sincere gratitude also extends to Paula Carder for her thoughtful comments and suggestions.

I would also like to express thanks to the members of the North West Research Group on Aging, with especial appreciation for Amy Cunningham for her outstanding effort in recruiting participants. I also gratefully acknowledge the support I received from 
the Alzheimer's Association, Western and Central Washington State Chapter in recruiting participants. I am specially thankful to Joanne Maher, Director Social Services and Linda Whiteside, Director Community Support. I would also like to thank Pauline Duffy, Ph.D. Program Assistant who brought a deep warm humanity to navigating organizational maze. Thank you also to Marjorie McGhee, Justine McGovern, and Lisa Bancroft for insisting that research and academia is the right path for me. I am indebted to my nursing and social work colleagues Debra O'Connor, Michiko Uto, James Knoll, and Christine Ingersoll for providing a clinician's perspective on my research. They have been terrific listeners and I greatly appreciate their insights and clarity. A special thanks to Sheila Barns and Linda Purdy who have supported me with their ongoing prayers.

My deepest gratitude and love extends to my parents, Helga and Helmut Conrad, gave me their full support in pursuing my doctoral studies from across the Atlantic Ocean. They have been wonderful role models and a source of encouragement and support throughout my life. I would like to convey my appreciation for my late motherin-law Pooran Ataie who always bolstered my confidence and thought I could do no wrong. My heartfelt thanks to my aunt Renate Thome for her gift of love and support. I would like to express my love and appreciation for my husband, Shahreyar Ataie, who is most responsible for my success in completing this dissertation. My journey as a doctoral student would not have been possible without his unending love, support, and patience. A very special thanks to Little Man and Gianni, our cats who saw me at my worst, never complained, and purred when I felt desperate. Finally, I would also like to thank all the people with cognitive impairment whom I have met and worked with over the years. They have taught me much and continue to be a source of inspiration. 


\section{TABLE OF CONTENTS}

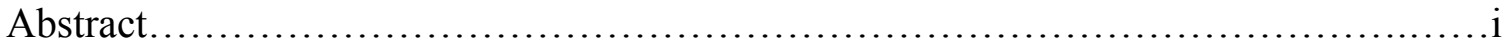

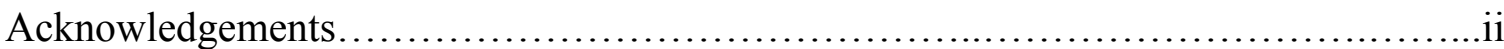

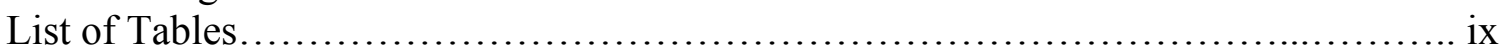

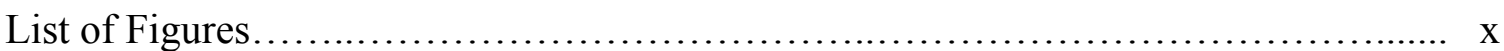

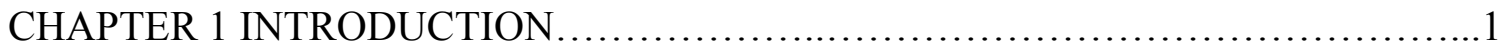

Purpose of the Study..................................................... 5

Research Questions......................................................6

Significance of the Study.................................................6

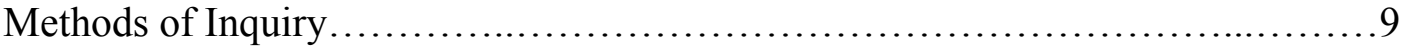

Definition of Important Constructs............................................. 9

Early-stage dementia............................................. 9

Well-being.................................................. 10

Strengths and resources..........................................10

Coping........................................................

Coping strategies.................................................... 11

Adaptation.....................................................11

Adaptive tasks..................................................11

CHAPTER 2 THEORETICAL FRAMEWORK...................................13

The Transactional Model of Stress, Appraisal, and Coping.....................13

Relevance to this study........................................16

The Strengths Perspective.............................................. 17

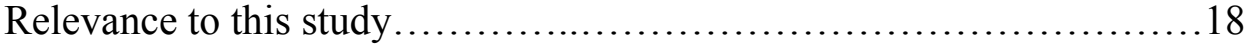

CHAPTER 3 LITERATURE REVIEW .......................................... 19

Dementia as a Social Construct................................................20

Historical representations of dementia............................22

Medical model.......................................................24

Bio-psycho-social-spiritual model....................................29

The Experience of Dementia............................................ 32

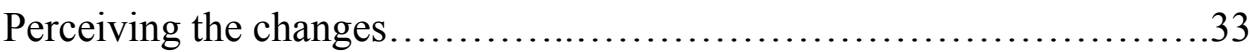

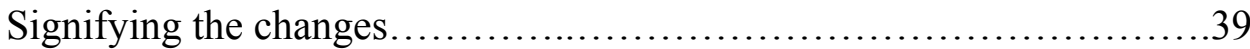

Emoting the changes............................................43

Coping with the changes..........................................46

Perceiving strengths and resources...................................52

Strength and coping......................................52

Strength and early-stage dementia..........................57

Implications for the present study...................................... 59

CHAPTER 4 RESEARCH METHODS, DESIGN, AND PROCESS ...................61

Rational for Methods, Design, \& Process........................................61

Qualitative methodology......................................61 


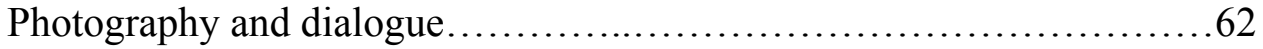

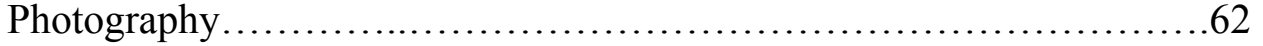

Participant-generated photographs..........................63

Dialogue...............................................................65

Photovoice methodology.......................................66

Traditional photovoice process ............................68

Modified photovoice process..............................69

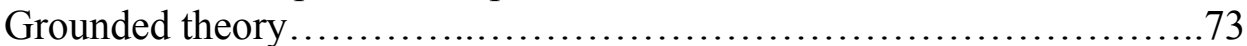

Combining photovoice and grounded theory...........................76

Quantitative measures..............................................76

Researcher as instrument.......................................... 79

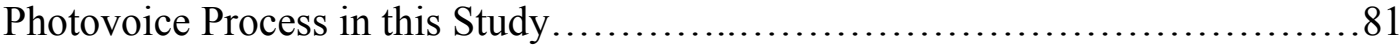

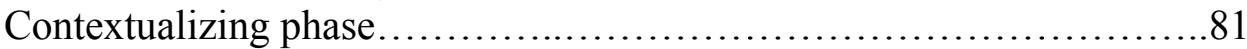

Building community support................................81

Identifying the project site...............................82

Specifying the methodology.............................82

Developing study-related tools........................82

Operationalizing strengths and resources................85

Conducting the pilot...............................85

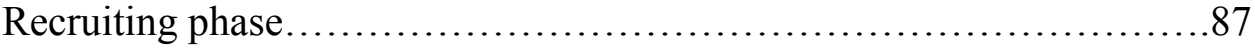

Eligibility criteria......................................... 87

Sampling strategy......................................... 87

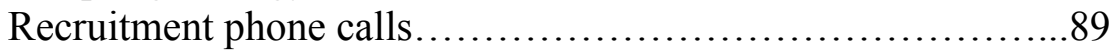

Sample size............................................89

Screening interviews....................................90

Consent procedure......................................93

Opening phase ...................................................... 95

Administering quantitative measures........................95

Photovoice training sessions..............................95

Discovery phase ................................................ 98

Photo-taking activity.....................................98

Researcher check-in phone calls............................98

Camera pick-up........................................98

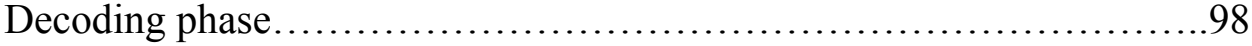

Brief individual interviews...............................99

In-depth individual interviews............................ 100

Interpretation phase ........................................... 102

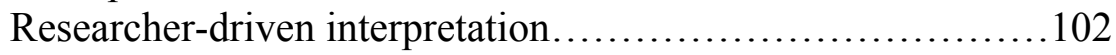

Data sources.................................... 102

Qualitative data analysis............................. 103

Quantitative data analysis..............................110

Participant-driven interpretation...........................110

Assembling the dyads...............................111

Dyadic interviews.................................. 112

Strategies for Ensuring Trustworthiness.................................. 115 
Credibility ................................................... 116

Prolonged engagement....................................116

Triangulation..............................................117

Reflexivity .......................................... 118

Member checking........................................121

Transferability and confirmability.................................121

Dependability .................................................

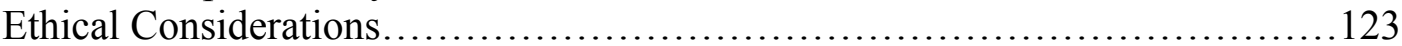

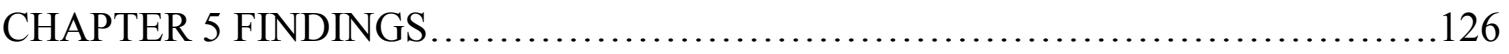

Snapshot of the Participants.............................................. 126

The Process of Coping and Adaptation.........................................129

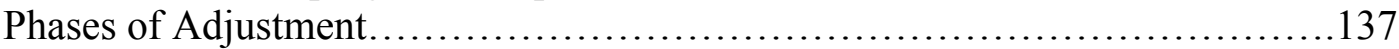

Living with a familiar sense of well-being............................138

Dementia intruding on the familiar sense of well-being..................140

Dementia disrupting the familiar sense of well-being...................146

Experiencing a disrupted sense of well-being..........................150

Disruption of sense of identity.............................. 151

Disruption of sense of control................................154

Disruption of sense of meaning \& purpose.....................156

Disruption of sense of connectedness..........................159

Disruption of healthy lifestyle..................................163

Discussion experiencing a disrupted sense of well-being........165

Defining an alternative sense of well-being............................166

Constructing an alternative sense of well-being.........................169

Reconstructing identity .................................... 171

Focusing on the here and now.........................172

Restorying the self.................................177

Assigning the illness a position..........................184

Contrasting experiences.............................187

Discussion reconstructing identity......................191

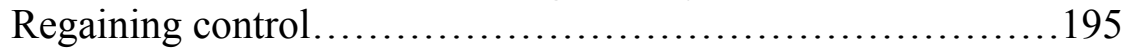

Managing the illness.................................195

Calibrating emotions..................................201

Simplifying life....................................208

Negotiating needs.................................218

Discussion regaining control...........................228

Restoring meaning and purpose.............................232

Finding meaning in goals.............................233

Finding meaning in activities..........................238

Finding meaning in simple pleasures...................249

Finding meaning in being part of larger whole...........256

Discussion restoring meaning and purpose...............262

Rebuilding connectedness.................................265

Defining one's inner circle of support...................266 
Defining one's outer circle of support...............273

Feeling valued.......................................281

Feeling understood...................................294

Discussion rebuilding connectedness..................304

Reestablishing a healthy lifestyle........................307

Managing one's overall Health.......................308

Resetting pace..................................... 310

Choosing a balanced diet.............................315

Maintaining a fitness program.........................316

Discussion reestablishing a healthy lifestyle.............320

Discussion Phases of Adjustment........................................ 322

Quantitative Findings................................................... 324

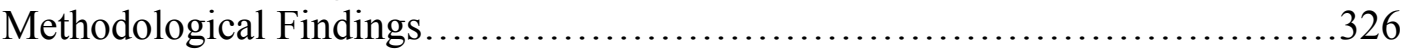

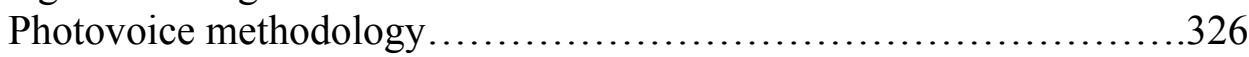

Dyadic interviews............................................. 330

CHAPTER 6 DISCUSSION, LIMITATIONS, IMPLICATIONS, AND

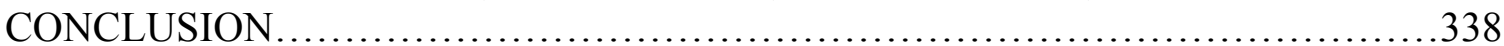

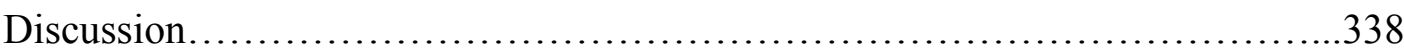

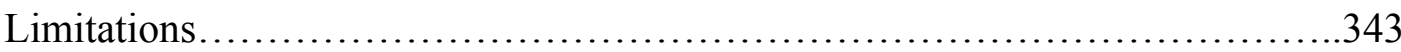

Implications Policy and Practice....................................... 346

Implications Research.................................................. 352

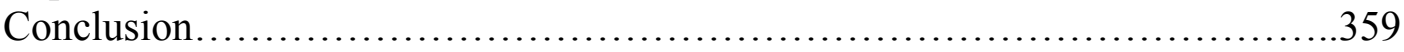

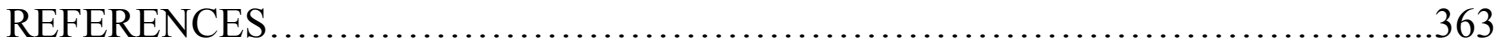

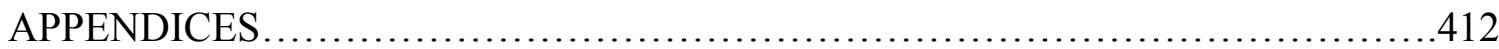

Appendix A: IRB Approval Letter......................................412

Appendix B: Recruitment Flyer..........................................413

Appendix C: Recruitment Letter NW Research Group on Aging..............415

Appendix D: Recruitment Letter Alzheimer's Association.....................416

Appendix E: Recruitment Interest Cards.................................417

Appendix F: Telephone Recruitment and Screening Script...................418

Appendix G: Demographic Data Sheet.................................. 425

Appendix H: Informed Consent Form..................................427

Appendix I: Photo Release Form........................................430

Appendix J: Screening Session Moderator Guide...........................431

Appendix K: Training Session Moderator Guide...........................4432

Appendix L: Brief Individual Interview Moderator Guide....................433

Appendix M: In-Depth Individual Photo Interview Moderator Guide..........4434

Appendix N: Dyadic Interview Moderator Guide............................435

Appendix O: Photo-Taking Toolkit.....................................436

Appendix P: Picture-Taking Plan......................................4437

Appendix Q: Photo-Diary ........................................... 438

Appendix R: Daily Instruction Sheet...................................439 
Appendix S: Refrigerator Magnets...................................440

Appendix T: Portraits of the Participants.................................. 441

Appendix U: Poster Boards.............................................4484

Appendix V: Participant-Generated Photographs and Photo-Diary Entries

(Figures 2-210) 


\section{LIST OF TABLES}

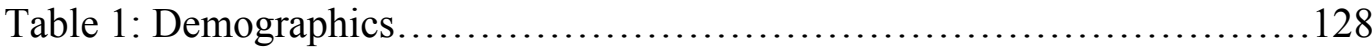




\section{LIST OF FIGURES}

Figure 1: Striving towards Well-being while Acknowledging the Inconceivable Notion of Losing One's Cognitive Abilities...........................130 


\section{CHAPTER 1}

\section{INTRODUCTION}

Longevity as a topic has had mesmerizing power throughout human history. It is not unexpected then that our leap in scientific progress in this century has also increased our knowledge of medicine, which has contributed greatly to a dramatic increase in human life expectancy resulting in longevity unknown to earlier generations. As a result, in the United States, the overwhelming majority of people who die are older adults (Hoyert, Kochanek, \& Murphy, 1999). Consequently, the United States and Western society as a whole is currently struggling with various topics associated with the increase in human lifespan. One of the biggest challenges associated with the increase in human life expectancy is the staggering and continuously growing number of older adults who are living with and dying from long-term progressive conditions such as dementia (Fisher $\&$ McCabe, 2005).

Dementia is a syndrome, a collection of signs and symptoms caused by a variety of diseases or conditions. Alzheimer's disease is the most common of these diseases, accounting for approximately $70 \%$ of cases of dementia in the United States. Vascular (or multi-infarct) dementia is estimated to account for $17 \%$ of cases and the remaining approximately $13 \%$ of cases are caused by diseases such as Lewy body disease, Parkinson's disease, frontotemporal dementia, Creutzfeld-Jakob disease, Huntington disease, Picks disease, HIV/AIDS, and normal pressure hydrocephalus or are due to head trauma, substance abuse, and multiple etiologies (Plassman et al., 2007).

These underlying diseases cause a progressive loss of cognitive abilities. In the early stages, dementia is primarily experienced as a decline in memory, judgment, and 
orientation (Reisberg, Ferris, de Leon, \& Crook, 1982). As the illness progresses, additional life domains are affected, with numerous aspects of quality of life impacted adversely. Although the duration of dementia varies, some people live for many years following the onset of the illness.

Over time, the decline in cognitive functioning affects all areas of life and ultimately, leaves people unable to care for themselves. While pharmacological interventions have been developed that temporarily slow the progression of the illness and/or provide symptomatic relief there is as yet no known medical treatment to cure or halt dementia (Aminzadeh, Byszewski, Molnar, \& Eisner, 2007; Fazio, 2008). Although dementia may occur at any stage of adulthood, the prevalence rate increases substantially with age.

Currently, an estimated 5.3 million individuals in the United States are affected by Alzheimer's disease and more than $90 \%$ of these individuals developed the illness after the age of 65 (Alzheimer's Association, 2009). It is projected that without further medical breakthroughs to treat or cure the disease, the number of cases of dementia will continue to increase dramatically in the next decades (Pressley, Trott, Tang, Durkin, \& Stern, 2003). "By 2050, the number of individuals aged 65 and older with Alzheimer's disease is projected to number between 11 million and 16 million" (Alzheimer's Association, 2009, p. 19).

Given the growing concerns about the costs involved in caring for the increasing number of older adults with the illness (Alzheimer's Association, 2009), dementia poses a significant challenge for the entire population and the healthcare system of the United States. With recent advances in diagnostic screening and growing emphasis on early 
detection, many people are now diagnosed at the very early stages of cognitive impairment and live in the early to middle stages of the illness for prolonged periods (Atri, Shaughnessy, Locascio, \& Growdon, 2008). As a result, dementia is increasingly viewed as a chronic illness.

Viewed through the lens of the stress, appraisal and coping perspective (Lazarus \& Folkman, 1984), the onset of chronic illness in general and dementia in particular can be viewed as a stressful event as it disrupts a person's life (Devins et al., 1983). It is the beginning of a chronically stressful condition characterized by numerous minor and major disruptions, which is reflected in a decreased sense of well-being. This decrease in well-being is thought to be associated with the extent to which the illness reduces a person's sense of satisfaction with important life domains (Bishop, 2005). Living with early-stage dementia places high demands on a person's coping capacity because he or she is required to negotiate challenging illness symptoms and their practical and psychosocial consequences in an attempt to increase or reconstruct his or her sense of well-being (Bishop, 2005). A person's response to illness-related changes (the process of adaptation) and his or her efforts to rebuild those areas of life that have been disrupted by the illness are influenced by psychosocial factors (Kitwood, 1990; Woods, 2001).

However, relatively little is known about the behavioral and attitudinal changes that result in adjustment to early-stage dementia and the factors that facilitate or hinder this change. The traditional biomedical conceptualization of dementia has led to research primarily focused on the biomedical nature of dementia neglecting the psycho-socialspiritual and environmental factors involved in experience and expression of the condition (Ballenger, 2006; Beard, 2004; Fox, 1989, 2000; Gubrium, 1986; Holstein, 
2000; Whitehouse, 2000).

Although the need for investigating the subjective experience of dementia was articulated almost two decades ago (Cohen, 1991; Cotrell \& Schulz, 1993; Foley, 1992; Froggatt, 1988; Herskovitz, 1995; Kitwood, 1987; Lyman, 1989), the scientific community had difficulties integrating this paradigm shift. People living with the condition continued to be generally discounted as active research participants under the misconception that they were poor informants of their own circumstances (Feinberg \& Whitlatch, 2001). Early studies exploring the subjective experience of dementia (e.g., Cheston \& Bender, 1999; Harris \& Sterin, 1999; Kitwood \& Bredin, 1992; Sabat \& Harre, 1992) provided evidence that people with dementia can both identify and describe their subjective experiences but received little or no attention in the scientific discourse.

The continued push for a more holistic bio-psycho-social-spiritual perspective of dementia has produced a small number of qualitative research studies that have examined appraisal and coping processes of people in the early stages of the illness (Clare, 2003; Harman \& Clare, 2006; Pearce, Clare, \& Pistrang, 2002). As the literature review will show, the findings of these studies have contributed valuable insight to our knowledge of the coping response to early-stage dementia. However, this body of research continues to be small and limited in scale and methods (Clarke \& Keady, 2002; Gillies, 2000) and researchers and clinicians are just beginning to develop an understanding of the complexities of this process (Beard, 2004). One area that remains poorly understood is the role that perceived strengths and resources play in the construction of the coping response. Who or what supports people with early-stage dementia in their attempt to increase their sense of well-being? Who or what helps them in the process of coping with 
and adapting to the illness and its practical and psychosocial consequences? Who or what assists them in rebuilding those areas of life that have been disrupted by the illness?

\section{Purpose of the Study}

The purpose for conducting this study was to develop a better understanding about the coping response to early-stage dementia, particularly the role that perceived strengths and resources play in this context. The stress, appraisal, and coping framework (Lazarus \& Folkman, 1984) posits that chronic illness poses challenges. At the same time, it illustrates through the process of appraisal and coping why some people successfully recognize and address the adaptive tasks that arise from the disruption of important life domains and others do not (De Ridder, Schreurs, \& Bensing, 1998). Subjective evaluations of the demands imposed by the illness and the available internal and external resources to address these demands are motivating forces crucial to the adaptive process. A person's response to illness-related changes (the process of adaptation) is largely influenced by the subjective perception of his or her strengths and resources. If a person has multiple strengths and resources available, he or she can more likely construct coping strategies that better fit the demands of the illness.

Knowing the perspective of people with early-stage dementia and their experience with this important aspect of the coping response is helpful in three ways: First, it will help to identify the role that perceived strengths and resources play in coping, adaptation, and attempts to reconstruct well-being. Second, it will help to explain some of the individual differences in coping response, adaptation, and well-being among people with the illness. Third, it will contribute to efforts to improve the quality of life for people with early-stage dementia by informing social workers and other healthcare providers of the 
adaptive potential and possibilities for support.

\section{Research Questions}

This study addressed the following research questions:

1. What do people with early-stage dementia perceive to be their strengths and resources?

2. How do people with early-stage dementia use these perceived strengths and resources in the process of coping with and adapting to the illness?

3. What is the process of coping with and adapting to dementia in the early stages of the illness?

\section{Significance of the Study}

Social workers are concerned with facilitating positive outcomes to maximize well-being in people with multiple complex needs (National Association for Social Workers, 2013). To achieve this goal, social workers assist people in coping with and adapting to illnesses or disabilities. This encouragement and strengthening of coping capacities often entails identifying factors inside and outside of the person that promote positive management of the demands posed by an illness or disability.

As knowledge about the implications of early diagnosis of dementia has increased, awareness of the need for psychosocial and spiritual interventions that respond to the emotional and practical needs arising from the onset of dementia has grown (Adams \& McClendon, 2006). People diagnosed in the early stages of the disease have longer to reflect on the illness and its consequences and thus experience longer periods of stress. As there is no cure for dementia at the present time, enhancing the ability of people with the illness to cope effectively with the disruptions in their lives is an 
important goal for social work practice. Although social workers are providing interventions to people with early-stage dementia, there is little known about the strengths and resources that are beneficial to this population. Social workers, like other professionals, are working with individuals with dementia predominantly from a medical model. This model does not reflect a person-centered, holistic approach to clinical practice. Instead, it focuses on deficits, dysfunction, and impairment and does not recognize remaining capabilities and capacities.

Knowing more about how people with early-stage dementia view, understand, recognize, and strategize their resources in the process of living with the illness will contribute to the identification of factors related to positive outcomes, which is a necessary precursor for the development of assessment and guidelines for social work intervention.

In short, strengths and resources enable people with early-stage dementia to cope with the illness in a way that has a positive impact on their well-being. Social workers can assist people with early-stage dementia in recognizing and utilizing available strengths and resources. Thus social workers have a vested interest in knowing more about the perception of strengths and resources of this population and investigating this aspect of the coping response systematically is an important focus of social work research.

Given the reality of managed health-care and cost containment, this information is important for making decisions about social support services for people with early-stage dementia and efficient ways to deliver them. Although person-centered care (Kitwood, 1997; Kitwood \& Bredin, 1992) and psychotherapeutic work with people experiencing 
early-stage dementia have gained some acceptance (Cheston, 1997), "the impact of Kitwood's ideas has so far been largely confined to the culture of care in institutions" (Cheston \& Bender, 1999, p. 207). Community-based health and social services continue to be focused on people experiencing the middle and later stages of dementia, and social workers and other healthcare professionals continue to have difficulties in describing "the task of dementia care (...) in a person-centered way" (Iliffe, Wilcock, \& Haworth, 2006, p. 335).

According to the strengths perspective, people generally perceive themselves as multifaceted and multidimensional constructs of abilities and assets. However, it is likely that people with early-stage dementia would benefit from assistance in recognizing and using their strengths and resources given their cognitive deficits. Knowing more about the perception of strengths and resources of this population will allow social workers to support their attempts to increase well-being in the context of their increasing difficulties and impairment.

Social workers are uniquely positioned to assume a leadership role in developing a continuum of interventions and services that target the changing needs of persons with early-stage dementia living in the community (Adams \& McClendon, 2006). However, the literature suggests that the role that social workers can play in providing support to persons with dementia remains under-developed. Social workers "have yet to know and to agree with what is expected of them in supporting people with early, recently diagnosed dementia" (Manthorpe \& Iliffe, 2005, p. 201). The findings of this study may provide knowledge that will lead to improved clinical practice and may help clarify the profession's role by describing and conceptualizing the experience of dementia in terms 
that are in line with social work values. Viewing and approaching complex needs from a holistic, strengths-based perspective are unique contributions of social work.

\section{Methods of Inquiry}

Qualitative research methods were identified as an appropriate vehicle for this study (Denzin \& Lincoln, 2005). The focus of this study was in the subjective realm of experience and because so little is known about the perception of strengths and resources

of people with early-stage dementia, inductive methods were needed to identify important constructs to pave the way for larger, more generalizable studies.

Eliciting the perspective of people with early-stage dementia is challenging due to the progressive loss of cognitive abilities, including increasing memory loss and decreasing discursive capabilities (Bond \& Corner, 2001; Cook, 2003; Phinney, 2002). Given these limitations, it was essential to use a research design that reduced the impact of these disease-specific difficulties and allowed the participants to convey their experiences through different forms of communication (Cook, 2003; Gilmour, Gibson, \& Campbell, 2003; MacQuarrie, 2005; Phinney, 2002). Thus, this study utilized photovoice methodology (Wang \& Burris, 1994, 1997) and grounded theory (Charmaz, 2006; Corbin \& Strauss, 1990, 2008; Glaser, 1978; Glaser \& Strauss, 1967; Strauss \& Corbin, 1998) to create a venue where the participants could express their experiences through images (their own photographs) and words (individual and dyadic interviews).

\section{Definition of Important Constructs}

Early-stage dementia. For the purpose of this study, early-stage dementia was defined as having a diagnosis of dementia and score no lower than 20 on the Mini Mental 
State Examination (Folstein, Folstein, \& McHugh, 1975).

Well-being. For the purpose of this study, subjective well-being is defined as "a person's cognitive and affective evaluation of his or her life. These evaluations include reactions to events as well as cognitive judgments of satisfaction and fulfillment. Thus, subjective well-being is a broad concept that includes experiencing pleasant emotions, low levels of negative moods, and high life satisfaction.” (Diener, 2002) (P. 63). Subsequent versions of the transactional framework of stress, appraisal and coping (Folkman, 1997; Folkman \& Greer, 2000; Park \& Folkman, 1997) propose that a person may experience stress and almost at the same time a sense of well-being when a particular event or situation does not allow for a favorable resolution as is the case of chronic and/or progressive illness.

Strengths and resources. Anything assisting a person in coping with challenges in his or her life can be regarded as a strength or resource, and this combination of assets varies from person to person. As such, it would be challenging to compose an exhaustive inventory of strengths, but Saleebey (Saleebey, 1992/2009) observes that some resources, capacities, and assets do appear regularly in any catalog of strengths. Strength will be discussed in greater depth in the next chapter.

Coping. White (1974) considers adaptation to be an overarching construct, with coping subsumed as a strategy of adaptation. In this perspective, coping refers to the activity of managing changes that defy familiar ways of thinking or behaving and demand the generation of new cognitive or behavioral responses, a process that is usually accompanied by negative affect. Within the transactional framework that underpins this dissertation, coping is defined as "constantly changing cognitive and behavioral efforts to 
manage specific external and/or internal demands that are appraised as taxing or exceeding the resources of a person" (Lazarus \& Folkman, 1984, p. 141).

Coping strategies. Lazarus and Folkman (1984) refer to coping strategies as the specific cognitions and behaviors that a person uses to alleviate disruption and its associated negative affect. The hallmark of coping strategies is that they require effort, whether conscious or unconscious, as their goal is to attempt to master, tolerate, reduce, or minimize stressful events. If adaptive tasks are understood as the general domains of adjustment, then coping strategies can be viewed as the specific means used to accomplish these tasks (Moos \& Tsu, 1977).

Adaptation. Given the multidimensional and subjective character of adaptation, there is an ongoing debate about as to what constitutes psychosocial adaptation (Livneh, 2001; Wright \& Kirby, 1999). At a basic level adaptation can be understood as a process of responding to changes. Drawing on the work of White (1974) and Bishop (2005), for the purpose of this dissertation, adaptation is defined as a person's unique response to illness-related disruptions across a wide range of life domains. According to White (1974), adaptation implies neither a total victory over the environment nor a total surrender to it; it suggests a striving towards an acceptable compromise. This striving is carried out by a person in interaction with his or her environment. As such, a person's response to illness-related changes (the process of adaptation) can be viewed as an attempt to increase or reconstruct his or her sense of well-being (Bishop, 2005).

Adaptive tasks. Corr (1992) defines adaptive tasks as the "work that may be undertaken by those who are coping', (p. 83) and considers such efforts vital to resolving life disruptions. Drawing on this perspective, in this dissertation, adaptive tasks are 
viewed as a person's efforts to rebuild those areas of life that have been disrupted by the onset of chronic illness in an attempt to reestablish a perceived equilibrium (Samson, 2008). This approach positions the person living with the illness at the core of the adaptation process because it puts the decision-making power in his or her hands. He or she decides which task to address given his or her reality. In other words, subjective evaluations of the demands imposed by the illness are crucial for understanding adaptive processes. Adaptive tasks become pivotal when chronic illness disrupts a person's familiar sense of well-being and thus move to the center of a person's coping efforts. In that sense, the concept of adaptive tasks fits into the transactional framework of stress, appraisal, and coping (Lazarus \& Folkman, 1984), which maintains that a stressor is appraised as such because a situational demand poses a threat or challenge to a person's sense of well-being.

In chapter 1, an overview of early-stage dementia was provided and the purpose of this study was stated. In addition, the potential significance of answering the three research questions was discussed and key terminology defined. The subsequent chapter provides an overview of the two theories that served as the theoretical framework in which this study was grounded. 


\section{CHAPTER 2}

\section{THEORETICAL FRAMEWORK}

The transactional model of stress, appraisal and coping (Folkman \& Greer, 2000; Lazarus \& Folkman, 1984) formed the theoretical foundation for this study and the strengths perspective (Saleebey, 1992/2009) served as a contextualizing lens which situates the study within the context of social work.

\section{The Transactional Model of Stress, Appraisal, and Coping}

Lazarus and Folkman (1984) proposed the transactional model of stress, appraisal and coping, stating, "psychological stress is a particular relationship between the person and environment that is appraised by the person as taxing or exceeding his or her resources and endangering his or her well-being" (p. 19). Coping in this model is defined as "constantly changing cognitive and behavioral efforts to manage specific external and/or internal demands that are appraised as taxing or exceeding the resources of a person" (Lazarus \& Folkman, 1984, p. 141). In other words, in this model the person and the environment are viewed in a "mutually reciprocal, bidirectional relationship" (Lazarus \& Folkman, 1984, p. 293). A person does not only react to an event or the environment but also has the capacity to change them through making choices and engaging in actions.

Pivotal to the model are two interrelated processes, appraisal and coping. Appraisal precedes coping and refers to the evaluative process by which a person determines the extent to which a particular event or situation impacts his or her wellbeing and/or current goals or concerns (primary appraisal) and what coping resources are available to manage the demands of a stressful situation (secondary appraisal). Primary 
appraisals categorize a given person-environment interaction according to its personal significance (benign-positive; harm/loss; threat; or challenge). Secondary appraisals evaluate what can be done about a specific event or situation and determine the extent to which it can be changed or controlled. In other words, the secondary appraisal process serves as an evaluation of available coping resources (personal and environmental strengths and resources) and the advantages and disadvantages of particular coping strategies given a person's goals and constraints. The outcome of these appraisal processes determines the intensity of the emotional reaction and the coping response that follows. Stress is only experienced when the evaluation of the event or situation causes the person to assign personal significance to it, question the adequacy of his or her resources to cope with it and its consequences, and worry about its potentially negative impact on his or her well-being and/or current goals and concerns.

Coping refers to a person's efforts to reduce, eliminate, or manage an event or situation perceived to be stressful and/or the emotions experienced as a consequence of this perception (Lazarus \& Folkman, 1984). Coping initially may take on two general forms, problem-focused and emotion-focused coping. Problem-focused coping strategies are likely to be used in situations that are appraised as controllable and amenable to change; these strategies attempt to alter the person-environment relationship directly. Emotion-focused coping strategies are likely to be prompted by situations that are appraised as less controllable and calling for acceptance; these strategies seek to regulate the emotional responses to the person-environment interaction. "Whether as a result of coping, changes in the environment, or changes in the individual, the relationship between the person and environment continues to unfold" (Folkman \& Greer, 2000, p. 
13) and leads to a reappraisal of the event or situation. Reappraisals evaluate the progress made in resolving the stressful event or situation, assess the effectiveness of the chosen coping strategies, and determine the level of satisfaction with the event outcome.

Reappraisal results can take one of three forms, favorable resolution, unfavorable resolution, and no resolution. Favorable resolution appraisals will likely end the coping activities.

However, unfavorable or no resolution appraisals, as is the case in chronic and progressive illnesses such as dementia, will initiate additional coping activities. Folkman and Greer (2000) suggest, "it is at this point that a third type of coping, meaning-based coping, is likely to come into play" (p. 13). Meaning-based coping does not seek to change or eliminate the event or situation perceived as stressful. Rather, this form of coping attempts to assign a different meaning to the event or situation - a meaning that is more in line and consistent with the person's overarching goals and beliefs. As such, meaning-based coping can facilitate a sense of well-being while facing events or situations that do not allow for a satisfactory resolution as is the case of chronic illness. The sense of well-being in turn enables the person to sustain the coping process. In situations that do not allow for a favorable solution such as early-stage dementia, the coping process continues and is sustained by feelings of emotional well-being that are achieved through meaning-based coping - despite persistence of the stressful event (Folkman \& Greer, 2000; Park \& Folkman, 1997). In other words, meaning-based coping strategies are assumed to allow a person to experience positive psychosocial functioning and psychological distress "perhaps not at the same moment, but certainly close in time" (Folkman \& Greer, 2000, p. 13). 
Relevance to this study. There is an extensive body of research that provides evidence to support the validity of the transactional model of stress, appraisal and coping (e.g., Folkman \& Lazarus, 1980; Folkman \& Lazarus, 1985; Krantz, 1983; Pearlin \& Schooler, 1978). Several studies have investigated and supported specific aspects of the model. For example, Folkman (1997) and Moskowitz, Folkman, Collette, and Vittinghoff (1996) investigated the role that coping plays in emotions; Folkman, Lazarus, DunkelSchetter, DeLongis, and Gruen (1986) explored the impact of appraisal on coping; Amirkhan (1990), Gottlieb and Gignac (1996) and Pearlin \& Schooler (1978) researched the difference between problem- and emotion-focused coping; and Folkman, Lazarus, Gruen, \& DeLongis (1986) studied the variability of coping across different situations. Although researchers and theorists are aware of and acknowledge the limitations of this research (e.g., Folkman, Lazarus, Pimley, \& Novacek, 1987; Lazarus \& Folkman, 1987; Moskowitz et al., 1996), in general, it is accepted that the transactional model of stress, appraisal, and coping provides a suitable framework for understanding the coping process and the role that the appraisal of internal and external resources plays in this context. In addition, the model has been used convincingly as a guiding framework by researchers investigating the subjective experience of early-stage dementia, and much of our current understanding of the coping response to the illness is grounded in it (Clare, 2002; Pearce et al., 2002; Sorensen, Waldorff, \& Waldemar, 2008; Van Dijkhuizen, Clare, \& Pearce, 2006). In line with the assumptions of grounded theory (Charmaz, 2006; Corbin \& Strauss, 1990, 2008; Glaser, 1978; Glaser \& Strauss, 1967; Strauss, 1987; Strauss \& Corbin, 1998), the basic tenets of the transactional model of stress, appraisal, and coping 
guided my thinking during data analysis, especially in understanding the meaning and subtlety of the data.

\section{The Strengths Perspective}

The strengths perspective, which served as a contextual background, is a philosophy and clinical practice model that emerged from within the field of social work. First articulated by Weick, Rapp, Sullivan, \& Kisthard (1989) and further defined and developed by Saleebey $(1992 / 2009 ; 1996)$, the strengths perspective emphasizes that the strengths and resources of people and their environments, rather than their weaknesses and deficits, should be the focus of social work practice.

Six core principles are at the heart of the strengths perspective (Saleebey 2009, pp. 15-19). First, all people (including those with cognitive impairment) possess strengths and resources and are capable of growth and change. Once recognized and acknowledged, these strengths and resource can be mobilized for the process of coping with and adapting to challenging life events. The dynamics that drive the process towards well-being are the awareness of hopes, dreams, and aspirations and the consistent focus on existing and available strengths and resources. Translated into realistic and attainable goals these desires are the building blocks for well-being. Second, a person's life is always considered bigger than his or her problem and every person is viewed as capable of growth and change. As such, challenging life situations are acknowledged as sources of discomfort and distress but they are also viewed as carrying the potential for growth and personal development. Third, the limits of a person's potential for growth and change remain generally unknown as people's capacities tend to be underestimated in the face of disrupting life events. Fourth, all people are viewed as experts of their own lives and 
considered to have the capacity to know what is best for them. A genuine interest and trust in people and their potential is understood as a key ingredient for empowering them to recognize, acknowledge, and use their own strengths and resources. Fifth, the notion of resilience is a vital component of the strengths perspective. People, no matter how challenging their circumstances, are viewed through a life-affirming rather than a lifedenying lens. All environments, even the most depressing and hostile, are viewed as containing resources that can be used in assisting people in their attempts to pursue wellbeing. Drawing attention to how people have managed to survive thus far in the face of adversity and disruption helps to discover these assets.

Relevance to this study. In the context of this study, the strengths perspective encouraged me to develop a research design that recognizes people with early-stage as experts of their own lives. During data collection and analysis, it served as a constant reminder for me to call into question the fascination with pathology with which we continue to view people with dementia and show appreciation for the whole person. This was particularly important because Steeman and colleagues (Steeman, Godderis, Grypdonck, De Bal, \& De Casterle, 2007) discovered that when interviewers focus exclusively on what participants with-early stage dementia have lost as a result of the illness and fail to show genuine interest, participants are more guarded in their responses. Conducting interviews from a standpoint of strength encouraged me to engage in a collaborative dialogue with the participants and to assist them in finding ways to express their insights. Taking a strengths approach reminded me of the importance of advocating for a language of appreciation for this population and the development of interventions to address their needs more effectively. 


\section{CHAPTER 3}

\section{LITERATURE REVIEW}

Chapter 3 provides a review of the literature relevant to strengths and resources and their relationship to the coping response to early-stage dementia. The use of literature in this study was reflexive, dialectic, and shaped by the emerging path of the study (Sandelowski, 1986) and therefore consistent with the paradigm, theoretical framework, and methodology underpinning the overall project. In other words, this study took advantage of the scientific literature but also of this researcher's theoretical knowledge and practical experience as a person and as a social worker familiar with the population under study (Corbin \& Strauss, 2008).

In general, the aim of a grounded theory study is to develop theory inductively from a body of data. Nevertheless, the review of existing literature and theory is considered important because it encourages theoretical sensitivity, enhances sensitivity to nuances in the data, stimulates questions, guides theoretical sampling, and provides supplementary validity (Corbin \& Strauss, 2008; Sandlowski, 1993). It was equally important for me to be conscious of the fact that my theoretical knowledge shaped the lens through which I viewed the overall project (reflexivity).

The meanings that are held by people about their illness are inseparable from the way in which people with chronic illness view and approach their lives and form coping responses (Kleinman, 1988). Given that the experience of illness is culturally situated, the literature review begins with a discussion of how the construct 'dementia' has changed over time. It then proceeds to explain how the social construction of dementia impacts the perception of illness-related changes and how this perception influences the way in which 
people with early-stage dementia cope with and adapt to the illness. Next, the small number of studies that have explored the coping response to early-stage dementia by asking people living with the condition directly is discussed. As there is as yet no study known to me that has investigated the perception of strengths and resources of people with early-stage dementia, this review proceeds with a discussion of what can be inferred from the current body of literature about what people with early-stage dementia perceive as strengths and resources. Lastly, the limitations of the current understanding will be highlighted.

The literature review provides information relevant to the research questions and topics that I thought were likely to be addressed in undertaking this study. However, additional literature was reviewed as new insights and topics emerged during the course of this study. This literature is integrated within the discussion chapter as it relates to the findings of this research.

\section{Dementia as a Social Construct}

Kuhn (1996) used the phrase 'paradigm shift' to portray periods in the history of a scientific field that resulted in a radical change or shift in ideas or methods. It can be argued that the field of dementia is currently undergoing such a paradigm shift. Until recently, dementia was exclusively viewed within the paradigm commonly known as the 'medical model' or 'biomedical model'. The medical model of dementia conceptualizes the experience of dementia as the direct consequence of an underlying brain disease caused by the neuropathological changes associated with Alzheimer's disease and other related disorders.

However, recently, there has been a shift away from the medical model to the bio- 
psycho-social-spiritual model, which is at the core of this proposal (Engel, 1977; Puchalski, 2007-2008; Saunders, 1999; Sulmasy, 2002). Although the scientific community has struggled to integrate this paradigm shift, there appears to be a promising tendency to view the illness as an experience that impacts and is impacted by internal and external factors. The way in which dementia is viewed, defined, and conceptualized is an expression of the Zeitgeist (the social, cultural, ethical, and political climate of an era) and is influenced by the prevailing constructs of and society's attitudes towards aging, death, and dying (Ballenger, 2006; Herskovitz, 1995; Holstein, 2000).

In Western societies, the social construction of dementia is largely influenced by advances in biomedical sciences and the availability of effective clinical interventions. Over time, the belief that biomedicine can solve human problems and the hope to ultimately achieve control over human mortality has led to a biomedicalization of aging and dying and, by extension, dementia (Beard \& Estes, 2002; Estes \& Binney, 1989; Lyman, 1989; Riper, 1995). Traditionally, the term medicalization is used to describe a process by which human conditions and/or social issues become defined as medical problems, which by extension are subject to medical research, diagnoses, and intervention and thus come under the auspices of health professionals (Conrad, 1992, 2007). The notion of biomedicalization reaches beyond this original conceptual framework by focusing on biomedical processes, located within an individual, that are assumed to be the cause of the condition and/or issue. The process of biomedicalization has been documented in countless fields and for numerous conditions (Cohen, 1993; Fishman \& Mamo, 2001; Mamo \& Fishman, 2001; Midanik, 2004; Pledger, 2003; Rotosky \& Travis, 1996) and it has been suggested that this process also plays an 
important role in the context of aging and dementia (Beard \& Estes, 2002; Estes \& Binney, 1989; Lyman, 1989). Categorizing and labeling aging processes and ailments that were previously considered a natural part of the human life cycle (including dementia) as a disease is viewed as an attempt to create a sense of control over them. As will discussed in the next section, the biomedicalization of dementia is the result of a number of societal forces.

Historical and socio-political representations of dementia. Historically, senile dementia (senility) was perceived as directly connected to old age and was viewed as part of the natural progression of physical and mental decline associated with aging. Alzheimer's disease, on the other hand, was originally characterized as presenile dementia with neuropathological hallmarks of senile plaques and neurofibrillary tangles. In other words Alzheimer's disease referred to the few cases where individuals under the age of 60 developed dementia.

However, the discovery that the neuropathological changes associated with Alzheimer's disease look the same at any age instigated a shift in the biomedical conceptualization of dementia (Fox, 1989). Both presenile and senile dementia were defined, categorized, and labeled Alzheimer's disease. One movement that benefited greatly from this shift in categorization is the positive aging movement in which gerontologists have engaged in efforts to change the negative image associated with aging and to promote a more optimistic attitude towards the later stages of life (Baltes \& Baltes, 1990; Rowe \& Kahn, 1987). While fostering a positive view of the aging experience was and continues to be timely, the concept of old age as a period of sustained health and meaningful activity interestingly also poses challenges in the context of frailty 
in late adulthood. The underlying assumption of lively aging is the idea of physical and mental well-being. Dementia is an illness that threatens this idea of lively aging the most because it undermines the individual's cognitive and physical abilities (Ballenger, 2006). It has been suggested that categorizing and labeling dementia as a disease is one way to keep the anxiety and fear associated with aging under control because it fosters hope. Putting dementia under the purview of medicine implies that it can be cured, that it is not an inevitable part of aging, and that a person is not in danger of developing dementia just because he or she is older (Beard \& Estes, 2002; Estes \& Binney, 1989; Lyman, 1989). The shift in the definition, classification, and labeling of dementia contributed to the burgeoning interest in dementia because it dramatically increased the number of people diagnosed with Alzheimer's disease. Alzheimer's disease no longer referred only to the few cases of individuals under the age of 60 who developed dementia but included a large number of people with cognitive impairment after the age of 60 (Holstein, 2000). As a result, dementia now receives attention as a social problem, a worldwide concern of epidemic proportion (Lyman, 1989).

In the United States, the Alzheimer's Association is a key player in constructing Alzheimer's disease as a social problem, thus shaping popular and scientific discourse as well as policies related to dementia (Beard, 2004; Bick, 2000; Fox, 1989; Whitehouse, Maurer, \& Ballenger, 2000). The historical foundation of the association is a mixture of family caregivers of individuals with dementia, biomedical researchers, and representatives of the National Institute on Aging who joined together to increase public awareness, provide caregiver support, and secure increased funding for caregiving and research into finding a cure for the disease (Beard, 2004; Bick, 2000; Fox, 1989; 
Whitehouse et al., 2000). In an effort to raise public awareness and secure federal funding for continuous biomedical research and needed support services the Alzheimer's Association emphasized the categorization of Alzheimer's disease as a distinct disease, de-emphasized age as a contributing factor, emphasized the economic burden that the disease would put on the aging society if sufficient funding were not granted for biomedical research, and persuasively conceptualized the search for a cure as a moral imperative (Fox, 2000). As a result of these societal forces, until recently, dementia has been viewed almost exclusively as a biomedical construct.

Medical model. Under the medical model dementia is considered an exclusively medical condition. The clinical manifestations associated with the illness are mostly considered symptoms of underlying neuropathology independent of psycho-socialspiritual factors. This focus on dysfunction and impairment causes both researchers and clinicians to center their attention on signs (which are observed by health care professionals) and symptoms (which are complained of by the person with the illness and his or her formal or informal caregivers) and not on the illness experience. This medicalized view of dementia has brought persons living with the condition within the sphere of the medical profession and considers medical practitioners experts in developing treatments for individuals who in turn are expected to comply with the prescribed treatment regime.

In this model, many of the behaviors associated with dementia are considered 'problem behaviors', which are viewed as the most stressful aspect of caregiving and perceived as the main contributor to caregiver burden (Davis, Buckwalter, \& Brugio, 1997; Ford, Goode, Barret, Harrel, \& Haley, 1997; Gilhooly \& Sweeting, 1994; 
Mittelman, Roth, Haley, \& Zarit, 2004). Family, friends, and health care professionals are inclined to view the changes in the person with dementia as exclusively burdensome. The medical view of dementia has been popularized by the Alzheimer's Association, which, in an effort to bring attention to the illness, emphasized the medical nature of the illness and its severity. One of the strategies employed was to give the illness a human face through popularizing accounts of family members reporting on their experiences of caring for a family member living with the illness. These personal narratives described persons living with dementia as victims of a dreadful disease that robs the person of their self as the illness progresses. This portrayal that emphasizes the slow and incremental losing of self has been the dominant view of dementia for many years.

The scientific and popular literature on dementia has also contributed to the 'monsterizing of senility' (Herskovitz, 1995) as the concept of annihilation of self is reflected in titles of books such as 'The loss of self' (Cohen \& Eisendorfer, 1986), ‘Alzheimer's disease: Coping with a living death' (Wood, 1989), or 'The vanishing mind: A practical guide to Alzheimer's disease and other dementias' (Heston \& White, 1983). Popular movies on Alzheimer's disease such as 'Iris' portraying the decline experienced by author Iris Murdock and media reports chronicling the late US President Ronald Reagan's experience of Alzheimer's disease have continued to reinforce the idea of losing one's status as a whole person (Goffman, 1963).

Conceptualizing dementia as an exclusively medical condition has had both positive and negative consequences. The understanding of dementia as a significant social and health problem has had policy implications for extending medical coverage for services associated with dementia. In addition, because of increased funding for 
biomedical research, there are numerous studies concentrated on describing the pathology of dementia, identifying its organic basis, and developing effective interventions targeting the underlying diseases and the clinical expressions of the condition. In a larger sense, the medical model is only marginally concerned with what dementia might mean to the person living with the condition or the demands that the illness poses for a person's coping skills and the complexities involved in responding to these challenges (Kitwood, 1990, 1997; Kitwood \& Benson, 1995; Kitwood \& Bredin, 1992; Woods, 2001). In addition, this model underestimates the important role that psycho-social-spiritual factors play in the course of the illness and does not consider the dynamic interaction of the person living with the illness and his or her environment (Cotrell \& Schulz, 1993; Lyman, 1989). In fact, it has been argued that understanding the subjective experience of dementia has been eclipsed by the biomedicalization of the disease (Cotrell \& Schulz, 1993; Lyman, 1989).

In addition, conceptualizing people with dementia as the 'living dead' (Aquilina \& Hughes, 2006) engenders assumptions that the caregiver and not the person with dementia is the primary victim of the disease, which in turn draws attention from the individual with the condition and makes the caregiver the focal point of interest. This contributed to the view of caregivers as the main beneficiaries of support and services rather than the individuals they care for (Beard, 2004; Downs, 1997; Goldsmith, 1996). As a result, 'treatment nihilism' (the belief that interventions for individuals with dementia are not beneficial) (Lawton \& Rubenstein, 2000) and 'clouds of passivity' (Pearlin, Harrington, Lawton, Montgomery, \& Zarit, 2001) have dominated society's view of people with dementia for many years. Post (1990a, 1990b, 1991, 1995) warns 
that at a time when activity and productivity are valued by society, the challenges and concerns about the costs involved in caring for those in the later stages of dementia have stimulated discussions about rationing healthcare, physician assisted dying, and more extremely, active euthanasia. He maintains that concepts such as 'biographically dead' and 'ceased to exist' could provide moral ground for developing a policy for geronticide specific to this population (Post, 1990a) and put individuals with dementia "at the cutting edge of the voluntary euthanasia debate" (Post, 1990b, p. 715).

Social psychological stigma theory (Link, Struening, Neese-Todd, Asmussen, \& Phelan, 2001) asserts that "people develop conceptions of mental illness early in life from family lore, personal experience, peer relations, and the media's portrayal of people with mental illness" (p. 1621). Based on these conceptions, people form beliefs about how people will react to people with mental illness. Once a person is diagnosed with a mental illness, these expectations have particular significance. A person with negative expectations about the way other people will treat a person with mental illness will likely worry that this negative expectation is personally relevant, which in turn impacts his or her self-esteem and coping response.

It can be argued that these dynamics also play an important role in the subjective experience of dementia because dementia "attracts the stigma of a psychiatric illness" (Benbow \& Reynolds, 2000, p. 174). As described above, the lay and professional discourse pertaining to dementia is dominated by a language of loss and decline. Living with dementia has been variously referred to as having a 'stolen mind' (Doernberg, 1989), or a 'slow death (Dodson, 1989). Following the assumptions of social psychological stigma theory people living in a sociocultural environment that portrays 
dementia as profound loss and gradual death would develop an unfavorable concept of dementia and of those living with the condition. Thus, it would come as no surprise if people would be fearful of developing dementia, have negative expectations about themselves and the way other people might treat them once they are diagnosed with dementia, and treat people with dementia in stigmatizing ways. In other words, while the strategy of portraying dementia as profound loss and gradual death may increase public awareness and encourage research on dementia, it also shapes the lens through which people with dementia view and treat themselves and are viewed and treated by others. It increases the likelihood of stigmatization and the fear of being stigmatized (Jolley \& Benbow, 2000) and thus impacts the coping response.

There is ample evidence in the literature to suggest the existence of these negative consequences. Society's negative construction of dementia (Snyder, 1999) and the 'conspiracy of silence' surrounding the disease (Dorenlot, 2005) has encouraged people to view dementia as being worse than death (Patrick, Starks, Cain, Uhlmann, \& Pearlman, 1994) and to treat people experiencing the disease as 'mute' or 'dead' (Davis, 1989). Empirical data on the stigma associated with dementia suggest that professionals as well as lay people stigmatize people with dementia (Vernooij-Dassen et al., 2005; Werner, 2005, 2006; Werner \& Davidson, 2004) and that people with dementia and family caregivers perceive being stigmatized and engaging in stigma managing activities (Blum, 1991; Burgener \& Berger, 2008; Cotrell \& Hooker, 2005; Keady \& Nolan, 1995a; Hazel MacRae, 1999). The self-regulation model (Leventhal, Nerez, \& Steele, 1984) proposes that people develop awareness about an illness based on symptoms and social messages. The incoming information is compared to existing beliefs about health and 
health threats and form an 'illness representation' (illness identity, time line, consequences, causes, and controllability). Based on this representation, people construct their coping response. Thus, it is likely that the popular portrayal of dementia impacts the subjective experience of living with the condition because dementia is experienced within the context of this negative discourse. The sociocultural and political representations of the condition have implications for the coping response as people view themselves as afflicted with a dreadful disease in the duration of which they lose themselves. In addition, when embracing a biomedical perspective a person cedes control of his or her experience to a medical professional. In the case of early-stage dementia, because of the limited available medical treatment options, it is possible for persons with dementia to be left with a feeling that 'little can be done'.

It has been challenging to introduce concepts such as 'wisdom, resilience and successful aging' into the dialogue about dementia. However, there are emerging efforts to change the 'master narrative' (Somers, 1994) on dementia that called into question the personhood of those exhibiting the signs and symptoms of the illness (Ballenger, 2006) and replace it with a discourse that emphasizes a more positive vision of living with the condition. In addition, there are a small but increasing number of publications that propose clinical practice models for working with people with dementia that embrace the bio-psycho-social-spiritual perspective of the dementia experience (Cox, 2007; Goldsmith, 1996; Marshall, 2006).

Bio-psycho-social-spiritual model. The bio-psycho-social-spiritual model puts forward the notion that illness denotes a complex interaction between biological, psychological, social, and spiritual factors (Engel, 1977; Puchalski, 2007-2008; Saunders, 
1999; Sulmasy, 2002). Disease influences and is influenced by these factors, and the interaction among these factors constructs the illness experience. In other words, illness is the multi-faceted experience associated with a disease. The illness experience depends on numerous elements such as perceptions of origin and significance of the condition, effects on behaviors and relationships, and efforts made to resolve the situation caused by the condition. As such, illness includes not only the experience of decreased well-being but also the meaning that is assigned to that experience by the person with the condition as well as family members, friends, and the wider social network (Charmaz, 1991; Eisenberg, 1977; Kleinman, Eisenberg, \& Good, 1978; Strauss, 1975). In short, illness is constructed from the psychological, social, and cultural responses to a disease (Kretlow, 1990).

The bio-psycho-social-spiritual framework implies that dementia is not only a biomedical disease but is also a lived experience for those who are affected by the condition. Conceptualized in this manner, the illness experience of dementia results from a complex interaction of (a) the neuropathological changes, (b) the symptoms associated with these changes, (c) the meaning that the person with dementia and his or her social network assign to these symptoms, and (d) the response available to and employed by the person with dementia to cope with these symptoms. In other words, the bio-psychosocial-spiritual perspective, most prominently represented by the person-centered care movement, maintains that people with dementia actively engage and cope with the condition as well as its consequences, that the sociocultural and political representations of the condition impact their experience, and that their perspective needs to be included in the research agenda (Kitwood, 1987, 1990, 1997; Kitwood \& Benson, 1995; Kitwood 
\& Bredin, 1992).

It can be argued that the following four developments contributed a great deal to the shift towards a more bio-psycho-social-spiritual understanding of the condition: the person-centered movement emerged in the mid-1980 and early 1990s (Kitwood, 1988, 1989, 1990, 1993, 1997; Kitwood \& Benson, 1995; Kitwood \& Bredin, 1992); the first autobiographical narratives written by people living with dementia were published (Davis, 1989; Dyer, 1996; Friel-McGowin, 1993; Rose, 1996); research commentary started to highlight the importance of integrating the perspective of individuals with dementia into our understanding of the condition (Cotrell \& Schulz, 1993); and initial efforts were made to explore issues related to dementia with qualitative research methods (Froggatt, 1988; Goldsmith, 1996; Pollitt, Anderson, \& O'Connor, 1991; Pollitt, O’Connor, \& Anderson, 1989; Wuest, Ericson, \& Stern, 1994). It has been suggested that one of the most important developments in providing support to people with dementia in the last two decades has been to acknowledge and value the perspective of those living with the condition and to recognize the significance of researching the lived experience (Woods, 2001).

Some researchers have started to document some of the processes and factors associated with the bio-psycho-social-spiritual experience of dementia. However, the number of research studies that embrace the view of the person with dementia as an active agent - a person with existing strengths and resources and the ability to use these capabilities and capacities to respond to and cope with the many challenges that dementia poses - continues to be small and limited in scale (Clarke \& Keady, 2002; Gillies, 2000). 


\section{The Experience of Dementia}

The purpose of this study was to develop a better understanding of the views of people with early-stage dementia as it applies to their perception of available strengths. Therefore, the search for literature focused on studies investigating the lived experience of the illness. To locate relevant studies, three different search strategies were employed. First, the reference lists of three recently published systematic reviews focused on the lived experience of dementia were searched (de Boer et al., 2007; Steeman, de Casterle, Godderis, \& Grypdonck, 2006; van der Roest et al., 2007). Second, a formal search of electronic databases (MedLINE, CINAHL, and PsychINFO) was performed. For each search the terms 'dement*' or 'Alzheimer*' and 'subjective experience*' or 'lived

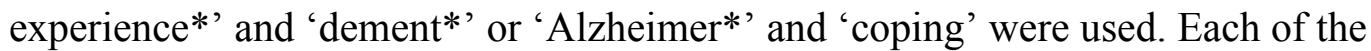
searches was restricted to articles published in English. Third, the peer reviewed journal 'dementia' was hand-searched (Keady, Harris, \& Wilkinson, 2002).

While reviewing the search results for the literature review the following development within the body of literature on the subjective experience of dementia became apparent. Early studies exploring the perspective of persons with dementia focused on gaining a general understanding of the subjective disease experience, while more recent studies investigate specific aspects of this experience and begin to fine-tune our knowledge. Current studies are broadly concerned with questions such as, how do assessment and diagnosis of dementia impact the experience of the condition?; what do persons with dementia consider important to their quality of life?; how do persons with dementia cope with and adapt to dementia?; how do personal and contextual factors such as illness awareness, personal identity constructions, personality traits, coping styles, 
gender, socioeconomic status, and relationships impact the experience of and adaptation to dementia?; and what types of outcomes do persons with dementia seek from and what experiences do they have with community support services?

The search for literature resulted in only a few studies that had explored the views of people with early-stage dementia and that provided information relevant to the role that perceived strengths and resources play in the coping process. These studies will be reviewed in terms of the stages of the coping response as proposed by the revised transactional model of stress, appraisal, and coping (Lazarus \& Folkman, 1984).

Perceiving the changes. The transactional model of stress, appraisal, and coping (Lazarus \& Folkman, 1984) proposes that in order to cope, people first appraise a lifeevent (primary appraisal), in this case the symptoms associated with early-stage dementia. This evaluation process is impacted by personal characteristics such as age, gender, and social class, the social construction of the illness, as well as a person's beliefs, values, and goals and his or her general life circumstances. The outcome of these evaluations impacts the significance that is attributed to the event and thus determines the level of stress that is experienced. In other words, how people with early-stage dementia perceive the changes they experience impacts how they feel about these changes and the amount of stress they experience.

A number of studies of the subjective experience of early-stage dementia have brought light to this evaluation process as it pertains to becoming aware of and recognizing the symptoms of the illness. These studies portray the 'becoming aware' of the changes associated with early-stage dementia as a highly individualized, multidimensional, and multifaceted process. This process starts long before a diagnosis is or 
can be given. People generally become slowly aware of their forgetfulness (the primary symptom in the early stages of dementia) through personal observations. Only few report a sudden onset of symptoms (Werezak \& Stewart, 2002).

Keady and Nolan (1995b) interviewed ten people with early-stage dementia and their family caregivers to elicit their views about living with the condition. In recounting their transition into the illness the participants described many 'lapses' and 'slips' (such as no longer being able to follow simple instructions and directions) that gradually became more pronounced and eventually led them to believe that something was wrong.

Robinson, Ekman, Meleis, Winblad, and Wahlund (1997) discovered similar patterns in the data she collected from eight individuals who entered a memory clinic for their initial medical consultation. Based on the data collected during interviews prior to the participants being told their diagnosis, Robinson found that the participants had "realized over time that they were experiencing many episodes of forgetfulness" (p. 112). The participants recounted how they gradually became aware of their difficulty in remembering appointments, names, phone numbers, and faces.

Beard (2004) combined the methods of participant observation and in-depth individual and focus group interviews and explored the experience of thirteen participants diagnosed with early-stage Alzheimer's disease. She discovered that most participants, prior to receiving a formal diagnosis, had sensed that 'things just weren't going right' ( $\mathrm{p}$. 421), which made them suspect that they were experiencing memory problems. Many had noticed changes in their ability to recall information, perform tasks previously considered routine (such as cooking, playing cards, walking a pet, or driving), or function well in unfamiliar surroundings (such as in a new place of residence or during vacation). 
Similar findings were reported by Sorensen, Waldorff, and Waldemar (2008) who, drawing on interviews with five women and six men, explored how people with early-stage Alzheimer's disease cope with the changes they face in everyday life and social relations. They found that the participants slowly became aware of the changes associated with the illness through the following four processes: "well-known places at the same time appeared both as something known and something totally unknown; words were recognized but not understood; actions, that previously were easy and natural, became difficult or impossible to perform; and relatives, especially spouses responded in new ways" (p. 293).

Parsons-Suhl, Johnson, McCann, and Solberg (2008) interviewed nine women and three men about how they experienced memory loss. They discovered that the participants started to recognize that they have memory problems "when they no longer remembered to do small everyday activities that were part of their daily routine, such as flushing the toilet, taking the pot off the stove, or removing clothes from the clothesline" (p. 34). When this forgetting happened repeatedly, they slowly became aware that something was wrong. Other significant events that contributed to the participants' realization that 'something was wrong' were "forgetting to do very meaningful activities or forgetting how to do these activities" (p. 35); having trouble finding words during conversations; and being unable to retain new information. One participant recounted that she was no longer able to dress herself and others noted that they became easily disoriented. Others "experienced themselves in an unfamiliar environment, although they were actually in a place that should have been very familiar" (p.35).

The evaluation of disruption is impacted by two main characteristics of the 
illness, the fluctuating awareness of symptoms (Cotrell \& Lein, 1993; Cotrell, 1997) and the insidious nature of the illness. Phinney (1998) explored how individuals with early Alzheimer's disease experience and interpret symptoms. Drawing on interviews with five individuals with the illness and their spouses she discovered that "several spoke of having a heightened awareness of their memory loss but at the same time, they realized that there were occasions when their spouse was aware of lapses when they were not" (p. 12).

Similar findings were also reported by Steeman, Godderis, Grypdonck, De Bal, and De Casterle (2007) who conducted 74 interviews with twenty people with dementia and their family to explore what it means for people to live with early-stage dementia. They found that all participants in their study were aware of their memory problems and the changes in their cognitive and behavioral functions. The participants also noted that their memory problems fluctuated day to day and that some days were better than others. At times, the presence of memory problems is initially determined through feedback from family members and friends who start to recognize and corroborate the person's observation (Parsons-Suhl et al., 2008). However, sometimes there is disagreement between the person with the illness and their family's perception of their memory problems and losses of competencies (Steeman et al., 2007). Initially, forgetfulness is only recognized as a problem if it presents itself as an extreme change from the norm. Because memory problems are also commonly related to other processes, such as increase in age, fatigue, or stress it is difficult to recognize them as a symptom of underlying pathology.

Robinson and colleagues (1997) found that initially, the participants explained away their difficulties as related to stress, age, or fatigue. Later, significant events led 
them to suspect that their symptoms were more than 'regular' forgetfulness. For example, feeling disoriented in well-known environments, being less and less able to focus and organize work, being hesitant about beginning new projects, and experiencing difficulties with problem solving and the management of multifaceted situations. Although many studies found that people in the early-stages of the illness acknowledge to a greater or lesser extent that they experienced memory problems, people develop different explanations for their forgetfulness.

Werezak and Stewart (2002) interviewed six people with early-stage dementia to explore how people in the early stages learn to live with the illness. They discovered that initially, some participants explained their failing memory as benign forgetfulness; others viewed it as a symptom of work-related stress. In addition, the participants provided several explanation for their difficulties in recognizing that they had memory problems, such as (1) a history of memory problems; (2) a family history of other health conditions that led them to believe that they would develop one of these illnesses rather than dementia; and (3) pre-existing health conditions, which made it difficult to distinguish the cause of symptoms.

Most participants in Steeman and colleagues' (2007) study thought of their memory problems as temporary and attributed them to external causes such as the loss of a spouse, loneliness, fatigue, changes in environment or habits, the weather, retirement, physical ailment, or hospitalization. Others viewed the changes as part of aging. "Only a few expected their memory problems to worsen, or they alluded to becoming demented in the future" (p. 123).

When conducting a phenomenological study aimed at identifying and 
conceptualizing the range of ways in which people with early-stage Alzheimer's disease cope with the changes they experience, Clare (2002) discovered similar explanations for perceived memory problems from nine women and five men. Some participants viewed the change as part of aging; others hoped that it was not Alzheimer's disease. Yet others observed that there was some improvement in their memory or hoped 'that they're all wrong' (p. 142) or that a cure will be found.

Similar findings were described by Pearce, Clare, and Pistrang (2002), who conducted separate interviews with twenty men with early-stage Alzheimer's disease and their wives to explore the ways in which men make sense of and cope with the illness. They discovered that many of the men thought that their memory was "worse than that of other people of a similar age" (p. 184). Some men attributed this difference to a more rapid aging process; others viewed it as an extension of the fact that their memory had never been good. Only two men spoke about their memory problems as related to having Alzheimer's disease.

These findings were also confirmed by Van Dijkhuizen, Clare, and Pearce (2006) who conducted semi-structured interviews with nine women who had a diagnosis of early-stage Alzheimer's disease and examined how these women made sense of, and attempted to cope with, their situation. The study participants voiced a number of different reasons for their memory problems, including "ageing, bereavement, loneliness, worry, a previous head injury, and, in the case of one woman, Alzheimer's disease" (p. 79). However, the majority of the women also expressed that they were unsure about what they were experiencing.

The findings of these studies suggest that the majority of people become gradually 
aware of the fluctuating symptoms of early-stage dementia. Initially, forgetfulness is perceived as normal and attributed to other factors until the frequency, quality, and context in which symptoms occur make it impossible to ignore the pathological nature of their experiences.

Signifying the changes. The transactional model of stress, appraisal, and coping (Lazarus \& Folkman, 1984) proposes that primary appraisals categorize a given personenvironment interaction according to its personal significance (benign/positive; harm/loss; threat; or challenge). The recognition of increasing symptoms prompts many to seek medical attention in an attempt to evaluate the significance of their observations.

Empirical data on the impact of diagnostic disclosure suggest both negative and positive consequences for both persons with dementia and their care partners (Bamford et al., 2004; Husband, 1999, 2000; McWilliams, 1998; Pearce et al., 2002; Pratt \& Wilkinson, 2003; Robinson, Ekman, \& Wahlund, 1998; Smith, et al., 1998; Smith \& Beattie, 2001; Young, 2002a). Generally, the perspective of people with the illness has been largely neglected in questions related to early diagnosis. However, there is a small number of qualitative studies that investigated aspects related to the early diagnosis of dementia from the perspective of those living with the illness.

Vernooij-Dassen, Derksen, Scheltens, and Moniz-Cook (2006) interviewed 18 people with early-stage dementia and their partners two weeks and twelve weeks after being diagnosed with dementia to examine the impact of receiving a diagnosis. They found that most participants experienced receiving the diagnosis of dementia as a confirmation of what they had already suspected. The participants reported that were selective in choosing with whom they shared their diagnosis. All participants had told 
their children but were more selective in disclosing the diagnosis to members of their wider social network. The decision depended on intensity of the relationship and likelihood of future support.

The participants in Beard's (2004) study also had strong opinions about with whom they would share their diagnosis, and why, and spoke openly about the different advantages of each choice. Some found that telling other people about their diagnosis "could be both personally empowering and foster a sense of compassion from others" ( $\mathrm{p}$. 423). For others it was "just easier not to hide it" (p. 423. In addition, being open about one's diagnosis, participants argued, provided the opportunity to elicit help and explain one's behavior to others. However, sharing one's diagnosis could also pose additional challenges. One participant voiced her irritation about the fact that "some people could not believe she had Alzheimer's disease" (p. 422).

Langdon, Eagle, and Warner (2007) interviewed six women and six men and explored how their perception of their condition and their understanding of how others reacted to them had changed since they had received a diagnosis of dementia. They discovered that the participants were apprehensive about how other people viewed the illness and how they would be treated based on that perception. They "seemed to have a heightened awareness of how others responded to them when discussing their diagnosis or when their new limitations were in evidence" (p. 993). As a result, the participants were selective in choosing with whom they shared this information and made a distinction between their 'inner and outer social circle'. They felt comfortable with disclosing their diagnosis to close family members and friends but were ambivalent in sharing this information with their wider social network. 
Whether a diagnosis is obtained or not the changes associated with early-stage dementia continue to create disruptions in many life domains. Snyder (2001), in summarizing the literature and her personal observations as a researcher and clinician working with people with early-stage dementia, identified three key areas of disruption: (1) changes in self-esteem and self concept; (2) family and social relationships; and (3) meaningful activities. These areas of disruptions have also been described by a number of studies, which have contributed to a better understanding of the level of significance that people living with the illness attribute to the changes they observe.

Harris and Sterin (1999) asked 17 individuals with early-stage Alzheimer's disease and their caregivers how they define their sense of self and how, from their perspective, this sense of self is impacted by dementia. They found that the majority of the participants felt that their sense of self had changed and was in a fluid state due to the impact of the illness. In addition, most participants reported a sense of loss on numerous levels such as loss of memory, loss of self-worth and respect, loss of sense of competency, loss of relationships that have been altered or terminated by the illness, loss of meaningful roles, and loss of independence.

Holst and Hallberg (2003) interviewed 11 people diagnosed with Alzheimer's disease about their experiences of living with the illness. They found that the participants' loss of memory impacted their relationships and their ability to play an active role in their social world. The participants portrayed their relationships with others in terms of suffering a lack of mutuality, openness and honesty and evoking feelings of anger, shame, sadness, insecurity, and disability. As a result, they felt devalued as people. The participants also communicated that they were frustrated about having lost their ability to 
decide "where to be or what to participate in" (p. 363). In addition, the participants felt that their inability to recall important aspects of their lives left them with a sense of dependence and emptiness. Lastly, many participants reported that they had to discontinue previously valued activities and interests, which in turn caused a sense of loss.

Parsons-Suhl, Johnson, McCann, and Solberg (2008) found that the participants in their study experienced their memory problems as utterly frightening and perceived their diagnosis as a 'death sentence'. The study reported that memory loss incrementally affected the overall shape of the participants' lives, altered the way they did things, and disrupted their sense of connectedness as it interfered with their ability to fulfill personal and/or social expectations. As a result, 'being-in-the-world' was experienced as increasingly unpredictable and burdensome.

Katsuno (2005) used a mixed method design to explore subjective and objective assessments of quality of life of twenty-three people with early-stage dementia, to describe their personal experiences of the condition, and to examine how and to what extend the negative public view of dementia affects their daily experiences and their quality of life. Katsuno found that the participants' sense of well-being was clearly affected by negative views of dementia. The participants reported that others sometimes treated them as if they did not exist. These experiences made them fearful of the illness itself and of the devaluing reactions of others and they engaged in efforts "to cover up the symptoms of their memory problems" (p. 206). Some participants expressed their anger about other people's responses and voiced their sadness about being left by long-term friends. 
The above studies show that people generally appraise the changes that the illness imposes on their lives as a loss or threat to their overall well-being with many lifedomains impacted. These losses evoke a wide range of feelings, which will be discussed in the next section.

Emoting the changes. The transactional model of stress, appraisal, and coping (Lazarus \& Folkman, 1984) proposes that loss and threat appraisals will evoke negative emotions. A number of studies have described the emotions that arise from the losses and threats associated with the early stages of dementia.

In Harris and Sterin's (1999) study, most participants reported difficulties in coping with their losses and recounted feelings of "frustration, embarrassment, humiliation, anger, dependency, fear, disempowerment, and uselessness" (p. 246). These feelings occurred most often when they were trying to accomplish a task, remember people, places, or things, or when they were treated in a way that called for "a total surrender of all independent thought and action" (p. 246). In addition, about half of the participants recounted that they were initially upset and surprised about receiving a diagnosis of Alzheimer's disease.

Similarly, Pearce, Clare, and Pistrang (2002) found that losses associated with memory problems, but also a general sense of uncertainty about the future and/or the failing of coping strategies, evoked feelings of sadness, frustration, and anger in their participants.

In Beard's (2004) study, most participants reported that they experienced the changes associated with the illness as frustrating. In addition, the study participants recounted different reactions to receiving their diagnosis. While one participant stated 
that she immediately decided that she would be able to cope with the illness by taking it one day at the time, another participant voiced that he could not believe it and felt really sad.

Similar findings were also reported by MacQuarrie (2005) who asked four women and nine men recently diagnosed with early-stage Alzheimer's disease about their experiences of living with the illness. She found that all participants expressed a variety of emotions ranging from "annoyance, anger, hurt, shock, sadness, and exasperation" (p. 434). The participants spoke about how frustrated they feel about the limitations imposed by the illness and their inability to accomplish familiar tasks. Many participants felt betrayed by the changes and recounted feelings of fear and dread. Many participants worried about losing their sense of orientation in public, and "feelings of humiliation in front of others were part of their experiences (p. 434).

The participants in Van Dijkhuizen, Clare, and Pearce's (2006) study experienced difficulties in remembering details about their family and losing their connection to the past as especially upsetting.

Steeman, Godderis, Grypdonck, De Bal, and De Casterle (2007) discovered that their study participants experienced feelings of "uncertainty, loneliness, shame, uselessness, losing one's mind, being disregarded, being restricted, being a burden, being a 'poor devil', and being 'written off'” (p. 125). In addition, some participants reported feelings of discontent about their past or present life, and others expressed concerns about their future well-being.

The participants in Sorensen, Waldorff, and Waldemar's (2008) study reported emotions on "a continuum from feelings of well-being to feelings of anxiety and sorrow" 
(p. 293). Feelings of anxiety and sorrow were mostly related to the increasing sense of personal insecurity that the participants experienced. In addition, the participants also worried about the lack of curative treatments for Alzheimer's disease, their increasing age, what to expect as the illness progresses, and the possibility of losing cherished abilities, such as reading. Some participants voiced concerns about the changes they experienced in their relationships and the impact the illness has on their partners.

For some of the participants in Parsons-Suhl, Johnson, McCann, and Solberg's (2008) study, forgetting to do very meaningful activities or forgetting how to do these activities was initially connected to feeling 'bad', 'frustrated', or 'useless' and gradually turned into living on a daily basis with the uncertainty and fear of not remembering. For two participants, not remembering left them with a feeling of losing time and caused them to wonder what death might be like.

In summary, the findings of these studies suggest that people with early-stage dementia experience a considerable amount of disruption in their lives, which in turn causes a variety of negative emotions and is perceived as stressful. That a large number of people with dementia experience a decreased sense of psychological well-being as a result of the illness has also been supported by the findings of studies that used quantitative methods. These studies suggest that as many as $80-90 \%$ of people living with the condition experience neuropsychiatric symptoms including depression, anxiety, delusion, hallucination, and insomnia during the course of the illness (Lyketsos, Gonzales-Salvador, Chin, Baker, \& Black, 2003; Steinberg et al., 2003; Tariot et al., 1995). Although the relationship between dementia and these symptoms remains somewhat unclear (Moretti, Torre, Antonello, Cazzato, \& Bava, 2002), research 
investigating the impact of these symptoms suggests a negative relationship with quality of life (Logsdon, Gibbons, McCurry, \& Teri, 2002) and with quality of relationship between persons with dementia and their care partners (Adams \& McClendon, 2006).

Coping with the changes. Although in the transactional model of stress, appraisal, and coping (Lazarus \& Folkman, 1984) loss appraisal initiate the secondary appraisal process, which determines the strengths and resources that will be used in the coping response, for the purpose of this literature review, coping strategies will be discussed next. The reason for introducing coping strategies before 'secondary appraisal' is the dearth of literature on this subject. The discussion on coping strategies will provide an initial, although limited, insight into the perception of strengths and resources as they form the ground in which coping strategies are rooted. The following section discusses types of coping strategies that people with-early stage dementia employed as they are presented in the literature of the lived experience of the illness.

In Harris and Sterin's (1999) study some of the participants coped with the changes they experienced by giving up some of their responsibilities and enjoying the attention they received as a patient. Others maintained that there was nothing wrong with them, perceived their memory difficulties not as a problem, and voiced anger about the way other people treated them. Yet others tried to hide their diagnosis from family, friends, and business partners. They engaged in efforts to maintain their professional and private life as it was before they experienced memory problems and used strategies such as continuously rehearsing names. Still others had seemingly given up totally and surrendered into being dependent on others. The researchers found that for seven participants being engaged in meaningful activity was of central importance. For some 
that meant to continue working, for others it meant to learn new skills or completing word puzzles. Some participants valued being independent above everything else; others assigned paramount importance to being comfortable and secure.

Clare (2002) found that most participants in her study coped with their memory problems by trying harder, maintaining a routine, and sticking to a medication regimen, although some participants were unsure about what the medication was for. Some participants managed the changes they experienced by avoiding situations that would exacerbate or reveal the challenges they faced such as social situations or complex conversations. In addition, most participants employed strategies to compensate for their difficulties such as relying on their partners and/or using a number of external memory aids including "diaries, calendars, lists, notes, written instructions, reminders on stickybacked notes, colored labels to make things more distinctive, alarms, and keeping systematic files of correspondence" (p. 144). Some participants dealt with the impact of the illness by seeking information about the illness. However, knowing more about the illness also posed challenges because the information presented in the media was experienced as mostly depressing. Most participants looked for opportunities to be 'useful' and participating in research was perceived as one way of making a contribution. Making comparisons with those they considered worse off and highlighting the absence of pain assisted participants in focusing on 'the good things in life' and enjoying pleasurable experiences such as walking, listening to music, and loving relationships. Some participants thought that talking to people with similar experiences might be helpful but had little opportunity to do so. Interestingly, some participants were able to find something positive in the experience of living with Alzheimer's disease and voiced 
that they "had learned a lot in this curious reaction thing" (p. 146).

Similarly, the men in Pearce, Clare, and Pistrang's (2002) study coped with their memory problems and their feelings by trying harder, modifying the way they went about doing things, and employing practical strategies such as "trying to look up information, setting themselves routines, using anxiety management techniques, and writing themselves notes or instructions" (p. 180). Most men viewed their wives as a source of both practical and emotional support and some discussed negotiating new roles within their relationship as one way of coping with the changes in their abilities and skills. Half of the men maintained a medication regimen, although only three men perceived the medication as effective. Only a few men perceived accessing health services as helpful and initially viewed it as another indicator of loss. Many thought of worrying as a 'waste of energy' (p. 181) and tried to 'make the best of things' (p.181). For some, getting on with life as best they could meant lowering their expectations and trying to accept their limitations.

Van Dijkhuizen, Clare, \& Pearce (2006) also found that the participants in their study employed active strategies to compensate for the changes they experienced, including "staying active by doing things, writing things down, keeping things at hand, checking, and taking medication" (p. 86).

Some participants in MacQuarrie's (2005) study “monitored their words to ensure they were communicating what they intended" (p. 434); others used a strategy of selfquestion and answer. In addition, participants relied on the support of others. A common strategy was to ask for help with recall from others. These others were expected to either provide the information directly or asked relevant questions. Reaching out for help, 
assistance, and support included talking about their memory problems with others (first with family members, then with friends) and accessing more formalized services. During the interview, the participants dealt with the challenges associated with communicating their experiences by using "household communication props such as family photos, mementos, and collections as reminders" (p. 434). Some participants clarified the ideas they wanted to share with body movements and gestures in addition to verbal communication. MacQuarrie also discovered that it was important for some of the participants to leave a legacy and make a contribution. They voiced that they considered donating their body to science and that they were interested in participating in research.

Most participants in Beard's (2004) study coped with the challenges they experienced by reducing activities that had the potential of revealing or exacerbating their difficulties such as "cooking, driving, and being at social events or in unfamiliar surroundings" (p. 424). As discussed above, the participants considered being in control of who knew about their diagnosis important. Regardless of the degree of disclosure, all participants engaged in a number of strategies to compensate for their difficulties such as writing notes or using phonetic cues. In addition, the majority of participants dealt with the impact of the illness by focusing on those things that they could do and used humor to lessen some of the stress they experienced in social interaction.

Sorensen, Waldorff, and Waldemar (2008) found that the participants in their study coped with the changes by maintaining a familiar structure in daily life and relying on supportive relationships with their family members and friends. In addition, being engaged in useful activities was considered important by the participants and facilitated a sense of well-being, "particularly when their efforts made a difference to other people" 
(p. 294). Study participants also dealt with the impact of the illness by focusing on and enjoying specific, everyday occupations such as bicycling, walking, watching TV, doing crossword puzzles, reading, and performing useful household tasks. The participants' efforts to cope with the changes included sharing "fragments from their life story during the interview without being asked directly" (p. 294). Study participants also noted that when they were no longer able to complete a task or were lacking the resources to complete mundane activities, they either adapted or no longer pursued these activities. Some participants talked about being engaged in new activities. The researchers found that the study participants viewed their spouse, children, and friends as a source of both practical and emotional support and that they played an increasingly important role in the participants' lives. Casual friends and more distant relations on the other hand had become less and less important in participants' lives.

Some participants in Parsons-Suhl and colleagues' (2008) study managed the changes in their ability to remember by utilizing the help of family members and relying on their cues, writing things down, and being mindful about what makes remembering easier and what makes it harder. Most participants voiced that they knew what helped them remember and mentioned relaxing as one way that assisted them in recalling information while others coped with their memory problems by using humor.

Steeman and colleagues (2007) reported that the participants used strategies such as avoiding confrontation, using humor, utilizing memory aids, highlighting remaining abilities, and exercising their memory. Most participants relied on family members for emotional and practical support but did not always agree with their family's perception of whether or not they were in need of assistance. The authors interpreted the participants' 
coping behavior as denying, minimizing, rationalizing, normalizing, or somatizing their memory problems (p. 127).

Keady and Nolan (1995b) identified a number of coping strategies used by the participants from which they created a 42-item scale. Constructed as an instrument to assess specific positive strategies used by people with dementia, the scale includes coping statements such as establishing routines, accepting memory loss and finding ways of overcoming it, using lists and other memory aids, constantly repeating things to self to help remember, staying in familiar surroundings, seeking information, exhibiting humor, writing in a personal diary, and practicing relaxation techniques (p. 311-312). Drawing on an in-depth examination of the coping statements, the authors discovered that the study participants exhibited all three types of coping strategies as proposed by Nolan, Grant, Caldock, and Keady (1994). The study participants were actively involved in 'finding creative solutions' to the difficulties they encountered during everyday life (e.g., using memory aids, establishing a routine), managed the meaning of these difficulties by 'creating alternative perceptions' of the situation (e.g., using humor, not dwelling on the problems that they face), and 'coped with the symptoms of stress' by using relaxation techniques (e.g., writing personal diary, trying to stay calm and relaxed).

In summary, the reviewed studies describe responses to dementia-related challenges, such as progressive neurological changes, changes in the way people react to them, and changes in how they view themselves in terms of self and identity. In their attempt to pursue well-being, the participants in these studies used a variety of thoughts and actions to alleviate disruption and its associated negative affect. They used problemfocused coping (e.g., using external memory aids, seeking information about the disease, 
enlisting informal and formal support); they tried to regulate the emotional response to the changes they experienced through emotion-focused coping (e.g., using relaxation techniques, distracting themselves, and finding ways to not let the symptoms of the illness bother them); and they attempted to assign a different meaning to the challenges they faced through meaning-based coping (e.g., finding value and purpose in their experiences, discovering positive aspects in the challenges they face, and lowering the level of importance originally assigned to the changes they experienced). These findings supports the notion that people with early-stage dementia actively engage with their illness, "that many individuals think a great deal about their illness" (Cotrell, 2007, p. 63), and that each person draws on a life time of personal experiences to design his or her coping response. In other words, living with dementia is not experienced as a passive enduring but an active constructing.

Perceiving strengths and resources. In the transactional model of stress, appraisal, and coping (Lazarus \& Folkman, 1984) secondary appraisals evaluate what can be done about the situation (the changes associated with early-stage dementia) and determine the adequacy of available internal and external strengths to cope with and adapt to the changes. In other words, strength forms the foundation upon which coping responses are built. Estimating the strengths and resources available to manage the disruption created by early-stage dementia precedes coping and by and large determines the choice of coping strategies. To that end, this section first discusses the general concept of strength as it relates to coping and then explores what the current literature on early-stage dementia suggests about the strengths and resources of this population.

Strength and coping. Strength is a term often used in everyday language. It 
appears regularly in the social science literature, permeates social work practice texts (e.g., Cohen, Phillips, \& Hanson, 2008; Longres, 2000; Shulman, 1999; Zastrow \& KirstAshman, 2004), and is generally considered an important component of well-being, fulfillment, and satisfaction with life. However, it remains an elusive, hard-to-define concept. In general, "strengths refer to the personal and environmental resources that serve to protect people and form the substance of their resistance and resilience" (Longres, 2000, p. 11). In other words, "resilience does not come from rare and special qualities, but from the everyday magic of ordinary, normative resources“" (Masten, 2001, p. 235). Almost anything can be considered a strength given the right conditions (Saleebey, 1992/2009). While researchers and theorists often use different names for strengths and resources, regardless of the terminology, "studies continue to corroborate the importance of a relatively small set of global factors associated with resilience" (Masten, 2001, p. 8).

It has been suggested that a positive sense of self and identity is associated with subjective well-being (Waterman, 1992), and that personal qualities, traits, and virtues can be considered strengths (Saleebey, 1992/2009). Moreover, Taylor (1983) argues that one of the coping responses following a personally stressful event such as serious illness (which often affects self-esteem negatively) is an effort to improve self-esteem.

Research suggests that being engaged in the successful pursuit of personally meaningful goals plays a major role in achieving and maintaining subjective well-being (Emmons, 1996; Lazarus \& Folkman, 1984; Taylor \& Brown, 1988, 1994). Brunstein (1993) found that a strong commitment to personal goals in combination with sympathetic conditions to achieve these goals was associated with a high degree of 
success in attaining the goals, which in turn contributed to higher levels of subjective well-being. Folkman (1997) reported that striving towards meaningful goals continues to be important even in situations that are seemingly uncontrollable and consistently changing. In her study investigating the coping responses of individuals caring for persons living with AIDS she found that participants were "able to identify realistic, attainable goals by focusing on specific, proximal tasks or problems related to caregiving or bereavement. (...) [This coping response] most probably contributed to positive psychological states by allowing participants to experience some personal control and sense of accomplishment, even during the horrific final stages of the partner's disease, when one loss after another is suffered" (Folkman, 1997, p. 1213).

Taylor (1983) argues that one of the components of the readjustment process after experiencing a personally stressful event is "to regain mastery over the event in particular and over one's life more generally" (p. 1170). It has been suggested that being confident and optimistic about the future, keeping a sense of humor, and maintaining the willingness to change enables people to appraise stressful situations or events as challenges to be resolved. Rather than feeling overwhelmed by the problem they discover positive aspects within the challenging circumstance (Frankl, 1963/1984; Scheier et al., 1989). Frankl (1963/1984) viewed humor as "another of the soul's weapons in the fight for self-preservation" (p. 62), and research on resilience puts forward the notion that "humor remains one of the most protective strengths a person can have in terms of physical and mental health" (Benard, 2006, p. 210).

Several theorists have highlighted the significance of finding meaning and purpose in stressful events and/or circumstances (Frankl, 1986; Frankl, 1963/1984; Moos 
$\&$ Tsu, 1977; Silver \& Wortman, 1983). They argue that, once perceived as meaningful, a challenging situation becomes less stressful to the person experiencing it. In Frankl's (1963/1984) words, "in some way, suffering ceases to be suffering at the moment it finds a meaning" (p. 135). Although the varied conceptualizations of 'meaning' have made systematic research of the construct difficult, the findings from several studies suggest a correlation between having a strong sense of meaning and purpose and lower levels of perceived stress (Lewis, 1989; Vickberg, Bovbjerg, DuHamel, Currie, \& Redd, 2000; Vickberg et al., 2001). Being able to engage in fulfilling activities is acknowledged as a way to reduce the negative impact of stressful events and facilitate a sense of well-being (Thoits, 1982, 2006; Werner \& Smith, 1982; 1992) and is viewed as an opportunity "to experience a new sense of control and mastery" (Cohen, 2006, p. 9). It has been found to improve the quality of life for older adults in general and for people with dementia in particular (Basting, 2006).

A sense of belonging and acceptance is considered a basic human need (Maslow, 1943) that can only be satisfied through meaningful and fulfilling relationships with other people. Having social support, which can be defined as "the interpersonal interactions and relationships that provide people with assistance or positive feelings" (Walsh, 2006, p. 278) is one way of establishing this sense of connectedness. In fact, seeking social support is recognized as a facilitator of well-being while experiencing a personally stressful event or situations (Taylor, 1983), and the beneficial effects of social support and its positive impact on health and adjustment for people coping with illness has been widely documented (e.g., Berkman, 1984; Cohen \& Wills, 1985; Funch \& Marshall, 1983; Hays, Chauncey, \& Tobey, 1990; House, Landis, \& Umberson, 1988). More 
specifically, "the simplest and most powerful measure of social support appears to be whether a person has an intimate, confiding relationship or not" (Thoits, 1995, p. 64). In fact, "perceived emotional support is associated directly with better physical and mental health" (Thoits, 1995, p. 64). However, the association between social support and stress is far more complex because relationships generally have positive and negative aspects (Morgan, 1989) and "both support and stressors often reside in the same set of interactions" (Lennon, 1989, p. 262).

It is widely acknowledge that religious and spiritual beliefs and practices assist people in coping with stressful events and situations (Aldwin, 1994; Bjorck \& Cohen, 1993; Carver, Scheier, \& Weintraub, 1989a; Koenig, George, \& Siegler, 1988; Pargament et al., 1990) and promote emotional well-being in people of all ages, but particularly in old age. The findings of several research studies suggest that older adults are highly involved and participate regularly in religious practices and services (Ainlay \& Smith, 1984; Taylor, 1986; Van Ness \& Larson, 2002) and report health benefits in participating in religious practices (Koenig, George, \& Siegler, 1988; Koenig, Kvale, \& Ferrel, 1988; Strawbridge, Shema, Cohen, Roberts, \& Kaplan, 1998; Taylor, 1986).

Lastly, having the financial means to afford and have access to needed services such as personal assistance, medical care, and specialized services is essential to the wellbeing of people with early-stage dementia and should be considered a strength.

However, although strengths generally play a positive role in a person's coping response, they could be easily threatened by the progression of dementia and, depending on the situational context, become weaknesses. For example, being confident about one's abilities is generally regarded as a strength. However, if confidence exceeds ability (e.g., 
driving a motor vehicle when no longer capable of doing so) it becomes a weakness. In addition, two people with similar personal and environmental resources may perceive their abilities and assets quite differently, thereby exhibiting a different level of functioning and experiencing a different sense of well-being (Frankl, 2001).

Strength and early-stage dementia. Although no study was found that directly investigated the strengths that people in the early stages of dementia draw on, the literature on dementia is interspersed with comments on and recommendations pertaining to strengths and resources. Some authors commented on the important role that past and remaining abilities and strengths of the person with dementia play in managing the uncertainty associated with the condition while preserving a sense of personal identity (Hellstroem, Nolan, \& Lundh, 2007; Koppel \& Dallos, 2007; Robinson, Clare, \& Evans, 2005). Others advocated for making the abilities and strengths of persons with dementia visible in the professional and popular discourse in order to foster a more positive and accurate understanding of the condition (Preston, Marshall, \& Bucks, 2007). Yet others highlighted the need for interventions and services for and approaches to clinical practice with persons with dementia that acknowledge and build on remaining strengths (Basting, 2006; Clare, 2008; Cotrell, 2007; Harris, 2004; Healy \& Walsh, 2007; Iliffe, Wilcock, \& Haworth, 2006; Moniz-Cook, Manthorpe, Carr, Gibson, \& Vernooij-Dassen, 2006; Moyle, McAllister, Venturato, \& Amdams, 2007; Pearce, Clare, \& Pistrang, 2002).

Furthermore, several authors commented on the role that the strengths of persons with dementia play in the research context. Clare (2003) described the research interview as an opportunity to get to know the strengths of the person with dementia. Van Dijkhuizen, Clare, and Pearce (2006) mentioned that care was taken to acknowledge the 
person's strengths throughout the interview. MacQuarrie (2005) highlighted the importance of interviewing persons with dementia in a setting that enhances their strengths. In addition, Sobel (2005) argued that the experience of dementia might provide the opportunity to "gain wisdom and knowledge of untapped strengths and inner resources not previously elicited" (p. 343).

In addition, some studies indirectly discuss this aspect of the coping response. Harris and Sterin (1999) noted three 'core values' that supported the participants in defining self-identity: (1) being productive; (2) being autonomous; and (3) being comfortable and secure (p. 249-251). Viewed from a strengths perspective, these 'core values' (productivity, autonomy, and comfort and security) were the foundation on which the participants' coping responses were built. Another way in which these values could be labeled is 'strengths'.

Werezak and Stewart (2002) explained that the process of 'fitting the dementia' into one's life was "facilitated by positive mediating experiences, such as feeling supported and accepted by significant others, retaining certain skills and abilities that gave life meaning and purpose, and being able to relate to others who were experiencing memory loss or other conditions" (p. 76). In addition, the participants in their study referred to certain 'factors' that supported them in accepting their memory loss, such as “others' acceptance of the illness, using humor to cope, and helping others" (p.78). Viewed through the lens of strengths, these 'mediators' and 'factors' can be construed as the ground in which the participants' coping strategies were rooted and thus could be understood as strengths.

In Sorensen, Waldorff, and Waldemar's (2008) study, the participants attributed 
feelings of well-being to enjoying a familiar routine; feeling useful; contributing to others' happiness; and benefitting from physical well-being. For the participants these components provided the foundation from which well-being could be extracted, which in this study is conceptualized as strength.

Implications for the present study. The body of literature reviewed here implies much for future research. I will first summarize what substantive knowledge can be drawn from the reviewed research reports and then highlight the implications for this study. The findings of the reviewed studies support the notion that that people with earlystage dementia are not inherently more fragile or predisposed to a more arduous illness experience than those living with other chronic conditions. The participants in the reviewed studies seemed to experience and view early-stage dementia as one of many disruptive problems that older adults encounter rather than the central problem. In other words, the illness competed for prominence in the global experience of aging.

The findings of the reviewed studies also suggest that people with early-stage dementia actively engage with their illness, and that each person draws on a life-time of personal experiences to design his or her coping response. The participants in the reviewed studies evaluated the impact the illness had on their wellbeing (primary appraisal) and used strategies to minimize the impact (coping).

However, the results of these studies tell us little about how people with earlystage dementia view the collection of building blocks (strengths and resources) from which they construct their coping response (secondary appraisal). The choices and decisions they made that eventually led to them to use the reported coping strategies remain unclear. Some questions that warrant further investigation in this context are: (a) 
How do people with early-stage dementia come to recognize their strengths and resources? (b) Do people with early-stage dementia use their habitual, customary strengths and resources?; (c) Does the experience of early-stage dementia change people's perception of their strengths and resources and if so, how?; (d) From their perspective, what strengths and resources make living with early-stage dementia easier or harder; (e) Do people with early-stage dementia perceive their resources as sufficient, and if not, how can we provide these?; and (f) How does the perception of strengths and resources interact with the illness experience?

The body of literature reviewed here also provides little information about the quality of well-being that the participants in these studies achieved through their coping efforts. There is scant knowledge about what 'feeling well' means for people with earlystage dementia. This study will contribute to knowledge in these areas by investigating what people with early-stage dementia perceive to be their strengths and resources and how they use these perceived abilities and assets in the process of living with the illness. By asking people with the illness directly about what they consider important, this study will also identify well-being using the perspective of the individual with the illness.

The findings of the reviewed studies also suggest a widespread shortcoming in situating current knowledge either within existing, appropriate theoretical or conceptual frames, or alternatively, acknowledging the shortage of suitable theory and engaging in theory-building efforts. This study will address this shortcoming by using grounded theory, a research approach that systematically generates theory from data. 


\section{CHAPTER 4}

\section{RESEARCH METHODS, DESIGN, AND PROCESS}

Qualitative methods, more specifically photovoice (Wang \& Burris, 1994, 1997) and grounded theory (Charmaz, 2006; Corbin \& Strauss, 1990, 2008; Glaser, 1978;

Glaser \& Strauss, 1967; Strauss \& Corbin, 1998) were used to explore what people with early-stage dementia believe and understand about their strengths and resources and how they use these abilities and assets in constructing their coping response. The first section of this chapter provides a rationale for the chosen methods of data collection and analysis. The second section provides an overview of the research design and how the process of data collection and analysis unfolded in this study. The third section discusses the steps that were taken to increase the rigor of this study, and the final section discusses how the participants' rights of confidentiality, privacy and protection from physical and psychological harm were assured.

\section{Rationale for Research Methods, Design, and Process}

Qualitative methodology. Denzin and Lincoln (2005) maintain “qualitative research involves an interpretative, naturalistic approach to the world. This means that qualitative researchers study things in their natural settings, attempting to make sense of, or interpret, phenomena in terms of the meaning people bring to them" (p.3). Given that the focus of this study, the perception and meaning of strengths, resides in the subjective realm of experience, and that no studies on this aspect of the coping response to earlystage dementia were identified, qualitative research methods were selected as the most appropriate vehicle for inquiry. Qualitative research methods are especially useful for exploratory studies in areas where little is known (Patton, 2002) and offer the means by 
which to investigate subjective social experiences (Guba \& Lincoln, 1994).

Photography and dialogue. Eliciting the perspective of people with early-stage dementia is challenging due to the progressive loss of cognitive abilities, including increasing memory loss and decreasing discursive capabilities (Hubbard, Downs, \& Tester, 2003). In fact, it has been suggested that words may be insufficient for communicating the experience of dementia and that even in the early stages of the illness and with the support of others in telling the story, "the experience is in some way beyond the power of language" (Phinney, 2002, p. 341). As a result, researchers have pointed out that there is a need to develop research methods and designs that provide the means to reduce the impact of these disease-specific difficulties (Bond \& Corner, 2001; Cook, 2003; Phinney, 2002).

Given this challenge, it was important to select a methodology that would provide an opportunity for people with early-stage dementia to express their views in creative, effective, and meaningful ways. To support this objective, I decided on a methodology that uses both photography and dialogue as vehicles for data collection as they offer different means for the participants to share their insights.

Photography. The idea of utilizing photographs as part of a research study is not novel (Close, 2007). As a method of data collection, photography has its roots in ethnographic studies where it was used to document observations on foreign cultures. More recently, a small but growing number of qualitative researchers have begun to use photographs in interviews as a means to encourage discussion, a process that became known as 'photo-elicitation'. Initially, these photographs were taken by either a professional photographer (Collier, 1967) or the researcher him- or herself (Harper, 
1984).

Since Collier's (1957) initial work, visual research methods are increasingly used in the social sciences, particularly in studies exploring psychosocial aspects of illness and well-being (Harrison, 2002; Knoblauch, Baer, Laurier, Petschke, \& Schnettler, 2008). This has led to a number of variations in the way photographs are utilized in the research process (Jenkings, 2008; Kolb, 2008). Essentially, photo-elicitation can be carried out along a continuum of participant-involvement, and research participants are increasingly invited to take photographs themselves. Photo-elicitation is considered a powerful research tool as it encourages the use of visual language, assists in building trust and rapport between the researcher and the participants, and fosters in-depth dialogues (Hurworth, 2003). I expected that photo-elicitation would bring the participants' perceptions and insights into clearer focus and thus enable the participants to reach deeper levels of reflection.

Participant-generated photographs. Making participant-generated photographs a central data source for this study was appropriate for a number of reasons. The decision to do so was grounded in both a review of the theoretical and empirical literature on dementia and my professional experience as a clinician working with people with cognitive impairments, in general, and early-stage dementia in particular. Given that the intention of this study was to capture the point of view of the participants, it was important to use participant-generated images because they invite the viewer to see the world through the same lens as the person behind the camera does; they convey the story of the person who clicked the shutter.

I initially discovered the usefulness of participant-generated images for people 
with early-stage dementia while conducting support groups for this population. I invited support group members to take theme-based photographs and discuss their images during the next meeting. The group members reported that not only had they enjoyed the phototaking activity but creating the images had allowed them to express what they wanted to say more powerfully than words alone. In addition, the photographs were helpful in a number of other ways. First, the photographs captured evocative aspects of the participants' lives; second, they facilitated the exploration of multifaceted, vague, and intricate concepts and ideas ; and third, they enabled the participants to speak in greater depths about their experiences as the photographs aided recall and reflection.

In addition to the above experiences, the literature suggests that photographs prompt storytelling and encourage participants to share perceptions that might otherwise be invisible or hidden from the researcher (Weiser, 2002); they also foster conversations about human experiences that are elusive and hard to describe (Miller \& Happell, 2006). Furthermore, the findings of earlier studies suggest that people with early-stage dementia benefit from visual cues during interviews. For example, MacQuarrie (2005) found that "during the interview, participants were resourceful in their communications. They used household communication props such as family photos, mementos, and collections as reminders" (p. 433). In Gilmour, Gibson, and Campbell's (2003) study “objects to hand, such as photographs and rosary beads, were used to encourage responses to simple questions" (p. 410). Thus, I concluded that using photographs as a means to document and stimulate the narration of experiences associated with early-stage dementia seemed appropriate. 
Dialogue. The decision for using individual and dyadic interviews (Morgan, Ataie, Carder, \& Hoffmann, in press) in addition to participant-generated photographs as a means of data collection was based on the following criteria. Photographic images are powerful data. The interpretation of a photograph is influenced by culture, which provides conventions for understanding and decoding images. However, there is a wide range of interpretation possibilities. To develop a better understanding of the meaning and experiences captured by a photograph, it is vital to enter into a dialogue about the image (Harrison, 2001). Engaging in dialogue about images creates shared meaning.

In this study, dialogue about the participant-generated photographs would allow me to investigate the participants' explanations of what they had chosen to make visible and why (Radley \& Taylor, 2003). The participants' verbal explanations of their images increase our understanding of what they had tried to document and facilitate a mutual interpretation. In addition to facilitating an understanding of the content and meaning of the participant-generated photographs, the dialogue would help me understand the larger context of these images. In other words, using dialogue as a means of decoding these images would allow me to capture a much wider range of experiences than the photographs alone. In addition, it would facilitate an exploration of what they chose not to portray and what they could not portray. Furthermore, I hoped that the dialogue would reveal some of the participants' personal values and that the process of examining the photographs together would uncover aspects of the photographs previously hidden from the photographer as well as the researcher (Wuggenig, 1990). In other words, dialoguing about the photographs has the potential to introduce new topics and aid in understanding the research phenomena, as photographs provide an opportunity to go "beyond that 
contained in the photographs themselves" (Collier \& Collier, 1991) p. 99.

Photovoice methodology. Although there are a number of research methods using photography and dialogue, I chose photovoice because it seemed the most empowering method of conducting research. Photovoice is an approach to inquiry that is grounded in the assumption that participant-generated photographs and group process can inspire dialogue in ways that spoken language alone often cannot. To that end, photovoice puts cameras in the hands of participants and asks them to explore the topic of interest from their social location and point of view.

By engaging the participants in a process that involves taking photographs, small group discussions, photo exhibits, and presentations, photovoice provides numerous opportunities for the participants to share their perspectives and concerns. What distinguishes photovoice from other research methods using photographs is its critical underpinnings, its emancipatory intent, and its participatory components (Wang, 1999). Choosing a methodology with participatory features was important to me because I wanted to conduct research with and not on people with early-stage dementia.

As a methodology photovoice was developed in 1992 by Wang and Burris (1994, 1997) while working on a project devoted to improving the health status of women in rural China. Grounded in the theoretical literature on education for critical consciousness, feminist theory and non-traditional approaches to photography (Wang, 1999), photovoice was developed to serve as a tool for assessing the needs of these women based on the assumption that women in developing countries rarely have the opportunity to make their needs known. In the original version of photovoice, the women in rural China were trained to operate cameras, encouraged to take photographs that represented their 
everyday health and work realities and invited to share their views of the world in large and small group discussions.

Photovoice studies generally have three goals. They aim to enable members of vulnerable populations to reflect on issues that are important to them, discuss their viewpoint on these issues with the researcher and/or other participants, and initiate social change by presenting the photovoice findings to the larger community including policy makers. The assumptions underlying photovoice methodology are that it is important to include the perspectives and perceptions of vulnerable populations in the policy-making process and that it is necessary to find ways for members of these populations to express their view. Building on the ideas of Paulo Freire (1970), the Brazilian educator who saw visual images as a way of helping people to think critically about their lives, cameras are handed to people who normally would not have the opportunity to communicate their perspective. In other words, photovoice invites the participants to take photographs and discuss their meaning as a way of empowering the participants and encouraging them to identify their own strengths and resources. Booth and Booth (2003) explain,

"Photovoice involves giving people cameras and using the pictures they take to amplify their place in the world. It puts people in charge of how they represent themselves and how they depict their situation. The process challenges the politics of representation by shifting control over the means for documenting lives from the powerful to the powerless, the expert to the lay-person, the professional to the client, the bureaucrat to the citizen, the observer to the observed" (p. 431).

In addition, photovoice aims to facilitate social change by encouraging participants to present their needs to policy and other decision makers (Wang \& Burris, 1997). To that end, the participants prepare presentations of the photovoice findings, which are then disseminated to both scientific and lay audiences including policy makers. 
Since Wang \& Burris' initial project, photovoice has been used in a wide range of health care research, including African American breast cancer survivors (Lopez, Eng, RandallDavid, \& Robinson, 2005), patients in a hospital ward (Radley \& Taylor, 2003), and hospital nursing staff (Riley \& Manias, 2006) and with a variety of vulnerable populations, including homeless people (Radley, Hodgetts, \& Cullen, 2005), senior citizens (LeClerc, Wells, Craig, \& Wilson, 2002), immigrant women (Bender, Harbour, Thorp, \& Morris, 2001), mothers with learning disabilities (Booth \& Booth, 2003), and people living with HIV/AIDS (Hergenrather, Rhodes, \& Clark, 2006).

Using photovoice methodology in this study seemed particularly appropriate because it puts those living with early-stage dementia in a position of power. By handing cameras to those living with the condition photovoice allows them to portray and talk about their strengths and resources in a new, unique, and different way. In other words, photovoice offers an opportunity to see the strengths and resources of people with earlystage dementia in a way not possible with other methods. In addition, given the role that creativity can play in facilitating, enhancing, or shaping well-being, it seemed possible that the participants might benefit from the activities inherent in a photovoice project as they provide opportunities for self-expression and self-exploration.

Traditional photovoice process. Although photovoice methodology varies according to the context in which it is used, photovoice projects generally progress through seven distinct and yet connected phases (Kolb, 2008). In the contextualizing phase, the researcher develops the research design, specifies the methodology, identifies the project site, and seeks community support. In the recruiting phase the researcher distributes information about the project and recruits participants. In the opening phase 
the researcher meets with the participants in a group session to describe the photovoice concepts, outline the process, and train the participants in how to use a camera. Then he or she introduces the photo-taking theme and facilitates a group dialogue in which the participants develop initial ideas about how to take photographs that would portray their perspective on the theme. This invitation to reflect on the photo-taking theme stimulates a cognitive process that continues throughout the remainder of the research project. An integral part of this dialogue is also a discussion of the ethical issues involved when taking photographs. At this point the participants decide whether or not they want to take part in the project and provide written informed consent if they wish to continue. In the discovery phase, the participants continue to contemplate the meaning of the photo-taking theme in the context of their lives and their life experiences and implement their reflections by taking photographs of people, things, and places that are meaningful to them and relate to the photo-taking theme. In the decoding phase, the participants reflect on their photo-taking experience and discuss their photographs in a group dialogue with the researcher and the other participants. During this dialogue the participants explore how their photographs relate to their life experiences and the photo-taking theme. In the interpretation phase, the participants and the researcher use group dialogue to categorize the photographs by themes and to discuss how the insights from these findings can be used to facilitate social change. A crucial element of this dialogue is a discussion about how the participants can become advocates for their own, personal well-being and the well-being of their communities. In the dissemination phase, the findings of the photovoice project are presented to the community at large including policy-makers.

Modified photovoice process. The biggest challenge of conducting a photovoice 
project with people with early-stage dementia is to modify the method in a way that it is 'safe' for the study participants to engage in the project, that participating in the project will empower the participant and not leave the participants with a sense of failure. Given these realities, I adhered as closely as possible to the intent, activities, and sequencing of the research procedures recommended by Wang and Burris (1997), but I adapted the overall process to accommodate the specific needs of people with early-stage dementia and ensure maximum empowerment. As recommended by Wang and Burris (1997), the study used two data sources: participant-generated photographs and dialogue about these images. However, unlike traditional photovoice projects that rely by and large on small group discussions as a means of collecting data, this study used a combination of individual and dyadic interviews to demystify the stories captured by the photographs and gain insight into the participant's perspective. More specifically, individual interviews were used to conduct the activities of the opening phase and the discovery phase of the project. Dyadic interviews were employed during the decoding phase of the project to elicit participant feedback on the preliminary findings and interpretations (member checking) and to further develop the understanding of the range of responses to the illness.

I decided to use individual interviews in the opening phase and discovery phase of the project because at the design stage of the project it remained unclear whether what I had learned from my support group participants about the abilities of people with earlystage dementia to successfully operate a camera was generally true for this population or whether people who attend support groups are a somewhat privileged, higher functioning subset of this population. Given this uncertainty, I felt that individual interviews would 
allow me more readily than a group environment to protect participants from potential harm. This more intimate environment would provide an opportunity to deflect embarrassment if the participants should not succeed in taking usable photographs, forget how to use the camera, or simply forget to take photographs altogether. It would also provide a safe space in which I could assist the participants in processing negative emotions should they arise. In addition, I felt confident that I could conduct the interviews in a way that would generate some data for answering the research questions even without the photographs.

The decision to use dyadic interviews (Morgan, Ataie, Carder, \& Hoffmann, in press) instead of focus groups during the decoding phase of the project was based on the following criteria. First, as dementia progresses, it becomes more difficult for people with the illness to express their thoughts and feelings verbally and/or understand what is being said. Thus, eliciting in-depth information from this population in a focus group setting is challenging as the moderator has to ensure that the participants not only understand him or her but also each other. Dyadic interviews seemed an appealing alternative as they not only increase the depths and breadth of information yielded by facilitating an exchange between people with similar experiences but also allow the moderator to attend to each participant's distinctive communication needs. Second, people with early-stage dementia feel easily overwhelmed when faced with groups of new people and/or unfamiliar surroundings. Dyadic interviews minimize the distractions of a group situation and yet create a space where views, beliefs, opinions, and perceptions can be discussed with someone in a similar situation. Third, discussing sensitive issues such as one's experiences with early-stage dementia in a focus group setting can potentially cause 
discomfort to participants. A carefully conducted pairing process for the dyadic interviews can eliminate this potential drawback of focus groups. The researcher can select participants who are able to easily relate to each other, which reduces the discomfort associated with self-disclosure. Fourth, the recruitment area for this study was geographically spread and participants lived in locations that were up to 200 miles apart. Given the geographical logistics, focus groups would have been difficult to arrange. The relatively low number of participants needed for dyadic interviews, increased the likelihood of finding pairs who lived in close proximity to each other and yet were homogenous enough to capitalize on their shared experiences. Finally, dyadic interviews added a participatory component to the data analysis and increased the trustworthiness of the study while maintaining a sense of privacy similar to individual interviews.

The modified process differed from the traditional process in a number of other ways. To ensure that the participants would be empowered to successfully progress through the photovoice project, I developed in the contextualizing phase a number of photo-taking tools, protocols, and memory aids that would compensate for the limitations resulting from cognitive impairment. I will discuss these tools, protocols, and memory aids in a later section of this chapter in more detail.

In the recruitment and opening phase, I used three diagnostic measures, the MiniMental State Examination (MMSE) (Folstein et al., 1975), the Geriatric Depression Scale-short form (GDS-short form) (Sheikh \& Yesavage, 1986), and the Quality of LifeAlzheimer's Disease measure (QOL-AD) (Logsdon, Gibbons, McCurry, \& Teri, 1999). The aim of using these diagnostic measures was to determine the participants' level of functioning and to more accurately identify the specific population under study. The data 
collected with these measures served as an additional guideline for interpreting the qualitative data.

I structured the discovery phase tightly. Taking into consideration that people with early-stage dementia benefit greatly from structure and routine, I assigned a certain number of photographs that had to be taken each day (4 photographs on 7 consecutive days). I also scheduled check-in phone calls to stay in contact with the participants throughout this phase. These check-in phone calls would remind the participants on the project, allow for trouble shooting, and establish a sense of familiarity that would be helpful for the interviews in the next phase. I also separated the dialogue about the participant's experiences of taking photographs (brief, individual interviews) from the dialogue about their photographs (in-depth, individual interviews). It seemed advantageous to interview the participants about their photo-taking experience immediately after they had completed this activity because, given that the participants experience memory problems, it seemed likely that they would forget some if not all of their experiences as time progressed.

In the interpretation phase, I used grounded theory analysis to analyze and interpret the visual and verbal data collected in the earlier phases of the study. In addition, dyadic-interviews were employed as a means of member checking and to further develop the understanding of the range of responses to the illness.

Grounded theory. Grounded theory is a research methodology that is used to investigate social processes and interactions (Charmaz, 2006; Corbin \& Strauss, 1990, 2008; Glaser, 1978; Glaser \& Strauss, 1967; Strauss \& Corbin, 1998). As a research method, it is considered to be most relevant for situations in which a phenomenon is 
marginally understood; additional detail is needed to augment existing knowledge of a phenomenon; a novel perspective of a phenomenon is desired; an "insider" perspective would add to understanding the complexities of a phenomenon; and understanding of the why and how of a phenomenon is needed (Stern, 1980). Grounded theory seemed a fitting method for this study because it aimed to identify the type of strengths and resources that people with early-stage dementia consider meaningful in coping with the illness, a topic about which little is known; it aimed to add detail to our current, rather general and limited understanding of the dynamics underlying the coping response to early-stage dementia; it aimed to add an insider perspective by investigating the topic from the perspective of those living with the condition; and it aimed to uncover details of the ways in which people with early-stage dementia construct their coping response.

In keeping with the assumptions of symbolic interactionism (Blumer, 1969), grounded theorists embrace the notion that knowledge is socially constructed and language is an important means by which experiences are interpreted. In other words, grounded theorists believe it is through social interactions that we come to know and understand a phenomenon. Observing and processing experiences that present themselves during social interactions are the means by which the self constructs realities, values, and beliefs. As such, grounded theorists believe that knowledge about the world is not independent of a person's beliefs, thoughts, and perceptions (Corbin \& Strauss, 2008; Strauss, 1987) and that values and interests are intrinsic to our observations and thus influence what we know. Grounded theorists subscribe to the notion that the researcher and the participants bring their own values to the inquiry (Corbin \& Strauss, 2008). These values are viewed as an intrinsic part of the research process and it is acknowledged that 
the researcher's values shape the research question and focus, influence the research purpose and methods, and affect the interpretation that the researcher offers during the data analysis process. As such, grounded theory embraces the idea that the research process is shaped by both the researcher's and the participants' attempt to engage in sense-making activities and that these activities occur from different standpoints (Glaser, 1978). The co-created data in turn shapes the findings. Given that people are considered inextricably linked to their environment through reciprocal relationships, the research participants' accounts and the way they present themselves are viewed as shaped by the research context and the interaction between the researcher and the participants. Acknowledging the important role that interaction plays in the research process, grounded theory asks researchers to be aware of and explicitly describe their influence on the data (reflexivity) (Corbin \& Strauss, 2008).

The goal of grounded theory is to generate a theory that explains, at a broad conceptual level, a process, an action, or an interaction. It aims to discover the central theme in the participants' photographs, words, and actions and the participants' approach in managing the unearthed issue. During the interviews, the researcher's role is to listen, probe, clarify, and repeat to ensure that the participant is accurately understood. The researcher develops an understanding of the data through constant comparison, writing memos, and engaging in conversations and interactions. Numerous encounters over time with the participants enable the researcher to develop and articulate an explanation for the participants' behavior and actions. Because meaning continues to change as a result of experiences and interactions, it is considered essential that participants and researcher interact in various ways. The way in which I used grounded theory to guide data 
collection and analysis in this study and how I influenced the data will be discussed in the relevant sections.

Combining photovoice and grounded theory. Photovoice methodology has thus far stopped short of providing guidance on how to analyze the data. Given these limitations, I decided to use photovoice methodology (Wang \& Burris, 1994; Wang \& Burris, 1997) in conjunction with grounded theory (Charmaz, 2006; Corbin \& Strauss, 1990, 2008; Glaser, 1978; Glaser \& Strauss, 1967; Strauss \& Corbin, 1998) for this study. Grounded theory provided structure and guidance for collection, analysis, and interpretation of the data. The combination of both methods allowed me to not only describe the perceived strengths and resources that the participants brought to the dementia experience but also develop a deeper understanding of the social and psychological processes related to coping with and adapting to the illness.

In summary, while grounded theory is committed to uncovering the participants' point of view (Glaser, 1978), photovoice provides the tools to draw out this perspective in innovative and creative ways. In keeping with the precepts of symbolic interactionism (Blumer, 1969), grounded theory relies on data generated in social interactions and thus is uniquely suited to analyze the words and actions of participants in a photovoice study. In other words, grounded theory facilitates the process of discovering themes and patterns relevant to the research question (Glaser, 1978) and assist in developing or discovering a theory, an abstract schema of the process under study (Corbin \& Strauss, 2008). Thus, combining the methods of photovoice and grounded theory seemed suitable for this study.

Quantitative measures. Dementia is an illness that contributes to high variations 
in levels of functioning, and there is no one single measure that adequately determines an individual's level of impairment. As such, one of the weaknesses of existing qualitative research is that it inadequately describes both important aspects of the population being investigated and how the findings relate to underlying conditions of interest (such as depression and/or low levels of perceived quality of life). The aim of using diagnostic measures in this study, therefore, was to more accurately identify the specific population under study and determine the participants' level of functioning in the areas of cognitive status, depression, and perceived quality of life. In addition, the collected information served as a context for interpreting the qualitative data. From the onset of the study, I aimed at gaining the best possible insight into the researched, the research context, and the researcher.

As mentioned earlier, three diagnostic measures were used in this study, the MiniMental State Examination (Folstein et al., 1975), the Quality of Life-Alzheimer's Disease measure (QOL-AD) (Logsdon et al., 1999), and the Geriatric Depression Scale-short form (GDS-short form) (Sheikh \& Yesavage, 1986). The MMSE (Folstein et al., 1975) is a short cognitive screening tool that measures item recognition, orientation, short-term memory, attention/concentration, language, and sentence formation capacity. The MMSE is one of the most widely used screening instruments for cognitive impairment and has well-established validity and test-retest reliability (Folstein et al., 1975). This instrument has a range of possible scores from 0-30. The score is the total number of correct answers with a maximum of 30 points for adults. In this study, the MMSE was used to gain a better understanding of the participants' level of cognitive functioning and exclude those who had progressed into the moderate stages of the illness. The QOL-AD (Logsdon et al., 
1999) is a 13-item questionnaire that can be completed by people with dementia, care partners, or both. This study will rely exclusively on the QOL data provided by the participants with dementia. The QOL-AD covers the following life domains identified to be of importance for older adults with cognitive impairment: physical health, energy, mood, living situation, family, marriage, friends, self as a whole, ability to do chores around the house, ability to do things for fun, and life as a whole. Ratings are made on a four-point scale: poor, fair, good, and excellent. Total scores range from 13 to 52, with higher scores indicating higher quality of life. The QOL-AD has good internal consistency reliability (0.89), test-retest reliability (0.92), and validity (Logsdon, Gibbons, McCurry, \& Teri, 2002), and has been used in individuals with MMSE scores as low as 3 (Hoe, Katona, Roch, \& Livingston, 2005; Thorgrimsen et al., 2003). In this study, the QOL-AD was used to gain a better understanding of how the participants viewed their specific life circumstance (e.g., physical health, living situation, marriage, functional abilities, etc.) as contextual factors have an effect on the responses to the research questions. The GDS-short version (Sheikh \& Yesavage, 1986) is a 15-item selfreport questionnaire designed to identify depressive symptoms in older adults (e.g., are you in good spirits most of the time; do you often feel helpless). Ratings are made in a yes/no format, with total scores ranging from $0-15$. Scores ranging from 0 to 4 indicate no depression; scores ranging from 5 to 10 are suggestive of mild depression; and scores 11 and higher are suggestive of severe depression. The simplicity of the yes/no scoring format allows for the scale to be used as a self-report measure with people with earlystage dementia. The GDS has good internal consistency reliability (0.83) for older adults, and cognitive impairment has not interfered with the measure in previous studies 
(Logsdon et al., 2002). Research suggests high incidence of depression among people with dementia (Lyketsos, Gonzales-Salvador, Chin, Baker, \& Black, 2003; Steinberg et al., 2003; Tariot et al., 1995) and a negative relationship between depression and quality of life (Logsdon et al., 2002). In this study, the GDS was used to determine the presence of depression, as it would influence the participants' responses to the research questions.

Researcher as instrument. In a qualitative research study the researcher serves as the primary instrument of data collection, analysis, and interpretation (Merriam, 2009). As such, it is important to become aware of and identify personal values, assumptions, and biases at the onset of a study and continue with this effort for the duration of the project. Awareness of personal bias mitigates the potential impact of preconceptions on the research process. It allows setting aside preconceived notions of what is considered strengths and resources and being receptive to the participants' point of view. In addition, describing the researcher's personal life circumstances and experiences allows the reader to determine how his or her culture (e.g., education, language, gender, social standing) may have impacted the interpretation of the data.

Personal experience and work as a clinician with people with cognitive impairment and/or at the end of their lives have led me to believe that it is essential to make the strengths and resources of people and their environments the focus of social work interventions and not their weaknesses and deficits. I have learned that all people (including those living with cognitive impairment) possess strengths and resources and are capable of growth and change, particularly when empowered to recognize, acknowledge, and use those strengths and resources. Once recognized and acknowledged, these strengths and resources can be mobilized for the process of achieving and/or 
maintaining a sense of well-being and satisfaction with life.

Clinicians are at risk of underestimating human capacity in the face of serious and/or life-threatening illness. During my clinical practice as a hospice social worker I have come to realize that the majority of people continue to be able to create and maintain a sense of well-being despite the challenges inherent in coping with a lifethreatening illness. Over time, I have come to think that the dynamics that drive the process towards well-being and life-satisfaction (even in the face of imminent death) are the presence of hopes and dreams and the consistent focus on existing capabilities and capacities. Once translated into realistic and attainable goals (no matter how small these goals may be), these aspirations become the building blocks for a more gratifying and enjoyable life.

In other words, as a social worker I am grounded in the belief that the most critical ingredient for clinical practice is viewing a person's life as always bigger than his or her problem. This means having and maintaining a genuine interest and trust in people. Learning from people who tend to be stereotyped and misunderstood and making their voices heard is my life's passion, which is what I bring to this research project. As a clinician who embraces an empowerment approach to social work practice I have also learned that it is important for people (including those living with cognitive impairment) to have an opportunity to explore, reflect on, and process their experiences in a supportive environment.

It was the lack of non-pharmacological/psychotherapeutic interventions for people with early-stage dementia that led me volunteer my time to the local Alzheimer's Association. I have facilitated support groups and educational programs for people with 
the illness for the past 6 years. I believe that each person's voice needs to be heard and that I have a responsibility to create an environment where people can process their experiences by sharing it, which is a first step to regaining a sense of control over their lives (empowerment) (Aujoulat, Luminet, \& Deccache, 2007). I chose photovoice methodology for this research because it creates an environment in which the participants can share their experiences with the researcher, other participants, and the community at large. Sharing their story with others fosters the participants' sense of self-efficacy and increases their self-esteem and at the same time, advocates for their needs by presenting their experiences to the community.

\section{Photovoice Process in this Study}

Contextualizing phase. Building community support. During this phase I consulted with several professionals who have extensive experience in the field of earlystage dementia either as clinicians and/or researchers to elicit their feedback on the overall research design and the specifics of the methodology and to discuss potential project sites. These experts offered not only valuable suggestions about how to adapt photovoice methodology to the needs of people with early-stage dementia but also helped me to gain access to the study population. Two professionals from the Alzheimer's Associations Western and Central Washington State Chapter (Joanne Maher, Director, Social Services; Linda Whiteside, Director, Community Support) and a researcher from the Northwest Research Group on Aging, University of Washington (Dr. Rebecca Logsdon, Research Professor) supported recruitment in this study by enlisting their organizations to distribute information about the study and identifying venues for later disseminating the study findings to lay audiences. 
Identifying the project site. The greater Seattle area (100 miles radius) was identified as the project site. There were two primary reasons for choosing this site. First, given the complexities of the research design which included meeting with each participant at least three or four times, the participants needed to live within a reasonable driving distance. Second, it was important for the participants to live close to each other so that I would to be able to pair participants for the three person dialogue sessions without putting unreasonable demands on their travel time.

Specifying the methodology. Developing study-related tools. A crucial component of using photovoice methodology with people with early-stage dementia was to develop a number of project-related tools that would assist them in volunteering for and progressing through the project. All tools discussed in this section can be found in appendices $\mathrm{O}-\mathrm{S}$.

The first step in terms of tools was to develop multiple options in which potential participants could voice their interest in the study, including one that would allow them to respond as soon as they received the announcement of the study. Given that memory problems are one of the challenges associated with early-stage dementia, it was essential to make it as easy as possible for potential participants to manage the steps from reading the flyer of the study, deciding that they wanted to participate in the study, and actually responding to the study announcement. Thus, I decided to not only provide my phone number and e-mail address in the announcement of the study but also attach an 'interest card' and a self-addressed, stamped envelope to each flyer. The 'interest card' stated the title of the study and provided a section where potential participants could note their phone number(s) so that I could contact them to arrange for a screening session. The 
second step in terms of project-related tools was to select the type of camera that the participants would use in the discovery phase. I decided on using old-fashioned, filmbased, single-use, disposable cameras for the project. The biggest benefit of using this type of point-and-shoot camera is that it is inexpensive and easy to use and more importantly, until recently, it was commonly in use. Thus, it is likely that the study participants are familiar with handling such a camera, and given the characteristics of dementia, it is likely that this sense of familiarity remains in the early stages of the illness. However, most older adults do not use a camera on a daily basis. Thus, it was important to re-familiarize the participants with the use of the camera. To that end, I developed a training protocol in which the rather complex activity of taking a photograph is broken into eight, simple steps. In addition, I designed a number of handouts to be available for the participants during the discovery phase. These handouts included a reminder on the photo-taking theme, instructions on how to use the camera (which mirrored the above 8-step training protocol), and information about how to contact me for troubleshooting.

The third step was to design a screening protocol that would allow me to screen for those participants who would indeed be able to successfully use the camera and thus succeed in taking photographs during the project. To accomplish this objective, I developed a four-step screening protocol that sandwiches an activity unrelated to phototaking (providing demographic information) between two practice pictures. It was necessary to develop such a protocol because none of the existing cognitive assessment tools provide reliable information regarding people's functional abilities, and thus it was impossible to know whether or not they were capable of operating the camera. 
The fourth step was to anticipate how 'forgetfulness' would potentially interfere with the photo-taking activity in phase 4 and develop a number of tools based on this prediction. As mentioned earlier, in order to function well, people with early-stage dementia rely heavily on structure and routine and benefit from storing everything in the same place, all the time. When thinking about the logistics of daily photo-taking in the context of early-stage dementia, I realized that if I wanted the participants to succeed in the project, it was important to honor and use this need for structure and order by providing each participant with the means to store all the tools needed for completing the project in one, easy-to-access location. Each 'photo-taking toolkit' contained, in addition to a film-based, disposable, single-use camera; a photo-taking plan; a daily instruction sheet; a photo diary; a photo-log; and two refrigerator magnets.

The photo-taking plan provided space for the participants to record initial ideas of the types of photographs they wanted to take, where they would keep the photo-taking toolkit, what reminders they created for themselves, and who might be able to help them if they ran into difficulties when taking photographs.

The photo-diary was designed to allow the participants to record each photograph and the reasons for taking the photograph. These photo-diaries supported self-reflection and self-exploration and at the same time, served as a memory aid. In other words, recording thoughts and reflections relevant to the photograph taken counteracted forgetting by allowing the participants to clarify how each photograph related to the photo-taking theme and by empowering them to share these insights during the dialogue sessions and express themselves more clearly. To increase the photo-diary's visibility and make it visually appealing I chose bright colors for the diary cover. I also built in a 
tracking system by using different color pages for each day of the photo-taking activity, which allowed the participants to evaluate their progress through the project. The photodiary consisted of twenty-seven pages, which were sorted in groups of four by color; each group of four (each color) represented one day of photo-taking activity.

The daily instruction sheet was developed because many people with early-stage dementia rely on tracking devices of some kind. In addition to serving as a tracking device in which the participants recorded the number of photos taken each day, the daily instruction sheet spelled out the photo-taking theme and provided simple instructions on how to use the camera and how to contact the researcher. The final items in the phototaking toolkit were two refrigerator magnets to serve as an additional memory aid and a pen.

Operationalizing strengths and resources. For the purpose of the photo-taking assignment the concept of 'strengths and resources' was operationalized as the phototaking theme:

"Picture what is important to you. What helps you keep on doing the things that give your life meaning and purpose? I want you to take photographs of the people, things, and places that help you continue the activities, relationships, and goals that are meaningful to you. These can be photographs of anything about you and your life anything you want."

Conducting the pilot. Prior to launching this study, I conducted a small pilot study with four of my co-workers at Providence Hospice of Seattle to try out the adapted photovoice process and see if the modified procedures perform as envisioned. Giving my research methods a test run with people who do not experience early-stage dementia was important because it allowed me to evaluate the efficiency of the adapted photovoice process without running the risk that the participants would interpret the potential short- 
comings of the method as a by-product of their cognitive impairment. The practice run gave me the opportunity to refine the research procedures, fine-tune the project-related tools, practice my dialogue skills, identify personal feelings arising during the dialogue, and begin to address my biases. It also allowed me to test the quality of the open-ended dialogue guide and analyze whether the questions were easy to understand and elicited rich responses. Practicing the dialogue questions and prompts before undertaking the actual research allowed me to learn the value of patience and become more confident in terms of silences. At the same time, I became aware of and learned to avoid situations where long and uncomfortable silences may arise from confusion about what was asked (Sorrel \& Redmond, 1995).

The practice run resulted in tightening some of the research procedures and in rewording those dialogue questions that the pilot-participants had answered differently than I had expected. For example, I had initially planned to have the photographs presorted by a third party with attention to the level of technical competency prior to meeting with the participants. However, the pilot revealed that sorting the photographs according to technical detail confused the participants and thus interrupted the flow of dialogue because the sequence of the photographs was important to the photographers and played an essential role in telling their story. Central to this study was that the participants would be able to successfully operate a film-based, disposable camera. Practicing with my colleagues allowed me to test-run two different camera brands and evaluate which of the two brands was most user-friendly and reliable. The findings of the pilot also resulted in minor adjustments of the camera training protocol and clarified the commonly made mistakes. For example it is easy for anyone to take a poor photograph if the distance 
between the object and the camera is too short, as was the case in one of the pilot participants' portfolio. Sharing this story with the study participants encouraged them to not be afraid of taking poor pictures because it could happen to anyone.

Recruiting phase. Eligibility criteria. To be eligible for participation in this study, participants had to fulfill the following criteria: (1) have a diagnosis of early-stage dementia and score no lower than 20 on the Mini-Mental State Examination (Folstein et al., 1975), (2) be able and willing to take photographs with a single-use, disposable camera, (3) be able and willing to express themselves verbally in English, and (4) live in the community. The process by which these criteria were evaluated is discussed as part of the screening interviews later on in this chapter.

Sampling strategy. The participants for this study were recruited using purposive (or purposeful) sampling. Purposive sampling focuses on selecting participants for their unique ability to provide information related to the research question(s). As Patton (1990) noted, "the logic and power of purposeful sampling lies in selecting information-rich cases" (p. 169). The decision to sample participants for this study purposively was motivated by my research questions, which called for a specific group of participants people with early-stage dementia. In other words, the participants for this study were selected because of their ability and willingness to provide insights about the experience of living with early-stage dementia.

Following approval for this study from the Portland State University Institutional Review Board, I delivered the research announcement with the attached 'interest card' and self-addressed, stamped envelope and a cover letter to the two organizations that had agreed to support this study, the Alzheimer's Association Western and Central 
Washington Chapter and the Northwest Research Group on Aging, University of Washington, School of Nursing. In their role as community 'gatekeepers', both organizations facilitated access to potential participants by mailing information about the study to people with early-stage dementia who lived within an approximately 100 mile radius from Seattle. The recipients of the mailing had attended educational classes and workshops about dementia and/or had participated in research studies investigating aspects of the dementia experience within the last year. The choice for the one-year timeframe was based on the assumption that the recipients would still be in the early stages of dementia, given the usual course of the illness. At the same time, the Alzheimer's Association distributed flyers about this study during support group meetings and/or educational seminars for people with early-stage dementia. Because of the unusually high response rate following the initial mailing and the speed with which the participants responded, the recruitment phase of the project was completed within a month and there was no need to employ 'snowball sampling' (Patton, 2002) as initially planned.

Twenty-seven potential participants responded to the initial mailing. As expected, the overwhelming majority of the respondents ( 22 of 27 ) used the immediate response option (interest cards and self-addressed stamped envelopes) to respond to study announcement. From the remaining five respondents, two phoned and three e-mailed. Twenty participants self-referred to the study; eighteen of these self-referring participants used the immediate response option; and two used e-mail. Seven caregivers responded to the study announcement; four of these caregivers used the immediate response option; two phoned; and one e-mailed. 
Recruitment phone calls. I conducted a total of 23 recruitment phone calls. From the 27 initial respondents, four did not receive a recruitment phone call. They had experienced a sharp decline in health and had moved to a nursing home, and family members had utilized the interest cards to inform me about this fact. During the recruitment phone calls, I first screened for the eligibility criteria 'community-dwelling'. All 23 respondents lived in the community. Next, I explained the study purpose and procedures and time commitment requirements and answered any questions raised by the potential participants. Next, I explained the basic elements of the informed consent form, such as risks, benefits, and safeguards. Lastly, I confirmed the respondents' interest in participating. All 23 respondents were interested in participating and scheduled a screening interview. Given that some people with early-stage dementia are acutely aware of their deficits and prefer to have their partner and/or care partner present during decision-making activities, I asked the potential participants if they would like to invite their partner and/or care partner to participate in the screening interview and consent procedure.

Sample size. Although I had a list of twenty-seven potential participants within two weeks, I decided to spread the data collection over a period of several months because grounded theory data analysis calls for 'constant comparison'. In 'constant comparison', data is collected and analyzed simultaneously. As concepts of interest are discovered, they are compared with other instances already collected and the incoming data for similarities and differences. In addition, emerging concepts from the ongoing data analysis are used to guide subsequent data collection, a process that is referred to as 'theoretical sampling' (Corbin \& Strauss, 2008). This process cannot be planned in 
advance because sampling decisions are grounded in the concepts emerging from and during the data analysis. The goal of 'theoretical sampling' is to discover concepts and their dimensions and properties to facilitate the emergence of a 'grounded theory'. When the emerging concepts are saturated and the connections between these concepts are understood, the theoretical sampling process is complete and data collection stops (Corbin \& Strauss, 2008). In other words, data collection for this study was guided by the developing analytic framework, and sample size ultimately depended on the saturation of the concepts comprising this framework.

Ultimately, 20 people with early-stage dementia participated in the study. From the 27 initial respondents, seven did not participate in the study. As mentioned earlier, four respondents had experienced a sharp decline in health and had moved to a nursing home before this study had started, and three participants had scheduled screening interviews but cancelled these appointments due to acute illness (two respondents) and sudden death in the family (one respondent).

Screening interviews. I conducted a total of 20 screening interviews. As expected, the overwhelming majority of respondents (17 out of 20) preferred to meet in the comfort of their own home due to a loss of ability to drive and lack of access to alternate methods of transportation, problems with orientation, and other limitations. Two screening interviews were conducted in restaurants chosen by the participants and one at the local Alzheimer's Association. Ten respondents chose for their partners to be present during the screening interviews and informed consent signing. Seven of these ten respondents also wanted their partners to be present during the photovoice training, which followed immediately after informed consent was obtained. 
To build rapport and equalize the power between me and the participants, I introduced myself as someone who has led support groups for people with early stage dementia for many years, highlighting that what I had learned from the participants in these support groups led me to conceive of this study. I emphasized the fact that (1) they are the experts, not me; (2) I do not have the answers, they do; and (3) their personal and valuable experiences with the illness could be useful to others who are at the beginning of the journey, people with early-stage dementia and caregivers.

Sixteen of the 20 participants responded to this introduction by sharing a detailed account of their journey with the illness in a more or less chronological order. It was not unlike a floodgate waiting to be opened. Once unlatched, the flow of information, emotion, and detail was immense. It was as if the participants had been waiting to be acknowledged and respected for who and what they were and what they had experienced. Initially, I worried that these important details of the participants' illness experience were not audio recorded. It quickly became clear however, that it was crucial to allow the participant this opportunity and space to share their stories as part of the rapport building process. When the participants were asked to repeat what they had shared as part of their introduction after they had signed the consent and the audio recorder was running, the narratives had a different quality. They lacked the sense of urgency and raw emotion that had been present in the introductory stories and many significant pieces of information did not appear the second time around. To document the critical details that were shared in the initial stories, I spend time immediately after each interview writing detailed field notes. The phasic nature of the illness experience that emerged during the data analysis is in part based on the initial, unsolicited narratives. 
Following the rapport building phase of the screening interviews, I provided indepth information about the nature of this study, introduced the photovoice concept and methodology, and invited the potential participants to ask questions about the study and the photovoice method. After all their questions were answered, I asked them if they were still interested in participating in the study. All 20 potential participants responded positively and I followed the screening protocol to determine eligibility.

First, the potential participants were trained in the use of the camera with the following, step-by-step instructions. I demonstrated the use of the camera by taking a photograph with the practice camera and speaking aloud each step of the photo-taking procedure: (1) I look at the counter (I have e.g., 27 pictures available); (2) I look through the viewer; (3) I decide whether I would like to take a vertical or horizontal picture; (4) I hold the camera still; (5) I press the button; (6) I listen to the sound, whether it clicks; (7) I turn the wheel to forward the film to next frame; (8) I look at the counter (now I have e.g., 26 pictures available).

Next, I handed the practice camera to the potential participants who practiced taking a photograph while I repeated the instructions. When I observed that the potential participants struggled with taking the first practice photograph, I repeated the training protocol until I felt confident that they were able to use the camera with ease. Following this practice run of picture taking, I collected demographic information from the potential participants by reading verbatim questions from the demographic questionnaire and marking participants' responses on the form. In addition, I administered the Mini-Mental State Examination (MMSE) (Folstein, 1975). All participants scored within the required range on the measure (between 20 and 28). After gathering this information, I asked the 
potential participants to take a second practice picture and recite each step involved. The reason for sandwiching the filling out of the demographic questionnaire between instruction in the use of the camera and the second practice picture was to evaluate the participants' ability to retain simple information and successfully use the camera after a distraction. People in the early stages of dementia are able to retain basic instructions and are generally able to learn a simple, new skill such as using a camera; people in the later stages of the illness no longer have that capacity. All 20 potential participants successfully took a second practice picture and thus were eligible to participate in this study. However, one participant asked if she could to use her own camera because it was easier for her, which I allowed. A moderator guide was prepared to facilitate these screening sessions (appendix J).

Consent procedure. After eligibility was established through the screening protocol, informed consent was obtained. Informed consent is at the heart of ethical research. Where there is clear understanding about the cognitive abilities of potential participants, obtaining informed consent is relatively straightforward. The situation becomes more complicated in instances where the cognitive abilities of potential participants are unknown to the researcher. Consent of people with dementia is always required when it is still possible (Alzheimer's Association, 2004), but the boundaries of who is and who is no longer able to understand the information about the study and/or to make reasoned judgment about participation are unclear. In general, allowing a person with dementia to feel that his or her autonomy is being respected is ethically important (Kim, Cox, \& Caine, 2002), and false assumptions of incompetence may have harmful consequences. As such, an important issue when inviting people with early-stage 
dementia to participate in a research project is how to determine whether or not capacity to consent to research has been lost due to cognitive impairment. This is especially true since cognitive impairment alone does not necessarily predict loss of decisional capacity. In other words, "cognitive impairment is not always associated with the lack of capacity for informed consent to research" (Alzheimer's Association, 2004, p. 172).

In the midst of ongoing debate and the absence of national guidelines for researchers to follow when including people with cognitive impairment in research, various advocacy groups and research teams investigating informed consent procedures with this population have articulated recommendations that provide at least limited protection for a low-risk study such as this (Alzheimer's Association, 2004; Black, Kass, Fogarty, \& Rabins, 2007; Slaughter, Cole, Jennings, \& Reimer, 2007). Using these guidelines, I assessed the potential participants' capacity to consent to this study.

I verbally described the purpose of the study to minimize misinterpretation of study benefits (e.g., that participants will have the opportunity to tell their stories and share their life experiences and that they will receive an enlarged version of their favorite photograph as a token of appreciation). I assessed understanding of important information by asking unstructured, open-ended questions, (e.g., "Can you tell me what you think I am asking you to do?"). I also assessed the appropriateness of the responses to questions during the discussion of informed consent. I verbally described fully the potential participant's rights of voluntarism and withdrawal and stressed the option to include rest breaks or continue the interview on a different day. I assessed the ability to consider alternatives, including the option not to participate. I explicitly asked the potential participants if they were willing to take part in the study after the discussion of informed 
consent and assessed their ability to make a reasoned choice. I maintained active and ongoing monitoring of the potential participants' willingness to participate and attended to verbal and non-verbal dissent/assent. Objections of individuals with cognitive impairment will usually be expressed indirectly as frustration, discomfort, unhappiness or passivity. The interview would have been discontinued if this was detected. Using the above steps, all 20 potential participants demonstrated the capacity to consent to this study.

Next, I read the consent form aloud and answered additional questions. During this process, I summarized more complex sections to minimize misinterpretations including the risks, safeguards, and benefits to participation. I emphasized the voluntary nature of participation in the study and reminded the potential participants that if they decided to become involved, they could withdraw from the research at any time without giving a reason. All 20 potential participants agreed to take part in this study and signed the consent form. Once written informed consent was obtained, the session progressed into the opening phase of this study.

Opening phase. Administering quantitative measures. The first step in the opening phase was to administer the QOL-AD (Logsdon et al., 1999) and the GDS-short form (Sheikh \& Yesavage, 1986). For 14 of the 20 participants, administering these measures entailed reading verbatim questions from the questionnaires and marking the participants' responses on the form. Six of the 20 participants preferred to read and mark themselves. The next step in the opening phase was photovoice training.

Photovoice training sessions. The first step of the photovoice training entailed familiarizing the participants with the photo-taking theme. As mentioned earlier, for the 
purpose of the photo-taking assignment the concept of 'strengths and resources' was operationalized as the photo-taking theme provided earlier in the text. I introduced participants to the photo-taking theme by reading it aloud and inviting them to ask questions. To ensure that they indeed understood the photo-taking theme, I initiated a discussion about it. During this discussion, I asked the participants to explain the phototaking theme in their own words and develop some initial ideas on how they could take photographs that could best portray their perspective of the theme. This initial, collaboratively developed list of photo-taking ideas was recorded on the 'picture-taking plan' (discussed earlier in the text) and handed to the participants as a preliminary guide for the photo-taking activity. Next, the participants were handed a 'photo-taking toolkit' and introduced to each item in the kit via a step-by-step process. To that end, I brought a practice toolkit, which I opened at the same time as the participants opened their toolkits. I modeled the handling and use of each item in the kit while helping the participants to understand how to use each item in their own toolkit.

As part of the training process, I showed the participants a set of my own pictures on a very general topic. I included some 'bad' pictures in the set to (a) show the participants that 'mistakes happen' so that they would not think they have to be 'perfect' and (b) help them avoid making some of the same mistakes, so that there would be a larger set of usable photographs. I also explained that although a reliable disposable camera had been selected, there was always the possibility that the camera would not work properly and that all or some photographs might not come out well. In this context, I emphasized the benefits of keeping the photo-diary: how it would keep track of each photograph and how it could serve as a substitute for photographs that may not come out. 
I explained that keeping a photo-diary was part of the research protocol, but if they experienced difficulty recording their thoughts in written language, they were not required to submit their journal.

Finally, a discussion about the following topics was initiated: (1) What are respectful ways of taking photographs (e.g., photos of personally relevant items, animals in outdoor and indoor environments, and people after respectfully obtaining their permission prior to taking the photograph)? (2) What strategies can you employ to remember that you wanted to take photographs (e.g., writing notes, placing the phototaking toolkit in a conspicuous area)? (3) How can the researcher assist you in remembering to take photographs (e.g., reminder phone calls, printing large notes)? (4) Who else can assist you in remembering to take photographs (e.g., family members, professional caregivers, friends)? (5) What can you do when you forget how to use the camera (e.g., call the researcher, ask a family member, read the instructions)? (6) How many pictures can you take (e.g., each camera has 27 exposures)? At the end of the meeting, the following information was reviewed in a brief summary: (1) what is the photo-taking theme?; (2) how can I make use of the items in the 'photo-taking toolkit'; and (3) how can I contact the researcher?. Finally, I assisted the participants to find a strategically visible place for the photo-taking toolkit. Most people with dementia have what I refer to as a 'memory center' - a place in their house that contains everything they need to attend to on a daily basis. Assisting the participants in identifying how the phototaking toolkit could become part of this 'memory center' increased the likelihood that the photo-taking activity would be integrated into their daily routine. A moderator guide was prepared to facilitate these training sessions (appendix K). At this point, the study 
transitioned from the opening phase to the discovery phase.

Discovery phase. Photo-taking activity. During this phase, the participants implemented their reflections on the photo-taking theme by taking four photographs a day on seven consecutive days.

Researcher check-in phone calls. During these seven days I called the participants at regular intervals (every second day) to remind them of the project, assist them if necessary with any technical problems associated with the use of the camera, troubleshoot other difficulties, and/or answer questions that had come up. The participants could also contact me at any time if they had questions about the phototaking activity or how to operate the camera.

Camera pick-up. At the end of the active photo-taking phase, I met with 16 of the 20 participants a second time to pick up the camera and engage them in a dialogue about their photo-taking experience. Due to distance, four participants mailed me their cameras in a pre-addressed and stamped envelope and did not participate in an interview about their photo-taking experience. Picking up the camera marked the end of the 'discovery phase' of this study, and engaging the participants in an interview about their phototaking experience introduced the decoding phase.

Decoding phase. The 'decoding phase' of this study consisted of 'brief individual interviews' and 'in-depth individual interviews'. Creating an environment in which the participants felt safe and secure was crucial to the decoding phase of this study. My attempt to create a comfortable environment conducive to conversation entailed making 'interviewing' an unobtrusive process that occurred in the context of ordinary conversation. To that end, I outlined the interview process at the beginning of each 
interview, which included stating my research goals, confirming the participants' permission to audio-record the interviews, assuring the participants of confidentiality, inviting the participants to turn off the recorder or refuse to answer my questions if they felt uncomfortable, and encouraging them to ask questions.

The interviews were conducted in an interactive and conversational style where I clarified, paraphrased, and summarized what the participants had told me. I listened carefully to their responses while attending to verbal and non-verbal cues as well as contextual issues. My responses included active listening, empathetic reflections, and minimal encouragers, and the questions I posed were generally linked to the dialogue and sought to create an opportunity for the participants to elaborate their stories, and expand their responses.

Intrinsically, the relationship between researcher and research participants is imbalanced. The researcher's role as the investigator of the participants' lives unavoidably places the researcher in a position of power. Aware of these differences, I remained committed to reducing power imbalances and establishing a research environment that put the participants at ease and empowered them to share their thoughts. To that end, I continued rapport building throughout the duration of the study. While I did not expect power differences to disappear, I hoped that they would be diminished. This was particularly important because of the vulnerability of the study participants (people with cognitive impairment).

Brief individual interviews. The brief interviews were conducted at the time I picked up the cameras. I conducted a total of 16 brief individual interviews. The individual sessions ranged in duration, from 15 minutes to one hour. All but one were 
conducted at the participants' homes; one took place at the Alzheimer's Association. Three participants chose for their partners to be present during the brief interview.

During the interviews, the participants were encouraged to discuss the phototaking activity (their photo-taking strategies and processes) as well as the overall research procedures (their experiences with the photo-taking toolkit and each item in the kit). In terms of the research procedures, the interview questions focused on what had worked and what didn't and what should be changed as well as any recommendations for future participants. To ensure that all topics were covered, a moderator guide had been developed (appendix L). At the end of each dialogue session, I scheduled an appointment for an in-depth interview.

Immediately after each brief interview, two sets of the participants' photographs were developed (one for the participant to keep and one for me). The next step in the decoding phase entailed meeting with the participants for an in-depth dialogue about their photographs. In keeping with the constructivist paradigm, I did not preview any of the photographs prior to the meeting with the participants so that the process of sharing and telling and uncovering themes could occur in a naturalistic manner (Creswell, 2005).

In-depth individual photo interviews. Each participant took part in an in-depth, open-ended interview. The individual sessions ranged in duration, from one to four hours with the majority of interviews being 60-120 minutes long. Eighteen interviews took place at the participants' homes; one at the Alzheimer's Association; and one at a restaurant selected by the participant. Five participants chose for their partners to be present during the in-depth interview.

At the beginning of the in-depth interviews, I provided the participants with a 
sealed envelope containing a set of their photographs and invited them to open the envelope, review their photographs, and comment on their meaning. In line with participatory approaches to research that aim to produce non-hierarchical research relationships, each interview began with an open-ended question: "Tell me about your photographs."

The decision to rely on the participants' photographs to structure and thematically guide the interviewing process was grounded in the belief that this approach would facilitate a communication of ideas and encourage the participants to create their own narratives by piecing together personal memories, interpretations, and perspectives. Inviting the participants to describe their photographs in their own words established a sense of trust and encouraged them to introduce me to their world. In other words, the photographs provided the means by which the participants were empowered to decide what they wanted to share and to take the lead in discussing their experiences. This open approach permitted an unencumbered flow of ideas and information and put the participants at ease, which allowed them to share insights they may not have shared otherwise. It aided not only in understanding what they had tried to capture in the photographs but also went beyond the immediate image into a more general discussion of their experiences and personal values. However, although the interviews were openended, unstructured, and led by the participants, I made sure that all topics relevant to the research questions were covered. To that end a moderator guide had been developed (appendix M).

All participants were eager to open the envelopes containing the photographs. They could hardly wait to show their photographs to me and their partner (if present) and 
discuss the details captured in the images. The freedom to select which of their photographs to share allowed them to frame the situation, determine the order of topics to be discussed, and feel respected. All participants chose to share their entire portfolio and discussed the photographs in the order they were taken. After reviewing their photographs, the participants were invited to choose the two that most accurately reflected the photo-taking theme and provide a title for them: "What did you intend these two photographs to depict, and what title would you like to give them?" At the end of the session, the participants were asked whether they still would be interested in participating in a dyadic interview, given their experiences with this interview. All participants expressed interest in taking part in a dyadic interview. In fact, their responses were so enthusiastic that I had to make sure that they understood that because of geographic distances some might not be chosen. Next, the participants were invited to decide which two photographs they would like to discuss in a dyadic interview with another study participant if they were chosen. All participants chose the same two photographs they had selected previously.

Interpretation phase. Researcher-driven interpretation. Data Sources. There are a number of different data sources for this study, quantitative, verbal, and visual. The quantitative data was obtained through the demographic questionnaire, the MMSE, the QOL-AD, and the GDS-short form. The verbal data was collected during 39 interviews (36 individual interviews and three dyadic interviews). The visual data, comprised of 482 participant-generated photographs and associated diary entries, was collected after the photo-taking activity was complete. The number of photographs taken by each participant ranged from 11 to 29 photographs (mean 24.1) with the majority of the participants (13 
out of 20) taking between 25 and 29 photographs. From the remaining seven participants, five took between 20 and 24 photographs, one participant took 14 photographs and one participant took 11 photographs.

Qualitative data analysis. To answer this study's three research questions, grounded theory methods (Charmaz, 2006; Corbin \& Strauss, 1990, 2008; Glaser, 1978; Glaser \& Strauss, 1967; Strauss \& Corbin, 1998) were used for the analysis and interpretation of the verbal and visual data. Presenting the steps involved in conducting data analysis and the manner in which I integrated these steps to achieve a level of abstraction (in other words, develop or discover theory) is a difficult task. For reasons of clarity, the data analysis process is discussed in a relatively linear fashion. However, the limitation inherent in this form of presentation is the activity of analyzing was far more organic and interactive than it is possible to present here.

The amount of data collected during the project (photographs, photo-diary entries, field notes, transcripts of the dialogue sessions, and memos) was vast. To avoid becoming overwhelmed, I devised an organizational process for handling the data (Miles \& Huberman, 1994). For example, during each dialogue session I made sure all photographs were labeled to correspond with the participant, the relevant photo-diary entries, and the recording of the dialogue session. Immediately after each dialogue session I made an entry in my 'project journal' and recorded my overall impressions of the session, peculiarities I had noted, and self-reflective observations. For example, I noted the emotions that came up during the interview, how I felt about my interviewing style, and what worked and what didn't work in terms of building rapport.

With permission of the participants all dialogue sessions were audio-recorded. I 
listened to the audio-recording of each dialogue session as soon as I was able to and started the process of transcribing, which transforms the audio-recording to 'raw data' for the analysis (Sandelowski, 1995). Familiarizing myself with the data by transcribing the audio-recordings myself allowed me to 're-live' the dialogue sessions and insert any observations I had made during the sessions as well as those that became apparent when listening to the audio-recordings (e.g., body language, emotions expressed, or voice inflections) (Miles \& Huberman, 1994). Later, when listening to the audio-recordings a second time, I evaluated the transcripts for accuracy by comparing the audio-recordings with the written transcripts. If necessary, the transcripts were corrected. I also created power point presentations from each participant's visual and written contributions by scanning each participant's photographs into my computer. I then added the corresponding photo-diary entries and highlighted the two photographs that the participants had identified as most important and that they wanted to use if they were chosen to participate in the member checking part of this study. Sorting the photographs and photo-diary entries in this manner allowed me to review each participant's contributions as a cohesive whole. Another part of this intra-case analysis involved developing a detailed description of each participant, summarizing my overall impressions and reflections (appendix T).

Being a 'hands-on' person I decided to work with both hardcopies and electronic versions of the data. Having both types of data available increased the data's accessibility and made it easier for me to immerse myself in it. The hardcopies of the data were organized in ring binders and stored securely in my home office; the electronic versions of the data were kept in my password-protected home computer. To enhance the 
efficiency of data storage and retrieval, the overall project was managed using Atlas TI. As mentioned earlier, one tenet of grounded theory is that the processes of data collection and analysis are interrelated and cyclical in that each influences the other. Thus, the data analysis started at the same time as the data collection. In fact, as discussed earlier, the first part of the process happened during the dialogue sessions when the participants reviewed their photographs. After all visual data was collected I organized the photographs into separate stacks following my general sense of 'look alike, feel alike'. After sorting the photographs into several stacks, I looked through each stack, decided on a preliminary rule of inclusion and assigned a preliminary concept name to each stack. I decided to use a mixture of 'in vivo codes' and paraphrases from the titles and photo-diary entries associated with each photograph that captured the overall sense of a particular stack of photographs. During the next step, I created a separate electronic poster presentation from each stack and titled it with the preliminary concept name. Sorting the photographs and photo-diary entries in this manner allowed me to review all visual contributions as a cohesive whole.

After reviewing each participant's visual and verbal data several times, I continued the data analysis with 'open coding', a process that seeks to identify, name, and categorize key concepts contained within the data (Corbin \& Strauss, 2008). In other words, 'open coding', also known as 'line-by-line coding', entails the discovery and labeling of concepts (also referred to as categories or themes) and their associated subconcepts relevant to the phenomenon under study. 'Open coding' scrutinizes the raw data; it explores the data, breaks the data apart, and yields numerous initial concepts that describe sections of raw data. I labeled these initial concepts using the participants' words 
(in-vivo codes) or my own terminology.

Strauss (1987) suggests adhering to a 'coding paradigm' when analyzing the data. He suggests "to code data for relevance to whatever phenomena are referenced by a given category, for the following: conditions, interaction among the actors, strategies and tactics, and consequences" (p. 27). In other words, when coding the data, I kept asking questions such as who, what, when, where, how, and with what consequences (Corbin \& Strauss, 2008). At times, pieces of data were given several codes reflecting as many concepts.

As I tried to remain as open as possible to what may emerge from the process of 'line-by-line coding', I generated a long list of initial codes. To better discover the processes hidden in the data, I followed Charmaz' (2006) recommendation and used gerunds when labeling data segments. Nevertheless, a few of these initial codes were rather descriptive and, in some cases, somewhat repetitive. As I continued data collection and analysis and constantly compared the evolving concepts with each other and the raw data, this list grew significantly smaller. Over time, a number of the earlier descriptive codes were integrated into newer conceptual codes, with only a small number of codes comprising the final conceptual coding list.

While still engaged in the process of 'open coding', I started consciously looking for 'core concepts' and as the process of 'open coding' continued, 'axial coding' emerged. 'Axial coding' aims at identifying the 'core concepts' in the study. Although 'axial coding' uses the results of 'open coding', more concepts can be identified through continued 'open coding' after the 'axial coding' has begun. In other words, 'axial coding' involved a regrouping of the data in which I used the open coding concepts to look for 
more analytic concepts to emerge. Later, 'axial coding' entailed intensive analysis around one identified core concept at a time. To that end, I returned to the raw data and started looking for and naming sub-concepts around each identified core concept (i.e., causal conditions, strategies, and intervening conditions). In other words, 'axial coding' reassembled the data through the connections that were made between concepts.

Building on the results of 'open coding' and 'axial coding' I started the process of 'selective coding' (Corbin \& Strauss, 2008; Glaser, 1978; Strauss, 1987). 'Selective coding' seeks to identify the central or core concept in the study: the one that organizes the other concepts; the one that all other concepts relate to. In this process some of the axial concepts were restructured as aspects of a more general concept. In Strauss' (1987) words, the researcher "delimits coding to only those codes that relate to the core code in sufficiently significant ways as to be used in a parsimonious theory" (p. 33). In this process, I took the initial, visual model and started to develop propositions that explain how the different concepts in the model relate to each other. Selective coding allowed me to identify the story behind the interplay between the concepts (the 'grounded theory') through clarifying and confirming the relationships between core and supporting concepts, including their properties, dimensions, and conditions. I adopted an inquiring attitude in reviewing the transcripts as I asked myself "What is going on here? What is happening here?"

Grounded theory relies heavily on what is known as 'constant comparison' (Glaser \& Strauss, 1967), a process in which "each incident in the data is compared with other incidents for similarities and differences" (Corbin \& Strauss 2008). As incoming information is compared to emerging concepts, it is increasingly possible to differentiate 
between one concept and another, recognize properties and dimensions relevant to each concept, and elevate common features to abstract representation. In line with the purpose of 'constant comparison', I used this process to investigate whether or not the data supported and continued to support the emerging concepts. At the same time, using 'constant comparison' allowed me to further build, substantiate, and refine the emerging concepts by clarifying their properties and dimensions. Data analysis continued until the emerging concepts accounted for all instances in the data (negative case analysis).

The central strength that the participants brought to the process of coping with and adapting to early-stage dementia, 'striving towards well-being while acknowledging the inconceivable notion of losing one's cognitive abilities', became apparent through examining the relationship among the conceptual categories (Glaser \& Strauss, 1967). When I felt relatively confident with the emerging framework, I began analyzing the data collected through quantitative measures (for a detailed description see the next section). The finding that four of the 20 participants had scores on the geriatric depression scale (Sheikh \& Yesavage, 1986) indicative of depression led me to investigate this aspect closely. I invited two of the four participants to take part in a dyadic interview and went back to the raw data and coded for incidents related to this particular facet. Ultimately, this step of the analysis led to a refinement of the core construct as significant process dimensions surfaced. I investigated these process dimensions more closely during the member-checking part of this study (dyadic interviews).

The entire data analysis process was documented through and guided by memoing, "a process in which the researcher writes down ideas about the evolving theory throughout the process of open, axial, and selective coding" (Creswell, 2007, p. 67). 
Memos (also referred to as notes) are written from the descriptive to the theoretical and later sorted to facilitate the process of forming grounded theory. "While the memo stage begins during the joint collection, coding, and analyzing the data, and peaks as coding saturates, it never is over" (Glaser, 1978, p. 88). As recommended by Straus and Corbin (1998), I started the process of writing memos early on in the research process and continued throughout the project. I wrote three different types of memos: code notes, theoretical notes, and operational notes. In my 'code notes' I recorded the code labels and their meanings so that I would have a clear understanding of what I meant by the codes that I used in the analysis. My 'theoretical notes' varied in length and covered a variety of topics such as reflections on the dimensions and deeper meanings of the emerging concepts, the relationships among the concepts, and theoretical ideas. To be able to organize these thoughts later, I created a new document for each thought. My 'operational notes' dealt primarily with methodological issues. Some drew attention to data collection circumstances relevant to understanding the data later; others consisted of notes directing future data collection. Thoughts calling for writing memos occurred as I listened to the audio-recordings, browsed through the photographs and photo-diary entries, read through the transcripts, or discussed my project with others. Over time, I developed the habit of writing down my thoughts as soon as possible so that elusive and hard to define concepts and ideas would not escape me.

Alternating data collection with conceptual memoing prevented me from collecting redundant data. Once a concept was 'saturated' (in other words, no new dimensions or properties were emerging), I ceased collecting additional data pertaining to that particular concept. In this way 'constant comparison' shaped and guided further data 
collection (Glaser \& Strauss, 1967; Corbin \& Strauss 2008).

Quantitative data analysis. All data obtained through the demographic questionnaire, the Mini-Mental State Examination (MMSE), the Quality of Life-AD measure (QOL-AD), and the Geriatric Depression Scale-short form (GDS-short form) were entered into SPSS Statistics version 19. To prepare the quantitative data, I developed a coding key for each tool. As discussed earlier, the MMSE instrument served to determine eligibility for this study. The MMSE instrument contains 11 components with sub-questions for a total of 30 questions. Each participant's response was scored as either correct or incorrect, depending on the answer; the MMSE total score reflects the number of correct responses. The QOL-AD instrument asks each participant to answer 13 questions (e.g., "Do you think your energy level is...?") related to quality of life on a scale of one to four (poor, fair, good, excellent). Possible scores on the QOL-AD range from 0 to 52 with higher scores indicating greater quality of life. A mean quality of life score was then created from the participants' responses, adjusting for non-responses to the question about 'marriage' for unmarried participants. The GDS-short form is a 15item self-report questionnaire designed to identify depressive symptoms in older adults. Ratings are made in a yes/no format (Sheikh \& Yesavage, 1986). Specific items on the GDS-short form were reverse coded: ten of the questions were 'negatively' worded such as 'do you feel that your life is empty?', five questions were worded positively (satisfied, good spirits, happy, wonderful to be alive, full of energy). A summed mood score was then created. In line with Sheikh and Yesavage's (1986) recommendations, participants scoring higher than five on the mood scale were categorized as 'depressed'.

Participant-driven interpretation. Dyadic interviews (Morgan, Ataie, Carder, \& 
Hoffmann, in press) were used as a means of member checking and to further develop the emerging findings.

Assembling the dyads. The process of assembling the dyads was guided by the ongoing theory development (theoretical sampling), direct clinical practice experience in the field of dementia, knowledge of related literature, and familiarity with the participants gained during the earlier phase of the project. The criteria considered in composing the dyads were gender, coping styles, interests, level of education, living alone or with a partner, time of diagnosis, and most significantly, level of functioning. In other words, I adopted the guidance provided in the literature on focus groups (Morgan, 1996) in that the dyad members were of the same gender and had similar characteristics. The primary aim of the pairing process was to create homogenous pairs because the sense of 'sameness' (the quality of being similar) would allow the participants to capitalize on their shared experiences.

In line with the participatory underpinnings of this study, the collaborative analysis sought out alternative explanations to those I had constructed. To develop a better understanding of the process dimensions of the core construct, dyad three was composed of two participants who had scores on the geriatric depression scale that were indicative of depression and yet had constructed life-affirming portfolios and reported positive coping strategies. I also aimed to compose the dyads in a way that would be beneficial for the participants. Familiarity with the participants and their characteristics gained during the earlier phases of the research project allowed me to compose dyads in a way that was stimulating for the participants. However, the pairing process was challenging and somewhat influenced by the proximity of participants to each other. The 
recruitment area for this study was geographically spread and participants lived in locations that were up to 200 miles apart.

A total of three dyads were formed; one was composed of men and two of women. Dyad one was composed of two men (Duncan, age 72 and Karl, age 82) who lived with their wives. Both men were diagnosed with early-stage dementia after they had retired and knew each other and me from attending a monthly support group. The sense of familiarity allowed the two men to bridge a difference in socioeconomic status and level of education (bachelor's degree in chemistry vs. physician). Dyad two consisted of two women (Corinne, age 69 and Tiffany, age 61), one holding a bachelor's degree in finance, the other a master's degree in nursing. Each attended a different support group for people with the illness. Although different in a number of ways (one woman was married and lived with her husband, the other was divorced and lived alone; one was diagnosed with early-stage dementia a few years after she had retired; the other was forced to retire as a result of the illness), both participants were fun-loving and outgoing, enjoyed traveling, and found meaning in educating others about the illness. Dyad three was made up of two women (Monica, age 59 and Kathryn, age 64) who were divorced and had lived alone for most of their adult lives. Both women were pushed into retirement by the symptoms of the illness. One of the women was a retired accountant, the other a retired mental health counselor. Both women were similar in that they enjoyed social interactions but experienced a lack of opportunities to engage in meaningful relationships. Both women had scores indicative of depression on the GDS-short. Dyadic interviews. In preparation for the dyadic interviews, I created two power point presentations for each participant. One captured the participant's entire contribution 
(their photographs and the corresponding photo-diary entries); the second showed the two photographs (and titles) that each participant had selected for sharing during the dyadic interview. To re-familiarize the participants with the overall project and help them prepare for the dyadic interview session, a hardcopy of these presentations was mailed to the participants prior to the dyadic interviews.

The dyadic interviews were held at three different sites. Dyad one met at a local church where both participants attend a monthly support group; dyad two met at the Alzheimer's Association where one of the participants volunteers her time; dyad three used one of the participant's homes as the meeting place. One of the characteristics of early-stage dementia is insecurity and disorientation in unfamiliar surroundings. Therefore, after introducing the members of the dyads to each other, I familiarized the participants with the meeting venue, pointing out the location of the restrooms. I then facilitated the settling-in process by offering refreshments and providing clear directions in terms of seating arrangements. To create a sense of privacy, I seated the participants facing each other with me as the moderator sitting at the head of the triangle, facing the laptop that would be used to view the participants' power points. This seating arrangement had the advantage that, while explaining their photographs shown on the laptop, the participants could, with a slight turn of the head, establish eye contact easily with each other but establishing eye contact with the moderator was much more difficult.

At the beginning of each dyadic interview I clarified my role and the purpose of the session; I explained the reason for composing the specific dyad in terms of similarities and differences and highlighted my interest in individual opinions. Finally, I reviewed the ground rules for the session and encouraged the dyad to talk to each other, 
not to me. To provide the context for the participants to talk about their experiences with early-stage dementia, I attempted to create an atmosphere that encouraged them to tell their stories captured by their photographs. To that end, I started each session by reviewing who I am and what brought me to be interested in their experiences and viewpoints.

To set the stage for the collaborative analysis, each interview started with a slide show of two photographs (and their corresponding titles) that the participants had chosen for this occasion, continued with a discussion of these images, and eventually progressed into a dialogue about the provisional analytic framework. Viewing their contributions on a 17 inch laptop served as an icebreaker and made it easier for the participants to decide who would start with sharing their photographs. In addition, the visually appealing presentation opened up a way for the members of the dyads to enter into an open exchange about their individual experiences, agreeing on some issues and disagreeing on others.

Next, I showed a visual representation of the emerging analytic framework to the dyads. This was in the form of 6 poster-boards showing the categories and sub-categories of the preliminary framework (represented by a selection of participant-generated photographs) positioned around the room (appendix U 1-6). After giving the dyads time to walk around the room and look at each poster-board, I explained in lay language how the categories and sub-categories relate to each other by describing the overall model of the findings. This presentation was conducted in a way that would not reinforce negative self-evaluations. I then invited the dyad members to share their reactions to the preliminary framework, comment on how it relates to their experiences, and voice any 
further reflections, comments, and suggestions. To facilitate this discussion, the dyad members were given a handout listing the associated and relevant categories and subcategories as a reminder.

As expected, the participants presented with some communication difficulties related to dementia. To overcome the barriers posed by these language difficulties, I conducted the dyadic interviews in an interactive and conversational style. I simplified my language and supplemented my speech content with orienting names or labels, such as 'your daughter Kim' to increase the likelihood that the participants understood what was said. However, I made an explicit effort that my use of simple language did not cause the participants to feel threatened, undermined, or confused.

Clarifying, paraphrasing, and summarizing in regular intervals what the participants had told each other assisted them with keeping the thread of the conversation and allowed me to verify that what I had understood was indeed what the participants had meant. Another useful skill was to be patient. Being patient was beneficial in two ways. It supported a flow of dialogue as it gave ample time for the participants to process what had been said and to formulate their responses. It also encouraged the participants to be patient with each other and validated their contributions. When the participants lost the thread of the conversation, I restated in a calm, gentle, and matter-of-fact way what had been said. During the sessions, I wrote down key points of the discussion and noted relevant observations (e.g., body language).

\section{Strategies for Ensuring Trustworthiness}

While the quality of quantitative research is evaluated on the basis of 'internal validity', 'external validity', 'reliability', and 'objectivity', Lincoln and Guba (1985) 
maintain that the quality of qualitative research should be evaluated on the basis of its 'trustworthiness'. The criteria for 'trustworthiness' are derived from the principles of the naturalistic paradigm (generally associated with qualitative research), which are different from the principles of the positivist paradigm (generally associated with quantitative research). The principles underlying naturalistic inquiry include (1) there are multiple, ever-changing interpretations and constructions of reality; (2) human behavior is contextbound and, as such, context-free generalizations are not possible; (3) thick and detailed descriptions are necessary for the reader to determine whether the findings are indeed transferable from one setting to another; (4) causality is multi-directional and multifactored; (5) it is impossible for researchers to be objective.

Lincoln and Guba (1985) and Rappaport (1992) assert that qualitative research should be evaluated using criteria that reflect these assumptions. They propose 'trustworthiness' should be assessed using the following criteria: credibility, transferability, dependability, and confirmability. Shenton (2004)) elaborated on Lincoln and Guba's (1985) earlier work by discussing these criteria in detail and proposing strategies that researchers can utilize to meet them. The following describes these criteria and how I addressed them in this study.

Credibility. The following provisions were made to promote confidence that the findings of this study are congruent with the reality that the participants portrayed.

Prolonged engagement. Lincoln and Guba (1985) maintain that ensuring credibility is an important factor in establishing trustworthiness. They recommend 'prolonged engagements' to establish a relationship of trust between the researcher and the participants for the former to gain an in-depth understanding of the population under 
study. I am familiar with the population under study from my work as a clinician and as a support group facilitator for people with early-stage dementia. Additionally, I personally collected all of the data for this study, transcribed the interview recordings, and analyzed the data. Furthermore, data collection entailed several interactions with each participant. I met each participant more than once with the following frequencies: six participants, four times; ten participants, three times; and four participants, twice. In addition, I spoke with each participant at least three times on the phone. Furthermore, five of the twenty participants were longtime members (five years) of a support group I facilitate.

To ensure openness and willingness to share, I reminded the participants often that their participation is voluntary and that they can withdraw from the project at any time without offering an explanation. In addition, I made ongoing efforts to establish rapport. In the beginning of each interaction with participants, I explained that there are no right or wrong answers to the questions they will be asked and stressed that I was interested in hearing their views and opinions.

The sum of these actions and interactions fostered a sense of familiarity between me and the participants, created an environment of trust, and increased the likelihood for me to properly identify and verify recurrent patterns in the data. In addition, I immersed myself in the verbal and visual data to see if my categories, themes, constructs, interpretations, and conclusions not only captured the nature of what I wanted to understand but indeed reflected what the participants had told me.

Triangulation. In this study multiple methods of data collection were used to provide in-depth understanding of the experience of the participants and compensate for the individual limitations of each data collection method. Using different sources of 
information (quantitative, verbal, and visual) allowed for innovative ways of understanding the phenomenon under study and increased the confidence in the findings. For example, the study used two types of interview formats for collecting verbal data, individual and dyadic. While individual and dyadic interviews have similar methodological shortcomings (both are in essence interviews), they each have particular strengths. Individual interviews provided in-depth information about the way the participants coped with and adapted to early-stage dementia while dyadic interviews (based on the notion of 'sharing and comparing') allowed me to develop an understanding of the sources of consensus and diversity in the coping response (breadth). In addition to verbal data, there was visual data. Arranging and rearranging the visual data (the participant-generated photographs) graphically and spatially facilitated a better understanding of the data as a whole.

Reflexivity. Trustworthiness demands heightened self-awareness as well as reflecting on events, before, during, and after (reflexivity). Reflexivity in the context of qualitative research is often linked to discussions of skill, perception, and introspection. In this study, I made continuous efforts to be respectful of and attend to the ways that the participants made meaning of their lives, which, in many instances, was different from my own experiences or ideas. Specifically, I used reflexive journaling throughout the research process so I would become aware of any feelings or thoughts (bias) that arose as a result of my interactions with the research participants and the literature. The following is a description of how reflexivity was used to increase the trustworthiness. As a clinician I treasure the notion that interviews do not just elicit stories already known, but often contribute to the construction of new narratives with a different perspective, which, in 
many ways, posed a dilemma for me as a researcher. One of the challenges during the interviews was to manage my positionality as a therapist and address those moments during which I felt drawn to follow up with feedback and/or questions to facilitate growth. Exploring these dynamics more closely during journaling, I discovered interesting patterns and connections.

My approach to research is rooted in the understanding and view that life is entirely interconnected and as such, I acknowledge the inherent interactive quality of interviewing. However, when reflecting on some of the discomfort I had experienced during the pilot interviews it became apparent to me that I had unconsciously embraced certain traditional research conventions. Even though intellectually I embraced the notion of subjectivity in the research process, in the actual 'doing research' I found myself striving for objectivity that I know does not exist.

During the pilot for this study, I adhered completely to my interview guides and used probes and follow-up questions only to elicit details or introduce other topics. As a result, there was relatively little interaction between me and the participants in the pilot. I often felt awkward in maintaining this position, but forced myself to stay 'in role' and allowed myself only to respond in a neutral or affirmative fashion to the participants' stories such as using affirmative utterances, i.e., uh-huh, mm-hmm, yeah. Somehow I was convinced that I had to do this so that the participants, their insights, and voices would remain the focus of the study. Trained in taking a non-judgmental stance rooted in respect for my clients, I was able to create a climate and space where the participants felt free and open to share their experiences and feelings during the pilot interviews. As they shared their joys, struggles, and fears, I empathized but suppressed my inclination to provide 
support and reassurance. Consequently, I kept many of my observations to myself in order to maintain my role as a researcher and missed some opportunities to 'dig deeper'. Struggling with my role as a researcher reminded me of the difficulties I had during my first years as a clinician.

Upon reflection, I realized that I had approached the pilot interviews the way I did out of a genuine concern for basic social work values. I wanted to respect the participants' autonomy and self-determination. As I struggled to accept that who I am as a person and a social worker will always be a part of my role as a researcher, I came to understand that the deeper reason for choosing photovoice methodology was that it looks at the research experience itself as a powerful change agent, which is in line with my goals as a social worker. As a result of these reflections, it became less and less appealing to treat the participants as 'passive objects' and consequently, during the next rounds of interviewing, I regarded the participants more clearly as partners in the dialogue. I made every attempt to respect the participants and to be thoughtful and sensitive about their needs and their life world. The power of this empathetic, validating, and empowering stance created a positive context for the exploration and construction of their experiences. As I progressed through the different stages of this study, I became intrigued with how grounded theory allowed me to tease out patterns of relationships from a large body of visual and verbal data. At times, the process of 'doing grounded theory' was mentally and emotionally draining. What made conceptualizing the findings especially difficult was that what emerged from the data was somewhat at odds with the prevailing frameworks of coping in the context of life-threatening illness. I learned to appreciate that 'doing grounded theory' is a multidimensional and complex process that benefits from 
contemplation, daily notebook writing, collegial exchange, and mentoring, all of which helped me to develop confidence in the emerging findings.

Member checking. Eliciting feedback from the participants on the preliminary analytic framework and verifying emerging patterns was another measure used to increase the credibility of this study. Lincoln and Guba (1985) view member checking as a critical component in ensuring credibility. The purpose of member checking is to ensure that the research findings accurately reflect the participants' perceptions and perspectives. In this study, the emerging findings were shared with six of the participants during the dyadic interviews to check whether my interpretation of their accounts indeed portray their experiences. The participants' commentary and feedback was integrated into the study findings and added richness to the final report. In other words, I used the contributions of the dyads to revise, refine, and validate the emerging framework. For example, one dyad felt that the initial categories forming the core concept 'identity' were too static. The core concept 'rebuilding identity' now emphasizes motion, process, and possibility.

Transferability and Confirmability. In qualitative research it is challenging to demonstrate that the findings and conclusions of one study are pertinent to other situations or populations. Lincoln and Guba (1985) suggest that it is essential for the researcher to provide the reader with enough contextual information that they can make such a transfer. Detailed and thick descriptions of the research design and its implementation, the specifics of data collection and analysis, and a reflective evaluation of the overall project were provided to allow the readers to determine whether the findings from this study are applicable to their specific situation. Comprehensive 
descriptions of the participants allow the reader to compare the study sample to other people with early-stage dementia. In addition, I made special efforts to include the viewpoints of all participants in the findings (Berg, 2007) and examined the results of this study in the context of previous research, which allows the reader to relate the findings of this study to the existing body of knowledge.

Dependability. Lincoln and Guba (1985) highlight the close connection between credibility and dependability. They argue that showing credibility to some extent will enhance dependability and recommend various audit strategies to confirm the findings. Since the researcher's perspective is naturally biased due to his or her close relationship with the participants, the data, and the methods, it is recommended to use a 'peer debriefer' who can offer a valuable second opinion on the meaning of the data, the proposed categories and sub-categories, and the emerging interpretive framework. In this study, I actively sought and welcomed opportunities for feedback on the research project from colleagues and peers. The peer debriefers critiqued the overall study process, challenged assumptions about the data, and provided feedback on how my biases influenced the overall development of the interpretive model.

During the three-year long period of data collection and analysis, I allowed my work to be evaluated by others familiar with qualitative methods. To that end, I met regularly with three fellow Ph.D. students to elicit their feedback on my overall data analysis process and my analytical decisions. I spent two seven-day retreats and had several half-day meetings with a member from my Ph.D. cohort from PSU scrutinizing each other's work. I also met with a fellow cohort from Hartford, a Ph.D. student from NYU, four times at gerontological conferences where we shared a room for the purpose 
of critiquing each other's work and presenting joint papers. Because she was in New York we also had extensive phone conversations for the specific purpose of verification and critique. In addition, I met several times with another fellow cohort from Hartford, a $\mathrm{Ph} . \mathrm{D}$ student from the UW, to present my analysis and ensure analytic rigor. I kept notes during these meetings and whenever appropriate incorporated the peer reviewers' feedback into the ongoing analysis. Preliminary feedback from members of my dissertation committee was also elicited.

\section{Ethical Considerations}

Ethical research practice requires that the researcher creates knowledge that has meaning for and is of importance to the researched population. It asks for an in-depth understanding of the perspectives, needs, and wishes of the participants and requires effort to identify all stakeholders early in the planning phase (Sieber, 1992). As mentioned earlier, I am familiar with the population under study as I have facilitated educational support groups for people with early-stage dementia for the past several years. In addition, as a hospice social worker, I have been exposed to the daily activities and environments of the population under study. The research questions for this study were developed as a direct result of these experiences in combination with the knowledge gained through the literature review.

The ethical issues that may arise in research with people with dementia center on the relationship with the participants. Measures to respect the participants' right to privacy included (1) making the participants aware that they could refuse to answer any questions; (2) providing them with the option to view their photographs alone prior to showing them to me; (3) refraining from probing and asking for further explanation or 
clarification during the interviews if I observed signs of unease, and (4) conducting the interviews at a location of their choice at a time that was convenient for them.

Confidentiality was upheld by ensuring that all records pertaining to the study were kept in a locked file cabinet and a password protected computer accessible only to me. Anonymity was preserved by assigning fictitious names to the participants and not revealing the identity of participants quoted when presenting the findings. Finally, the participants were informed that the direct benefits from participating in this study were minimal as was any potential harm that might result from their participation.

Ethical research requires the researcher to think carefully before entering into multiple relationships with any person. The major ethical concern related to multiple role relationships is that unintentional harm, risk, or exploitation can be potentially experienced by the participant in the research. In this context, the approach to recruitment is an important element in assuring voluntariness. In particular, how, when, and where participants are approached, and who recruits them are important elements.

To avoid ethical dilemmas that may arise from playing two roles, i.e., researcher with a pre-existing relationship as a support group leader, I decided not to personally recruit participants for my study and did not announce the study at my work or any support groups that I facilitated. I did not want to use my influence as a social work professional or volunteer support group facilitator to impact my clients' or support group member's decision about participation in this research. Instead, I relied on the Alzheimer's Association and the Northwest Research Group on Aging to distribute the information about my study to the members of their mailing lists.

I noticed that five of the 27 respondents were members of an ongoing support 
group that I was facilitating at the time. Two of the five used the interest card to express their interest and initially did not realize that the researcher of the study was their support group facilitator. During the screening and recruitment phone call I made them aware that I was their support group facilitator, which was a great surprise to them. Three of the five were made aware that I was their support group facilitator by their care partners when they voiced interest in the study to their care partners. Initially, I considered omitting these five participants in my study sample, but after consulting with social work colleagues it became clear that the risk of harm by denying participation was greater than including them in the study.

Although I did not expect for my dual role to have an adverse effect on these participants, I made special effort to ensure that they understood that their participation in the study was voluntary and that their decision to participate or not would not impact their continued membership in the support group. I also paid special attention to the ethical issues of confidentiality and privacy when obtaining informed consent from these five respondents. I emphasized that I would not share any information contributed by them during the research project with other support group members. In addition, at the beginning of each data collection event, I reiterated the importance of confidentiality and asked the participants not to discuss our meeting with other support group members.

In chapter 4 an overview of the research methods, design, and processes that were used in this study was provided. The next chapter presents the participants in this study and the key findings that emerged when analyzing their visual and verbal contributions (photographs, photo-diary entries, and individual and dyadic interview transcripts) using grounded theory. 


\section{CHAPTER 5}

\section{FINDINGS}

This study sought to reveal what people with early-stage dementia perceive to be their strengths and resources and how they use these abilities and assets in the process of coping with and adapting to their illness. In answering these questions a model of adjustment to early-stage dementia emerged in which the different strengths and resources that the participants identified are inextricably linked to each aspect of this model. The remainder of this chapter will first introduce the participants of this study and then provide a general overview of the proposed model, explaining each component and how they are linked to each other. Each component will be introduced in detail and in a manner that is grounded in the voice of the participants. Given the amount of content in the findings, a discussion will follow each major section. Next, the quantitative findings are discussed and their relationship with the qualitative findings will be presented. Lastly, methodological findings related to photovoice and dyadic interviews in this study will be presented.

\section{Snapshot of the Participants}

Thirteen women and seven men, age 57 to 90 (mean 73.4) took part in this study. All participants lived in the community and their primary residences were in one of the following counties of Washington State: Clallam, King, Kitsap, Lewis, Pierce, and Snohomish. Each participant had a diagnosis suggestive of early-stage dementia with mini-mental state examination scores (MMSE) (Folstein et al., 1975) ranging from 20 to 28 (mean 25.6). Scores on the quality of life measure (QOL-AD) (Logsdon et al., 1999) ranged from 29 to 48 (mean 33.5) for those participants who, at the time of this study, 
lived with their spouse and from 24 to 41 (mean 40.6) for those who did not, indicating relatively high levels of quality of life. The ratings for the individual questions on the scale ranged from 2 to 4 with a mean of 3.1 for those participants who were married and 2.7 for those who were not, indicating slightly lower levels of quality of life for those who were not married. Responses on the question 'life as a whole' on the quality of lifeAlzheimer's disease scale (QOL-AD) (Logsdon et al., 1999) ranged from fair to excellent. Eleven participants rated the quality of their life as a whole as good; six rated it excellent; three rated it as fair. Scores on the geriatric depression scale (GDS), short form (Sheikh \& Yesavage, 1986) ranged from 0 to 12 (mean 3.55), with the majority of the participants (70\%) scoring 3 or less indicating normal mood. Four of the 20 participants (30\%) had scores indicative of depressive mood, ranging from 8 to 12 .

The context of the participants' lives was important background to understanding the losses they had experienced and the strengths and resources they brought to the process of learning to live with the illness. The study attracted almost exclusively Caucasian Americans (only one participant was of African American descent), and the overwhelming majority of participants were well educated (all but one participant held degrees beyond high school) and reported adequate financial resources (only one participant disclosed financial difficulties due to the onset of early-stage dementia). Nine of the 20 participants lived on their own in the community and thus had to negotiate many aspects of daily life, from the simple to the complex. Nine participants lived with their spouses; one lived with her daughter, and one with her sister. At the time of this study, the participants had lived with memory problems and the knowledge of their illness for a number of years (approximately 3 to 6 years). Although the symptoms of the 
illness had increased, the acute awareness of and focus on their illness had quieted. The disruption from frequent medical appointments and cognitive testing had abated for all of them.

\section{Table 1}

'Sample Demographics ‘

\begin{tabular}{|c|c|c|c|c|c|c|c|c|}
\hline Name & Age & $\begin{array}{l}\text { MMSE } \\
\text { Scores }\end{array}$ & $\begin{array}{c}\text { GDS } \\
\text { Scores }\end{array}$ & QOL & $\begin{array}{c}\text { Diagnosis type } \\
(\mathrm{dx})\end{array}$ & $\begin{array}{c}\text { Years } \\
\text { since } d x\end{array}$ & $\begin{array}{c}\text { Marital } \\
\text { status }\end{array}$ & $\begin{array}{l}\text { Lived } \\
\text { alone }\end{array}$ \\
\hline Chester & 66 & 21 & 1 & excellent & early-onset AD & 6 & married & no \\
\hline Corinne & 69 & 26 & 2 & excellent & early AD & 5 & married & no \\
\hline Duncan & 72 & 26 & 1 & excellent & early-stage AD & 6 & married & no \\
\hline Fanny & 78 & 25 & 3 & good & Alzheimer's & 5 & married & no \\
\hline Fay & 75 & 24 & 1 & excellent & early-stage AD & 5 & widowed & no \\
\hline Frank & 82 & 26 & 3 & good & $\begin{array}{c}\text { early symptoms } \\
\text { of } A D\end{array}$ & 5 & married & no \\
\hline Irene & 57 & 20 & 2 & good & early-onset AD & 6 & single & yes \\
\hline Karl & 82 & 25 & 4 & good & Alzheimer's & 5 & married & no \\
\hline Kathryn & 64 & $\mathrm{n} / \mathrm{a}$ & 10 & Fair & early-onset AD & 5 & divorced & yes \\
\hline Kevin & 76 & 28 & 2 & excellent & $\begin{array}{c}\text { probable } \\
\text { dementia of the } \\
\text { Alzheimer's } \\
\text { type }\end{array}$ & 3 & married & no \\
\hline Monica & 59 & 28 & 9 & fair & early-onset AD & 5 & divorced & yes \\
\hline Odette & 82 & 27 & 3 & good & $\begin{array}{c}\text { early stages of } \\
\text { senile dementia }\end{array}$ & 3 & widowed & yes \\
\hline Romilda & 76 & 27 & 0 & good & $\begin{array}{c}\text { Mild } \\
\text { Alzheimer's }\end{array}$ & 3 & widowed & yes \\
\hline Rosabelle & 82 & 25 & 0 & excellent & $\begin{array}{l}\text { mild senile } \\
\text { dementia }\end{array}$ & 4 & married & no \\
\hline Ruby & 90 & 24 & 8 & good & $\begin{array}{l}\text { suggestive of } \\
\text { early AD }\end{array}$ & 6 & widowed & no \\
\hline Sam & 80 & 28 & 5 & good & benign senility & 6 & single & yes \\
\hline Sima & 79 & 28 & 2 & good & $\begin{array}{l}\text { suspected early- } \\
\text { stage AD }\end{array}$ & 3 & divorced & yes \\
\hline Tiffany & 61 & 28 & 1 & good & $\begin{array}{c}\text { early-onset } \\
\text { dementia of the } \\
\text { Alzheimer's } \\
\text { type }\end{array}$ & 5 & divorced & yes \\
\hline Uma & 58 & 27 & 2 & good & $\begin{array}{c}\text { mild } \\
\text { Alzheimer's }\end{array}$ & 1 & single & yes \\
\hline Vincent & 74 & 24 & 12 & fair & $\begin{array}{l}\text { early stages of } \\
\text { vascular } \\
\text { dementia }\end{array}$ & 4 & married & no \\
\hline
\end{tabular}


All participant have given me permission to use their photographic images, photodiary entries, and the material they provided during the interviews without attempting to obscure their identity. However, following IRB regulations and because the findings of this study are based on personal accounts, I have used pseudonyms, removed identifying details, and blurred faces in photographs. For a detailed description of each participant summarizing my impressions and reflections see 'participants portraits' (appendix T).

\section{The Process of Coping and Adaptation}

The model presented in figure 1 schematically portrays what emerged when grounded theory analysis was applied to the data provided by the participants in this study and serves as a guide for the discussion of the components of the process as they are presented in this chapter. The components of the model will be described in this section and further developed in later sections. The model conveys how the participants in this study experienced early-stage dementia as a condition that had a profound impact on their sense of well-being. Coping with and adapting to early stage dementia was an ongoing, continually shifting process that unfolded in a series of interrelated phases in which the participants moved from a familiar sense of well-being to a new, alternative sense of well-being.

Learning to live with the early stages of dementia meant for the participants to initially experience a collapse of their familiar sense of well-being, then endure a period of intense disruption, which gradually subsided as they found ways to feel well again. Finding ways to feel well again meant to discover the strength and resources necessary to resolve relevant adaptive tasks, which in turn provided the necessary conditions to redefine, rebuild, and maintain an alternative sense of well-being. The adjustment process 
itself is a complex interplay between the person living with the illness, the illness symptoms, and the external environment, which is reflected in the sense of well-being that is experienced.

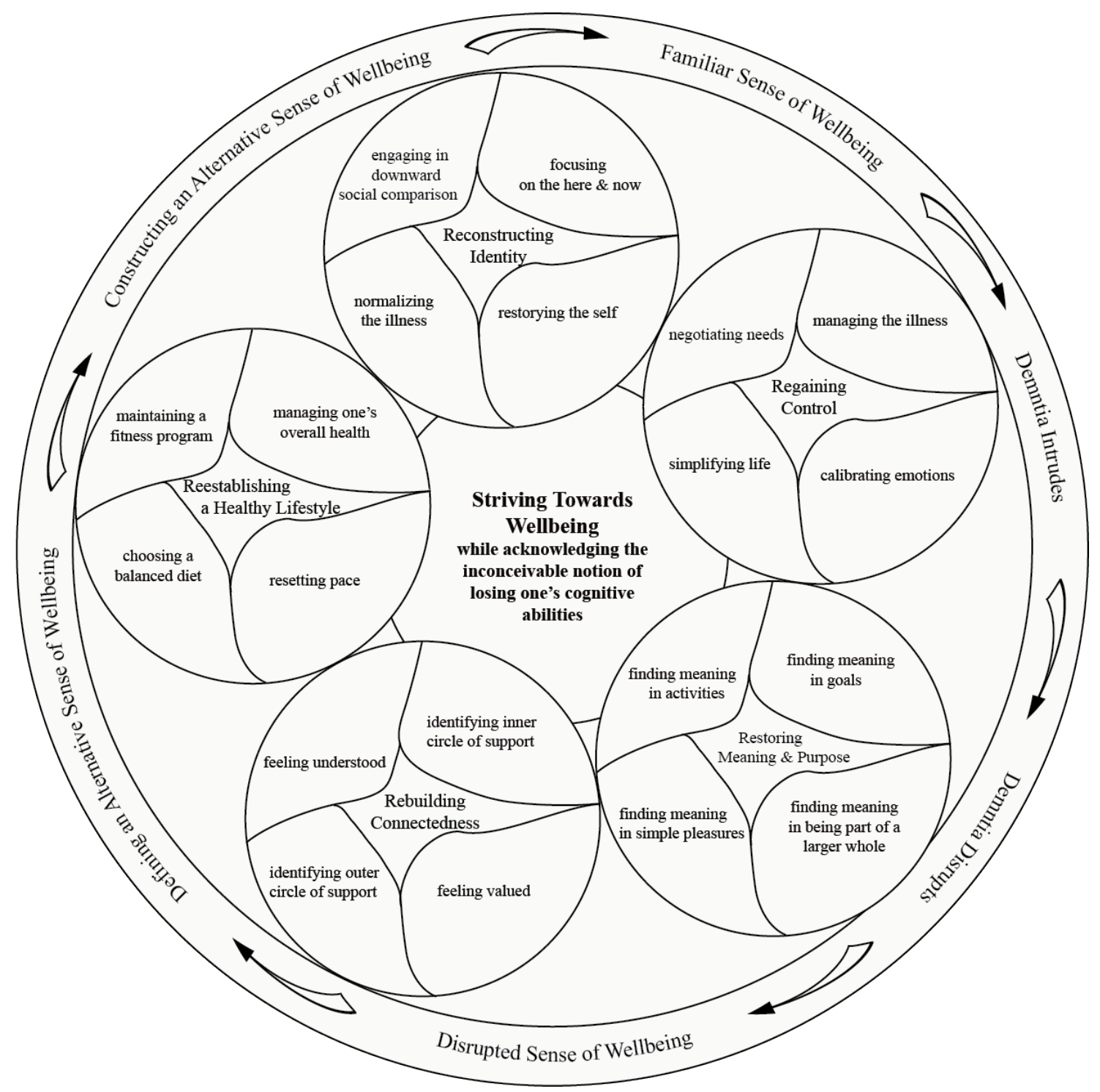

Figure 1. Striving towards Well-being while Acknowledging the Inconceivable Notion of Losing One's Cognitive Abilities

The model in figure 1 shows the core construct in the center of the model. The core construct became evident early on in the data analysis and was substantiated as the analysis continued and the relationship among the conceptual categories examined. It is 
critical in understanding the participants' response to the illness and has strong connections to the other components in the model.

'Striving towards well-being while acknowledging the inconceivable notion of losing one's cognitive abilities' is the central strength that the participants brought to the process of coping with and adapting to early-stage dementia. It captures what early-stage dementia means for a specific person and how he or she finds and uses his or her strength and resources to move through the phases of adjustment. It captures that learning to live with early-stage dementia is an ongoing process of negotiation between the will to experience well-being and the reality of a progressive, irreversible brain disease. It also speaks to the paradox and the dynamic challenge inherent in living with an illness that causes ongoing cognitive decline and therefore impacts every domain of a person's sense of well-being. The ongoing challenge is that the participants in this study were impelled to relegate the illness to the background of their lives while managing its symptoms in the foreground. In other words, they attended to the demands of the illness while striving to carry out what was possible in their lives.

'Striving towards well-being while acknowledging the inconceivable notion of losing one's cognitive abilities' appeared to be a cyclical and continuous process that is characterized by a simultaneous and concurrent acknowledgment of and resistance to the illness and the co-existence of positive and negative emotions. What emerged from the data was a pattern of coping in which the attention to the illness fluctuates. At times, the participants focused on well-being (with the illness in the background); at other times, they focused on the illness (with well-being in the background). What comprised the illness experience was on ongoing cycling of perspectives with the illness shifting from 
foreground to background and vice versa.

Feeling well, as a whole, meant for the participants finding richness in the details of their daily doings and cherishing the interconnection between their lives and the lives of others. In other words, living a normal, everyday life while being engaged in fulfilling and supportive relationships and pursuing mundane activities of daily living created the sense of well-being that they were striving for. Adopting an attitude of personal responsibility and maintaining a focus on everyday experiences allowed the participants to achieve a sense of well-being that was rooted in ordinary events. The findings suggest the relationship between well-being and coping is symbiotic. This mutually beneficial relationship between well-being and coping is illustrated by the fact that an enhanced sense of well-being increased the participants' ability to cope and enhanced coping increased their sense of well-being.

The remainder of the model shows the process through which the participants defined and formed their new, alternative sense of well-being. In the model, coping, the way a person responds to early-stage dementia, is understood as a threefold process. It includes acknowledging the changes that were experienced in one's familiar sense of well-being, accepting the responsibility for looking beyond the perceived losses, and looking at the possibilities of what remains today to define and construct a new, alternative sense of well-being. As time progresses and a person grows accustomed to this new, alternative sense of well-being, it becomes the person's familiar sense of wellbeing. The process of adjustment repeats itself when threats that cannot be easily controlled propel the illness back into the foreground of a person's life and make it impossible to maintain the familiar sense of well-being at that time. Although a person 
can receive a diagnosis of early-stage dementia only once, the disruption in well-being is similar and just as powerful when events that trigger uncertainty disturb the delicate illbeing/well-being balance that constitutes alternative sense of well-being. This could be a sudden awareness of illness progression or changes in symptoms. In other words, the phases of adjustment are iterative, repeated as symptoms occur and losses come about. For people with early-stage dementia the process of adjustment never ends as their abilities continue to decline. Although familiarity with the erratic and unpredictable nature of the illness helps to some extent, it does not guarantee that what worked today will necessarily work tomorrow.

Although the experience of the illness is a highly individualized process, there was a pattern that seemed to unfold in each participant's life. The model shows learning to live with early-stage dementia as a progression of six phases of adjustment: (1) living with a familiar sense of well-being; (2) dementia intruding on the familiar sense of wellbeing; (3) dementia disrupting the familiar sense of well-being; (4) experiencing a disrupted sense of well-being; (5) defining an alternative sense of well-being; and (6) constructing an alternative sense of well-being. However, portraying the process as a sequence of well-defined phases does not capture the complexities of living with dementia. The phases are not mutually exclusive and do not progress in a linear fashion; rather, they are interwoven and reciprocally determined and the transitions between them are fluid. Furthermore, the participants moved through the phases at different rates and frequently experienced some or all of the phases more than once. In short, early-stage dementia was not experienced as having a specific onset or stable symptoms. Rather, the course of the illness was experienced as a waxing and waning process that was marked 
by a gradual onset and ebb and flow of symptoms. Each phase had a number of characteristics and posed a series of challenges and adaptive tasks.

Initially I thought that there would be a clear distinction between 'strengths and resources' and 'adaptive outcomes'; however, as the data analysis progressed it became clear that they are interconnected and often serve more than one purpose. For the purpose of clarity, the adaptive outcomes and the strength and resources to achieve these outcomes are presented separately in this model. In other words, this model has a circular momentum, with each component feeding back into the others, strengthening them even as it gains strength from them. Adaptive outcomes are understood as a person's successful efforts to address relevant adaptive tasks and reconstruct aspects of his or her life that have been affected by early-stage dementia, which in turn provide the necessary conditions for him or her to define and form a new, alternative sense of well-being. Adaptive tasks focus on those areas of life that a person identifies as central to his or her well-being. The adaptive tasks that played a role in the adjustment process to early-stage dementia for the participants in this study were: (1) reconstructing identity, (2) regaining control, (3) restoring meaning and purpose, (4) rebuilding relationships, and (5) reestablishing healthy living. Through their experience of living with early-stage dementia, the participants discovered a number of strengths and resources that assisted them in achieving these personally relevant adaptive outcomes.

The model shows the strengths and resources that were relevant to the adjustment process of the participants in this study. Not all participants used all the strength and resources shown in the model but the majority did. The strength and resources that the participants discovered are the personal and social tools that helped them resolve relevant 
adaptive tasks as they moved through the phases of adjustment. Each participant discovered personally relevant strength and resources at different times of the process and through different means, including word of mouth, recommendations from other people with dementia, face-to-face encounters with healthcare professionals, and written information. Although all participants used a similar set of strength and resources including some that were long-standing coping tools and others that were new, each participant's set of social and personal tools unfolded in a unique way. Each strength or resource was discovered at one point in the process; once discovered, the majority of these abilities and assets played a role in each phase of the adjustment process but varied in their importance. Eventually they move from the foreground to the background only to move into the foreground once again. The discovered strength and resources remain available to be used at different times for different purposes while new ones are added. In other words, the use of each individual strength and resource varies across time and individuals.

The basic strengths that emerged as primarily linked to reconstructing identity were: focusing on the here and now; restorying the self; assigning the illness a position; and contrasting experiences. The basic strengths that surfaced as most relevant to regaining control were: managing the illness; calibrating emotions; simplifying life; and negotiating needs. The basic strengths that were mostly relevant to restoring meaning and purpose were: finding meaning in goals; finding meaning in activities; finding meaning in simple pleasures; and finding meaning in religion and spirituality. The basic strengths that became visible as mainly associated with rebuilding connectedness were: feeling supported; feeling valued; feeling enabled; and feeling understood. The basic strengths 
that appeared as predominately connected to reestablishing healthy lifestyle were:

managing one's overall health; resetting pace; choosing a balanced diet; and maintaining a fitness program.

The overlapping areas in the model show the interconnectedness of the components and how each component is related with the others. At times, one strength or resource served more than one adaptive task. In addition, a successfully resolved adaptive task (adaptive outcome) had the potential to serve as a strength or resource. Finding a new, alternative sense of well-being out of the possibilities that remained today meant for the participants to actively give way to the interplay of the above components to find a new, harmonic equilibrium.

In summary, early-stage dementia is a condition that cannot be overcome but can be integrated into one's life. It was in continuing to strive for a sense of well-being that the participants found meaning and purpose in life even if it meant fighting an uphill battle. In other words, well-being was a journey not a goal. At the time of this study, the participants had managed to integrate the illness into the pattern of their lives and used their strength and resources to maintain the delicate balance of 'ill-being' and 'wellbeing' that is characteristic of the alternative sense of well-being that they had constructed. They had woven a new pattern where the illness was just one thread in the bigger tapestry of life. However, early-stage dementia continued to pose ongoing challenges for the participants, each with the possibility of fostering a shift from 'wellbeing' to 'ill-being' taking center stage and for the adjustment process to re-occur.

The remainder of this chapter will first present the different phases of adjustment and then explore in-depth what 'feeling well' meant to the participants and the strength 
and resources they used to achieve relevant adaptive outcomes and live with a new, alternative sense of well-being. The participants showed with their photographs the kinds of people, things, and places that contributed to and helped them to construct their new, alternative sense of well-being. Their narratives revealed the perseverance with which they chose to strive towards feeling well in the face of dementia and the courageous and resourceful nature of their strategies. Direct quotes are heavily woven into the presentation of the findings as they ground the findings in the stories that the participants shared during the interviews. The participants' voices convey attitudes and emotions in a way that only they themselves can communicate. It should be noted that while each theme has unique characteristics that distinguish it from others, the themes are not mutually exclusive and sometimes overlap in meaning and content. Therefore, some photographs, photo-diary entries, and quotes are at times relevant to more than one theme. All relevant photographs and photo-diary entries can be found in appendix V (figure 2 - figure 210).

\section{Phases of Adjustment}

As discussed earlier, much of what emerged about the phasic nature of the illness is based on information that the participants shared with me during the rapport-building phase before consent was signed and audio recorder was turned on. Although these unsolicited narratives provided rich data, which I captured in my field notes written immediately after each encounter, there are fewer participant quotes available for this section. The first five phases of adjustment are based on the retrospective accounts of the participants and they describe the participants' experience of the past however many years they have been living with the illness (approximately 3 to 6 years). It is likely that 
the participants did not remember exactly how events had unfolded and that I was told their interpretation of what had actually happened. The last phase of the adjustment process ('constructing an alternative sense of well-being') pertains to their current life, the way they live with the illness at the time of the study and how they use strengths and resources during this part of the journey.

Living with a familiar sense of well-being. 'Living with a familiar sense of wellbeing' is the phase that precedes the onset of early-stage dementia and provides the conditions and background essential to the process of learning to live with the illness. All participants spoke openly about the time that preceded the onset of the illness. In their stories the participants used their familiar sense of well-being as a reference point to explain how things had changed and what they had lost and compared it to how things were now and what they were trying to rebuild. A person's familiar sense of well-being is extracted from his or her life experiences, personal values and beliefs, routines and habits, and long-standing ways of coping with and adapting to losses. It includes a certain, taken-for-granted sense of security and stability in terms of overall health, belonging, and ability to plan and do things. Essentially, 'familiar sense of well-being' captures how a person knew him or herself and his or her life to be before dementia intruded. It constitutes the internal recollection of a continuum of personal history through time. The below quote from Frank who used to be a fire scout for the forestry service and spent many weeks alone in the forest illustrates the above construct.

I've never been very social, I've never been a small talk person [...] I've always been a maverick and sometimes I think maybe that one summer on the fire lookout by myself wasn't good for me. Maybe, I don't know. I didn't have anybody to talk to. They called in at 9 o'clock in the morning and said, are you alive? And I said yes, and went out and cut wood. [...] It was then that I found out 
that I could get along by myself. I didn't need people and I still don't. I am happy when I'm by myself. (Frank Photo-Interview)

A familiar sense of well-being is created from the interplay of what is accepted as normal in a social community and how a person sees him or herself fitting within that range. It is the satisfaction of knowing one's place within an agreed upon social range and the recollection of the history of that satisfaction.

So I resigned my position, and, I mean, it was really hard when it was time for me to renew my nursing license and I thought no, I can't do it. You know, 'cause I had malpractice insurance, but I did not want to do anybody any harm, so I thought well, okay, handwriting's on the wall. (Tiffany, Individual Interview)

In this phase, there is an accepted equilibrium in the aspects of life that are important to a person; a comfortable balance in the way he or she sees the world and the way the world sees them. Generally speaking, well-being is in the foreground of a person's life. Once dementia intrudes, a person's familiar sense of well-being is permanently changed and relegated to the past but previous patterns of living become symbolic of a person's sense of security and stability. Nevertheless, the life experiences from which the familiar sense of well-being was extracted, become supporting conditions for defining and forming a new, alternative sense of well-being. In other words, a person's familiar sense of well-being influences how a person copes with early-stage dementia and yet, at the same time, is irrevocably changed by the illness.

Kathryn:

I think being independent has been always very important to me; being independent and being professionally competent [...] because I grew up in a household where my job was to not have any needs. [...] And so I think my identity has been build up around being professionally competent and taking care of things myself and I feel a lot of loss in that area.

Monica:

I hear what you are saying

Kathryn: 
And it's hard to know who to be if you have never been somebody else - because that's the role I learned to play and if I can't play it I am often not quite sure who I should be. [...]

Monica:

Part of it for me is - I was never alone - and now I don't have anybody to take care of. [...] That and what you [Kathryn] said - being competent at my workplace - these two things were who I was; they were the two sides of me one was the softer side and one was the side where I was on task all the time. And now I don't have either one of these. And that makes me cry sometimes. And I am not quite sure what to replace it with. You know, anything that I can do to replace it with.

In essence, a person's familiar sense of well-being sets the stage for the process of coping with and adapting to the illness and provides the condition for the next phase.

And when you are used to being with people, I mean specially with you [Kathryn], you were with people all the time because of what you were doing. But basically, I was too because I had people who reported to me and people that I reported to. And I talked to all kinds of outside people too, you know, attorneys. And so at work, I was very busy all the time and so I have to learn how to deal with being alone and not having anything to do. (Monica, Dyadic Interview)

Dementia intruding on the familiar sense of well-being. 'Dementia intruding

the familiar sense of well-being' is the phase when dementia starts to slowly and insidiously invade the participants' lives. It is the period when the illness gradually appears. Most participants experienced a period when they noticed occasional symptoms of forgetfulness (the primary symptom of early-stage dementia). However, the fluctuating nature of the symptoms made it difficult for the participants to recognize that they were experiencing the beginning of an illness. "Probably it [getting mad] was more then [before the diagnosis]. Well, I was kind of in denial. Yeah, but not too much.” (Irene, Photo-Interview) In other words, these clues were initially discounted because the participants believed that they were experiencing 'normal' forgetfulness associated with aging or stressful life circumstances. 
Life continued by and large in its familiar pattern with mild forgetfulness being the primary obstacles that the participants faced. "Oh, I think I knew I had it. You know, I'd know I'd forget things, but I didn't cry about it. Never, I never...I just...kept on going." (Chester, Photo-Interview) Only in retrospect did some of the participants understand that they had experienced memory problems.

It was just, you know, it wasn't something that I'd expected. It never occurred to me that I would ever have any problems with my memory. And when I was about, let's see, I'm almost 60 now, I would have been about 50 the first time that I started having trouble remembering on a job. But, you know, it wasn't real bad. I just thought it was stress, you know. (Monica, Photo- Interview)

Some participants were able to recall the day when they recognized the first symptom, even though the illness was not diagnosed until some time later.

Well, the first thing that really struck me, it was up here [at the couple's beach house]. It was probably four or five years ago, and we'd be all around the campfire, and we always would play games, like 'I'm going on a trip and I'm taking, one would say an apple, and then pajamas and then, and I, I wouldn't know. And I'd get stuck. I'd get stuck and people would laugh. I was at the end of the line, probably, and people thought that's why. And I thought it was the glass of wine I was having. I got excuses for everything. But, I didn't drink too much wine. I just couldn't play the game. And I thought, this, this is weird. But, for a couple years that went on, but nothing, but that was so clear. I would say, nothing seriously for about two years or so. I started forgetting keys or something, I don't know, but nothing serious. You know, you don't recognize. (Frank, Photo Interview)

As time progressed, dementia impacted the participants' familiar sense of wellbeing more vigorously; forgetfulness became increasingly pervasive and invaded multiple aspects of their lives. The recognition of the severity, frequency, and quality of symptoms as well as the context in which they occurred made rationalizing and ignoring the symptoms no longer possible as well-being was threatened.

Well, I do remember when I discovered that I have problems with my memory. My husband was talking with me, and I couldn't, I couldn't get together what he 
was saying. And he said something about, well, we talked about this two weeks ago, or something like that. And I had no recollection of that at all. And it was something that I would normally remember, because it had to do with family. And, but I couldn't, I just, I couldn't bring it up. (Rosabelle, Photo Interview)

The participants recognized considerable change in their abilities to complete job responsibilities and more complex activities of daily living, most notably managing finances and driving.

I first became aware of having short-term memory when after 28 years of nursing, we had to go from paper charts to electronic charts, and I couldn't follow the steps on how to access the information that I needed. And I kept asking the same nurses over and over again, how do I get in patient blah blah blah's chart? [...] And about that same time, my son and daughter-in-law moved to a part of Seattle that I'm not familiar with, and I kept getting lost trying to find their house. [...] And those two things at work and in my home life, I thought, what's going on here? (Tiffany, Photo Interview)

Increasing symptoms kept the participants from living the way they once did which they experienced as a change in their normal routine. This change was perceived as a threat because they could not explain why their minds no longer cooperated as before.

Tiffany:

Well that's the piece that was just so hard for me because I was always the A student and it was my brain that kept me functional in a really dysfunctional childhood - I knew that my brain was what was going to save me Corinne:

And here it's letting you down - hey

Tiffany:

And here it's letting me down - that pisses me off. [...]

Bonny:

That's exactly what you go through. (Dyadic Interview)

Not understanding the meaning of the symptoms led to a sense of uncertainty and powerlessness and was a source of frustration, anger, sadness, anxiety, resentment, worry, and discouragement for a number of participants. "I noticed it - it was 
embarrassing to me because I couldn't remember people's names and things like I used

to.” (Fanny, Photo-Interview)

And after a while, I started hearing people at work say, well I've already told you that. [...] So I went through being really angry about it or just trying to deal with it but if I would ask somebody a question and they would say - I've already told you that? - Okay - so, will you tell me again? - And I found myself gritting my teeth in conversations

(Tiffany, Dyadic Interview)

I think that in situations that could turn into where you have to defend your position, anything that creates some anxiety I just don't trust myself because I lost my ability to think something through. And I can tolerate that kind of fumbling around a bit if I am with somebody who I know well and I feel comfortable with them but to be put on the spot with strangers is scary. (Kathryn, Dyadic Interview)

In terms of emotion management, most participants simply endured their negative

feelings and did not deal with them in any specific way. One participant self-medicated

and sought relief through drinking increasing amounts of alcohol.

There was a period of time that I was just frustrated. You know, I didn't really know what was going on, and I thought it was, I thought it was me. I thought it, well I, so I drank. You know, not lots and lots, but I mean it was, became my friend. But, you know, that wasn't, I mean, I was just trying to get some relief. You know, I thought of myself as being a kind of a fuck-up, sort of. But I wasn't really. (Irene, Photo-Interview)

Three participants sought medical advice for sadness and anxiety and were treated

for depression. Their memory concerns, however, were not taken seriously by their physician.

And the psychiatrist, actually, she's the one I started seeing when I thought what was going on was just anxiety. And I had actually seen her for, I don't know, probably at least six months before the diagnosis or something. And I, you know, she just would see me every, well for a while it was like every month, but now it's more like every two to three months or something like that I see her. And, you know, she prescribed an anti-anxiety medication, but it's one of those that you take when you start to feel anxious. But she didn't suspect that there was anything wrong with my memory. (Kathryn, Photo Interview) 
I think that [depression] kind of cloaked the Alzheimer's for a while, because it has its own symptoms of, of, you know, problems with thinking processes and that sort of thing. So there was some disagreement among the doctors as to which was causing the problems. And so that's why the first testing, they said, well this could be just the, you know, the depression and the medications that you're taking for the depression. [...] And, and so it wasn't until there was such a dramatic difference in the next year and a half that, that they were, where there'd been no change in medications, and my situation had improved as far as the depression were concerned. And, and then it made it, you know, a much clearer diagnosis. (Monica, Photo-Interview)

The coping response during this phase was characterized by a constant striving to reestablish 'old' normality and familiar sense of well-being. Many participants tried to find new ways to perform old routines. "And so I started getting a little bit better about taking notes and that sort of thing, instead of just relying on my memory." (Monica, Brief Interview)

Others tried to cover up difficulties by limiting or discontinuing challenging activities and interactions. "So I thought, okay, so I have to remember who to ask next time that I haven't asked before." (Tiffany, Photo Interview)

Having the support of and collaborating with family members and/or friends was important during this phase. There were two types of family members and/or friends; those who recognized and those who did not recognize the changes. Those who were aware of the changes initially supported the participants in their striving to maintain normality by helping them to manage and cover up the symptoms. "She's, oh, she's been great during that time, yeah. Except chewing me out for forgetting where I put my glasses a few minutes ago, you know, and that kind of stuff.” (Frank, Photo-Interview)

However, they eventually encouraged the participants to seek medical advice.

And so a year and a half later, my sister insisted that I have it tested again with someone else, and I had it tested again. And the difference was dramatic in the 
test results, that my memory had really, my ability to remember, to recall, had degenerated sharply in that year and a half, year and three quarters. (Monica, Photo-Interview)

Those who were not aware of the changes, failed to validate the participants' complaints or assist them with their difficulties.

And my son started getting really impatient with me when I would call and say, I'm on the corner of blah blah blah and blah blah blah. How do I get to you? And he said, you're lost again? I said, don't lecture me. Just tell me how to get to your house. (Tiffany, Photo Interview)

Some participants felt they lacked empathic understanding and emotional support from others.

My family started noticing that I was repeating myself a lot. And they started bringing it to my attention, which was extremely irritating, you know, to have someone say, well you just told me that, well you just told me that, well you told me that yesterday when we talked on the phone. [Monique, Photo-Interview]

Upon recognition of the severity, frequency, quality and context in which the symptoms occurred a medical condition was suspected and all participants in this study sought medical attention. The deciding factor for seeking medical advice and undergoing cognitive testing varied from participant to participant. For some, it was a specific event that led them to believe that 'something was wrong' and to consult their physician about the changes they had observed.

I thought, well, this is what memory loss is, if something happened only two weeks ago, and I can't remember something. And I thought, if there's anything to be done about it, I ought to do something about it. [...] And I called my doctor. (Rosabelle, Photo Interview)

For other participants, it was the increasing number of events that indicated a discrepancy in their ability to remember or process information as they once had that led them eventually to seek medical attention. 
I was concerned about my memory when I was still in practice because I was occasionally forgetting the names of some of the people that I worked with when I didn't see them very often and we ended up going on an Alzheimer's walk and in that walk they said that if we were concerned about our memory we should have memory testing done and so I ended up at the VA hospital and had that done. (Duncan, Photo-Interview)

For a number of participants this gradual process was less noticeable and they were encouraged by family or friends to seek medical consultation. "So I just feel kind of blessed that people cared enough about me to bring it to my attention, I think that really saved me.” (Tiffany, Brief Interview)

Three participants learned about their illness from volunteering for studies investigating the lives of family members of people with Alzheimer's disease. These participants did not recognize any changes in their well-being prior to volunteering for these studies.

I volunteered for a study; that was it. There was a thing in the paper for family members from, of people with Alzheimer's. You know, they wanted to do a generation study. And through the VA hospital and the University of Washington. Cause it was, we went down to someplace in south Tacoma, I think it was the VA. (Corinne, Photo-Interview)

Dementia disrupting the familiar sense of well-being. 'Dementia disrupting the familiar sense of well-being' refers to a period where the lives of the participants centered around the illness. The decision to seek medical advice and undergo cognitive testing formalized the disruption in the participants' familiar sense of well-being because it moved the illness to the foreground of their lives. It initiated a flurry of activity which included extensive diagnostic procedures as well as follow-up appointments with a number of different physicians which culminated in receiving a diagnosis suggestive of early-stage dementia. 
I went to the memory testing at the University of Washington, the VA, and I have had their hour one and I have had their two-hour one and then later went back for their four hour one and I was told I have 'suspected early-stage Alzheimer's Disease'. (Sima, Photo-Interview)

Some participants received their diagnosis from their primary care physician.

Others obtained their diagnosis from healthcare professionals who specialized in the field of dementia.

A couple of years ago, I got referred to some medical people in the area that specialize in this and went through sitting down for testing which lasted about 4 hours and I just went through another one again - maybe a couple of weeks ago and the diagnosis was that there was no appreciable change. (Kevin, Brief Interview)

Accessing specialized healthcare services and navigating the healthcare system was challenging for a number of participants. They reported difficulties with obtaining referrals.

In fact, when I asked her [primary care physician] for a referral, she was pretty dismissive of it. She didn't think there was any issue. You know, at the time I was still working and seeing clients and all that kind of stuff. And I just think that that made her think that it couldn't be that issue. [...] But I kind of pressed a little bit, because I knew there was something different. And so she finally gave me the name of a referral, and that sort of began the process. (Kathryn, Photo Interview)

Some participants were no longer able to recall the exact circumstances of their diagnosis but remembered the gist and the emotion of the event. Others were keenly aware of the circumstances surrounding their diagnosis. "The picture showed inadequate blood flow to the parietal and frontal lobes, which I know is early signs of early memory loss, and the pictures look very similar to an Alzheimer's picture.” (Tiffany, Photo Interview)

Yet others had vivid memories of their initial encounters with the medical profession. They were frustrated with the way medical care was provided and described 
their interactions with healthcare professionals in negative terms. Their stories also point to a lack of healthcare professionals who were compassionate, respectful, and skilled communicators, demonstrating engagement with the particular needs of each person.

Some complained about the way in which the diagnosis was delivered.

They did a few tests and then we went back to have it explained to us. Man, that guy was so 'positive' - he laid it on "you got Alzheimer's" - just like that. That is a shocker. You know, I, I - if you work with people - there is better ways to do it, I think, you know. You have some loss of memory, you have memory retention problems that may develop into Alzheimer's or something like that - that is a hell of lot easier to accept than if you say flat "you got it". I thought, I have always remembered that as a very poor way to do it. It was a shocker particularly because I hadn't noticed that I had that much problems. (Fanny, Photo-Interview)

Others identified that healthcare professionals were interested during the

assessment period but, after a diagnosis was established, appeared rushed or showed little consideration during follow-up appointments.

Yeah. And then you go like you know, every few weeks for quite a while; for a while she just sort of checked in with you, and then I guess she decides, okay, well this person seems to have been stable long enough that there's no point in them seeing them. You know, so then, it's sad. I don't even know when the next one is. I think there's one out in the future sometime. But I don't know when it is, it's kinda sad. (Kathryn, Photo-Interview)

In general, cognitive testing was perceived as a major source of distress and a number of participants were irritated that testing continued on annually despite the fact that there were no treatment options.

I mean I just, you know, that business about going back to the doctor every month or whatever it was for, you know it just felt like an insult, almost. And you know, that they're just, they were just watching me, basically. And oop, well, you're still the same. You can't think any better than you did last week. I mean, you know, what's the point? And you know, and I think that goes along with the feelings of humiliation. (Kathryn, Photo-Interview)

The confirmation that their condition was not improving or was worse than 
before, precipitated the recurrence of sadness and grief.

And then I would go back every year, and they'd do the tests and of course, you know, it gets worse and worse all the time. In fact this year, I was so frustrated. One of the things they do is they tell the story about, so and so lived on Elm Street and they, you know, went, this story thing. And I just, I was so frustrated at that time. I said I don't remember any of it. But I did remember some of it. But I was so frustrated, because you get, you know, this probably goes on for several hours, these tests, different tests. (Corinne, Photo-Interview)

Receiving a diagnosis was an important milestone for all participants. "It was like, 'shit' when they, they told me I had early onset Alzheimer's." (Irene, Photo-Interview)

For those who had felt that their symptoms had impinged considerably on their normal way of life, receiving a diagnosis of early-stage dementia was less of a surprise and more of an explanation for what they had not been able to comprehend. It was a confirmation of what they had observed and helped them make sense of the changes they had noticed. "Getting the diagnosis was more a confirmation - no it wasn't a shock out of the blue because I knew I forgot things.” (Kevin, Photo-Interview)

For the participants for whom the gradual intrusion of dementia had been less noticeable, receiving a diagnosis was an unexpected surprise and invariably, came as a shock.

I may have been a little upset about things, you know, when I couldn't remember. But I don't think I noticed much change. But I was, I was, but I was of course devastated when they told me the diagnosis. (Corinne, Photo-Interview)

Some recalled feeling a sense of panic when they were informed of their illness.

So I participated in this study and they basically gave me a memory test. [...] I just thought I could learn more about myself, basically. But you know, then I find out I've got this horrible condition. It was horrible. (Uma, Photo-Interview)

For some, the confirmation that they had a medical condition provided some comfort because it produced hard, tangible evidence that family members and friends 
could no longer dispute. "So I was able to go to my son to say, you know, this is why I can't find your house. You know, it's not a, it's not logical to me. So cut me some slack [Laughs]." (Tiffany, Brief Interview)

Nevertheless, being diagnosed with early-stage dementia was a distressing event for all participants and inevitably provoked a profound disruption in their sense of wellbeing. Although the participants received different labels for their condition (see table 1), it was clear to them that they had received a diagnosis of a progressive brain disease for which there was no cure. After the initial shock of receiving a diagnosis of early-stage dementia, a certain numbness protected the participants from the full impact of their losses. The coping response during this phase was focused on living one day at the time, managing the practicalities of medical appointments, and marshaling strength and resources to face the reality of what had occurred.

In summary, receiving a diagnosis of early-stage dementia signified for the participants the beginning of a progression from healthy to ill. At the same time, it also provided them with a base from which to start to actively cope with and adapt to the illness.

I mean, knowledge is power. And once I knew that there is a reason, you know, there is an organic reason that I'm losing my memory. And so with that knowledge, the question became, how do I deal with it? This is how I'm dealing with it. (Tiffany Photo Interview)

Experiencing a disrupted sense of well-being. 'Experiencing a disrupted sense of well-being' signifies the transition from the flurry of activity associated with diagnostic procedures and follow-up appointments to the relative inactivity of routine medical care. It is the period where the focus is on the illness, its symptoms and its 
negative trajectory. A plateau was reached, and the participants slowly realized that they had been diagnosed with a condition with long-term coping implications. The inescapable certainty that the illness could not be cured brought the losses incrementally to the foreground. The persistence of symptoms and the uncertainty about the future forced the participants to acknowledge that their lives had changed permanently and in unexpected ways. They realized on a deeper level that the losses had impacted many facets of their being and that fundamental parts of their lives had been lost and could not be regained. As the numbness of the previous phase began to wear off, it was replaced with intense emotional discomfort. Grieving their losses and the fragmentation of their lives led the participants to experience a wide range of feelings including anger, resentment, sadness, depression, fear, and anxiety. They mourned the parts of their lives that had been lost, and grieving took up much of their time and energy.

I think I've been grieving losses ever since the diagnosis came. I mean I just, right now it probably doesn't feel quite as intense as it did before. But I just have felt like I've been grieving ever since the diagnosis. (Kathryn, Photo-Interview)

The painful realization that life would never be the same made the participants keenly aware of the disruption and fragmentation that early-stage dementia had brought to a number of life domains.

Well I wasn't as surprised [about the diagnosis] as I, you know, looking back, would have thought I would have been, because it explained what was happening. But I did a lot of mourning, "cause it changed my life completely from what I had expected it to be. And I still really get pissed sometimes and very sad. (Monica, Photo-Interview)

The participants' accounts revealed five recurring themes related to the losses that they had experienced. Essentially, early-stage dementia prompted a disruption in the participants' familiar sense of well-being through its effect on (1) identity; (2) control; (3) 
connectedness; (4) healthy lifestyle; and (5) meaning and purpose.

Disruption of sense of identity. The diagnosis of early-stage dementia disrupted the participants' positive sense of self and activated a negative perception of self. Many participants claimed that immediately after receiving their diagnosis, some facets of their pre-illness identity were no longer available to them. They experienced stigmatization. Others spoke of how their sense of self-worth and personal power had been gradually undermined by the illness which left them with little or no confidence in their own value. The loss of control over memory had resulted in a sense of shame and vulnerability, both of which eroded the participants' sense of being a capable and functioning human being. During this phase, it was not uncommon for the participants to struggle with their sense of identity, as they no longer knew who they were.

I think I spent most of my adult life not realizing how I trusted in my own brain or skills - but now I can't trust myself. [...] And that's so different - and I wonder happened to that person that I knew. (Tiffany, Dyadic Interview)

In fact, some participants lived in a constant state of grief. They mourned aspects of themselves that they could not reclaim and spoke of a devalued sense of self.

Several factors that are critical to helping individuals define themselves were lost. The illness had forced a number of participants to relinquish valued roles and responsibilities. As social roles and status were increasingly limited and social involvement became restricted, many participants experienced feelings of inadequacy. Those who were laid off or had to quit their jobs as a result of early-stage dementia identified a loss of identity on three levels. First, losing employment meant it was no longer possible for the participants to define themselves by their job, which is particularly difficult in a culture that uses a person's occupation to establish identity. 
I think I'm much more uncertain of myself now, because I don't do anything interesting anymore. And so it makes me feel less of an importance, you know, to not have a job and be busy. (Monica, Photo-Interview)

Second, losing their job meant an erosion of financial stability, which, living in a culture that uses money to define a person, further chipped away at the participants' sense of identity.

I mean I ended up here [living with her ex-husband's grandmother] because I lost my job and I couldn't work anymore. [...] Well actually, my unemployment hadn't run out. I didn't, I actually, I didn't qualify for unemployment because I got fired from the job I worked at for a long time. [...] They said that my firing was justified, and so I didn't qualify. [...] Well, and just the length of time it takes to get a diagnosis that the government will accept, you know, there's, I mean if I hadn't had my friend Sue to live with, and then Nana to live with, I literally would be out on the street, because the state doesn't give you enough money for you to stay anywhere. And, and you're not, you have to be officially declared disabled by the federal government before you can get subsidized housing. And that took four years. You know, so, so, so there's, I mean, if I hadn't had strong social relationships with people, I'd, I would have been screwed. I mean I would have been one of those people out there on the corners, begging for money, because there's, there's no way, even, even getting the state, you know, 339 dollars and food stamps, I mean you can't stay anywhere for a month for 339 dollars. (Monica, Photo-Interview)

Third, being forced to give up their professional life, the participants also lost several important, work-related friendships, which is also an area where identity is formed. "Most, well I don’t really see any of my old colleagues." (Monica, PhotoInterview)

The participants' positive sense of self was further undermined as the illness created dependency on others. Feeling dependent on others decreased the participants' self-esteem and self-image. This devalued sense of self was often reinforced by other people who the participants came in contact with in their day-to-day lives. Several participants felt that other people thought they were stupid. "My experience out and about 
in the world is that if somebody finds out that I have this diagnosis, they treat me as if I am stupid." (Kathryn, Dyadic-Interview)

Given these forced and unwanted revisions of their beliefs about the world and their place in it, it was not uncommon for the participants to experience a negative sense of self.

Disruption of sense of control. Viewing the illness as controlling one's life and perceiving the future in terms of progression of the illness was a common reaction to the diagnosis. The uncertainty inherent in the illness and the shortage of treatment options fostered a sense of insecurity and powerlessness. "I've liked my life. But I, this isn't what I envisioned for the end. I don't get to be in charge.” (Kathryn, Photo-Interview)

The participants lived with considerable uncertainty regarding the condition's stability, severity, and manageability and were faced with the task of managing the illness on the one hand while dealing with the uncertainties of the illness progression on the other. All participants were well aware of and understood the as yet incurable nature and downward trajectory of the illness. "I know what it's like, you know, and I lived the life with mother, so I, I know what it’s gonna be.” (Corinne, Photo-Interview)

Although all participants had started on a prescribed medication regimen immediately after the diagnosis, the lack of curative treatment options exacerbated feelings of helplessness and hopelessness. "So, but, you know, I just... and I know what's coming. I don't like it, but you know, what do I do about it?" (Corinne, Photo-Interview)

Given the lack of curative treatment options, many participants worried about how the illness may unfold in the future and pondered about the extent to which they may become incapacitated. The inability to make predictions about the course of the illness 
contributed to the participants' sense of powerlessness and was perceived as one of the most difficult aspects of living with early-stage dementia. "Well, I don’t, I mean there is uncertainty about the timing. I mean I think, I know the, I know what's coming. I mean I've watched it in my sister." (Kathryn, Photo-Interview)

In addition, the participants continued to experience the manifestations and intensity of illness symptoms as highly fluctuating, unforeseeable, and erratic, which exacerbated feelings of uncertainty and loss of control. In other words, the intermittent and unpredictable symptomatology not only continued to be frustrating but also had consequences for their sense of confidence and competence and triggered ongoing concerns about the increase in illness severity.

You know there are days when I wake up and my memory is perfect there are days when I wake up and boy, I have trouble. Insert, take it away, insert, take it away. And that's difficult when that happens. I don't think there is ever a time where it lasts the whole day. But the times when it inserts itself - boy, my memory will be perfect or as good as it used to be - but it doesn't last. (Vincent, Photo-Interview)

The uncertainty that the participants experienced because of the unpredictability and inconsistency of symptoms involved many areas of their lives and influenced daily routines and activities. Fluctuating memory function led to day-to-day uncertainties involving frustrating, frightening, and upsetting experiences of getting lost or misplacing objects.

Or you don't, you get there [breakfast table] and all of a sudden, I don't know where I'm at, I don't know what's going on, you know. [...] Well, you're just a blank, you know. You wonder well who am I? What am I supposed to do? (Chester, Photo-Interview)

The participants' sense of frustration was increased when they were forced to ask family members for help in managing simple tasks. 
I know my sister would do all of this [medical insurance paperwork] for me but you know, she works these long hours and she and her partner have rescue dogs. [...] So, I really hate to ask her because she has such a full life anyway but I know she would add it on to everything else if I would ask her. But, I am going to give it one more shot. (Monica, Photo-Interview)

In summary, the participants encountered a number of unpleasant experiences and unexpected changes as a result of the illness, which in turn led them to feel a lack of control over their lives. They were faced with the task of managing the illness on the one hand while, at the same time, coping with the uncertainties of the illness progression on the other. Witnessing the spread of uncertainty and facing a highly unclear and restricted future fostered feelings of insecurity and powerlessness. This was particularly difficult in a cultural context where predictability and control are valued.

Disruption of sense of meaning and purpose. The diagnosis of early-stage dementia also disrupted the participants' sense of meaning and purpose. Crucial parts of a world model that the participants had developed during their lives were nullified or called into question by the illness. The illness had shaken up the participants' well-organized and highly-detailed set of beliefs about who they were, where they were in their lives, and what they could and could not do. They felt limited by how much and for how long they were able to do the things they wanted to do, as they believed they were racing against time. The illness deterred them from what they treasured in life and keeping true to their values and beliefs was not easy as familiar ways of expressing them were no longer accessible. "No, I have had to pull back on a lot of things, 'cause I used to work a lot with doing dinners [for my church] and doing these kind of things, and I can't do that anymore.” (Corinne, Photo-Interview)

Cognitive changes obliged the participants to give up their pre-illness hobbies and 
recreational activities. They lost many opportunities to engage in activities that would have allowed them to express their uniqueness.

Yeah, some of the things that I gave up are still important to me. But it's selfpreservation, you know, you give up things that are pretty dangerous to your health. [laughs] It's like being an alcoholic. (Rosabelle, Photo-Interview)

Intermittent and unpredictable symptomatology prevented the participants from engaging in valued activities and life itself. "Well, I quit volunteering because of my memory problems. I wasn't, I couldn't follow, I wasn't following through on my obligations, and so I stopped then.” (Monica, Photo-Interview)

They felt unable to use their past and current life experiences in a way that would contribute to the well-being of other people because they could no longer trust their abilities and did not want to risk failure.

Well and especially since I don't know what I can still do. You know, it's, it's, I mean, I have always known what I can do and what I can bring to an organization and even though I don't think that I was ever a real prideful person or anything, I knew what I could do and I knew what I was willing to do and now I know what I might be willing to do but I don't know if I can do it. And what I can do today, can I do it six months from now? So, it makes me kind of hold back from anything that requires very much brainpower. And I know that part of is that I am - I don't want to fail. At least, I'd much prefer not to. (Monica, Dyadic-Interview)

The illness had constricted their social world to such an extent that they thought they had nothing to contribute that was of value to others.

Oh yes, yeah I think there are barriers that keep me from being involved in the world. From not wanting to look stupid or, you know, not certain that I have anything to contribute anymore and that sort of thing, you know. (Monica, PhotoInterview)

Those participants who had lost their jobs as result of the illness missed the opportunity to perform the multiple roles they had played in the past. "I miss that part; my work." (Duncan, Photo-Interview) 
The expression of personal beliefs and values that is connected to professional life was greatly diminished or lost. "But anyway, it was just hard. It was, I mean I just couldn't do what I should be able to do." (Irene, Photo-Interview)

Pervasive role changes and the knowledge that they had relinquished some of their duties triggered the fear that they would never again be of value to themselves or others.

And you know, there, I mean losing your job, not being able to do this thing that I love to do, not being able to teach, you know, not feeling really useful to anybody, is such a huge loss. (Kathryn Photo-Interview)

In addition to the loss of immediate competency, the participants were also deprived of their hopes and expectation for the future. By altering the expected trajectory of their lives the diagnosis called into question taken-for-granted certainties and visions about the future. Many participants mourned the loss of dreams of an ideal future; one that did not include illness.

Well I just, I mean I expected to work until I was like 65 or 68 , something, you know. I expected to have a home that my son could come home to. And my grandchildren could come to. And those things won't happen now. (Monica, Photo-Interview)

What was lost was not only every imaginable future plan for retirement and old age but also the efforts expended in preparing for it. The illness had not only changed their life plans but also took the enjoyment out of thinking about the future.

Well, I think I, you know, envisioned retiring and moving up to Lummi and maybe keeping a, like an apartment or something here and kind of going back and forth so that I'd have the best of both, you know. [...] Now I won't be able to do those things. (Kathryn, Photo-Interview)

Being faced with the reality of having to relinquish numerous hopes and dreams was particularly challenging living in a culture where it is normal to be focused on the 
future. No future is ever guaranteed, but most people are comfortable with reasonable odds for fulfilling their dreams. However, when early-stage dementia intervened, all past efforts seemed irrelevant. Being unable to do what they wanted to do and not remembering what they had done contributed to a sense of meaninglessness and boredom. "Every day looks the same and sometimes I am really bored because I can never remember what I did the day before.” (Ruby, Photo-Interview)

Intermittent sadness seemed to be the result of two interacting feelings. One was the recognition of the limitations that occurred several times a day every day. The second feeling was the sense of loss felt when the limitations evoked a vision of how life would have been if the illness had not intruded. If this vision had strong emotional meaning for the participants, sadness seemed to be the likely outcome. "But it, but it's one of those things that I'm aware of feeling grief about [...] when I'm thinking about stuff and what I won't get to do anymore, that kind of stuff." (Kathryn, Photo-Interview)

Disruption of sense of connectedness. Early-stage dementia impacted the participants' sense of connectedness and belonging in the world in two ways. There was a disruption due to symptoms and there was a disruption due to the decision of whether or not to share one's diagnosis with others. A major challenge that the participants encountered during this phase was to determine whom they wanted to inform about their condition, how, and when. The decision whether to reveal or conceal their illness had both positive and negative consequences. Diagnostic disclosure at once altered the participants' relationships and social life due to the reaction of others. Some family members and friends were sympathetic and understanding as the diagnosis helped them to make sense of the changes they had noticed. "I told my wife and children (two 
daughters and one son) about my diagnosis. It was like - 'oh my - I thought you were kind of forgetful'." (Kevin, Photo-Interview)

Others met the diagnosis with disbelief and rejection. They refused to believe that the participants had an illness since they 'appeared' well. "My father acts like it's never happened. [...] And I mentioned it a couple times, and it just was like it, he didn't even hear it, you know? Like his ears were stopped up.” (Monica, Photo-Interview)

Yet others dropped away and gave up on the participants or significantly reduced contact after being informed of the diagnosis, which left the participants with another loss. A few participants speculated that many of their relationships fell apart because family members and friends did not know how to respond to their illness and how to interact with them. They did not know what to do or what to say and did not understand the participants' daily struggles or their grieving of lifelong hopes and dreams. To illustrate the uniqueness of their struggles, Corinne compared her experiences of living with early-stage dementia with those of people living with other life-limiting illnesses.

And there're the kind of people, I get real frustrated because people know I have a problem, but they never, never ever, you know, if somebody's got cancer, first thing you say, how are you doing, how are you feeling? And nobody ever says anything about Alzheimer's. They know that we have it, but it's just, nobody ever talks about it. (Corinne, Photo-Interview)

Kathryn expressed a similar sentiment,

I wonder if a lot of it has to do with, you know, like I think that people might be more willing to, or have an easier time imagining that, okay, well this is still a human being who happens to have cancer. But their cancer doesn't compromise their thinking or doesn't raise the possibility of compromising their thinking. And so, you know, people, people who are healthy will go visit somebody with cancer or spend time with somebody with cancer and not think a thing of it, because they'll expect this person, well they have cancer but they still have their brain and it's functioning, and so they're still really a human being, after all. But I think that a lot of people assume that once you get Alzheimer's, you start losing it. And 
you're not there in the same way. (Kathryn, Photo-Interview)

These changes in relationships were experienced as painful and were another source of disruption in well-being.

Most, well I don't really see many of my old colleagues, family and friends, you know. Some of my friends have been okay. I don't get to see a lot of them. You know, I don't get to see them often, so they haven't really had a chance to get used to the whole thing. But they don't call as often, partly I suppose my life's pretty boring, but I'm more than willing to listen about their life, you know. So it's just small changes, but you notice them. (Monica, Photo-Interview)

The perception of a potential or real reduction in status also generated a sense of disconnectedness. The following quote illustrates the reduction in status that the participants experienced when they were left out of an activity or talked about behind their back.

You know, I told my neighbor lady that works here, lives here up the hill. And she's a younger woman than I am, and I, I think that she's kind of backed away some when I told her about my diagnosis, yeah. Yeah, I mean it, we weren't like close or anything, but we used to talk out in the yard some and stuff. And it, I don't, you know, it, we weren't that connected that I like really notice a difference. I can't say that. So I don't know whether I'm imagining it or too sensitive about it or, you know, whatever. But, but it does feel somewhat different to me. I think it scares a lot of people. (Kathryn, Photo-Interview)

Fear of rejection made information control essential for a number of participants and the decision to conceal their illness had both positive and negative consequences. On the one hand, control over what other people knew about them contributed to maintaining a sense of autonomy and integrity. On the other hand, withholding information about the illness required looking and behaving as a healthy, 'normal' person, which became increasingly difficult and feared or actual embarrassment was a primary reason for disengaging socially. Appearing dumb or stupid when interacting with others was one of the motivating factors for staying home more often. The specifics of these dynamics will 
be discussed in the 'constructing an alternative sense of well-being phase' because the decision about whom to trust and what is possible to reveal was not a onetime choice; it was a decision that the participants had to make repeatedly in old and new relationships. "I mean I wouldn't even say anything to her - she right away would say "there is nothing wrong with you". (Fanny Interview)

In addition to the impact that diagnosis disclosure or non-disclosure had on the participants' sense of connectedness, the illness symptoms also constricted the participants' social world. Although the participants tried to perform the multiple roles they had played in the past, they met their social obligations with great difficulty or not at all. Relationships with family members and friends changed because they were no longer able to perform prescribed social roles. In other words, the symptoms of dementia created new interaction patterns. "I feel like when I get involved in a dialogue with another person, I lack the trust in my own ability to keep up the conversation and keep to my guns.” (Kathryn, Dyadic Interview)

For many participants, finding the energy or drive to be social was a challenge. Some felt overwhelmed because much of their attention went into meeting the demands of the illness, which caused physical and mental fatigue. "I don't know if this is me or if this is the Alzheimer's or whatever - but too much chaos just feels like - I just want to back out the door.” (Kathryn, Dyadic Interview)

Others experienced a general decline in motivation to stay socially engaged and active.

I don't know whether I hold back or not. I think I have, I think I'm much more uncertain of myself now, because I don't do anything interesting to talk about, and so it makes me feel less of an importance, you know, to not have a job and a 
busy life, 'cause I, I used to have a really busy work life and a really busy volunteer life and a really busy being a mom life, and now I don't do anything. So, so yeah I haven't reached out. (Monica, Photo-Interview)

Yet others considered the frustration they experienced due to their cognitive difficulties in social encounters a major reason for cutting back on social engagements. They discovered that memory loss or word finding problems made interactions with others more challenging and tiring. "I think that it's harder to communicate, because you're not sure whether you've said it before, especially around strangers." (Corinne, Photo-Interview)

In any case, the participants were faced with changing roles in family circles and social situations. Many participants felt alone and isolated during this phase of the adjustment. In summary, the disruption in well-being that the participants experienced was further complicated by the dropping off of close family members or friends, stigmatization, changes in social roles and functioning, and increasing dependence that followed the onset of the illness and/or diagnostic disclosure. Illness symptoms curtailed social engagement but the reactions of family and friends left the participants with feelings of abandonment, rejection, and loneliness.

Disruption of healthy lifestyle. Early-stage dementia forced many stressful lifestyle changes, such as adapting to limitations, giving up cherished activities, and paying for medications. Some changes were immediate; others gradual; some were anticipated but the majority were not and appeared not to be connected to the illness. However, no matter the pace, lifestyle changes seemed to be inevitable and occurred at some time. As the illness progressed, the participants encountered a number of barriers to maintaining a healthy lifestyle. Some developed poor sleep habits. 
Sometimes I get up at $2 \mathrm{AM}$; sometimes I get up at $4 \mathrm{AM}$; sometimes I get up at 5 AM. I go downstairs, rest on the couch, watch a little TV - I wake up about every two hours. It attacks you during the day - it really gets you tired. But I learned to live with it. I sleep a lot during the day. (Vincent, Photo-Interview)

Others encountered difficulties in planning and preparing healthy meals.

And there would be, in the beginning there would be two and three, four days at a time where I didn't fill anything out, or I'd do breakfast and then there's be nothing for the rest of the day. And so I had no way of remembering whether I had eaten, you know, whether I had taken my pills and that sort of thing. (Monica, Photo-Interview)

Overwhelming mental fatigue and insurmountable practical difficulties when trying to be active led many participants to pursue a lifestyle that lacked social activity and sufficient self-care.

Yeah, it's good because before she [sister] moved in with me, I stayed home and didn't do anything for a week or whatever, was in there in the room. And so I was here by myself all the time. [...] I just know that my son came in my bedroom and said mom... and he had a tray ....and he said, I made you some fresh soup. You haven't eaten in, I thought he said two days. And I said what? And he said, that's right. And I said, well how could I go two days without eating? And he said, well just eat it and we can talk later . (Fay, Photo-Interview)

Due to the disabling nature of the illness symptoms, they spent their time at home not doing much of anything.

I don't have the energy to do it every day. It had to do with energy, and it's like I don't have the necessary energy to read my mail every day. I mean I just, you know, it is a task. And, you know, basically I have to psych myself up. (Uma, Photo-Interview)

This inactivity increased the likelihood of social isolation and negative emotions. Furthermore, the participants' desire to care for themselves was superseded by thoughts of their future and their poor state of health. They felt depleted of the emotional energy necessary to move forward and be engaged in life.

Food and exercise and pills and stuff - I think there was a part of me that was 
resistant to that - and I just rebelled against anything that sounded like that. Life is too short. I got so tired of all that - vitamin this, vitamin that... (Kathryn, Dyadic Interview)

Another barrier to a healthy lifestyle was the participants' focus on managing the symptoms of their illness. As day-to-day living became increasingly difficult, it was not uncommon for the participants to focus entirely on symptom management and pay little or no attention to other areas of their lives. In doing so, the participants neglected their long-standing and well-established healthy routines and developed a sedentary lifestyle. The participants experienced a disruption in lifestyle and faced many challenges in remaining physically active and maintaining a healthy eating regimen. The primary disruption appeared to be caused by the illness symptoms. However, secondary disruptions such as depression, lack of physical activity, and poor nutrition also played a major role.

Discussion experiencing a disrupted sense of well-being. In summary, during this phase, the participants became keenly aware of the profound disruption that earlystage dementia had brought to their familiar sense of well-being and grieved the fragmentation of their lives. Kathryn captured the essence of this feeling by taking a photograph of a lifeguard sign (figure 2).

Well I think, I mean I think, I mean in some ways I think well, you know, our, I mean I, this is sort of like, I don't know, naïve or whatever, but you know we have all this gazillion dollars that we spend on healthcare and you know these amazing tests that we can do, and you know, scans and all these things that cost thousands of dollars for a scan, and I don't know how many thousand dollars both I and my insurance company have paid since I've had this diagnosis, but it's a lot of money. And you know, and they can't do really anything. So the irony of all that, or the frustration of all that, what it all comes down to is, the lifeguard's off duty and they can't really help you with this. I mean I just, you know, that business about going back to the doctor every month or whatever it was for, you know it just felt like an insult, almost. (Kathryn, Photo-Interview) 
It was easy for the participants to be consumed by the effects of the illness and the losses it had generated. Their world appeared to be spinning out of control as a result of their emotional reactions to the disruption in well-being. The coping response during this phase was characterized by an angry sadness and fading hope for their familiar sense of well-being to re-emerge. At the same time, the participants started to face the responsibility of moving beyond their losses. They slowly took charge of their lives and the new reality by establishing initial, basic routines for managing the illness.

Defining an alternative sense of well-being. 'Defining an alternative sense of well-being' refers to the phase where the participants slowly assumed a different stance; they changed the perspective from which they viewed their situation and started to focus on feeling well. It is the period where the illness began to move into the background of the participants' lives. Well-being became more and more the center of their focus as it slowly moved into the foreground. Corinne captured the essence of this phase when she stated, "Well of course it's depressing that there's nothing they can do about it, of course it's depressing. But, you know, you just learn to live with it." (Corinne, Photo-Interview)

During the 'defining well-being' phase, the participants learned to recognize new limits and defined well-being in ways that accommodated these changes. "Hetti [sister] got me to see somebody and then the meds and, and there's just, I mean it's Hetti and my other sister and of course my brother, but there's other people too, you know. "(Irene, Photo-Interview)

It is an internal dialogue with the self, which promotes the process of solving problem and resolving emotions, which in time leads to the definition of an alternative 
sense of well-being. The participants had experienced symptoms and relapses, and there was a growing recognition that a return to their familiar sense of well-being is not possible. The realization that they will not be able to regain their familiar sense of wellbeing provided the ground for the participants to develop a definition of well-being that included the illness.

You don't quit. ... Well, I did for the first couple of years. I didn't, you know, well I guess I can't say that, because I went walking every day. I didn't belong to a gym but I went to counseling and went to groups, and, you know, did what had to be done. (Monica, Photo-Interview)

The participants gradually became aware that they had a lot of life to live and that it was possible to feel well again only if they adjusted their lives to meet the demands of the illness. They started to actively think about the changes they would have to make to their longstanding, habitual ways of living to accommodate the illness. The participants found themselves at a crossroad in the road of life and made the decision not to sit back and let life go by. The following quote illustrates this shift in perspective:

Yeah, I was kind of angry. So it's kind of like Kübler-Ross' stages of grief, you know, anger, denial. You know, I was just angry with everybody cause they were telling me that I was repeating myself and then it's, it's like no, I'm not doing that. And then the bargaining piece of, okay if I stop talking so much and stop asking the same questions, maybe nobody'll notice that I'm repeating myself. And then the denial of, you know, this can't be happening to me. I can't be losing my brain. You know, why don't I have kidney problems or liver problems? Why does it have to be my brain? And then it was like, then the acceptance piece of okay, got a diagnosis, let's get on with it. Are you familiar with Kübler-Ross' stages? [...] And those are the things that were so helpful to me. I mean I've been a nurse forever. Never did I think I would need to go and pull that out, to be my guide to know what to do next and to see, okay, this is a normal phase. It's going to pass. (Tiffany, Individual Interview)

During this phase, the 'chronicity' of the symptoms became even more the focus of attention and the participants realized that they had to cope with the effects of the 
illness long-term. Making room and shifting behavior to accommodate the illness was the first step to integrate the illness in their lives. The initial, basic routines for managing life with the illness developed during the previous phase provided the participants with some preliminary ideas about how feeling well with the illness might look like and how it may be possible to achieve this new sense of well-being. Having lived with the illness for some time provided the condition for moving forward in the adjustment process. Hope resurfaced, there was less worry, and the participants did not feel quite as bothered by the uncertainties that are characteristic of the illness. Although the participants continued to be unsure about many aspects of their lives, the redefinition of what it meant to do well allowed them to feel less ill and less distressed; essentially, it contributed to adopting a more positive outlook on life. Although questions about how they would be able to take care of themselves in the future never ceased, the future was not seen exclusively in terms of illness trajectory. Instead, the focus shifted onto practicalities of planning for the future.

And at the first, when I, you know, I was so rattled by everything that was happening, you know, I was taking more. And, but you know once, once I, you know, they gave me this diagnosis and I started figuring out what happened, and then we started making changes so that I wasn't having high pressure situations or something, then, I don't, you know, I'm not anxious most of the time at all. (Kathryn, Photo-Interview)

However, restructuring the way in which the participants defined themselves also included considerable sadness. The participants came to realize that the process of adaptation was ongoing and that adjusting habitual activities and thinking takes time. Redefining well-being was like a patchwork process where the participants tried to assemble familiar segments with newly found segments of well-being to piece together 
an alternative sense of well-being. Initially, this project appeared untenable as none of the pieces seemed to connect or relate to each other until they were stitched together to form a bigger quilt. Each calamity and complication and each success and accomplishment was a new color added to the work.

In summary, during this phase the participants sought answers to the following questions: How can I feel well again? How can I live daily life differently? What are new ways to live meaningfully? They started to restructure their life patterns and perceptions so that they could move on with the illness as part of their lives and not all of it. Developing the courage and determination to face the paradox inherent in living with early-stage dementia and starting to see the possibilities in what remained today was the prerequisite condition to progress to the next phase in the adjustment process. Moving forward meant to translate the newly gained definition into a practical approach to life.

The 'defining an alternative sense of well-being phase' concludes the participants' description of their experiences from the onset of the illness to the present and transitions into the here and now of their lives. The 'constructing an alternative sense of well-being phase' describes how the participants currently use their strengths and resources to cope with and adapt to the illness.

Constructing an alternative sense of well-being. 'Constructing an alternative sense of well-being' refers to the phase in which the participants live with the complex co-existence of 'ill-being' and 'well-being'. By balancing these opposing forces in their daily lives the participants achieve a sense of well-being. It is the period when well-being is generally in the foreground and the illness in the background of the participants' lives. "So it's kind of like a new beginning. [...] So this is a really good moment in my life, and 
isn't it weird that it took this diagnosis to get me to focus my attention on myself?" (Tiffany, Photo-Interview)

However, any event that triggers uncertainty has the potential to transition the illness back into the foreground of the participants' lives. This could be a sudden awareness of illness progression, changes in symptoms, or negative interactions with others. 'Constructing an alternative sense of well-being' is the time in which the participants became familiar with a world where the illness is ever-present but its presence does not dominate. It is the process of discovering life as it is now. The participants restructured profoundly the way in which they interacted with the world. They recognized that the restrictions imposed by the illness also allowed for alternatives to surface and took on the responsibility of creating well-being out of a changed reality. They played a more active role in shaping their lives. Although illness symptoms and the psychological pain associated with these symptoms continued to have a presence in the participants' lives, the participants translated the understanding that there is still 'a lot of life to live' into practical steps. They realized that given the right tools, they could be in charge of their lives. Reconstructing well-being meant to actively utilize the possibilities that unfolded as a result of their losses. It entailed saying yes to a life with dementia and using available strength and resources to create a new sense of well-being on a day-today basis.

I don't know, I'm failing pretty fast. But, you know, I'm just, I'll just take it and cry sometimes but make the best of it. You know, if you don't, then you just, you know, you just lose life. And I don't want to do that. (Corinne, Photo-Interview)

At times, the experience carried the participants closer to recognizing their strengths and resources, as they felt strong, able to cope, and determined not to let their 
illness get the better of them. Other times, the experience made it difficult for the participants to see their strengths and resources and they felt fragile, despondent, defeated, and worn down.

The following section describes how the participants used their strengths and resources to juggle the competing priorities of managing the illness and striving towards well-being. It illustrates that the strengths and resources that the participants brought to the adjustment process are the active ingredients for the construction of a cogent sense of well-being despite early-stage dementia. The participants' accounts revealed five recurring themes related to the adaptive tasks that the participants encountered in reconstructing well-being: (1) reconstructing identity; (2) regaining control; (3) rebuilding connectedness; (4) restoring meaning and purpose; and (5) reestablishing healthy lifestyle. Successful completion of these adaptive tasks was a crucial condition for adjustment. The basic strengths and resources that the participants used to achieve these adaptive tasks are presented under the adaptive task for which they are most relevant. This is not to suggest that these strengths and resources are exclusively relevant to this particular adaptive task rather, they are associated with a number of adaptive tasks. However, for the purpose of clarity they are presented under the adaptive task they are primarily linked to.

Reconstructing identity. A recurring theme within the verbal and visual data was the importance of feeling good about oneself and the life that one leads. Positive selfregard was a necessary prerequisite for an alternate sense of well-being. 'Reconstructing identity' meant for the participants to develop a new sense of self, a self that differed from their pre-illness, familiar sense of self but was satisfying enough to support their 
alternative sense of well-being. Monica included a self-portrait in her portfolio to show that positive perceptions of self support her in reclaiming a sense of well-being (figure 3).

In a similar vein, Karl captured the essence of this theme and expanded it to include the illness. Karl's favorable constructions of self included the notion of dementia. "I love myself. [Laughs] No, I've accepted it [Alzheimer's disease]." (Karl, PhotoInterview)

Irene expressed a similar sentiment by taking a photograph of her mother's photograph (figure 4) and sharing her thoughts about the importance of maintaining a sense of personhood.

I loved my mother very much. She thought kids were smarter than adults. Mama had MS [multiple sclerosis] when I was four. She taught me you're always a real person - no matter what. And that's so important! That's what it really, really I mean, I mean that's really important! I think, I think some people with Alzheimer's forget that. (Irene, Photo-Interview)

The participants proved to be resourceful in terms of pursing this adaptive task and four strengths associated with the process of reconstructing identity surfaced when grounded theory was applied to the verbal and visual data: (1) focusing on the here and now; (2) restorying the self; (3) assigning the illness a position; and (4) engaging in social downward comparison. The common thread within these strengths is the importance of positive self regard. This theme speaks to the significance of a positive sense of self and how the outcome of self-evaluation has profound effects on the participants' sense of well-being.

Focusing on the here and now. 'Focusing on the here and now' and not comparing it to how things were before the illness was one of four strengths that helped the participants see themselves more positively. The specific resources that the 
participants employed to remain in the present were: valuing remaining abilities and keeping a daily journal.

The notion of 'focusing on the here and now' emerged as a theme through the repeated use of the term 'still' in the participants' accounts. Many participants used the term 'still' when referring to those abilities that, until now, were not impacted or were only partially impacted by the illness and thus signaled an unchanged identity. Monica captured the essence of this strength and its connection to positive perceptions of self when she took a photograph of an absentee ballot for the primary election (figure 5) and shared, "It just kind of makes me feel like I'm still part of the world. You know, I can still vote. So I'm still a US citizen.” (Monica Photo-Interview) Irene shared a similar sentiment, "But you can be still somebody, that's for sure." (Irene, Photo-Interview)

By highlighting the ways in which they were 'still able' rather than how they were 'dis-abled' the participants rekindled their sense of confidence, which in turn supported the process of rebuilding positive perceptions of self. Kathryn included a photograph taken during a ferry ride to Lummi Island (figure 6) and shared, "I sort of figure, as long, like right now I can still go up to Lummi and sort of have a certain amount of independence, and so life is worth being alive.” (Kathryn, Photo-Interview)

The term 'still' marked remaining abilities and functioned as a claim of competence asserting present possibilities and things to look forward to.

I don't feel like I am 79 - okay - yeah, I have slowed down a little bit but my mind is still there - I still want to do things - I am not ready for the grave. I mean, I want to see things and I want to do things. (Sima, Photo-Interview)

In some cases, the use of the term 'still' portrayed the participants' strategies of reconstructing identity as grounded in long-standing values. Emphasizing remaining 
abilities served as a springboard for coping with the illness while preserving a sense of identity. For Fay, continuing competence was maintained through her remaining ability to practice religion. Fay's ongoing ability to pray helped manage her life with early-stage dementia while preserving and enhancing her identity as a good, honorable Christian. Fay included a photograph of her church in her portfolio (figure 7) and explained, "I still pray, that's the one thing that gets me through, yeah.” (Fay, Photo-Interview)

Frequently when the participants used the term 'still', it was followed by the qualifier 'but' which is an indicator that they were both acknowledging and accommodating the changes that the illness had brought into their lives. Chester included a photograph of one of his son's dogs (figure 8) and explained, "I still walk them [dogs]. They walk me. But I don't go very far anymore, just around the patio and going down the street or something." (Chester, Photo-Interview)

'Focusing on the here and now' also meant to incorporate necessary changes and precautions. Tiffany spoke with pride about her remaining ability to handle her own finances but did not present an undiluted tale of success. Instead she shared her strategy for limiting the effects of the illness on identity and well-being.

I am still able to balance my checkbook but I'm getting a little nervous about it, cause I used to be very sharp. So now I sort of double-check things. And I got myself a calculator, so instead of, you know, just doing it in my head, you know, I double check that I have everything right. So I know I need to have that secondary check. (Tiffany, Photo-Interview)

The use of the term 'still' in the narratives also shows the participants' awareness of possible decline in abilities in the future while simultaneously constructing a positive sense of self through being proud. "I can still walk the whole mile - but I pay attention to what I am doing - and so far, it works.” (Odette, Photo-Interview) 
The above sections of the narratives not only provide evidence for the participants' focus on remaining abilities but also show the awareness with which the participants contrasted current with previous levels of functioning. 'Still ... but' constructions point to the central theme that emerged during data analysis, namely the dual process of striving towards well-being while acknowledging decline. Another way in which the participants focus on the here and now became apparent through their celebration of everyday successes, whether big or small. Small victories confirmed the continuity of competence and skill, which reinforced a positive perception of self. Kathryn's photograph of a lake (and the associated story) (figure 9) provides evidence of the extent to which everyday accomplishments become important victories which are linked to feelings of self-respect and self-esteem.

Well this actually is a place on the trail that I had taken a photograph of with my own camera. And I loved the photograph, and I think it's actually stuck on the refrigerator still. But then I couldn't find it again down at this place down at Green Lake. And you know, I've looking for it, and I'm thinking well, I know where, what part of the lake has this slope to it and everything, so I thought I should know. But I kept looking for it and looking for it and I kept, where is that spot? Why can't I find that spot? This made me feel a little crazy. And then I don't know what prompted me, if I got suspicious or if I just wandered over there. But there's a, I was going to say boathouse but it's not a boathouse. It's a building that the parks department uses for their lifeguards and stuff. [...] And this, so you kinda had, that kinda blocks, that building blocks your view from the walking trail of this section. And that's why I wasn't seeing it. But you know, in some ways, that made it, I mean, you know cause I was searching for it really hard. And it became more and more important that I find it and sort of understand, why wasn't I finding it, you know, when I started looking for it again. And, and I did, I figured it out! (Kathryn, Photo-Interview)

Kathryn presented her re-discovery with a sense of pride as indicated by using a high case and exclamation mark in her photo-diary and the language she used when sharing the associated story. 
For a number of participants, 'focusing on the here and now' also meant to keep a daily journal. By doing so the participants remained in the present. They not only created a written record of their lives but also developed clearer perceptions of self. Journaling was a way of documenting and preserving their personal stories. Another benefit of journaling was that the recorded daily activities would serve as a reference for the future. In other words, journaling created a sense of identity that was intimately bound to their daily doings and present life events, which in turn helped them feel better about themselves. The participants were amazingly inventive when it came to taking full advantage of this resource. In order to create a reliable and trustworthy record of their lives, some participants developed a habit of jotting down relevant events or ideas as the day progressed. At the end of the day, they created memorable journal entries by expanding on these notes. Monica illustrates this dynamic when talking about the photograph she took of her notebooks (figure 10).

And the one that says private on it, is like a diary. I write in it almost every night when I go to bed. And I just, during the day, I kind of jot down notes on my life $\log$, and then at night, if I can't remember, you know, what happened during the day, I can use that to jog my memory. And then that way I can, and I have done this, where I've gone back and read over it, you know, just to kind of remind myself what's been going on. And then I'll remember more details, when I do that. (Monica, Photo Interview)

Keeping a daily journal helped the participants 'remember' what their day had looked like, reflect on their experiences, and think about alternative ways with which they could pursue well-being. Focusing on their daily experiences also provided a way for the participants to integrate seemingly unconnected life events and create new insights. They learned what increased or decreased their sense of well-being during this time of their lives. 'Focusing on the here and now' through writing helped clarify 
emotions and reactions which in turn helped them to identify their triggers. Over time, these collections of memories provided the participants with a sense of personal continuity. The journal entries were evidence of who they were now. Tiffany used her long-standing habit of journaling as a tool to form her own perspective, views, and opinions. It helped her to develop a better sense of who she has been in the past, who she was in the present, and who she would like to become in the future.

Writing in my journal feels like I'm answering the questions that I want to be asked. You know, it's like, you know sometimes it's just telling the story of what Igor and I did. Or, you know, if I'm upset about something, I can get it all out. You know, I would love to have somebody asking me the questions, but since I don't, I have my journal. And it's been, I've started to go back [...] and it's amazing how I recall those things because I've written them down. And I see where I made some errors in judgment and how I rectified them. [...] And so I think, well, maybe I'll learn from it. (Tiffany, Photo Interview)

Going back to old journal entries was a way for Tiffany to reflect on her life and her accomplishments untainted by other people's views. "So I feel like I'm getting to know me, not from people telling me stories about things that I've done, but I've a written record; I'm so glad I wrote all that stuff down.” (Tiffany, Photo-Interview)

Recording their daily lives was a way for the participants to enjoy their present experiences and occasionally, share them with others. 'Focusing on the here and now' was a strength that supported the participants in their efforts to reconstruct a positive sense of self, which was a crucial prerequisite for an alternative sense of well-being to emerge. To remain in the present the participants employed two specific resources valuing remaining abilities and keeping a daily journal.

Restorying the self. 'Restorying the self' was another strength that the participants drew on to construct their sense of identity and rekindle positive self-regard. What 
supported the participants in moving from negative perceptions of self towards positive self-regard was taking responsibility for the stories they told about themselves. The participants seemed keenly aware that the way in which they viewed and described themselves to self and others made a big difference in how they themselves and others saw them. Defining themselves instead of letting other people label them was an important act of self-advocacy for the participants. This self-definition in turn increased their positive self-regard. All participants put much effort into the production of a favorable narrative, a story that described them in positive terms. The specific resources that the participants used to construct a favorable self narrative were drawing on home as a source of positive self-regard, drawing on internal qualities as a source of positive selfregard, drawing on pre-illness identities as a source of positive self-regard, and drawing on family history as a source of positive self-regard.

One way that the participants chose to portray themselves in a positive manner was by including photographs of their homes in their portfolios. 'Home' not only referred to the physical place where they lived but also acted as a container to support the participants' sense of identity. The participants were proud of their homes, and 'home' provided the physical and social context of their life experiences. 'Home' allowed the participants to experience a fundamental sense of belonging and identity as it showed who they were as individuals and reflected their histories. It provided a sense of continuity and stability and reinforced the participants' sense of uniqueness (figures 11, $12, \& 13)$.

Sima drew on photographs she had taken of the interior of her house (e.g., figures $14 \& 15$ ) to present a positive view of herself and her life story. Much of Sima's sense of 
identity was stored in her furniture and mementos, which she had carefully collected over the years. The interior design of her home stored memories of important events, specific life experiences and associated emotions, and thus reflected her life story. Harvesting and sharing the stories embedded in her home allowed Sima to express personal preferences and make her sense of self visible.

Another way of presenting themselves in a positive light was by referring to their internal qualities. Attributes that the participants used to describe themselves included being 'appreciative, optimistic, happy, sociable patriotic, and funny'. "Oh, I've always been appreciative of things." (Corinne, Photo-Interview) "I have always been an optimist; I had to be scolded at school all the time because I was too happy." (Odette, PhotoInterview) "I am generally happy with the way things are going." (Duncan, PhotoInterview) “I am very patriotic.” (Fanny, Photo-Interview) "Eric [son], is a good looking guy and a real pest like me. [...] Like me, yeah. Yeah, I mean he's a joker, you know. (Chester, Photo-Interview)

So, so yeah, I've been very aware of how fortunate I am that I had, you know, that I didn't have the kind of problems that kept me from having social relationships, personal relationships with people that would be willing to, you know, to take me in. And fortunate enough to have people in my life who cared enough about me to take me in. (Monica, Photo-Interview)

Many participants defined themselves in terms of the way they had always been by detailing salient events, behaviors, and feelings, which they thought characterized them. This dynamic became apparent through the repeated use of the term 'always' in the participants' accounts.

And I think, I mean one of the ways that I think I've always coped with difficult feelings is to work, you know, like work in the yard, or to do something physical that I could feel good about having accomplished once it's done. (Kathryn, Photo- 
Interview)

I've always kind of hoarded money [...] Yeah, I've always been very neurotic about money, always. Cause I grew up in a very impoverished home. So I always wanted to make sure that I had some stashed away, you know, for a rainy day fund. So I've always sort of had that. (Tiffany, Photo-Interview)

I like to surround myself with nice things - I have always been that way and now that I am alone I don't have to account to anybody. (Sima, Photo-Interview)

In a similar vein, Frank constructed a favorable self narrative by explaining the changes he had observed as he aged. He was consciously aware of the fluid nature of the self and yet the continuity of self in the process of life. Positioning himself as part of the stream of life helped Frank to feel content with who he is.

I never was a real social butterfly [...] I didn't need people and it became more so as I got older. (Frank, Photo-Interview)

Another way of regaining the sense of being capable and functioning individuals was for the participants to draw on earlier identities and reflect on their accomplishments. "It is important to me to connect with my past. I wanted to visit my childhood neighborhoods and so we did.” (Ruby Photo-Interview)

All participants showed pride in their pre-illness identity; they demonstrated positive self-regard when talking about who they had been and what they had done. Some of the presentation of a past self focused on the participants' identities and accomplishments in the work force. Kevin constructed a positive sense of self by including a photograph of his previous place of work (figure 16).

Chester took a photograph of a framed picture of a crane (figure 17) to symbolize his identity as a longshoreman. Chester was proud that he had continued the family 
tradition of long-shoring and much of his positive sense of self was connected to the profession.

I was about the best crane operator, and I'm not bragging. I was pretty good, you know. [...] Being a longshoreman was a great job. I think it was the greatest job, for me anyway. You know? I'm glad my dad caught me one time to tell me, don't work down at the glass plant, be a longshoreman. (Chester, Photo-Interview)

Karl's positive sense of self was connected to two patents he had recorded while working as a chemist.

And I was fortunate enough to be brilliant enough to have developed two patents for 'em, which got me five thousand bucks a piece. And one of em was when they, well [name of the company] was out here doing all kinds of things with Douglas fir and all that crap. Well, then they developed in the south, and there was pine. Then they tried to make their big beams, and they'd go to test 'em, but they'd just go all to hell. These glues out here would not hold together pine. Well, so I developed a glue. I don't know how or I don't remember what I did, but it definitely glued pine. So that was one of my rewards. (Karl, Photo-Interview)

Kathryn referred to her identity as a mental health counselor by pointing out her

preference of working with adults. It could be implied that being aware of one's potential to change helped Kathryn view herself in a more positive way. "I liked, I've liked working with adults, cause they can make changes if they want to.” (Kathryn, PhotoInterview)

Interestingly, some spoke about their professional identities in the present tense, "I am a nurse." (Rosabelle, Photo-Interview) Others referred to them in the past tense. I was an oncology nurse. (Tiffany, Photo-Interview)

The reference to a past self also encompassed volunteer work. The participants spoke about their contributions in a variety of settings.

I used to belong to organizations, like I was a member of Zonta, which is an organization of women that was started back in Syracuse, New York, in the 1800 's, that's dedicated to education and other ways of furthering the well-being 
of women. And I was pre-, you know, went through all the offices, president in charge of the main fundraiser and all that sort of thing. (Monica, Photo-Interview)

Others constructed positive perceptions of self by referencing their past

recreational activities. Duncan showed that it was important for him to hold on to some aspects of his pre-illness self by taking a photograph of a picture of Mount Rainier (figure 18) and sharing the story of how his carefully planned climb led to a successful ascent.

I wanted to get a picture of Mount Rainier but I couldn't so I took a picture of a photo that was taken by the son of my dentist. So I ended up just taking a picture of the picture. It's important to me because I look at it all the time if you live here and because I kind of fell in love with the place so that I eventually decided 'okay, I want to try to climb that' and then got all that training and then eventually was successful. (Duncan, Photo-Interview)

Similarly, Karl's identity is rooted in his past recreational activities. He is a proud huntsman. The following quote shows that he had not only enjoyed the activity but that he is also proud that he had wisely invested the money he had saved in the stock market.

Oh yeah, I always have enjoyed being in nature, because like I say I was hunting and fishing. I was in the woods all the time. All my buddies and whatnot were in the taverns getting drunk, and we took the same money that they were getting drunk on, unfortunately, and we bought a whole different bunch of stocks and stuff, which really paid off in the long run. So we have quite a portfolio of various investments. (Karl, Photo-Interview)

Another way in which the participants presented themselves in a positive light was to position themselves as part of a long ancestral line. This historical dimension of self was linked to notions of what the participants considered a valuable personality trait. Monica viewed her approach to coping with adversity as a personality trait that runs through her family. "I come from a long line of strong women. You don't quit." (Monica, Photo-Interview)

Kevin took a photograph of a photograph of his family of origin (figure 19) to 
express that he views himself as part of a tightly-knit family system, something of which he is proud. Similarly, Frank took a photograph of a painting hanging in his living room (figure 20) to show that he is proud of his family history, which he can trace back to the Mexican-American War.

Frank welcomed any opportunity to share facts about his ancestral research, which made up a large part of his sense of identity.

In addition, a number of participants produced a favorable self-narrative by referencing their children and grandchildren. "I have five sons - each in his own way is really special." (Odette, Brief-Interview). "You know, I mean, he's [son] just such a wonderful person." (Uma, Photo-Interview). "Now that's my son. That's the person I can depend on. That's the guy. Yes, he's the one. He's his mama's protection, and he knows it.” (Fay, Photo-Interview) “That's Isabelle [granddaughter]. I am proud of her, yeah. It's at the academy, and she's in the Naval Academy, yeah. And she's a pretty girl, too. And she's very smart." (Frank, Photo-Interview)

So that's Ingo [son]. And he is, to me, one of the most unique people I have ever known. And the fact that he's my son, I just feel honored that he came to me and gave my life meaning and purpose and focus. And he was just a god-send. And I respect him. I admire him. But I try not to smother him. [laughs] (Tiffany, PhotoInterview)

Lastly, another strategy that the participants used to establish a positive sense of self was by calling attention to their role as a guide in establishing a roadway for those yet to come. Tiffany used her membership in a support group for people with early-stage memory loss and the reciprocity associated with that role to aid in her quest towards a positive sense of self (figure 22).

By weaving together historic and present expressions of self the participants 
communicated who they were and what they appreciated about themselves and their lives. The participants constructed a positive sense of self through a narrative process in which they stitched together memories of a past, pre-illness self and an emerging, current self that encompassed the illness. Contrasting their historic view of self, of who they had been prior to the onset of the illness with the contemporary reality of who they were now allowed the participants to portray their uniqueness and experience continuity of self.

Assigning the illness a position. Another strength that the participants used to renew positive perceptions of self was to normalize and universalize their condition. ‘Assigning the illness a position' meant for the participants to view 'illness' (including early-stage dementia) as an inevitable part of life and accept the fact that everyone (including themselves) is confronted with adversity. Embracing such a perspective minimized the difference between the participants as 'the ill' and generalized others as 'the healthy'. It allowed the participants to view the illness as an unfortunate but normal and inescapable part of life.

I don't feel sorry for me. I feel sorry for her. I mean I got it, I mean I just, I gotta live with it, so I can't do nothing. I can't cry over it, so, it's how it goes. It's a bummer, you know, but it's, she takes care of me, so she's a doll. So, and I don't feel sorry for myself, I just, course I wish it never happened, you know, whatever, you know. I don't know what it's about, you know. I guess it just happens to some people, disease I guess. (Chester, Photo-Interview)

One way of 'assigning the illness a position' was to face, accept, and adapt to the limitations that the illness imposed and try to do the best possible given the circumstances. “I guess I don’t let it bother me, you know, I keep trying, I keep trying. I have probably always been like that." (Fay, Photo-Interview) "I just live with it and joke about it, because otherwise I cry. So we have a good time.” (Corinne, Photo-Interview) 
Another way of 'assigning the illness a position' was for the participants to remove it from the center of their attention and highlight those areas of their lives in which they considered themselves lucky, fortunate, or blessed. Some participants assigned the illness a background position by considering themselves lucky for being alive and feeling grateful for the life they had lived so far. "I thank the Lord for all my blessings each and every day, and just for letting me wake up to behold another day." (Fay, Photo-Interview)

And we've been, I, we've been so blessed, because we have, always had, we've had, you know, some times that were pretty tough, when Ken was going through this illness that he had. But, you know, you just live with it and go on with life and take a little detour and stay in line. (Corinne Photo-Interview)

Others considered themselves fortunate in terms of family and financial security. "I am kind of lucky especially that I have such a wonderful daughter who has helped me all these years." (Ruby, Photo-Interview). "I think I'm very fortunate. I got a beautiful family, you know.” (Chester, Photo-Interview). "And I am lucky, I have enough money to get by - not rich but not, you know, poverty and then I got my family nearby." (Romilda, Photo-Interview)

'Assigning the illness a position' was also accomplished by minimizing the severity and unpredictability of the illness. "I'm alright. I just forget things, but that's all right." (Irene, Photo-Interview)

Minimizing the perceived impact of the illness provided a sense of security and comfort for the participants because it allowed them to proceed with their lives as if the illness did not exist. They viewed the illness as inconsequential to their sense of self.

I would say that my life hasn't changed much since I have memory problems. It's annoying not to remember something but I usually move on to something else or I 
ask my wife - and she has a retentive memory that won't quit. (Kevin, PhotoInterview)

Reducing the sense of alienation and victimization that accompanied the diagnosis of early-stage dementia by arguing that their condition was not an outcome of life style choices or negligent healthcare was another means by which the participants normalized the illness.

And it's not something I did myself. You know, cause I come from a long line of alcoholics. This isn't alcoholism. This isn't something that I did to me. This is something that is a challenge for me. And I'm going to work with it as best I can for as long as I can. (Tiffany, Photo-Interview)

In addition, many participants assigned the illness a position by highlighting their current independence. Constructing a positive identity meant to see oneself as someone who has functioned, is functioning, and will continue to function more or less independently in the future. "I tend to be pretty independent. So I don't need them to take care of me. I mean, sometimes I might need them to help me remember something, but that's about it." (Kathryn, Photo-Interview) "It's kind of hard to explain but I want to be independent as long as I can.” (Odette, Photo-Interview)

So I think my family really helps to keep my independence, which is very important, you know. And some day maybe I won't be able to do that, and that's just the way it's going to be. (Irene, Photo-Interview)

None of the participants fully accepted the social identity associated with a diagnosis of early-stage dementia as a means of assigning the illness a position. They did not embrace the role as a 'care recipient' exclusively or adopt a lifestyle that would have been congruent with this role. Instead, the participants normalized their need for support by emphasizing the reciprocity inherent in their interactions with family members and friends who supported them and expressed open affection and appreciation. "And she 
does a good job in taking care of me, loving me, and making sure that I get the 'honey-do list' to do and all that sort of stuff." (Duncan, Photo-Interview)

And you know, several of my neighbors have agreed, if they don't see me and Igor [dog] for a couple of days, they'll come and knock on my door. And so I've said, you know, and in return, if you guys want a couple of days off, I'll take care of your dogs, you know, whatever. You know, and they said, well this is our neighborhood. This is what we do for each other. And I went wow. So I feel kind of like, you know, like things are, it really gave me a chance to hone in on the particulars and how good things are right now. (Tiffany, Photo-Interview)

He [husband] has sort of taken over and I just let him - it's easier than arguing with him - 'if you want to cook [laughs] - fine, I'll help you'. I did it for many years, you can do it now. I don't agree with the way you do things but ... (Fanny, Photo-Interview)

Constructing and living a story of 'illness as normal' was one of the strengths that the participants used to achieve positive perceptions of self, which in turn supported their efforts to reclaim well-being.

Contrasting experiences. 'Contrasting experiences' was another strength that the participants drew on to construct positive perceptions of self. To restore a favorable sense of self, the participants compared themselves to other people who they viewed as less fortunate than themselves. "I would imagine it's much harder for somebody with a high status symbol in life to have this [Alzheimer's disease], like a doctor for example. [laughs] [...] It must be terrible for them to get it.” (Irene, Photo-Interview)

Looking to others who they considered 'worse off' allowed the participants to dissociate themselves from perceived similarities and feel better about themselves and their situation. A common theme was the notion of 'being different' from other people with dementia, particularly those in the later stages of the illness who were generally evaluated negatively. The participants felt good about themselves when they established 
an identity hierarchy by highlighting that they did not possess the characteristics associated with the later stages of the illness. The participants referred to themselves as 'not like them', not as far along in the disease process, not as impaired, and not as alone. “I get mixed up with stuff; I am sure I am not as far along as other people are - I don't, I just feel like I get mixed up in different things.” (Fanny, Photo-Interview)

I go to an adult day care program once a week. [...] There are very nice people there and some of the other people who come are a little bit worse off than me so I can appreciate my abilities a little bit as few as they are. A lot of them don't understand, some can't walk and their mental status is poor. That makes me realize that I am kind of lucky especially that I have such a wonderful daughter who has helped me all these years. (Ruby, Photo-Interview)

By drawing a distinction between themselves and people in the later stages of the illness, the participants constructed an 'us-them' dichotomy which not only portrayed the participants' present self as 'other than' but also as 'better than'. The following excerpts of the participants' accounts illustrate this dynamic.

One of the people from the senior center [...] she's real different, she forgets more and more - I hope her family is noticing, I am sure they're noticing that she's a little bit different. And I am feeling good about, really, how my mental capacity - I think I am on hold. (Odette, Photo-Interview)

Odette overtly differentiates herself from another person with dementia whom she encounters in the senior center by discussing her as struggling with forgetfulness and in need of family support. Offering a critique of this 'other' allowed her to construct a positive sense of self by stating 'I am different'. Irene also distanced herself from other people with dementia by pointing out that those who do not have as much support as she has and do not have the benefit of a mother who had taught them well are in a worse position. She further disassociated herself from these generalized other, less fortunate individuals with dementia by discussing them as being unable to maintain a sense of 
personhood and having suicidal ideation.

Well yeah, and when you're not, you know, and you have support, and you, you know. And that's why my mother was so important, cause that's the way she taught about herself, you know. And a lot of people don't have that. And that helps me right now. I mean, absolutely helps me right now. What am I going to do? Kill myself? I don't want to kill myself. But I'm sure a lot of people who have Alzheimer's are thinking about it. Like I'm not a person anymore. But they are, you know? That's really so sad. Yeah. (Irene, Photo-Interview)

Similarly, Corinne differentiated herself from other people with dementia by feeling sorry for 'them' because unlike her, they don't have anybody to care for them. Interestingly, Corinne's concept of self contained both a statement about what makes her similar to other people with dementia and what makes her different.

Jack is just, oh, he is so patient, you know. And just does so much to accommodate whatever I feel like doing, or places to go, and I just think of people who don't have somebody to, that cares. It's got to be so lonely, 'cause you feel yourself going down. (Corinne)

Sima draws a distinction between herself and 'others' at the senior center by pointing out that, compared to these women, she feels much younger in mind.

Because what I see and I don't know if this is an accurate impression but what I see is that a lot of the women [at the senior center] are younger than me age wise but are older than me mentally. Does that make sense? (Sima, Photo-Interview)

Voicing disappointment in the Alzheimer's Association, the very institution that is meant to offer aid and support to people with dementia, also offered a path for the participants to feel good about themselves, as it established a sense of self that is removed from the identity associated with the organization. Kathryn used us-them language to verbally distance herself from those she considers further along in the disease process when she expressed her opinions about how the Alzheimer's Association should change the language of its procedures and policies regarding early-stage memory loss support 
groups.

And, you know, I also think the Alzheimer's Association should be different too, and I don't think that telling people, you know, saying okay we're going to have a support group for you, but by the way, we want you to sign this really demeaning document that we've picked the language for, and written it in the most demeaning way possible. [...] Well the part that, the part that really offended me, and that I said, I think this is offensive, I'm not going to sign this, was that I had to promise by signing this document that I wouldn't poop on their floor. That was the offensive part. I mean, nobody in that group was that far along, I mean this was all early onset Alzheimer's. And these were people who, I mean most of us, I don't know how many, but I think a good number of the people in the group were driving there. But they wanted us to sign this document that promised we wouldn't, they used a different word, but poop on their floor. (Kathryn, PhotoInterview)

Upon closer analysis, this statement is not only a criticism of the Alzheimer's Association's bureaucracy, but rather an articulated desire for differentiation from those 'other' people with dementia who are 'worse off'. With her symptoms being less invasive compared to those of individuals in the later stages of the illness, Kathryn felt she should be treated differently. When asked whether she continued going to the support group, Kathryn replied,

I wanted to; if they would have a discussion, you know, about what's wrong with it, what's offensive in this group of people who have been, two months before, you know, professionals doing surgery on people. [...] You know, doing all kinds of things. But it's just, that's the way it was. And, and I don't need this kind of humiliation, that I feel like crap every time I'm going there. [...] So, I haven't been back. (Kathryn, Dyadic-Interview)

This distancing strategy helped Kathryn alleviate the incongruity between her assigned identity through illness and her preferred identity as a professional. Another strategy that the participants used to restore a positive sense of self was by comparing themselves to people living with other life-threatening conditions whom they deemed 'worse off'. Corinne drew a distinction between herself and those hypothetical others by 
pointing out that their symptoms are more unpleasant and incapacitating than hers.

You know, I don't hurt. You know, I think of those people with cancer that are, they hurt so bad and they want to die. I don't have any of that. You know, so I really feel blessed about that. (Corinne, Photo-Interview)

Karl compared himself to his classmates who had already died and pointed out that, unlike them, he is lucky to be alive. "I look at some of the old pictures back and half of em are dead, I feel I'm lucky.” (Karl, Photo-Interview) 'Contrasting experiences' helped the participants to view their circumstances as less threatening. Self-reminders such as 'it could be worse' were emotionally reassuring and allowed the participants to feel less victimized and more in control of the situation.

\section{Discussion reconstructing identity. Consistent with Lazarus and Folkman's}

(1984) view, feeling good about oneself and the life that one leads emerged as an important factor in reclaiming and maintaining a sense of well-being. Data analysis revealed that early-stage dementia, like other chronic and progressive illnesses, is an experience in which, as Bury (1982) and Charmaz (1983) suggest, a person's self-concept is profoundly disrupted. For the participants in this study the changes in identity associated with early-stage dementia were exclusively negative and activated unfavorable perceptions of self, which in turn disrupted their familiar sense of well-being. As such, early-stage dementia presented the participants with the coping challenge of reconstructing a satisfactory self-image. In line with Moos and colleagues (Moos \& Holahan, 2007; Moos \& Tsu, 1977), who argued that the successful performance of the adaptive task 'maintaining a positive self-image' is a key element of adjustment to chronic illness, reconstructing favorable perceptions of self was a necessary step in creating an alternative sense of well-being. 
Lazarus and Folkman (1984) maintain that internal and external resources play a vital role in the way in which people cope, and a person is considered resourceful "when he or she has many resources and/or is clever in finding ways of using them to counter demands" (p.158). The participants in this study proved resourceful in terms of pursing the adaptive task 'reconstructing identity' and drew on the following four strengths and resources to construct their coping response: (1) focusing on the here and now; (2) restorying the self; (3) assigning the illness a position; and (4) contrasting experiences. Some of the capabilities and capacities that facilitated the construction of a favorable sense of self were readily available to the participants. The majority however existed as competencies that the participants needed to cultivate in order for them to function as coping resources. Using the above mentioned strengths and resources required complex cognitive processes that entailed not only recognizing their potential but also translating them into coping strategies. As the participants practiced their coping skills, each strength or resource not only served as a positive factor in reconstructing favorable perceptions of self but also reinforced the strength or resource itself. In other words, the participants were engaged concurrently in recognizing, utilizing, and cultivating their strengths and resources.

Consistent with the transactional model of stress, appraisal, and coping (Lazarus and Folkman, 1984), all participants used a variety of strategies to cope with the consequences of the illness for self-perceptions and reconstruct a positive sense of self. Some of these strategies were problem-focused, that is they were directed at managing the disruption in identity directly. Examples of this type of coping strategy included finding alternative ways of remembering (such as keeping a daily journal) and altering 
procedures (such as using a calculator when balancing the checkbook). The majority of the coping strategies, however, were emotion-focused, that is they targeted the emotional responses to the changes in identity. Examples of this type of coping-strategy included practicing selective attention (such as valuing remaining abilities rather than focusing on what was lost as a result of the illness), minimization (such as positioning oneself and the illness as part of the stream of life), distancing (such as not paying much attention to the illness), positive comparison (such as considering oneself lucky or blessed compared to others less fortunate), avoidance (such as staying away from thoughts about the illness), distraction (such as engaging in cognitions that draw attention away from the illness), and making sustained efforts (such as consciously harvesting and sharing the stories embedded in one's home and/or personal belongings). Certain cognitive forms of this type of coping (positive reappraisal), in later work referred to as meaning-based copingstrategies (Folkman, 1997; Moskowitz et al., 1996), allowed the participants to see the problem in a different light (such as viewing the impact of the illness as inconsequential to one's sense of self) or wrestle positive values from negative events (such as celebrating the rediscovery of what was previously lost). The palliative effect of these emotionregulating strategies allowed the participants to cope with irreversible changes in their sense of identity by altering how they thought or felt about them. Lazarus and Folkman (1984) argue that coping strategies in and by themselves are neither negative nor positive. Instead, their value depends on the situation in which they are used and the function they serve.

The 'shifting perspective model' (Paterson, 2001) illustrates that at times, people with chronic, progressive illness will focus on well-being and consider themselves 'well', 
assigning the illness a background position in their lives ('wellness-in the foreground perspective'). At other times, they will view their lives through the lens of illness and perceive themselves 'ill', with the illness taking a foreground position ('illness-in the foreground perspective'). For the most part, the participants in this study used the above coping strategies to accentuate 'well-being' and maintain hope and optimism (Dewar \& Morse, 1995) while straddling the paradox inherent in living with chronic illness, which is that, although the illness is distant, the management of its symptoms requires ongoing attention. Traditional staging models of emotion processing in long-term illness (KüblerRoss, 1969) conceptualize healthy coping as progressing from denial to the ultimate outcome of acceptance. Viewed from that perspective some of the strategies that the participants used to sustain their focus on well-being could be interpreted as defense mechanisms and view the participants as 'locked' in this stage. However, the chosen strategies may be an indication that the participants realized that their pre-illness sense of identity was no longer available and that focusing on well-being meant that they had to learn to live with a changed sense of identity, one that included but did not focus on the illness (Paterson, 2001). In fact, the participant's approach to coping served a useful function. For example, when the participants embraced favorable perceptions of self, their behavior included activities that facilitated participation in life and promoted interaction, which in turn increased their sense of self-esteem. The findings of earlier research suggest that the shift from illness in the foreground to illness in the background is gradual and related to the duration of the illness (Loomis \& Conco, 1991). "Over time, the ill person becomes more resilient and increases their endurance by learning to manage the assaults" (Dewar \& Morse, 1995, p. 962). 
The findings of this study suggest that, at the time of data collection, the participants had accepted that the presence of early-stage dementia was unchangeable but that it was possible to regulate the impact of the illness on their sense of self by employing both problem-focused and emotion-focus coping strategies. Although all coping strategies targeted the relationship between the participants and their environment (Lazarus and Folkman, 1984), some aimed primarily at modifying the environment (assimilative coping), others at modifying the self (accommodative coping) (Brandtstädter \& Greve, 1994). For the most part, limiting the effects of the illness on identity involved reframing the situation so that positive perceptions of self could emerge. Jensen and Allen (1994) summarize these processes by arguing that illness can bring about "the recognition and redefinition of one's self, roles, and goals. Considerable energy is expended in re-framing one's place in the world, $[\ldots]$ in learning to live again with a "new me"' (p. 353).

Regaining control. Another theme that emerged from the participants' accounts was the importance of taking charge of the areas of life that still remained under one's control. Having the greatest possible control in shaping one's life was a crucial condition for the construction of an alternate sense of well-being. 'Regaining control' meant for the participants to find positive ways of maximizing personal power and taking back control over their lives in small, task-oriented steps. Through accepting personal responsibility, persistence, and hard work the participants took charge of those areas of life that they considered important and made themselves feel relatively certain of their world. The participants proved to be resourceful in terms of pursuing this adaptive task. The strengths that surfaced as most relevant to regaining control were: (1) managing the 
illness ; (2) calibrating emotions; (3) simplifying life; and (4) negotiating needs. The common thread within these strengths is the importance of being in charge of one's life and how having the opportunity to exercise control over personally relevant life domains is significant to the participants' sense of well-being.

Managing the illness. Actively 'managing the illness' was one of four strengths that the participants drew on to enhance their sense of control. One of the characteristics of early-stage dementia is that committed involvement in medical treatments and healthy behaviors cannot guarantee an arrest or a slow-down in the progression of the illness. As such, efforts to manage the illness focus mainly on developing a sense of control over its symptoms and limiting its impact. The specific resources that the participants used to manage the illness were being informed about the illness and adhering to a prescribed medication regimen.

Being informed about dementia and up-to-date on research was one resource that the participants considered important in terms of illness management. Being informed referred to finding and reviewing material about their condition, medications, and treatments. Illness management required that the participants first educate themselves about the illness and then apply the information to specific situations. A number of participants felt that once they had received their diagnosis they wanted to know everything there was to know about their illness. They felt that having a 'label' allowed them to actively seek information about the most up-date research and the latest treatment options. Knowing that they had explored all options open to them and learned what is 'normal' or 'to be expected' provided a sense of predictability. Some participants relied on public libraries to find relevant information. Others took advantage of information 
available on the internet. Yet others attended programs offered through the Alzheimer's

Association and other organization. For the majority, it was a combination of all.

Yeah I read about Alzheimer's disease. In fact I've got one book on order right now. It's just a brand new one that I heard about. [...] But yeah, I get, yeah, I read everything I can about Alzheimer's, and some of it is good, and part of is just yuck, you know. But yeah, but, and I do a lot of online research too. Yeah, I find it important, you know. [...] And I get stuff from the Alzheimer's Association every day, and I get stuff from all different sources. I don't do just that on the computer. (Corinne, Photo-Interview)

Kathryn particularly appreciated publications by people with the illness.

Richard Taylor's was absolutely the best book and I probably read - I don't know at least six to eight books that I could find about Alzheimer's when I first was diagnosed and I, what I appreciated most was his, he's got a blog - that's free. (Kathryn, Dyadic-Interview)

Uma found it helpful to watch dementia-related movies. "I'm actually watching

an Alzheimer's movie right now." (Uma Photo-Interview)

Reading up also helped to determine whether their symptoms were related to the medications they were taking or the illness itself. The participants became more critical of their prescribed medication because they were able to evaluate the balance between side effects and perceived value.

Now the Aricept, when I got started on that, I was told to take it at night, but I was having Cecil B. DeMille dreams. I mean, they were so involved and so intense that I would wake up from them. And so I wasn't getting any rest. So I read about it and talked to the pharmacist, and he said, 'why don't you start taking it in the morning?' I thought okay, I'll do that. So I take it in the morning. And so it, I feel like it really keeps me alert. But by the evening it's sort of worn off. So I still dream, which I know is important, you know, cause that says that blood flow is getting to those areas. After I'd been on the Namenda for about a month, I felt like I regained a lot of time. (Tiffany, Photo Interview)

One participant felt that staying informed was important because she wanted to be the first to know about a cure. "I read everything I can about Alzheimer's, because I'd 
love them to say we finally found a cure! [laughs] But I haven't found anything like that yet." (Corinne, Photo-Interview)

Gathering information about the illness also included seeking organizations that provide services to people with dementia. Support groups and educational seminars were considered important educational resources and played a vital role throughout the entire adjustment process. One participant reported that the moment she received her diagnosis she started looking for information to help her take charge of her situation and make decisions for the future.

I went to a support group from the Alzheimer's Association; I went to the class they had at Saint John's church because I felt it was pertinent that I do it because I was really concerned and I needed information so I could plan for the future. (Sima, Brief Interview)

Drawing on experiences of others with dementia was another resource that the participants drew on to find out what worked.

I told her [doctor] that I had read this article about this medication that was, it wouldn't get back any memory that you'd lost, but it would keep, it would stop memory loss where it was. And I think, it was a drug I couldn't take at the time. I can't remember what it was. It started with an A. [...] Yes, yes, Aricept. And I couldn't tolerate that. And then my longtime friend in Richmond, Virginia - we talked on the telephone fairly often - she told me that she was taking Celexa. And so I had my doctor, talked to her about that and she gave me a prescription for that. And I've been taking that and I have had no memory loss. And I'm pretty clear on things. (Rosabelle, Photo-Interview)

Lastly, one participant hoped to learn about the illness by participating in this study. "I hope you will teach me some more about the mind problems, and mental, memory problems that I need to know as I watch my own mind gradually disintegrate." (Sam, Photo Interview)

However, a few participants shared that nearly all information about the illness 
and its management was primarily written for caregivers. "There is only one [online] group I belong to, are people that have it. DASNI it's called. But the rest of them are not written for people that have it. But I don't care; I read it anyway." (Corinne, PhotoInterview)

For some this literature was frightening to an extent that they decided to stop reading, as the information about the progression of the illness was too much for them to handle.

I have read about Alzheimer's - but it's a puzzle - in fact it scared me. So I thought, I'd better stop reading. As you get deeper into it - so I just wait for it to happen and don't worry about it. Live day by day. (Fanny, Photo Interview)

Adhering to a prescribed medication regimen and following through on recommended treatments was one of two resources that the participants drew on to manage the illness and was considered an important means to achieve well-being. All Participants followed strictly the advice or instructions of their healthcare providers. "I take my pills religiously." (Odette, Photo Interview)

Although the participants were aware that the currently available dementia drugs do not cure the illness, they were appreciative of the improvement in their quality of their life as a result of taking these drugs. A number of participants claimed that their very ability to participate meaningfully in life was due to these drugs. Tiffany shared, "And I was able to get started on the medications early. I think that really saved me." (Tiffany, Photo-Interview) Monica expressed this concept by taking a photograph of her pill bottles (figure 23).

Several participants felt thankful that there are better options for treating dementia available today than ever before. 
Oh, I think it's wonderful these kinds of drugs because my mother had such tremendous memory loss. I mean, she reverted all the way back to childhood, and having had a very painful childhood, she was just, kind of in a state of anger and misery all the time, which was really sad to see. (Rosabelle, Photo-Interview)

Although all participants were appreciative of the potential benefits of these drugs, the way in which they were involved in managing these medications differed. For some participants it was very important to be informed about and participate in the management of their medication. Uma took a photograph of her pill bottles (figure 24) to show that she takes great pride in being able to fill her own prescriptions and oversee her day-to-day medication intake.

I took a picture of my pills. Something I have to remember every day. Well actually I have to remember to take two pills in the morning, and, well actually three pills in the morning and four pills every night. (Uma, Photo-Interview)

Others found it helpful to collaborate with family members. They preferred that their care partners fill their prescriptions but took pride in and drew a sense of satisfaction from filling their medication manager and remembering to take their daily medications. Frank took a photograph of his mediset (figure 25) to show the active role he plays in managing his medications and noted,

Yeah. Karen [wife] goes and gets the prescriptions. My daughter, who is in this kind of thing kinda, gave me the box, or told me about it. And so this is morning, and this is evening, and it's a big deal; I take seven in the morning and then another one of the ones in the morning in the evening. However, it's easy to forget the evening one. But I am supposed to remember, see? (Frank, PhotoInterview)

Still others trusted their physicians and care partners to make the right decision about their medications and deferred to their judgment. These participants felt unsure about the different medications they were taking and considered medication management not important enough to waste energy on. Chester took a photograph of his pills (figure 
16) and shared, "She usually helps me, watches me anyway. She watches me and stuff about the pills. She sets them out, you know. [...] Yeah. It helps me, I guess, you know, the pills." (Chester Photo-Interview)

A number of participants said that finding the right drug and/or combination of drugs had required a period of trial and error. "I'm doing better. The Namenda, I'm almost at full strength on that, and I have noticed a difference since I started that in addition to the Aricept." (Monica, Photo-Interview)

Some participants were interested in both conventional and non-conventional dementia treatments. Alternative or complementary therapies ran the gamut from vitamins and herbs to eating special foods.

And I think oh, you know, and you'll hear something like, 'drink 36 ounces of orange juice' kind of thing, and if it doesn't hurt me I'll try it. Or you know, whatever it would be. But if it's something really far out, I won't do it, but if it's just regular stuff, I'll do it. (Corinne, Photo-Interview)

Actively managing the illness was one of four strengths that the participants drew on to enhance their sense of control. Illness self-management helped the participants curtail the threat to their taken-for-granted assumptions about the future as it minimized uncertainty and maximized certainty. Regaining control through illness management meant to be persistent and diligent. By knowing everything there is to know about dementia and adhering to a prescribed medication regimen while hoping against hope for a cure during their lifetime the participants were able to take back control in small, task-oriented steps. Being relatively certain of their world allowed for an alternate sense of well-being to emerge.

Calibrating emotions. Another strength that the participants drew on to exercise 
control over their lives was to take responsibility for managing their feelings and being mindful of their overall mood. Rosabelle captured the essence of this strength when she explained,

I mean, you know, you know you have just one life, right? And you have the, you're in control most of the time. You can make decisions about whether you live it well or sink into it and live it badly. And knowing that I, in my head that I have that choice, I guess I'm some-, something of a philosopher, because if the choice is mine and I mess it up, that's mine too. I'm, I would rather have it go well, not just for me, but for everybody around me. (Rosabelle, Photo Interview)

'Calibrating emotions' speaks to the notion of acknowledging and accepting that the quality of one's emotions impacts thinking, desires, values, and goals and influences one's overall sense of personal power. The participants felt they have the power to choose their attitude and the responsibility develop a habit of maintaining a positive outlook. "Well I always try to look on the positive side of things." (Uma, PhotoInterview) The participants utilized a number of resources that they found helpful in the process of calibrating their emotions including choosing an attitude, managing feelings, and benefit finding.

All participants emphasized the general notion of having a positive attitude as a way of coping with early-stage dementia and its effect on control, certainty, and predictability. They felt that they were in control of their emotions and that it was their responsibility to make necessary adjustments in their thoughts and behaviors so that they could think positively. “I guess I don't let it bother me, you know, I keep trying, I keep trying to stay positive.” (Fanny, Photo-Interview) Feeling well was related to being mindful and maintaining a life-affirming state of mind.

'Cause I want to be positive about this. I've always been a positive person. I want to enjoy this time of my life when I don't have to work, when I can get my little 
Orca card and go someplace that I've never been and don't have to worry about coming back and cooking dinner for anybody or, I don't have responsibility to anybody else other than me and my dog and my cat. And it's so freeing that I feel like most of my life I've had to be responsible. And now it's all about me. So I'm enjoying this time. So for however long it lasts, I'm enjoying this time. (Tiffany, Dyadic-Interview)

It involved initiating a change of perspective and developing the habit of maintaining a positive frame of mind. "I just try not to let it bother me, so... and keep happy... especially on a nice day like this, yeah." (Frank, Photo-Interview) It also entailed choosing to have a positive outlook for the future. "Anyway, life goes on. [...] I am aiming for a hundred." [laughs] (Odette, Photo-Interview)

Trying to achieve a greater emotional balance was a process that ebbed and flowed. 'But, and you do get frustrated. So you just can't, I mean I'll just have a fitty, a fist, a, I'll just go, goddammit! Then I go, that's enough of that. But anyway it's not bad." (Irene, Photo-Interview) In the participants' opinion, allowing and processing negative feelings was important but dwelling on worries served no good purpose. "So sometimes I do do a pity party for myself for a little while and I go 'oh, get over it girl'. It's not gonna change unless you do something." (Monica, Dyadic-Interview) Although the participants moved back and forth in their level of acceptance and adjustment, they were motivated to cope with and overcome their negative emotions simply because it was the right thing to do.

I had some pity parties there for a while. Poor, poor pitiful me. Then I thought, okay, get on with it. You know, don't sit around feeling sorry for yourself, and life isn't ending, and you got a dog to feed and you got a kid to raise and you got stuff to do. Get on with it. (Tiffany, Photo-Interview)

An attitude of personal responsibility becomes also apparent in the following quote in which Uma describes her efforts to make adjustments in her thoughts and 
behaviors. She made conscious efforts to avoid patterns of thinking that had negative effects on her.

I try to remember to always walk around with a half-smile on my face, you know? So I'm not, I try not to be one of those stony-faced people, you know? I try instead to look like I'm pleased with myself and my life. [...] I always try to look on the positive side of things. (Uma, Photo Interview)

Many participants attributed their perseverance and ability to cope with negative

emotions to a lifelong habit of coping with adversity. "I didn't have a good childhood so I learned to, you know - what do you call it - you can dwell on it - you make the best of everything you can.” (Fanny, Photo-Interview)

I have pretty much handled things that way all my life, yeah. You do what you have to do, but you have, you know, you're not going to let it [the illness] take over. I laugh a lot. I choose to. Yeah. And I'm, I'm happy, you know, where... And sure I have times when I just cry like mad. But not very often. I just choose to be happy, and make life fun. (Corinne, Photo-Interview)

Threaded through all accounts was the notion of individual responsibility for the quality of one's emotions. The participants felt that they had the power to adopt an optimist stance and develop positive ways of thinking. In other word, 'choosing an attitude' was a coping strategy that entailed making adjustments in thought and behavior.

A number of participants experienced 'nature' as a mood lifter, at least to some degree, and as helping them to tolerate the limitations imposed by the illness. Spending time in nature elicited positive emotions and was one of the ways in which the participants rediscovered their love for life.

And I'm, have been prone to depression at times when things aren't going well. I always feel like it's my fault, you know? And so if I can go out and walk, it really helps me to shed that feeling. And helps me to love life. (Rosabelle, PhotoInterview)

There seemed to be something peaceful about working in and with nature. It 
showed the participants that even in the chaos of life, there is a natural flow, a sense of order and helped them achieve a sense of accomplishment.

And I think, I mean one of the ways that I think I've always coped with difficult feelings is to work, you know, like work in the yard, or to do something physical that I could feel good about having accomplished once it's done. (Kathryn, PhotoInterview)

Some participants commented on the influence of colorful flowers on mood. They felt that simply looking at flowering plants reduced feelings of stress and fostered positive emotional reactions. Irene took two photographs of the flowers on her deck (figures $27 \& 28$ ) and commented on the emotional influence of blossoming flowers. Enjoying colors in nature increased her sense of happiness.

Similarly, Sam reflected on the balancing quality of nature images by taking several photographs of his container garden (e.g., figures $29 \& 30$ ). For him, looking at his container garden of flowering plants was one way of coping with lingering negative emotions following conflict. It reestablished a sense of calmness, inner peace, and overall tranquility. Kathryn also commented on the balancing effect of nature images. She captured the view of Green Lake out of her bedroom window (figure 31) to show that seeing nature every day increased positive emotions.

Oh, and then this is out of my window upstairs in my bedroom. This is my, part of my view, anyway. And you see I can see Green Lake. And, you know, I've always just so liked that view. I mean I can lay in bed and enjoy the view, and it's a, you know, in the winter when it's all snowy and it's just, it means a lot to me. [...] And the reason I took this [photograph] is because the sunshine in the morning is so helpful. I know it helps my mood, and it makes me a lot more motivated to you know, get up and get ready to walk and, you know, get out of the house. (Kathryn, Photo-Interview)

Others experienced positive emotions when spending time alone in nature. They described the effects of observing nature's patterns as rejuvenating. It helped them focus 
on the present and experience a sense of peace and harmony.

Sometimes, when I go for a walk, I just look at things on both sides of me. And I love to look up at the sky and see the clouds. And the, anything, anything that is, anything involving nature, which is everything around me, I somehow, I just kind of seem to sink into it. And everything else goes except what's, what I'm seeing around me. And that's a lovely feeling. (Rosabelle, Photo-Interview)

Other strategies to manage negative emotions focused on finding activities that redirected attention or made light of the situation. Kevin reported that one activity that helped him to feel good was watching favorite TV shows or interesting movies.

I watch a movie when I get into those dark places. I think Netflix has been really helpful and if it's not raining, I can do something in the yard - but to just have to be in the house - I just get antsy. (Kevin, Photo-Interview)

Rosabelle felt that listening to classical music and reading helped her relieve some stress and balance emotions. "I love classical music. Sometimes I listen to music as a way of turning my head around, and reading." (Rosabelle Photo-Interview) A number of participants used humor to alleviate the pressure of negative thought patterns and reframe them into more positive ways of thinking.

But, you know, but you just make the best of life. You could either sit down and cry, not that I don't, but, but I just choose to laugh about it and make fun about it and have my friends tease me, and you know. (Corinne, Photo-Interview)

Managing feelings was an important strategy that the participants used to cope with early-stage dementia and its negative impact on feelings of control, certainty, and predictability. A number of participants discovered that spending time outside or looking at nature images even from the confines of their home fostered positive emotional states. It increased their curiosity about life and rejuvenated their will to live and feel well. Other means of achieving positive emotional states were watching favorite TV shows and movies, listening to music, and humor. 
Another resource identified by the participants as beneficial to viewing their situation favorably was to highlight the positive changes that had come into their lives as a result of the illness. For some participants, the limitations imposed by the illness gave occasion to realize and appreciate all the things they previously had taken for granted.

And so, so these elements of my life that bring me contentment, you know, being at Lummi or walking around Green Lake, or you know, looking over the horizon on a sunny day or something like that, is, I don't take it for granted anymore, you know, it's like it's so important, and I just feel like I'm trying to suck up gratifying activities at this point, you know. (Kathryn, Photo-Interview)

For others, the experience of living with early-stage dementia had brought about a greater capacity to be understanding of the hardship of others.

I have no pain! You know, and I think of our friends that have cancer, and oh, I just, we've got one that, they've got a feeding tube that they've got to pour stuff down this feeding tube, and oh, I think, oh, I'm so blessed. (Corinne, PhotoInterview)

Yet others felt that the changes that the illness had introduced into their lives had forced them to focus on the most important things in their lives.

I ain't gonna cry about it. I mean, I don't want to be glory about it, what I have. I'd say I'm, I'm feeling very lucky I'm alive. I feel lucky for my health. Lucky I have a beautiful wife. I don't know if it's lucky, but you know. You know, I don't, you know, I don't mean to be, that's nasty saying lucky. [...] But I'm happy with my life. I have fun. I mean, I enjoy life. I enjoy laughing and giving people a bad time, just a little bit bad time, as a joke, you know. (Chester, PhotoInterview)

Some participants felt that as a result of the illness they had changed for the better as human beings. One participant, who had been forced to retire due to the onset of the illness, felt that she had experienced positive personality changes. She was grateful for the opportunity to do a lot more thinking, be more peaceful and less perfectionistic, and focus on her own needs rather than those of others. 
I feel like I'm kind of living my life backwards. You know, I left all of that behind, and now I'm starting my life again. So it's kind of like a new beginning. $[\ldots]$ So this is a really good moment in my life, and isn't it weird that it took this diagnosis to get me to focus my attention on myself? (Tiffany, Photo-Interview)

Looking for any tangible positive that had come out of the illness experience was a useful coping approach for the participants in this study. Making intentional efforts to acknowledge these benefits assisted the participants in recognizing personal growth in patience and empathy and having a greater appreciation for life and a better sense of what really mattered. 'Calibrating emotions' was a strength that helped the participants regain a sense of control over their lives. The participants by and large felt responsible for the quality of their psychological states and made efforts to avoid patterns of thinking that had negative effects on their emotions while developing the habit of staying positive and maintaining a good attitude.

Simplifying life. 'Simplifying life' was another strength that the participants drew on to regain a sense of control. Simplifying required breaking up previous life structures and establishing habits and life patterns of a thinner texture and lighter complexity. Dissolving fixed designs and creating simpler patterns was not easy because it had a flavor of failure and a sense of growing dependence. Nevertheless the participants embraced the task with a certain enthusiasm and found the following resources useful in simplifying their lives, being proactive, cultivating routines, and reducing belongings.

Being proactive was a resource that the participants found helpful in simplifying their life. The participants took control of their lives by actively managing their memory failure. They used compensatory strategies to keep themselves on track to meet social demands and attend to their own needs. Many of the participants developed or maintained 
the long-term habit of writing themselves reminders such as Post-It notes and/or to-do lists. "I write notes, you know, when, for example 'Thursday, Jutta's coming', you know." (Frank, Photo-Interview)

To be effective, the reminders had to be strategically placed or carried around and contain just enough detailed information to keep the participants on track. Writing reminder notes about everyday activities alleviated the stress of having to remember them or worrying about forgetting them. It also focused attention and eliminated some everyday distractions. Kathryn considered Post-It notes an integral tool in her everyday life and links her current use of reminders to her longstanding habit of writing notes to herself.

And I, I've always been somebody that writes myself notes. And I think I do it more now, and I think the notes have a little more detail now. But that's just a habit I've had forever. [...] Every night - because I have trouble more in the morning when I wake up - like what was I going to do today - it takes a while for it to come around again. So, at nighttime I write out my schedule on a little post-it note - the date, the day of the week - I have trouble keeping track of what day it is - and stick it at the side of my bed - so it's the first thing I see when I wake up. (Kathryn, Dyadic Interview)

Sima took a photograph of her to-do list (figure 32) and explained how she creates to-do lists to simplify her life and add order.

I write my to-do list in the morning and I take it with me. For example, if I have to go to more than one place, I put down all the different things that I need to do. For me, writing down helps me remember. [...] I also started making a list when I go seeing the doctor of what I wanted to talk about. (Sima, Photo-Interview)

Almost all participants used some type of daily planner and/or calendar to compensate for their deficit in memory functions. It helped them remain organized and establish a record of their daily doings. Irene took a photograph of her calendars (figures $33 \& 34)$ to describe her complex system of keeping two calendars, one at home and the 
other in her purse. She shared,

This is my desk calendar. That's where I put down my appointments. Mhmm, it's sitting on the desk, as a, like that. So it's right, yeah. So I have it for the whole year. So, so I can put in the things that are coming up in those spaces, you know. And I keep em, just to remind me what I did do. But anyway, so I have that. So I always have a good calendar on my desk. [...] And I have one in my purse, yeah. So if I'm someplace and I, you know, I make an appointment or something like that, then I write it into the one that I have in my purse. And then it will go onto my calendar at home. [...] Yeah, and sometimes it gets confusing, but you know, I usually can find somebody to help me straighten it out, yeah. (Irene, PhotoInterview)

The above quote illustrates that although Irene is diligent in her effort to be independent she is also aware that at times, she is in need of help. Romilda photographed her daily notebook (figure 35) and her calendar (36) to describe how she uses a combination of both to keep her life manageable. Whereas her calendar provided room for noting important appointments and keeping track of other obligations, her daily notebook served as a catch-all for the usual randomness of life - to-do lists, reminders, thoughts, ideas, and doodles.

My Daily Diary - I jot down anything that springs to my mind whether it is shopping, taking a library book back, writing a letter, a lunch date - whatever. Everything goes in that - pick up meds. [...] I have to have a reminder. For example, if you ask me what did you do on the weekend - it's sort of "oh, what did I do on the weekend?" It's kind of a blank what I did. And that's where the diary and the calendar help a great deal. (Romilda, Photo-Interview)

Monica included photographs of her daily planner (figure 37) in her portfolio to express a similar sentiment. She shared that she uses her daily planner as both a forwardlooking planner but also as a backward-looking diary. A short calendar entry helped bring back to life where she had been and what had happened. It enabled her to have a comprehensive and detailed record of her life.

My daily planner is there to go back to, so I don't have this feeling that today is 
happening but everything behind me is gone. [...] And I don't really know what is going to happen going forward - you know, other than what's in my daily planner and so, that gives me some feeling of continuity. I can look back and I can look forward - both ways - with that daily planner. Because, I used to have a really good memory and even after I quit working for company they would call me and ask but now I can't even remember something without notes that it happened. And it's hard to lose that. (Monica, Dyadic-Interview)

This quote shows that simple tools such as daily planners and calendars can become powerful instruments of personal agency. That daily notebooks were more than a tool for keeping track of what to do and where to go became also apparent when several participants referred to their notebooks to find the threads with which to weave their stories during the interviews. Daily notebooks provided a sense of continuity by keeping a record of the participants' daily lives and personally relevant information. For some participants it was just as important to go back over their notes as it was to write them in the first place. They reread their notebooks on a semi-regular basis to find a sense of continuity, notice patterns of behavior, moments of triumph, or points of change.

I make notes for everything. I've been using a notebook like this for years and years and years - maybe $20-25$ years. And it's - it goes from - it has the last bit of August and it goes to the next August. Most of our [religious] life runs from September to September. We don't really have that many appointments in the summertime. And I write everything down. See like I have to pick up my friend for temple services - she broke her wrist and can't drive - and I just make note of all my appointments - everything. And then I like to review my notes and look at what I've done and plan something new. (Sima, Photo-Interview)

Keeping a daily notebook also helped bring focus and clarity in terms of decision-

making. It helped the participants decide on which action to take or option to choose.

And when, like here I needed to go to the social security office, and so, you know, I have the information that I needed to find out and the directions and everything are in here. And this is notes about what to do about whether I'm going to have a garage sale or not, you know, what are the pros and cons of whether I'm going to do a garage sale. And talking to the people at my attorney's office. And I'm looking into doing a special needs trust with my back social security money. And 
so the notes from the attorney that I talked to about that, and, you know, so. (Monica, Photo-Interview)

In addition to handwritten notes, several participants used the alarm function on their cell phones to create reminders. Monica took a photograph of her cell phone (figure 38) and noted,

I set my cell phone alarm throughout the day for different things. I have permanently set for my medications, morning and night. Always it goes off at $8 \mathrm{am}$ and 8pm every day. And then I can set three alarms on here, and so the third one I use for different things throughout the day, you know, to remind me of an appointment coming up and that sort of thing, yeah. Monica, Photo-Interview

Anther innovative way of remembering to do things was to create visual hints. Romilda took a photograph of a shopping bag hanging on her front door handle (figure 39) to show one of the visual cues that she used. Similarly, Sam photographed his bathtub with a bathmat (figure 40) to show one of his visual reminders. For him, the position of the bathmat indicated whether or not he had taken a shower that day. Some participants considered family members and friends as an important resource in terms of reminders. They found it helpful when others double-checked that all events were added to their calendars and reminded them on the day of the event.

Cultivating routines was another resource that the participants drew on to simplify and take charge of their lives. If we understand a schedule to be a plan that structures a day by time, activity, etc. and routines to be the repeated physicat execution of the schedule, then if a schedule is consistent, it becomes a routine and after some time, a pattern is established. Through repeated performance of a consistent schedule (routine) a person becomes accustomed to what will or will not happen next. This way, there are not too many unknowns. Once a pattern is set a person can infer, for instance, that lunch will 
be at 11:30 am after completing the daily household chores. In other words, having a predictable schedule fosters a sense of certainty, which in turn decreases feelings of anxiety and worry. On the contrary, if a schedule is unpredictable and it is uncertain if there will be lunch and at what time, there are many unknowns that provide ground upon which anxiety and worry build.

Adhering to schedules and routines establishes a sense of security that comes not only from knowing what to expect but also from knowing what has already been accomplished. In other words, eating at about the same time every day will not only help a person understand when the next meal is coming, but also that the last meal has occurred. He or she can be certain that they have eaten without having to remember it. A schedule establishes the security of having eaten. Given the uncertainties associated with early-stage dementia, maintaining consistent schedules and routines was important for the participants. It decreased their sense of anxiety and worry and increased their sense of comfort by letting them know what was coming next. Karl described the sense of security he derives from the predictability and consistency of his routine by saying,

I am very routine. I am in a set way and I do walking with the dog and what not and don't really do much out of the way. Occasionally or so we'll go and see my brother across the mountain or something but everyday is very similar and I enjoy it. [...] I just feel very fortunate that I am able to enjoy my days and I don't have to worry about 'what the hell am I going to do tomorrow?' - Wake up alive - I hope (laughs) but I mean I am just - I am just routine and my life might bore somebody else but I am happy with it, I am surely happy with my life. (Karl, Dyadic Interview)

Although the routines differed from person to person, all participants maintained schedules that provided structure for their days. Routines around mornings, evenings, meals, chores, and personal care offered the repetitiveness needed to succeed. For many 
participants a morning routine was especially important. Kathryn included a photograph of her morning walking route (figure 41) to show that going for a walk every morning helps her set the mood for the day.

So I want to call this picture, 'calibrating my day'. So this is my, you know, early morning walk in this peaceful place where these, you know, where I've sort of made friends, just walking around, I don't know them very well, but they're such a regular, predictable part of my day. And they're warm and friendly, and there's something just very, something very reassuring or comforting or, you know, whatever about that ritual of walking and seeing the same people. And it kind of feels like home in some way. (Kathryn, Photo-Interview)

Uma took a photograph of three dogs (figure 42) to show that her morning routine not only establishes an orderly and efficient atmosphere but also fosters a sense of responsibility for the day. "But you know, then the first working thing I do is I go walk these dogs $[\ldots]$ it's like a good way to start the day, you know?" (Uma, Photo-Interview) Tiffany included a photograph of her dog 'Igor' in her portfolio (figure 43) and described how having a morning routine that included her calendar served as an anchoring event.

I need to know what, I don't know why, but I feel like I need to know what day it is. So I stand in front of my calendar and, you know, it's one of those big wall calendars, and I tell Igor [dog], you know, today is such and such a day, and this is the date. And that helps it stick for me. [...] Every morning. So he always knows what day it is. [laughs] (Tiffany, Photo-Interview)

One of Sam's photographs (figure 44) illustrates how doing something consistently - even if it is a seemingly mundane and ordinary act such as admiring the view out of one's window - can become an important routine. He included a photograph of Mount Rainier in his portfolio to show that for him looking at the distant mountain range is an integral part of starting the day. "It's a picture of my volcano. I have a volcano outside that I look at every morning on a clear day." (Sam, Photo-Interview) 
However, adhering to a routine did not mean to have no flexibility. Most participants followed a relaxed routine, a routine that had a basic structure and yet allowed enough freedom to vary activities. Monica took a photograph of a newspaper (figure 44) to express the important role that a relaxed routine plays in her life.

Well, I get up and eat my breakfast and do my UV light and read the paper and do the crossword puzzle. And I always, first thing in the morning, when I get up in my room, I check my book, you know, my planner, to see what I have for the day. And then I check it again as soon as I'm done to make sure that I remember what I looked at. And then some, the rest of the day doesn't have a solid structure, although it's something that I'm working on. Basically, I have things that I want to have happen, and that's the eating of the three meals, to make sure that I'm, you know, eating properly, and working out. Monica, Photo-Interview

Rosabelle described her relaxed routine as having some basic structure that allowed her enough freedom to vary the time for her daily walk.

When we're here, when we're going to be here all day, we, of course our morning routine is to have breakfast, coffee. And then I do whatever housework I need to do after breakfast. And I, sometime during the day I go for a walk. Usually it's either before or after lunch. (Rosabelle, Photo-Interview)

Marking the end of the day with an evening routine was equally important for a number of participants. Frank included a photograph of his fireplace to show the importance of his year-round routine of making a fire at night. For him, sitting around a fire with his wife was something greatly pleasing and helped him transition out of the day and relax. "Oh, we have the fire, a beach fire every night, always. And we'll even put an umbrella out if it's raining and sit by the beach fire and talk. It's peaceful; it's an important part of our evenings." (Frank, Photo-Interview) For a number of participants TV shows served as building blocks for structuring the day. For Sima, her simple, everyday routine of watching Nightly News helped her transition to a restful night. "I have dinner with Brian Williams every night.” (Sima, Photo-Interview) 
Schedules and routines also provided structure, which ensured that things were getting done. To establish structure, many participants prepared detailed lists of what needed to be completed each day. The participants referred to these lists as daily life logs or charts. These charts captured relevant information relating to the performance of personal tasks and activities. Karl took a photograph of his daily chart (figure 46) to show how by establishing his own unique routines, he kept a record of his personal tasks and ensured that progress was made in what he considered most important. In addition, by documenting important events of the days, he created a record of his current life, which also served the important function of a backward looking diary.

And this is my chart, the little list of things I have to do. Okay, when I, the pills, this is my pill taking. This is when I had my, pardon the expression, daily crap. And this is, I forget what the hell that...right this when I take a walk, I usually write it down - where I went. You see here there's Des Moines or whatever and whether Betty went with me. Sky was blue, tide was out, just the kind of crap which I don't really need to know, but... That's all in there. Yeah, this is my, what do you call it, back, whatever. Between that and the clock and the thing, you know, I try to organize, because with Alzheimer's I'm so screwed up, I need to. (Karl, Photo-Interview)

However, establishing and maintaining a routine involved diligence, persistence, and self-discipline. Practice was critical to any routine and it usually took several weeks for these to become firmly established. Monica took a photograph of her daily life log (figure 47) to show her rather sophisticated charting system. She shared that she had started the practice of keeping a life log because it was important to her to be able to track the completion of personal tasks. Her life log also served as a place where she noted important moments and events as they occurred throughout the day. As part of her evening routine Monica wrote a diary, which included looking at the day's notes and fleshing them out. Over time Monica's life log became a tool that she used to analyze 
how she spent her time, what changes she needed to make, and what goals she wanted to establish. In fact, keeping a life log was one of Monica's major goals. It was a commitment that she presumed would serve her well during the later stages of the illness.

"So I've been doing my life log for at least three and a half years, I think. Maybe it's only three. Doesn't matter. Somewhere around three. And there would be, in the beginning there would be two and three, four days at a time where I didn't fill anything out, or I'd do breakfast and then there'd be nothing for the rest of the day. And so I had no way of remembering whether I had eaten, you know, whether I had taken my pills and that sort of thing. And I was, there's this part of my brain that doesn't quit, you know, that won't give up. And by sticking to this, I got so that, so that my blanks are only one or two days, you know, they're not most of a week. And I haven't had, and now the blanks are only like part of a day, like I'll forget to mark down whether I had dinner or not. But it's become such a habit now, that even as I get worse, I know I'll keep doing it, because it's just, it's become a daily part of life. Yeah. So... [laughs].” (Monique, Photo Interview)

The above quote illustrates that Monica is clearly aware of how profoundly the illness had changed her life. She seemed, however, to have made the deliberate decision to remain an active agent in her own life and worked hard in doing so. The quote also shows that regaining control is not simply a matter of implementing coping strategies. Instead, there is often a real struggle to resist the forces of the illness. It takes time, patience, and diligence to implement new patterns and rhythms of living. Success in this instance is about consistency, and one way to establish consistency is through daily routine and habit. The quote also highlights the central theme of this study, namely the complex co-occurrence of striving towards well-being while acknowledging one's inevitable decline. Routines around mornings, evenings, meals, chores, and personal care built structure into the participants' days and offered the repetitiveness needed to succeed. Routines were the backbone of the participants' daily lives. "And I'm, well, set in a rut, I guess, but it's the way that I want it to be. I don't want to change." (Karl, 


\section{Photo-Interview)}

Routines created an environment where small victories could be celebrated as they provided proof of what had been already accomplished and that new habits could be learned. This security helped the participants learn to trust that they have some control over their lives and increased their confidence in their own ability to change things. The variety of resources and the creativity with which they were used indicates the determination that the participants brought to the process of regaining control.

A number of participants, particularly those living alone, felt that it was important to reduce their personal belongings and eliminate non-essentials. Sima took a photograph of one of her bookshelves (figure 48) to show that she was in the process of downsizing. Similarly, Odette expressed plans to take inventory of items in and around her house and reduce her belongings.

This [motioning to the living room] is such an embarrassment [laughs] because I got so many things. I got stuff I need to get rid of and give away and make up my mind what I am going to do with it, you know. (Odette, Photo-Interview)

By being proactive the participants were able to be in charge of their everyday life and thus meet the demands of their personal and social world along a varying continuum of support.

Negotiating needs. 'Negotiating needs' was another basic strength that surfaced as most relevant to regaining control. To reclaim a sense of well-being, the participants needed to negotiate many things with many people and used the following resources to do so: asking for help, feeling empowered, securing material resources, securing access, and planning for the future.

All participants were aware of the seriousness of their condition and conscious of 
their need for support. There were a number of things they could no longer do alone.

Nevertheless, seeking help and learning to say yes if others offered was not easy as most participants were used to handling things themselves.

Well I think people, I mean, I don't know if it's that they feel like they have to take care of me or not. I'm not a very good, I'm not very good at being taken care of. I tend to be pretty independent. So I don't need them to take care of me. It doesn't make me feel good. I mean, sometimes I might need them to help me remember something, but that's about it. (Kathryn, Photo-Interview)

For Kathryn to accept and appreciate help, meant to be valued as a whole person, not as a 'care recipient'. Another way for the participants to negotiate their needs was to reduce the necessity of asking for help. Tiffany's photograph of her living room (figure 49) illustrates this strategy.

It drives my son crazy that I'm renting an apartment, because I have bought and sold a whole lot of real estate. But at this point, I don't want to be responsible for anything, and if I decide in the middle of the night to pack up and move out, I can just pack up and move out. (Tiffany, Photo-Interview)

Negotiating needs also meant for the participants to assert themselves and define

the boundaries on what they needed help with and what they were able to do.

My sister is very worried about me, and she's getting very motherly and bossy, which is, you know, the motherly part doesn't bother me as much as the bossy part does, you know, thinking that she has, you know, a huge say in my decisionmaking processes, and she doesn't yet. You know, I'm not at that point. [...] And she had very decided opinions about whether I should be keeping these things or giving these things, you know, which I should be keeping and which I should be giving away, and whether I should be moving into an apartment of my own, and why wasn't I moving in down in Portland near her so that she could take care of me and, you know, all of that. And I finally just had to say look, I can make my own decisions. I don't need to be taken care of yet. And she was pretty perturbed by that. And I managed my sister and my step-daughter at the same time, because she was kind of pushing me too, you know. They both thought that I was just nuts for staying up here, that I should be, you know, closer to my sister. So anyway, they'll get over it. (Monica, Photo-Interview)

The above quote illustrates the tension that could arise from negotiating needs. 
Monica clearly understands her sisters and stepdaughter's viewpoint but for her, negotiating boundaries and setting personal limits was important. Standing up for herself allowed her to achieve what she wanted and increased her sense of control. However, providing direction and asserting their viewpoint was difficult for the participants as it required effort and trust that they will not be deserted. Negotiating needs also meant for the participants to carefully prepare for their meetings with healthcare professionals. To have a sense of competency during the meetings, they made lists of things they wanted to discuss. "I started making a list when I go seeing the doctor of what I wanted to talk about." (Sima, Photo-Interview)

Negotiations for support also included the notion of feeling empowered. In describing what type of support they appreciated, the participants highlighted qualities such as sympathy, understanding, and cooperation. Feeling empowered meant for the participants to feel a level of supportive energy and commitment from others that anticipated and accommodated their needs without bringing too much attention to it. It meant to be involved in interactions that compensated for their weaknesses while drawing out their strengths. In other words, relationships in which the participants were able to successfully negotiate their needs were those in which they not only felt assured and comforted but in which they also felt empowered to participate. Particularly successful were those relationships in which supportive others were willing to be flexible with their plans to meet the participants' needs.

When talking about the photograph she had taken of her friend (figure 50), Kathryn highlighted the empowering qualities of her friendship with Gisela. She explained that Gisela planned activities in a way that did not require Kathryn to use a lot 
of mental energy. For example, she picked out convenient meeting places and took the lead on outings. Kathryn felt empowered because the social encounters with Gisela focused on her current level of abilities and facilitated access to valued social activities.

And then the other person that I wanted to be sure to take a picture of is a good friend who also meets me several times a month to walk. And she works in Madison Valley, and I'd get totally lost down there. But I just drive to this one spot on Madison and then we go on these great walks that are beautiful, that she...but I have no idea where they are, 'cause she just says okay, we're going here and there and that really works for me. (Kathryn, Photo-Interview)

Being empowered meant to be with others regularly, at predetermined intervals. Giving up work and pre-illness recreational activities had taken away the regularity of meeting people without the need for appointments. The participants encountered the added challenge that every meeting needed to be scheduled, which took a considerable amount of organizational skill and thus made it less likely to happen. As such, feeling empowered meant for the participants that other people took the initiative and provided them with means and opportunity. Irene reiterated the importance of being included in activities that she genuinely enjoyed but was no longer able to pursue on her own. "Yeah, my friends come up. They come and pick me, make sure I get rides to, you know, go to meetings and stuff that I would be, that I would like to go to." (Irene, Photo-Interview)

Recognizing that it is sometimes challenging to have their needs met, the participants became creative in accommodating others. They were always available, always ready, always grateful to be included, and always okay with everything others suggested, joining in whatever activity that others were engaged in.

I have a very good friend and she has two young grandkids. When she baby-sits them, she calls and asks whether I want to come over and want to play with Eric. So I always say yes. I say yes because it's a way to be with human beings. (Kathryn, Dyadic Interview) 
Even when the participants were not motivated to participate in an activity, they felt obliged to do so to accommodate others so as to make sure that they would be asked again when the occasion arose.

And I wouldn't, I would have to be awfully sick not to go [to the movies]. I would have to be awfully sick not to go because he looks forward to it [...] I think he would be crushed, he would be absolutely crushed if I wouldn't go with him to the movies. (Odette, Photo- Interview)

Financial security was raised as a key issue by many of the participants, more extensively expressed by those who had lost their job due to early-stage dementia and were not or only marginally eligible for pension, social security, or government subsidies. Kevin took a photograph of a $\$ 20$ bill (figure 51) to illustrate this resource. Duncan and Karl echoed Kevin's sentiment during the dyadic interview and expanded its boundaries.

Duncan: I would like there to be plenty of money to take care of me and my wife and then have a fair amount of money to give to the kids when we both pass on. Karl: That's something I started early. When I graduated from college, I invested in all kind of stocks - so if we ever would be in financial trouble there would be some back-up. (Dyadic interview)

Many participants did not give up control of their finances but made efforts to safeguard against possible mistakes by using checkbooks or ATM machines to balance their accounts. Tiffany took a photograph of an ATM machine (figure 52) to show the importance of being financially secure and being able to manage her assets.

My financial security. I love that machine. It gives me money. It tells me how much I have. It doesn't tell me, I've already told you that. So this is black and white concrete. And I feel like as long as I can keep my finances straight, I'm okay. (Tiffany, Photo-Interview)

Irene thought it was a good idea to use a credit card or a checkbook (figure 53) rather than carrying large amounts of cash when making a purchase. Monica took a 
photograph of a business card of an attorney (figure 54) and explained how she had taken responsibility of her finances by investigating how to open a 'special needs trust'.

I'm looking into doing a special needs trust with my back social security money. $[\ldots]$ You can take money that you've received as a result of that disability, whether it's from a lawsuit or back payment from social security qualifies, and set it up in a trust, and it can't be used for regular, ordinary, everyday needs. It has to be something special, you know, like going on a vacation once a year, or going out to dinner a couple times a month, or, you know, that sort of thing. [...] So I read up on that, and I understood it, and I followed through. And so I was proud of myself. (Monica, Photo-Interview)

Others thought that it was a good idea to have a trusted person take over their financial tasks, such as balancing checkbooks and making sure that bills were paid. "She [sister] just takes care of it. What I did is I took her to the bank and I got a form. And I put her on it, my check, and my son on the check." (Fay, Photo-Interview) They appreciated support with navigating the bureaucratic maze of financial paperwork. "She does all the financial stuff. I'm lost in that, so that's a big one." (Karl, Photo-Interview)

However, transferring responsibilities and giving up control was not always easy. The quote below illustrates some of the tension that arose for the participants in the process of deciding whether and to whom to transfer financial responsibilities. Corinne had decided to transfer the responsibilities for the couple's finances to her husband. However, she was also aware of her husband's limitation and worried that he might not do as good a job as she had done given her background in finance. Nevertheless, acknowledging her decline, she followed through with her decision.

Well Ken's [husband] taken over all that [finances]. I have done all the travel stuff, all the checkbook stuff. And here he is, you know. In fact he turned 70 this year, and he's never done it before. And we, we think so differently, that, cause I would have a chart, you know, with all the bills and, and I'd divide it, you know, the 12 months, and I'd put the amount of the bill, the date that it's due, and, I remember three things. I don't remember what the other was. But I had this chart. 
And he doesn't. And one time we got a late notice and I just, oh, never late with bills. I was really upset that he doesn't like to do my way. So, and he doesn't have a method of doing it. And I, that's not uncommon, I guess, but I don't know, I just, I'm so afraid of getting my credit messed up. But I guess I just have to let him do it his way. And what difference does it make if our credit's messed up a little bit, I guess? But having worked in that, in that work force that takes care of bills and stuff, it really, cause I worked for General Motors in their financing division. So I was really involved in some car payments and stuff. So it's, it's really hard for me to let Ken do it. But I know I'm not going to be able to do it eventually, and I wanted him to, so I could, so I could talk to him about it. (Corinne, Photo-Interview)

Those who had lost their jobs as a result of the illness identified the challenges that came with having lost a regular paycheck; they experienced economic hardship.

And, and if you're not, you have to be officially declared disabled by the federal government before you can get subsidized housing. And that took four years. You know, so, so, so there's, I mean, if I hadn't had strong social relationships with people, I'd, I would have been screwed. I mean I would have been one of those people out there on the corners, begging for money, because there's, there's no way, even, even getting the state, you know, 339 dollars and food stamps, I mean you can't stay anywhere for a month for 339 dollars. (Monica, Photo-Interview)

Accessing government benefits was confusing and challenging. "I had to find my way to DSHS." (Monica, Photo-Interview) It required the support of an attorney specialized in accessing disability benefits and it taught the participants not to take no for an answer.

I applied for Social Security Disability, and they denied me the first time, which I guess is par for the course. So I had to get all my documents together and meet with an attorney, and then they finally did issue me my social security disability. (Tiffany, Photo-Interview)

Finding a physician knowledgeable enough to explain the impact that the illness had on their lives and work and document decline was challenging and delayed the process of accessing needed funds.

Well, and just the length of time it takes to get a diagnosis that the government will accept is difficult. [...] They [Department of Social and Health Services 
(DSHS)] turned me down two times. And that I was accepted the third time was a result of the last testing that the other doctor did. They, I had showed the results but they had kicked me off the state Medicare or Medicaid, state, DSHS had kicked me off the rolls, whatever you call it anyway. And, and I went to the northwest justice center for help with that. And when they saw the results from the doctor, they said you have, they had me send them directly to my caseworker. And everything went really fast after that. I mean, within six months. (Monica, Photo-Interview)

Tiffany's story illustrates how arduous accessing benefits could be. She was well aware that she no longer had the ability to fulfill the duties of a nurse. Nevertheless, Tiffany was forced to apply for and be documented to fail in several nursing jobs before she was eventually granted Social Security Disability Insurance (SSDI) benefits. Along with losing a regular paycheck, the participants also lost the benefits that came with the job. Those participants who were not old enough to access Medicare benefits experienced difficulty in buying adequate, affordable health insurance. Some were able to continue their health coverage for up to 18 months under the Consolidated Omnibus Budget Reconciliation Act (COBRA), which gave them temporary access to health coverage at group rates. However, being insured under COBRA was expensive and did not eliminate the threat of being without health insurance for periods of time.

Yeah, the part that's kind of hard for me though is the healthcare insurance. Cause I was able to get Cobra from my previous employer for 18 months. And then there was some sort of caveat that if during that 18 months I had a secondary diagnosis that would make me eligible for Social Security Disability, it's extended another nine months. So I still have some wiggle room before that runs out. And then my understanding is at that point, I'm eligible for early Medicare with 62. [...] It's supposed to be at 65, but because I'm on SSDI they sort of make a, an allowance for that. [...] And that's why, I brought all of my lists of medications and everything to the attorney and he said, you know, why are you on Wellbutrin? And that's because I have the diagnosis of depression. And he said, so now you have two diagnoses. (Tiffany, Photo-Interview)

Negotiating the practical barriers to social life was another means to regain a 
sense of control. A number of participants took photographs of their cars (e.g., figures 55 \& 56) to show that they considered having access to transportation important. Many of the activities that they enjoyed were connected to navigating transportation, such as seeing friends, attending exercise classes, or simply walking on the beach. At the time of this study, 11 of the 20 participants were still able to operate a car but limited their car use to driving to or from familiar places such as grocery stores, post offices, family members' or friends' homes, doctor's offices, or senior centers.

I have a car, but it's like, not the freeways. It's kind of just these little circuits that I know. [...] But I go on, when I go, you know, like from here to the university district, I do side streets. And I'm not going past 30 miles an hour. And it's fine. And you've got stop signs, and you know. And I'm so familiar to it now, it's not such a problem, you know. (Irene, Photo-Interview)

Tiffany was the only participant who took advantage of public transportation resources in the community. She included photographs of both, her car (figure 57) and her bus card (figure 58) in her portfolio and shared her thoughts about giving up driving.

So this is my car that I drive about once a week. [...] And I'm just wondering if I should keep my car, if I should sell it. And I haven't quite decided yet. I'm not quite ready to give it up. But I'm sort of thinking in that, you know, that's a possibility down the road. (Tiffany, Photo-Interview)

The following two quotes illustrate that those participants who had few other means of transportation were particularly impacted by the threat of losing their ability to drive since public transportation resources were limited in their area.

Well except I grew up driving long distances, and I'm still good at driving. My son rode with me and didn't say a single word. Cause they had started making noises about, well do you think you should be driving? Oh yes. Driving is one of those things that I've been doing, you know, since I was 21 years old, and, or 18 years old, and I still am okay. I used to be a crazy driver but I'm not a crazy driver anymore. I'm a very careful driver, but I'm a good driver, so... (Monica, PhotoInterview) 
Monica reiterated her fear of not being able to drive any more once more in the dyadic interview.

Well, there is a bus that runs once an hour during the daytime hours - I am not sure what the nighttime schedule is - but I think I probably qualify for the ACCESS services and I suppose once my children are going to hide my car keys, that's what I am going to use. (Monica, Dyadic-Interview)

Others had already lost their ability to drive. Most of these participants could not

help feel disappointed, as they did not want to depend on others to pursue valued activities. "They pulled my driver's license. [...] So I'm limited. [...] I stay closer to home and she [wife] leads me around with a chain on my nose when we go anywhere." (Karl, Photo-Interview)

Many participants relied on family members or friends for help.

I go once a month to a support group. [...] It gets me out of the house but I feel bad because he [husband] sits in the car for an hour and a half because he has to drive me. I don't drive anymore. But he doesn't mind it. (Fanny, Photo-Interview)

However, the value of driving did not only lie in the getting from one place to another, which could be provided by someone else, but also in having control over choosing when to drive and perhaps enjoying the activity of driving itself (figure 59).

Planning for the future was another means to exercise control. It involved for the participants to put their preference into an advance directive, a legal document that would go into effect if they were incapacitated and unable to speak for themselves. To that end they had to learn about the types of decisions that might need to be made and consider those decisions ahead of time. A number of participants had completed a durable power of attorney for health care, a legal document naming a healthcare proxy. This document ensured that they would get the medical care they wanted at a time when others were making the decisions for them. 
I went to the class they had at Saint John's church because I felt it was pertinent that I do it because I was really concerned and I needed - if this was going to be a problem I needed to make arrangements because I live alone and I cannot rely on my kids for any help. (Sima, Photo-Interview)

However, effectively communicating and negotiating needs was not always easy for the participants, particularly when it concerned their future. Some worried that people may get tired of helping them.

Kathryn: Do you ever worry that if you get more needy that he may disappear? Monica: Well, I haven't really thought about it that way - I don't think - but maybe I should. I feel like my problem is an imposition in his life. Not that he made me feel that way but I feel that way. When I was 25 , I wouldn't have liked to deal with a mother who is losing it.

Kathryn: I don't think I think about this real consciously very often but I think that - one of the fears that goes on in my head - what if my kids get tired of helping me? (Interview)

Discussion regaining control. As argued by Lazarus and Folkman's (1984), perceived control played an important role in the process of coping with early-stage dementia. Regaining a sense of control emerged as a key element in reclaiming and maintaining a sense of well-being. When grounded theory was applied to the visual and verbal data provided by the participants of this study, early-stage dementia emerged as a condition that, like other chronic and progressive illnesses (Bury, 1982; Bury, 1991; Charmaz, 2000), exposed the participants to a loss of control and a sense of powerlessness and uncertainty, which in turn disrupted their familiar sense of well-being. Three characteristics of early-stage dementia appeared to be associated with the experience of uncertainty, (1) the fluctuating illness awareness; (2) the erratic nature of the illness symptoms; and (3) the unpredictable trajectory of the illness.

In line with Moos and colleagues (Moos \& Holahan, 2007; Moos \& Tsu, 1977) who identify coping with uncertainty as a vital adaptive task, the participants engaged in 
cognitive and behavioral efforts aimed at regaining a sense of control over themselves and their emotions as well as the illness and its symptoms. While all participants felt that early-stage dementia was a stressor that disrupted their sense of control and direction in life, they believed that they have the power to choose how to respond to their situation and reported an attitude of having to get on with their lives and 'deal with it'. They portrayed themselves as people who have choices and felt that they can do much to restore a sense of control. To cope with the uncertainties evoked by the illness, all participants adopted a problem-solving attitude and learned ways in which they could take charge of the areas of their lives that still remained under their control. To construct their coping strategies the participants drew on internal and external assets available to them such as (1) managing the illness; (2) calibrating emotions; (3) simplifying life; and (4) negotiating needs, and by and large judged their resources as adequate for the task at hand. Although most of these strengths and resources were readily available, translating these capabilities and capacities into specific coping strategies required intricate cognitive processes and ongoing efforts.

As described by the transactional model of stress, appraisal, and coping (Lazarus and Folkman, 1984), the participants used a variety of problem- and emotion-focused coping strategies to overcome the challenges associated with early-stage dementia. Lazarus and Folkman's (1984) problem-focused and emotion-focused coping is similar to the conceptualization of primary and secondary control (Heckhausen \& Schulz, 1995; Rothbaum, Weisz, \& Snyder, 1982) and Brandtstädter \& Greve's (1994) conceptual distinction of assimilative and accommodative coping. In other words, the two strategies implemented by the participants involved changing the conditions created by the illness 
(primary control or assimilative coping), while secondary control strategies involved changing themselves to suit the circumstances created by the illness (accommodative coping) (Brandtstädter \& Greve, 1994; Rothbaum et al., 1982).

All participants evoked a variety of task-oriented coping strategies to prevent or resolve challenges perceived to be the cause of the disruption in their sense of well-being. In line with Moos (Moos \& Holahan, 2007; Moos \& Tsu, 1977), who argued that the successful performance of three illness-related adaptive tasks is a key element of adjustment to chronic illness, the participants tried to regain a sense of control over the illness and its symptoms. All participants were aware that they had little or no control over the most important aspect of the illness (its inevitable progression), but that they could minimize its consequences. However, they felt that it was possible to mostly manage the illness symptoms and their impact on their daily lives. One example of a problem-focused strategy used by the participants was gathering information about the illness, which is consistent with Thompson (2002) who argued, "because predictability enhances control (Thompson, 1981), just getting information on the causes and course of one's disease and treatment options can increase perceived control" (p. 204).

However, the findings of this study also support Lazarus and Folkman's notion that "ambiguity is not always more threatening than clarity" (p. 107). Some participants preferred the uncertainty of the illness trajectory over knowing the depressing details of the later stages of the illness. Another example of a problem-focused strategy was seeking and accepting the support of family members and friends and, to a variable degree, healthcare professionals, support groups, and spiritual communities. To manage the sense of uncertainty created by the unforeseeable nature of the symptoms, the 
participants employed illness self-management techniques (day-to-day activities that the participants adopted to follow a prescription regimen) and managed forgetfulness in practical ways by establishing routines and using reminders, to-do-lists, calendars, diaries, and daily notebooks. Although some disruptions could be acted on, others needed to be endured, and many of the strategies that the participants used aimed to alter how they thought or felt about the experience. In other words, consistent with the theoretical framework underpinning this study, the participants used emotion-focused or meaningbased strategies to cope with irreversible changes. A number of these emotion-regulating strategies aimed at learning to live with uncertainty and coming to terms with the paradoxical nature of life with dementia.

Regaining a sense of control meant for the participants to embrace the paradox of accepting uncertainty as a reality of life and yet striving to find ways to feel relatively certain in the world. Complex cognitive processes enabled the participants to navigate the experience of living with the ebb and flow of unpredictability while at the same time creating patterns in living that established a sense of predictability. Ultimately, it was by straddling the interstices of certainty/uncertainty that the participants found the greatest possible control. Becoming aware of and accepting the limitations of their control in turn allowed them to be flexible within these boundaries.

Other ways with which the participants limited the emotional impact of the illness were, putting the illness and its symptoms in perspective and treating the illness as a problem to be managed in a depersonalized way (externalizing rather than internalizing the illness), processing how they felt (by talking with others or writing), limiting the time spent in negative thinking, and increasing the time spent pursuing soothing activities. 
Although emotion-focused coping strategies have historically been viewed as inferior to active coping (Stanton, Parsa, \& Austenfeld, 2002), the participants in this study used this approach to coping in a way that elicited beneficial responses. This finding is consistent with the position that coping with stressors that are unchangeable or irreversible (such as chronic, progressive illnesses) is best accomplished through acceptance and reinterpretation (Schulz \& Mohamed, 2004). As argued by Folkman and colleagues (Folkman \& Greer, 2000; Folkman \& Moskowitz, 2000), the palliative effect of these strategies enabled the participants to experience a sense of well-being despite unfavorable circumstances, a well-being that was based on the co-existence of positive and negative emotions. Creating new avenues for control while accepting unchangeable circumstances was a key element for the participants to find well-being.

Restoring meaning and purpose. Another leading theme within the interviews related to meaningful living. 'Restoring a sense of meaning and purpose' speaks to the notion of making life meaningful at a time when illness challenges one to rethink priorities, revise and remodel expectations, and find new directions in life. It captures the importance of knowing that one's life has value. Constructing meaning is a unique and life-long undertaking, and a person's sense of purpose is reflected in the way he or she pursues that endeavor. This includes a person's relationships and goals, work and leisure activities, and religious and spiritual practices. Meaning is not permanent; meaning is negotiated continually and therefore is never the same.

Living purposefully was important for the participants and a necessary prerequisite for an alternative sense of well-being to emerge. The participants proved to be resourceful in terms of pursuing this adaptive task. Although it took time for them to 
rethink what brought a sense of purpose to their lives, over time, they found many ways to add meaning to their day-to-day lives as they tried to reorganize their lives around new circumstances. The strengths that surfaced as most relevant to restoring meaning and purpose were: (1) finding meaning in goals; (2) finding meaning in activities; (3); finding meaning in simple pleasures; and (4) finding meaning in being part of a larger whole. The common thread within these strengths is the will to adopt an intention to live purposefully and look for meaning in old and new places. This theme speaks to how clarifying what continues to be of value and actively attending to these areas of one's life provides a sense of meaning. What brought meaning to the participants' lives was to feel fully alive and live life to the fullest or as Rosabelle pointed out, the meaning of life was to live. "Life is to live, you know? That's what it's for. And I do my best to stay in it." (Rosabelle, Photo-Interview)

Finding meaning in goals. Finding meaning in goals was one of four strengths that the participants drew on to cope with the threat that early-stage dementia posed to their meaningful existence. Although the illness had completely changed the participants' life plans, having goals continued to be important to them. Focusing on what they wanted to achieve and honoring their personal choices was an important step to making life meaningful. Monica captured the essence of this dynamic when she shared how having the goal of moving into her own apartment had given her a sense of future, which gave her life meaning.

Well I just, I mean I expected to work until I was like 65 or 68 , something, you know. I expected to have a house that my son could come home to. And my grandchildren could come to. And those things won't happen now. But I will have a home. It won't be a house. It won't be my own house. But it'll be my own apartment. Mhmm. And I will learn that that can be enough. (Monica, Photo- 
Interview)

For some participants having goals in and by itself was fulfilling enough to make life meaningful. Setting and pursuing goals was more important than achieving them. The path on which they traveled was what mattered most and the journey was more important than the goal. "It's important to me to always have goals. But, you can't always win." (Odette, Photo-Interview)

A few months ago I took a course at the local college on abnormal psychology and I got credit for it. But it wasn't important for me to get the credit; I simply enjoyed the challenge. There were about thirty of us. I was about three times the age of the oldest one there. [Laughs] (Kevin, Photo-Interview)

For others a sense of purpose arose from finding new ways to pursue old goals.

Being successful in reaching old goals using different paths added meaning to their lives.

Yeah, so, you know I don't know how long I'm going to be able to live on my own. It's hard to say. It could be five years; it could be ten years. There's no way to know. But I definitely, I've lived alone very seldom during my life, and so I don't really know if I'm going to like it or not, but I want to try it. So I'm doing that. (Monica, Photo-Interview)

Yet others construed meaning from reevaluating and actively changing some of their goals. This process entailed for the participants to acknowledge that their priorities had changed over the years as different stages of life had posed new questions and challenges.

I don't feel like I am 79 - okay - yeah, I have slowed down a little bit but my motivation is still there. I still want to do things - I am not ready for the grave. I mean, I want to see things and I want to do things. (Sima, Photo-Interview)

No matter whether the participants pursued longstanding or newly developed goals, reaching these goals contributed to a sense of meaning as each experience of success served as a building block for the next step of reconstruction. Based on these 
successes the participants developed a growing sense of competency. The following quote provides evidence of the extent to which some of the participants had become committed to acknowledging small accomplishments. When talking about the photograph she had taken of weeds (figure 60), Monica explained, "This is a picture of weeds, 'cause I weed, and it gives me satisfaction. I can see what I've done." (Photo-Interview)

For Monica, weeding was not only a worthy pursuit because she wanted to contribute to her family's well-being but also because it confirmed her ability to reach what she set out to achieve. It was an attainable goal and progress and success were immediately and readily visible and easily measured. Kathryn had similar reasons for gardening. Gardening activities were measurable and allowed her to carry out her goals to a successful completion, which facilitated a sense of accomplishment and increased feelings of confidence, optimism, and control. "I like working in the yard, or do something physical that I can feel good about having accomplished once it's done." (Kathryn, Photo-Interview)

One way in which the participants made success more likely was by recruiting support for their plans from everyone they considered important. Sharing their wishes, helped them to accomplish their goals. However, not everyone supported their plans in the way the participants had envisioned.

Anyway, they are all helpful in their own ways but there are other times - 'yeah, I'll take care of it, mom' and then you don't see them again and they have forgotten about it and if it is trying enough - some of it I can fix myself - my mother was a widow for a long time - you learn a lot when your mother is a widow [laughs]. But anyway, I would just poke along and then 'what are you doing there, mom' and I would say 'well this is leaking or this needs fixing' and then 'well, let me fix it' [laughs]. [...] Well, I don't know, you don't get mad at fifty year old men [laughs]. (Odette, Photo-Interview) 
The above quote is an intriguing example of the determination with which some participants pursue their goals. They try different means to achieve their objective and do not give up until they reach some sort of resolution, which in Odette's case was something as simple as having things fixed. To increase the likelihood of success, the participants put much effort into choosing realistic goals and carefully planned a roadmap to achieve these goals. They broke larger, long-term goals into smaller, short-term goals. They wrote down options with the pros and cons for each and chose the one that they thought would be best fit. The following example illustrates this process. At the time of this study, Monica's long-term goal was to move into her own apartment. It was her central focus, something large and grand to work towards. That she never lost sight of this objective became apparent when, during the photo-interview, she showed me the intricate system she had developed to determine and plan for the small, realistic, everyday goals she needed to achieve to reach her dream. "And this is notes about what to do about whether I'm going to have a garage sale or not, you know, what are the pros and cons of whether I'm going to do a garage sale." (Monica, Photo-Interview)

For Monica, these short-term objectives were markers along the journey to her long-term goal; they kept her on track, served as building blocks, and boosted her morale, even if progress was small. For many participants early-stage dementia brought to awareness that time is limited. The perception that the future is not open-ended motivated the participants to focus on goals that were emotionally meaningful and relevant to the present. Living meaningfully in the temporal context of perceived endings meant for the participants to pursue goals that were not only realistic but also harmonized their feelings, provided emotional fulfillment, and evoked a sense of connectedness. In other words, 
feeling positive emotions on a day-to-day basis was a goal for many participants. They found it important to do something simple and pleasurable every day that they could look forward to and that provided emotional satisfaction. Sima expressed the essence of this approach to life by taking a photograph of a wall-hanging in her bedroom (figure 61).

Karl and Kevin took photographs of their evening drinks (figures $62 \& 63$ ) to show that they are devotees of the cocktail hour as it makes their day more satisfying. Both men added meaning to their lives by seizing the moment and taking full advantage of the present instead of focusing on the future by enjoying an evening drink with their wives. Spending the time it takes to make and enjoy a drink was very valuable to them. Similarly, Duncan included a photograph of his favorite non-alcoholic beverage in his portfolio to show that for him getting a Starbucks frappuccino (figure 64) is more than drinking coffee; it is an opportunity to have fun and enjoy life.

And this is my Starbucks frappuccino. If it's cold outside, I probably get the coffee with mocha in it but if it's at all warm, then I like to get this [frappuccino]. And what they do with that is they put some coffee in there and then flavoring and then some ice and they have a machine and they grind it up and then put it in this container. Ruth and I go together to Starbucks although she usually gets a mocha light frappuccino and I usually get the java chip frappuccino. We get different ones. I have a Starbucks card and I usually pay for that and they end up - once or twice a year - sending me a freebie card to get in and get a free drink because I am using that on a regular basis - probably every other day. Usually it's when we're headed some place, and we'll stop and get Starbucks. The grocery store that we go to is right next to Starbucks. (Duncan, Photo-Interview)

For a number of participants being mindful of the attitude with which they approached their circumstances was a goal that brought value to their lives. Their sense of meaning and purpose was connected to how they conducted their lives and doing their best was what mattered. "It's always easier to say 'I am too tired to go out' but if you make the effort, oftentimes it is rewarding. So am trying to push myself as far as I can 
and as I am comfortable.” (Romilda, Photo-Interview)

The determination with which they struggled to do their best was an inspiring demonstration of courage and fortitude. What brought meaning to their lives was to not give up, do their best, and maintain a positive outlook.

You get up in the morning and you make it through the day and then you get up and do it again. [...] Well there are other options. We can fall down on a little heap and woo 'poor me' and just stay in that little heap. I mean some people do that but it doesn't sound like very much fun. But I think probably, I'm going to generalize here, but it sounds to me like it's been a very full life for you where you had work that you enjoyed, you had family that you enjoyed, and you had friends that you enjoyed, and you didn't just sit around waiting for things to happen to you. You went out and did them and I think that pretty much is what I have done with my life to and even though I feel like I am not doing anything because I am not doing the kinds of things at the level of activity that I used to do, I know that I still have not given up. And all I can do is get up every day and do my best. (Monica, Dyadic-Interview)

Setting, pursuing, and attaining goals added meaning to the participants' life which in turn was a major contributor to their sense of well-being. Having goals gave the participants and their efforts focus.

Finding meaning in activities. Another way in which the participants added meaning to their lives was to identify and sustain meaningful engagement. "I'm not just a hermit in the closet, no. I'm out doing different things. And that helps me." (Karl, PhotoInterview)

But it's, you know I still, I don't want to just be one of those people that just sits aside and, okay, here I am in the nursing home. I'm going to sit in my rocking chair and watch TV all day long. You know, I want to keep active. (Corinne, Photo-Interview)

In 'doing' the participants defined their values and created meaning for their lives.

Although being engaged in meaningful activity contributed to a sense of well-being, meaningful engagement meant something different for each participant. For some, it 
meant to enjoy solitary activities; for others, it entailed being involved with other people in more structured venues. The key in making life meaningful through 'doing' was for the participants to seek out activities that brought some measure of enjoyment and helped them feel useful, needed, and fulfilled. The activities that were most important were those that reflected best the participants and their personal values. The specific resources that the participants employed to find meaning in activities were having and maintaining a home, being home, pursuing recreational activities, engaging in creative activities, doing things with others, and adapting activities and interests.

Much of the participants' lives happened at home. Living purposefully meant being proud of one's home and the ability to care for it. Sima took a photograph of her front door (figure 65) to symbolize that personalizing her home and enjoying the feeling it evoked in her as well as guests was meaningful for her. Kathryn expressed a similar sentiment by taking a photograph of her front door (figure 66). Having and maintaining her home was a meaningful activity for her.

I had my house painted while I was doing this project. I'm so happy with the new colors, but it's particularly the door, 'cause they made it a darker red. It isn't so bright red anymore, and it looks more dignified. And it just, every time I drive home now I'm just happy because my house looks so dignified. I feel so much better about my house now. You know, that it was looking pretty shabby, and I didn't like it. And it was such a relief to finally get it done. (Kathryn, Photointerview)

Romilda took a photograph of her half-painted deck (figure 67) to show that maintaining a beautiful home is important to her.

Being engaged in everyday activities at home served as a source of meaning and purpose. Vincent took a photograph of his mailbox (figure 68) and patch of hay (figure 69) to show that his sense of meaning was intimately bound to life at home. For him, 
home was a place of great familiarity where daily tasks and responsibilities (such as getting the mail and feeding the family's animals) let him know that his life has value. "I love my home. I feed the animals in the morning and she [wife] feeds them in the afternoon." (Vincent, Photo-Interview)

To show that for her, tidying up her home brings a sense of satisfaction and fulfillment, Romilda included a photograph of her scarves and beads in her portfolio (figure 70) and shared, “This is my jewelry. I do everything in colors. My scarves I hang in sort of color range. I love just looking at them.” (Romilda, Photo-Interview)

Finding meaning in having and maintaining a home also meant to draw meaning from being engaged in everyday activities. Odette expressed this sentiment by stating, "Well, there are things that are just almost like everyday routines but all of a sudden they are important to me." (Odette, Photo-Interview)

Being home was another activity that brought meaning to the participants' lives. For several participants, 'being home' meant to be able to withdraw from the demanding and overwhelming reality of their social and cultural environment and yet find meaning. These participants referred to their home as an inner sanctum, a place where they feel secure and protected and where they can be themselves. Uma took a photograph of her front door (figure 71) to convey that for her, 'being home' meant taking time out, escaping the stress of social interactions, and enjoying the comfort of a familiar environment. "This is the place where I feel most centered. You know, I'm really in control of things here. [...] You know - it's meaningful for me to be here." (Uma, PhotoInterview)

Similarly, Tiffany photographed her living room (figure 49) to show that 'being 
home' allowed her to enjoy her space. Being in her space was meaningful because it allowed her to be herself without having to pretend she was different or having to worry about the consequences of her impairment.

So I guess having lost my sense of smell is one of the reasons I don't want people coming into my apartment, cause I have a big hairy dog, and I have wall to wall carpeting. And so I don't know if my apartment smells. So as long as nobody comes in, it doesn't matter. (Tiffany, Photo-Interview)

Rosabelle took a photograph of her home (figure 72) and reiterated how much she appreciates living in a house that allows her to enjoy one of the things she values most nature.

And that's, that's our house. I love my home. And it's, you know, it's here in the woods, and there's no other houses that we can see right around us. So we feel like we have complete privacy here. And when I get up in the morning and I see the sunrise, and sometimes when it's clear you can see all the way to the coast from here. And so I really love living here. (Rosabelle, Photo-Interview)

Similarly, Corinne photographed the view outside of her living room window (figure 73) to show that for her 'being home' meant to relax and watch people play golf. Sima took several photographs of the interior of her house (e.g., figures $74 \& 75$ ) to show that spending time at home was meaningful because it evoked memories of past experiences. Enjoying these memories and the positive feelings attached to them was an activity that Sima valued tremendously as they allowed her to experience a sense of connectedness and resurrected the meaning she assigned to her life. "I became more aware that I have nice things - they are not necessarily expensive things because cost to me doesn't equate nicer - it's things that have meaning for me." (Sima, Photo-Interview) Karl expressed a similar sentiment when he photographed parts of his home that were meaningful to him (e.g., figures $76 \& 77$ ). For him, the value of life was also connected 
to appreciating memories, many of which were at home. Kevin took photographs of mementos and works of art that he and his wife had collected over the years (e.g., figures $78 \& 79$ ). Spending time at home allowed him to treasure personally meaningful memories and shared histories.

For the participants, living purposefully also meant to pursue recreational activities. For several their long-standing interest in gardening served as a vital resource for meaning and purpose. Romilda took three photographs of her garden (e.g., figures 80 $\& 81)$ to convey that gardening is an activity she values. Kathryn expressed the important role that gardening plays in her life through photographs of flowers in her backyard (e.g., figures $82 \& 83)$.

I like, particularly at Lummi, I like to garden. I work in the yard a lot when it's not raining. And that's, I know that that's become more important and I have more time to spend on it and, like that. (Kathryn, Photo-Interview)

Sima included a photograph of her Hydrangea bush (figure 84) to express the joy that her garden brings into her life. Sima not only enjoyed working in the garden but also appreciated the fruits of her labor and the fond memories that they evoked. How meaningful her garden was for Sima became also apparent when, at the end of the photointerview, she went into the garden to cut me some flowers as a thank you for the opportunity to participate in this study. Uma took a photograph of her indoor plants to show that she enjoys gardening (figure 85). For her, growing flowers brought meaning not only by creating visually pleasant experiences but also by reminding her to keep up with her responsibilities and enjoy the present.

Kevin took several photographs related to cooking (e.g., figures $86 \& 87$ ) to show that gourmet cooking is an activity that adds meaning to his life. Chester shared that 
listening to music or audio stories is an activity that he values. For him, putting on his favorite recordings, laying back, and listening to them provided a sense of joy and satisfaction. "Yeah, yeah. Good music, you know. I like to listen to music, so I turn the TV on with the music or turn some stories on. I've got some tapes of Lake Wobegon. Have you ever heard those?" (Chester, Photo-Interview)

Watching TV or movies at home was another activity that many participants considered meaningful. Vincent took a photograph of his TV (figure 88) and asked his wife to take a photograph of him while lying in bed (figure 89) to show the interconnectedness of two activities that he treasures. "I enjoy watching TV and then watching TV goes into a nap." (Vincent, Photo-Interview) Karl took photographs of his TV while his two favorite TV shows were running (figures $90 \& 91$ ) to show that watching TV on a daily basis added value to his life.

And this is the idiot box. [...] I enjoy watching the Seahawks playing on TV. And of course every night also, maybe I already put it in there [photo-diary], Wheel of Fortune and Jeopardy are a must. Well, I mean we're not giving up other things to do, but I mean when we're here, this is what we do. [...] Yeah, and it's what I'm looking forward to every day. (Karl, Photo-Interview)

Kevin took a photograph of a Netflix envelope (figure 92) to show that watching movies with his wife is something he values. Both Tiffany and Sima included a photograph of their favorite movie theaters (figures $93 \& 94$ ) to show that they considered going to the movies a valuable activity.

Engaging in creative activities was meaningful for a number of participants. It provided an opportunity for them to express their talents, knowledge, and experience, and to create new connections to life. Kathryn took a photograph of her artwork (figure 95) to portray the role that creative expression plays in her life. Her engagement in artistic 
activities deepened the meaning of her life by allowing her to experience a sense of pride and connectedness. Knowing that other people appreciated and treasured her work reinforced the sense that her life has value.

And it's sort of a sculpture kind of thing that I make out of driftwood. And so I just go pick up all these little pieces on the beach at Lummi, and then I drill a hole in them and string them. And when Lisa and Steve were going to get married, Lisa said to me, Steve has a specific wedding present he'd like from you. And I thought, oh, okay. And then she said, he wants one of those things that you have, that you made up at Lummi that you have hanging off the back of your house. And he noticed it and commented it to her. [...] So I made him one and Lisa said they have it hanging up at their house. (Kathryn, Photo-Interview)

Romilda included several photographs of her artwork in her portfolio (e.g., figure $96 \& 97)$ and shared that her passion for painting was grounded in a life-long interest in art. Painting was not only something she really enjoyed but it also provided continuity in identity. In contrast, Irene presented painting as a newly discovered hobby and shared that she had developed an interest artistic expression after being diagnosed with earlystage dementia. She included a photograph of her artwork in her portfolio (figure 98) to show that she enjoys the opportunity to explore her creativity by painting watercolors. In her photo-interview, Irene also showed me a large portfolio of artwork, which provided further evidence of Irene's commitment to her creative expression. Irene preferred to paint in a group setting which allowed her to not only enjoy the expressive process individually but also to share her experience with others.

I go to Elderwise. And I go once, once a week. [...] I really enjoy that, because painting by myself is not that much fun. It's, I mean everybody's painting at the same time, and you're not really looking at everybody's else's. You're kinda getting engrossed in what you're doing. And so, and what really amazes me about it is that I paint something and then I go, ouh. You know, when I get finished I go ouh. And then I come back, and it's up on the wall. And I go, did I do that? Yeah, that looks like the one I did. Wow, that's pretty nice. [...] And when I come back and I look at it and I go, is that really mine? I'm not sure. Oh yeah, remember that 
little piece. That is mine. It's ama-, isn't it amazing? (Irene, Photo-Interview)

Getting out of the house, at least once a day and spending time with others was important to many participants. "I do need some, something outside of the house; that's just something I need to do." (Rosabelle, Photo-Interview) "I like to go down to the senior center and if nothing else, sweep somewhere - just do something and say hello; I know a lot of these people." (Odette, Photo-Interview) Ruby took a photograph of the bus that takes her to the adult day care center (figure 99) and several photographs of fellow day care participants (e.g., figure 100) to show the important role that being involved in activities outside of home played for her. Being meaningfully engaged meant for her not only pleasure, enjoyment, and happiness but a welcomed change from her daily routine.

I go to an adult day care program once a week. There are lots of people and I enjoy the change. I don't talk much but they - I can't remember what we do there besides have lunch but I know I enjoy going there because it is a change. Anything, any change, whether I like it or not, is good. (Ruby, Photo-Interview)

Duncan took several photographs showing different groups, activities, and programs that he started to attend weekly (e.g., figures $101 \& 102$ ) after being diagnosed with early-stage dementia. For him, living a full life meant keeping busy and socializing and trying out new activities not restricted by the illness. Rosabelle expressed her joy of being a member of a hiking club by including several images of nature she had taken during one of the club's outings (e.g., figures $103 \&$ 104). Participating in outdoor adventures was meaningful to her in several ways. It provided an opportunity to socialize with people who share the same passion, motivated her to hike to new places, and allowed her to appreciate nature, something that was very important to her.

Romilda shared that working on beading projects is something she enjoys. The 
beading activity as well as the colorful finished product brings happiness into her life and brightens up her day (figure 70).

There is a place down in Kirkland, which I have taken one or two of my friends to, called 'bead world' or something. It's a room and it is floor to ceiling beads, all beautifully hung and there is a table in the middles and you can just sit and pick out what you want and they'll help you and they got all tools there and you can sit there for an hour or two hours. It's such fun - and the results are really amazing. (Romilda, Photo-Interview)

Fay took several photographs of fellow bridge players to portray that playing card games is an activity she values (e.g., figures $105 \& 106$ ). Fay played bridge three times a week and had lunch with the other players afterwards. Playing cards, having lunch, interacting, conversing and discussing all added meaning to her life by fostering a sense of connectedness. Rosabelle took a photograph of a waterfall (figure 107) to symbolize the important role that friends with whom she shares a common interest play in her life. Similarly, Chester took a photograph of his favorite card game (figure 108) to show that simply being with others and pursuing a shared interest was a much-valued component of his life.

Although all participants drew on their longstanding skills and interests to construct meaning, some pre-illness activities needed to be discontinued or modified. The following quotes illustrate that being meaningfully engaged meant to let go of those activities that were no longer of interest or required a different skill level. "I used to just volunteer there at the senior center but I don't volunteer anymore." (Fay, PhotoInterview) "I don't do everything in the yard and around the house now, not like I used to do, but I do some things all right like make sure we have enough firewood every night." (Frank, Photo-Interview) 
Other pre-illness activities needed to be adapted or replaced with different kinds of engagements. When adapting old or finding new activities, it was important to keep the same quality of satisfaction and fulfillment as the old activities.

Well, I still go to meetings from the radical women and the freedom socialist party, you know, like we have events and stuff. They have food and then they have, people give presentations and then there's discussion from the floor of whatever people want to talk about. But I find that, I mean, I just can't absorb it, and I just kind of zone out, cause it's too hard for me to figure out what's going on but I'm there and that's important. (Irene, Photo-Interview)

Fanny took several photographs of quilts she had made (e.g., figures $109 \& 110$ ) and shared how adapting old skills and interests allowed her to continue to express who she was and what she valued by using her unique skills gained over a lifetime - only differently. How meaningful sewing was for Fanny became apparent when in our last meeting, she gifted me with a handmade pillowcase - an appreciation for the opportunity to participate in this study.

Quilting has been an important part of my life for the past 25 years. [...] Now I don't quilt anymore, but I still sew. I sew about 6 hours a day. That's what I like to do - it is relaxing to me. (Fanny, Photo-Interview)

However, adapting meaningful, pre-illness activities was not always easy. The following photographs and quotes illustrate the effort, imagination, innovation, and creativity that it required to pursue these activities that sometimes yielded a diminished sense of pleasure and satisfaction because they were much harder to do. Frank photographed a wreath made out of crabs (figure 111) to show that he is determined to continue to crab fish, despite his growing limitations.

I have done crab fishing everywhere, yeah, a lot of places. [...] I like catching 'em and cleaning 'em and eating them. [...] Oh yeah. Yeah, we're going to have a little crab cocktail when we're done with, with this [interview] and I caught them. That's an interesting thing, actually, that I went out and the boat tipped over. I 
guess you heard about that? Yeah. [...] And then our daughter Eileen is, she's at school, and she's, “you, you're, you can't take my boat out. I won't allow it. You can't take it out unless I'm here. You cannot take my boat out." Hmm, how am I gonna get any crabs? And I thought ah, yeah. I got a 150-foot-long rope, and the tide's not going out, but it'll go way out there another couple hundred feet when it's low. Anyway, so I go out there with the crab trap, put the bait in it, tie the rope to it, bring it way back in to where the next tide's going to be, drive a stake in bottom. There isn't any float. But it's out where the tide's not going to go out to, and the crabs come in to feed. So the next day I go out there and I got eight crabs, or nine crabs, I think. (Frank, Photo-Interview)

Corinne took a photograph of the local library (figure 112) to show that reading is something she values and described how she manages to continue this meaningful activity.

Well for instance now in reading, I'm an avid reader. I read books all the time. [...] Well, this book I'm reading is short stories. And I switched to short stories, because I have trouble. And I write down the plot of it, but I still have trouble forgetting, you know. So it's been a little frustrating. [...] Yeah, I write the plot, yeah, so I can pick it up. So I thought, this short story is working a little better, because I... And some of the short stories are 30 pages, you know. So it's not that it's, you know, I can always remember everything if I put the book down. But I've started, but I take pretty good notes, you know, to explain. And it's hard, because that's always been one of my favorite. In the past, when I was, I've been a little down, I've read read read read read. (Corinne, Photo-Interview).

The below quote illustrates how meaningful activities are first adapted to fit one's current skill level but over time, become too difficult and eventually, hold no meaning.

Well, I can still follow a plot but I know I'm not reading as much as I used to. That's pretty clear. I don't feel quite as motivated, and I know that's because I'm not enjoying it as much 'cause it's harder. [...] But I, you know, and I can still read the paper and that kind of stuff, and enjoy that. (Kathryn, Photo-Interview)

Although all participants considered meaningful engagement essential, identifying activities that expressed significant parts of who they were was sometimes difficult.

Nevertheless, the participants found it important to keep trying.

I've just always done a lot of things at once, and that's kind of hard for me now, actually, because I'm not doing lots of things at once, so I do, I mean I actually 
get bored sometimes, which in the rest of my life, I always told my kids there's no excuse for saying you're bored. If you're bored, that's just a failure on your part to act. So go do something, you know. Go think, do anything. But it's even more important now, because, because if I don't, if I don't keep doing, I will stop. You know, I mean, I don't know if I can get going again if I stop, and I don't intend to stop until I have no other choice. (Monica, Photo-Interview)

Interestingly, Tiffany pointed out that she was in some ways grateful to earlystage dementia for presenting her with an opportunity to engage in activities that she would not have had time for, had the illness not led to early retirement. "I feel like I am getting away with something because I don't have to work 10 hours a day and I am kind of enjoying this time.” (Tiffany, Dyadic Interview)

Finding meaning in simple pleasures. Many participants achieved a sense of meaning by paying attention to simple joys and valuing everyday experiences. The participants describing this resource had noted that early-stage dementia, despite its sometimes devastating effects, did not prevent positive moments from occurring, and that savoring these pleasurable experiences added meaning to their lives. The specific coping strategies that the participants used were valuing positive moments, appreciating nature, and spending time with pets.

Having gratitude for special moments that are generally taken for granted helped the participants appreciate life. Kathryn included a photograph of one of her neighbor's children (figure 113) to show that she enjoys observing and playing with children. "And I just had to get little kids in there somewhere because I just, they're just too wonderful." (Kathryn, Photo-Interview) She described children as having a cheerfulness that spreads joy to people around them.

The last time we went [playing with my friend's grandson], she'd heard about this deal they have in a high school. They have this great big gym and they have all this great toys for little kids and so you can bring your kid in and a dollar and 
they have all these little cars and tractors so they can drive all over this great floor. And I wasn't just watching Eric play - it was watching this whole room full of kids play. I just loved it. (Kathryn, Dyadic-Interview)

Furthermore, Kathryn believed that people with early-stage dementia could contribute to the community by engaging in volunteer work with children. "I think to a certain stage anyway, we'd be great with kids. [...] I can't be the only person with Alzheimer's that is infatuated with little kids and their playfulness and all that stuff." (Kathryn, Dyadic-Interview)

Many participants valued engagement in activities for the sake of the experience, for how they felt when undertaking the activity, rather than for its outcome or its purpose. Irene asked her sister to take two photographs of her on a swing set (figures $114 \& 115$ ) to show that she cultivated experiences that are enriching and pleasurable. Finding happiness in the small things of life such as swinging motivated her and gave her energy.

In a similar vein, Vincent included two photographs of car rides in his portfolio to show his love of road trips. Although Vincent was no longer able to drive, he enjoyed being a passenger. In one photograph that he had asked his wife to take, Vincent was seen getting into a car (figure 116) and in the other, he captured an image during his ride (figure 117). Tiffany took a photograph of her 'Orca' card (figure 58) to express a similar sentiment. She enjoys sitting on a bus going for bus rides. "Yeah. When I just want to get out and do something. So I have, here's my card, let's go.” (Tiffany, Photo-Interview)

For the most part, valuing precious moments and enjoying life did not require much; it was woven into the fabric of everyday life. A number of participants included photographs of their mailboxes (e.g., figures 118 - Uma \& 199 - Rosabelle) to express the pleasure they derived from looking forward to and getting their daily mail. The 
quotations below also reveal the ways in which seeking meaning, valuing precious

moments, and enjoying a sense of connectedness are intertwined.

And this is our mailbox. It's down at the end of the driveway here. And I love going down to get the mail. I, I, some people still write letters, you know? [...] I have friends back east. I still have my best friend from high school. We stay in touch. And my best friend from college, we stay in touch. And they both live in North Carolina. And we correspond, and I have a friend in Tasmania who corresponds with me. (Rosabelle, Photo-Interview)

The pleasure and sense of control inherent in pursuing ordinary activities, even those as basic as buying groceries and preparing meals, becomes also apparent in the following quote. "I value that I can still buy my own food, cook my own food, and clean up after I cooked my own food.” (Monica, Dyadic Interview)

Being meaningfully engaged also meant to have fun. Corinne photographed a singing-dancing doll (figure 120), a gift that she had received from her grandchildren, to show that amusing herself and laughing adds value to her day-to-day life. It was so much fun for her that she had to show me the doll during the photo-interview. "I think just having fun, you know like stuff like this. That's so important to me to, to, you know, really have a good time.” (Corinne, Photo-Interview)

In contrast, for some, there was an acute realization of the temporary nature of human existence, a renewed sense of urgency with thoughts of having little time left, especially in comparison to healthy others. They felt that they had to do everything now. For Kathryn, the issue of how to make the best use of her time became paramount in the face of an uncertain future.

I don't want to get to the point where I can't go anywhere and then wish I had done this, wish I had done that, kind of thing. But I, you know, I did something this year. I mean I, before your assignment, so I couldn't take pictures of it. [...] I found the new QFC $[\ldots]$ and they had this great magazine section at this store, at 
the QFC. And one of them was on Cannon Beach. And I picked that thing up and, Cannon Beach. I haven't been there in a long time. And I brought it home, and I was looking through it, and it had an ad about the sandcastle festival coming up. And I thought, I'm going to do that. I'm going to finally go, cause this'll be the last year I can go. So I made a reservation, and Dora [dog] and I went together. And the weather was not good at all, but we went out walking in the rain. And he sandcastles were a lot smaller than normal because of the weather. But I finally got to be there. (Kathryn, Photo-Interview)

Valuing positive moments was an important coping strategy for the participants.

Making their days more pleasant by identifying gratifying moments and sprinkling instances of happiness helped them experience feelings of joy, love, and hope and fostered the realization that their life had value. It increased their overall sense of purpose and gave them reasons to think that life was worth living.

Another way in which many participants infused their lives with meaning was by appreciating nature and enjoying the outdoors. A number of participants gave emphasis to enjoying the outdoors from within the boundaries of their homes. In other words, contact with nature was not always achieved in a physical sense, but through sharpening their appreciation for the environment immediately outside of their homes. Ruby took a photograph of a hanging basket with flowers (figure 121) to show that watching and observing nature from the confines of her wheelchair played a vital role in her life. In a similar vein, Karl took a photograph of 'his' humming bird and explained,

And this is a little friend of mine, and if you look long enough and have the right position, you will see one out here every day. It's a hummingbird. (Karl, PhotoInterview)

Similarly, Rosabelle took a photograph of a spider web (figure 122) and a pack of dogs (figure 123) to show her love for watching animals and shared her appreciation for observing patterns in nature. Likewise, Odette took advantage of the slower pace that the 
illness had obliged her to take and appreciated the natural beauty around her. "Oh, I like to garden $[\ldots]$ Have you ever seen onions sprout - it is so neat." (Odette, Brief-Interview)

Getting outdoors and using all the senses (not only the sense of sight) to appreciate nature was another way in which the participants made their lives meaningful. Walking, even if it was only in the immediate neighborhood, provided a change of scenery and brief moments of 'connectedness' with the natural world. Uma included several photographs of trees in her portfolio (e.g., figures $125 \& 126$ ) to express her love for walks in the neighborhood and her appreciation for different types of vegetation. In contrast to many activities that the participants perceived as requiring too much cognitive effort nature walks in familiar areas were perceived as a manageable and enjoyable activity within the constraints of the illness. "Well, there's no problem down walking around Green Lake, because you never get lost. You just, you won't get lost, cause you just follow that yellow line. Cause it's a circle, you know, around the lake.” (Kathryn, Photo-Interview)

Rosabelle included several photographs (e.g., figures $127 \& 128$ ) to express that she enjoys walking in nature, both alone and with others. She explained,

So I go walking up the hill here. I have a good place to walk. There's a dirt road that goes across the top of the hill here. And when you walk up to the top, you can see all the way to the coast and all over the valley. And it's, it's a, lovely places to walk around here. And sometimes I just walk up to the water tank and around it and back down. (Rosabelle, Photo-Interview)

Sometimes, walking with other people who shared their love for the outdoors enhanced the quality of the experience. Karl took a photograph of one of his favorite spot for nature walks (figure 129) and explained, "Usually we go together down to Seahurst and walk along the beach. And once in a while we go to Des Moines. [...] No, unless we 
go together, I don't. I don't enjoy it as much.” (Karl, Photo-Interview)

How meaningful spending time in nature was for Frank became apparent when at the end of the photo-interview, Frank went to his rock collection and gifted me a rock he had found on the beach as a thank you for the opportunity to participate in this study. Appreciating nature was another pivotal coping strategy for the participants. Spending time and exploring favorite places in nature helped the participants draw out those elements that were important to them and engage in an internal dialogue of meaningmaking. Observing nature patterns fostered the realization that meaning is fluid and changes over time. In this way, experiencing precious moments in nature helped offset some of the paralyzing meaninglessness that accompanied the illness and made the context of daily life richer.

Spending time with pets was another means in which the participants derived a sense of meaning and purpose in life. Some participants simply liked pets, enjoyed watching them, and considered them part of the family. However, most participants spoke about the calming effect of caring for and/or spending time with pets. Pets were perceived as an unconditional source of love, companionship, and friendship. Uma captured this sentiment by taking photographs of her boyfriend's pets (figures $132 \&$ 133). Similarly, Monica shared, "My cat - he is a lot of company [laughs]." (Monica, Dyadic Interview)". Odette also took photographs of her dog (figure 134) and cat (figure 135) to show how much she appreciates the companionship of her pets and explained, Anyway, I took a picture of my dog - she is, she is my good buddy - she keeps me company. [...] Like when I took my shower this afternoon, she was watching me and knew I was taking a bath [...] and the cat was there too. [...] The three of us are the three musketeers or something because we are very close and very decidedly taking care of each other. (Odette, Photo-Interview) 
Others felt that they derived benefits from the relationship with their animal companions that went beyond brief moments of joy. Many participants liked talking to their pets and appreciated them for being good listeners.

And that's why I need him [dog]. I need him 'cause I can talk. I can tell him stories. He never interrupts. He's never once said, you've already told me that. And he's just, to me, kind of this little guardian angel. And I know he loves me. (Tiffany, Photo-Interview)

Others confided their frustrations to their pets. "And when I am frustrated, I talk to the dog [laughs], 'this is a lousy day' [laughs]." (Odette, Photo-Interview) By providing boundless measures of acceptance, attention, and unconditional love, pets improved the participants' sense of self-esteem and self-respect. "I know she's just a cat. But there's something in her that gives me a feeling that I am precious and special [laughs]." (Tiffany, Photo-Interview) Pets also infused the participants' lives with meaning and purpose by serving as a 'time clock' for those who had few scheduled activities. Their presence brought rhythm and structure into the participants' daily lives. "Dora [dog] wakes me up every morning and we go for a walk." (Judy, DyadicInterview) Dog walking also facilitated brief social interactions with other dog owners. These brief moments of contacts with other human beings, in the presence of each other's animals, were perceived as non-threatening as pet-ownership provided a mutual interest and simple conversation topics.

And I enjoy talking with the people and their dogs. We always meet the same people because ... And a lot of them know, 'oh, here's Queenie [dog]' again. And I know some of their dog's name, because we've been, you know, doing it so much. (Karl, Photo-Interview)

Talking to fellow dog walkers created an opportunity for the participants, 
particularly those who lived alone, to practice social skills, thereby increasing a sense of connectedness with others.

It was so fascinating for me when I got Dora [dog] and started walking every morning, I found out that there is that community down there of all these people who walk at the same time every morning. And so if you walk for a number of days, people start saying hallo to you and sometimes you stop and talk about the weather or whatever. (Judy, Dyadic-Interviews)

Communicating and spending time with pets helped the participants cope with and make meaning of their illness experiences. Pets were an important source of love and affection and increased the participants' sense of belonging through comforting humananimal interactions. Their presence also facilitated non-threatening social interactions with other human beings and reduced the amount of stress that the participants generally experienced in encounters with strangers. In addition, performing tasks such as feeding, brushing, walking, or talking to their pet seemed to foster the participant's sense of selfrespect and independence and promote feelings of perceived control. Finding meaning in simple pleasures was an important strength that the participants drew on to construct coping strategies aimed at managing the threats to their sense of direction and purpose in life. Living with a restored sense of meaning in turn served as a supporting factor in the participants' striving towards an alternative sense of well-being.

Finding meaning in being part of a larger whole. 'Finding meaning in being part of a larger whole' was another strength that the participants drew on to cope with the impact of the illness and re-establish a sense of well-being. For those who followed a traditional religious or spiritual path, 'being part of a larger whole' meant to actively carry out religious or spiritual practices as an outward expression of their beliefs or faith. Turning to longstanding religious and spiritual practices offered solace and considerable 
comfort during a time when not much else made sense. Prayer was a tool that some participants used extensively. It was the foundation that supported their lives and provided a sense of stability.

Interviewer: So tell me what makes it possible for you to feel so well? Fay: Faith being at the top of the list; just trust in God. [...] I pray to him. [...] Yes, I, praying that's the one thing that gets me through, yeah. [...] You know I'm faithful. I am, I believe, and my faith is so strong that I know all I have to do is ask. [...] And you know, I mostly pray for me; I thank the Lord for all my blessings each and every day, and just for letting me wake up to behold another day. And I thank him for being so faithful. [...] Thank you Lord. I just love the way you solved that problem for me. Thank you for the inner peace. (Fay, PhotoInterview)

Fay viewed prayer as a means of 'talking to God'. For her, praying was a dynamic, personal friendship with God and this special connection helped guide her as she went through her days. Praying served to seek God's wisdom and provided a stabilizing effect in her life as she trusted that her prayers would be answered. Odette echoed this sentiment when she explained that God is constantly and always available to help navigate her life.

I say daily prayers that I have been saying ever since I became Catholic; I say prayers in the morning and prayers at night. [...] There was a song they used to sing and it, it's sustained me all these years 'Jesus loves me I know because the bible tells me so'. I don't know how many times a day I am humming that and so I know Jesus is with me. It's just God and me and I am comfortable, real comfortable. (Odette, Photo-Interview)

For her, God provided the necessities of life and [was] a loving soundboard when problems arose. Her personal closeness with God empowered her and provided comfort. Corinne also took great comfort in her faith. She believed that there was a larger purpose behind her illness and looked for ways to find gratitude for her life and current circumstances. 
Well, I, I don't, I don't blame him [God]. I'm sorry I have it, and I might rant and rave, but you know, why, why not... You know, I'm sorry they have to have the disease, but why shouldn't I be, you know, why should I not be? And he gives me so much other relief. You know, I don't hurt. (Corinne, Photo-Interview)

Coping meant for Corinne to integrate her illness within a spiritual framework.

Yeah, my faith helps me a lot, absolutely. Oh yes, for sure. [...] And I've sure cussed God out a few times. [laughs] [...] Our faith is so essential in our lives. But you know, you just hang in there. And there's got to be a reason. [...] You know, and that's why I try to encourage people to accept it like another disease and not... (Corinne, Photo-Interview)

Contrary to Corinne, Fanny struggled to find a meaning for her illness. For Fanny, the diagnosis of early-stage dementia created an inner struggle as she searched for an answer to the 'why me?' question. "I talk to God all the time 'why did you do this to me?' [laughs] No, I am just kidding." (Fanny, Photo-Interview)

The interpersonal dimension of spiritual and/or religious practices also played a vital role in the participants' lives. For many participants it was important to be part of a faith community and attend religious services weekly. Corinne included several photographs of her church in her portfolio (e.g., figures $136 \& 137$ ) to show that she not only took great comfort in her faith and belief in God but also in the support she received from her religious community.

She explained,

Oh yes, yeah, we're real active in our church. [...] And it's a loving, comforting church, you know. We have a lot of fun people, you know. [...] And I've had to pull back on a lot of things, 'cause I used to work a lot with doing dinners and doing these kind of things, and I can't do that anymore, so, but people accept it and understand, and, you know, they're just as loving as they would be had I been still as active as I am, as I was. (Corinne, Photo-Interview)

Similarly, Fay photographed the interior and exterior of her church (e.g., figures $138 \& 139)$ to show that being part of a religious community, participating in services at 
least once a week, and holding a church position was a coping resource and a key

component of her sense of well-being.

This is in my church. [...] And my sister and I sing in the choir. [...] And we go every Sunday. Yeah, I go every Sunday. [...] And that's important to me, my church family, and my pastor. (Fay, Photo-Interview)

Duncan photographed the choir director and organist of his church (figure 140).

For him, singing in the church choir brought satisfaction as it allowed him to both

participate in and contribute to his church community.

Sally [wife] and I sing in the church choir. I think I have been singing in the choir since I retired; I don't think I was doing that - may be I was doing that before I retired also. I am probably retired four years. I think I retired at 68 . [...] We sing almost every Sunday and sometimes we have practice on Thursday night and sometimes they have it set up so you just show up on Sunday and practice for half an hour before church. [...] I really enjoy singing. (Duncan, Photo-Interview)

For others, being an active member of a religious community was less important

than it had been in the past. These participants had developed an intimate and individual relationship with God that no longer required attending church services. God was involved in and accessible during their day-to-day process of living.

There is something about Sundays but I don't go to church anymore. I used to go to church like just 'had to be there' and I know where my God is. [...] I don't go [to church] any more at all. [...] I can muddle along, I have my beliefs, I am content. (Odette, Photo-Interview)

We go in, probably once a month we go in and have church with my daughter. [...] I just feel I can read the bible when I want to. I can watch church on TV, I don't have to go to church every Sunday to be a good Christian. (Fanny, PhotoInterview)

Others were not or no longer on a specific religious or spiritual path. "We are not Hindu or Jewish, or ... just normal, everyday, moderate religious affiliation - but we don't go to church.” (Kevin, Photo-Interview) "My religious community does not play as 
much of a role as it used - no, not as much as it used to but I do go for the high holidays." (Sima, Photo-Interview)

Well, I spent a good part of my life functioning in a kind of a Protestant religious setting. But I've kind of, that's not so much important to me anymore, and hasn't been for several years now. And I don't give it a lot of thought anymore, like do I still believe that or do I not, or you know, whatever. And I don't know. I suppose in some ways, life just seemed like it became more important. (Kathryn, PhotoInterview)

Some did not follow a specific religious or spiritual path but felt a sense of connectedness. "I definitely believe that there's more than just us, and I think that it's all connected. You know, everything's connected.” (Monica, Photo-Interview) For many of those participants, 'being part of a larger whole' involved having a contemplative connection with nature.

As far as religion, I mean, personally, nature is my religion. It has always been. I've said that all, since I was a kid, I think. [...] Well that's why I've spent a lot of time outdoors, and I bought timber. (Frank, Photo-Interview)

Being in nature fostered the awareness that they were not alone. "When I'm weeding, I have a connection to nature, which sounds weird. You're killing nature, and you're having a connection with it at the same time." (Monica, Photo-Interview) For Monica, 'communing with the dirt' provided a pathway to becoming attune to natural rhythms (including her own) and experiencing a sense of stability and continuity.

Yes, communing with the dirt is relaxing. I need these growing things. Something to go out every day - pick out the day leafs, look at the flowers, and poke around in the dirt and make sure that it is still - that it got enough water and it's warm enough - all that kind of stuff. And it gives me continuity that you don't always get in other things. (Monica, Dyadic-Interview)

Many participants experienced being in or watching nature as soothing and calming. They felt that by being present and tapping into the timelessness of nature they 
could experience a sense of peace and connectedness. Kathryn included several photographs of nature images and large bodies of water to express that her relationship with nature was something to fall back on, a connection that provided her with sense of rest and peacefulness.

And then I started thinking about how many calming, restful moments I spend sort of looking at those things and enjoying them. [...] And I love being up there [at Lummi Island]. I like the water. I like walking on the beach, and it's just peaceful and, you know, pretty laid back and there's always places to walk. [...] There's just, there's this sort of comfort. (Kathryn, Photo-Interview)

Through spending time in nature some participants realized that there was a timelessness that was greater than themselves, greater than their worries, and greater than their symptoms.

[I feel well] when I'm in the woods and have nature read me. Sunshine and nature, of course sunshine is nature too, but those are my just, they're my healing things. (Rosabelle, Photo-Interview)

Maintaining a contemplative relationship with nature meant for some participants to regularly hike in the mountains (figure 141) or walk on the beach (figure 142). For others, appreciating nature in its many expressions meant to take frequent walks and/or to visit meaningful places in their neighborhoods (figure 143). "And we were hiking. [...] But I stopped and took a picture of this, because I love Lake Crescent. It's just wonderful." (Rosabelle, Photo-Interview) For Irene who shared “I don’t believe in God. [laughs] I'm an atheist” (Irene, Photo-Interview), 'being part of a larger whole' meant being a member of an organization that was dedicated to a higher purpose.

It was a card from a Clara Frasier, which is a friend of mine. [...]Clara Frasier was the founder of 'Radical Women' and the 'Freedom Socialist Party'. And she grew up in Los Angeles, and she, her origins, she came from the Jewish people $[\ldots]$ but there were others that looked at life as a broader concept of it all. And that's the way Clara Frasier was. She was a wonder-, she was a wonderful 
woman, just incredible. [...] I mean her, her theory was, if you take care of those who are the bottom of the class, then that's mostly women, it, which it is women. Then you bring up everybody, you know? And I have been a member of that organization for 30 years. (Irene, Photo-Interview)

'Being part of a larger whole' promoted a sense of well-being in various ways.

For the participants a sense of well-being was a spectrum of qualities that made them feel well, such as calmness, restfulness, peacefulness, and contentment. These qualities or components of well-being were encouraged by the experience of being part of something larger than themselves. 'Being part of a larger whole' also assisted the participants in coping with the illness as it facilitated a sense of inner security and stability. Praying, being in nature, or contributing to a life larger than oneself helped regulate emotions, increased the awareness of the impermanence of feelings, and/or reduced reactivity.

Discussion restoring meaning and purpose. The participant's coping response to the disruption of their sense of meaning and purpose was analogous to what Bury (1991) conceptualizes as "maintaining a sense of value and meaning in life, in spite of symptoms and their effects" (p. 461). For many participants, the illness experience brought to the foreground a sense of mortality and acute awareness of the aging process. This was evidenced by the participants' perception that their illness had called into question their hopes and expectation for the future, one that did not include cognitive impairment. As such, developing a new sense of purpose and making one's current life meaningful surfaced as an important step in the process of creating an alternative sense of well-being. To perform the adaptive task 'restoring a sense of meaning and purpose', the participants drew primarily on the following strengths: (1) finding meaning in goals; (2) finding meaning in activities; (3); finding meaning in simple pleasures; and (4) finding meaning 
in being part of a larger whole.

To cope with the threats to their well-organized and highly-detailed set of beliefs about the world and their place in it, all participants used a variety of problem- and emotion-focused coping strategies, which is consistent with the transactional model of stress, appraisal, and coping (Lazarus and Folkman, 1984). The problems addressed by the participants using solution-oriented coping strategies were those that were amenable to change. Examples of this type of task-oriented coping included making sustained effort, modifying activities, and seeking out new activities that they could share with others. For those problems resistant to change, the participants used numerous strategies to regulate their emotions (emotion-focused strategies). For example, they engaged in cognitive processes whereby they learned to tolerate the illness and simultaneously bracket off the impact of its symptoms so that the effects on their sense of meaning and purpose was minimized.

Consistent with Bury (2001), the participants engaged in two types of normalizing coping strategies. They tried to maintain their familiar sense of meaning and purpose by pursuing as many pre-illness activities as possible while at the same time trying to find ways to incorporate early-stage dementia into an altered lifestyle, one that was inclusive of the illness but did not focus on it. In addition, they were mindful of the attitude with which they approached life; they distracted themselves; and they prayed, contemplated, or engaged in calming activities such as spending time in nature or with pets.

Lazarus and Folkman (1984) argue, "the persistence of a chronic stressor can give the person the opportunity to deal with its demands, or to deal with it by avoidance or distancing. New skills can be developed, commitments reordered, old goals abandoned, 
and new ones created." (P. 100). Consistent with the above description, the symptoms of early-stage dementia were persistent stressors in the participants' lives as evidenced by the fact that all participants alluded to coping processes that reflect Lazarus and Folkman's conceptualization. Many participants reported that they were no longer able to pursue many of the goals they had had prior to the onset of early-stage dementia and described coping processes that closely matched what Folkman and Moskowitz (Folkman, 1997; Moskowitz et al., 1996) termed meaning-based coping. They reevaluated and actively changed some of their goals and substituted unrealistic hopes and dreams with realistic and meaningful objectives or developed favorable interpretations of events.

Although behavioral and mental disengagement is viewed as dysfunctional in many circumstances (Carver, Scheier, \& Weintraub, 1989b), giving up efforts to achieve goals with which the illness was interfering and pursuing activities that distracted from thinking about these unattainable goals surfaced as highly adaptive responses in this study. This finding is consistent with theories that have begun to recognize that misguided persistence might have undesirable consequences (Taylor, 1991). In addition to withdrawing effort and commitment from an unattainable goal and formulating new goals, the participants ascribed positive meaning to everyday moments (such as appreciating the view out of the bedroom window) or conceptualized their situation within the larger context of religious or spiritual beliefs (such as seeing God in control of their lives and the illness as part of his larger plan).

As discussed earlier, some of these coping strategies could be interpreted as 'denial', if viewed through the lens of traditional frameworks of loss and grief (Kübler- 
Ross, 1969); however, if looked at within the context of the 'shifting perspective model' (Paterson, 2001), these same strategies have a healthy function. According to Frankl (1963/1984), the essence of life is the meaning that each person ascribes to life in a given moment. From this perspective, there are unlimited choices and always an opportunity to decide how to approach unchangeable circumstances. As agents of meaning-making, the participants used many of the above coping strategies not only to deal with problems but also to negotiate and shape their sense of meaning and purpose.

Rebuilding connectedness. Another significant theme that surfaced was the importance of feeling connected to other people and the world. Kathryn captured the essence of this theme when she stated,

Well, I think being connected to other people is what makes it easier. You know, whether it's my kids or whether it's friends or, or, you know, or even if it's just sort of making some sort of a brief connection, whether it's playing baseball with those little guys or whether it's saying hello to somebody down at the lake or whatever. But connecting with other people and, you know, I think that the, you know I've lived alone for quite a few years now, and I didn't feel lonely. Since the diagnosis, I feel lonely. And I, and I think it has to do with going through this alone, in a lot of ways. And so to be with other people is a break from that feeling. So being with people makes it easier. (Kathryn, Photo- Interview)

In a similar vein, Irene explained,

I feel so lucky 'cause so many people in this society have been alienated. And they're not connected to anybody, a human being. And then when you get Alzheimer's, gosh, it's just gotta be hell. It's just got to be hell. (Irene, PhotoInterview)

Interactions with others served as an environment in which an alternative sense of well-being was constructed and sustained. It was through a process of repeated contact and experiences with others that the new sense of well-being emerged.

For the participants, 'rebuilding connectedness' meant to acknowledge their 
relationships had changed and engage in efforts to construct new forms of relatedness. Relatedness was construed as having a sense of safety with others and spending time in rich and rewarding interactions. How others responded to the changes associated with early-stage dementia determined to a large extent the participants' sense of belonging and well-being. With family members and long-term friends new ways of relating were established or the relationships dissolved. New friends and acquaintances were selected on how well they related to the participants' current level of functioning. Most meaningful and thus preferred and sought out were those relationships that increased the participants' sense of (1) identifying one's inner circle of support; (2) identifying one's outer circle of support; (3) feeling valued; and (4) feeling understood. The feature common to these strengths is the importance of attending to the interpersonal dimension of well-being by honing one's social network so that one's available social partners fulfill one's emotional and practical needs, which in turn support one's striving towards wellbeing.

Defining one's inner circle of support. One of the strengths that surfaced as being relevant to the participants' sense of connectedness was 'defining one's inner circle of support'. 'Defining one's inner circle of support' meant for the participants to acknowledge the profound sense of loneliness that accompanied the experience of earlystage dementia and identify caring and sympathetic others that gave them both tangible assistance and the feeling of being vitally connected with others.

Feeling connected meant to perceive oneself as being part of a supportive social network and having help available. Being surrounded by supportive people was a crucial part of the process of reclaiming well-being as it allowed the participants to both have a 
sense of belonging and to manage the changes that the illness introduced into their lives more effectively. When talking about the photograph she had taken of her sister (figure 144), Irene captured the essence of this strength and its many aspects by sharing the way in which her sister supported her.

I mean she [sister] makes sure I get the bills paid, and you know, and she doesn't let me, you know, be alone very long, you know, by myself, although I've got a lot of friends who know I have Alzheimer's, and they pick me up and do stuff. So she's, she's really on top of it in terms of taking care of my needs, which is very important, and taking care of the things like finances and, you know, decisions and, you know, things like that, which I've always, part of the, the, I'm always, part of that discussion. It's not just somebody telling me that's going to do it. (Irene, Photo-Interview)

All participants had (consciously or unconsciously) identified one or more persons within their network of family members and friends who they could call on for assistance and support. All participants included photographs of these supportive others in their portfolios to show that they could count on them for help on a day-to-day basis and in emergencies. Karl (figure 145), Chester (figure 146), and Duncan (figure 147) included photographs of their spouses to illustrate that having support from their wives made their journey with early-stage dementia easier. "She's my guardian angel, so to speak. And the love of my life." (Karl, Photo-Interview) "She takes care of me as good as she can. [...] She does everything for me, just about. She takes care of me. She cooks. She's a doll. She's a beautiful, I'm glad I got her." (Chester, Photo-Interview)

Our roles have changed a bit but I don't really know what changed. I love her as much as when we got married. I think when she tells me she wants me to do something I better listen to her. (Duncan, Photo-Interview)

Monica included a photograph of her ex-husband's grandmother (figure 148) to illustrate that sometimes the sense of being supported was all she needed to get through 
tough times. Ruby included a photograph of her daughter (figure 149) and Uma took a photograph of her long-term boyfriend (figure 150).

These spouses, significant others, siblings, children, stepchildren, grandmothers, and friends provided needed support by playing one or both of two central roles. They were a source of emotional or practical support or both. Those whose role was to provide emotional support assisted the participants in managing emotional responses to the illness through ordinary conversations and shared activities as well as specific conversations on how to cope with stress. Although there was nothing these supportive others could do that would alleviate the feeling of loss that the participants experienced, just being there, willing to listen helped the participants regulate emotions and feel connected. Others were appreciated because they listened to what the participants shared about their illness journey and the ideas they had about coping, learning, and adapting. Kathryn captured the essence of this resource in a photo-diary entry that accompanied the photograph she had taken of one of her friends (figure 151). "You know, being a therapist, my friends are therapists. And they, I don't know whether this is true or not, but my experience has been that they're very understanding." (Kathryn, Photo-Interview)

Among other things, these supportive others were warm, empathic, and affectionate and were not afraid to ask the participants directly about their feelings and experiences.

She [daughter] is there for me. She will call on the worst day that you've had in six months and say something like 'something is going on - isn't it?' And you think to yourself 'how the hell did you know that?' (Monica, Dyadic-Interview)

They were not easily shocked and accepted the participants' feelings as natural. Walking, being outdoors, and talking to somebody that will listen to me without 
arguing with me [is important to me]. [...] I have some friends, that I can be very up-front with. And I have one friend, one friend who says, well Rosabelle, just get over it. [laughs] But she listens well. (Rosabelle, Photo-Interview)

Being able to share one's experiences with someone who was willing to listen was perceived as a 'real gift'. There was a sense of relief in knowing that they could give voice to what was frightening and painful to them.

And I feel like that's a real gift to be able to do that. I don't know if everybody can do that or not. But if they can, I'm very grateful, because I think it's a wonderful feeling just to empty yourself. (Rosabelle, Photo-Interview)

Knowing that there was a sympathetic listener made it easier for the participants to express themselves. They were able to verbalize their feelings and through verbalizing, discover and acknowledge them. For some, the illness invoked a feeling of profound loneliness that expressed itself in bouts of insecurity and intense helplessness. The calming presence of others relieved some of the feelings of anxiety and fear.

When she's gone and I'm by myself at home, it's like, like I get scared, you know. You know. I don't know, which is stupid, but I do, you know. You know, start thinking that people are going to come in or something like that. I think I do that. (Chester, Photo-Interview)

Those whose role was to provide practical support offered some type of tangible assistance and guidance that helped the participants navigate life in a complex society. They helped the participants work out practical problems by providing transportation, handling financial matters and attending to other types of paperwork, organizing calendars and securing access to recreational activities as well as providing assistance with activities of daily living. Practical support from others was seen as a vital resource and over time, the participants learned to say yes when somebody asked if they could be of assistance. 
Both, Fanny (figure 152) and Kevin (figure 153) took photographs of their spouses to highlight the important role they played in terms of transportation. "He [husband] drives me. I don’t drive anymore.” (Fanny, Photo-Interview)

I drive to places that I am familiar with but there are some out-of-the-way places that Beatrice [wife] has to go with me because there are so many turns and curves - then I get to the point 'how the hell do I get back' and I start feeling freaky about it. (Kevin, Photo-Interview)

Likewise, Kathryn's daughter assists her mother by driving her to medical appointments and other meetings. "I have one daughter that still lives in the Seattle area, and she's the one that drives me to appointments and stuff if I need that." (Kathryn, Photo-Interview) When talking about the photograph she had taken of her husband (figure 154), Corinne explained that one of the many things that Ken does to support her is managing their finances. "Well, Ken's taken over all of that [finances]." (Corinne, Photo-Interview) Similarly, Kathryn explained that she relies on her daughter for doing paperwork related to insurance and finances. "My smart daughter down in Oregon - she has taken over all that insurance stuff - I don't have to do any of that." (Kathryn, DyadicInterview) Others highlighted the assistance they receive in organizing their calendars and securing access to recreational activities. "And sometimes it [calendar] gets confusing, but you know, I usually can find somebody to help me to straighten it out. Mostly Hetty [sister], you know.” (Irene, Photo-Interview)

Identifying supportive others involved for the participants to carefully examine their own needs and their family members' and friends' strengths. This evaluation allowed them to identify people who could provide them with specific assistance if needed. The below quotes illustrate some of the complexities involved in defining one's 
circle of support. Some participants composed circles of support in which one person had more than one function. When Karl talked about the photograph he had taken of his wife (figure 145), he described the multi-faceted role that Betty plays for him in terms of emotional and practical support. "Yes, she's the love of my life and keeps me out of trouble. Keeps me clean. Keeps, well she does all the financial stuff. I'm lost in that, so that's a big one." (Karl, Photo-Interview)

Others created support networks where a combination of several family members met the needs they could no longer address themselves. Monica shared how she relies on several family members and how each person in her support network has been carefully selected for a specific reason to perform distinct tasks.

I don't know whether it is one person because I rely on my daughter for different things than I rely on Nana for. And I do rely on my sister and I haven't brought her up very much but she calls me almost every day to see how I am doing. (Monica, Dyadic-Interview)

Similarly, Odette shared how two of her five sons (figures $162 \& 162$ ) provide her with different types of practical support. Practical and emotional support often went hand in hand. Odette's son takes the opportunity to have lunch with his mother weekly after he takes her for her medical appointments.

My older son has my power of attorney. [...] My youngest takes me to my doctor's appointment every Monday and then we go out to lunch and then he brings me home and then I don't see him again until next Monday unless there is some real reason to come. (Odette, Photo-Interview)

Others selected a group of family members and friends to provide assistance. Irene considered her sister (figure 144) her primary support but relied on others to supplement her sister's support.

Yes, yeah I've got a lot of friends. They come up. They come and pick me, make 
sure I get rides to, you know, go to meetings and stuff that I would be, that I would like to go to. And so, you know, there's a, it's just, it's not just me here and, and just my sister Hetty. But, you know I've got close friends that also look out for me and engage me, which is really important. It's very important. (Irene, Photo-Interview)

Some participants particularly appreciated the support they received in terms of activities of daily living. "And my son-in-law came over the other day and helped me do something. I forget what it was, but something in the house.” (Kathryn, Photo-Interview) Odette took a photograph of her daughter-in-law who had surprised her with a warm dinner (figure 155) to show that she appreciates the support she receives from her children. In a similar vein, Vincent and Ella felt supported by others taking over the cooking responsibilities. "My son cooks a lot for us lately - since my illness. He moved back to live with us to and help us. He is a really good cook." (Vincent, Photo-Interview)

He [husband] has sort of taken over the cooking - I, I hesitate - I, I - and I am depending on him a lot - I, I get a little mixed up, you know. [...] I don't catch things as fast as I used to. (Fanny, Photo-Interview)

Whom the participants chose to be included in their circle of support depended primarily on the following criteria: trust and sense of safety, proximity and availability, and collaboration in decision-making. Fay included photographs of her son and sister in her portfolio (figures $156 \& 157$ ) to show that to be included in her circle of support she needed to feel that she could trust that person implicitly.

My sister and my son. Two people that truly love me. I don't worry about anything. I don't care what they ask me. The answer is yes. I don't care what it is. I would trust the two of them with anything. (Fay, Photo-Interview)

Similarly, Sima took a photograph during one of her outings (figure 158) to symbolize that she had included her friend Nathan into her circle of support because she trusts him completely. Monica spoke about the photograph she had taken of one of her 
primary support persons (figure 148) to illustrate that she values 'being accepted'. "She

[Nana] just accepts me. She just loves me." (Monica, Photo-Interview) Kathryn explained how proximity and availability played a major role in defining her circle of support.

I mean one thing is, one of my daughters lives in Oregon - so, what can she do? I mean she does other kinds of things that she doesn't need to be around for - she keeps track of financial stuff but my other daughter - she works full-time. My friend Kelly is the one who supports me most. (Kathryn, Photo-Interview)

For Irene, collaborative decision-making and a sense of safety were the determining criteria in assembling her network of support.

Hetty's [sister] very important because she, she looks out for me. And at the same time she's looking out for me, she's also very aware of my own independence, and giving me that space, which is that, you know, then I'm still a person. You know? And I think, and it's tw-, it's a tween-, it's a, we're a team together doing that, you know? (Irene, Photo-Interview)

Establishing one's circle of support was not an easy task. Tiffany included a

photograph of her son in her portfolio (figure 159) to illustrate that it required a great

effort from her to ask him to function as her primary support person.

And the hardest thing for me in all of this was, you know, I'm, I'm the parent and he's the child. And he should be coming to me for help. But for me to have to turn the tables and tell him, you know, that yes, I know you've noticed, you know, my short term memory loss, but this is the reason, and, you know, I'm going to need somebody to back me up. So he without hesitation agreed to be my care partner. So he comes to the support groups here and, you know, we don't talk about what happens in my groups and what happens in his group. But he comes here faithfully, and again, I'm just, I'm so glad. (Tiffany, Photo-Interview)

Defining one's outer circle of support. 'Defining a circle of support' not only

entailed identifying those who would be able to provide emotional and/or practical support. It also entailed being aware of and seeking contact with those who facilitated connectedness by providing a sense of joyful companionship and nurturing togetherness. The specific resources that the participants used to identify their outer circle of support 
were nurturing family relations, nourishing relationships with friends, neighbors, peers, and strangers, and participating in online communities.

Being part of a family system was important to all participants as can be inferred from the fact that images portraying family life were part of each participant's portfolio. Many participants found that the regularity with which they had contact with family members played an important role in their sense of connectedness. Ruby (figure 160) and Fanny (figure 161) included photographs of family members to show that to them daily contact with their children and grandchildren was particularly valuable. "My children, especially the oldest one calls me almost every morning." (Fanny, Photo- Interview) "My granddaughter comes to visit every day.” (Ruby, Photo-Interview)

What also contributed to the participants' sense of connectedness was when family members were supportive of their goals. Odette include photographs of two of her five sons (figures $162 \& 163$ ) and shared,

They [children] have been good about, you know, just keeping an eye on me and they encourage me to walk 'you need to walk more, mom'; 'you need to do this more, mom' - that sort of thing but anyway, we get along and I appreciate their help. (Odette, Photo-Interview)

Similarly, Uma took a photograph of her son's photograph (figure 164) to show that she values his support. "I mean, he [son] totally helps me when he's here. I mean it's wonderful, you know? He just, he's like okay mom, I'm here, what can I do? You know? Just totally supportive in what I want." (Uma, Photo-Interview) Irene expressed the vital role that family relations play in her life by sharing her concerns for people who may lack this resource. "Yeah, we are close as a family. I would think for people who, who don't have a family it must be really horrible to have Alzheimer's." (Irene, Photo-Interview) 
Interestingly, some of the participants noted that experiencing the symptoms of early-stage dementia had positive interpersonal consequences. They explained how family members and friends had become closer and more supportive since their diagnosis. Fanny took a photograph of her son (figure 165) to show that her children have become more affectionate since her diagnosis. She shared, "I am getting kisses and hugs from him now and all these years I never have. [laughs] [...] Oh yeah, it has to do with the Alzheimer's. They [children] are nicer than they have ever been." (Fanny, PhotoInterview) Similarly, when talking about the photograph she had taken of her husband (see figure 154), Corinne shared that she is surprised about the dedication with which her husband cares for her. "It's just, and Ken [husband] is, I just cannot believe. He's always been kind of not caring much about things. But now he's just, just been remarkable.” (Corinne, Photo-Interview)

Pre-illness friendships played a role in many of the participants' lives. Although generally not as essential as family relations, feeling connected with friends was important for those participants who lived alone and/or had no family nearby. Kathryn took photographs of two of her friends (figure $151 \& 166$ ) to show that she genuinely appreciated the approach that these friends had taken in maintaining their friendship by being a supportive and reliable presence in her life. For her, what sometimes seemed harder to bear than the illness itself, was feeling alone in the daily struggle. Kathryn's friends played an important role in this context by reducing her sense of loneliness.

And I have two good friends. And, and they're doing a good job of taking care of me. And it makes living alone a lot easier, and I like living alone. But it's harder now, I think, in some ways, you know. I mean if too much time goes by that I'm not with somebody else, I get kind of lonesome. But my friends have been great about it. (Kathryn, Photo-Interview) 
Similarly, Irene took a photograph of a photograph of her friends and former coworkers (figure 167) to show that having a support network is important to her as it facilitates being engaged and having days out with friends. "Well and I have a lot of friends, like in radical women and, you know, other people come by and take me out, and, you know, there's a very strong support system for me.” (Irene, Photo-Interview)

Nevertheless, even those who lived with partners considered pre-illness friendships important. Getting together with friends occasionally served as supplemental support and facilitated a sense of connectedness. Chester and Fanny took photographs of friends (figures $168 \& 169$ ) to show that occasional visits from long-term friends support their sense of relatedness. Romilda (figure 170) and Sam (figure 171) took photographs of their friends to show that meeting with friends for lunch is an important resource for them.

For some, pre-illness friendships no longer contributed to their sense of connectedness. Tiffany deliberately decided to end friendships she had with some of her co-workers after receiving her diagnosis. She felt that it was important to turn the page, start a new chapter in her life, and develop relationships with people who know her for who she was now, someone living with the symptoms of early-stage dementia.

No, I don't have any contact with my old colleagues and I think I'm doing that intentionally, cause I think that's who I was. And so when I left, I left. You know, it's like, the book's over. Put it on the shelf. And now this is a new book. This is a new journey. So I don't need to pull from old. I don't want people to know that they're comparing me to who I was, cause this is who I am now. And I like it. I really like it. [...] I was seeing a counselor for a while who told me I have to stop compartmentalizing. And when I left that day, I thought, he doesn't get it. He doesn't get who I am, so I'm not going to go back. That's what I do. (Tiffany, Photo- Interview) 
Support groups and peer support also played an important role in rebuilding the participants' sense of connectedness. At the time of this study, 11 participants were attending support groups for people with early-stage dementia. For the past five years, Chester, Corinne, Duncan, Fay, and Karl were members of a support group that I facilitate. They found that contact with these peers facilitated a fundamental sense of belonging and drew strengths from knowing that they were not alone, that others were coping with similar challenges. When Monica talked about her support group, she expressed the sense of kinship she felt when interacting with others with the illness.

I went online looking for research projects, and so that's how I ended up with an Alzheimer's disease group where they helped you come up with coping mechanisms and that sort of thing. [...] And we're still meeting [...] I've got a relationship with these people. We're meeting once a month and it's quite a support and encouragement for me. (Monica, Photo-Interview)

Being a member of a support group and having an opportunity to talk about her experiences was an empathetic, encouraging, and empowering source of power for Monica. Another way of rebuilding a sense of connectedness was for the participants to spend time with peers outside of a support group setting. Kathryn took a photograph of a woman she had met in a support group (figure 172) and explained how her feeling of being socially connected increases by spending time with someone who can relate to and validate her experiences.

And then I took a photograph of a woman from the Alzheimer group that I met there. And she's a really bright young woman. She was the head, she was a nurse, head of a burn unit in one of the major hospitals here in town. And this has, you know, been quite disrupting and dismaying and all kinds of things, and she's certainly younger than I am. So I just think I have a good feeling about what this is like for her. And we, you know, we walk a lot together and talk about what it's like and all that kind of stuff. So I wanted a picture of her. [...] I mean some of our symptoms at this point are different, but we're pretty overlapping. And so, you know, we can have a lot of good conversations and talk about our experiences and 
that sort of thing, and it's very gratifying. (Kathryn, Photo-Interview)

Joining other types of support groups also helped the participants to decrease feelings of social isolation. Actively processing their experiences in interactions with others in similar situations provided a sense of security and relatedness. Sima included photographs of two other support groups she had joined (figures $173 \& 174$ ). She found that support groups were a good place to receive practical tips and get confirmation that her feelings were 'normal'. It was a source of acceptance and respect. Uma took a photograph of the support group facilitator (figure 175) to show that being a member of a support group is a good way for her to stay in touch with other people. Tiffany photographed fellow support group members (figure 22) to show that for her, participating in a support group allowed her to share information and experiences. Meeting others in similar situations provided reassurance as it reminded her that she was not alone and fostered her motivation to cope positively with the changes to her lifestyle.

To know I'm not alone, and to hear how other people are experiencing this in our lives, you know, 'cause we have been highly functional people, and how are we coping with it, and you know, just to get the experience knowing I'm not alone, I'm not the only pioneer, you know, trying to forge out, you know, a path about all of this. And so we, you know, we sort of check each other. (Tiffany, PhotoInterview)

Giving and receiving support, encouragement, and guidance meant for Tiffany to be able to stand up for herself and others. A number of participants were unable to attend a support group because no appropriate group was offered in their community. For those who lived alone and were no longer able to drive, attending support groups was also challenging due to lack of public transportation. New relationships focused mostly on whom the participants met at organized activities, namely senior centers and/or adult 
daycares, and they did not translate beyond these settings. Outside of these organized activities only Kathryn maintained a friendship (figure 172) with a woman whom she had met in a support group. "I go to the groups at the senior center but it is very hard when you are a senior to break in and get friends. I mean I've got friends but there are friends and there are friends." (Sima, Photo-Interview)

Neighbors were also an important component of many participants' outer circle of support. These participants found that their neighbors not only offered practical help but the positive interactions with them facilitated a sense of being in contact with the world. Tiffany included several photographs of her neighborhood and neighbors (e.g., figures $176 \& 177$ ) to show that being part of a close-knit neighborhood provided rewarding opportunities for interactions. To be known in the neighborhood at least well enough to be greeted by people she met in the street was a natural part of rebuilding a sense of connectedness.

And so there's this little park that, 'cause I live on a street that's, you know, one way here and then one way that way and so the whole park is right there. And it's grassy and there's a little park bench there and we all take our dogs there and get to know each other and, it was either last week or the weekend before that somebody hired a band and we had a potluck barbecue. So I feel like I know my neighbors. So I know the big world and I know the small world, and I've told everybody, you know, my situation. [...] I live in the best neighborhood. [...] It's important to me to know who's around me. (Tiffany, Photo-Interview)

Corinne took a photograph of her neighbor to show the importance of living in her old neighborhood and the generous support she receives from her neighbors.

And our neighborhood. That's this, you know, we don't need this big house, but we don't want to leave the neighborhood. They're so good. [...] Well I was by myself in the house while Jack was gone, but I'll tell you, every friend and neighbor I know came by. I was always like, enough, enough. Just let me be. But they were pretty considerate. But they, you know, they didn't actually bug me very often, but I just was, I just couldn't believe how supportive they were. They 
came by or invited me over all the time, you know. (Corinne, Photo-Interview)

Odette enjoyed regular walks with her neighbor and felt supported in her effort to maintain a normal and regular friendship. "And there is a lady in the neighborhood that doesn't drive anymore - so I pick her up and - we have a nice friendship and she is very patient." (Odette, Photo-Interview) Several participants enjoyed the loose community of fellow 'dog walkers'.

And oh, the other thing, I don't know if I said this already, that if you start walking at Green Lake, and you go routinely about the same time of day, you get to know all the regulars. And, you know, I've lived here for 40 some years, and, yeah, a little over 40, and it's pretty close to Green Lake. But I didn't know until I started walking down there on a regular basis when I got her that there's this community of people that are connected down there, and it's just this wonderful, nice surprise in the middle of a big city, you know, that these people are as connected as they are. And they're the regulars, and they all walk about the same time, you know, all that kind of stuff. And that sense of community is real important to me. (Kathryn, Photo- Interview)

Through involvement in their immediate community the participants established a sense of connectedness that allowed them to focus their attention on their day-to-day activities and enjoy regular informal and predictable exchange with their neighbors. Sometimes it was easier to establish a sense of connectedness with 'strangers'.

I mean I, I just, it's a pleasure to go to a store and be waited on when they don't know what's going on, cause they treat me like a normal person. But other places, it just doesn't feel quite the same. (Kathryn, Photo-Interview)

When interacting with strangers, it was easier to control the flow of information and avoid disclosure as these encounters were short and the likelihood that the participants' limitations would be discovered was less. As such, meeting strangers was generally viewed positively as it helped the participants to interact socially.

Meeting other people via virtual communities helped two of the participants to 
feel connected. Corinne shared that she regularly visits the Dementia Advocacy and Support Network International (DASNI) website where she finds interesting articles and/or connects with others living with the symptoms of dementia. Kathryn felt supported by Richard Taylor's contributions. She reported that she frequently visits his website, has signed up for his blog, and off and on exchanges e-mails with him.

And he's [Richard Taylor] written, well, he wrote my favorite book about Alzheimer's ... 'Alzheimer's from the inside out'. And when I read his, when I finished his book, I sent him an email.... [...] And then I signed up for his blog. (Kathryn, Photo-Interview)

Feeling valued. What also strengthened the participants' sense of connectedness was the experience of feeling valued. Feeling valued is about having a sense of being recognized as a human being, as someone who is a thread woven into the fabric of humanity, intrinsically the same and part of the same weave. As such, a person's sense of value is maintained and promoted when his or her basic humanity is recognized not only by him- or herself but also by others. The sense of being a valued human being is reinforced and strengthened in social settings. The perception of oneself as a valued human being is tied to the perception that one is cut from the same cloth and recognized as such. The specific resources that assisted the participants to feel valued were enjoying mutuality and making contributions.

Meaningful relationships were those that allowed the participants to perceive themselves as valued human beings. The participants placed a premium not just on family members and friends responding to their needs but also on the quality of the relationships, as their feelings of worth were linked to a great extent to how they were looked upon by these supportive others. A number of participants noted that knowing 
'somebody cares', in and by itself, re-affirmed the value of and their motivation for life. Knowing that others were concerned or cared reinforced the feeling that they were worth something, sufficiently valuable to be in receipt of this benevolence. Chester captures this sentiment in the photo-diary entry that accompanied the photograph of his wife (figure 146).

Relationships with a high level of closeness increased the chances that the participants felt positive and good about themselves. Duncan included a photograph of his wife (figure 147) to show the importance of having a mutual partnership in which both partners seek what is best for the other. Being valued for Duncan was visible in the way he and his wife related to one another. "There is nobody in the world more important than Sally [wife] - not even me. I mean, she is the most important person in the world, and if I'd lost her I'd be totally devastated." (Duncan, Photo-Interview)

As such, much of the participants' sense of feeling valued was connected to being close to someone, to be loved and having someone to love. When people reached out to the participants, it was taken as a signal that they were appreciated and that others valued their presence. Kathryn talked about the photograph she had taken of her friend Kamil (see figure 151) to highlight the value-affirming characteristics of their relationship. Kathryn appreciated Kamil's initiative in reaching out and the consistency and the predictability of their get-togethers.

There are certain, have been a few certain people in my life who have been a reliable presence. One is a friend that I've had for years that as soon as she found out about my diagnosis, she just has made an extreme effort to include me in a lot of things. And she and her dog come over and walk with us every Tuesday morning, 'cause that's the one day she doesn't have to be at work as early. And she just has, you know, really showed up ever since this diagnosis, you know, just making sure that she's in contact frequently, several times a week, and, you know, 
just stayed, keeps in touch with me and keeps track of where I, what's going on and how I'm feeling about it. (Kathryn, Photo-Interview)

For Kathryn, the act of meeting for a walk every Tuesday morning became a personal ritual. It showed that Gisela was willing to invest a specific time each week in a relationship-fostering activity that fitted Kathryn's needs as it did not require taking the initiate and scheduling a meeting time, something with which Kathryn struggled. For most participants, feeling valued meant to be respected as a 'normal', ordinary person. To be perceived as an ordinary person meant to share humanness with others, to be essentially the same as other people. Monica captured this sentiment by including a photograph of her cousin in her portfolio (figure 178). "She still treats me the same as she always has, you know, and there's not very many people that do that anymore. People, it, it tinges their response to you when they know. And, and it doesn't with her." (Monica, Photo-Interview)

What confirmed Monica's sense of being a valuable person in this instance was to be treated the way she had been treated prior to the onset of the illness and to participate in an ordinary activity with another human being. Feeling respected and regarded positively by others also included the notion of reciprocity. Relationships based on reciprocity served as an environment in which the participants felt recognized as fellow human beings. Being involved in relationships where each partner played an active role and where both parties talked and listened and acknowledged and respected each other's perspective affirmed the participants' sense of personal integrity. When talking about the photograph she had taken of her sister (see figure 144), Irene described that her sense of being a valuable person is confirmed in encounters where she is considered and treated as 
an equal partner.

So... And that's, I mean, and I rely on her [sister] for decision-making. You know, for, you know, for many things... but not everything. I mean I can be, I can make up my mind to do something or whatever. But it's helpful, you know, as a team. And what is going on and, you know, but anyway it, she helps. She helps, but she lets me make my decision too. So that's very important. (Irene, Photo-Interview)

Feeling valued meant to be respected as the author and agent of one's own life.

For Irene, this respect became visible in the way she was included in decision-making processes. Similarly, Corinne took a photograph of her husband (figure 154) to show that relationships that offered needed support but also included healthy challenges served as an environment in which her sense of value was promoted and sustained.

The above excerpts allude to a special kind of reciprocity in the context of earlystage dementia. Reciprocity here means that the partners in a relationship are equal in principle but that the goal of the relationship at that time is the best interest of the person with the illness. Reciprocity as a core quality for a relationship meant that others included the person with the illness in deciding on any agenda and more importantly, that others showed their own individuality and preferences. Valued interactions were described as those where others involved themselves and gave of themselves in relation to the participants. When discussing the photograph she had taken of her friend Gisela (see figure 166), Kathryn explained the importance of being accepted as 'me' despite the limitations.

But like my friend Gisela and her former office partner and I for years, we would get together every Tuesday and have lunch. [...] It was sort of like our own sort of support group. But once the diagnosis occurred, that became very uncomfortable for me, because they didn't talk about their own issues anymore. You know, their expectation was that I would be talking about what was going on for me. [...] And I finally stopped meeting with them, because I felt too on display. You know, they would wait for me to talk and they didn't share with me in the same way that they 
used to. That's why it's been encouraging for me that Gisela has started talking more about her dating and stuff like that, because it's a little bit of a vulnerable issue, not major, but somewhat, and she's doing it. (Kathryn, Photo-Interview)

When others revealed personal details about themselves the participants felt valued because they sensed they were appreciated enough to be invited into the lives of others. It provided a sense of being equal partners in a relationship, a feeling of essentially being like the other, of sharing humaneness. Reciprocity also involved being asked for one's opinion. Monica noted that for her being an equal partner in a relationship meant to be involved in a fair exchange where her perspective is sought out and respected. "She [sister] still trusts my instincts in parenting so she'll call me and we talk about what's going on although she is not real open about suggestions. But sometimes, when we are discussing it, I can get something in." (Monica, Dyadic-Interview) For Monica, reciprocity meant that both parties have a voice and both parties are willing to listen to the other and change their perspectives if needed. What strengthened Monica's sense of value was the feeling that her sister wanted something from her, something which perhaps only she could give.

What feeling valued meant for the participants can also be understood by contrasting value-affirming with value-diminishing interactions. In other words, it is possible to discover the meaning of 'feeling valued' by carefully examining situations in which the participants experienced a lack of respect or were at risk of losing it. That feeling valued is associated with feeling equal becomes visible in the following quote from Rosabelle where she describes an incident in which she was not recognized as a human being in her own right.

It was kind of sweet and kind of her and it kind of made me angry at the same 
time, like they think I can't do this. And so the woman [hiking group member] stayed right with me all the time. [...] You know, I was trying to give her permission not to stay with me. But she did. (Rosabelle, Photo-Interview)

For Rosabelle, being part of a reciprocal relationship would have meant that she and the other person would have had the same right to promote their interests. Being around people who were 'in tune' with the participants' condition and their needs helped the participants to feel positive and good about themselves. This becomes evident through the below quote in which Kathryn explains how not being seen for who and what she was decreased her sense of self-worth.

I've noticed that people used to talk to me a lot more about what's going on in their life and stuff like that. And, and then, people kind of stopped doing that when they found out, you know. Like, oh well, I'm not, you know, that, I don't know if it's about feeling like they need to take care of me or, or if it's about, you know, that they assume that I'm not going to be able to sort of process stuff with them in the same way I used to be, or, you know. Anyway, it doesn't feel good. (Kathryn, Photo-Interview)

For Kathryn who used to be a therapist, to be valued would have meant to be appreciated as a whole, which for her included her emotion-processing skills. The value that the participants placed on others being respectful in speaking to them was conveyed by Fanny who describes encounters that lack this quality.

"My daughters quite often say to me when I talk to them on the phone 'repeat mother, repeat' - 'oh okay, I already told you this'. So, I just try to ignore it and don't let it bother me. I mean, they are nice about it and stuff; they don't call me names or say 'you are stupid' or something like that [laughs] - they just say 'repeat mother, repeat'. So, I 'okay, okay'. But still... (Fanny, Photo-Interview)

For Fanny, to feel that she was taken seriously would have meant that others accepted and acknowledged her limitations in a respectful and non-judgmental way. Although being connected with others was a truly essential resource, minimizing negative feedback from others was equally important. Feeling valued meant for the participants to 
spend less time with people who tended to be negative or critical and could not accept their illness or comprehend their symptoms. Kathryn described how she maintained a sense of being a valuable person by avoiding those who judged, irritated, or annoyed her.

And it, and then she [physician], at one point she, I've had trouble with my eyes being kind of dry the last couple of years, and she said, she said, do you have a tic? And I just thought man, get out of here. [...] Yeah, 'cause I, you know, was trying to get them moist again. And it was usually this eye that was the driest, and you know it wasn't all the time, but I think that it probably was going on while I was sitting in that thing and she just jumped to this conclusion and, you know, and I think if it was somebody else or another setting or whatever, nobody would have ever said that to me. But, you know, here I am with this, you know, diagnosis, and she would jump to that conclusion. And it just felt like I was being pathologized, and didn't feel good. And I just, you know at this stage of my life, I don't put up with that kind of treatment anymore. It's just, it's like, no. And I didn't go back to her. (Kathryn, Photo-Interview)

By refusing to let her physician treat her as an incomplete or damaged person, Kathryn emphasized that everyone (including her) is a full and complete human being entitled to be treated as such regardless of a diagnosis of dementia. Relationships in which the participants felt valued and recognized as fellow human beings were a necessary condition for a sense of connectedness to emerge. It was important for others to acknowledge the relationship as mutually beneficial. This mutuality was grounded in the belief that the participants' role was as important as the 'helper's' role. While the participants needed 'helpers' to support their striving towards well-being, the 'helpers' needed the participants to develop their capacity as human beings, their skills at being human.

'Feeling valued' was about particular relationships with specific others, but it was also about feeling appreciated in general, which was connected to living up to one's own and other's expectations. In other words, the participants' sense of value was linked to 
doing what was expected. In many ways the participants were not able to live up to normative expectations. For example, they could no longer hold down a job, drive a car, or manage their finances. As such, making contributions to one's family or community was an important means for the participants to experience their own worth as human beings.

Many participants embraced the notion that living is giving. They did not feel truly alive unless they contributed in meaningful ways. They wanted to use their talents, compassion, knowledge, and experience in a way that makes a difference to others. There was the consistent desire, at varying levels, to make a contribution. The participants felt valued when they had opportunities to pursue activities that were meaningful to others. Monica captured the essence of this resource in the photo-diary entry that accompanied a photograph that she had taken of weeds (figure 60). It shows that although early-stage dementia had made 'doing something' for others more difficult, Monica considered it important to do whatever she could to contribute to family life. What contributed to her sense of value was to use her current abilities in a way that would benefit her family. Similarly, Kevin included a number of photographs to show that he made many efforts to contribute to the couple's life by cutting wood (figure 179), grocery shopping (figure 180), and cooking (figure 181).

Making contributions beyond their immediate family was also important. Many participants commented on the value of joining in with social activities in their communities. They volunteered, contributed to research, shared their experiences, and joined the cause. Many participants volunteered at places where they could meet other people or in organizations that catered to senior citizens. Rosabelle felt that now was a 
critical time to discover new ways of engaging in and contributing to life. She wanted to keep herself open to experiencing and enjoying new people and activities and shared her plans of taking on a different kind of volunteer work.

And I used to belong to the League of Women Voters for many years, and that was my thing that I did. Now I'm going to take a volunteer job manning the book sale, used book sale at the library. [...] It's a very pleasant place, and you meet people, and people who like books. People who like books are sitting, sitting there selling books, and people who want books are coming into the library, so you have conversations about books. (Rosabelle, Photo-Interview)

Odette shared her plans of wanting to explore other volunteer options at the senior center.

They just re-opened the senior center and I go there and they are looking for people to do some volunteer work and I know I don't feel well but I thought if I could do two hours a week - maybe one day helping the dining room helping set the tables or something - I am going to go down and talk to them about it because I don't want to not be able to get there you know - and - but I would like to go and like to get out with people a little bit because I need that - I, I don't have, I, I don't have friends except at the senior center. (Odette, Photo-Interview)

Both women acknowledged that they were no longer able to perform their previous volunteer responsibilities but were confident that they would find other opportunities and be able to be successful at it. When talking about the photographs he had taken of his exercise class (figures $181 \& 182$ )), Duncan shared what he considered an important contribution - keeping the attendance sheet.

There are a lot of things that she [instructor] has everybody do that I can't do; like on Mondays and Fridays they start out kind of lying on mats down on the floor and doing exercises and then getting up and I have so much trouble with the arthritis getting up that I usually sit on the bench while the rest of them are doing that but then I also - when I am doing that - I take the attendance and fill that out for her [instructor]. (Duncan, Photo-Interview)

In addition, he noted his ongoing effort to help others by providing financial support to organizations that he considered having a worthy cause. 
Duncan: Well, I am really impressed with what the Gates are doing. It impresses me that there all kinds of situations that they give money to. That's something I would like to do. We give some money to various charitable groups but not as much as they do because we don't have as much money as they do but there is a variety of things, organizations that I belong to, church and rotary, that I volunteer and give time, and I really enjoy doing that. And we still have a lot of wine that came out of the wine cellar that I used to have and that is stored at some friend's cellars. And when some annual event comes up where you can donate something we may end up donating a bottle of that wine, go online on the computer to see what it would cost you there if you'd buy a bottle of that today.

Karl: [laughs]

Duncan: And there is a lot of wine in the wine cellar that I'd paid 20 to 30 dollars a bottle for that right now if you went online on the computer to see what it would cost to track down one of those bottles of wine it's like a 100 dollars or more.

Karl: Probably more.

Duncan: So I can donate it to one of these organizations and they can put it on their list with the price that's listed on the computer and sell it. And then, we get the 100 dollars tax write-off.

Karl: That's good. (Dyadic-Interview)

By contributing to causes that benefitted others, the participants experienced a sense of competence. Being active in dementia-related advocacy was another means for the participants to improve the well-being of others. Some participants used their personal experiences to help change public opinion about the illness and influence dementia-related policy. They hoped that their contributions would encourage people not living with dementia to recognize that people living with the illness are and always will be whole and complete human beings. They spoke about their experiences at town-hall meetings, told their story publicly to the media and state legislators, volunteered their time at a local chapter of the Alzheimer's Association, and contributed to research on dementia.

I'm just very lucky that I've had the life I've had. That's the way I think. I mean it could be really bad if you... And you know that's so, people, so many people are so alienated in this culture. It's really, it's really, it's not right not to be, I mean it's important to be a person [pause] to re-, to be respected that way. [...] Yeah, I'm a fighter; I will always be. [...] Well yeah, what else? What else do you need 
to do? I can't give any speeches, but I'll be there. [laughs] I'm going to lobby. I just think I'll go [...] I might not be able to read a speech but I'll be there... (Irene, Photo-Interview)

For Irene, who had been politically active all her adult life, advocating for dementia-related causes allowed her to continue a longstanding passion of working towards social change. Others joined the cause by contributing to organizations providing services to people with the illness and their caregivers. Tiffany took two photographs of a fellow volunteer worker to symbolize the value that contributing to the Alzheimer's Association has for her (figures $183 \&$ 184). For Tiffany, 'joining the cause' was a primary means of 'giving back'. It allowed her to have a role in society, to be someone who is needed and can contribute.

And there's my buddy, that's Don. [...] Well he and I volunteer here [at the Alzheimer's Association] on Mondays and Wednesdays. He's 92 years old. And his wife had Alzheimer's, and so she came here and he was her caregiver. And after she died, he just kept coming. Now he lives in Ballard, and we, I take the bus here, and then he drops me off at home on his way home. [...] Oh, we do mailings. We're doing a lot of stuff to get ready for the walk. You know, whatever they need. [...] I come twice a week. Mondays and Wednesdays. So we're here from like 9 or 10 until 2 or 3, till the job gets done. [...] Uh huh, because I'm a contributor. I want to be doing. I don't want to be taking. I want to be contributing. And so I feel like I'm giving back what I can. (Tiffany, PhotoInterview)

The determination with which the participants tried to continue making contributions becomes apparent in the following excerpt. Like Tiffany, Monica considered volunteering a vital part of her life but, contrary to Tiffany, Monica does not meet her own expectations. Nevertheless, making a difference to those around her was more important to Monica than her feelings of inadequacy.

You know, I don't really know what else to do but stay involved in some organizations that I believe in and that sort of thing - and I volunteer for the Alzheimer's Association. I am on the steering committee for the South Sound 
Walk and things like that. So, I am still out there, I am still trying but I definitely feel inadequacies that I have now. (Monica, Dyadic-Interview)

Having the opportunity to contribute to research on dementia also increased the participant's sense of value. All participants considered taking part in research on dementia as a primary mean for 'giving back'. They hoped that their contributions would further the effort to find a cure as well as advance support programs for future generations.

And I really enjoy it [participating in research]. I enjoy it because it's for my daughter. Everything I look at, I think this is for my daughter. 'Cause she's, I'm the third generation [with Alzheimer's disease], so, you know, she's next in line. All my, so I'm, this is, everything I do is for her. (Corinne, Photo-Interview)

The desire to help others through participating in research became apparent when the participants voiced the hope that I would distill their stories into information that could be used to educate health care professionals to help others.

After our conversation the other day when you were here, you know, sort of hearing what you're hoping for, hoping to be able to do with some of this information that you're gathering and that sort of thing, and one of the things that I sort of took away from that conversation, I hope this accurately reflects what you said, but it sounded to me like part of your overall goal is to help people in the healthcare industry learn something about people with Alzheimer's. You know, and my assumption would be so that they would be able to provide better help or more support or have a greater understanding of these human beings that go in and out of their offices and so, you know, that's, that made it very, very worthwhile to, you know, do this project. (Kathryn, Photo-Interview)

Sharing some of the knowledge they had gained from living with the illness with others was also important to a number of participants. Having experienced early-stage dementia themselves, they believed, put them in an empathetic position and enabled them to help others who are at different stages of the journey or in a different position (caregiver). Frank felt that having learned how to live with early-stage dementia gave him 
a deeper understanding of how to cope with challenges of the illness, which he wanted to share with caregivers.

Matter of fact, in some of those groups, yeah, support groups, I guess, I've been the only one there. I am the only one, the rest of them are caregivers, a lot of times. [...] And I think it's important to let 'em know the other side of the story, you know. [...] So I think, I feel that my contribution is that sometimes that they're, don't know what, the caregiver doesn't know what the person getting the care is looking for or understands and so on. And I try to help that way if I can. [...] Because some of these meetings that I go to, some of the people that are the care..., giving the care are really not all that smart either. So [laughs], that's a little different too. (Frank, Photo-Interview)

Nancy was equally eager to pass on her experiences. She thought that I, as a researcher, would be a good conduit for conveying her insights for the benefit of others.

You should encourage any elderly person to go the movies. If they have a group of people to go with or somehow get there - that's just ideal for your imagination and for your thinking. (Odette, Photo-Interview)

However, sometimes it was not easy for the participants to recognize their own contributions. Although they used their abilities and skills in ways that benefitted others in a number of ways, they overlooked the little things and had to remind themselves that they were unique individuals and therefore had something to give.

And I realized [during the photo-taking activity] I do still have things to contribute, I think, and I think the world has things to contribute to me still, too. So I need to, I want to start, you know, pushing some of those a little bit more. (Monica, Photo-Interview

Reaching out, giving, and helping other people was important to all participants. Contributing to the lives of others provided a sense of value in two ways. It increased social recognition, and it fostered the participants' awareness of their own worth. Some used their talents and skills in the family context while others chose the community setting. Some used volunteer work as their primary means to 'give back'. Others 
participated in research on dementia hoping that their contributions will benefit future generation. Yet others supported efforts to raise awareness about dementia or tried to help people with the illness and their care partners directly drawing on their own knowledge and experience of the illness. The participants worked hard to maintain a sense of value. Two components were described as playing an important role in the process of feeling valued. Social recognition and the opportunity to contribute to the well-being of both, one's own family and the community at large. What increased the participants' sense of value was to experience that they mattered as persons, that their presence made a difference to others and that their input was appreciated. A valuable life was a life that they enjoyed but that also allowed them to make contributions.

Feeling understood. What also strengthened the participants' sense of connectedness was if others 'understood' the participants' situation Tiffany took two photographs related to the Alzheimer's Association to symbolize the importance of 'feeling understood' (figures 185 \& 186). She shared,

One of my photos of something that's important to me is this building. I come here to volunteer. I come here for support. Everybody who works here understands what we're going through and is supportive, and it's, it's such a safe, nourishing place to come. It has absolutely been a god-send. (Tiffany, PhotoInterview)

Being connected to the Alzheimer's Association meant for Tiffany to be supported by people who understand what she was saying and who were willing to work with her in a partnership. However, gaining understanding from others was usually not simple as it required involvement and courage from both the participants and others. The specific resources that the participants used to feel understood included telling or not telling, protecting others, and educating others. 
Telling or not telling required that the participants revealed only what they

thought others would be able to understand. This was not always a conscious act, but was something that the participants had learned by experience. Some participants recounted that once they told someone of their diagnosis, their relationship with that person changed dramatically.

People treat me differently just because they know about the diagnosis, yeah. [...] Well, people pull back. You can, I mean, they actually will physically move back. They don't touch you as much. The way they talk to you is a little bit different. And I'm sure they have no idea. I'm sure they have no idea, you know, that there's a difference there, but there is. (Monica, Photo-Interview)

However, deciding whether or not to disclose their diagnosis or continue to disguise their limitations was difficult. In other words, the participants had to make a choice about if, when, and to whom to disclose their diagnosis and/or limitations, a choice they had to make repeatedly in old and new relationships. Some participants were very open about their diagnosis and shared it with everybody immediately after they had learned about it. "Oh, of course. I tell everybody. You know, if you have cancer you tell everybody too." (Corinne, Photo-Interview) They assumed that others would be supportive and provide needed assistance and help. Others decided to tell their immediate family as soon as possible but were hesitant to inform their friends or strangers about their diagnosis. They felt that sharing the diagnosis helped others to make sense of the changes they had noticed. "I told my wife and children about my diagnosis. It was like 'oh my - I thought you were kind of forgetful'.’ (Kevin, Photo-Interview)

Again others were less comfortable with sharing this information because they feared adverse reactions of others. They wondered how family members and friends would react and decided to keep their diagnosis to themselves, as they feared stigma and 
rejection.

I haven't really told many people in my life about it, because I'm afraid to have people brand me, you know? [...] I was originally thinking about telling my son, but I'm like, you know I don't want to tell my son, even though I'm close to my son, and, you know, he's probably the closest person to me in my life. But basically, it didn't seem like anything good would come of it. You know? And really, that was the starting of it. I mean I did tell my boyfriend. He's the only person besides you that I've ever told. [...] basically because I'm too afraid of being labeled. (Uma, Photo-Interview)

Avoiding disclosure meant maintaining distance, controlling the flow of information, and therefore preserving one's control over the situation. Many participants thought that when meeting new people, it was advantageous to hide what others would not be able to comprehend. They explained that if they were not asked about their illness, it was helpful not to volunteer the information.

I'm not bragging about it. I don't think I have to do that, I think I'm just as good as you, you know what I mean. But I know what my memory is. But that's the way it goes, you know. I ain't worried about that. That's just little things, you know. (Chester, Photo-Interview)

Some family members and friends met the diagnosis with disbelief and rejection.

They refused to believe that the participants had an illness since they 'appeared' well.

I told people about my diagnosis - I mean I don't say it unless something comes up in the conversation. To me, it's nothing to be ashamed of [laughs]. [...] But I have this one friend who thinks there is nothing wrong with me. [...] I mean I don't even say anything to her anymore - she'd right away would say 'there is nothing wrong with you'. (Fanny, Photo-Interview)

Most participants tried to mask their declining abilities and cover up gaps and lapses in memory during social encounters. They seemed to think that there was no sense in sharing things that would not be interesting to and understood by others.

I feel like that with my son - more so than with my daughter. I feel like I have to keep up a façade and I am not sure whether I do it because I feel like he is so young [25 years]. It still seems important to me to try to maintain a façade that I 
am who I used to be with him, which I don't feel with my daughter at all. I still feel I have to maintain that façade of being a parent with him although I understand that he has his own life and he is happy with his own life. And although I don't know what his plans are - all I know is that he has plans in terms of what he intends to do. It's not that I have to do this but I feel like I have to do this - it's totally illogical. And maybe sometimes I think it's just because I don't want to lose my place. (Monica, Dyadic-Interview)

Not to disclose their diagnosis and/or limitations was a source of security and comfort as it allowed them to continue valued social roles and occupations. 'Feeling understood' was about revealing and hiding, and depending on chance for being understood and accepted or rejected. It required the participants to guard themselves by either 'rationing' themselves or making adjustments in the relationship by playing a role or pretending to be someone else. The importance of maintaining dignity and wholeness through setting limits to what others know about oneself becomes apparent through the following two excerpts.

My son was not doing well at all. He did help me move, you know, go through the storage unit this last weekend. And after the day was over, he said, you know that wasn't so bad. And I knew, because he's, I knew that he was having a really hard time with the diagnosis, and almost in a way was mad at me, which is not an unusual reaction for people to have when moms and dads don't act the way they're supposed to. And, you know, and it hurt. But I also understood it. So, so it was nice to spend that time with him this weekend and have, you know, things go well, and have him feel more comfortable with me. (Monica, Photo Interview)

This is an intriguing extract; Monica explains that she simultaneously feels hurt by and yet understands her son's reaction to the diagnosis. It seems that Monica is mindful of the dynamics underlying her son's behavior. She explains it and has apparently decided to accept it in order to continue the relationship with her son. At the same time, she tries to make her son more comfortable by continuing to play her expected role as a mother. She tries to minimize the intrusiveness of the illness because she values 
her relationship with her son more than being able to be vocal about her illness. For her,

the importance of this relationship far outweighs her need to talk about her concerns.

Kathryn shared a similar experience.

I spent a day or a better part of a day at a grade school and what I had wanted to do was work with kids individually who have trouble with reading skills - you know, where they can really benefit from one-on-one. And I found a volunteer coordinator's name and I told her what I was interested in and wanted to do and then she wanted me to come in and have a day where you sort of meet people, see the facility and whatever, which I kind of found kind of a waste of time but, it seemed to make her feel really good. [Laughter] But, you know, the places she took me didn't really have anything at all to do with what I had asked about. And towards the end of the time that she was going to chauffeur me around through the building, she took me into a math class that was facilitated by a man who was in his thirties somewhere. And I am sure that he was a great math teacher and that he has this very booming voice and very dynamic gesturing and a lot of energy in the room and stuff like that - but it was all math. And I don't know if you have noticed - well, I don't know if this is me or if this is the Alzheimer's or whatever - but too much chaos just feels like - I just want to back out the door. And that's what it felt like in that classroom because of the loud voice and the chaos and all that stuff. And so the volunteer lady sort of left me there and well, 'help him for a while'. I was so discouraged with that. So I just came home and thought - it's too complicated and I sort of feel damned if I do and damned if I don't. I just wanted to tell her, these are my limitations but this is what I can do but my experience out and about in the world is that if somebody finds out that I have this diagnosis, they treat me as if I am stupid. (Kathryn, Dyadic-Interview)

The above quote presents the predicament that some participants face when trying to communicate with others. Even if the misunderstanding is with the other person, the person with the illness has become through experience apprehensive about a direct confrontation. In this instance, Kathryn chose to ration herself and go along with the volunteer coordinator's obvious misunderstanding because she wanted to pass as normal. Even though Kathryn explained that she could not deal with chaos very well she chose to stay in the noisy classroom. Kathryn was afraid to disclose her limitations because past experience had shown her that once her diagnosis was revealed people no longer treated 
her the same way. She felt that she was less in the eyes of others. They doubted and scrutinized her once they found out about her diagnosis. The irony of this situation was not lost on Kathryn. She was both disappointed and angered by how events had unfolded. The result of her effort was that she could not volunteer. The above quote clearly show that rationing, role playing, and pretending have clear limitations. Despite the ongoing efforts of the participants to contribute in some way, there are limits as to what others are willing or prepared to understand.

All participants spoke about partially concealing or rationing what they shared with others in the context of protecting others. They conceived their lives as intertwined with the lives of others. Conscious of the fact that they needed their family members and friends to do many things for them, the participants worried that those providing them with assistance and support may be dragged down because of their needs and dependencies.

You know, so that's the bad news [having a diagnosis of early-stage dementia], but I can live with it, but I don't know about her, you know, if I don't drive her crazy, you know. And I don't want to lose her, I'll tell you that, but I hate to cramp her stuff, whatever, you know, cramp on her too, you know. It's hard on her. She takes care of me all the time, so very fortunate to have her. That's why I wish I didn't have it, you know, my Alzheimer's, you know. (Chester, PhotoInterview)

A number of participants voiced concerns about becoming or being a burden to their family or friends and engaged in active efforts to protect them.

I guess I just don't think that it's an option to not do anything. If I'm not doing anything, then I'm a burden on my kids. And I'm not going to be a burden. And so I, and I have to do something. I mean I can't just sit all day. That would be, yeah. So, so you just do things. (Monica, Photo-Interview)

The practical steps employed by the participants to minimize the burden their care 
put on family members and friends included concealing information that would be upsetting to them, securing alone time, and educating others. By regulating the amount they revealed of their emotions, needs, and preferences they hoped to unburden family members and friends. Many participants did not want family members or close friends to know about their difficulties because they felt that it caused anxiety in other people when confronted by their problems. “I don't want people to be worried, you know. That's one of my big things. I don't want them to worry." (Irene, Photo-Interview)

They did not feel confident in sharing their negative emotions with close family members and friends. They avoided talking about negative things in general, about the changes they had experienced, and how they felt about what was happening to them. They tried to hide the aspect of their experience that they perceived as stressful for others. Rosabelle explained how she consciously chose to forgo talking about her concerns and/or the illness experience to avoid being a burden. "I seldom talk to anybody. I don't want to stick my whatever, my depression, my negative stuff on somebody else. And I try not to talk about it with people in my family, because it affects them too." (Rosabelle, Photo-Interview)

For the participants, balancing their needs with the needs of others also included securing 'time outs' for their partners. They recognized that it was crucial for their partners to take time for themselves. They were conscious of the time that family members and friends devoted to their support and encouraged them to continue pursuing the little things they did to nurture themselves. That Duncan was mindful of his wife's needs became apparent when he discussed the photographs he had taken of his weekly activities (see figures $101 \& 102$ ). 
I enjoy it [attending the senior social] and I think Sally [wife] enjoys it because it gets me out of her hair. She's got so many 'girlie' groups that she belongs to that she's always off running around doing things - I'd otherwise be sitting here at home falling asleep or reading a book. (Duncan, Photo-Interview)

He tried to be conscious of his wife's needs and by attending senior socials created a situation where his wife did not have to feel guilty about spending time with her friends. Similarly, Karl seemed to have recognized that too much togetherness may result in him expecting too much from his wife. When he talked about the photograph he had taken of his radio and earphones (see figure 187), it became apparent that he not only enjoyed listening to the programs at night but that he intentionally went to bed early to give his wife some space. "Well I go to bed earlier than Betty [wife] - so she can have her boyfriend in. [laughs] No, I am joking - she needs some time without me around." (Karl, Photo Interview) For Karl, being attentive to his wife's needs meant to encourage her to do something fun for herself after he retires for the day. Interestingly, neither Duncan nor Karl had shared the reason behind their behaviors with their wives who were under the assumption that both men did what they did exclusively because they liked it. Likewise, Corinne was attentive to her husband's (figure 154) needs when she encouraged him to visit their daughter by himself while she volunteered to stay with the support of friends and neighbors.

He [husband] just went back to his family. Our daughter met him in Minnesota. And I thought, you know, he just needs a little time, just to be himself and not have to think about me all the time. Course he called me a lot, you know. But I just thought that was so good. [...] And I, and I loved it [the support from the neighbors], and I was so glad because he didn't have to worry about me, because all, I'm sure he called all the neighbors all the time. (Corinne, Photo-Interview)

She also realized that her husband was not able to completely leave his responsibilities behind but she was glad that he at least had some time off for himself. 
Corinne's concerns underscore the participants' efforts to adjust what they present of themselves in order to maintain good relations. Corinne probably would have liked to see her daughter too but felt that accompanying her husband would put additional stress on him. Similarly, Ruby tried to care for her daughter by not having preferences or special wishes. Additionally, Ruby took a photograph of her weekend caregiver (figure 188), explaining that she tries to minimize the burden her care puts on her daughter by hiring a weekend caregiver so as to give her daughter a regular break.

I don't make any request - she [daughter] does as much as she can. I don't want her to do too much and I feel sorry for her that she is stuck with her old mother. [...] My caregiver - she comes on the weekend - she spends one night and two days. So my poor daughter gets a break. (Ruby, Photo- Interview)

Ruby is keenly aware of the day-to-day responsibilities her daughter is faced with. She therefore rations her needs to spare her daughter additional work and has agreed to let a stranger care for her.

Feeling understood also included the notion of educating others. Many participants found it important to share information about the illness with others. Talking about the illness with other people served a specific function in the overall social network of the participants; it communicated their experiences and explained their behaviors and feelings.

And when I read his book [Taylor, R. (2007). Alzheimer's from the Inside Out] [...], I had been highlighting the sections that I really identified with, and then I went back and had typed in some of those sections into my computer, and then shared those with my daughters. And I would say, this is what it's like, because this is the stuff I identified with. And he had already come up with the language. (Kathryn, Photo-Interview)

Sharing with others what they had learned about the illness not only helped family members, friends, and acquaintances to understand what it means to live with progressive 
memory loss but also prepared them for what to watch for. :I said [to my neighbors] I'm in the early stages, you know, and it's going to progress, and I don't know how it's going to progress, so keep an eye on me." (Tiffany, Photo-Interview) Using themselves as the source, they spoke with others about the disabling ways well-intended people treated them as they coped with the illness.

I try to encourage people to accept it like another disease. And it's really hard for them, 'cause they don't quite know what to say. And I have friends that tease me, but very few 'cause most people wouldn't like it. You know, it's a strange thing. (Corinne, Photo-Interview)

People who wanted to learn about the illness and the participants' experiences were appreciated.

But it's this, you know, I want to know. I want to know what this is like. I want to know, you know, how you're doing. I want to hear about it. She's just right there. And, and my experience has been that most people don't really ask me much about the Alzheimer's. They don't, you know, they don't ask how I'm doing. They don't, you know, they talk about other things, which is fine. But not very many people really ask you questions about it. Kamil asks absolutely everything, which is very nice. (Kathryn, Photo-Interview)

Feeling understood meant to be treated 'like the rest of us', as individuals with strengths and weaknesses and positive and negative characteristic and not be treated with suspicion, discrimination, and disrespect. Disclosing information about diagnosis and/or limitations was a double-edged sword. There were arguments that could convincingly be made for revealing or not revealing the condition. Some participants had experienced a positive openness when they shared their diagnosis and/or difficulties with others; telling others resulted in 'understanding' and they continued to feel respected. Several participants who had negative experiences of other people knowing about their diagnosis had a strong desire to keep the information about their illness and/or difficulties private. 
Once their illness was revealed, there were indications that they were relegated to a stigmatized group rather than considered individuals, second-rate citizens to be kept at arm's length. Rationing, role play, or pretense were the pieces of an elaborate web woven by the participants to be rewoven daily in order to keep being understood and respected. These efforts show that the participants possess contextual sensitivity; they reflect on their role in relationships and make adjustments as needed. That it was difficult for the participants to feel understood becomes apparent in the following excerpts.

Kathryn: I have been so impressed how much you get about this disease and not have it because my experience everywhere else is that nobody understands it. They don't quite get it. I mean they try to get it but you obviously have done some thinking about this at a level that is deeper than most people are able to go to or put themselves. You know I have some pretty caring friends, I think, who ask 'what is that like for you', but you can complete sentences and know what words to use - and that's such a nice thing.

Monica: Uhuhm - yes, you feel like you are being heard. (Dyadic-Interview)

Similarly, Vincent shared,

Like right now, I feel good. When I am around you [interviewer], you make me feel good but there are times that it doesn't work for me to be with people. (Vincent, Photo-Interview)

Discussion rebuilding connectedness. In this study I use the term connectedness to describe the important role that social interaction, support, and sense of belonging play in the participants' sense of well-being. A sense of belonging (the knowing that one is loved and that one's life has value - for oneself as well as for others) and acceptance is considered a basic human need (Maslow, 1943) that can only be satisfied through meaningful and fulfilling relationships with other people. "Social belonging is a sense of "relatedness" (Ryan \& Deci, 2000, p. 73) that arises from "lasting, positive, and significant interpersonal relationships" (Baumeister \& Leary, 1995, p. 497)" (Walton, 
2012, p. 514). However, a common thread throughout the participants' accounts was the notion that early-stage dementia, like other chronic and progressive illnesses $(1982,1991$; Charmaz, 2000), had disrupted or threatened to disrupt the participants' relationships. This disruption in their social environment impacted their sense of well-being negatively. This finding is consistent with the results from a number of studies indicating that difficulties with social relationships can have negative implications for well-being (O’Brian \& DeLongis, 1996). Thus, 'rebuilding a sense of connectedness' emerged as a necessary prerequisite for finding a sense of well-being, which is consistent with Moos and colleagues (Moos \& Holahan, 2007; Moos \& Tsu, 1977) who maintain that the successful completion of the adaptive task of 'relating to family members and friends' is pivotal to adjustment to chronic illness. To cope with the disruption to their relationships and rebuild social ties, the participants drew strength from their inner and outer circle of support and from interactions in which they felt valued and understood to develop a blend of different coping strategies as outlined by Lazarus and Folkman (1984). Problemfocused strategies (task-oriented coping) were typically evoked to prevent or resolve challenges perceived to be under their control. For example, seeking and accepting social support, "the interpersonal interactions and relationships that provide people with assistance or positive feelings" (Walsh, 2006, p. 278), was one problem-focused strategy that the participants utilized to reestablish their sense of connectedness.

Consistent with Taylor (1983) who argues that seeking social support is a means of achieving and/or maintaining well-being while experiencing personally stressful events or situations, it was through a process of repeated contact and experiences with supportive others that the participants managed to feel well again despite early-stage 
dementia. Supportive interactions with others served as an environment in which the participants experienced a sense of connectedness. As described by Carver, Scheier, and Weintraub (1989) the participants sought social support for instrumental reasons (seeking advice, assistance, and information) and for emotional reasons (seeking moral support, sympathy, and understanding). They felt connected when they were in the company of those who used empathy-based strategies (O’Brian \& DeLongis, 1996) to respond to their needs. The transactional coping literature (e.g., Amirkhan, 1990; Folkman et al., 1987; Lazarus \& Folkman, 1984) acknowledges social support as an important coping resource, but views support by and large as a uni-directional process originating from those without illness and extending to those with illness. Interestingly, in this study, social support emerged as a reciprocal process, and the notion of caring for supportive others was woven into the visual and verbal narratives of the participants. The participants used strategies such as minimizing the intrusiveness of the illness (e.g., covering up their symptoms) and believed that it was important to relieve family members and friends from time to time from their supporting responsibilities.

In other words, the participants used relationship-focused coping, which refers to "modes of coping aimed at managing, regulating, or preserving relationships during stressful periods" (O’Brian \& DeLongis, 1996, p. 782). Strategies aimed at maintaining and protecting their social relationships included making excuses for others, educating others about the illness, being always available, hiding potentially distressing symptoms from others, and treating embarrassing incidents with humor. It also meant for the participants to engage in 'anticipatory coping' (Schwarzer \& Knoll, 2003). In other words, the participants developed strategies that focused on managing pending threats. 
For example, they carefully planned social interactions to compensate for their limitations. In addition, the participants used emotion-focused (cognitive processes directed at reducing emotional distress) and meaning-based coping strategies (cognitive maneuvers aimed at changing the meaning of a situation) to cope with changes in their relationships with the social environment that they perceived as impossible to repair. Examples of how these coping-strategies were used included (1) practicing understanding and finding rational explanations or excuses for the reactions and behaviors of others (e.g., explaining a negative reaction with the fact that the illness somehow had undermined their roles as mothers, spouses, or friends); (2) distancing themselves from or making light of the changes (e.g., normalizing the changes in relationships by citing age as a contributing factor); or (3) finding benefit from the experience (e.g., highlighting the positive effects of the illness on relationships).

Reestablishing a healthy lifestyle. Another recurrent theme that emerged when grounded theory was applied to the visual and verbal data provided by the participants was the importance of healthy living. 'Reestablishing a healthy lifestyle' speaks to the notion of acknowledging the contemporary reality of early-stage dementia while preserving one's overall health so that the symptoms of the illness could be better controlled. It captures the importance of caring for the body as a whole. Protecting one's overall health by managing health stressors, establishing a slower rhythm, having proper nutrition, and engaging in regular physical activity was a key element for the construction of an alternative sense of well-being.

Knowing that a weak and frail body could aggravate the symptoms of early-stage dementia, the participants proved to be fierce protectors of their overall health and were 
creative in pursuing this adaptive task. All participants devised and engaged in healthpromoting activities and strategies. The strengths that surfaced as most relevant to healthy living were: (1) managing one's overall health; (2) resetting pace; (3) choosing a balanced diet; and (4) maintaining a fitness program. The common thread within these strengths is the importance of caring for the parts of the body that are functioning well and that can be influenced and supported by adopting healthy habits. This theme speaks to the significance of paying attention to one's overall health and how a healthy lifestyle plays a pivotal role in the participants' sense of well-being.

Managing one's overall health. 'Managing one's overall health' was one of four basic strengths that surfaced as most relevant to reestablishing a healthy lifestyle. The two resources that the participants drew on were redefining health and dealing with stress, minor ailments, and other illnesses.

A number of participants developed a new definition of health; they saw themselves as healthy if they did not have other medical conditions. "And my health [knocks on wood] - I am pretty healthy and that dominates everything. If you are not healthy, you can't do anything." (Romilda, Photo-Interview) In similar vein Karl explained,

[Interviewer: What would you say are the two things that are most important to you at this moment in your life?] Staying alive would be the first one - there is nothing bigger than that - and healthy so I can enjoy being alive. (Karl, DyadicInterview)

Nevertheless, the new definition of 'healthy' did not mean the participants saw themselves as 'free of illness'. Rather, they seemed to understand that there was more to their lives than dis-ease, impairment, and loss. "I mean, I'm glad I'm alive and healthy, 
except for this, you know.” (Chester, Photo-Interview) Others claimed that the illness made them appreciate the value of health and adopt a more positive life style.

Consequently, they felt 'healthier' than before their diagnosis.

And so it [the photo-taking activity] made me realize how, you know, how focused I was on me and my health and what was right for me to do and, you know, eating the right things, because that was good for me, and sleeping because that was good for me, and working out because that was, you know, helps my mind, and you know, all that kind of stuff. (Monica, Photo-Interview)

Dealing with stress, minor ailments, and other illnesses speaks to the notion of paying close attention to and addressing general health concerns and overall stress. Corinne captures the essence of this approach when she describes how she seeks and incorporates the advice of her physician in her efforts to develop healthier sleep habits. The quote below illustrates that Corinne is well aware that lack of sleep impacts her symptoms of dementia and her efforts to remedy the stressor.

I have a very poor sleep habit. I just can't get to sleep. And 2:30 is my normal time for my body to shut down, but sometimes it isn't, and I'll only sleep a couple hours a night. [...] Yeah, so we've been talking to the doctor about this, cause I really do need, and of course, that's one of the best things I need, you know. For Alzheimer's you need to have a lot of sleep, you know, enough to, or at least enough. He recommended walking. So I walk. When we come home, I have Ken drop, I didn't today, but I have Ken drop me off, and then I walk through, or and sometimes back again and then down the cul-de-sac. So it's, and it's quite a large cul-de-sac. (Corinne, Photo-Interview)

Three participants shared that they use prescription medications to reduce the symptoms of depression (Monica, Rosabelle, and Tiffany) and one participant (Kathryn) reported that she treats her anxiety with prescription medication. Additionally, Monica shared how she follows the recommendation of her physician and uses light therapy to combat depression by including a photograph of a sun lamp in her portfolio (figure 189),

I have a problem with not getting enough light, you know, enough sunlight. 
Seasonal affective disorder, that's what it's called. So I have a lamp that I use daily. [...] A doctor finally told me about the light. And so that's a big part of my life because of that. [...] It makes a huge difference in my mood. I do it for 45 minutes to an hour every day, and it turned my life around. (Monica, PhotoInterview)

Vincent shared his plans of losing weight.

I went to the doctor. He put me on a whole new regime. My first goal is to lose 40 pounds. That will put me at 200. And how I am going to do that? I don't know how but I will. One of the ways is walking, doing more things around the yard, and eating the right foods. You are not going to recognize me. The first 6 weeks, I will lose about 6 pounds. (Vincent, Photo-Interview)

Many participants described the importance of alleviating overall stress and finding an atmosphere of rest and relaxation. Karl took a photograph of his radio and earphones (figure 187) to portray that having an end-of-the day ritual of listening to his favorite radio shows is an important source of relaxation for him. It helps him wind down, achieve a peaceful state of mind, and eventually fall asleep.

I go to bed earlier and go to the twilight zone. Those ship programs from yesteryear - the old-time radio shows. [...] And so I listen to that crap. And then I get tired, go to sleep. [...] It's something I've started since I have Alzheimer's. I didn't used to. (Karl, Photo-Interview)

Other participants expressed that spending time in or observing nature helps them reduce stress and anxiety. Sam photographed his container garden to show that looking at nature eases his stress and helps him relax (figures $29 \& 30$ ). Kevin took a photograph of his favorite spot in the garden (figure 190) and shared, "This is a very tranquil place to sit and enjoy the flowers, shrubbery, and the water." (Kevin, Photo-Interview)

Other participants used stress management techniques such as reading or going for a walk to enjoy stillness and silence. "I need some time to just kind of sit down, read, and relax." (Corinne, Photo-Interview) Managing other health stressors was an important 
strength in the participants' efforts to reestablish a healthy lifestyle. By managing their overall health the participants cared for their body as a whole so that controlling the symptoms of early-stage dementia would be easier and an alternative sense of well-being could emerge.

Resetting pace. Another fundamental strength that the participants drew on to establish a healthy lifestyle was to adjust their pace and find ways to slow down. 'Resetting pace' speaks to the notion of acknowledging and accepting that a slower pace lowers the level of stress one experiences which in turn influences one's overall health. Resetting pace allowed the flexibility to adjust the way they spend their time to suit their needs. Learning to pace themselves was essential as it created a more stable energy level which in turn allowed for a more successful management of symptoms. The participants identified a number of resources that they found helpful in the process of resetting their pace were establishing a reasonable schedule, balancing activity and rest, and prioritizing commitments.

Establishing a reasonable schedule and not fitting too much into one day was one resource that the participants found helpful in establishing a healthier living. Uma captured the essence of this approach by taking a photograph of a storefront covered with graffiti (figure 191). She shared,

I want my life to be peaceful and serene. You know, to tell you the truth, that's not how my life is. But when I see something like this, and I'm like no. I mean it's totally not what I want in my life. [...] And, you know, I do my best to not have a life like this picture looks. (Uma, Photo-Interview)

Reasonable meant something different for each participant. For some having a reasonable schedule meant to attend organized activities daily. 
I go to a Senior Social up at the church on Tuesdays and Thursdays [...] and on Tuesdays, the Rotary meeting is also up there at the Community Center so I eat lunch with this group and then cut out and go to the Rotary meeting. [...] And then I go to exercise class at the Community Center Monday, Wednesday, and Friday. (Duncan, Photo-Interview)

Others found that maintaining an active schedule at home and a less active schedule outside of their home kept them functioning at a higher level. "Since I've had Alzheimer's, yeah, I don't go out as much. I used to. [...] but this kind of slowed me down, but...well it's, I also, I also have my schedule here at home." (Karl, PhotoInterview) Nevertheless everyone considered having a schedule that structured their time provided a sense of security and continuity.

When I was working, you know, so there was structure there. And losing that structure sort of concerns me. [...] and I so volunteer here [Alzheimer's Association] on Mondays and Wednesdays. [Tiffany, Photo-Interview]

The participants experienced creating limitations and boundaries for themselves as one of the hardest tasks; it went against everything that they had hoped for. They spoke about their struggles to give up certain things and not to expect too much of themselves.

They just re-opened the senior center and I go there and they are looking for people to do some volunteer work. [...] I thought that maybe I could do it for two hours a week. [...] I used to work at the desk of the senior center all the time. (Odette, Photo-Interview)

Some recounted that it had taken some time to figure out what their threshold was and develop a sense for and decide on a more reasonable schedule. Cognitive overload and exhaustion was recognized by the participants and they made efforts to pace themselves accordingly during their day-to-day living. Odette shared,

It's what you do, sort of. I would like to work more in my garden and in my yard and be more independent in mowing my lawn and I haven't played with my new 
lawn-mower but you know, I went out there last night just before dark and cut away a whole pile of grass and I felt so good about it but I am only good for about 35 minutes and then I have to quit and go in. And the cat's sitting over here and the dog's sitting over there - I mean [laughs] - I've got company - it is just, I don't know, I am happy with what I've got. (Odette, Photo-Interview)

Some participants related that they had days where they felt especially energetic. However, when they tried to take advantage of it by doing too much, they paid for it the next day by being totally exhausted. To feel well, they found that pacing themselves was just as important on 'good' days as it was on 'bad' days. If they agreed to one very busy day, they had to schedule less for the next day to give them some time to recover from cognitive over-stimulation. "I can't believe we talked for 5 hours yesterday [during the dyadic interviews]. I went home and slept til morning! I'll be taking it easy today. [...] Again, thank you for all your time and encouragement.” (Monica, E-mail)

Others recounted that the erratic and unpredictable nature of the illness symptoms precipitated a process of trial and error to get to know their limitations and learn to function within them. Many participants had grown accustomed to the need for constant adjustment. "I don't have a perfect day - I never have a perfect day. [...] I learned to flow with it - just go along with it." (Vincent, Photo-Interview)

Another resource that the participants found helpful in establishing a healthier pace was finding a balance between 'relaxation' and 'keeping busy'. Many participants reported that they needed more rest than before the illness.

I sleep more now. I never used to. I always liked to be busy, I've never been able to sit down. I was going. I liked, I was moving, you know. But now, I rest more often - I need it. (Frank, Photo-Interview)

To feel well the participants actively changed the way they spent their days to include longer and more periods of rest and 'alone time'. They found that sometimes, 
structuring the day too much was not a good idea. Incorporating timeouts into their schedule and balancing stimulating activity with free time was important and made a big difference. "I have to have sort of open boundaries of, you know, where I want to go and what I want to do. And if I don't want to do anything, I can grab a book and sit on the couch and pour myself a cup of coffee.” (Tiffany, Photo-Interview) Vincent took a photographs of hammock to portray the importance of finding ways to relax and energize (figure 192). Similarly, Ruby portrayed the strength she draws from adequate rest by taking a photograph of her bed (figure 193). Kathryn included a photograph of an evening sky in her portfolio to express the importance of taking breaks when she becomes over-stimulated (figure 193).

Prioritizing commitments and conserving energy was another resource that the participants found helpful in establishing a healthier pace. This meant for the participants to carefully plan their daily activities so that they would be able to enjoy the most meaningful ones and not waste their energy on necessary chores or draining tasks. Some participants reported that they had an honest look at their lives to decide what they could cut back on. Corinne took a photograph of a burned pizza to portray that it was important for her (and her husband) not to waste energy on household chores that could be accomplished in a different way (figure 194). She recalled,

I don't cook anymore. I was cooking one day, and I got really frustrated. Ken walked in. I was just sobbing. I was just crying, cause I couldn't figure out what I had done or something. He said, that's it, you're never going to have to cook again. So we eat out all the time. (Corinne, Photo-Interview)

Frank photographed a maple tree in his backyard to portray that he considers hiring help an important resource (figure 195) and shared, "I used to do this kind of 
pruning, but we had a guy come and do it this year." (Frank, Photo-Interview)

Saying 'no' was a strategy that all participants had to employ at one time or another. For a number of participants putting themselves first meant cutting back on social engagements.

Resetting pace and adopting a lifestyle that allowed for a lighter schedule, less commitments, and a healthy balance between activity and rest was an important strength that the participants drew on to construct their sense of well-being.

Choosing a balanced diet. Many participants alluded to the notion of eating healthy foods. They considered 'choosing a balanced diet' an important strength in their journey back toward a healthier lifestyle. Although all participants considered good nutrition important, a well-balanced diet, however, meant something different for each participant. Some participants paid special attention to the nutritional value of their meals. Corinne photographed a plate of salad to portray that she manages to eat well despite eating out almost daily (figure 196). Kevin showed he considers eating well important by taking a photograph of the vegetable displays in a grocery shop (figure 197).

Tiffany shared that because she has lost her ability to remember taste and has trouble identifying flavors, it was easy for her to eat well and have a healthy diet. "And since I have no taste buds, I eat things that are healthy for me, since I can't taste them anyway." (Tiffany, Photo-Interview) Others put their trust into special foods. Monica included a photograph of her breakfast table (figure 198) and shared, "I swear my breakfast is one of the things that keep me going. I was down at my dad's and I didn't have my breakfast. I felt like my days weren't going right.” (Monica, Photo-Interview) 
Others described that they ate well but still wanted to make changes to their diet. Odette described how she manages to prepare her own food and eat well and that she was thinking about becoming a vegetarian.

I still cook my own meals. Sometimes I make enough to last 2-3 days but for the most part I do, I make mostly like stir-fries, you know, simple stuff but I, I like to eat well and I - ehm - I, I am thinking all the time of becoming a vegetarian - I like that idea. [...] I pay attention to what foods are good and healthy for you and things like that "(Odette, Photo-Interview)

Others considered it important to incorporate emotionally soothing foods. Karl took a photograph of a bowl of strawberry ice cream (figure 199) to show that he views food as a source of enjoyment and that he includes favorite snacks into his daily meal plans. To him, looking forward to his snacks was more important than eating healthy foods.

For most participants eating healthy foods was an important element in reestablishing a healthy lifestyle. However, for some the nutritional value was of primary importance whereas for others the emotional value of the food was the focus of attention.

Maintaining a fitness program. 'Maintaining a fitness program' was another strength that surfaced as highly relevant to reestablishing a healthy lifestyle. For most participants regular workouts included exercise routines for both mind and body.

Regular physical activity meant to participate in exercise classes, maintain personally designed exercise programs or enjoy walks, hikes, or bicycle tours regularly. The majority of participants incorporated regular physical activity into their daily routine because they believed that by optimizing their overall fitness the symptoms of early-stage dementia could be managed better. Monica expressed the important role that exercise plays in her life by including several photographs of exercise equipment and workout 
clothes in her portfolio (e.g., figure 200). She noted,

And this is a picture of my workout clothes, because working out is something that's supposed to be good for people with Alzheimer's, and it's good for every person anyway, and it's, you know, I do it three or four times a week, sometimes five. And so it's just an important part of my daily life. Monica, Photo-Interview

Uma detailed her belief in regular physical activities by sharing her plans of changing her workout routine and taking rowing classes.

Well I was, I was a member of Curves, you know, exercise place, and actually, you know, I really believe in getting good exercise, so I used to do that three times a week. But I'm actually giving that up now, because I want to start taking rowing classes. (Uma, Photo-Interview)

Kevin took a photograph of a wood pile (figure 179) to show that he considers cutting and splitting wood part of his exercise routine. Chester took a photograph of two bicycles to show that he rides his bike for exercise (figure 201).

A number of participants reported that being responsible and caring for a pet was a great stimulus for being active. They felt they benefitted from the increased exercise that resulted from owning a pet. Kathryn took a photograph of her dog (figure 202) and shared, "I mean there is that build-in being commitment to it because of Dora [dog] - it's kind of like - I don't care if I don't get the exercise but it's important for her - so, that's been really good for me." (Kathryn, Dyadic-Interview) Duncan included a photograph of his dog in his portfolio (figure 203) and explained, "Nicolas [dog] is important to me because I take him out for a walk every day.” (Duncan, Photo-Interview)

In addition to the fitness component, several participants emphasized the role that the weekly routine of participating in an exercise classes plays in their life. Duncan included three photographs of his exercise class in his portfolio (e.g., figures $181 \& 182$ ) and noted, "I go to exercise class at the Community Center Monday, Wednesday, and 
Friday from 8 to 9 o'clock in the morning." (Duncan, Photo-Photo-Interview)

Several participants considered exercise as a resource for managing stress and depression. Others highlighted the social component of an ongoing exercise class. Being a member was an opportunity to interact with other people and provided a source of support. Romilda included a photograph of her exercise class in her portfolio (figure 204) and shared, "My exercise class - apart from the exercise it's very social. The people in the class are very supportive. [...] It's a great support group - yeah it's just amazing." (Romilda, Photo Interview) Others considered exercise as a resource for managing stress and depression.

However, being aware of the benefits of physical exercise did not prevent the participants from struggling with keeping up a workout routine. Fay took a photograph of her exercise class (figure 205) to show that she is proud of her accomplishment but also how she struggles to maintain an exercise routine without her sister's support. "A lot of times I get up and I don't want to be bothered with my exercise. My poor sister, cause she's trying to get me moving." (Fay, Photo-Interview) Odette thought she did not exercise as much as she thought she should.

There is that lady-friend here in the neighborhood [...] that I walk with once a week. [...] I think it's real important -I think partly that's what I am not doing not getting enough walking exercises and taking care of myself." (Odette, Brief Interview)

Fanny talked about her failed efforts to add exercise to her daily routine. "My exercise bike. This was given to me as a gift by my daughter and my son. [whispers] And I don't ride it enough and he [husband] is always making remarks 'why aren't you riding it'.” (Fanny, Photo-Interview) 
The participants believed that keeping mentally active can, to a degree, protect against cognitive decline and followed the advice from experts who recommend daily brain workouts, or mental stimulation, as part of a regular routine to stay healthy. In other words, the participants embraced the view that memory is a cognitive skill and exerted efforts to maintain their memory function. As such, regular brain workouts were an important part of their fitness program despite the awareness that it might not help. "Having an active and healthy mind is important to me but it's unrealistic I feel at this point although trying to keep it active is a worthwhile goal." (Monica, Dyadic Interview)

Some of the brain wellness strategies considered by the participants to be helpful were solving hocus focus, sudoko, and crossword puzzles, playing computer and video games, and going to the movies. Kevin took a photograph of a Nintendo game (figure 206) to show that he plays video games to challenge his brain and fight off further cognitive decline. Karl took a photograph of a hocus focus game printed in a newspaper (figure 207) to portray his daily brain fitness exercise and shared,

Oh, this is hocus focus. I do it every day to get these gray cells moving. [...] It's two pictures side by side, and you find the differences. Most of the time I find ' $m$ all, but sometimes I have five and I'd look and look and look and it'd be some little thing or something I shoulda gotten. (Karl, Photo-Interview)

Tiffany included a photograph of her computer to show how she exercises her brain daily (figure 208). Monica photographed a crossword puzzle in a newspaper (figure 209) to show that she continues to challenge her brain daily while acknowledging her growing limitations. She shared,

I red the whole paper every day and then I do the crossword puzzle. I can't do the New York Times or the LA... I can still kind of do the LA Times, the first part of the week, but New York Times is out. [...] I also play solitaire. That's my other game that I play to keep my brain working. (Monica, Photo-Interview) 
Odette felt that her weekly routine of going to the movies and being exposed to different images and new concepts provided the mental stimulation she needed to keep her brain functioning well.

Kevin [son] takes me to some wild movies every Friday, you know, but still, if you don't like the movie, you look at the background and you look at - there are so many things that go with the movie that I get a lot out of it. And a lot of the artwork, you know, and it is just, it's been a real privilege. [...] Going to the movies really does something to you - to your mind - it's like reading a fast book, I guess. (Odette, Photo-Interview)

Maintaining a program of regular exercises for both mind and body was an important strength for all participants. It helped them to develop coping strategies aimed at reestablishing a healthy lifestyle, which in turn supported their efforts to construct an alternative sense of well-being.

Discussion reestablishing a healthy lifestyle. According to the transactional model of stress, appraisal, and coping (Lazarus and Folkman, 1984), "it is easier to cope when one is feeling well than when one is not" (p. 159). In other words, a person who experiences physical well-being has more energy available for coping than a person who experiences physical dis-ease. Data analysis revealed that the participants had experienced a number of unanticipated lifestyle changes, all of which had decreased their overall sense of physical well-being. Initially, the participants thought only of memory loss and forgetfulness as the cause of their problems. As the disease progressed, they realized that their difficulties were also due to fatigue and other tangible and intangible factors. The findings of this study suggest that a portion of the disruption in well-being was not due to the illness itself, but to the sedentary lifestyle that the participants engaged in as result of the illness. Some of the limitations imposed by the illness (e.g., increased 
fatigue, decreased mobility, reduced ability to participate in favorite activities, and changes in interpersonal relationships) fostered an unhealthy lifestyle, including poor eating and exercise habits.

However, over time, the participants had come to the understanding (cognitive reappraisal) that when they felt unwell, they had less energy to expend on coping and that caring for their overall health was important. As such, consistent with findings investigating the coping response to other chronic illnesses (Reynolds \& Prior, 2003), 'reestablishing a healthy lifestyle' emerged as an adaptive task pivotal to adjustment to early-stage dementia. To cope with the threat to their overall health and reduce the chances of secondary illnesses, the participants drew on the following strengths: (1) managing one's overall health; (2) resetting pace; (3) choosing a balanced diet; and (4) maintaining a fitness program.

Consistent with the theoretical framework underpinning this study (Lazarus and Folkman, 1984), strategies for caring for their physical and mental health as a whole included problem- and emotion-focused approaches. The participants chose problemfocused coping strategies to influence a variety of health-related outcomes, including incorporating health-promoting behaviors into their daily routines (e.g., eating properly and exercising) and consciously taking care of their overall health needs (e.g., seeking and accepting the advice of health care professionals for ailments or other illnesses). Although the participants felt that the responsibility for looking after their physical and mental well-being was theirs, one of the strategies that a number of participants employed was seeking the support and encouragement from other people (e.g., joining an exercise class or choosing a walking buddy). Other behavioral coping strategies included adopting 
a slower pace and seeking a balance between activity and rest.

In addition, emotion-focused and meaning-based coping approaches were used to prevent worries about the illness from getting in the way of pursuing health-related goals (such as eating properly, getting rest, exercising, and having fun). To that end, the participants engaged in cognitive restructuring. They saw themselves as healthy if they did not have other medical conditions and it was common for the participants to be concerned about the progression of the illness, while at the same time viewing their illness as less invasive than other illnesses. Other cognitive coping strategies included efforts to prioritize commitments and balance personal needs with the needs of others.

\section{Discussion Phases of Adjustment}

The findings of this study suggest that the participants tried to live life as fully as possible in spite of early-stage dementia and, although disappointing, frustrating and discouraging, the palpable and visible decline did not to stop them from doing their best every day. It was the participants' incredible courage, perseverance, and persistence that made well-being possible despite growing impairment.

For the participants, reconstructing well-being meant to recognize and accept the limits early-stage dementia imposed on their lives. To an extent, they learned to live with a sense of uncertainty and not feel overwhelmed by the things they did not know and could not control. Being realistic about what to expect from themselves and setting reasonable goals allowed the participants to adapt and make substitutions and modifications so that they could participate in a meaningful life. Certainly, life was not like it was before the illness. Instead, what the participants had reconstructed was a sense of themselves, their lives, and the illness as a cohesive whole. Over time, their striving 
towards well-being produced meaningful experiences and understandings, which emphasized that a person is more than just a diseased body.

Maintaining this alternative sense of well-being was challenging for the participants because the delicate balance of 'ill-being' and 'well-being' was easily disturbed. Oftentimes, the participants did well for a few weeks and then were thrown off balance by some unexpected incident. They learned that it was not good to completely forget that they had early-stage dementia as it made them do things that they regretted later. Intense grief no longer dominated their lives but a general sense of sorrow was folded in their daily experiences. For the participants, the losses and the sadness they caused never went away entirely. Dementia left no area of life untouched, and there was a sense of lingering regret for all the capacity and potential that had been lost.

Maintaining this alternative sense of well-being meant to face the paradox of early-stage dementia daily. The paradox is to recognize the unchangeable reality of ongoing losses and simultaneously be willing to interact with that changed reality. Although it was challenging to view their current situation as something that allowed them to unfold their full potential, if the challenge was met, the participants recognized a multitude of possibilities to find well-being. Essentially, maintaining this alternative sense of well-being meant that the participants acknowledged the ever-present nature of dementia and attended to its symptoms while at the same time viewing the illness as only one aspect of the life experience. In short, it required the participants not to waste the present by mourning the past or fearing the future. Feeling well meant to juggle the ongoing tension between the desire to feel well and the noticeable and ongoing decline. As time progressed and the participants grew accustomed to this new, alternative sense of 
well-being, it became their familiar sense of well-being, and the process of adjustment and reconstruction repeated itself when new symptoms disrupted the fragile equilibrium.

'Striving towards well-being while acknowledging the inconceivable notion of losing one's cognitive abilities' was not easy for the participants. It was an ongoing process that required adjustment and readjustment on a daily basis. It required viewing the illness as only one part of life and having the courage to face the loss of the familiar sense of well-being and take that changed reality as an opportunity to create a new and different sense of well-being.

A balancing act of sorts was always in play where the sense of illness was juggled with the alternative sense of well-being, with the condition that the illness and the wellbeing were never the same at any given time.

\section{Quantitative Findings}

A closer examination of the quantitative data revealed that MMSE scores were not correlated with either mean quality of life as measured by the QOL-AD, nor depression as measured by the GDS-short form. As expected, the total score on the GDSshort form was correlated with the mean quality of life score $(r=-0.756, p<.001)$. Among the 13 items making up the QOL-AD, eight were negatively correlated with scores on the depression scale [physical health $(\mathrm{r}=-.614, \mathrm{p}<.01)$, $\operatorname{mood}(\mathrm{r}=-.556 \mathrm{p}<.05)$, family $(\mathrm{r}=-.516$, $\mathrm{p}<.05)$, friends $(\mathrm{r}=-.635, \mathrm{p}<.01)$, self as whole $(\mathrm{r}=-.610, \mathrm{p}<.01),(\mathrm{r}=-.749, \mathrm{p}<.01)$, money $(\mathrm{r}=-.538, \mathrm{p}<.05)$, and life as a whole $(\mathrm{r}=-.783, \mathrm{p}<.01)]$.

Four participants had scores indicative of depressive symptoms on the GDS-short form: Kathryn, Monica, and Ruby scored suggestive of mild depression; Vincent scored suggestive of severe depression. An in-depth examination of these participants' 
characteristics revealed noteworthy patterns. Three participants (Kathryn, Monica, and Vincent) rated their overall quality of life as fair; one participant (Ruby) rated it good. All four participants had experienced more abrupt changes than most of the other participants. Two participants (Kathryn and Monica) had lost their jobs unexpectedly due to onset of the illness. One participant (Ruby) was involved in a car accident about five years ago, which killed her husband and left her a paraplegic. One participant (Vincent) had suffered a stroke two years ago. Another similarity is that three of the four participants were either divorced (Kathryn and Monica) or widowed (Ruby), suggesting the importance of intimate partnership.

In addition, the participants scored in the range of mild (Kathryn, Monica, and Ruby) to severe (Vincent) depression at the beginning of the study and yet composed lifeaffirming portfolios and reported positive coping strategies. This raises the question of whether participating in the photo-taking activity of this study, which focused on discovering personal strengths and resources, may have, at least temporarily, had a moodenhancing effect and changed their perspective on life. For example, Vincent expressed contentment with his current life; he has accepted who he is right now and is happy. "I feel that at 75 and with the things that are going on in my life right now, I am happy - it's part of the deal and I accept it." (Vincent, Photo-Interview) Similarly, Kathryn views her independence as something to enjoy and expressed satisfaction with being able to express it. "I sort of figure, as long, like right now I can still go up to Lummi Island and sort of have a certain amount of independence, and so life is worth being alive." (Kathryn, Photo-Interview) In a similar vein, Monica has plans for her new apartments and enjoys the activity of shopping for it. 
This is my cousin. We went shopping together [...] and I bought some seat cushions for my new apartment that I just love. [...] We had so much fun. It was nice to just have a day like I was normal. (Monica, Photo-Interview)

It is important to me to connect with my past. I wanted to visit my childhood neighborhoods and so we did and I really enjoyed that. (Ruby, Photo-Interview)

The difference in quantitative and qualitative findings as it relates to these four participants also supports the notion of positive and negative emotions co-existing and appearing almost simultaneously while having different and shifting weights. This dynamic appears to be central to the experience of the illness and the movement towards adaptation. In addition, the ongoing, dynamic motion of ill-being in the foreground/wellbeing in the foreground discovered during data-analysis led me to become more sensitive about the type of questions that are asked in the quantitative measures. I noticed that many of the questions on the GDS-short are focused on the losses that people have experienced as they require a comparison between the present and the past. The general tendency of these questions and the focus on loss may have initiated a shift of ill-being to the foreground. That shift to ill-being might have influenced their overall score. As mentioned earlier, I decided to invite two of these four participants to take part in the dyadic interviews, the results of which will be presented in the section of methodological findings.

\section{Methodological Findings}

This section presents the findings as they pertain to the methodology that was used in this study.

Photovoice methodology. One picture is worth a thousand words. This timeworn cliché proved true for this study. Photography allowed the participants to capture some of 
the pivotal elements of the dementia experience. "Well, they aren't great photos, but they sure tell a lot about my life, that's for sure...." (Corinne, Photo-Interview) They were able to genuinely get in touch with what was important to them and what may have been beyond the reach of normal discourse.

This was good for me, a very uplifting kind of thing. [...] I mean, I became aware of the things I sort of took for granted [...] So it's, this was a good experience for me. $[\ldots]$ Thank you for giving me this opportunity. You know, how often do we get to talk about us? (Tiffany, Photo-Interview)

Taking photographs helped the participants to expose the deeper structure of what it means to experience the illness and cope with and adapt to its symptoms. "Well, there were things that are just almost like every day routines but all of a sudden they are important, you know, and that was enlightening to me." (Odette, Photo-Interview) Although I did not observe the participants during the photo-taking phase of this study, their images indicate that they were sincerely engaged in the research process. They acted freely outside the sphere of my influence within their own social environment with its own rules and normative behaviors.

It was kind of interesting to see how your whole day went and to record it. Yeah, I found it quite interesting to do. It's a nice memory. It makes you sort of remember what went on in the day, which is good. (Romilda, Photo-Interview)

Photography put the participants in charge of how they wanted to represent themselves and their situation. Frank portrayed his circumstances creatively by taking a photograph of a photograph of his family and included himself as a shadow image (figure 210). Photography stimulated the participants' creativity, as it obliged them to think about abstract constructs and how to represent them visually. Uma, for example, portrayed her wish for a simple and uncluttered life by taking a photograph of something 
symbolizing the opposite (figure 191).

The participants proved inventive in taking photographs of things that were not available. Duncan took a photograph of a photograph of Mount Rainier on a sunny day. He explained that on the days he wanted to take the photograph of Mount Rainier, the sun had not been out so he decided to take a photograph of a photograph instead. "I wanted to get a picture of Mount Rainier but I couldn't, so I took a picture of a photo that was taken by the son of my dentist." (Duncan, Photo-Interview)

By giving the participants a relatively free reign in deciding what photographs to take, the images were personally meaningful to each participant and painted a vivid picture of the people, things, and places that assisted them in striving towards and achieving a new, alternative sense of well-being. "I mean this was really an enjoyable project. I was always thinking that week, $[\ldots]$ during that whole week I was always thinking, okay, what's important to you, you know?" (Uma, Photo-Interview)

The act of taking photographs of their experiences allowed the participants access to specific and general memories and reflections. "I enjoyed it, because it brought back memories. [...] This has been fun, making me remember all this stuff." (Fay, PhotoInterview) Images invite the viewer to look at the world through the lens of the photographer. Taking photographs was a way for the participants to amplify their place in the world for themselves and others.

This project has been very, very widening for me, you know, made me think about some things that I hadn't really thought about at all. [...] It made me realize that I need to reach out to people more than I do, you know, that people aren't going to reach out to me, that I'm going to have to push those little boundaries or barriers or whatever myself and get a little more involved in the world again. (Monica, Photo-Interview) 
Participation in this study may have contributed to an enhanced self-image,

personal learning, and growth.

Well it was very fun to have that assignment, you know. 'Cause I mean it wasn't heavy duty thinking, but it gave some structure to it. And, and it made me much more conscious about what I care about. [...] It surprised me how quickly I knew. I mean, you know, that I had, I mean, if somebody would have said, you know, what's the most, what are the most important things to you right now, I would have come up with that list. But, you know, most people don't ask that. And so none of them were a surprise, but nobody ever asked me that question before either. And you know, I hadn't sat down and really thought about it, you know, like okay, well what makes me feel good, or what, you know, what do I look forward to or what's important, keep me going and keep me motivated and that kind of stuff. But as soon as the question was posed in this, you know, it was like oh, I have the list, that I hadn't put together, but part of me was certainly aware of it. (Kathryn, Photo-Interview)

Photovoice methodology equalized the distinction between the researcher and the

participants and facilitated a more symmetrical dialogue.

Well, I think you're undervaluing your contribution to this process. I mean, listen to how horrible those experiences with other people were for me. And then you come in and you say, I want to know what's important to you, and I want you to talk about it with me. And you've spent a lot of time getting me to think about what's been important, and providing me a venue in which I get to talk about it and explain it to another human being who's doing a really good job of listening. [...] You know, I'm just, this has been a very delightful thing, and you're very pleasant to talk to, and I like how smart you are. I feel like I'm the winner. [...] I mean, your, I mean, most people treat us patients as if we're worthless already. And you don't. You don't treat me like that, and I doubt that you treat your other interviewee people like that. I mean you come and you say, you have something to tell us. And that's affirmation. (Kathryn, Photo-Interview)

It made participation in this study a joyful and pleasant experience. "I am not interested in a lot of social stuff, I don't know [...] but I enjoy talking to you." (Frank, Photo-Interview) "I enjoyed it; it, it was a stimulating thing for me." (Odette, PhotoInterview) "That was one of the things I was telling them [church group members]; what an eye-opening experience this project was. Because normally, I just look at things as, 
not thinking about it. So I really have enjoyed it." (Corinne, photo-Interview) In addition, it provided the participants with a sense of accomplishment. "Yeah I enjoyed it because I accomplished something and I got my kudos for it." (Karl, Photo-Interview)

The below quotes are excerpts of personal thank you notes from some of the participants sent to me after completion of the study. In these handwritten postcards the participants express gratitude for the opportunity to take part in this study and reiterate how much they enjoyed the process. "I had such an interesting time with the camera study." (Odette, Handwritten Postcard) "I truly enjoyed the time, conversation with you." (Rosabelle, Handwritten Postcard) "Thank you again for the opportunity to be part of this wonderful project.” (Sam, Handwritten Postcard)

Other indicators that speak to the value that this study held for the participants were that all but one participant decided quickly to take part in this study. In addition, the participants had a high level of commitment once the decision was made to participate as indicated by the fact that nobody dropped out. Both of these factors are generally issues in recruiting and retaining this population in research. Furthermore, most participants wondered about the need to disguise their identities; they were proud of their contributions and would have rather owned their comments. Discussing their photographs had created a new role for the participants. As they shared their expertise about coping with early-stage dementia, they took on the proud role of experts of their lives.

Dyadic interviews. Dyadic interviews were employed during the 'interpretative phase' of this study as a means of member checking and to further develop the understanding of the range of responses to the illness. These interviews generated rich data, which refined the points that the participants had made during the individual 
interviews and clarified the emerging model. The dyadic interview format promoted a sense of safety and openness that facilitated a flow of shared stories. The dyads entered readily into candid dialogues disclosing in-depth thoughts and discussing emotionally challenging topics almost immediately. "And we had a son who died." (Corinne, 2 minutes into the interview). They felt safe enough to share their concerns early on in the conversation.

Monica: I haven't really told anybody but I do worry about what is going to happen when I start needing help. (8 minutes into the interview) Kathryn: I know what you mean.

Open dialogue about 'taboo' topics emerged without the moderator initiating these lines of inquiry.

Corinne: What are you planning to do when you are getting older - I don't mean getting older - I mean getting worse? What will you be doing?

Tiffany: I don't know.

Corinne: Are you planning like a home?

Tiffany: I haven't thought that far - I mean that's something I have to deal with but right now I feel like - I feel like I am safe living alone right now.

The dyadic dialogue provided space for the participants to ponder questions pertaining to their specific situation.

Tiffany: Now when will we know that it is not safe to live alone? I don't do anything dangerous, I don't turn on my stove. [...] I just wonder - will I know when it is time?

Corinne: How about your son - can he help you with that?

In addition, observing the dyadic interactions provided vital insight into how strength and resources were used in social encounters to cope with the symptoms of the illness. For example, I was familiar with Corinne from a support group setting, and she had a well-rehearsed synopsis of her life that included the illness. However, when she introduced herself in the dyadic interview, her life story included tragic events that other 
people can relate to but excluded the illness. To cope with the stress of meeting a new person in a new environment Corinne chose not to mention her illness although she knew full well that the other participant also had a diagnosis of early-stage dementia. It seemed as if she was feeling out the openness of her dialogue partners to talk about the illness.

One particular strength of the dyadic interview format was the reduced effort that the moderator needed to clarify, paraphrase, and summarize what the participants had told each other, without interrupting the flow of communication. It was easier to notice and bridge gaps in understanding between the dyad members when they occurred.

Karl: I forgot what we were talking about.

Moderator: We were talking about what is important to us. Health is important and family is important - what else is important?

Karl: I like coming here, talking; how we get along here.

Duncan: Well, and being careful with our assets, to invest them properly so that we don't end up running out of money.

As expected, the participants presented with some dementia-specific

communication difficulties; however, the interactions between the participants remained vibrant. There were long segments of conversations between dyad members with little interruption or participation from the moderator. The dyadic interviews were especially useful for participants who needed additional time to process what had been said and formulate their responses.

Moderator: How about your relationship with your wives?

Karl: We've always stuck to each other Duncan: Ours is the same thing.

Karl: I am glad we met; we met in kindergarten [laughs]; my wife knows that I have the memory problems and she realizes that I am sometimes off base and that I have to ask her stuff.

Duncan: Same for us.

Karl: I feel very fortunate with my wife.

[Pause - Duncan looking through his day runner]

Moderator: What are you looking for, Duncan? 
Duncan: I was just looking to see if in here it says what year we got married - so I can figure out how long we have been married. Let's see, we got married in June of 1965.

Karl: We got married in 57.

All three dyads engaged in intense discussions that were characterized by sincere

interest, respect, and curiosity as well as a high level of interaction.

Tiffany: Are you still driving?

Corinne: I - drive just off time. [...] And I haven't had a problem yet - but that's what happens - you never know when you'll have a problem.

Tiffany: Yeah - I am trying not to but this morning I had to. [...] So driving that's kind of an issue on the table right now - should I sell my car, should I keep my car? [...]

Corinne: I think even if I don't drive a car, I would want one there.

Tiffany: Yeah - it's kind of a security thing.

Corinne: Yeah - it's a dilemma - when do you stop?

Tiffany: And I met with an attorney who said that if I am involved in an accident even if I am not at fault, I am liable because...

Corinne: ... you have this diagnosis.

Tiffany: Of course that's what went through my head when I got lost this morning [laughing] - so I am sort of sitting on the fence.

Corinne: The problem with this is that everybody is so different.

The participants encouraged each other to express their perspectives even if that

meant struggling to find the right word for the concept they wanted to express.

Corinne: $[\ldots]$ - what are we supposed to be talking about?

Tiffany: No, I am following what you were saying. [...]

Furthermore, the participants felt secure enough in the dyadic interview

environment to agree on some issues and disagree on others.

Kathryn: Well, I would say, well, I care about my house and I want it to be comfortable and that sort of thing but I don't, to me that isn't about self-identity it's about what kind of environment do I want to be in, what's comfortable for me. $[\ldots]$

Monica: For me it's different. Having my own home is important to me and it is part of my self-identity because once I was divorced, providing a home for me and my son has been my responsibility and after rebuilding that one house I just always felt a more visceral connection to the place where I live than I have before that. And so, having my own place, I guess that is very important to me. 
The dyadic interviews fostered a sense of camaraderie and collective identity

among the participants as indicated by their growing confidence to tease the moderator.

Moderator: I am sorry but I just lost my train of thought - I forgot what I wanted to ask you.

Corinne: Is it catching? [Laughing]

Tiffany: [Laughing] You've got to be careful. You spend too much time with people that got it.

Corinne: [Laughing] Join the club.

More importantly, the dyad members felt comfortable to voice their perspective

on the emerging model during the member-checking portion of the discussion. Their

responses directly and indirectly confirmed the emerging framework. An example of a

direct confirmation is the following transcript excerpt.

Moderator: It sounds to me as if you are coping exceptionally well. What do you think makes that possible?

Tiffany: 'Cause I want to. I made that decision. I've always been a positive person. I want to enjoy this time of my life when I don't have to work, where I can get my little Orca card and go someplace that I've never been. [...] Now it's all about me. So I'm enjoying this time. So, for however long it lasts, I'm enjoying this time. (Tiffany, Individual Interview)

An example of an indirect confirmation is the following transcript excerpt.

Moderator: So how about if tomorrow you could do anything you wanted. What would that be?

Karl: Well, I am very regimental; I have my - I have my calendars on the wall, I know what day it is, and I know my tablets - I know when I have taken this or taken that - and when I take my crap - I am very regimental. And I know what I have planned for the next day - I am very set.

Moderator: So that would be what you would like to do?

Karl: Yeah, I like my day how it is. I am set in my way.

Moderator: How about you Duncan? If tomorrow you could do anything you wanted to do. What would that be?

Duncan: Well, I am really impressed with what the Gates are doing. It impresses me that there all kinds of situations that they give money to. That's something I would like to do.

Furthermore, the dyad members felt comfortable to disagree with some of the 
findings.

Kathryn: I don't like to cook anymore. I have this great grocery store and they fix dinner for me every day and I just walk up and pick it up.

Monica: Yeah, see, I feel the same way - 'Lean Cuisine' and I are close friends. [Laughing]

Kathryn: We got our priorities right. [Laughing]

Moderator: I do have to defend that category by pointing out that there were a couple of gourmet cooks in the group. [Everybody laughing]

Monica: I think we would both like to be invited to their homes. [Everybody laughing]

Kathryn: Definitely - yeah - [Everybody laughing]

Monica: Obviously, we're both failing that category. [Everybody laughing]

Kathryn: We are not even related to that category. [Everybody laughing]

Sometimes the dyad members not only confirmed the emerging model but made

suggestions about changing the labels of the categories and subcategories.

Monica: I think the items are all very valid. I think it's probably just a difference in the way your brain works and how my brain does and your brain does - it's just in the headings - because I think we all agree with the individual items but the headings don't necessarily indicate to a person what is underneath.

Kathryn: I would agree with that. That fits my experience.

Moderator: So I may have to think about labeling the categories differently.

Monica: Yeah, the headings don't match the subcategories

Kathryn: I would agree with that.

Overall, the dyad members confirmed my interpretations but given that English is not my first language, I took the suggestions regarding relabeling seriously. This led to a renaming of the categories and sub-categories, which ultimately resulted in a more parsimonious model (reducing the conceptual categories from six to five). As for the process dimensions of the core model, the dyadic interviews helped to confirm the finding that living with early-stage dementia is a dynamic process that is characterized by a cycling of attention to ill-being and well-being.

I mean part of it has to do with I can feel like one person one day and like somebody totally different the next. [...] There are days where I feel more confident and therefore feel like I have more competency and then I feel better 
about myself and I feel not so worried about what am I going to do if this or that. (Kathryn, Dyadic Interview)

In addition, the dyadic interviews allowed me to further investigate noteworthy patterns about the notion of 'abrupt change' that emerged in connection to those participants who had scores indicative of depressive symptoms on the GDS-short. Both women confirmed that coping with the sudden changes of losing their job had been challenging and that reconstructing a new sense of identity continued to be demanding task.

Furthermore, the interaction in the dyadic interviews created a sense of ease and relaxation for the participants, which in turn allowed topics to emerge that were not discussed in the individual interviews. For example, the theme of contemplating, wrapping up, and coming to terms with one's life was introduced toward the end of the first dyadic interviews. This theme opened up a new line of inquiry, which was pursued in the subsequent interviews and prompted me to review the transcripts for evidence in support of the new theme.

Moderator: What else is important?

Karl: I'd like to go to heaven - rather than down the other way. [Laughing] You know, living a good life - being okay - you know. Some people get into terrible messes - in and out of prison and - not for me. Yeah, when I look back - I've been very pleased with my life.

Duncan: Yeah - being happy with the way you'd lived your life.

Karl: That pretty much covers it.

Taking part in dyadic interviews was an enjoyable experience for the participants, as it provided a venue for them to share their perspectives with confidence.

Kathryn: This was so much more of a pleasure than I even thought it could be. Monica: Yeah, I totally enjoyed it.

The participants viewed the dialogue as an opportunity to learn from each other. 
Tiffany: You know - this [dyadic interview] has been very helpful - just you and I.

Corinne: I think it really is - I really enjoy this - this is really helpful.

The smaller, more personal format of the dyadic interviews reduced the stress of meeting new people and assisted in overcoming dementia-specific communication barriers.

Kathryn: [Turning to interviewer] I really like her [Monica] - she's the real deal. Monica: [Laughing] The real deal.

Kathryn: [Laughing] I think we speak the same language. Monica: [Laughing] Yeah.

The dyad members supported my findings and increased my sense that I have described the experience of the participants in this study somewhat accurately. In addition, even if member-checking would have served no other purpose, it was a powerful way of validating the participants by showing respect for their opinions. 


\section{CHAPTER 6}

\section{DISCUSSION, LIMITATIONS, IMPLICATIONS, AND CONCLUSION}

In this chapter, I discuss the most important points that emerged from this study and how they relate to previous research, address study limitations, make suggestions for future research, and provide implication for policy and practice. The discussion section of this chapter focuses on the overall model that emerged from this study since the individual components of the model were already discussed as part of the previous chapter.

\section{Discussion}

Medical progress in making a diagnosis of dementia has created a new experience of living with the illness. This experience is no longer dominated by the final stages of the illness; rather, it is a prolonged uncertain state of living with ongoing cognitive decline, which can last for many years. Studies reporting the experience of this period of decline from the perspective of the individual with the illness have attempted to shift away from a predominantly medical view of dementia. Those studies have informed our understanding of the psycho-social-spiritual experience of the illness, and the results of the current study are largely consistent with their findings. However, no previous study has been grounded directly in visual and verbal data provided by people with early-stage dementia in a wholly participatory manner; this was the first study to use photovoice and dyadic interviews with people with early-stage dementia. It expanded the pool of methodologies with which to investigate the subjective experience of people with cognitive impairment, amplifying the voices of individuals who are increasingly deprived of verbal abilities to express their experience with dementing illness. In this way, the 
study has demonstrated the unique contribution of these methods in research with older adults with dementia.

This study also makes a distinctive contribution to the understanding of the subjective experience of early-stage dementia by identifying and describing phases of adjustment used by older individuals with the illness. These phases organize the perceptions of strengths and resources reported by the participants, while the ways in which those resources are used to adapt to the illness are detailed in the phase, "constructing an alternative sense of well-being." A discussion of each phase of adjustment is located at the end of the corresponding section in Chapter Five of the findings, and a summary discussion of all phases is located on page 322 of the findings. A third contribution of this study addresses the research question, "What is the process of coping with and adapting to dementia in the early stages of the illness?" In addressing how the various phases of the model are related, a conceptual model for understanding this experience emerged from this study (figure 1). This model of adaptation is organized by the core category. 'Striving towards well-being while acknowledging the inconceivable notion of losing one's cognitive abilities' offers a conceptual understanding of the coping response to early-stage dementia and the place of perceived strengths and resources in it. The model suggests that people with early-stage dementia are able to identify their internal and external resources and make conscious choices about how to use these abilities and assets in the process of coping with the illness and its consequences.

It also suggests that attention to the illness fluctuates over time, and that at times a person with early-stage dementia experiences a sense of well-being (with the illness in 
the background), while at other times he or she experiences a disruption in well-being (with the illness in the foreground). Furthermore, 'striving towards well-being while acknowledging the inconceivable notion of losing one's cognitive abilities' reveals a simultaneous and concurrent acknowledgment of and resistance to the illness and the coexistence of positive and negative emotions in a cyclical and continuous process. In the model, a mix of positive and negative emotions characterize the experience of well-being, suggesting that well-being may be the result of a fragile and complex interplay between two opposing forces. A lack of complete acceptance may help to sustain feelings of hope and optimism, resulting in a positive influence on a person's ability to cope.

Arriving at a new place of well-being for the participants of this study did not mean that is was easy for them to stay there. As the illness progressed and new symptoms developed, the illness transitioned back into the foreground of the participants' lives, and many of the challenges that the participants had previously coped with were 'recycled'. The process of adjustment is never complete, as a person with early-stage dementia is constantly challenged to decide on, develop, and incorporate coping strategies aimed at dealing with new difficulties as they appear. The participants in a study conducted by Atkin, Stapley, and Easton (2010) described this adjustment not "as an achievable, finite outcome, but rather as an aspiration, partially realized through daily living” (p. 390). In other words, the process of adjustment is conceptualized as repeating itself when threats to wellbeing resurface.

A fourth contribution of this study is that it provides an organizing framework for what is currently known about the experience of early-stage dementia as it refines and confirms what has been reported in earlier studies. The model integrates the many levels 
of context that other authors have proposed as important (e.g., Kitwood, 1997), including observations that the processes informing identity construction in early-stage dementia are best portrayed as an interplay of opposing forces that aim to preserve a sense of self congruent with the sense of self prior to the onset of dementia and self-adjusting strategies aimed at facing the challenges associated with dementia in order to integrate them within a changing sense of self. (Clare, 2002). These layers of context reveal a richer understanding of the adjustment process and the experience of people with dementia.

The model departs from previous conceptualizations of the dementia experience that do not account for the 'striving towards' that exist in duality with ongoing loss and decline (Keady \& Nolan, 1994; Cohen; 1984). 'Constructing a new, alternative sense of well-being' is a beginning not found elsewhere in the literature. It suggests that it is possible to achieve a sense of well-being despite the losses encountered as a result of the illness and highlights the potential for self-management in the context of early-stage dementia. All participants had not only lived with the illness for many years but by employing self-management strategies had been able to adapt and rebuild a life with satisfying quality. They had assumed responsibility for identifying and creatively using available internal and external assets. Looking at the coping response to early-stage dementia through a lens of strength provided a different understanding of the subjective illness experience than has been proposed by earlier research.

Although in this study the experience of early-stage dementia was investigated with a strengths perspective (Saleebey, 1992/2009), the focus on capabilities and capacities is not to suggest that there was no scope for 'loss'; it suggests quite the 
opposite. Data analysis revealed that it would undoubtedly be an exaggeration to suggest that the participants felt well all the time. The onset of early-stage dementia had torn a hole in the fabric of their lives, and there was nothing easy about coping with the illness. However, threaded through the participants' accounts was also the notion that there was no need to give up.

The central thesis of the present model of adjustment is that the participants' desire and motivation to feel well was the basis on which they made decisions about their responses to the illness, which did not always make immediate sense to an outsider. This may be in part due to the fact that the participants' coping response contradicted prevailing beliefs that learning to live with loss is a sequential response that occurs in stages that culminate in acceptance (Kübler-Ross, 1969). Contrary to this understanding of a more or less predictable coping trajectory of phases, what emerged from the data was a pattern of coping in which a person's attention to early-stage dementia fluctuates over time (Patterson, 2001). In other words, the current model allows for the simultaneous and concurrent presence of acceptance and denial and the co-existence of positive and negative emotions.

In line with the transactional framework of stress, appraisal, and coping (Lazarus \& Folkman, 1984), which underpinned the study, the participants experienced coping with the illness as a process that unfolded over time and left room for individual choice. In fact, taking responsibility for one's response to specific circumstances and emotions surfaced as a strong current in the adjustment process. The motivation for moving towards adaptation was the participants' determination to achieve and maintain a maximum level of well-being, a well-being that was inclusive of the illness but that did 
not situate it center stage. The participants in this study clearly did not want to give up; they were determined to continue their striving towards well-being even if it seemed to be an uphill battle.

This striving suggests the existence of an ever-present force, which corresponds with notions of existential and humanistic theories. It is similar to Frankl's (1959/1993 notion of the 'will for meaning' as a basic motivational force, suggesting that potential meaning is 'inherent and dormant' in every situation throughout a person' life. "The perception of meaning $[. .$.$] boils down to becoming aware of a possibility against the$ background of reality, $[\ldots]$ to becoming aware of what can be done about a given situation” (p. 145). It is also resonates with Roger's (1980) concept of the 'actualizing tendency", defined as "one central source of energy in the human organism, $[\ldots]$ most simply conceptualized as a tendency towards fulfillment, towards actualization, involving not only the maintenance but also the enhancement of the organism" (p. 123). This tendency to self-actualize manifests regardless of the circumstances. Both writers view this innate motivation to fulfill one's potential and live purposefully as the most basic human drive. The model proposes that this striving towards well-being is not stopped by the onset of dementia. It is informed but not halted by the challenges of the illness. Further, it dispels popular myths of predisposition and tendency to disengagement among people with dementia and expands and elaborates the notion that people living with the illness "seek outcomes for themselves as human beings rather than as "people with dementia"” (Dabbs, 1999, p. 18).

\section{Limitations}

While the proposed model appears adequate for understanding the coping 
response to early-stage dementia and the role that perceived strengths and resources play in this process, it is still limited by several factors. There are limitations in the research design and study sample. The cross-sectional design made it impossible to observe the coping process over time. The participants provided a retrospective account of their journey with the illness and I was tasked with piecing together these 'memory snap shots' into a 'movie'. It is likely that the retrospective data collection was influenced by the participants' recollection and recall bias. For example, it has been proposed that people with an optimist outlook on life are more likely to recall past successes and gloss over failures (Seligman, 2002).

Data collection was also influenced by the participants' cognitive limitations and the topics they wished to discuss. Although triangulation of methods was used to increase the trustworthiness of the findings, another caveat to the method used in this study is that it was not possible to verify the self-reported information. In addition, it is likely that my strength-oriented perspective influenced the research design, interview questions, and data analysis.

The study sample also needs to be taken into consideration. This study was based on a small sample, although similar in size to other qualitative studies. In line with the tenets of qualitative research the sample was not in any sense random. Rather, the participants were chosen purposefully. Nevertheless, it is unlikely that the participants in this study were representative of the general population of people with early-stage dementia. The participants represent a special group of people with early-stage dementia due to the following characteristics, which may have fostered coping and adaptation. All participants were well educated and reported adequate financial resources. All but one 
participant held degrees beyond high school. Individuals with advanced degrees may be more inclined to volunteer for research projects due to familiarity with the process. They may have a stronger desire to learn about their illness and how to self-manage.

The overwhelming majority of participants reported adequate financial resources; only one participant disclosed financial difficulties due to the onset of early-stage dementia. In addition, all participants enjoyed positive interpersonal relationships and a strong network of support. Positive interpersonal relationships and social support are considered important factors to buffer stress and promote positive coping (Hinson Langford, Bowsher, Maloney, \& Lillis, 1997; Salovey et al., 2000). Eleven participants lived with a spouse or other family member. The nine participants who lived alone were able to rely on their children, siblings, or friends for support.

Another limitation of the study sample is that all participants were recruited through the Alzheimer's Association and the Northwest Research Group on Aging. Affiliation with an organization offering support to people with the illness can be a key factor in coping with and adapting to an illness. The possibility that more positive and self-reliant individuals join organizations is a confounding variable (Smith, Curtas, Werkowitch, Kleinbeck, \& Howard, 2002). Twelve participants were members of ongoing support groups, which provided them with access to resources and probably influenced their views on their coping abilities and strategies.

Several other variables could have influenced the participants' perception of their strengths and resources and their ability to use them. The participants self-selected for the study, which suggests that they had a degree of awareness about their illness. At the time of this study, they had lived with memory problems and the knowledge of their illness for 
a number of years (approximately 3 to 6 years) and felt that they were at a relatively stable disease state.

Bury (1982) argues that the unfolding character of chronic illness requires understanding experiences within a temporal framework and that the experience of time is a significant aspect of the adjustment process. For the participants in this study, a longer duration of living with the illness allowed additional time to identify strengths and resources. The study population was drawn from a relatively small area in Washington State where shared values and beliefs are likely (Creswell, 1998). Therefore, the results of this study cannot be generalized to other geographic locations. The study attracted almost exclusively Caucasian Americans. Only one participant was of African American descent, which precludes an evaluation of how ethnic diversity influences the perception of strength and resources and coping. Any of the above-discussed characteristics could have increased the participants' motivation and capacity to construct a new, alternative sense of well-being compared to the general population of people with early-stage dementia. In other words, all participants in this study had access to internal and external strengths and resources that contributed to the likelihood of successful coping and achieving of well-being compared to other people with early-stage dementia who lack similar assets. Nonetheless, this exploratory study broke ground by providing a first step into an inquiry that links the perception of strengths and resources to coping with earlystage dementia and the findings hold several implications for social work policy, practice, and research.

\section{Implications for Policy and Practice}

This exploratory study produced many possible avenues for social workers and 
other healthcare professionals to enhance the ability of people with early-stage dementia to cope with and adapt to the illness. The findings of this study support a holistic, personcentered approach to clinical practice emphasizing the influence that internal and external resources have on the coping response to early-stage dementia and the construction of well-being. Incorporating aspects of a person's lifestyle, emotional needs, spiritual preferences, and availability of a support system into services and interventions is part of this approach.

At the time of this study, the participants had not only lived for many years with the illness but had taken just as many years to rebuild a sense of well-being. This process most likely could have been shortened if ongoing professional support would have been available to them. As such, the findings of this study emphasize the need to expand the scope of education and psychosocial support provided to people with early-stage dementia. These services should ideally begin at the time of diagnosis and more importantly continue throughout the progression of the illness as new concerns and questions arise. The findings of this study suggest that the participants' educational and emotional needs changed as they adapted to early-stage dementia. It seems prudent therefore that interventions should correspond to their changing needs and skills. Examples of interventions that would provide this type of support are: ongoing counseling that combines creative expressions with psychotherapy and life coaching; stage-specific psycho-educational support groups for people living with the illness as well as their family members and friends; opportunities for people living with the illness to get together in an informal setting; programming that focuses on strengthening ties with family members and friends, and health promotion activities such as walking, 
dancing, and swimming.

The participants' strong desire to actively participate in managing their illness highlights the need for a shift in the role that social workers and other healthcare professionals play and the services that they offer to this population. This shift repositions the focus from primarily educating and supporting caregivers to an approach that is inclusive of the person with the illness. In fact, the findings of this study support emerging discussions on self-management in the context of early-stage dementia (Baehrer-Kohler \& Krebs-Roubicek, 2009; Mountain, 2006) and point to the need for the development of self-management training protocols for this population. "The commonly held view that people with dementia cannot learn new skills and therefore any intervention will at best fail and at worst have an adverse effect upon the person and their carer, is now being challenged" (Mountain, 2006, p. 434).

Research in the context of chronic illness shows that education programs that include teaching self-management skills improve clinical outcomes and can reduce costs when compared with programs that provide information only (Bodenheimer, Lorig, Holman, \& Grumbach, 2002; Coleman et al., 2004). The findings of this study support a holistic approach to self-management in which social workers and other healthcare professionals would take on the responsibility to (1) initiate the process of selfmanagement; (2) foster empowerment to participate in this process; (3) teach selfmanagement skills to people with the illness; (4) educate care partners on how to support self-management efforts; and (5) help identify and resolve barriers to self-management. Improving the education and support processes will undoubtedly require not only policy changes to ensure reimbursement for these services but also improvements in the 
education of social workers and other healthcare professionals. This education should include an understanding of the coping response to early-stage dementia that prioritizes actual experiences and recognizes the complexity and uniqueness of each person's journey. Stanford University's chronic disease self-management program (CDSMP) has shown to be effective in empowering individuals with various chronic conditions to make needed lifestyle changes to cope successfully with their illnesses. Given the established infrastructure of this program and its effectiveness, it might be useful to develop a disease-specific version targeting people with early-stage dementia and their care partners.

Contrary to current conceptualizations of the coping response, in the proposed model, acceptance and denial are not separate and distinct phases of a process in which acceptance is the desired, positive outcome. Instead the coping response is characterized by a simultaneous and concurrent presence of acceptance and denial. 'Striving towards well-being while acknowledging the inconceivable notion of losing one's cognitive abilities' allows that well-being and ill-being co-exist and oscillate between foreground and background. People who have a 'well-being in the foreground perspective' could easily be misunderstood as being in denial by social workers and other healthcare professionals whose conceptualizations of the dementia experience are based on the acceptance-denial framework (Telford, Kralik, \& Koch, 2006).

Another part of this education should involve fostering the awareness that people with early-stage dementia are capable of and have an interest in the self-management of their illness. This improvement in knowledge about the coping response to early-stage dementia would put social workers and other healthcare professionals in the position to 
help people with the condition to live more fully within the constraints of their illness. Another implication for clinical practice is the awareness that each person has a distinctive mixture of internal and external strengths and resources and consequently, utilizes some abilities and assets more than others. As a whole, the strengths and resources used by the participants in this study may not be transferrable to the general population of people with early-stage dementia. However, components of the proposed model and many of the strengths and resources and coping strategies discussed within the five main categories could be integrated into interventions targeting this population.

In addition, educational materials for people with the illness and their care partners could be improved to include and enhance the use of a person's internal and external resources. Based on the findings of this study, there is a need to teach and facilitate both problem-focused and emotion-focused coping strategies because the effective use of multiple coping styles was critical in the participants' ability to successfully adapt and ultimately reconstruct a sense of well-being.

In the past, psychotherapeutic interventions have been largely underutilized possibly due to the prevailing stereotype that people with the illness are incapable of sharing and processing thoughts and emotions. However, a review article by Cheston (1998) has documented promising results of various psychotherapeutic interventions with this population, including individual and group approaches. Within the context of these much-needed interventions and support services, photovoice methodology can play a vital role. Realistically, judging one's strengths and resources is at times difficult given the stresses associated with the illness, particularly in a counseling setting. The use of photography allows people with early-stage dementia to identify and capture their 
strengths and resources at home and use their photographs to convey their abilities and assets in a counseling setting. As such, social workers and other healthcare professional could explore the usefulness of photography as a way of empowerment in the context of self-management.

Self-regulation lies at the heart of illness self-management. As such, it is critical to create an environment that supports and sustains internal motivation. One of the necessary conditions for self-regulation is self-observation (Bandura, 1991). Systematic self-observation yields not only important information about a person but also promotes a sense of control and has a self-motivating effect. The self-motivating effect arises from the fact that one sets more challenging objectives if one monitors one's successes and failures. As such, one of the objectives of self-management trainings is to teach people to consciously observe their own process of change. The findings of this study suggest that people with early-stage dementia enjoy the process of self-monitoring and in the absence of available tools develop their own. Photography could serve as such a tool. In addition, photography could facilitate a better understanding of the subjective illness experience for social workers and other healthcare professionals because visual representations can communicate an idea or concept non-verbally.

The findings of this study also suggest a need to provide more venues where people with the illness can meet and interact with people with similar challenges. This could also take the form of panel discussions where people with the illness, caregivers, and healthcare professionals can share their perspective. Instigating and supporting selfadvocacy efforts of people with the illness similar to the 'early-stage town hall meetings' organized by the Alzheimer's Association would be another avenue to explore. 
Lastly, the findings of this study highlight that people with early-stage dementia manage some of the psychosocial challenges of the illness through the pursuit of personally meaningful activities. In other words, there is need to develop programming that empowers people with the illness to utilize this avenue of coping. Helpful programs could include courses that encourage creative expression through arts and crafts, photography, painting, dance, music, sculpture, writing, and improvisational storytelling.

\section{Implications for Research}

Based on data collected during the 'MacArthur Project on Healthy Aging', Rowe and Kahn produced a number of publications (e.g., Rowe \& Kahn, 1987, 1997, 1998) demonstrating the importance of widening the research agenda from predominantly investigating losses associated with aging to exploring psycho-social and environmental factors that facilitate and maintain healthy aging. The findings of this study extends that body of research to include psycho-social factors that support a more successful negotiation of early-stage dementia, a condition associated with aging that is usually addressed with a disease or deficit focus.

Further it would be important to research how people with moderate to severe dementia cope with and adapt to the illness. Although it is likely that one loses many coping and adapting skills as the illness progresses, investigating the extent to which late stage symptoms are affected by psycho-social-environmental factors would be a critical line of inquiry. Findings from such research would increase our understanding about how to more effectively support people in the later stages in their efforts to cope and adapt.

Clearly, the proposed model is a beginning, and there is much work to be done to 
verify the adequacy of 'striving towards well-being while acknowledging the inconceivable notion of losing one's cognitive abilities'. There is a need for future studies to examine, refine, and confirm the overall process, phases of adjustment, adaptive tasks, strengths and resources, and behavioral outcomes suggested by the data of this study. Future research investigating the experience of coping with and adapting to early-stage dementia should likely go in the following directions.

The proposed model suggests that attention to the illness fluctuates over time and with circumstances. At times, people experience a sense of well-being (with the illness in the background); at other times, people experience a disruption in well-being (with the illness in the foreground). Sometimes the choice to experience well-being is consciously determined even though illness symptoms are clearly competing for a foreground position. As such, conceptualization of well-being for people with early-stage dementia found in many of the current quality of life models and evaluation instruments may not adequately capture the experience of people living with the illness. To help improve current quality of life measurement, the components of the proposed model could be used to develop questions that would capture this oscillation between foreground/background. The use of this research instrument would increase our knowledge by including the phasic and fluctuating nature of the illness experience into quality of life investigations.

Future research could also investigate the relationships among the adaptive tasks depicted in the proposed model and the strengths and resources used to pursue these tasks. This research would provide information to refine or improve the proposed model, and thus, result in a better understanding of the experience in the early stages of the illness. 
Another area of inquiry would be to investigate how people with early-stage dementia manage their relationships. The findings of the present study suggest that to establish a sense of connectedness, people with early-stage dementia position themselves for acceptance. They try to anticipate which future actions will result in either acceptance or rejection, and based on this appraisal select their strengths and resources and their course of action. One line of inquiry in this context would be to investigate the mechanisms through which the strengths and resources identified in the proposed model facilitate the forming and maintaining of supportive relationships. This research would provide information about the cognitive and behavioral strategies intended to increase and decrease rejection. Furthermore, it would help identify the characteristics of the types of relationships that people with early-stage dementia consider supportive or unsupportive and thus provide insight into how to educate family members and friends accordingly.

A related line of inquiry could investigate how social forces such as stigmatizing beliefs about dementia impact the quality of relationships between people with the illness and others. This research could explore how family members and friends form their attitudes and beliefs about dementia and their perceptions of the needs of people living with the illness. Knowledge gained through this research could be used to develop education programs and support services for care partners and to address social forces that perpetuate nihilistic views of dementia and thus make it difficult for people with the illness to receive adequate support.

Most participants in the present study believed that in general, people with dementia are devalued and rejected. Future research could explore how individuals living 
with the illness are affected by and navigate these negative stereotypes. Initially, an important line of inquiry would be to investigate the degree to which people living with early-stage dementia are aware of the stigma associated with the illness and whether they actually believe these stereotypes (internalized stigma). A follow-up inquiry would be to measure the prevalence and intensity of internalized stigma among people with earlystage dementia and examine whether, and if so to which degree, internalized stigma is associated with levels of self-esteem and depressive symptoms. Findings from such research would allow for the development of interventions that focus on reducing internalized stigma. Assisting people living with early-stage dementia to overcome the aspect of stigma that is within themselves would facilitate self-esteem which in turn would empower them to more effectively fight the aspects of stigma that are external.

Positive perceptions of self were important to the participants in the present study. Future research could explore what interactions and activities facilitate a positive sense of self when customary avenue for identity construction are less or no longer available.

The findings of the present study suggest that people with early-stage dementia continue to have goals and anticipate a future, which points to another area of needed inquiry. Building on previous work (Cotrell \& Hooker, 2005; Fazier, Cotrell, \& Hooker, 2003), future research could further explore the aspirations of people with early-stage dementia and their appraisal of their abilities to reach these goals (self-efficacy). This research could continue to examine the difference in goal content of adults with and without cognitive impairment. Findings from such research would generate more knowledge about planned and future directed behaviors of people with early-stage dementia and how to support them in these personally meaningful endeavors. 
Given that the participant in the present study showed great motivation and interest in proactively addressing challenges that impact their well-being, future research should ask people with dementia directly about the type of support services they consider meaningful and work in partnership with them to develop such programs and services. Social workers and organizations such as the Alzheimer's Association should advocate for the inclusion of persons with dementia in publicly funded studies testing service models and interventions used to improve cognitive functioning and to increase independence. Individuals with dementia should be encouraged to organize and advocate for their concerns, which creates another important option for creating efficacy and adapting more successfully to the illness as well as making a contribution.

There is a need for future studies to include a larger and more representative sample of people with early-stage dementia. Most research on the coping response to early-stage dementia has been conducted with samples from Caucasian American and African American backgrounds. Further, study on the role of perceived strengths and resources in the lives of people with early-stage dementia should include people with different ethnic backgrounds, allowing a broader understanding of the coping response across diverse populations. Efforts to increase diversity might include those living in less nurturing environments and with limited access to material and psychosocial resources as well as different age groups. Their unique perspective could add to a growing base of knowledge.

More research is needed to investigate gender differences in coping with and adapting to early-stage dementia. Although gender differences have been noted in making medical decisions, use of support systems, and adjustment to illness (Stewart, Abbey, 
Shnek, Irvine, \& Grace, 2004; van der Molem, 1999), possible gender differences in the coping response to early-stage dementia remain to be explored. As of this date no such studies have been conducted. However, there have been two studies, one investigating women (Van Dijkhuizen et al., 2006) and the other men (Pearce et al., 2002). Their findings suggest differences in the adjustment process. The sample of the current study consisting of 13 women and seven men was inadequate to identify patterns of behavior based on gender. The age range in this study was wide (57 to 90 ; mean 73.4 ). The perception of strength and resources and their use in the coping response might be different for those experiencing the onset while still working than those in retirement. Previous research suggests that people's patterns of coping change with different life stages and as they face age-related changes (Folkman, Lazarus, Pimley, \& Novacek, 1987; Wagnild \& Young, 1990).

The present study sample consisting of 20 participants with early-onset was inadequate to identify coping patterns based on age. A longitudinal study design could provide important information by investigating noteworthy changes over time. Longitudinal research would allow the examination of within-subject changes and potentially offer insight into causality (Lazarus, 2000). The study period should preferably begin at the time of diagnosis to reflect the variation in perceived strengths and resources and coping responses and their changes over time. This study was essentially a retrospective self-view of the participants' lives, which may not precisely portray their overall experience over the course of the disease (Paterson, 2003).

The participants' interest in self-management highlights the need for an intervention study where one group of people with early-stage dementia receives 
comprehensive education, self-management training, and ongoing psychosocial support and another group serves as a control receiving a standard level of education and support. This study design would assist in understanding the extent to which coping and adaptation can be enhanced in this population (Lazarus 2000). Using measures of quality of life, depression, and other outcomes would allow for quantifying the effectiveness of the intervention.

Data collection in this study was limited to the viewpoint of the person with the illness. More in-depth research investigating the context in which the appraisal of strengths and resources and coping occurs is needed (including interactions with family members and friends and the broader social environment). A more holistic inquiry into the environment in which people with early-stage dementia interact would be useful to increase our knowledge about how strengths and resources unfold in the everyday process of living with the illness and how variations in the environment (e.g., types of spousal support) affect the coping response.

The findings of this study also suggest the need for a comprehensive evaluation of current educational programs and support services available to people with early-stage dementia. The general dissatisfaction articulated by the participants in this study points to the need for participatory action research to design programs and interventions that are more effective in addressing the needs of this population.

Photovoice emerged as a promising methodology for investigating aspects of the dementia experience. Taking photographs and engaging in a dialogue about their images was not only a joyful experience for the participants but more importantly it allowed them to communicate and elaborate their perceptions of an elusive concept. This finding 
suggests that photovoice methodology may be a valuable tool for researchers

investigating hard-to define concepts with people with cognitive impairment.

Furthermore, the role of meaningful activity in coping with early-stage dementia, and the process whereby new interests are discovered, should be explored further.

Another direction for future research is our need to better understand the potential of dyadic interviews in investigating the subjective experience of dementia. In this study, dyadic interviews were used as a means of member checking and successfully addressed the weakness of individual and focus group interviews by increasing the breadth of the data while decreasing the complexity of interaction in group discussions. As such, dyadic interviews may offer new opportunities to investigate the experience of early-stage dementia in a more interactive way. The findings of this study suggest that using a combination of individual and dyadic interviews within the same study is advantageous. Individual interviews were valuable not only for providing a measure of the depth and detail in the data and but also for allowing the researcher to achieve a sense of familiarity with the participants, which in turn allowed for a careful selection of the participants for the dyadic interviews.

\section{Conclusion}

This study is the first to explore the experience of early-stage dementia using photovoice and grounded theory methodology. Through their photographs and verbal contributions, the 20 women and men who participated in this study provided new insights into the coping response to the illness. As such, the findings of this study contribute to the body of research that, building on the pioneering work of Glaser and Strauss $(1966,1967)$ seeks to understand the meaning and experience of chronic illness 
from the perspective of those living with it.

More specifically, this study furthers what is currently known about living with early-stage dementia by highlighting the importance of looking beyond the losses and searching for the strengths and resources that people with the condition bring to the illness experience. Although some similarities can be found between the findings of previous studies and the findings of this study, investigating the illness experience with a participatory research approach and from a strength perspective allowed for finer nuances and a different emphasis to emerge.

The transactional framework of stress, appraisal, and coping (Lazarus \& Folkman, 1984) that underpinned the overall study and its components offered a comprehensive fit with the qualitative data and facilitated an in-depth understanding of the coping response. 'Striving towards well-being while acknowledging the inconceivable notion of losing one's cognitive abilities' was the core construct that emerged from the data, along with five categories representing the adaptive tasks relevant to the adjustment process together with the perceived strengths and resources that surfaced as connected to each adaptive task. The findings of this study add to this framework by suggesting that it can be used in research investigating the experience of people with cognitive impairment.

The tone of the visual and verbal data, the voice in which the participants recounted life-fragmenting events, reflected something beyond mere coping. It suggests a coping response in which the will to experience well-being and an attitude of personal responsibility are the driving forces in the process of adaptation. This coping response supports the notion of acceptance and denial and positive and negative emotions coexisting in the face of early-stage dementia. Although the thought of living with dementia 
was never free of negative emotions, the participants succeeded in recognizing that, while dementia is an undeniable part of their lives, it is not the only part.

Coping with early-stage dementia emerged as a dynamic process in which the participants oscillated between viewing life through the lens of well-being (with the illness in the background) or viewing life through the lens of ill-being (with well-being in the background). Embracing this paradox of managing the symptoms of the illness in the foreground while relegating the illness to the background of their lives was the main strength of the participants. The findings of this study suggest that the participants experienced early-stage dementia as a condition that persisted over time without an easily definable beginning, middle, or end and that learning to live with the illness was an ongoing process of adjustment. As such, the proposed model supports the notion that people with early-stage dementia are active agents (Keady \& Gilliard, 2001; Kitwood, 1990) who do not passively endure but actively engage with their illness and its consequences. As agents they actively seek to bring to bear whatever strengths and resources are available in order to cope and adapt (Cotrell \& Schulz, 1993) and by doing so construct a new, alternative sense of well-being.

Feeling well meant for the participants to willingly face and interact with the changes the illness had brought into their lives and take on the responsibility of creating well-being out of the changed reality. Having strong internal and external resources, as well as the ability to use a variety of problem and emotion-focused coping strategies, helped the participants in this study to successfully cope with and adapt to the ongoing changes that are characteristic of life with early-stage dementia and achieve an acceptable sense of well-being. 
What also emerged was that despite the best of intentions, the current health and social services offered to people with early-stage dementia are inadequate, which further exacerbates the vulnerability of this population. There is a need for health and social services to improve and expand the current education and support processes to reduce the risk of life disruption and foster a wide range of effective coping strategies, including self-management skills. Self-management skills are an essential and currently neglected component of education for people with early-stage dementia. Although these interventions can be challenging to implement and integrate into existing practices, they are important strategies to maximize a sense of normalcy and well-being. An environment of person-centered care that identifies strengths and resources could help people to cope and adapt more effectively with the illness and its wide-ranging consequences. Although not every person with early-stage dementia may be capable of reaching the extent to which the participants in this study coped with and adapted to the illness, all people with the condition should be given the opportunity to maximize their own well-being. 


\section{REFERENCES}

Adams, K. B., \& McClendon, M. J. (2006). Early-stage cognitive impairment: A social work practice and research agenda. Families in Society, 87(4), 590-600.

Aggarwal, N., Vass, A. A., Minardi, H. A., Ward, R., Garfield, C., \& Cybyk, B. (2003). People with dementia and their relatives: Personal experiences of Alzheimer's and of the provision of care. Journal of Psychiatric and Mental Health Nursing, 10(2), 187-197.

Ainlay, S. C., \& Smith, D. R. (1984). Aging and religious participation. Journal of Gerontology, 39(3), 367-363.

Aldwin, C. (1994). Stress, coping, and development. New York: Guilford.

Alzheimer's Association. (2004). Research consent for cognitively impaired adults: Recommendations for institutional review boards and investigators. Alzheimer's Disease and Associated Disorders, 18(3), 171-171.

Alzheimer's Association. (2009). 2009 Alzheimer's disease: Facts and figures. Retrieved 02.25, 2009, from http://www.alz.org/alzheimers_disease facts figures.asp

Aminzadeh, F., Byszewski, A., Molnar, F. J., \& Eisner, M. (2007). Emotional impact of dementia diagnosis: Exploring persons with dementia and caregivers' perspectives. Aging \& Mental Health, 11(3), 281-290.

Amirkhan, J. H. (1990). A factor analytically derived measure of coping: The coping strategy indicator. Journal of Personality \& Social Psychology, 59(5), 1066-1074.

Aquilina, C., \& Hughes, J. C. (2006). The return of the living dead: Agency lost and found. In J. C. Hughes, S. J. Louw \& S. R. Sabat (Eds.), Dementia: Mind, meaning, and the person (pp. 143-162). Oxford: Oxford University Press. 
Atkin, K., Stapley, S., \& Easton, A. (2010). No one listens to me, nobody believes me: Self management and the experience of living with encephalitis. Social Science \& Medicine, 71(2), 386-393.

Atri, A., Shaughnessy, L. W., Locascio, J. L., \& Growdon, J. H. (2008). Long-term course and effectiveness of combination therapy in Alzheimer Disease. Alzheimer Disease and Associated Disorders, 22(3), 209-221.

Aubin, G., Hachey, R., \& Mercier, C. (1999). Meaning of daily activities and subjective quality of life in people with severe mental illness. Scandinavian Journal of Occupational Therapy, 6, 53-62.

Audulv, A., Asplund, K., \& Norbergh, K.-G. (2010). Who's in charge? The role of responsibility attribution in self-management among people with chronic illness. Patient Education and Counseling, 81, 94-100.

Aujoulat, I., Luminet, O., \& Deccache, A. (2007). The Perspective of patients on their experience of powerlessness. Qualitative Health Research, 17(6), 772-785.

Baehrer-Kohler, S., \& Krebs-Roubicek, E. (2009). Self management of chronic disease: Alzheimer's disease. Switzerland: Springer.

Ballenger, J. F. (2006). Self, senility, and Alzheimer's disease: A history. Baltimore, MD: The John Hopkins University Press.

Baltes, P. B., \& Baltes, M. (Eds.). (1990). Successful ageing: Perspectives from the behavioural sciences. New York: Cambridge University Press.

Bamford, C., Lamont, S., Eccles, M., Robinson, L., May, C., \& Bond, J. (2004). Disclosing a diagnosis of dementia: A systematic review. International Journal of Geriatric Psychiatry, 19(2), 151-169. 
Bandura, A. (1977). Self-efficacy: Toward a unifying theory of behavioral change. Psychological Review, 84, 191-215.

Bandura, A. (1982). Self-efficacy mechanism in human agency. American Psychologist, $37(2), 122-147$.

Bandura, A. (1997). Self-efficacy. New York: Freeman.

Bandura, Albert. (1991). Social cognitive theory of self-regulation. Organizational Behavior and Human Decision Processes, 50(2), 248-287.

Barrett, J. J., Haley, W.E., Harrell, L. E., \& Powers, R. E. (1997). Knowledge about Alzheimer disease among primary care physicians, psychologists, nurses, and social workers. Alzheimer Disease \& Associated Disorders, 11(2), 99-106.

Basting, A. D. (2006). Arts in dementia care: 'This is not the end ... it's the end of this chapter.'. Generations, 30(1), 16-20.

Beard, R. L. (2004). Advocating voice: Organisational, historical and social milieux of the Alzheimer's disease movement. Sociology of Health \& Illness, 26(6), 797-819.

Beard, R. L. (2004). In their voices: Identity preservation and experiences of Alzheimer's disease. Journal of Aging Studies, 18(4), 415-428.

Beard, R. L., \& Estes, C. L. Medicalization of aging. Retrieved 03. 23, 2013, from http://www.encyclopedia.com/doc/1G2-3402200254.html

Becker, G. (1997). Disrupted lives: How people create meaning in a chaotic world. Berkeley, CA: University of California Press.

Benard, B. (2006). Using strengths-based practice to tap the resilience of families. In D. Saleebey (Ed.), Strengths perspective in social work practice (pp. 197-220). Boston, MA: Allyn \& Bacon. 
Benbow, S. M., \& Reynolds, D. (2000). Challenging the Stigma of Alzheimer's disease. Hospital Medicine, 6(3), 174-177.

Bender, D., Harbour, C., Thorp, J., \& Morris, P. (2001). Tell me what you mean by "Si"': Perceptions of quality of prenatal care among immigrant Latina women. Qualitative Health Research, 11(6), 780-794.

Berg, B. L. (2007). Qualitative research methods for the social sciences. Long Beach, CA: Pearson Education.

Berkman, L. (1984). Assessing the physical health effects of social networks and social suppport. Annual Review of Public Health, 5, 413-432.

Beuscher, L., \& Beck, C. (2008). A literature review of spirituality in coping with earlystage Alzheimer's disease. Journal of Clinical Nursing, 17(5a), 88-97.

Bick, K. L. (2000). The history of the Alzheimer's Asoociation: Future public policy implications. In P. J. Whitehouse, K. Maurer \& J. F. Ballenger (Eds.), Concepts of Alzheimer's disease: Biological, clinical, and cultural perspectives (pp. 234-244). Baltimore, MD: The John Hopkins University Press.

Bishop, M. (2005). Quality of life and psychosocial adaptation to chronic illness and acquired disability: A conceptual and theoretical synthesiss. Journal of Rehabilitation, 71(2), 5-13.

Bishop, M. (2005). Quality of life and psychosocial adaptation to chronic illness and disability. Rehabilitation Counseling Bulletin, 48(4), 219-231.

Bjorck, J. P., \& Cohen, L. H. (1993). Coping with threats, losses and challenges. Journal of Social and Clinical Psychology, 12(1), 56-72. 
Black, B., Kass, N., Fogarty, L., \& Rabins, P. (2007). Informed consent for dementia research. IRB: Ethics and Human Research, July/August, 7-14.

Blum, N. S. (1991). The management of stigma by Alzheimer's family caregivers. Journal of Contemporary Ethnography, 20(3), 263-284.

Blumer, H. (1969). Symobolic interaction: Perspective and method. Englewood Cliffs, NJ: Prentice Hall.

Bodenheimer, T., Lorig, K., Holman, H., \& Grumbach, K. (2002). Patient selfmanagement of chronic illness in primary care. Journal of the American Medical Association, 288, 2469-2475.

Bond, J., \& Corner, L. (2001). Researching dementia: Are there unique methodological challenges for health services research? Ageing \& Society, 21(01), 95-116.

Booth, T., \& Booth, W. (2003). In the frame: Photovoice and mothers with learning difficulties. Disability \& Society, 18(4), 431-442.

Brandtstädter, J., \& Greve, W. (1994). The aging self: Stabilizing and protective processes. Developmental Review, 14(2), 52-80.

Brunstein, J. C. (1993). Personal goals and subjective well-being: A longitudinal study. Journal of Personality \& Social Psychology, 65(5), 1061-1070.

Burgener, S. C., \& Berger, B. (2008). Measuring perceived stigma in persons with progressive neurological disease; Alzheimer's dementia and Parkinson's disease. Dementia, 7(1), 31(23).

Burgener, S. C., \& Dickerson-Putman, J. (1999). Assessing patients in the early stages of irreversible dementia: The relevance of patient perspectives. Journal of Gerontological Nursing, 25(2), 33-41. 
Bury, M. (1982). Chronic illness as biographical disruption. Sociology of Health and Illness, 4(2), 167-182.

Bury, M. (1991). The sociology of chronic illness: A review of research and prospects. Sociology of Health and Illness, 13, 451-468.

Bury, M. (2001). Illness narratives: Fact or fiction? Sociology of Health and Illness, 23, 263-285.

Bury, M. (1982). Chronic illness as biographical disruption. Sociology of Health and Illness, 4(2), 167-182.

Cahill, S., Begley, E., Topo, P., Saarikalle, K., Macijauskiene, J., Budraitiene, A., . . . Jones, K. (2004). 'I know where this is going and I know it won't go back': Hearing the individual's voice in dementia quality of life assessments. Dementia, 3(3), 313-330.

Cahill, S. M., Gibb, M., Bruce, I., Headon, M., \& Drury, M. (2008). 'I was worried coming in because I don't really know why it was arranged': The subjective experience of new patients and their primary caregivers attending a memory clinic. Dementia, 7(2), 175-189.

Carstensen, L. L., Isaacowitz, D. M., \& Charles, S. T. (1999). Taking time seriously: Atheory of socioemotional selectivity. American Psychologist, 54(3), 165-181.

Carver, C. S., Scheier, M. F., \& Weintraub, J. K. (1989). Assessing coping strategies: A theoreticallly based approach. Journal of Personality and Social Psychology, 56(2), 267-283.

Charmaz, K. (1983). Loss of self: A fundamental form of suffering in the chronically ill. Sociology of Health \& Illness, 5(2), 168-195. 
Charmaz, K. (1991). Good days, bad days: Chronic illnesses and time. New Brunswick, NJ: Rutgers University Press.

Charmaz, K. (2000). Experiencing chronic illness. In L. Albrecht, R. Fitzpatrick \& S. C. Scrimshaw (Eds.). The handbook of social studies in health and medicine (pp. 277-292). London: Sage.

Charmaz, K. (2006). Constructing grounded theory. Thousand Oaks, CA: Sage.

Cheston, R. (1998). Psychotherapeutic work with people with dementia: A review of the literature review. British Journal of Medical Psychology, 71(3), 211-231.

Cheston, R., \& Bender, M. (1999). Brains, minds and selves: Changing conceptions of the losses involved in dementia. British Journal of Medical Psychology, 72(2), 203-216.

Clare, L. (2002). We'll fight it as long as we can: Coping with the onset of Alzheimer's disease. Aging \& Mental Health, 6(2), 139-148.

Clare, L. (2003). Managing threats to self: Awareness in early stage Alzheimer's disease. Social Science \& Medicine, 57(6), 1017-1029.

Clare, L., Goater, T., \& Woods, B. (2006). Illness representations in early-stage dementia: A preliminary investigation. International Journal of Geriatric Psychiatry, 21(8), 761-767.

Clare, L., Roth, I., \& Pratt, R. (2005). Perceptions of change over time in early-stage Alzheimer's disease: Implications for understanding awareness and coping style. Dementia, 4(4), 487-520.

Clare, L., Rowlands, J. M., \& Quin, R. (2008). Collective strength: The impact of developing a shared social identity in early-stage dementia. Dementia, 7(1), 9(22). 
Clare, L., \& Shakespeare, P. (2004). Negotiating the impact of forgetting: Dimensions of resistance in task-oriented conversations between people with early-stage dementia and their partners. Dementia, 3(2), 211-232.

Clarke, C., \& Keady, J. (2002). Getting down to brass tacks: A discussion of data collection with people with dementia. In H. Wilkinson (Ed.), The perspectives of people with dementia: Research methods and motivations (pp. 25-46). London: Jessica Kingsley Publishers.

Close, H. (2007). The use of photography as a qualitative research tool. Nurse Researcher, 15(1), 27-36.

Cohen, C. I. (1993). The biomedicalization of psychiatry: A critical review. Community Mental Health, 29(6), 509-521.

Cohen, C. S., Phillips, M. H., \& Hanson, M. (Eds.). (2008). Strength and diversity in social work with groups: Think group. New York, NY: Routledge.

Cohen, D. (1991). The subjective experience of Alzheimer's disease: The anatomy of an illness as perceived by patients and families. American Journal of Alzheimer's Care and Related Disease and Research, 6(3), 6-11.

Cohen, D., \& Eisendorfer, C. (1986). The loss of self: A family resource for the care of Alzheimer's disease and related disorders. London: Norton.

Cohen, D., Kennedy, G., \& Eisendorfer, C. (1984). Phases of changes in the patient with Alzheimer's disease: A conceptual dimension for defining health care management. Journal of the American Geriatrics Society, 32(1), 11-15.

Cohen, G. D. (2006). Research on creativity and aging: The positive impact of the arts on health and illness. Generations, 30(1), 7-15. 
Cohen, S., \& Wills, T. A. (1985). Stress, social support, and the buffering hypothesis. Psychological Bulletin, 98(2), 310-357.

Collier, J. Jr., \& Collier, M., Hall, E. T. (1967/1991). Visual anthropology: Photography as a research method. Albuquerque: University of New Mexiko Press.

Conrad, P. (1992). Medicalization and social control. Annual Review of Sociology, 18, 209-232.

Conrad, P. (2007). The medicalization of society: On the transformation of human conditions into medical disorders. Baltimore, MD: John Hopkins University Press.

Cook, A. (2003). Using video to include the experiences of people with dementia in research. Research Policy and Planning, 21(2), 23-32.

Corbin, J., \& Strauss, A. (1990). Grounded theory research: Procedures, canons, and evaluative criteria. Qualitative Sociology, 13(1), 3-21.

Corbin, J., \& Strauss, A. (2008). Basics of qualitative research. Thousand Oaks: Sage Publications.

Corr, C. (1992). A task-based approach to coping with dying. OMEGA--Journal of Death and Dying, 24(2), 81-94.

Cotrell, V. C. (2007). Assessment of individuals with dementia. In C. B. Cox (Ed.), Dementia and social work practice: Research and interventions (pp. 45-68). New York: Springer.

Cotrell, V. C., \& Hooker, K. (2005). Possible selves of individuals with Alzheimer's disease. Psychology and Aging, 20(2), 285-294. 
Cotrell, V. C., \& Schulz, R. (1993). The perspective of the patient with Alzheimer's disease: A neglected dimension of dementia research. The Gerontologist, 33(2), 205-212.

Cotrell, V. C., \& Lein, L. . (1993). Awareness and denial in the Alzheimer's disease victim. Journal of Gerontological Social Work, 19(3-4), 115-132.

Cotrell, V. C. (1997). Awareness deficits in Alzheimer's disease: Issues in assessment and intervention. Journal of Applied Gerontology, 16(1), 71-91.

Cox, C. B. (2007). Dementia and social work practice: Research and interventions. New York: Springer.

Creswell, J. W. (1998). Qualitative inquiry and research design: Choosing among five approaches. Thousand Oaks, CA: Sage.

Creswell, J. W. (2005). Educational research: Planning, conducting, and evaluating quantitative and qualitative research (2nd Ed.). Columbus, OH: Pearson Prentice Hall.

Creswell, J. W. (2007). Qualitative inquiry and research design. Thousand Oaks, CA: Sage.

Crossley, M. L. (2000). Narrative psychology, trauma and the study of self/identity. Theory \& Psychology, 10, 527-546.

Dabbs, C. (1999). What do people with dementia most value in life. Journal of Dementia Care: For all Who Work with People with Dementia, July/August, 16-19.

Dahlberg, K., Todres, L., \& Galvin, K.. (2009). Lifeworld-led healthcare is more than patient-led care: An existential view of well-being. Medicine, Health Care and Philosophy, 12(3), 265-271. 
Davis, L. L., Buckwalter, K., \& Brugio, L. D. (1997). Measuring problem behaviors in dementia: Developing a methodological approach. Advances in Nurses Science, $920(1), 40-56$.

Davis, R. (1989). My journey into Alzheimer's disease. Wheaton, IL: Tyndale House Publisher.

De Boer, M. E., Hertogh, C., Droes, R. M., Riphagen, I. I., Jonker, C., \& Eefsting, J. A. (2007). Suffering from dementia - the patient's perspective: A review of the literature. International Psychogeriatrics, 19(6), 1021-1039.

De Ridder, D. T. D., Schreurs, K. M. G., \& Bensing, J. M. (1998). Adaptive tasks, coping and quality of life of chronically ill patients: The cases of Parkinson's disease and Chronic Fatigue Syndrome. Journal of Health Psychology, 3(1), 87-101.

Denzin, N. K., \& Lincoln, Y. (2005). Introduction: The discipline and practice of qualitative research. In N. K. Denzin \& Y. Lincoln (Eds.), The sage handbook of qualitative research. Thousand Oaks, CA: Sage.

Devins, G. M., Blinik, Y. M., Hutchinson, T. A., Hollomby, D. J., Barre, P. E., \& Guttmann, R. D. (1983). The emotional impact of end-stage renal disease: Importance of patients' perceptions of intrusiveness and control. International Journal of Psychiatry in Medicine, 13(4), 327-343.

Dewar, A. L., \& Morse, J. M. (1995). 'Unbearable incidents': Failure to endure the experience of illness. Journal of Advanced Nursing, 22(5), 957-964.

Diener, E. (2002). Subjective wellbeing: The science of happiness and life satisfaction. In C. R. Snyder \& S. J. Lopez (Eds.), Handbook of positive psychology (pp. 63-73). Oxford: Oxford University Press US. 
Dodson, J. (1989). The slow death: Alzheimer's disease. Journal of Neurosurgical Nursing, 16, 270-274.

Doernberg, M. (1989). Stolen mind: The slow disappearance of Ray Doernberg. Chapel Hill, NC: Alonquin Books of Chapel Hill.

Dorenlot, P. (2005). Editorial: Applying the social model of disability to dementia: Present-day challenges. Dementia, 4(4), 459-461.

Downs, M. (1997). Evaluating dementia services. In M. Marshall (Ed.), State of the art in dementia care. London: Centre for Policy on Ageing.

Droes, R. M. (1997). Psychomotor group therapy for demented patients in the nursing home. In B. M. L. Miesen \& G. M. M. Jones (Eds.), Care-giving in dementia: Research and applications (Vol. 2, pp. 95-118). London: Routledge.

Droes, R. M., Boelens-Van Der Knoop, E.C. C., Bos, J., Meihuizen, L., Ettema, T. P., Gerritsen, D. L., Hoogeveen, F., De Lange, J., Scoelzel-Dorenbos, C. J. M. (2006). Quality of life in dementia in perspective: An explorative study of variations in opinions among people with dementia and their professional caregivers, and in literature. Dementia, 5(4), 533-

Dyck, I. (1995). Hidden geographies: The changing lifeworlds of women with multiple sclerosis. Social Science \& Medicine, 40(3), 307-320.

Dyer, J. (1996). In a tangled wood. Dallas, TX: South Methodist University Press.

Eisenberg, L. (1977). Disease and illness: Distinctions between professional and popular idea of sickness. Culture, Medicine, and Psychiatry, 1(1), 9-23. 
Elliott, T. R., Kurylo, M., \& Rivera, P. (2002). Positive growth following acquired disability. In C. R. Snyder \& S. J. Lopez (Eds.), Handbook of positive psychology (pp. 687-699). New York: Oxford University Press.

Emmons, R. A. (1996). Striving and feeling: Personal goals and subjective well-being. In P. M. Gollwitzer \& J. A. Bargh (Eds.), The psychology of action: Linking cognition and motivation to behavior (pp. 313-337). New York: Guilford Press.

Engel, G. (1977). The need of a new medial model: A challenge for biomedicine. Science, 195(4286), 129-136.

Estes, C. L., \& Binney, E. A. (1989). The biomedicalization of aging: Dangers and dilemmas. Gerontologist, 29(5), 587-596.

Fazio, S. (2008). The enduring self in people with Alzheimer's: Getting to the heart of individualized care. Baltimore, MD: Health Professions Press.

Feinberg, Lynn Friss, \& Whitlatch, Carol J. (2001). Are persons with cognitive impairment able to state consistent choices? Gerontologist, 41(3), 374-382.

Finfgeld-Connet, D. (2005). Clarification of social support. Journal of nursing scholarship, 37(1), 4-9.

Fisher, M. H., \& McCabe, S. (2005). Managing chronic conditions for elderly adults: The VNS CHOICE model. Health Care Financing Review, 27(2), 33-45.

Fishman, J. R. , \& Mamo, L. (2001). What's in a disorder? A cultural analysis of the medical and pharmaceuttical construction of male and female sexual dysfunction. Women and Therapy, 24(1/2), 179-193. 
Foley, J. (1992). The experience of being demented. In R. Binstock, S. Post \& P. J. Whitehouse (Eds.), Dementia and aging: Ethics, values, and policy choices (pp. 30-43). Baltimore, MD: The John Hopkins University Press.

Folkman, S. (1997). Positive psychological states and coping with severe stress. Social Science \& Medicine, 45(8), 1207-1221.

Folkman, S., \& Greer, S. (2000). Promoting psychological well-being in the face of serious illness: When theory, research and practice inform each other. PsychoOncology, 9(1), 11-19.

Folkman, S., \& Lazarus, R. S. (1980). An analysis of coping in a middle-aged community sample. Journal of Health and Social Behavior, 21(3), 219-239.

Folkman, S., \& Lazarus, R. S. (1985). If it changes it must be a process: Study of emotion and coping during three stages of a college examination. Journal of Personality \& Social Psychology, 48(1), 150-170.

Folkman, S., Lazarus, R. S., Dunkel-Schetter, C., DeLongis, A., \& Gruen, R. J. (1986). Dynamics of a stressful encounter: Cognitive appraisal, coping, and encounter outcomes. Journal of Personality \& Social Psychology, 50(5), 992-1003.

Folkman, S., Lazarus, R. S., Gruen, R. J., \& DeLongis, A. (1986). Appraisal, coping, health status, and psychological symptoms. Journal of Personality \& Social Psychology, 50(3), 571-579.

Folkman, S., Lazarus, R. S., Pimley, S., \& Novacek, J.. (1987). Age differences in stress and coping processes. Aging \& Psychology, 2(2), 171-184.

Folkman, S., \& Moskowitz, J. T. (2000). Positive affect and the other side of coping. American Psychologist, 55(6), 647-654. 
Folstein, M. F., Folstein, S. E., \& McHugh, P. R. (1975). 'Mini Mental State': A practical method for grading the cognitive state of patients for the clinician. Journal of Psychiatric Research, 12(3), 189-198.

Ford, G. R., Goode, K. T., Barret, J. J., Harrel, L. E., \& Haley, W. E. (1997). Gender roles and caregiving stress: An examination of subjective appraisals of specific primary stressors in Alzheimer's caregivers. Aging and Mental Health, 1(2), 158165.

Fox, P. J. (1989). From senility to Alzheimer's disease: The rise of the Alzheimer's disease movement. The Milbank Quarterly, 67(1), 50-102.

Fox, P. J. (2000). The role of the concept of Alzheimer's disease in the development of the Alzheimer's Association in the United States. In P. J. Whitehouse, K. Maurer \& J. F. Ballenger (Eds.), Concepts of Alzheimer's disease: Biological, clincial, and cultural perspectives (pp. 158-180). Baltimore, MD: The John Hopkins University Press.

Frankl, V. E. (1986). The doctor and the soul: From psychotherapy to logotherapy. New York: Vintage books.

Frankl, V. E. (1963/1984). Man's search for meaning (3rd ed.). New York: First Washington Square Press.

Frazier, L. D., Cotrell, V. C., \& Hooker, K. (2003). Possible selves and illness: A comparison of individuals with Parkinson's disease, early-stage Alzheimer's disease, and healthy older adults. International Journal of Behavioral Development, 27(1), 1-11. 
Freire, P. (1970). Pedagogy of the oppressed. New York: Continuum.

Friel-McGowin, D. F. (1993). Living in the labyrinth: A personal journey through the maze of Alzheimer's New YorK: Dell Publishing.

Froggatt, A. (1988). Self-awareness in early dementia. In B. Gearing, M. Johnson \& T. Heller (Eds.), Mental health problems in old age: A reader (pp. 131-136). Buckingham: Open University Press.

Funch, D. P., \& Marshall, J. (1983). The role of stress, social support and age in survival from breast cancer. Journal of Psychosomatic Research, 27(1), 77-83.

Gilhooly, M. L. M., \& Sweeting, H. N. (1994). Family care of the dementing elderly. International Review of Psychiatry, 6(1), 29.

Gillies, B. (2000). A memory like clockwork: Accounts of living through dementia. Aging \& Mental Health, 4(4), 366-374.

Gilmour, H., Gibson, F., \& Campbell, J. (2003). Living alone with dementia: A case study approach to understanding risk. Dementia, 2(3), 403-420.

Gilmour, J., \& Huntington, A. D. (2005). Finding the balance: Living with memory loss. International Journal of Nursing Practice, 11(3), 118-124.

Gitterman, A. (2001). Social work practice with vulnerable and resilient populations. In A. Gitterman (Ed.), Handbook of social work practice with vulnerable and resilient populations (pp. 1-38). New York: Columbia University Press.

Glaser, B. (1978). Theoretical sensitivity: Advances in the methodology of grounded theory. Mill Valley, CA: Sociology Press.

Glaser, B., \& Strauss, A. (1966). Awareness of Dying. New York: Aldine.

Glaser, B., \& Strauss, A. (1967). The discovery of grounded theory. Chicago, IL: Aldine. 
Glaser, B., \& Strauss, A. (1971). Status passage: A formal theory. Chicago: Aldine.

Goffman, E. (1963). Stigma: notes on the management of spoiled identity. Englewood Cliffs, NJ: Prentice Hall.

Goldsmith, M. (1996). Hearing the voice of people with dementia: Opportunities and obstacles. London: Jessica Kingsley.

Goode, D. (1994). The national quality of life for persons with disabilities project: A quality of life agenda for the United States. In D. Goode (Ed.), Quality of life for persons with disabilities: International perspectives and issues (pp. 139-161). Cambridge, MA: Brookline.

Gottlieb, B. H., \& Gignac, M. A. M. (1996). Content and domain specificity of coping among family caregivers of persons with dementia. Journal of Aging Studies, 10(2), 137-155.

Guba, E. G., \& Lincoln, Y. S. (1994). Competing Paradigms in Qualitative Research. In N. K. Denzin \& Y. S. Lincoln (Eds.), Handbook of Qualitative Research, Thousand Oaks, CA: Sage (pp. 105-117). Thousand Oaks, CA: Sage.

Harman, G., \& Clare, L. (2006). Illness representations and lived experience in earlystage dementia. Qualitative Health Research, 16(4), 484-502.

Harper, D. (1984). Meaning and work: A study in photo elicitation. International Journal of Visual Sociology, 2(1), 20-43.

Harris, P. B. (2004). The perspective of younger people with dementia: Still an overlooked population. Social Work in Mental Health, 2(4), 17-36. 
Harris, P. B., \& Durkin, C. (2002). Building resilience through coping and adapting. In P. Harris (Ed.), The Person with Alzheimer's Disease: Pathways to Understanding the Experience (pp. 165-184). Baltimore: The John Hopkins University Press.

Harris, P. B., \& Sterin, G. J. (1999). Insider's perspective: Defining and preserving the self of dementia. Journal of Mental Health and Aging, 5(3), 241-256.

Harrison, B. (2001). Photographic images and narrative inquiry. Narrative Inquiry, 12(1), 87-111.

Harrison, B. E. (2002). Seeing health and illness worlds - using visual methodologies in a sociology of health and illness: a methodological review. Sociology of Health \& Illness, 24(6), 856-872.

Harvey, J. H., Orbuch, T. L., \& Fink, K. (1990). The social psychology of account-making: Meaning, hope, and generativity. New Zealand Journal of Psychology, 19, 46-57.

Hays, R. B., Chauncey, S., \& Tobey, L. A. (1990). The social support networks of gay men with AIDS. American Journal of Commmunity Psychology, 18(4), 374-384.

Healy, D., \& Walsh, P. N. (2007). Communication among nurses and adults with severe and profound intellectual disabilities: Predicted and observed strategies. Journal of Intellectual Disability Research, 11(2), 127-141.

Heckhausen, J., \& Schulz, R.. (1995). A life-span theory of control. Psychological Review, 102(2), 284-304.

Hellstroem, I., Nolan, M., \& Lundh, U.. (2007). Sustaining 'couplehood': Spouses' strategies for living positively with dementia. Dementia, 6(3), 383-409. 
Hergenrather, K., Rhodes, S., \& Clark, G. (2006). Windows to work: Exploring employment-seeking behaviors of persons with HIV/ AIDS through photovoice. AIDS Education and Prevention, 18(3), 243-258.

Herskovitz, E. (1995). Struggling over subjectivity: Debates about the "self" and Alzheimer's disease. Medical Anthropology Quarterly, 9(2), 146-164.

Heston, L. L., \& White, J. A. (1983). The vanishing mind: A practical guide to Alzheimer's disease and other dementias. New York: W. H. Freeman and Company.

Hinson Langford, C. P., Bowsher, J., Maloney, J. P., \& Lillis, P. P. (1997). Social support: A conceptual analysis. Journal of Advanced Nursing, 25(1), 95-100.

Hoe, J., Katona, C., Roch, B., \& Livingston, G. (2005). Use of the QOL-AD for measuring quality of life in people with severe dementia--the LASER-AD study. Age Ageing, 34(2), 130-135.

Holst, G., \& Hallberg, I. R. (2003). Exploring the meaning of everyday life, for those suffering from dementia. American Journal of Alzheimer's Disease and Other Dementias, 18(6), 359-365.

Holstein, M. (2000). Aging, culture, and the framing of Alzheimer's disease. In P. J. Whitehouse, K. Maurer \& J. F. Ballenger (Eds.), Concepts of Alzheimer's disease: Biological, clinical, and cultural perspectives (pp. 158-180). Baltimore, MD: The John Hopkins University Press.

House, J. S., Landis, K. R., \& Umberson, D. (1988). Social relationships and health. Science, 241(4865), 540-545. 
Hoyert, D. L., Kochanek, K. D., \& Murphy, S. . (1999). Deaths: Final data for 1997. National Vital Statistics Report, 47(19).

Hubbard, G., Downs, M., \& Tester, S. (2003). Including older people with dementia in research: Challenges and strategies. Aging \& Mental Health, 7(5), 351-351.

Hurworth, R. (2003). Photo-interviewing for research. Social Resesarch Update, 40, 1-4.

Husband, H. J. (1999). The psychological consequences of learning a diagnosis of dementia: Three case examples. Aging \& Mental Health, 3(2), 179-183.

Husband, H. J. (2000). Diagnostic disclosure in dementia: An opportunity for intervention? International Journal of Geriatric Psychiatry, 15(6), 544-547.

Hutchinson, S. A., Leger-Krall, S., \& Skodol, W. H. (1997). Early probable Alzheimer's disease and awareness context theory. Social Science \& Medicine, 45(9), 13991409.

Iliffe, S., Wilcock, J., \& Haworth, D. (2006). Delivering psychosocial interventions for people with dementia in primary care: Jobs or skills? Dementia, 5(3), 327-338.

Jenkings, N. K., Woodward, R., \& Winter, T. (2008). The emergent production of analysis in photo elicitation: Pictures of military identity. Forum: Qualitative Social Research, 9(3).

Jensen, L. A., \& Allen, M. N. (1994). A synthesis of qualitative research on wellnessillness. Qualitative Health Research, 4(4), 349-369.

Jolley, D. J., \& Benbow, S. M. (2000). Stigma and Alzheimer's disease: Causes, consequences and a constructive approach. International Journal of Clinical Practice, 54(2), 117-119. 
Jumisko, E., Lexell, J., \& So derberg, S. (2005). The meaning of living with traumatic brain injury in people with moderate to severe traumatic brain injury. Journal of Neuroscience Nursing, 37(1), 42-50.

Jumisko, E., Lexell, J., \& So derberg, S. (2009). The meaning of feeling well in people with moderate or severe traumatic brain injury. Journal of Clinical Nursing, 18(16), 2273-2281.

Katsuno, T. (2003). Personal spirituality of persons with early-stage dementia: Is it related to perceived quality of life? Dementia, 2(3), 315-335.

Katsuno, T. (2005). Dementia from the inside: How people with early-stage dementia evaluate their quality of life. Ageing and Society, 25(2), 197-214.

Keady, J. (1996). The experience of dementia: A review of the literature and implications for nursing practice. Journal of Clinical Nursing, 5(5), 275-288.

Keady, J., \& Gilliard, J. (2001). The early experience of Alzheimer's disease: Implications for partnership and practice. In T. Adams \& C. Clarke (Eds.), Dementia care: Developing partnerships in practice ((pp. 227-256). London: Ballie`re Tindall.

Keady, J., Harris, P., \& Wilkinson, H. . (2002). Dementia: The International Journal of Social Research and Practice (February 2002 - January 2009 ed.). London: Sage.

Keady, J., \& Nolan, M. (1994). Younger onset dementia: Developing a longitudinal model as the basis for a research agenda and as a guide to interventions with sufferes and carers. Journal of Advanced Nursing, 19(4), 659-669.

Keady, J., \& Nolan, M. (1995a). IMMEL 2: Working to augment coping responses in early dementia. British Journal of Nursing, 4(7), 377-380. 
Keady, J., \& Nolan, M. (1995b). IMMEL: Assessing coping responses in early dementia. British Journal of Nursing (BJN), 4(6), 309-314.

Keady, J., Nolan, M., \& Gilliard, J. (1995). Listen to the voices of experience. Journal of Dementia Care, May/June, 15-17.

Kim, S. Y. H., Cox, C., \& Caine, E. D. (2002). Impaired decision-making ability in subjects with Alzheimer's disease and willingness to participate in research. American Journal of Psychiatry, 159(5), 797-802.

King, G., Cathers, T., Brown, E., Specht, J. A., Willoughby, C., Polgar, J. M., MacKinnon, E., Smith, L. K., Havens, L. (2003). Turning points and protective processes in the lives of people with chronic disabilities. Qualitative Health Research, 13(2), 184-206.

Kishon-Barash, R., Midlarsky, E., \& Johnson, D. R. (1999). Altruism and the Vietnam War veteran: The relationship of helping to symptomatology. Journal of Traumatic Stress, 12(4), 655-662.

Kitwood, T. (1987). Explaining senile dementia: The limits of neuropathological research. Free Associations, 10, 117-140.

Kitwood, T. (1988). The technical, the personal, and the framing of dementia. Social Behaviour, 3, 161-179.

Kitwood, T. (1989). Brain, mind, and dementia: With particular reference to Alzheimer's disease. Ageing and Society, 9(1), 1-15.

Kitwood, T. (1990). The dialectics of dementia: With particular reference to Alzheimer's disease. Ageing and Society, 10(2), 177-196. 
Kitwood, T. (1993). Person and process in dementia. International Journal of Geriatric Psychiatry, 8(7), 541-546.

Kitwood, T. (1997). Dementia reconsidered: The person comes first. Buckingham: Open University Press.

Kitwood, T., \& Benson, S. (1995). The new culture of dementia care. London: Hawker.

Kitwood, T., \& Bredin, K. (1992). Towards a theory of dementia care: Personhood and well-being. Ageing \& Society, 12(3), 269-287.

Kleinman, A. (1988). The illness narratives: Suffering, healing, and the human condition. New York: Basic Books.

Kleinman, A., Eisenberg, L., \& Good, B. (1978). Culture, illness, and care: Clinical lessons from anthropolic and cross-cultural research. Annals of Internal Medicine, $88(2), 251-258$.

Knoblauch, H., Baer, A., Laurier, E., Petschke, S., \& Schnettler, B. (2008). Visual Analysis: New Developments in the Interpretive Analysis of Video and Photography. Qualitative Social Research, 9(3).

Koenig, H. G., George, L. K., \& Siegler, I. C. (1988). The use of religion and other emotion-regulating coping strategies among older adults. The Gerontologist, 28(3), 303-310.

Koenig, H. G., Kvale, J. N., \& Ferrel, C. (1988). Religion and well being in later life. The Gerontologist, 28(3), 18-28.

Kolb, B. (2008). Involving, sharing, analysing: Potential of the participatory photo interview. Qualitative Social Research, 9(3). 
Koppel, O. S. B., \& Dallos, R. (2007). The development of memory difficulties: A journey into the unknown. Dementia, 6(2), 193-213.

Kralik, D., Koch, T., Price, K., \& Howard, N. (2004). Chronic illness self-management: Taking action to create order. Journal of Clinical Nursing, 13(2), 259-267.

Krantz, S. E. (1983). Cognitive appraisals and problem-directed coping: A prospective study of stress. Journal of Personality \& Social Psychology, 44(3), 638-643.

Kretlow, F. (1990). A phenomenological view of illness Australian Journal of Advanced Nursing, 7(2), 8-10.

Kübler-Ross, E. (1969). On death and dying. New York, N.Y.: Macmillan.

Kuhn, T. S. (1996). The structure of scientific revolutions (3 ed.). London: University of Chicago Press.

Langdon, S. A., Eagle, A., \& Warner, J. (2007). Making sense of dementia in the social world: A qualitative study. Social Science \& Medicine, 64(4), 989-1000.

Langer, E. (2002). Mindfulness versus positive evaluation. In C. R. Snyder \& S. J. Lopez (Eds.), Handbook of positive psychology. New York: Oxford University Press.

Lawton, M. P., \& Rubenstein, R. L. (2000). Interventions in dementia care. New York: Springer.

Lazarus, R. S. (1991). Emotion and adaptation. New York: Oxford Univeristy Press.

Lazarus, R. S. (2000). Toward better research on stress and coping. American Psychologist, 55(6), 665-673.

Lazarus, R. S. (2001). Relational meaning and discrete emotions. In K. R. Scherer \& A. Schorr (Eds.), Appraisal processes in emotion: Theory, methods, research (pp. 37-67). New York: Oxford University Press. 
Lazarus, R. S., \& Folkman, S. (1984). Stress, appraisal, and coping. New York: Springer.

Lazarus, R. S., \& Folkman, S. (1987). Transactional theory and research on emotions and coping. European Journal of Personality, 1(3), 141-169.

LeClerc, C. M., Wells, D. L., Craig, D., \& Wilson, J. L. (2002). Falling short of the mark: Tales of life after hospital discharge. Clinical Nursing Research, 11(3), 242-263.

Lennon, M. C. (1989). The structural contexts of stress. Journal of Health and Social Behavior, 30(3), 261-268.

Leventhal, H., Nerez, D., \& Steele, D. J. (1984). Illness representations and coping with health threats. In S. Baum, S. E. Taylor \& J. E. Singer (Eds.), Handbook of Health Psychology (Vol. 4, pp. 219-252). Hillsdale, NJ: Lawrence Erlbaum.

Lewis, F. M. (1989). Attributions of control, experienced meaning, and psychosocial well-being in patients with advanced cancer. Journal of Psychosocial Oncology, $7(1-2), 105-119$.

Lincoln, Y. S., \& Guba, E. G. (1985). Naturatlistic Inquiry. Newbury Park, CA: Sage.

Link, B. G., \& Phelan, J. C. (2001). Conceptualizing Stigma. Annual Review of Sociology, 27, 363-385.

Link, B. G., Struening, E. L., Neese-Todd, S., Asmussen, S., \& Phelan, J. C. (2001). Stigma as a barrier to recovery: The consequences of stigma for the self-esteem of people with mental illnesses. Psychiatric Services, 52(12), 1621-1626.

Livneh, H. (2001). Psychosocial adaptation to chronic illness and disability. Rehabilitation Counseling Bulletin, 44(3), 151-160. 
Loeckenhoff, C. E., \& Carstensen, L. L. (2004). Socioemotional selectivity theory, aging, and health: The increasingly delicate balance between regulating emotions and making tough choices. Journal of Personality, 72(6), 1395-1424.

Logsdon, R. G., Gibbons, L. E., McCurry, S. M., \& Teri, L. (1999). Quality of life in Alzheimer's disease: Patient and caregiver reports. Journal of Mental Health and Aging, 5(1), 21-32.

Logsdon, R. G., Gibbons, L. E., McCurry, S. M., \& Teri, L. (2002). Assessing quality of life in older adults with cognitive impairment. Psychosomatic Medicine, 64(3), $510-519$.

Longres, J. F. (2000). Human behavior in the social environment (3rd ed.). Belmont, CA: Wadsworth.

Loomis, M. E., \& Conco, D. (1991). Patients perception of health, chronic illness, and nursing diagnosis. Nursing Diagnosis, 2(4), 162-170.

Lopez, E. D. S., Eng, E., Randall-David, E., \& Robinson, N. (2005). Quality-of-life concerns of African American breast cancer survivors within rural North Carolina: Blending the techniques of photovoice and grounded theory. Qualitative Health Research, 15(1), 99-115.

Ludwig, F. M. (1997). How routine facilitates wellbeing in older women. Occupational Therapy International, 4(3), 215-230.

Lyketsos, C., G., Gonzales-Salvador, T., Chin, J. J., Baker, A., \& Black, B. (2003). A follow-up study of change in quality of life among persons with dementia residing in a long-term care facility. International Journal of Geriatric Psychiatry, 18(4), $275-281$. 
Lyman, K. A. (1989). Bringing the social back in: A critique of the biomedicalization of dementia. The Gerontologist, 29(5), 597.

MacKian, S. (2000). Contours of coping: Mapping the subject world of long-term illness. Health and Place, 6(2), 95-104.

MacQuarrie, C. R. (2005). Experiences in early stage Alzheimer's disease: Understanding the paradox of acceptance and denial. Aging \& Mental Health, 9(5), 430-441.

MacRae, H. (1995). Women and caring: Constructing self through others. Journal of Women \& Aging, 7(1-2), 145-167.

MacRae, H. (1999). Managing courtesy stigma: The case of Alzheimer's disease. Sociology of Health and Illness, 21(1), 54-70.

MacRae, H. (2008). Making the best you can of it: Living with early-stage Alzheimer's disease. Sociology of Health and Illness, 30(3), 396-412.

MacRae, H. (2010). Managing identity while living with alzheimer's disease. Qualitative Health Research, 20(3), 293-305.

Maddi, S. R. (1970). The search for meaning. In M. Page \& W. J. Arnold (Eds.), Nebraska symposium on motivation. Lincoln, NE: University of Nebraska Press.

Mamo, L., \& Fishman, J. R. (2001). Potency in all the right places: Viagra as a technology of the gendered body. Body and Society, 7(4), 13-35.

Manthorpe, J., \& Iliffe, S. (2005). Timely responses to dementia: Exploring the social work role. Journal of Social Work, 5(2), 191-203.

Marshall, M., \& Tibbs, M.-A. (2006). Social work and people with dementia: Partnership, practice and persistence. Bristol, UK: The Policy Press.

Maslow, A. H. (1943). A theory of human motivation. Psychological Review, 50(4), 370- 
390.

Maslow, A. H. (1970). Motivation and personality (3 ed.). New York: Harper and Row.

Masten, A. (2001). Ordinary magic: Resilience processes in development. American Psychologist, 56(3), 227-238.

McNicoll, P., \& Young, J. M. (1998). Against all odds: Positive life experiences of people with advanced amyotrophic lateral sclerosis. Health and Social Work, 23(1), 35-43.

McWilliams, E. (1998). The process of giving and receiving of a diagnosis of dementia: An in-depth study of sufferes', carers', and consultants' experiences. PSIGE Newsletter, 64, 18-25.

Mead, G. (1934). Self and society. Chicago, IL: Chicago University Press.

Menne, H. L., Kinney, J. M., \& Morhardt, D. J. (2002). 'Trying to continue to do as much as they can do': Theoretical insights regarding continuity and meaning making in the face of dementia. Dementia, 1(3), 367-382.

Merriam, S. B. (2009). Qualitative research: A guide to design and implementation. San Francisco, CA: Jossey-Bass.

Midanik, L. T. (2004). Biomedicalization and alcohol studies: Implications for policy. Journal of Public Health Policy, 25(2), 211-228.

Miles, M. B., \& Huberman, A. M. (1994). Qualitative data analysis: An extended source book. Thousand Oaks: Sage.

Miller, G., \& Happell, B. (2006). Talking about hope: The use of participant photography. Issues in Mental Health Nursing, 27(10), 1051-1065. 
Mittelman, M. S., Roth, D. L., Haley, W. E., \& Zarit, S. H. (2004). Effects of a caregiver intervention on negative caregiver appraisals of behavior problems in patients with Alzheimer's disease: Results of a randomized trail. Journal of Gerontology: Psychological Sciences, 59B(1), 27-43.

Moniz-Cook, E., Manthorpe, J., Carr, I., Gibson, G., \& Vernooij-Dassen, M. (2006). Facing the future: A qualitative study of older people referred to a memory clinic prior to assessment and diagnosis. Dementia, 5(3), 375-395.

Moos, R. H., \& Holahan, C.J. (2007). Adaptive tasks and methods of coping with illness and disability. In E. Martz \& H. Livneh (Eds.), Coping with chronic illness and disability (pp. 107-128). New York: Springer.

Moos, R. H., \& Tsu, V. D. (1977). The crisis of physical illness: An overview. In R. H. Moos (Ed.), Coping with physical illness. New York: Plenum.

Moretti, R., Torre, P., Antonello, R. M., Cazzato, G., \& Bava, A. (2002). Depression and Alzheimer's disease: Symptom or comorbidity? American Journal of Alzheimer's Disease and Other Dementias, 17(6), 338-344.

Morgan, D. L. (1989). Adjusting to widowhood: Do social networks reallly make it easier? The Gerontologist, 29(1), 101-107.

Morgan, D. L. (1996). Focus Groups. Annual Review of Sociology, 22, 129-152.

Morgan, D. L. (1997). Focus groups as qualitative research. Thousand Oaks, CA: Sage.

Morgan, D. L., Ataie, J. E., Carder, P., \& Hoffman, K. (in press). Introducing dyadic interviews as a method for collecting qualitative data. Qualitative Health Research. 
Moskowitz, J., Folkman, S., Collette, L., \& Vittinghoff, E. (1996). Coping and mood during AIDS-related caregiving and bereavement. Annals of Behavioral Medicine, $18(1), 49-57$.

Mountain, G. A. (2006). Self-management for people with early dementia: An exploration of concepts and supporting evidence. Dementia, 5(3), 429-446.

Moyle, W., McAllister, M., Venturato, L., \& Amdams, T. (2007). Quality of life and dementia: The voice of the person with dementia. Dementia, 6(2), 175-191.

National Association for Social Workers. (2013). NASW standard for social work case management. Retrieved 02.10, 2013, from http://www.naswdc.org/practice/naswstandards/CaseManagementStandards2013. pdf

Nolan, M. R., Grant, G., Caldock, K., \& Keady, J. (1994). A framework for assessing the needs of family carers: A multidisciplinary guide. Stroke-on-Trent: BASE Publications.

Nygard, L, \& Borell, L. (1998). A life-world of altering meaning: Expressions of the illness experience of dementia in everyday life over three years. Occupational Therapy Journal of Research, 18, 109-136.

Nygard, L., Borell, L., \& Gustavsson, A. (1995). Managing images of occupational self in early stage dementia. Scandinavian Journal of Occupational Therapy, 2, 129137.

O’Brian, T. B., \& DeLongis, A. (1996). The interactional context of problem-, emotion-, and relationship-focused coping: The role of the big five personality factors. Journal of Personality, 64(4), 775-813. 
Öhman, M., Söderberg, S., \& Lundman, B. (2003). Hovering between suffering and enduring: The meaning of living with serious chronic illness. Qualitative Health Research, 13(4), 528-542.

Olsson, L. E., Nystrom, A. E. M., Karlsson, J., \& Ekman, I. (2006). Admitted with a hip fracture: Patient perceptions of rehabilitation. Journal of Clinical Nursing, 16(5), $853-859$.

Olsson, M., Ska r, L , \& So derberg, S. (2010). Meanings of feeling well for women with multiple sclerosis. Qualitative Health Research, 20(9), 1254-1261.

Ostwald, S. K., Duggleby, W., \& Hepburn, K. W. (2002). The stress of dementia: View from the inside. American Journal of Alzheimer's Disease and Other Dementias, 17(5), 303-312.

Pargament, K., Ensing, D. S., Falgout, K., Olsen, H., Reilly, B., Van Haitsma, K. V., \& Warren, R. (1990). God help me: Religious coping efforts as predictors of the outcomes to significant negative life events. American Journal of Community Psychology, 18(6), 793-824.

Park, C. L., \& Folkman, S. (1997). Meaning in the context of stress and coping. Review of General Psychology, 1(2), 115-144.

Parsons-Suhl, K., Johnson, M. E., McCann, J. J., \& Solberg, S. (2008). Losing one's memory in early Alzheimer's Disease. Qualitative Health Research, 18(1), 31-42.

Seligman, B. L. (2001). The shifting perspectives model of chronic Illness. Journal of Nursing Scholarship, 33(1), 21-26. 
Paterson, B. L. (2003). The Koala has claws: Application of the shifting perspective model in research on chronic illness. Qualitative Health Research, 13(7), 987994.

Patrick, D. L., Starks, H. E., Cain, K. C., Uhlmann, R. F., \& Pearlman, R. A. (1994). Measuring preferences for health states worse than death. Medical Decision Making, 14(1), 9-18.

Patton, M. Q. (1990). Qualitative evaluation and research methods (2nd ed.). Newbury Park, CA: Sage Publications.

Patton, M. Q. (2002). Qualitative research and evaluation methods (3rd ed.). Thousand Oaks, CA: Sage Publications.

Pearce, A., Clare, L., \& Pistrang, N. (2002). Managing sense of self: Coping in the early stages of Alzheimer's disease. Dementia, 1(2), 173-192.

Pearlin, L. I., Harrington, C., Lawton, M. P., Montgomery, R. J. V., \& Zarit, S. H. (2001). An overview of the social and behavioural consequences of Alzheimer's disease. Aging and Mental Health, 5(1), S3-S6.

Pearlin, L. I., \& Schooler, C. (1978). The structure of coping. Journal of Health and Social Behavior, 19(1), 2-21.

Phinney, A. (1998). Living with dementia from the patient's perspective. Journal of Gerontological Nursing, 24(6), 8-15.

Phinney, A. (2002). Living with the symptoms of Alzheimer's disease. In P. Harris (Ed.), The person with Alzheimer's disesae: Pathways to understanding the experience (pp. 49-74). Baltimore: The John Hopkins University Press. 
Phinney, A. (2002). Fluctuating awareness and the breakdown of the illness narrative in dementia. Dementia, 1(3), 329-344.

Phinney, A. \& Chesla, C. A. (2003). The lived body in dementia. Journal of Aging Studies, 17(3), 283-299.

Plassman, B. L., Langa, K. M., Fisher, G. G., Herringa, S. G., Weir, D. R., Ofsteda, M. B., ...Wallace, R. B. (2007). Prevalence of dementia in the United States: The aging, demographics, and memory study. Neuroepidemiology, 29(1-2), 125-132.

Pledger, C. (2003). Discourse on disability and rehabilitation issues: Opportunities for psychology. American Psychologist, 58(4), 279-284.

Pollitt, P. A., Anderson, I., \& O’Connor, D. W. (1991). For better or for worse: The experience of caring for an elderly dementing spouse. Ageing and Society, 11(4), 443-469.

Pollitt, P. A., O’Connor, D. W., \& Anderson, I. (1989). Mild dementia: Perceptions and problems. Ageing and Society, 9(3), 261-275.

Post, S. G. (1990a). Infanticide and geronticide. Ageing and Society, 10(3), 317-328.

Post, S. G. (1990b). Severely demented elderly people: A case against senicide. Journal of the American Geriatric Society, 38(6), 715-718.

Post, S. G. (1991). Euthanasia, senicide, and the ageing society. Journal of Religious Gerontology, 8(1), 57-65.

Post, S. G. (1995). The moral challenge of Alzheimer's disease. Baltimore, MD: John Hopkins Press. 
Pratt, R., \& Wilkinson, H. (2001). Tell me the truth: The effect of being told the diagnosis of dementia from the perspective of the person with dementia. London: The Mental Health Foundation.

Pratt, R., \& Wilkinson, H. (2003). A psychosocial model of understanding the experience of receiving a diagnosis of dementia. Dementia, 2(2), 181-199.

Pressley, J. C., Trott, C., Tang, M., Durkin, M., \& Stern, Y. (2003). Dementia in community-dwelling elderly patients: A comparison of survey data, medicare claims, cognitive screening, reported symptoms, and activity limitations. Journal of Clinical Epidemiology, 56(9), 896-905.

Preston, L., Marshall, A., \& Bucks, R. S. (2007). Investigating the ways that older people cope with dementia: A qualitative study. Aging \& Mental Health, 11(2), 131-143.

Puchalski, C. M. (2007-2008). Spirituality and the care f patients at the end-of-life: An essential component to care. OMEGA--Journal of Death and Dying, 56(1), 33-46.

Radley, A., Hodgetts, D., \& Cullen, A. (2005). Visualizing homelessness: A study in photography and estrangement. Journal of Community \& Applied Social Psychology, 15(4), 273-295.

Radley, A., \& Taylor, D. (2003). Images of recovery: A photo-elicitation study on the hospital ward. Qualitative Health Research, 13(1), 77-99.

Rappaport, J. (1992). Research goals and the empowerment social agenda. In P. Tolan, C. Keys, F. Chertok \& L. Jason (Eds.), Researching community psychology: Issues of theory and methods (pp. 51-63). Washinton, DC: American Psychological Association. 
Reisberg, B., Ferris, S. H., de Leon, M.J., \& Crook, T. (1982). The global deterioration scale for assessment of primary degenerative dementia. American Journal of Psychiatry, 139, 1136-1139.

Reker, G. T., \& Chamberlain, K. (2000). Introduction. In G. T. Reker \& K. Chamberlain (Eds.), Exploring existential meaning: Optimizing human development across the life span (pp. 1-22). Thousand Oaks, CA: Sage.

Reynolds, F. (2002). Stitching together past and present: Narratives of biographical reconstruction during chronic illness. Retrieved from http://eprints.hud.ac.uk/5139/.

Reynolds, F., \& Lim, K.H. (2007). Turning to art as a positive way of living with cancer: A qualitative study of personal motives and contextual influences. Journal of Positive Psychology, 2(1), 66-75.

Reynolds, F., \& Prior, S. (2003). "Sticking jewels in your life": Exploring women's strategies for negotiating an acceptable quality of life with multiple sclerosis. Qualitative Health Research, 13(9), 1225-1251.

Riley, R., \& Manias, E. (2006). Governance in operating room nursing: Nurses' knowledge of individual surgeons. Social Science \& Medicine, 62(6), 1541-1551.

Riper, H. (1995). Shifting models and methods in the study of death and dying. Retrieved 02/21, 2010, from http://www.iol.ie/ mazzoldi/toolsforchange/postmet/dying.html

Robinson, L., Clare, L., \& Evans, K. (2005). Making sense of dementia and adjusting to loss: Psychological reactions to a diagnosis of dementia in couples. Aging \& Mental Health, 9(4), 337-347. 
Robinson, P., Ekman, S. L. , \& Wahlund, L. O. (1998). Unsettled, uncertain and striving to understand: Towards and understanding of the situation of persons with suspected dementia. International Journal of Aging and Human Development, 47(2), 143-161.

Robinson, P., Ekman, S. L., Meleis, I., Winblad, B., \& Wahlund, L. O. (1997). Suffering in silence: The experience of early memory loss. Health Care in Later Life, 2(2), 107-120.

Rogers, C. (1980). A way of being. Boston, MA: Houghton Mifflin.

Rose, L. (1996). Show me the way to go home. San Francisco, CA: Elder Books.

Rothbaum, F., Weisz, J. R., \& Snyder, S. S. (1982). Changing the world and changing the self: A two-process model of perceived control. Journal of Personality and Social Psychology, 42(1), 5-37.

Rotosky, S. S., \& Travis, C. B. (1996). Menopause research and the dominance of the biomedical model. Psychology of Women Quarterly, 20(2), 285-312.

Rowe, J.W., \& Kahn, R. L. (1987). Human aging: Usual and successful. Sciences, 237(4811), 143-149.

Rowe, J. W. \& Kahn, R. L. (1997). Successful Aging. The Gerontologist 37(4): 433-440. Rowe, J. W. \& Kahn, R. L. (1998). Successful Aging. NY: Random House.

Sabat, S. R. (2001). The experience of Alzheimer's disease: Life through a tangled veil. Oxford: Blackwell.

Sabat, S. R., \& Harre, R. (1992). The construction and deconstruction of self in Alzheimer's disease. Ageing and Society, 12(4), 443-461. 
Sadeh, N., \& Karniol, R. (2012). The sense of self-continuity as a resource in adaptive coping with job loss. Journal of Vocational Behavior, 80(1), 93-99.

Saleebey, D. (1992/2009). The strengths perspective in social work practice (5th ed.). Boston, MA: Pearson Education.

Saleebey, D. (1996). The strengths perspective in social work practice: Extensions and cautions. Social Work, 41(3), 296-305.

Salovey, P., Rothman, A. J., Detweiler, J. B., \& Steward, W. T. (2000). Emotional states and physical health. American Psychologist, 55(1), 110-121.

Samson, A., \& Siam, H. (2008). Adapting to major chronic illness: A proposal for a comprehensive task-model approach. Patient Education and Counseling, 70(3), 426-429.

Sandelowski, M. (1993). Rigor or rigor mortis: The problem of rigor in qualitative research revisited. Advances in Nursing Science Methods of Inquiry, 16(2), 1-8.

Sandelowski, M. (1995). Focus on qualitative methods: Sample size in qualitative methods. Research in Nursing and Health, 18(2), 179-183.

Sandelowski, M. (1986). The problem of rigor in qualitative research. Advances in Nursing Science, 8(3), 27-37.

Saunders, C. (1999). Origins: International perspectives, then and now. In I. B. Corless \& Z. Foster (Eds.), The hospice heritage: Celebrating our future. Binghamton, NY: Haworth Press.

Scheier, M. F., Magovern, J., Abbott, R. A., Matthews, K. A., Owens, J. F., Lefebvre, R. C., \& Carver, C. S. (1989). Dispositional optimism and recovery from coronary 
artery bypass surgery: The beneficial effects of physical and psychological wellbeing. Journal of Personality and Social Psychology, 57, 1024-1040.

Schroevers, M. J., Ranchor, A. V., \& Sanderman, R. (2003). The role of social support and self-esteem in the presence and course of depressive symptoms: A comparison of cancer patients and individuals from the general population. Social Science \& Medicine, 57(2), 375-385.

Schulz, U., \& Mohamed, N. E. (2004). Turning the tide: Benefit finding after cancer surgery. Social Science \& Medicine, 59(3), 653-662.

Schwarzer, R., \& Knoll, N. (2003). Positive coping: Mastering demands and searching for meaning. In S. J. Lopez \& C. R. Snyder (Eds.), Handbook of positive psychological assessment. Washington, DC: American Psychological Association.

Schroevers, Maya J., Ranchor, Adelita V., \& Sanderman, Robbert. (2003). The role of social support and self-esteem in the presence and course of depressive symptoms: a comparison of cancer patients and individuals from the general population. Social Science \& Medicine, 57(2), 375-385.

Seligman, M. E. P. (2002). Authentic happiness. New York: Free Press.

Shakespeare, P., \& Clare, L. (2005). Focusing on task-oriented talk as a way of exploring the interaction between people with early-onset dementia and their carers. Qualitative Research in Psychology, 2(4), 327-340.

Shannon, C., \& Shaw, S. (2005). 'If the dishes don't get done today, they'll get done tomorrow': A breast cancer experience as a catalyst for changes to women's leisure. Journal of Leisure Research, 37(2), 195-215. 
Sheikh, J. I., \& Yesavage, J. A. (1986). Geriatric Depression Scale (GDS): Recent evidence and development of a shorter version. Clinical Gerontologist, 5(1-2), $165-173$.

Shenton, A. K. . (2004). Strategies for ensuring trustworthiness in qualitative research projects. Education for Information, 22(2), 63-75.

Shulman, L. (1999). The skills of helping individuals, families, groups, and communities (4th ed.). Melmont, CA: Wadsworth.

Sieber, J. (1992). Planning ethically responsible research. Newbury Park, CA: Sage.

Silver, R. L., \& Wortman, C. B. (1983). Coping with undesirable life events. In J. Garber \& Seligman, M. E. P. (Eds.), Human helplessness: Theory and application. New York: Academic Press.

Skaggs, B. , Barron, C. R. (2006). Searching for meaning in negative events: Concept analysis. Journal of Advanced Nursing, 53(5), 559-570.

Slaughter, S., Cole, D., Jennings, E., \& Reimer, M. . (2007). Consent and assent to participate in research from people with dementia. Nursing Ethics, 14(1), 27-40.

Smith, A., King, E., Hindley, N., Barnetson, L., Barton, J., \& Jobst, K. A. (1998). The experience of research participation and the value of diagnosis in dementia: Implications for practice. Journal of Mental Health, 7(3), 309-321.

Smith, A. P., \& Beattie, B. Lynn. (2001). Disclosing a diagnosis of Alzheimer's Disease: Patient and family experiences. The Canadian Journal of Neurological Sciences, 21(1), 67-71.

Smith, C. E., Curtas, S., Werkowitch, M., Kleinbeck, S. V., \& Howard, L. (2002). Home parenteral nutrition: Does affiliation with a national support and educational 
organization improve patient outcomes? Journal of Parenteral and Enteral Nutrition, 26(3), 159-163.

Snyder, L. (1999). Speaking our mind: Personal reflections from individuals with Alzheimer's disease. New York: Freeman.

Snyder, L. (2001). The lived experience of Alzheimer's: Understanding the feelings and subjective accounts of persons with the disease. Alzheimer's Care Quarterly, 2(2), $8-22$.

Snyder, L. (2002). Social and family relationships: Establishing and maintaining connections. In P. Harris (Ed.), The Person with Alzheimer's Disease: Pathways to Understanding the Experience (pp. 112-133). Baltimore: The John Hopkins University Press.

Snyder, L. (2003). Satisfactions and challenges in spiritual faith and practice for persons with dementia. Dementia, 2(3), 299-313.

Sobel, S. (2005). Thursdays with Marion: The therapist's tale. Journal of Genetic Counseling, 14(5), 341-347.

Söderberg, S., Lundman, B., \& Norberg, A. (1999). Struggling for dignity: The meaning of women's experiences of living with Fibromyalgia. Qualitative Health Research, 9(5), 575-587.

Somers, M. R. (1994). The narrative constitution of identity: A relational and network approach. Theory and Society, 23(5), 605-649.

Sorensen, L., Waldorff, F., \& Waldemar, G. (2008). Coping with mild Alzheimer's disease. Dementia, 7(3), 287-299. 
Sorrel, J. M., \& Redmond, G. M. (1995). Interviews in qualitative nursing research: Differing approaches for ethnographic and phenomenological studies. Journal of Advanced Nursing, 21(6), 1117-1122.

Stanton, A.L., Parsa, A., \& Austenfeld, J.L. (2002). The adaptive potential of coping through emotional approach. In C. R. Lopez \& S. J. Lopez (Eds.), Handbook of positive psychology (pp. 148-158). New York: Oxford University Press.

Steeman, E., de Casterle, B. D., Godderis, J., \& Grypdonck, M. (2006). Living with early-stage dementia: A review of qualitative studies. Journal of Advanced Nursing, 54(6), 722-738.

Steeman, E., Godderis, J., Grypdonck, M., De Bal, N., \& De Casterle, B. D. (2007). Living with dementia from the perspective of older people: Is it a positive story? Aging \& Mental Health, 11(2), 119-130.

Steinberg, M., Sheppard, J. M., Tschanz, J. T., Norton, M. C., Steffens, D. C., Breitner, J. C. S., \& G., Lyketsos C. (2003). The incidence of mental and behavioral disturbances in dementia: The Cache County study. The Journal of Neurosychiatry \& Clinical Neuroscience, 15(3), 340-345.

Stern, P. (1980). Grounded theory methodology: Its uses and processes. Image, 12(1), 2023.

Stewart, D. E., Abbey, S. E., Shnek, Z. M., Irvine, J., \& Grace, S. L. (2004). Gender differences in ealth information needs and decisional prefernces in patients recovering from an acute ischemic coronary event. Psychosomatic Medicine, $66(1), 42-48$. 
Stocker, K. B., \& Turner, B. F. (2004). The personal experience of coping with brain disease: A multimethod validation and assessment. Journal of Mental Health and Aging, 10(1), 7-29.

Stoll, S. (1979). Guidelines for spiritual assessment. American Journal of Nursing, 79(9), $1574-1577$.

Strauss, A. (Ed.). (1975). Chronic illness and the quality of life. St. Louis, MO: Mosby.

Strauss, A. (1987). Qualitative analysis for social scientists. Cambridge, UK: : Cambridge University Press.

Strauss, A., \& Corbin, J. (1998). Basics of qualitative research (2nd ed.). Thousand Oaks, CA: Sage.

Strawbridge, W. J., Shema, S. J., Cohen, R. D., Roberts, R. E., \& Kaplan, G. A. (1998). Religiosity buffers effects of some stressors on depression but excacerbates others. Journal of Gerontology, 53B(3), S118-S126.

Stuckey, J. C., Post, S. G., Ollerton, S., FallCreek, S. J., \& Whitehouse, P. J. (2002). Alzheimer's disease, religion, and the ethics of respect for spirituality: A community dialogue. Alzheimer's Care Quarterly, 3(3), 199-207.

Stuifbergen, A., \& Rogers, S. (1997). Health promotion: An essential component of rehabilitation for persons with chronic disabling conditions. Advances in Nursing Science, 19(4), 1-20.

Sulmasy, D. P. (2002). A biopsychosocial-spiritual model for the care of patients at the end of life. Gerontologist, 42(3), 24-33.

Svanstroem, R., \& Dahlberg, K. (2004). Living with dementia yields a heteronomous and lost existence. Western Journal of Nursing Research, 26(6), 671-687. 
Tariot, P. N., Mack, J. L., Patterson, M. B., Edland, S. D., Weiner, M. F., Fillenbaum, G., . . Mortimer, J. A. (1995). The behaviour rating scale for dementia of the consortium to establish a registry for Alzheimer's disease. The American Journal of Psychiatry, 152(9), 1349-1357.

Taylor, R. J. (1986). Religious participation among elderly black. The Gerontologist, 26(6), 630-636.

Taylor, S. E. (1983). Adjustment to threatening events: A theory of cognitive adaptation. American Psychologist, 38(11), 1161-1173.

Taylor, S. E. (1991). Asymmetrical effects of positive and negative events: The mobilization-minimization hypothesis. Psychological Bulleting, 110(1), 67-85.

Taylor, S. E., \& Brown, J. D. (1988). Illusion and well-being: A social psychological perspective on mental health. Psychological Bulletin, 103(2), 193-210.

Taylor, S. E., \& Brown, J. D. (1994). Positive illusions and well-being revisited: Separating fiction from fact. Psychological Bulletin, 116(1), 28.

Telford, K., Kralik, D., \& Koch, T. (2006). Acceptance and denial: Implications for people adapting to chronic illness: Literature review. Journal of Advanced Nursing, 55(4), 457-464.

Thoits, P. A. (1982). Conceptual, methodological, and theoretical problems in studying social support as a buffer against life stress. Journal of Health \& Social Behavior, 23(2), 145-159.

Thoits, P. A. (1995). Stress, coping, and social support processes: Where are we? What next? Journal of Health \& Social Behavior, 36, 53-79. 
Thoits, P. A. (2006). Personal Agency in the Stress Process. Journal of Health \& Social Behavior, 47(4), 309-323.

Thompson, S. C. (2002). The role of personal control in adaptive functioning. In C. R.

Lopez \& S. J. Lopez (Eds.), Handbook of positive psychology (pp. 202-213). New York, NY: Oxford University Press.

Thorgrimsen, L., Selwood, A., Spector, A., Royan, L., de Madariaga Lopez, M., Woods, R., \& Orrell, M. (2003). Whose quality of life is it anyway?: The validity and reliability of the quality of life-Alzheimer's disease (QoL-AD) scale. Alzheimer Disease \& Associated Disorders, 17(4), 201-208.

van der Molem, B. (1999). Relating information needs to the cancer experience: Information as a key coping strategy. European Journal of Cancer Care, 8(4), 238-244.

van der Roest, H. G. , Meiland, F. J., Maroccini, R., Comijs, H. C., Jonker, C., \& Dröes, R. M. (2007). Subjective needs of people with dementia: A review of the literature. International Psychogeriatrics, 19(3), 559-592.

Van Dijkhuizen, M., Clare, L., \& Pearce, A. (2006). Striving for connection: Appraisal and coping among women with early-stage Alzheimer's disease. Dementia, 5(1), 73-94.

van Hout, H., Vernooij-Dassen, M., Hoefnagels, W., \& Grol, R. (2001). Measuring the opinions of memory clinic users: Patients, relatives and general practitioners. International Journal of Geriatric Psychiatry, 16(9), 846-851. 
Van Ness, P. H., \& Larson, D. B. (2002). Religion, senescence, and mental health: The end of life is not the end of hope. American Journal of Geriatric Psychiatry, 10(4), 386-397.

Vernooij-Dassen, M. J. F., Moniz-Cook, E. D., Woods, R. T., De Lepeleire, J., Leuschner, A., Zanetti, O., . . . Iliffe, S. (2005). Factors affecting timely recognition and diagnosis of dementia across Europe: From awareness to stigma. International Journal of Geriatric Psychiatry, 20(4), 377-386.

Vernooij-Dassen, M. J., Van Hout, H. P., Hund, K. L., Hoefnagels, W. H., \& Grol, R. P. (2003). Information for dementia patients and their caregivers: What information does a memory clinic pass on, and to whom? Aging \& Mental Health, 7(1), 34 38.

Vernooij-Dassen, M., Derksen, E., Scheltens, P., \& Moniz-Cook, E. (2006). Receiving a diagnosis of dementia: The experience over time. Dementia, 5(3), 397-410.

Vickberg, S. M. J., Bovbjerg, D. H., DuHamel, K. N., Currie, V., \& Redd, W. H. (2000). Intrusive thoughts and psychological distress among breast cancer survivors: Global meaning as a possible protective factor. Behavioral Medicine, 25(4), 152160.

Vickberg, S. M. J., DuHamel, K. N., Y., Smith M., L., Manne S., G., Winkel, B., Papadopoulos E., \& Redd, W. H. (2001). Global meaning and psychological adjustment among survivors of bone marrow transplant. Psycho-Oncology, 10(1), 29-39.

Wagnild, G. M., \& Young, H. M. (1990). Resilience among older women. Journal of Nursing Scholarship, 22(4), 252-255. 
Walsh, J. (2006). Theories for direct social work practice. Belmont, CA: Brooks/Cole.

Walton, G. M., Cohen, G. L., Cwir, D., \& Spencer, S. J. (2012). Mere belonging; The power of social connections. Journal of Personality and Social Psychology, 102(3), 513-532.

Walton, J., Craig, C., Derwinski-Robinson, B., \& Weinert, C. (2004). I am not alone: Spirituality of chronically ill rural dwellers. Rehabilitation Nursing, 29(5), 164168.

Wang, C. (1999). Photovoice: A participatory action research strategy applied to women's health. Journal of Women's Health, 8(2), 185-192.

Wang, C., \& Burris, M. A. (1994). Empowerment through photo novella: Portraits of participation. Health Education Quarterly, 21(2), 171-186.

Wang, C., \& Burris, M. A. (1997). Photovoice: Concept, methodology, and use for participatory needs assessment. Health Education Behavior, 24(3), 369-387.

Waterman, A. (1992). Identity as an aspect of optimal psychological functioning. In G. Adams, T. Bulotta \& R. Montemayor (Eds.), Adolesence identity formation (pp. 50-72). Newbury Park, CA: Sage.

Weick, A., Rapp, C., Sullivan, W. P., \& Kisthardt, W. (1989). A strengths perspective for social work practice. Social Work, 34(4), 350-354.

Weiser, J. (2002). Phototherapy techniques: Exploring the secrets of personal snapshots and family albums. Child and Family, 5(3), 16-25.

Weitzenkamp, D., Gerhart, K., Charlifue, S., Whiteneck, G., Glass, C., \& Kennedy, P. (2000)). Ranking the criteria for assessing quality of life after disability: Evidence 
for priority shifting among long-term spinal cord injury survivors. British Journal of Health Psychology,, 5(1), 57-70.

Werezak, L., \& Stewart, N. (2002). Learning to live with early dementia. The Canadian Journal of Nursing Research, 34(1), 67-85.

Werner, E., \& Smith, R. (1982). Vulnerable but invincible: A longitudinal study of resilient children and youth. New York: McGraw Hill.

Werner, E., \& Smith, R. (1992). Overcoming the odds: High risk children from birth to adulthood. New York: Cornell University Press.

Werner, P. (2005). Social distance towards a person with Alzheimer's disease. International Journal of Geriatric Psychiatry, 20(2), 182-188.

Werner, P. (2006). Lay perceptions regarding the competence of persons with Alzheimer's disease. International Journal of Geriatric Psychiatry, 21(7), 674680.

Werner, P., \& Davidson, M. (2004). Emotional reactions of lay persons to someone with Alzheimer's disease. International Journal of Geriatric Psychiatry, 19(4), 391397.

White, M., \& Epston, D. (1990). Narrative means to therapeutic ends. New York, NY: Norton and Company.

White, R. (1974). Strategies of adaptation: An attempt at systematic description. In G. V. Coelho, D. A. Hamburg \& J. E. Adams (Eds.), Coping and adaptation (pp. 4768). New York: Basic Books. 
Whitehead, L. (2006). Toward a trajectory of identity reconstruction in chronic fatigue syndrome/myalgic encephalomyelitis: A longitudinal qualitative study. International Journal of Nursing Studies, 43(8), 1023-1031.

Whitehouse, P. J., Maurer, K., \& Ballenger, J. F. (Eds.). (2000). Concepts of Alzheimer's disease. Baltimore, MD: The john Hopkins University Press.

Williams, G. (1984). The genesis of chronic illness: Narrative reconstruction. Sociology of Health and Illness, 6(2), 175-200.

Williams, S. J. (2000). Chronic illness as biographical disruption or biographical disruption as chronic illness? Reflections on a core concept. Sociology of Health \& Illness, 22(1), 40-67.

Wills, T. A. (1981). Downward comparison principles in social psychology. Psychological Bulleting, 90(2), 245-271.

Wilson, P. M. (2008). The UK Expert Patient Programme: lessons learned and implications for cancer survivors' self-care support programmes. Journal of Cancer Survivorship, 2(1), 45-52.

Wood, J. V. (1989). Theory and research concerning social comparisons of personal attributes. Psychological Bulleting, 106(2), 231-248.

Woods, R. T. (1989). Alzheimer's disease: Coping with a living death. London: Souvenir Press.

Woods, R. T. (2001). Discovering the person with Alzheimer's disease: Cognitive, emotional and behavioural aspects. Aging \& Mental Health, 5(1), 7-16. 
Wright, S. J., \& Kirby, A. (1999). Deconstructing conceptualizations of "adjustment" to chronic illness: A proposed integrative framework. Journal of Health Psychology,, 4(2), 259-272.

Wuest, J., Ericson, P. K., \& Stern, P. N. (1994). Becoming strangers: The changing family caregiving relationship in Alzheimer's disease. Journal of Advanced Nursing, 20(3), 437-443.

Wuggenig, U. (1990). Die Photobefragung als projektives Verfahren. Angewandte Sozialforschung, 16(1/2), 109-129.

Young, R. (2002). Medical experiences and concerns of people with ALzheimer's disease. In P. Harris (Ed.), The Person with Alzheimer's Disease: Pathways to Understanding the Experience (pp. 29-46). Baltimore: The John Hopkins University Press.

Zastrow, C., \& Kirst-Ashman, K. K. (2004). Understanding human behavior and the soical environment (6th ed.). Belmont, CA: Brooks/Cole. 


\title{
Appendix A: IRB Approval Letter
}

\author{
胥 Portland State \\ Human Subjects Research Review Committee \\ Post Office Box 751 \\ $503-725-4288$ tel \\ 503-725-3416 fax \\ hsrrc@lists.pdx.edu
}

June 2, 2010

To: Jutta Ataie

From: Nancy Koroloff, HSRRC Chair

Re: Approval of your application titled, "The Role of Perceived Strength and Resources in Coping with Early-stage Dementia" (HSRRC Proposal \# 101324).

Dear Jutta,

In accordance with your request, the Human Subjects Research Review Committee has reviewed your proposal referenced above for compliance with DHHS policies and regulations covering the protection of human subjects. The committee is satisfied that your provisions for protecting the rights and welfare of all subjects participating in the research are adequate, and your project is approved. Please note the following requirements:

Changes to Protocol: Any changes in the proposed study, whether to procedures, survey instruments, consent forms or cover letters, must be outlined and submitted to the Chair of the HSRRC immediately. The proposed changes cannot be implemented before they have been reviewed and approved by the Committee.

Continuing Review: This approval will expire on June 2, 2011. It is the investigator's responsibility to ensure that a Continuing Review Report (available in ORSP) of the status of the project is submitted to the HSRRC two months before the expiration date, and that approval of the study is kept current.

Adverse Reactions: If any adverse reactions occur as a result of this study, you are required to notify the Chair of the HSRRC immediately. If the problem is serious, approval may be withdrawn pending an investigation by the Committee.

Completion of Study: Please notify the Chair of the Human Subjects Research Review Committee (campus mail code ORSP) as soon as your research has been completed. Study records, including protocols and signed consent forms for each participant, must be kept by the investigator in a secure location for three years following completion of the study.

If you have questions or concerns, please contact the HSRRC in the Office of Research and Sponsored Projects (ORSP), (503) 725-4288, 6th Floor, Unitus Building, 4th \& Lincoln.

Cc: Vicki Cotrell 


\section{Appendix B: Recruitment Flyer}
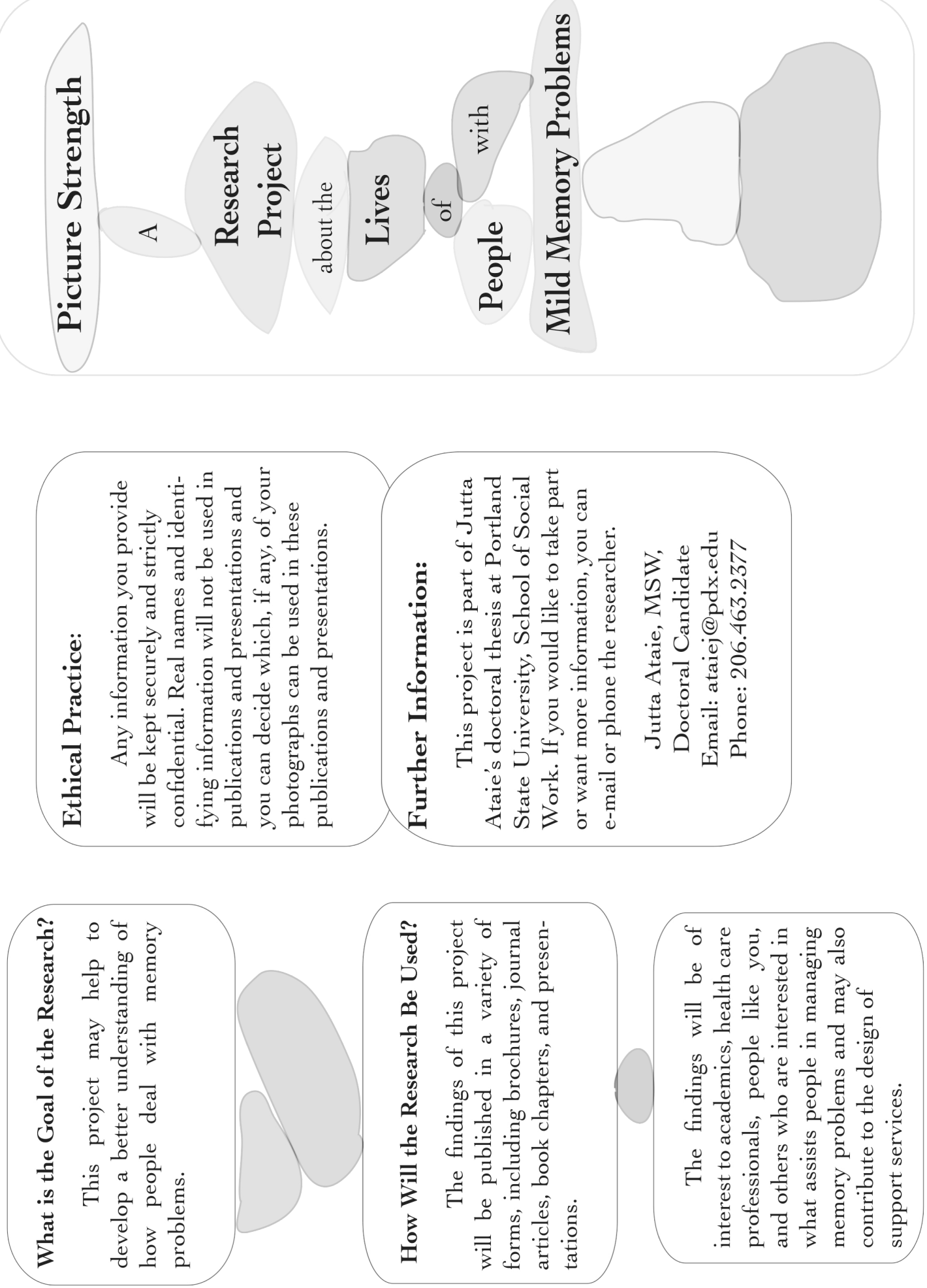

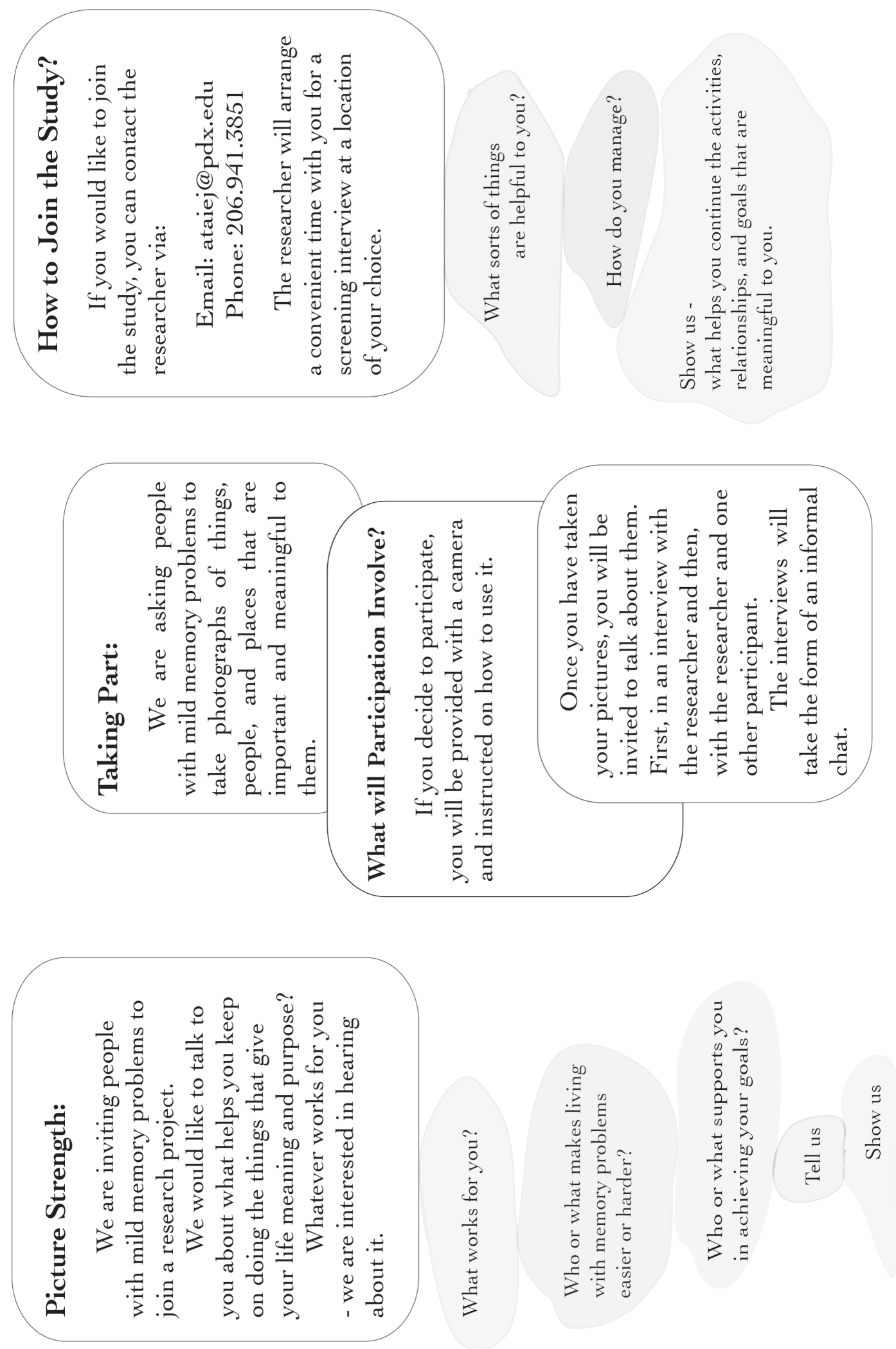


\title{
Appendix C: Recruitment Letter NW Research Group on Aging
}

\author{
School of Nursing \\ UNIVERSITY OF WASHINGTON \\ Northwest Research Group on Aging \\ Psychosocial and Community Health \\ University of Washington \\ 9709 3rd Ave. NE, Suite 507 \\ Seattle, WA 98115
}

Dear (care partner or participant's name),

Thank you again for participating in the Early Stage Seminar Research Study conducted by the UW School of Nursing and the Alzheimer's Association Western and Central Washington chapter. Your contributions are helping us develop better and more effective early stage services both here in Western Washington and around the country.

When you volunteered for the Early Stage Seminar study, you indicated that you were interested in being contacted about future research. I want to let you know about a study being conducted here in Seattle this summer by Jutta Ataie, MSW, for her doctoral studies at Portland State University.

The goal of the "Picture Strength" study is to develop a better understanding about how people with memory problems manage to maintain a fulfilling and active life and invites them to share their perspective. Study participants will be provided with and trained in the use of a disposable camera and invited to take photographs of things, people, and places that are important and meaningful to them. The photographs will provide the means by which participants can take the lead in a dialogue about their experiences, first in an individual interview with Jutta and then in a two-person interview with Jutta and one other study participant. I have enclosed a brochure with more information about the research study.

If you are interested in learning more about the study, I encourage you to contact Jutta directly, by phone 206.941.3851 or email juttaataie@pdx.edu

Sincerely,

Rebecca Logsdon, PhD

Research Professor

Psychosocial \& Community Health

University of Washington School of Nursing | Box 357260, Seattle, Washington 98195-7260 | 206-543-8736, Fax 206-543-3624 


\section{Appendix D: Recruitment Letter Alzheimer's Association}

June 28,2010

Dear:

The Western \& Central Washington Chapter of the Alzheimer's Association is pleased to support one of our long-time volunteers, Jutta Ataie, MSW, a doctoral candidate atPortland State University. She is currently recruiting participants for her doctoral research study entitled, "Picture Strengths: A Research Projectabout the Lives of People with Mild Memory Problems".

The goal of the "Picture Strength" study is to develop a better understanding about how people with memory problems manage to maintain a fulfilling and activelife and invites them to share their perspective. Study participants will be provided with a disposable camera and invited to take photographs of things, people, and places thatare importantand meaningful to them. The photographs will provide the means by which participants can take the lead in a dialogue about their experiences, firstin an individual interview with Jutta and then in a two-person interview with Juttaand one other study participant.

Please see the enclosed brochure formore information about the research project Ifyou have questions please contactJuttaAtaieat(206)941-3851 or by email atataiej@pdx.edu.

Sincerely,

Joanne Maher,MSW

Director of Social Services

Alzheimer's Association

Western \&Central Washington StateChapter

100W.HarrisonStreet

NorthTower-Suite200

Seattle,WA98119 


\section{Appendix E: Recruitment Interest Cards}

\begin{tabular}{l} 
I am interested in participating in the Picture Strength Study. \\
Interest Cards \\
Please contact me: \\
Name: \\
Home Phone Number: \\
Cell Phone Number: \\
\hline
\end{tabular}

\section{Please contact my care partner:}

Name:

Home Phone Number:

Cell Phone Number: 


\section{Appendix F: Telephone Recruitment and Screening Script}

*Making a call in response to a message left by a potential study participant

If not available:

Investigator: Hello, may I please speak to ?

Response: No, he/she is not in right now. Can I take a message?

Investigator: Sure, could you tell him/her Jutta Ataie returned her phone call. She can be reached at 206.463.2377. Thank you!

If available:

Investigator: Hello, may I please speak to $?$

Response: This is he/she.

Investigator: This is Jutta Ataie and I am calling you back regarding the 'Picture Strength' research study. You left a message for me yesterday about being interested in hearing more about this study. Is this a good time to talk?

If no, not a good time to talk:

Response: No, actually, it isn't a good time to talk.

Investigator: When would be a better time to call you back?

Response: (Day) and (Time) will work better.

Investigator: Okay, I will call you back on (Day) at (Time).

If no, not interested at all anymore:

Response: I changed my mind; I don't want to be in the study.

Investigator: Oh, okay. Thank you for your time. Good-bye.

If, yes, a good time to speak:

Response: Yes, actually this is a good time to talk.

Investigator: Wonderful. Let me tell you a little bit about myself and the research study. I am a graduate student at Portland State University, School of Social Work and I am looking for people with mild memory problems to participate in my doctoral research study. The purpose of this study is to develop a better understanding of who or what assists people in living with mild memory problems and what makes living with memory problems easier or harder. We are inviting people experiencing mild memory 
problems to takes photographs of things, people, and places that are important and meaningful to them in their everyday lives. Each participant will be provided with a disposable camera and instructed on how to use it. Once the participant has taken his or her photographs, he or she will be invited to talk about his or her photographs. First, in a one-on-one interview with the researcher and then, with the researcher and one other participant. Does this sound like something you might be interested in?

If no, not interested at all anymore:

Response: Actually, this study isn't really something I am interested in.

Investigator: Oh, okay. Thank you for your time. Good-bye.

If, yes, still interested:

Response: Yes, this sounds interesting. Go on...

Investigator: Great. Let me ask you a question to make sure that you fit the criteria for this study. Would you be willing to answer a question about your living arrangement? Please know that you are free to say no and not answer my question.

If he/she does not want to answer the question:

Investigator: It's okay; I understand your decision not to answer my question. Would you prefer I continue to describe the research project or would you prefer not to answer my question at all?

If he/she would rather not answer my question at all:

I will thank him/her for his/her time.

If he/she wants me to continue describing the study:

I will provide more information, and then return to the eligibility question.

If he/she is willing to answer the question:

Response: $\quad$ Sure, I don’t mind answering your question.

Investigator: Thank you. Do you live in an Assisted Living Facility or a Nursing Home? Do you live in a house, an apartment, or a mobile home?

Do you live alone or with a partner?

If he/she lives in an Assisted Living Facility or a Nursing Home.

Investigator: Thank you. Unfortunately, this study is not a good fit since I am looking for people with mild memory problems who meet a specific set of eligibility criteria. Thank you for taking the time to talk to me today. I appreciate your interest in this study and your willingness to help me. 
If he/she meets the eligibility criteria:

Investigator: Thank you. Based on your answer, we can go to the next step. I would like to talk to you a little bit more about this study and what your participation would include. If you have any questions, please feel free to ask them at any time.

If you decide that you are interested in participating in the study, we will schedule a screening interview with you at a location of your choice. During the screening interview, I will ask you some more questions about yourself and your memory problems and train you in how to use a disposable camera. If you meet the specific set of eligibility criteria for this study and if you decide to take part in this project, I will review an informed consent form with you. If you agree to become involved in the project, I will ask you to sign the consent form.

After you sign the consent form, I will invite you to develop some ideas about what you might want to photograph. Next, I will hand you a 'phototaking toolkit' and train you in the use of each item in the toolkit. Then, we will work together to develop a picture-taking plan for you and develop ideas on how you can record your photographs in a picture log. Next, we will discuss ethical ways of taking photographs and develop ideas about who or what might remind you on taking the photographs. You will have 7 days to take photographs. During these 7 days, I will check in with you daily to troubleshoot any difficulties that may arise. After 7 days, I will meet with you at a location of your choice, collect the camera and the picture log, interview you briefly (approximately 15 minutes) about your picture taking experience, and we will schedule an appointment for an individual interview.

Investigator: Does this still sound like something you might be interested in? Do you have any questions so far?

If no, not interested at all anymore:

Response: Actually, this isn't really something I am interested in.

Investigator: Oh, okay. Thank you for your time. Good-bye.

If, yes, still interested:

Response: Yes, this sounds interesting. Go on...

Investigator: Great. Let me fill in some of the details of the study.

I will have two sets of photographs developed (one for you to keep and one for me). I will not preview your photographs prior to our meeting. When we meet, I will invite you to open the envelope, review your photographs, comment on their meaning, and talk about the experience of 
taking the photographs. The interview will take the form of an informal chat about your photographs and their significance and will last approximately 45 minutes. Next, you will decide which two photographs you would like to discuss in a meeting with me and one other participant and choose a title for these photographs. I will note which two photographs you selected and store them in a separate envelope. Then we will schedule an appointment for the meeting with me and another study participant.

Investigator: Does this still sound like a study you might want to participate in? Do you have any questions so far?

If no, not interested at all anymore:

Response: Actually, this isn't really something I am interested in.

Investigator: Oh, okay. Thank you for your time. Good-bye.

If, yes, still interested:

Response: Yes, this sounds interesting. Go on...

Investigator: At the beginning of the meeting with me and the other study participant I will hand out the envelopes containing the two photographs each of you had chosen previously. Then, I will ask you and the other participant to open your envelopes, share your photographs with each other, give a short explanation why you took the photographs, and talk about the photographs and the experience of taking them. Again, the interview will take the form of an informal chat about your photographs and their significance and again will last approximately 45 minutes.

At the end of this meeting, I will ask you which of your photographs, if any, can be used in publications and presentations reporting on the findings of the study. If you agree, you will sign a photo release form consenting that your photographs can be used in publications and presentations. If there are people in your photographs, I will collect their contact information from you so that I can also obtain their consent.

Investigator: Do you have any questions?

If, yes,:

I will answer his or her questions

If no:

Response: No, go on...

Investigator: I just want to make sure that you understand that even if you decide to participate, you can withdraw from the study at any time without giving an 
explanation. Also, it is possible that taking photographs of things, people, and places that are important and meaningful to you and that symbolize your life experiences will be emotionally challenging. At times, you may experience feelings of joy or happiness. At other times, you may experience sadness or frustration. However, your comfort is most important to us, which means that we do not want you to share anything that makes you uncomfortable or you do not wish to discuss. Also, you are free to stop the dialogue at any time. In addition, if you experience any stress or discomfort during the participation in this study, I will provide you with referrals to agencies that support people with mild memory problems.

What you have to say during the interviews is important to us; with your permission, I will audio-record the interviews and have them professionally transcribed. However, if you ever want to have the audio recorder turned off during the interviews, please tell me and I will turn off the recorder.

All information that you provide in this study will be kept securely and strictly confidential. Your name will not be used in publications and presentations and you can decide which, if any, of your photographs can be used in these publications and presentations. If you would like, you can choose a fictitious name and use this name during the meeting with me and the other study participant. Only I will know your real name and I will keep this information confidential. While we ask all study participants to keep what is said during the meeting confidential, I cannot guarantee that the participant with whom you will meet will not share the content of the meeting with others. Again, I want to emphasize that your participation in this study is voluntary and that you may stop participating at any time. I also want to add that you are free to contact me throughout this study at any time. Do you have any questions?

If, yes,:

I will answer his or her questions

If no:

Response: No, go on...

Investigator: Does this study sound like something you would like to participate in?

If, no, not interested after hearing about the study:

Investigator: Thank you for your time. I appreciate your taking the time to listen. Goodbye.

If, yes, interested after hearing about the study:

Investigator: The next step is for us to schedule a screening interview so that we can determine whether the study is a good fit for you. If we determine during 
the screening interview that the study is a good fit and you decide that would like to participate in this study, we can go over the consent form in person so that you can sign it.

Before scheduling an appointment, let me ask you one more thing. Some people feel more comfortable if their partner is present during a decisionmaking activity such as whether or not to participate in a research project. Would you like to invite your partner to be present during the screening interview and/or consent procedure?

If, no, does not want partner to be present:

Response: $\quad$ No, I feel comfortable meeting with you by myself.

Investigator: Great. Would (date) at (time) work for you? Where would you like to meet?

Response: $\quad$ Sure, I can meet you at (time) on (date) where (location).

Investigator: Great! Do you have any other questions for me? Do you have my phone number so that you can call me if you have any questions? What is the best way to contact you?

Response: No, I don't have any questions right now and I have your phone number so I call you if I have any questions. Also, I can be reached at one of these two numbers...

Investigator: Great! I will call the day before our appointment to confirm (date/time). Thanks again for your time! I am looking forward to meeting you.

If, yes, wants partner to be present:

Response: $\quad$ Yes, I would like for my partner to be present during the screening interview.

Investigator: Great, would you like to schedule an appointment now or would you like to talk to your partner first?

Response: I would like to talk to my partner first.

Investigator: Okay, when do you think you will have a chance to do that?

Response: On (day of the week).

Investigator: Great, I will call you on (day of the week) to schedule the appointment. Investigator calls on that day. 
Investigator: $\mathrm{Hi}$, this is Jutta Ataie, the principal investigator of the Picture Strengths project. I am calling to set up an appointment with you and your partner for a screening interview. Is this a convenient time to talk?

Response: $\mathrm{Hi}$, sure. It is.

Investigator: Did you decide on a date and time for the screening interview?

Response: $\quad$ Sure, we can meet you at (time) on (date) where (location).

Investigator: Great! Do you have any other questions for me? Do you have my phone number so that you can call me if you have any questions? What is the best way to contact you?

Response: No, I don't have any questions right now and I have your phone number so I call you if I have any questions. Also, I can be reached at one of these two numbers...

Investigator: Great! I will call the day before our appointment to confirm (date/time). Thanks again for your time! I am looking forward to meeting you. 


\section{Appendix G: Demographic Data Sheet}

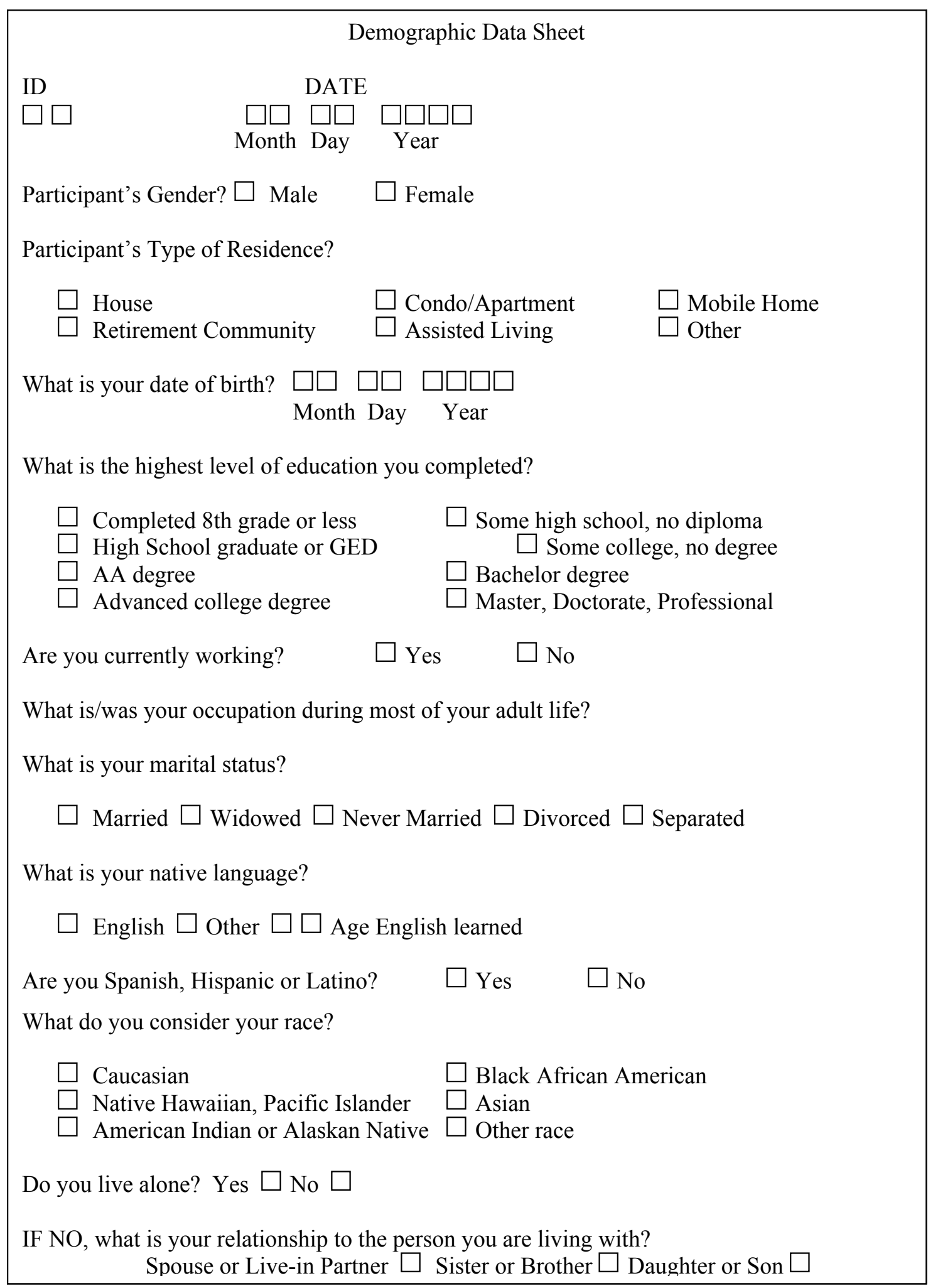


ID

$\square \square$

Do you live alone? $\square$ Yes $\quad \square$ No

IF NO, what is your relationship to the person you are living with?
Spouse or Live-in Partner
Daughter or Son
$\square$ Sister or Brother
Niece or Nephew
Daughter-in-law or Son-in-law
Parent
$\square$ Granddaughter or Grandson
$\square$ Friend

Do you have problems with your memory? $\quad \square$ Yes $\quad \square$ No

IF YES, how long have you had problems with your memory? (best estimate)
Weeks
Months
Years

IF YES, have you ever seen a doctor for your memory problems?

Weeks $\square$ Months $\square$ Years

IF YES, what did the doctor tell you about your memory problems?

IF YES, did you ever have a formal diagnosis? $\quad \square$ Yes $\quad \square$ No

IF YES, what diagnosis were you given? 


\title{
Appendix H: Informed Consent Form
}

\author{
Informed Consent Form \\ (HSRRC Proposal \#101324) \\ Picture Strength
}

A Research Project about the Lives of People with Mild Memory Problems

\section{Principal Investigator:}

Jutta Ataie, MSW, Doctoral Candidate; Portland State University, School of Social

Work; P.O. Box 751; Portland, Oregon, 97207; phone 206.941.3851, email ataiej@pdx.edu

\section{Purpose of the Study:}

The purpose of this project is to develop a better understanding of who or what assists people in living with mild memory problems and what makes living with memory problems easier or harder. The findings of this project will be of interest to academics, healthcare professionals, people like you, and others who are interested in how people deal with memory problems and may also contribute to the design of support services.

\section{Study Procedures:}

If you decide to take part in this project, you will be asked to take photographs of things, people, and places that are important to you in your life. You will be given a 'photo-taking toolkit' by the researcher and trained in the use of each item in the toolkit. Next, you will work with the researcher to develop a picture-taking plan and brainstorm about some of the ways you might want to take photographs of the things that are meaningful and important to you. You will have 7 days to take photographs and the researcher will check in with you daily. After 7 days, the researcher will meet with you, collect the camera and interview you briefly (approximately 15 minutes) about your picture taking experience, and schedule an appointment for an individual interview.

During the individual interview, you will be invited to review your photographs and comment on their meaning (approximately 45 minutes). Next, you will be invited to decide which two photographs you would like to discuss in another meeting with the researcher and one other participant and choose a title for these photographs. The researcher will contact you and schedule an appointment for the meeting with her and another study participant. During the meeting, you and the other participants will be invited to share your photographs with each other and talk about the photographs (approximately 45 minutes).

The interviews will take the form of an informal chat and the combined time for all interviews is about two and a half hours. With your permission, the researcher will audio-record the interviews and have them transcribed. However, if you ever want to have the audio recorder turned off, please tell the researcher and it will be turned off. 


\section{Risk, Stress, and Discomfort}

Taking photographs that symbolize your life experiences may be emotionally challenging. However, your comfort is most important to us, which means that we do not want you to share anything that makes you feel uncomfortable or you do not wish to discuss. Also, you are free to stop the dialogue at any time.

\section{Benefits}

(1) Your participation will contribute to a better understanding of who or what assists people in living with mild memory problems. (2) You will have the opportunity to voice your experiences through your photographs and engage in a dialogue with another participant who shares similar experiences. (3) You will receive an enlarged version of your favorite photograph after completing the study.

\section{Costs}

There are no costs associated with participating in this study.

\section{Confidentiality}

All information that you provide in this study will be kept securely and strictly confidential. Your name will not be used in publications and presentations and you can decide which, if any, of your photographs can be used in these publications and presentations. While we ask all study participants to keep what is said during the meeting with the researcher and one other participant confidential, we cannot guarantee that the participant with whom you will meet will not share the content of the meeting with others.

All identifying information will be removed from the audio-recording transcripts. The information you provide, including demographic information, photographs, audio-recordings, and transcripts will be kept in a locked storage cabinet in the School of Social Works and on a password protected computer. The exceptions of confidentiality are if you share information with me about abuse and neglect and/or voice suicidal ideation, which by law, have to be reported to the appropriate agency.

The findings of this project will be published in a variety of forms, in print and electronically, including brochures, journal articles, book chapters, and presentations. Some of your comments may be included in these reports written to summarize what has been learned from this study. However, these summaries will not include any identifying information about you.

\section{Voluntary Participation and/or Withdrawal from the Study}

Your decision to participate in this study is strictly voluntary. You may refuse to participate or, if you decide to take part, you are free to withdraw from the research at any time without giving a reason. 


\section{Statement of Consent}

The study described above and the risks and benefits associated with participation in the study have been explained to me and my questions have been answered in a language I understand.

I understand that the information regarding my personal identity will be kept confidential. I agree not to disclose information that the other participant shares during our meeting with the researcher.

I understand that I will be given a copy of this consent form after signing it. I freely agree to release my photographs for use in this research study. I understand that I can choose which of my photographs, if any, I want to share with another participant. I also understand that none of my photographs will be used in publications or presentations without me signing a separate photo-release form, and that I may withdraw my permission at any time.

I have read this consent form and I authorize the Portland State University, Institutional Review Board to view or copy the research records.

If I have questions later on about the research I can ask the principal investigator, Jutta Ataie, 206.941.3851 or I can contact the Human Subjects Research Review Committee, Office of Research and Sponsored Projects, 600 Unitus Building, Portland State University, 1.503.725.4288 or toll free 1.877.480.4400.

Signature of Participant

Printed Name of Participant
Date

\author{
Researcher Signature
}




\section{Appendix I: Photo Release Form \\ Photo Release Form \\ Picture Strength \\ (HSRRC Proposal \#101324)}

I, , give my consent to Jutta Ataie (Doctoral

Candidate, Portland State University, School of Social Work) to use

$\square \quad$ All my photographs

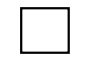

Some of my photograph

Number(s)

in publications, both in printed and electronic forms, including brochures, journal articles, book chapters, and presentations.

Signature of Participant

Printed Name of Participant
Date

Researcher Signature 


\section{Appendix J: Screening Session Moderator Guide}

\section{Screening Session Moderator Guide}

Interviewer provides information about the nature of the study and introduces the photovoice concept and methodology.

Interviewer trains potential participant in how to use the camera by demonstrating the process of taking a photograph with the practice camera and speaking out loud each step of the phototaking procedure:

(1) I look at the counter (I have e.g., 27 pictures available). (2) I look through the viewer. (3) I decide whether I would like to take a vertical or horizontal picture. (4) I hold the camera still. (5) I press the button. (6) I listen to the sound whether it clicks. (7) I turn the wheel to forward the film to next frame. (8) I look at the counter (now I have e.g., 26 pictures available).

Interviewer hands practice camera to potential participant. While interviewer repeats the instructions, potential participant practices taking a photograph.

Interviewer obtains demographic information and administers the MMSE.

Interviewer asks potential participant to take a second practice picture and recite out loud each step involved

Accomplished

YES No

$\square \quad \square \quad$ I look at the counter (I have e.g., 27 pictures available)

I look through the viewer

I decide if I would like to take a vertical or horizontal picture

I hold the camera still

I press the button

I listen to the sound whether it clicks

I turn the wheel to forward the film to next frame

I look at the counter (now I have e.g., 26 pictures available)

Participant successfully accomplished to take a practice picture

Yes $\square \quad$ No

IF NO, interviewer informs potential participant that the study is not a good fit for him or her and potential participant will be excluded from participation in the study. The collected information will be destroyed immediately after the session.

IF YES, interview continues with training session. 


\section{Appendix K: Training Session Moderator Guide}

\section{Training Session Moderator Guide}

Interviewer first administers the QOL-AD and the GDS- short form and then familiarizes participant with the photo-taking theme.

"Picture what is important to you. What helps you keep on doing the things that give your life meaning and purpose? I want you to take photographs of the things, people, and places that help you continue the activities, relationships, and goals that are meaningful to you. These can be photographs of anything about you and your life -- anything you want."

Interviewer hands a 'photo-taking toolkit' to participants and invites participants to open their toolkit. Interviewer opens her toolkit simultaneously with the participants and models the handling and use of each item in her toolkit while helping the participants to understand how to use each item in their toolkit.

Interviewer explains that although a reliable disposable camera had been selected, there was always the possibility that the camera would not work properly and that all or some photographs might not come out well. Interviewer explains that in this case, participants could choose to repeat the process with a different camera or use some of their own photographs of things, people, and places that are important and meaningful to them. Interviewer shows a set of her own pictures on a very general topic, which includes some 'bad' pictures to show the participants that bad pictures are 'normal'.

Interviewer reviews use of camera, explains how she can be of assistance during the study, and works with participants to develop a plan for picture taking. Participants will be invited to brainstorm about the photo-taking theme and come up with ideas about where to keep their photo-taking toolkit, potential memory aids, and who could assist them during the study. Interviewer records these ideas on participants' picture-taking plan. Interviewer teaches participants how to record their photographs in picture log.

Interviewer initiates a discussion about the following topics: (1) What are respectful ways of taking photographs; (2) What strategies can you employ to remember that you wanted to take photographs; (3) How can the researcher assist you in remembering to take photographs; (4) Who else can assist you in remembering to take photographs; (5) What can you do when you forget how to use the camera; (6) How many pictures can you take (27 exposures)?

Interviewer reviews the following information and encourages participants to invite their partner and/or care partner to participate in the following brief review so they can be of assistance during the photo-taking phase if need be: (1) Photo-taking theme; (2) Items in the 'photo-taking toolkit'; (3) Contact information for the researcher.

Did the participant invite his/her care partner to participate in the brief review at the end of the session?

$$
\text { Yes } \square \text { No }
$$

IF YES, name of the care partner: 


\section{Appendix L: Brief Individual Interview Moderator Guide}

\section{Brief Individual Interview Moderator Guide}

Interviewer collects camera and picture log from participant and interviews participant briefly about his/her experience of taking photographs:

(a) Tell me about your experience of taking photographs for the study.

(b) What did you like about it?

(c) Was there anything about the photo-taking process that you particularly enjoyed liked?

(d) What didn't you like?

(e) Was there anything about the photo-taking process that was difficult for you?

(f) Did you find the daily instruction sheet helpful?

(g) Did you find the picture log helpful?

(h) Is there anything that didn't work for you?

(i) Is there anything we should be doing differently?

(j) Is there anything that worked really well for you and that you would like me to tell future participants? 


\section{Appendix M: In-Depth Individual Photo Interview Moderator Guide}

\section{In-Depth Individual Photo Interview Moderator Guide}

Interviewer invites participant to open the envelope, review his/her photographs, comment on their meaning, and discuss the experience of taking photographs.

a. Tell me about your pictures.

b. Tell me about your experiences in taking theses pictures.

c. Probes:
a. What are some of the things that give your life meaning and purpose?
b. Who or what is important and meaningful to you?
c. Who or what helps you keep on doing the things that give your life meaning and purpose?
d. Who or what supports you in achieving your goals?
e. What sorts of things are helpful to you?
f. What makes living with memory problems easier?
g. What makes it harder?
h. How do you manage?
i. What works for you?
j. Who or what help you do well?

Interviewer reviews the photo-taking theme:

"Picture what is important to you. What helps you keep on doing the things that give your life meaning and purpose? I want you to take photographs of the things, people, and places that help you continue the activities, relationships, and goals that are meaningful to you. These can be photographs of anything about you and your life -- anything you want."

d. Choose the two photographs that most accurately reflect the photo-taking theme.

e. Since photographs do not always turn out the way we intend to, what did you intend these photographs to depict?

f. Choose the two photographs that you would like to discuss in a two-person interview with another study participant.

g. Since photographs do not always turn out the way we intend to, tell me what you intend these photographs to depict?

h. What title would you like to give these two photographs?

i. The researcher notes which two photographs the participant chooses and selects them from her set and puts them in a separate envelope.

j. Tell me what you would like me take away from our conversation today, what you would really like me to remember. 


\section{Appendix N: Dyadic Interview Moderator Guide}

\section{Dyadic Interview Moderator Guide}

Interviewer invites participant to open the envelope, review his/her photographs, comment on their meaning, and discuss the experience of taking photographs (show and tell).

(a) Tell each other about your pictures.

(b) While one of you is talking about his/her photographs, I want the other person to think about how his/her photographs are similar or different from those of the other participants. What are some of the similarities and differences?

(c) Tell each other about your experiences during the photo-taking process.

(d) While one of you is talking about his/her photographs, I want the other person to think about how his/her photographs are similar or different from those of the other participants. What are some of the similarities and differences?

Interviewer reviews the photo-taking theme:

"Picture what is important to you. What helps you keep on doing the things that give your life meaning and purpose? I want you to take photographs of the things, people, and places that help you continue the activities, relationships, and goals that are meaningful to you. These can be photographs of anything about you and your life -- anything you want."

(e) Since photographs do not always turn out the way we intend to, tell each other what you intend these photographs to depict?

(f) While one of you is talking about his/her photographs, I want the other person to think about how his/her photographs are similar or different from those of the other participants. What are some of the similarities and differences?

(g) Probes: (a) What are some of the things that give your life meaning and purpose? (b) Who or what is important and meaningful to you? (c) Who or what helps you keep on doing the things that give your life meaning and purpose? (d) Who or what supports you in achieving your goals? (e) What sorts of things are helpful to you? (f) What makes living with memory problems easier? (g) What makes it harder? (h) How do you manage? (i) What works for you? (j) Who or what help you do well?

Wrap-up:

(h) Have you noticed any changes as a result of participating in this project? If so, how would you describe these changes?

(i) I want you to tell me what you would like me take away from our conversation today, what you would really like me to remember. 


\section{Appendix O: Photo-Taking Toolkit}

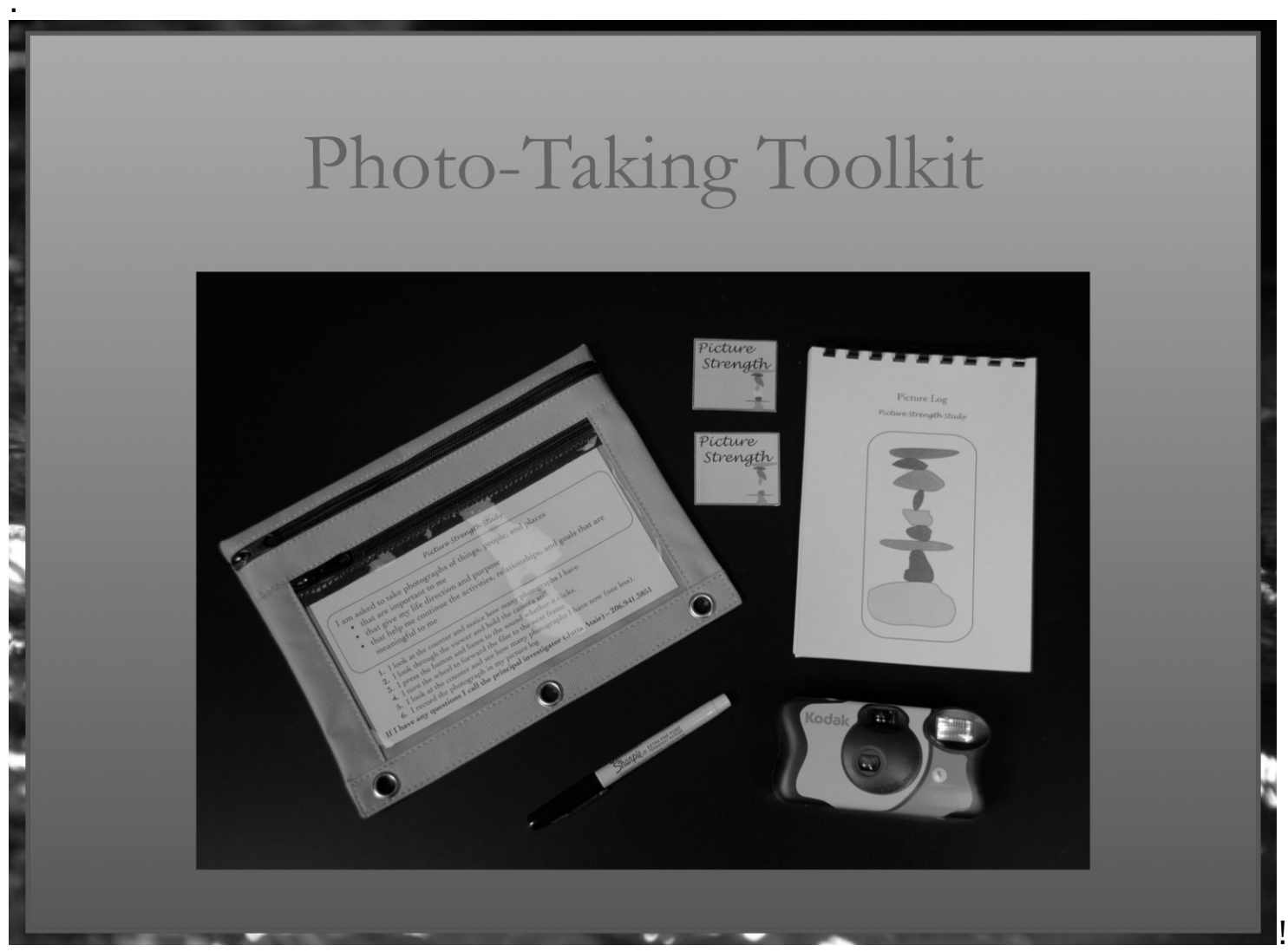




\section{Appendix P: Picture-Taking Plan}

Picture-Taking Plan

Picture Strength Study

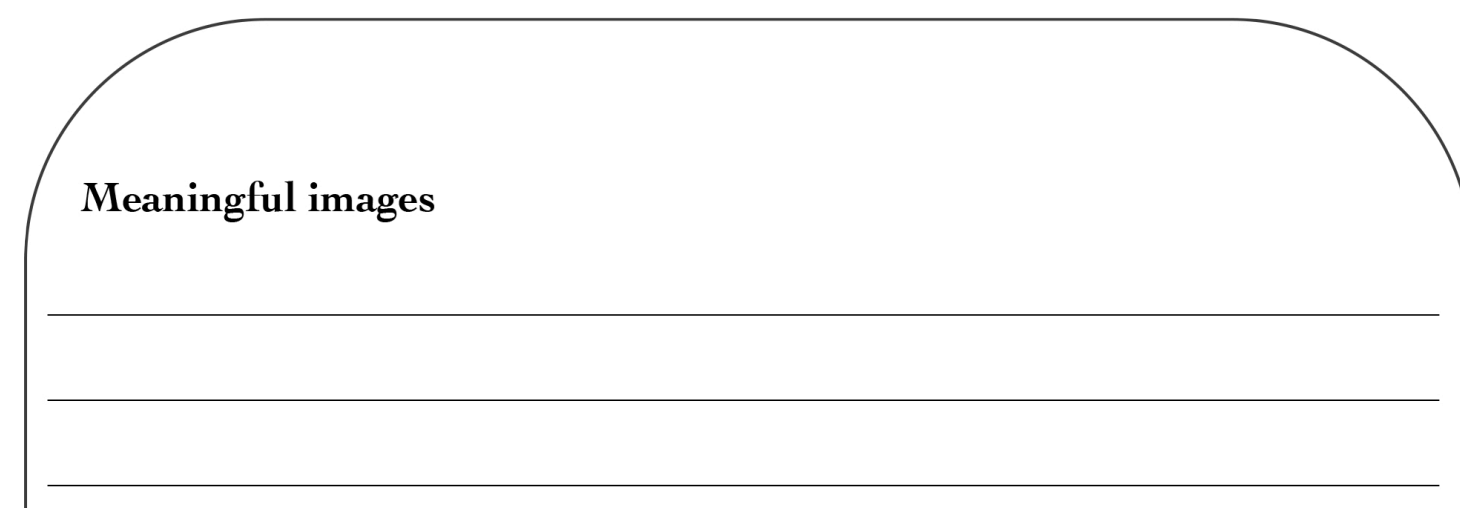

Where will I keep the photo-taking toolkit?

What reminders will I create for myself?

Who will help me if I run into difficulties when taking photographs?

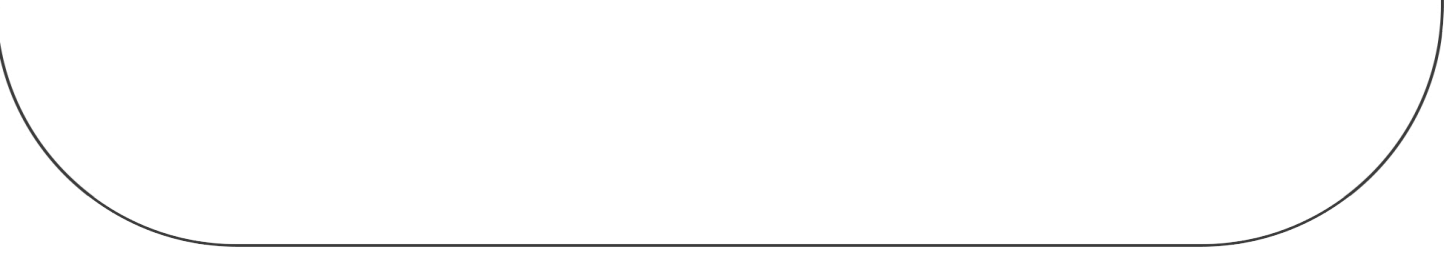




\section{Appendix Q: Photo-Diary}
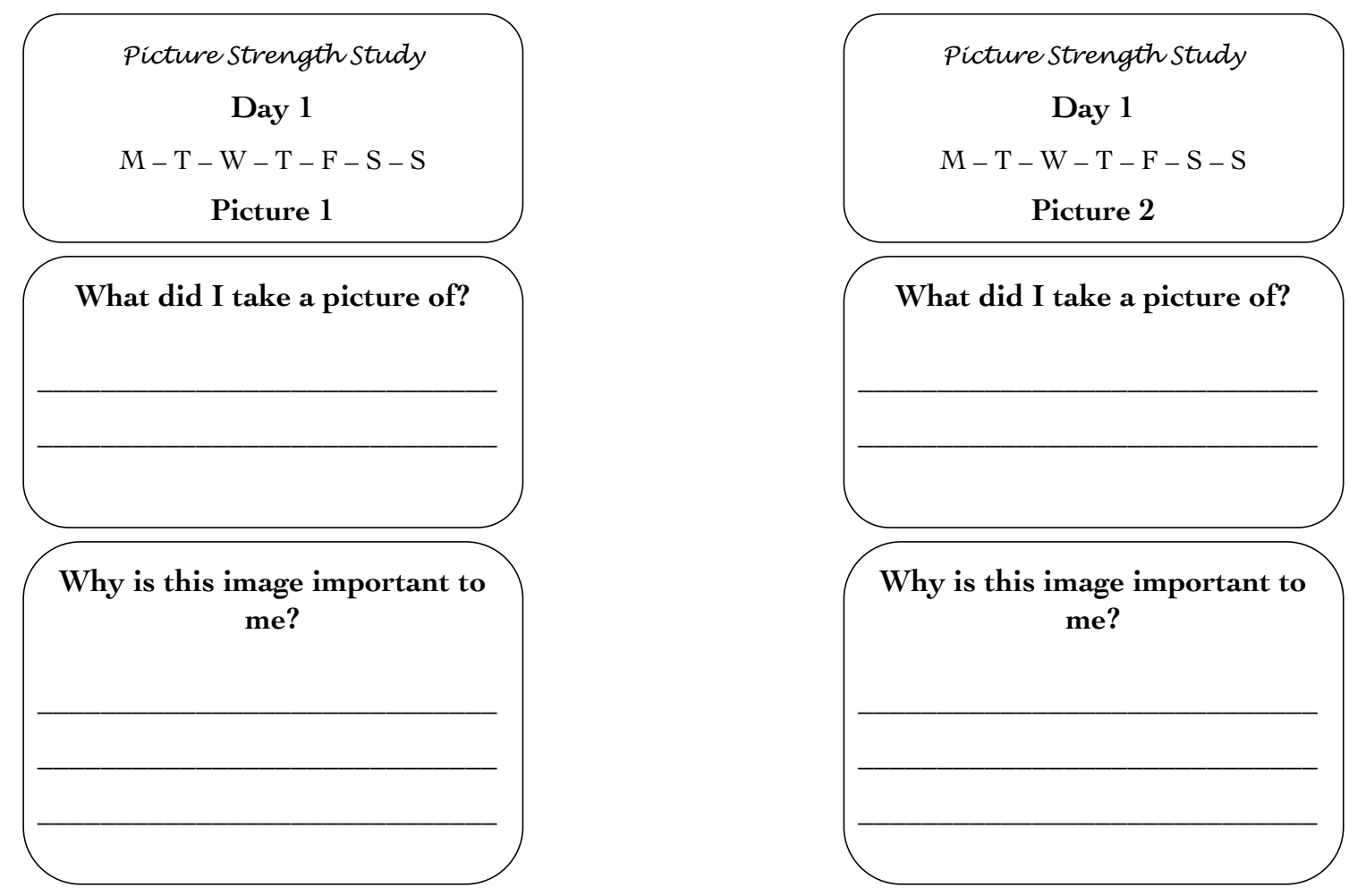

What did I take a picture of?
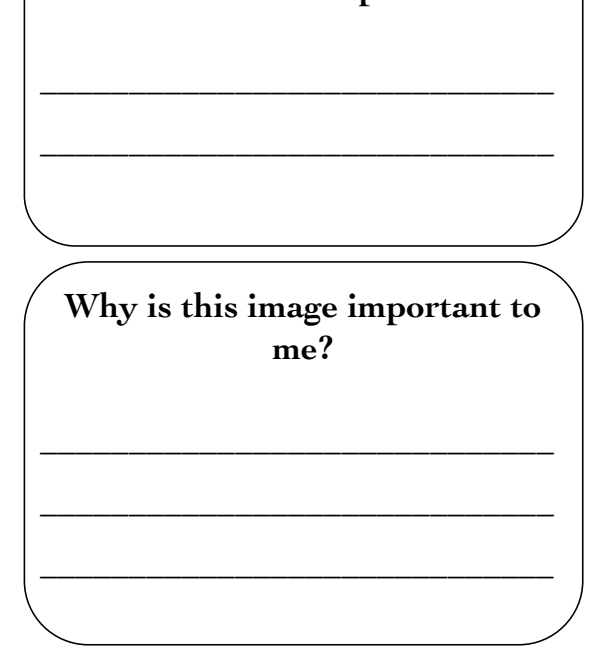


\section{Appendix R: Daily Instruction Sheet}

Daily Instruction Sheet

Picture Strength Study

I am asked to take photographs of things, people, and places

- that are important to me

- that give my life direction and purpose

- that help me continue the activities, relationships, and goals that are meaningful to me

When I want to take a photograph,

1. I look at the counter and notice how many photographs I have.

2. I look through the viewer and hold the camera still.

3. I press the button and listen to the sound whether it clicks.

4. I turn the wheel to forward the film to the next frame.

5. I look at the counter and see how many photographs I have now

6. I record the photograph in my picture log.

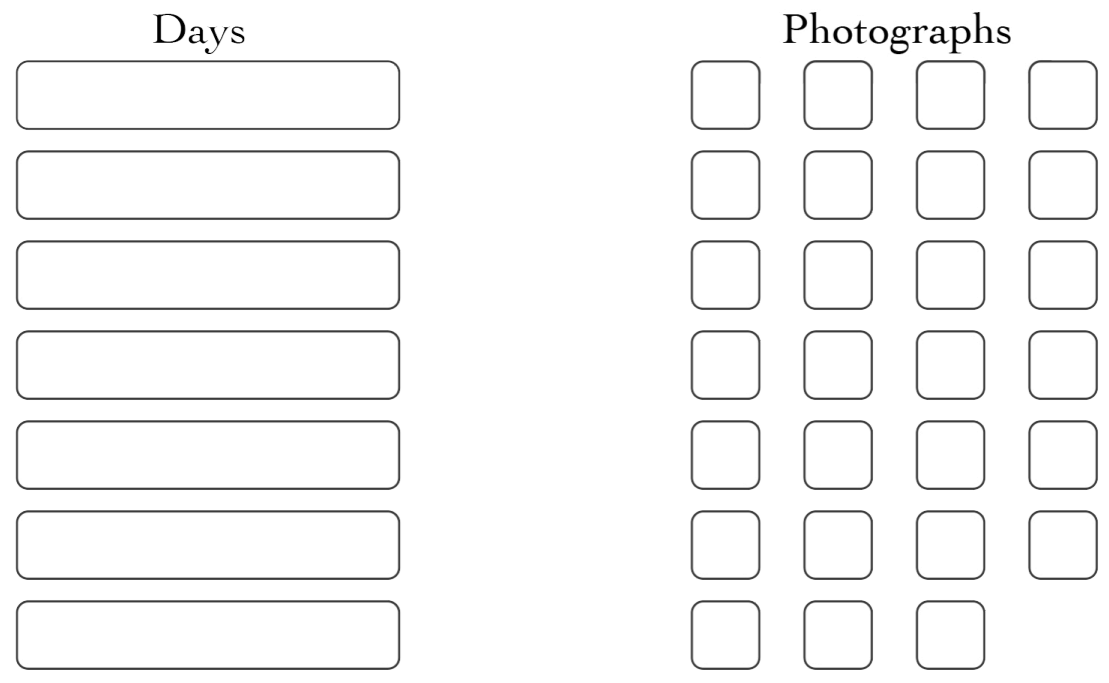

If I have any questions or want to talk to the researcher about the photo-taking theme or the photo-taking process, I call 206.941.3851 and the principal investigator (Jutta Ataie) will call me as soon as possible.

Thank you for participating in this important study,

Jutta Ataie, MSW, PhD Candidate

(Principal Investigator, 206.941.3851) 
Appendix S: Refrigerator Magnets

Refrigerator Magnets
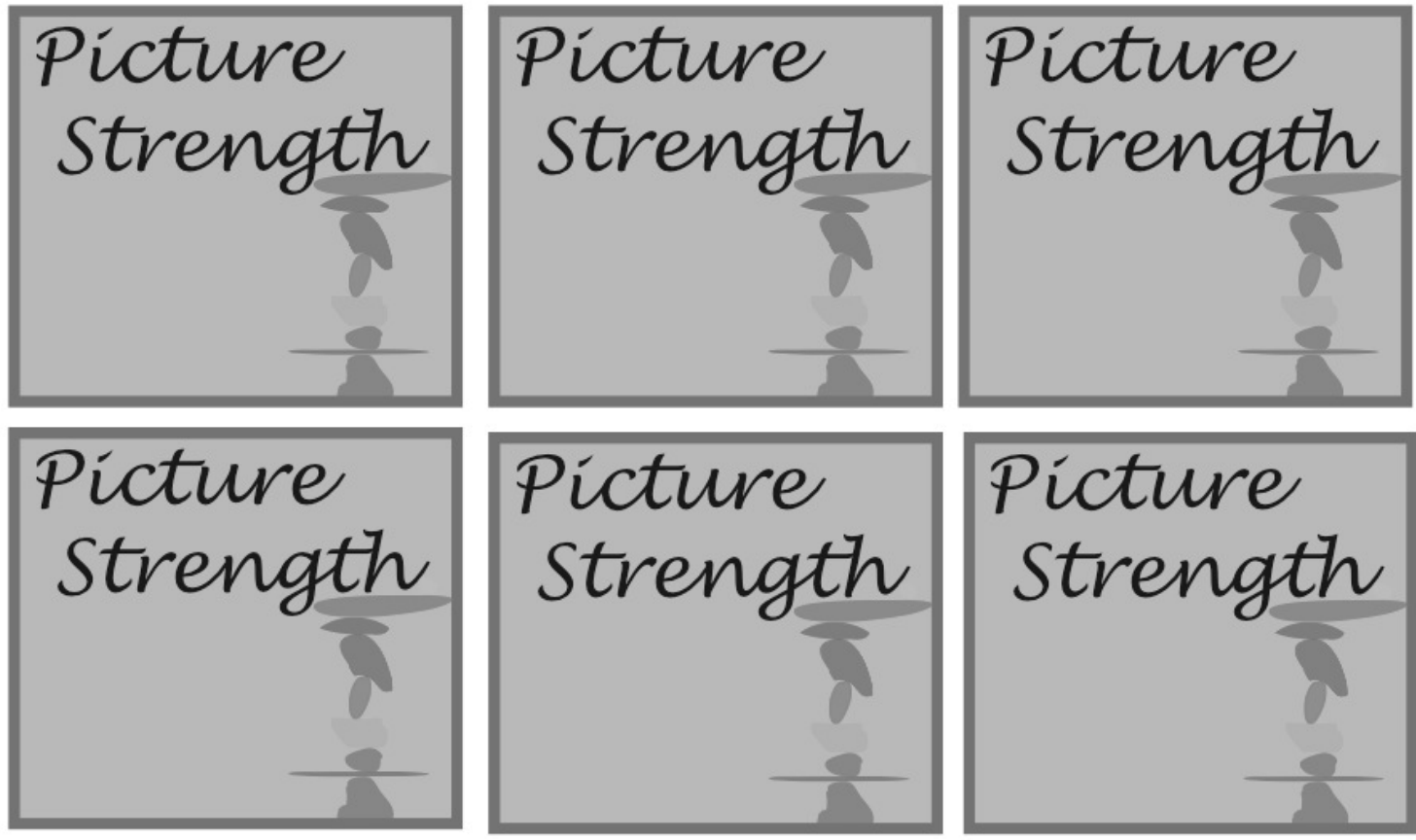

Picture
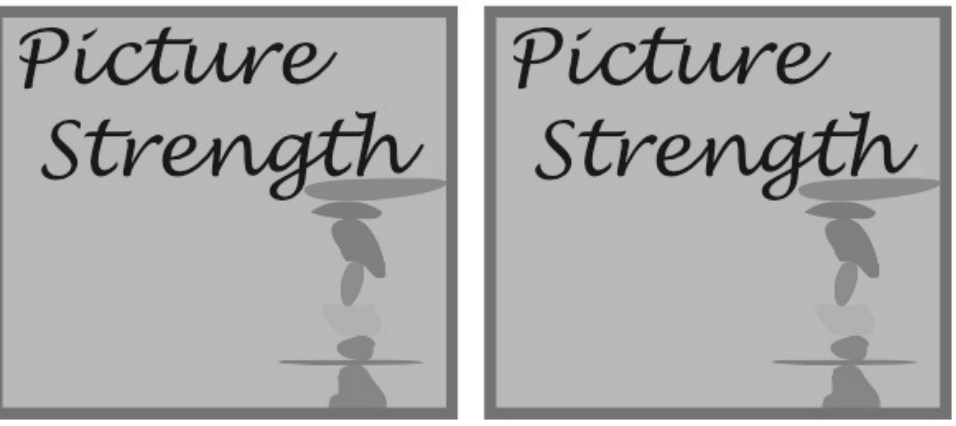

Strength
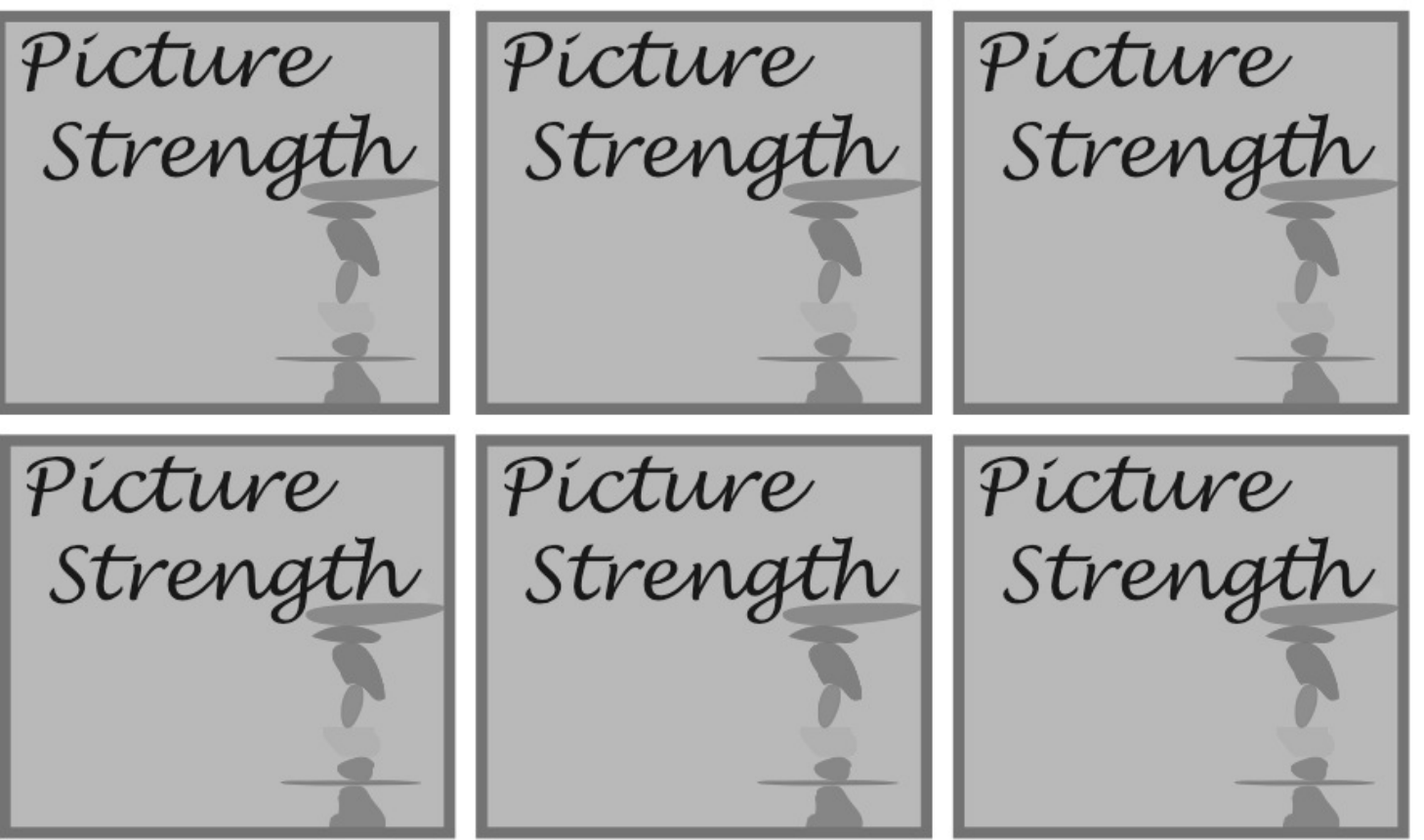

Picture

Strength 


\section{Appendix T: Portraits of the Participants}

\section{Chester}

\section{Portraits of the Participants}

Chester is a 66-year old Caucasian man who lives with his wife Tina in the house in which the couple raised their son and daughter. Both children as well as Chester's father and step-mother live close by and spend much time with Chester and Tina. Chester was 60 years old and working as a longshoreman when he received his diagnosis of 'early-onset Alzheimer's disease'. Chester was not able to recall the exact circumstances of his diagnosis. However, he remembered that it was his inability to perform his job and angry outbursts at home and at work that had eventually led to his diagnosis. Chester has accepted that the illness has irrevocably changed his life and he no longer feels angry or sorry for himself but it has been a process for him. However, at the time of this study, he seemed to have integrated the illness into his life and considered himself lucky not to suffer from other health concerns. Chester felt that he was living a full, rewarding life and was grateful for the times he was able to spend with his family. In fact, Chester's main strength and priority in life was his family, primarily his relationship with Tina. It seemed that for Chester, neither time nor the progression of his illness could erase the beauty of his relationship with his wife. Chester seemed to have discovered that everyday life has its own richness. For him, wellbeing was woven within the fabric of his everyday life. Volunteering for this study was important for Chester because it provided him with the possibility to contribute to research on dementia. In addition, he felt grateful to 'finally' have an opportunity to give something back to me for 'being there every month' to facilitate the support group that he and Tina had attended for the past five years. However, he felt uncertain whether his current level of functioning would allow him to operate the camera successfully. To be sure he could participate successfully, he asked his wife Tina to assist him during the phototaking activity and be present during our three dialogue sessions.

Chester took 27 photographs. His portfolio emanated a sense of contentment and happiness and covered a wide range of strength and resources that contributed to his sense of wellbeing. Chester photographed his wife Tina to show that she was the love of his life and that he considered her his primary caregiver. In fact, much of Chester's sense of wellbeing seemed to come from the strength he drew from his relationship with Tina. He relied on her and liked to join her in most of her activities. At the time of this study, Tina made sure that Chester's physical and emotional needs were addressed and that the rhythm of his everyday life followed an even pace. Tina had also taken over all of Chester's responsibilities in the couple's day-to-day affairs. For Chester, this role change was neither good nor bad; he simply accepted it. Chester was very appreciative of everything that Tina did but was also genuinely concerned about becoming a burden to his wife. Chester relied on Tina to bring structure to his life, something he considered important to his sense of wellbeing. Tina's carefully constructed schedules for 
Chester's days included a balanced mix of routine activities at home interspersed with social interactions and ample amounts of rest. Chester dedicated a number of photographs to showing that he considered family support an important resource. He felt that being connected to his larger family made it possible for him to cope successfully with his illness. He was proud of his children and their accomplishments and felt that he had done a good job raising them. He drew strength from knowing that his memory will live on in them. In addition, he was happy that they regularly spent time with him. They shared meals, reminisced, played games, took him on outings, or simply told him how much they loved him. At the time of this study, Chester also met with his father and step-mother regularly, mostly to play cribbage, his favorite card game. Chester's life seemed full of activities that were meant to have an emotional value. He often did not remember what he had done but the emotions associated with the activity remained. For example, although Chester could not recall when he had played cribbage last, he did remember that he won often, and that made him happy. In other words, Chester liked to celebrate small successes. Chester viewed spending time with the family pets in a similar way. It was an activity that brought him joy and facilitated a state of calm and relaxation. Chester also used a photograph of the family pet and a photograph of two bikes to symbolize that regular exercise and a healthy lifestyle are important resources for him. At the time of this study, Tina and Chester went for daily walks with the dog, took regular bike rides, and attended a weekly exercise class. Chester also took photographs of his medications to show that he considered following a prescribed medication regimen an important resource. Although he could not remember the names of the medications that he took, he knew that among others he was taking two dementia drugs. Chester included three photographs in his portfolio that symbolized his past life. These photographs captured his professional life as a glass blower and a longshoreman, as well as his hobby of collecting and fixing up antique cars and going to shows. When discussing these photographs, Chester seemed proud but at the same time content. He seemed to have come to terms with how his life had unfolded. Chester did not include a photograph that symbolized the role that attending a support group for people with early-stage memory loss played in his life because the group did not meet within the timeframe of the photo-taking activity. However, he did mention during one of our conversations that he enjoyed the monthly meetings because they provided an opportunity for him to listen to the experiences of others and to share his own story.

This study provided an opportunity for Chester to contribute to research on dementia, something he felt strongly about. In addition, it allowed him to capture and document the richness of his life to this point on the journey. Chester felt so satisfied with the quality of his contribution that he was planning on sharing his portfolio with his children. He felt that he had produced something of value, something that would help others to understand him better. 


\section{Corinne}

Corinne is a 69-year-old Caucasian woman who is happily married to Ken, her greatest source of support. At the time of this study, the couple lived in a local golf community, travelled extensively for months at a time, and was in the process of acquiring a second home in Sarasota, Florida. They have one daughter who lives out of town. Corinne's mother died of Alzheimer's disease about a year ago, and Corinne received her diagnosis of 'early Alzheimer's disease' about 5 years ago while participating in a study investigating family members of individuals living with the illness. At that time, Corinne, who had worked in the financial department of a car dealership, had already retired. Corinne described the limitations she experienced as a result of the illness primarily in terms of social encounters. Struggling to find the right words, losing her train of thought, and repeating herself while interacting with others were some of her major concerns. Whenever the couple is in town, Corinne participates in one of the support groups I facilitate for the local chapter of the Alzheimer's Association. Given this familiarity, our dialogue was characterized by mutual respect and trust and the tacit understanding that it was okay to challenge conventional wisdom and knowledge. Corinne liked to tease and joke. In fact, she considered a sense humor a strength that she draws on frequently and that helps her to keep the illness in perspective. Focusing on wellbeing was equally important to Corinne. Although she spoke about her anger regarding the nature of the illness and its annoying symptoms and how frustrating events can bring her to sudden tears, deep down, Corinne believed that there must be a reason for her illness. She drew strength from the belief that the illness was part of God's plan, which provided her with a great sense of comfort. In other words, Corinne's greatest strength in coping with the illness was her faith.

Corinne approached the photo-taking activity for this study with great enthusiasm. She took 27 photographs but forgot to keep the photo-diary. Consistent with her general approach of drawing strength from focusing on the positive, many of Corinne's photographs consisted of nature images that she considered beautiful and/or inspiring. When discussing her photographs she highlighted the fact that she felt grateful for having an illness that does not cause pain. Corinne took one photograph of her husband to relay the important role he plays in coping with the illness. For Corinne, being able to relinquish roles and responsibilities was both, frustrating and a source of relief. She considered herself fortunate to be able to concentrate on the things in her life that she considered valuable without having to pay much attention to cumbersome duties. Interestingly, Corinne did not take photographs of medications and/or memory aids. She explained that not including these images was a conscious decision since she considered them necessities of life and not a resource to help her cope with the illness. For Corinne, connections with other people were an important resource that had a profound effect on her coping skills. She considered the connection to her neighbors a major source of strength and was greatly loved and respected by many people in her community. To show the extent to which she feels integrated 
into her community, she took two photographs of her neighbor. In addition, to symbolize how much she relies on help from her neighbors, she included an image of a burned pizza (her last attempt at cooking) and explained that her neighbors look after her when her husband Ken is out of town. When talking about her relationships, Corinne explained that it was important not to tire people by repeating herself and asking the same questions over and over again.

She considered being able to cover up her forgetfulness a strength that she draws on to avoid being a burden to family members and friends. Corinne also said/explained/reported that making connections with other people sometimes is difficult for her. It seemed to her as if close friends and family members did not know what to say or do in her company. They seemed so worried about saying the wrong thing that they did not say anything at all. Corinne found humor an important resource as it eased tension and allowed her friends and family members to be more encouraging and supportive. Corinne took three photographs of her church to highlight the important role that religious resources play in her life. At the time of the study, she attended weekly church services, socialized after the services with some of the church members, and regularly participated in different church groups. Corinne also included a photograph of the local library in her portfolio to illustrate the strength that she gains from reading both fiction for pleasure and informational material about the illness for advice and guidance. She explained that if she is in a cheerful mood, her joy is increased by reading. When she feels depressed and dejected, books console and soothe her. Being reasonably comfortable with the subject of the illness is a strength that supported Corinne in two ways. It provided a sense of comfort, and it increased her ability to educate others about the illness, which was very important to her.

This study provided Corinne with a deep sense of enjoyment. She was enamored with the idea of capturing what is important to her visually and then sharing it with others. She was planning on engaging her entire family in a photo-taking and photo-sharing activity similar to this study for a Christmas project. In addition, this study provided Corinne with an opportunity to make a contribution to research on dementia, something that was very important to her.

Corinne was one of four women who participated in the dyadic interviews. She was excited about the prospect of meeting other study participants because she viewed the opportunity to exchange ideas with people living with the illness a valuable asset.

\section{Duncan}

At the time of this study, Duncan was a 72-year-old Caucasian man and lived with his wife Sally and his dog 'Nicolas' in their new home. Duncan and Sally had decided that they wanted to downsize and simplify their life and had recently sold their house in which the couple had brought up their two children. They saw their daughter who lived near-by on a weekly basis; their son lived in another 
town but visited regularly. Neither child was involved in the couple's day-to-day life.

Duncan and I knew each other from a support group that I facilitate for the local chapter of the Alzheimer's Association, which he and Sally had attended regularly for five years. Duncan began his story at medical school where he had specialized in cardiology, followed by a successful career in medicine. Duncan recalled the exact circumstances of his diagnosis and remembered that he was diagnosed with 'early-stage Alzheimer's disease' six years ago while he was still working as a cardiologist. He described that, prior to his diagnosis, he had not suspected that anything was wrong but his wife had noticed the first signs of his forgetfulness and convinced him to undergo medical testing. Duncan responded to his diagnosis with shock and disbelief followed by anger because he still did not feel that he had memory problems, and yet, being diagnosed meant that he had to give up his practice as a physician and retire. Transitioning into retirement was difficult for Duncan because it meant a tremendous change in lifestyle. As a cardiologist, he had maintained a rigorous work schedule that left him with little or no free time. Forced to retire, he suddenly found himself with a huge amount of free time and no satisfactory plans of how to use it. In time, he developed new interests and established a daily schedule outside the home, which provide some stability and helped with the role change within the marriage.

While listening to his story, I came to understand that for Duncan, nothing had changed much. He viewed his illness as if it had not happened. Although he initially missed his medical practice, he did not feel that the illness had put many restrictions on him or that he needed to integrate his experiences with the illness into a new philosophy of life. His attitude toward the illness was to proceed as though it did not exist. In fact, he thought of the illness as more a hurdle to be cleared than an event requiring course adjustment. His work post-diagnosis was indicative of this attitude. He had volunteered on two medical missions to thirdworld countries because he felt that he could still contribute his medical knowledge without endangering patients by being part of a medical team. At home, he had found plenty of large and small projects requiring his attention. In a way, he had resumed his normal life and felt productive and happy. At the time of this study, Duncan still had little symptom awareness. He recognized occasional forgetfulness but generally thought that not much had changed in this cognitive functioning. In terms of lifestyle, Duncan had re-established a rigorous schedule and maintained a very active social life.

Duncan approached his participation in this study with lots of energy and took 25 photographs. His portfolio conveyed the enthusiasm with which he engages with people and yielded a rich array of examples of activities that he considered important to his wellbeing. Duncan dedicated a series of photographs to express that he considered being connected to organizations whose programming focused on older adults to be the most important resource. At the time of this study, 
Duncan attended the local senior center Monday through Friday where he participated in a variety of activities such as singing, playing cards, and having coffee or lunch with a group of other members. When talking about his activities at the local senior center, Duncan gave the impression that he was well liked by both staff and fellow seniors, which seemed to contribute to his positive selfimage. Duncan took several photographs to show that being loved and respected by his wife and children was a major source of strength to him. When discussing his photographs, he confided that Sally was the love of his life and that without her, life would have no meaning for him. He was concerned about becoming a burden to her and had made arrangements for the future. He had purchased long term care insurance and established a stock portfolio with the goal of insuring that his care needs would be addressed without impinging on Sally's comfort and lifestyle. Duncan took a photograph of church members to show that a religious community was also an important strength in his life. At the time of this study, he and Sally regularly attended services at a large Presbyterian church in their community and socialized with some of the church members after services. In addition, Duncan sang in the church choir and attended weekly choir practice. Simple pleasures also played an important role in Duncan's life. He took a photograph of a frappuchino to show how much he enjoys the stops at Starbucks that he and Sally make while running errands. During one interview, Duncan showed me his wine cellar to explain that he enjoys choosing the right wine that he and Sally enjoy having with dinner every night. Duncan took three photographs of members of his exercise class to show that he considered caring for his body an important strength. He had exercised all his life. Receiving a diagnosis of early-stage dementia had not changed that habit. However, he no longer used the fitness studio but took advantage of the exercise class at the senior center. Duncan did not take photographs of resources that helped him manage his life, perhaps because he had referred to them during our first meeting. At the time of this study, he used a calendar and a medication manager to keep himself organized and had transferred some of his responsibilities involving finances and other instrumental activities of daily living to his wife. Duncan's portfolio also did not include a photograph that showed the important role that the senior center bus plays in his life. However, in our conversations he referred to the senior center bus as a lifeline because he was no longer able to drive on his own. At the time of this study, Duncan was still a proud member of the local Rotary club and believed in the importance of contributing to a good cause. He felt proud of his contributions to the local charities.

This study provided Duncan with an opportunity to engage in an activity that brought him joy. In addition, he loved the idea of contributing to research on dementia, something he considered very important. He was one of the two men chosen for the dyadic interviews. He was paired with Karl. 
Duncan passed away January $12^{\text {th }} 2012$, ten days after he was diagnosed with stage four stomach cancer which had metastasized to his liver, approximately a year after he had participated in the dyadic interview.

Fanny

Fanny is a 78-year-old Caucasian woman who, at the time of this study, lived with her husband Ben in the house that they had built years ago. Fanny was unable to recall the exact circumstances of her diagnosis but was certain that she was diagnosed with 'Alzheimer's' about five years ago during one of her routine check-ups at the Veterans Affairs medical center. She remembered that she had reacted to the diagnosis with surprise and disbelief since she had not observed any lapses in her memory. For Fanny, the changes had been so gradual that she had already redefined her 'normal' and was living somewhat comfortably with the changes. In other words, her everyday life did not feel much different to her.

Fanny was both fascinated and intimidated with the prospect of taking part in this study. Initially, she was concerned about completing the photo-taking activity because she had not used a camera for years but she quickly convinced herself because she was certain of Ben's support. To get started and overcome her doubts, Fanny took her first photograph even before our first meeting was over.

Fanny took 28 photographs and the notion of 'normalcy' figured prominently in her portfolio. Although Fanny acknowledged that she was experiencing the symptoms of early-stage dementia, she continued to believe that her everyday life had not changed much. Fanny took a photograph of Ben to symbolize the strength she draws from the relationship with her husband. She explained that she had transferred a number of her everyday responsibilities to Ben and that she depends on him to a large extent. Interestingly, Fanny did not perceive giving up some of her former duties as a loss. Instead, she felt the freedom from these obligations was well deserved. Fanny dedicated a series of photographs to her family. Fanny thought her illness had brought positive changes because it had pulled her family closer together; her family members were willing to extend more love, affection, and care than they had in the past. She drew much strength from her children's daily calls and the increased attention she received during family get-togethers. Fanny also felt that a burden had been lifted from her shoulders because her children had assumed all responsibilities for hosting gettogethers, which allowed her to simply be there and enjoy herself.

Meeting other people with early-stage dementia in a group setting was a major source of strength for Fanny. However, the logistics of attending a support group were challenging. The couple lived in a rural area where no support group was offered locally and the next available group was thirty miles away. At the time of this study, Fanny was no longer able to drive and, with no access to a public transit system, depended on her husband for all her transportation needs. Fanny's 
husband Ben drove her to the support group meeting thirty miles away once a month and waited in the car for her. Fanny had discovered the benefits of support groups when she was diagnosed with breast cancer fifteen years ago. What she appreciated most about her current support group is the exchange of practical information and knowing that she is not alone in living with the illness. Fanny felt that meeting people who face similar challenges provided her with the motivation to restructure her life and establish a new routine. Fanny took several photographs to show the important role that quilting and sewing plays in her life. When discussing her photographs it became clear that, long before being diagnosed with early-stage dementia, Fanny had made a deliberate decision to cultivate interests she thought would serve her well in her later years. Quilting and sewing were the two activities she found most satisfying and she had put much time and effort into learning the craft and refining her skills. In fact, Fanny's anchoring activity in retirement had been quilting. However, at the time of this study, she no longer quilted because of the demanding nature of the craft. Instead, she sewed and worked on smaller patchwork projects. Fanny was very proud of her work. The couple's house was filled with beautiful quilts and, during our first meeting, Fanny took me to her sewing room to show me what she was currently working on and tell me of her plans for future projects. Her sewing room was a delight, pleasantly covered with half-finished projects and starts of new ones. The room radiated happiness and joy. At our last meeting, Fanny surprised me by presenting me with a beautiful, handmade pillowcase. Fanny dedicated a series of photographs to her other hobby, collecting birdhouses. Fanny was fond of birdhouses because they were beautiful and brightened her home, of which she was very proud, but she also liked the fact that they brought 'nature' to her doorstep. She enjoyed providing a safe place for the birds to nest, and watching them enhanced her sense of connectedness to nature. Fanny included a photograph of her favorite store to show that shopping is a valuable strength to her. She clarified that statement by commenting that only shopping for her sewing hobby was important to her and that she did not miss the responsibility of grocery shopping, which Ben had assumed. In fact, she abdicated all her cooking responsibilities as she interpreted preparing meals as an extension of grocery shopping. For her, not cooking and grocery shopping was not a loss but freedom from a lifelong burden. Fanny's portfolio also included images that showed the important role that simple pleasures played in her life. When talking about the photograph she had taken of the American flag in her front yard, it became clear that for Fanny simply looking at the flag contributed to her wellbeing in a number of ways. Most importantly, it helped her in maintaining a positive sense of identity because it reminded her that she is proud to be an American. It also symbolized past accomplishments; Fanny had been a flag bearer in the military, something which she was very proud of. Fanny's photograph of her gardening bench symbolized one of her failed efforts to add exercise and healthy food to her daily routine. She had purchased several pieces of exercise equipment and received others as gifts in a conscious decision to maintain a healthy lifestyle. At the time of this study, Fanny had several lifestyle- 
related goals. However, following a balanced regimen of exercise and healthy food remained a challenge.

Fay

Fay is a 75-year-old African American widow who lives with her son Derek and her sister Kacie in the house that Fay and her husband had built. At the time of this study, Fay considered Kacie her primary support person. Although Derek lived in the same house, he was not involved in the lives of either of the women, but Fay considered him her 'number one protector'. Fay holds a Masters degree in public administration and had worked for most of her life as a manager for the federal government. I have known Fay for five years from a support group that I facilitate for the local chapter of the Alzheimer's Association. For this study, we met three times. Fay was approximately 70 years old and retired when she received her diagnosis of 'early-stage Alzheimer's disease'. She did not remember the exact circumstances of her diagnosis but recalled that it had taken a while for her primary care physician to take her complaints about memory problems seriously. He had initially brushed off her concerns by normalizing her increasing forgetfulness as the natural by-product of aging. It was not until her symptoms seriously affected her daily life and her forgetfulness had caused financial problems that her sister insisted on comprehensive cognitive testing. Fay did not worry about her diagnosis or her illness because she believed that God had put her on this earth for a purpose and although she did not understand the intention, she viewed her illness as part of a plan. Nevertheless, it had taken Fay a while to accept that her mental, emotional, and physical energy was not what it used to be. Prior to experiencing memory problems, she had thought of herself as sturdy, vigorous, and healthy and assumed that she would stay that way until her death of old age.

Fay's contribution to this study revealed that she eventually discovered a number of strengths and resources that helped her adjust to her current level of functioning. Fay was excited about the prospect of participating in this study but was initially worried about her ability to take photographs. However, after several test runs with the practice camera, Fay felt confident of her ability to manage the camera and took the first two photographs even before the end of our first meeting. Her portfolio of 20 photographs conveyed the enthusiasm with which she engaged in life and the strength she drew from leading a life based on family support and faith. Fay took a series of photographs to show that she attributed much of her current sense of wellbeing to her reliance on faith and her trust in God. In other words, Fay used her faith as a resource to cope with the illness and found spiritual richness in everyday life. For Fay, faith was the pivot of her life and provided her with meaning and purpose. Being able to call on God when needed was Fay's most significant strength. For Fay, trusting in God was a lifelong pattern of coping. Her photo-diary entry read "I was raised in church; we attended church six days a week. Saturday night was 'off night"', In addition to 
providing her with a sense of comfort and continuity, Fay's faith also facilitated a number of other resources through her involvement in church. At the time of this study, Fay's involvement in the church included attending weekly church services, singing in the church choir, and maintaining friendships with a number of fellow parishioners. Fay took several photographs of her sister Kacie to portray the important role that she played in her life. Kacie made sure that Fay's physical needs as well as the broader needs of her day-to-day life were addressed so that she could continue maintaining the active social life she was accustomed to. Fay had also transferred all responsibilities and duties associated with living in a complex society to Kacie and had designated her as durable power of attorney for finances and healthcare. At the time of this study, the two sisters did everything together; they followed a shared weekly routine which provided Fay with much strength. Her portfolio included a number of photographs symbolizing activities that occurred at regular intervals. Having a schedule and knowing what to expect each day of the week not only created a sense of certainty and continuity but also allowed Fay to experience 'pre-joy' (finding joy in anticipation). She was looking forward to her weekly church services and her weekly appointment with the hairdresser as much as her three times a week bridge game and exercise class at the senior center.

Fay took a photograph of her television to express her love of football in particular and sports in general and the anticipation of the games every week. Fay's portfolio also included photographs that showed that she drew strength from remembering past experiences. For example, she took a photograph of a picture that showed Fay and her husband and said that although her husband had died many years ago, she still misses him and draws strength from remembering their shared life. Another example is a photograph that Fay took of a lake. Fay said that she loves water and that she likes to remember the times when she used to operate her boat on that lake. Providing support to other family members was also important to Fay's sense of wellbeing. She symbolized this by including a photograph of her 97-year-old uncle and explained that she and Kacie support him by visiting him daily. Fay reiterated that she draws strength from meeting once a month with the support group because it provides a venue for her to give and receive practical and emotional support and get confirmation that her experiences are 'normal'. However, she did not have an opportunity to take a photograph of fellow members because the group did not meet within the time span of the phototaking activity. For Fay, participating in this study provided an opportunity to recall and share cherished memories. The photo-taking activity allowed her to visually document her present life, something that she considered very important. Fay was very proud of the portfolio she had created and was planning to share it with her extended family. In fact, Fay was so taken by the quality of the photographs she had taken for this study that she was planning on buying a disposable camera so that she would be able to continue taking photographs [of her experiences. To support her enthusiasm, I sent her two cameras by mail. 
Frank

Frank is an 82-year-old Caucasian man who is happily married to Karen, his primary source of support. The couple has four children all of whom live in the area and visit frequently but are not involved in the couple's everyday life. At the time of this study, the couple's primary residence was located in a golf course community. In addition, the couple owned a beach house and a small cabin on a large parcel of forested land. Frank was very proud of the cabin since he had constructed it from scratch as part of his retirement plans. Both, Frank and Karen come from families that were in the paper/forest industry and Frank had worked in forest management all his life. Frank drew much strength from knowing and honoring the couple's ancestral roots and family history and expressed this by including a photograph of a painting of two of his ancestors in his portfolio. Frank first noticed his memory problems during a family get-together sitting around the campfire playing 'I pack a suitcase'. It then took another two or three years for his symptoms to become so intrusive that he decided to seek medical advice.

About five years ago, after extensive testing, Frank was told that he had 'the early symptoms of Alzheimer's'. For Frank, receiving his diagnosis was a confirmation of his suspicion and evoked feelings of sadness and confusion. He approached coping with his emotions and managing his illness in a practical way. He read about his illness, attended a number of educational seminars and support groups, and followed a prescribed medication regimen.

He also arranged a family-get-together to inform everybody about his diagnosis and brainstorm how to cope as a family. Due to distance, I met with Frank twice; our first meeting took place at the couple's primary residence and the second time we met at the couple's beach house. Sitting in the couple's beach home I came to understand what had given the greatest meaning to Frank's life were his deep care and concern for his family, his profound connectedness with nature, and his genuine love for work. Frank's overall approach to life could be described as anticipating the interesting. He told me at one point that he goes to bed at night looking forward to the next morning, so that he can do those things that he was unable to accomplish that day.

Frank approached the photo-taking activity for this study in much the same way and produced an interesting portfolio that included 28 photographs capturing both a wide range of resources and ongoing construction of identity. He experimented with different camera angles and infused a sense of timeline in his photographs conveying a feeling of continuity between his past and present life. Frank drew strength from connecting his current activities to past accomplishments. Viewing his life and work as temporal patterning, provided a sense of continuity, stability, and contentment for him. It allowed him to see how his interests had changed over the years and view the process as a natural progression and not necessarily a loss. For example, he took several photographs of his vineyard and his winemaking machinery. Frank started this business in 1974 and was proud that he had one of the first wineries in the State of Washington. He happily recounted that the honors he had received for his award-winning wines. Frank still treasures 
growing grapes and making wine but over the years, as his energy declined, the activity had become less and less important to him. Another example of seeing the temporal sequencing of his life was when Frank spoke about how he drew strength from his relationship with nature. Frank took great pleasure in spending time alone observing in nature. He included two photographs of rabbits in his front yard, a discovery he had made recently. Frank traced his love for solitude to his young adulthood as a forest worker when he was sent into the forest by himself for weeks at the time to spot wild fires. Frank also took a number of photographs to show that he likes working in and with nature. For example, he included photographs of a well-pruned tree, a grape sprayer and mildew-covered grapes, and a wreath that Karen had arranged from crabs he had caught.

Participating in some constructive activity that keeps his mind and body active had been and continued to be an important resource for him. He enjoyed looking back on his career, remembered fondly the projects that had filled his retirement, and reviewed his current undertakings. Frank was not discouraged by the roadblock that his illness produced. Instead, he took them as an opportunity to be creative and drew strength from the challenge. He explained that he had been able to overcome most of the challenges through determination and hard work and was planning to do so in the future. At the time of this study, he was in the process of developing an innovative way of crab fishing as he had just discovered that he was no longer able to safely operate his boat.

Frank included a photograph of his truck in his portfolio to show that he considers having access to transportation an important resource. At the time of this study, he drove rarely but liked to know that the opportunity to drive was there - just in case he came up with another project that would require the transportation of heavy materials. Frank included several photographs that symbolized his love and appreciation for Karen and the strength he drew from the relationship. At the time of this study, Frank had transferred some of his responsibilities to Karen but tried to contribute as much as he could to the couple's everyday chores. He also photographed family pictures from a photo album to show that he was proud of his children and their accomplishments and that it was comforting for him to know that his life's work will continue through them. Frank took a photograph of his mediset to symbolize a number of resources that he considered important, such as having access to dementia drugs, self-managing his medications, and having tools that assist him in staying organized. At the time of this study, Karen managed his prescription refills but Frank had the responsibility for filling the mediset and taking his medication on a day-to-day basis. Frank did not include a photograph of the couple's efforts to simplify their lifestyle. However, at the time of this study, Frank and Karen were thinking about downsizing and moving closer to their children.

This study prompted Frank to examine his life narrative, the stories he tells himself about who he is and how he had come to be that person. For Frank, taking photographs of his experiences initiated a kind of biographical dialogue, an 
engagement between his family history, his personal sense of identity (past and present) and social standards of success and accomplishment.

Irene

Irene is a 57-year-old, single Caucasian woman who, at the time of this study, lived in her own apartment. Irene has two siblings; Hetti, her sister who lives near-by and is in daily contact with her and Victor, her brother who lives out of town but visits her regularly. Irene worked for the local power company all her life. At age 20, Irene was one of the first women hired into the electrical trade and following a landmark verdict three years later, became one of the first female line workers in Washington State. She worked on the line crew until suffering a broken back in a near-fatal fall from the top of an electrical pole at age 24. Her injuries left Irene walking with a cane for the rest of her life but did not prevent her from returning to work at the power company where she held a position as a power station operator until she retired at age 50 due to 'early-onset Alzheimer's disease'. As a feminist activist, Irene helped women break into the trades and stood up for their labor rights, social and economic justice, and disability rights. Irene was no longer able to recall the circumstances of her diagnosis but remembered that when she was experiencing the early symptoms of the illness, she had felt as if she had lost herself. She remembered feeling empty, angry, and unable to share what was going on with her. After her diagnosis, Irene felt much calmer because she had an explanation for what was going on and became resourceful in reclaiming her sense of wellbeing. I met with Irene three times in her apartment. Hetti, her sister, was present for our first meeting. Irene was the only participant who used her own camera as she was unable to retain the instructions for operating the disposable camera.

Irene's approach to this study was grounded in political activism and the tenets of feminism. Although completing the photo-taking activity was difficult for Irene, she committed herself to this study fully. She hoped that her contribution would help change current, negative perspectives on dementia and improve the conditions for people living with the illness. Irene had her sister write a note, which she kept as a daily reminder explaining why her participation is important. Irene built an intriguing portfolio that included 30 photographs symbolizing past and present life experiences as well as personal values and political opinions. The notion 'the personal is political' figured prominently in Irene's portfolio. Irene clearly viewed her personal experience of early-stage dementia as embedded in the current political context and social realities. Her portfolio addressed themes such as empowerment and independence; equal mutual relationships of caring and support; and resilience. Irene emphasized the need for social and political change so that affordable and adequate services for people with dementia would be available. For Irene, overcoming the challenges and difficulties she had experienced as a result of early-stage dementia was an expression of her desire to live. She did not want to be seen as a negative by-products of unfortunate 
circumstances. Irene had recognized the limitations that the illness imposed on her and had found ways to be flexible within these limitations. Irene portrayed a number of resources that helped her stay organized and manage her life and the illness. At the time of this study, Irene used calendars, medication organizers, and a chart that kept her on track with personal activities of daily living. Irene took several photographs to show that social support and being loved and respected by family and friends was a major source of strength to her. Her sister Hetti was a daily presence in Irene's life. The two shared many friends and fellow feminists whom they visited often. In addition, Hetti provided gentle guidance in areas that were increasingly difficult for Irene. Her support focused on streamlining Irene's calendars, making sure she had her medications, and keeping track of her finances as well as other instrumental activities of daily living. Irene felt that Hetti provided just the right amount of assistance so that she could remain independent without getting frustrated or facing undue risks. In planning for the future, Irene had designated Hetti as her durable power of attorney and felt that she could trust her with making decisions pertaining to her life in the spirit of equal partnership. Irene had come to understand that frustration is one of the most disruptive forces in her life and had discovered a number of resources that helped her avoid feeling frustrated as much as possible. She added new activities to her routine and discovered the beauty of simple pleasures which brought joy and a greater sense of serenity to her life. As was evident from her portfolio, her favorite past-times were swinging on a swing set and enjoying the vibrancy of colors in nature. The notion of living purposefully played a major role in Irene's life. She followed a routine that structured each day with meaningful activities and needed rest. Irene took a photograph of one of her recent watercolor paintings to show that she considers attending an adult day care program with community, art, and cultural activities an important resource. She explained that attending a weekly art program provides her with the opportunity to engage in social interactions and allows her to enjoy the creative and expressive process of painting. For Irene, finding different forms of expressing herself has become increasingly important as she has experienced a noticeable decline in her discursive abilities. She described that exploring creative expressions has had a positive effect on her emotional and mental wellbeing. Although Irene would have liked to attend the adult day program more often, her financial means did not allow for it. However, she was grateful for what she was able to do and felt sorry for others with dementia who did not have the financial resources to enjoy a similar program. Irene's inclusion of a photograph of her support group members showed how much she values being connected to the Alzheimer's Association. For Irene, the monthly support group meeting is a place where her experiences are validated and a place where she can contribute and reach out to help others and in the process, lift up her own spirit. Irene's role model was her mother who lived with multiple sclerosis for most of her life. She drew strength from remembering her mother's resilience, her fighting spirit and her love for life. Irene photographed her car to show that being able to get around is an important resource to her. At the time of this study, Irene was able to operate her own car but she limited herself to driving 
the side streets and staying in familiar surroundings. Irene enjoyed the freedom that being able to drive brought because it allowed her to pursue the activities that she considered essential to her wellbeing.

For Irene, this study provided an opportunity to once again, become an agent for change and express the feminist principles and values that have guided her life.

Taking the stance that social and political action are in the realm of each person's individual responsibility, Irene saw her participation in this study as a chance to stand united with other people with dementia and advocate for the allocation of scarce resources.

\section{Karl}

Karl is a 82-year-old Caucasian man who lives with his wife Betty and their dog 'Queenie' in the house they had built 52 years ago. The couple has two sons both of whom visit regularly but are not involved in the couple's day-to-day life. For most of his professional life, Karl worked as a chemist, something he is very proud of. Karl was unable to recall the exact circumstances of his diagnosis but was certain that he was diagnosed with 'Alzheimer's'. He remembered that he had been somewhat surprised when he learned about his illness but that he had settled into a new routine shortly thereafter. He established new boundaries in his life and it seemed that everything within those boundaries became more precious than before. Karl coped with the illness by watching his horizon come closer but he felt comforted by allowing it to do precisely that. Initially, Karl felt uncertain about participating in this study. His wife explained that, although the activities associated with the study sounded appealing to him, Karl worried he may lack the skills to complete the required tasks and feared the routine he maintained and enjoyed may be considered 'boring' by other people. Given his ambivalence, it came as no surprise that he opted for Betty to join us in the dialogue sessions. Karl, Betty, and I know each other from a support group that I facilitate for the local chapter of the Alzheimer's Association, which they have attended regularly for the past five years.

Karl approached the photo-taking activity in much the same way as he had approached the decision about participating in this study; he began taking photographs with a sense of frustration. However, his sense of frustration decreased the more he engaged in the activity and he produced an interesting, diversified portfolio that captured not only his present life but also showed parts of his past life.

Karl took 27 photographs. The notion of 'familiarity' and 'routine' figured prominently in his portfolio. Knowing what to expect and how to behave provided Karl with a sense of wellbeing that came from feeling in control. He took a series of photographs of the inside and outside of the couple's house to convey that he drew strength from the sheltering comfort of familiar surroundings 
and everyday things. Within the small rooms of the couple's home, Karl found meaning in the details of daily life. He kept a tightly-structured routine that eliminated most of the stress in his life and allowed him to relax. In other words, for Karl, the security of a predictable schedule provided a wholesome channel into which he could funnel his energy and time. Karl included images of his calendar and daily chart in his portfolio to show that he considers memory aids and keeping track of his daily tasks important resources. Karl considered being able to spend parts of his days to reminisce and enjoy memories of the past by looking at mementos and favorite works of art a resource that contributes immensely to his sense of wellbeing and satisfaction with life. He included a couple of photographs that symbolized some of his past hobbies; hobbies that he once actively pursued and that now brighten his days by means of the fond memories they evoke.

Overall, Karl's portfolio emanated a sense of 'pre-joy' (finding joy in anticipation). He was looking forward to his daily walks with Queenie, his daily TV programs, his daily bedtime ritual, his daily bowl of strawberry ice cream, and his daily glass of Martini as much as he was looking forward to the monthly lunch with his wife in their favorite Chinese restaurant and the frequent visits of the hummingbirds in his backyard. Karl took a photograph of Betty to symbolize the strength he draws from his relationship with his wife. When discussing his photographs, he explained that he not only values Betty's companionship but that he also depends on her to a large extent. He had transferred a number of roles and responsibilities associated with life in a complex society to Betty. At the time of this study, Betty was the only person with whom he interacted regularly as he had also relinquished most of his social activities. However, Karl did not miss any of his former duties nor did he miss the human interaction that comes from being involved in social activities. In fact, he liked being alone for parts of the day and enjoyed and drew comfort from the company of his immediate family, his wife and his dog. What he missed, however, was his ability to drive as it prevented him from enjoying solitary walks on the beach with Queenie. Karl dedicated a number of photographs to portray the important role Queenie plays in his life. He listed Queenie as a family members and explained that he values her not only for the unconditional love and affection he receives from her but also for the sense of consistence and structure she brings to his life. To Karl, Queenie's companionship was enormously comforting. She provided him with a reason to remain active and life-focused by taking care of her, interacting with her, and going for walks with her. Karl did not include a photograph symbolizing his attendance in the monthly support group for people with early-stage memory loss. When I asked him about it, he explained, that he felt ambivalent about his participation in the group. Although he enjoyed being part of the group and having the opportunity to exchange ideas with the other group members, his attendance in the group caused a disruption in Karl's daily routine, which he did not appreciate. It kept him from watching two of his favorite TV programs and shortened his bedtime ritual, both of which he valued immensely. Although there 
was no photograph symbolizing his work ethic, it became apparent through our conversations that early-stage dementia had not changed Karl's values. He continued to believe in hard work as the way to ensure one's place in the world and to avoid the dreaded existence of being poor and/or on welfare.

This study provided Karl with the opportunity to take stock of his life. He realized that his approach to coping with his illness by adhering to a well-designed daily routine is far from dull and something people may disregard as boring. Instead, he realized that his approach is a creative way of dealing with the challenges of a progressive brain disease. This understanding changed the way in which he presents his coping strategy in the support group. He no longer excuses himself for maintaining a daily routine. In keeping with Karl's appreciation for mementos and the important role that reminiscing played in his life, I gave Karl a flip-style photo stand as a gift at the end the study. This photo-stand kept the twenty-seven photographs he had taken for this study easily accessible and to this day, Karl looks at his contributions to this study at least once or twice a week.

Karl was one of the two men chosen for the dyadic interviews. He was paired with Duncan.

\section{Kathryn}

Kathryn is a 64-year old, divorced Caucasian woman. At the time of this study, she lived with her dog 'Dora' in the house in which she had raised her two daughters. One of her daughters lived close-by, the other in Portland. Kathryn holds a Masters degree in mental health counseling and was one of the two participants who contacted me via e-mail about a week after the study announcement had been mailed out. During our first meeting, she was very inquisitive about who I was, why I was interested in the lives and views of people with early-stage dementia, and what my views were on professional organizations providing services to people with the illness. She explained that since her diagnosis of 'early onset Alzheimer's disease' about 5 years ago she had had a number of negative experiences with healthcare professionals and organizations and that she wanted to make sure that I indeed would respect her and her perspective. After careful consideration, Kathryn decided that she indeed wanted to participate in this study and offered the following explanation for her decision. She was pleased that somebody was actually interested in what was important to her (she did not think anybody else was); she saw participating in this study as an opportunity to discover for herself what she values and what contributes to her sense of wellbeing; and she loved photography. I met with Kathryn several times during this project. Kathryn was very talkative and we connected easily through our mutual love for counseling and self-improvement. Our meetings took place in the privacy of her home where we sat at the dinning room table talking for several hours each time. Kathryn not only enjoyed sharing her approach to living and coping with early-stage dementia but also found it valuable to discuss her 
experiences from a socio-cultural-critical perspective. During our first meeting, Kathryn shared that she had received her diagnosis after experiencing several distressing episodes with memory problems while working as a mental health counselor. She explained that although she is fairly knowledgeable about the signs and symptoms of dementia, noticing that something was wrong with her took some time.

Initially, she did not associate her problems with 'illness' but interpreted her difficulties within the general context of being stressed and having too much to do even though she had watched her sister progress through the various stages of the illness for the past 10 years. At the time of the study, Kathryn visited her sister weekly in the adult family home where she lived. Kathryn responded to her own diagnosis with shock, disbelief, and anger followed by a deep sense of loss and a prevailing sense of uncertainty. She talked at length about how the illness had not only forced her to retire prematurely but also destroyed her hopes and dreams for retirement. For Kathryn, losing her ability to practice as a mental health counselor was one of the hardest things. It threatened her sense of identity and meaning and purpose in life because 'doing therapy' was what she loved doing and what defined her sense of self-worth. By forcing her to give up her professional life, the illness also put a strain on several important relationships because most of Kathryn's friendships were work-related. For a while, Kathryn tried to maintain these long-term friendships but discovered that meeting with her former coworkers threatened her sense of self-worth because she felt that they no longer treated as an equal, as somebody who has something to contribute. These meeting that used to be an essential part of Kathryn's wellbeing became meaningless to her because she and her friends no longer shared the same experiences and her friends were unable to adjust to a different role. Losing her professional life and her friends and having to deal with the symptoms and longterm trajectory of the illness left Kathryn with a sense of 'illbeing'. However, Kathryn's contribution to this study showed that she had discovered a number of strengths and resources that helped her cope with the illness and feel well again.

Kathryn approached taking photographs for this study with a great sense of purpose. Even before deciding whether to participate in this study or not she had already composed a list of images she wanted to include in her portfolio and negotiated an extension based on the fact that some of the photographs she wanted to take required her to travel. Kathryn's portfolio included 20, carefully selected images. Each photograph showed that she had put a lot of thought into the compositions of her images. Kathryn dedicated a number of photographs to capture images of nature: landscapes, bodies of water, flowers, and trees. Many of her images speak to the inner work, the internal processes that Kathryn considers necessary to achieve a sense of wellbeing. Being in nature, particularly around calming bodies of water is a strength that she draws on daily. In describing her photographs Kathryn explained that spending time in nature balances her emotions and replaces negative ways of thinking with more life affirming thought patterns. Kathryn also photographed her dog 'Dora'. Having a 
pet is a resource that Kathryn considers essential to her sense of wellbeing for several reasons. Dora's non-judgmental companionship has been enormously comforting to Kathryn. It helped her cope when she felt distressed and assisted her in overcoming the loneliness she experienced as a result of the illness. When talking about Dora, Kathryn eluded to the interconnectedness and the intricate connection between the different strengths and resources that help her cope with the illness. She explained that in addition to the emotional benefit, Dora also facilitates a number of other valuable strengths. For example, walking Dora provides structure to her day and ensures that she exercises daily. Kathryn did not take any photographs of family members. Instead, she photographed her house from the outside to represent two resources: living independently in the privacy of her own home and the support she received from her daughters, which ultimately enabled her to do so. Kathryn described that her family took over the paperwork related to living in a complex society. Kathryn viewed living in her own home as an important resource because it facilitated a sense of wellbeing by providing a space where she could be herself and did not have to worry about managing her appearance.

Although Kathryn felt betrayed by many of her friends, friends who she could trust undoubtedly were a central strength in her life. Kathryn took several photographs of her friends. She explained that she appreciated her friends for companionship, conversations, and laughter, but that the real value of these friendships rested on the support she received from her friends and the concern and respect they showed. Kathryn took a photograph of a fellow support group member to portray two resources that she considered important: access to organizations that provide services to individuals with dementia and interactions with people who are living with the illness. By the time of our second meeting, Kathryn no longer participated in this support group offered by the local chapter of the Alzheimer's Association. She explained that she did not want to be part of an organization whose policies treat people like her, who are in the early stages of the illness, with disrespect. To avoid future affronts to her sense of self-worth and self-esteem, she had discontinued her membership in the organization. As someone who valued friendships as an important resource, Kathryn believed that she had reason to maintain her current friendships and cultivate new ones. As a result, she made efforts to develop a friendship with her former support group member. Being inspired was another resource that Kathryn treasured. She expressed the important role that being inspired plays in coping with the illness by including a photograph of a man living with disability who she met regularly during her early-morning dog walks and whom she admired for his positive outlook. For Kathryn, Richard Taylor's (2007) book and website were also inspiring resources. She felt that he had been able to describe the dementia experience in a way that she could not and, with his permission, had copied passages from his book to hand out to family members and friends. For Kathryn, 'doing art' was another important strength because it facilitated a sense of meaning and purpose which in turn supported her striving towards wellbeing. 
She included a picture of one of her driftwood sculptures. Kathryn was the only participant who considered the Washington Death with Dignity Act an important resource. She felt that having the option to attain a peaceful, dignified death helped her to live meaningfully.

This study served as a space for Kathryn to explore her life deeply, the sense of wellbeing she aspires to achieve, and what supports her in striving towards that goal. Kathryn was one of the four women chosen for the dyadic interviews. She was especially interested in exchanging ideas with another participant and volunteered her home for that occasion. Kathryn was paired with Monica.

$\underline{\text { Kevin }}$

Kevin is a 76-year-old Caucasian man who lives with his wife Beatrice in their home on the Olympic peninsula where the couple has lived for several years. The couple has two daughters and one son all of whom live out of town. Kevin considered living in a familiar environment a valuable resource because it increased the ease with which he was able to pursue some of his favorite activities (such as shopping). For most of his professional life, Kevin had worked as a researcher in the Navy. After retiring from the Navy in his fifties, he had started an Alpaca business which, at the time of this study, he was in the process of phasing out.

Kevin had undergone and extensive tests designed to evaluate cognitive performance because Beatrice had noticed changes in his memory and overall functioning. Kevin coped with the illness by normalizing it. He did not feel that his diagnosis of 'probable dementia of the Alzheimer type' or the symptoms of the illness had impacted his life and relationships much. He felt that his values had remained the same and that he was able to live more or less the same way he used to before the illness.

Kevin's participation in this study was driven by his wish to make a contribution to research on dementia. He did not seem to want to know much about me or engage in a discussion about this study. Being primarily trained in quantitative research methods, given his professional background as a Navy researcher, he questioned the value of this study because he could not understand how taking photographs and then talking about them would produce research data. However, he decided to participate anyway because contributing to research on dementia was important to him, and since living in rural Olympic peninsula presented little opportunity to do so, he was grateful for the chance. Kevin approached the phototaking activity as an assignment, something that needed to be done. After making a list of the things that he wanted to photograph, he took all twenty-four photographs in one day. Having completed the photo-taking assignment in a much shorter timeframe than was allocated gave him sense of accomplishment and pride. Due to distance, I met with Kevin only twice. Kevin was very 
organized. When I arrived for the photo-dialogue session, Kevin was prepared. The photo-taking toolkit together with a copy of the photo-diary that he had made for himself neatly sitting on the dining room table. He was not much interested in sharing or talking about his experience. The project, he explained "gave me something to do". Drawing out his feelings, observations, and commentary was a project in and by itself. However, Kevin produced an interesting, diversified portfolio that included 24 photographs; his choice of images, relating the past to the present, communicating past and present experiences, produced a comprehensive rendering of his sense of identity.

Among Kevin's photographs were several images that portrayed the important role that his wife plays in his life. As our conversations about his photographs unfolded, I came to conclude that the relationship with Beatrice was Kevin's most significant strength. Kevin told me at one point that he went to bed each night looking forward to the next morning hoping that he could spend one more day with his wife. Kevin took several photographs to portray that he considered engaging in meaningful activities an important resource. When discussing his photographs he explained that in addition to breeding and raising Alpaca, retirement had brought a number of other activities into his life (such as cooking, gardening, and wood working) that continue to provide him with much enjoyment. More importantly, Kevin felt that these activities made a positive contribution to the couple's day-to-day life. Sharing household duties and bringing joy to his wife's life were some of his core values from which he drew most of his strength. Kevin took a series of photographs to symbolize his passion for gourmet cooking. He enjoyed finding and trying out new recipes, shopping for ingredients, using his own garden-fresh vegetables and most importantly, dinning in the company of his wife. Among his cherished possessions was a meticulously maintained collection of favorite recipes which he produced proudly during the interview. Kevin considered himself fortunate because he was still able to drive. He limited his driving to short daytime trips in familiar surroundings; he used his driving ability primarily for food shopping. He also photographed the inside and outside of the couple's home to show the important role that spending time at home played for him in coping with the illness. Time at home meant for Kevin enjoying tranquility, watching nature, looking at artwork that the couple had acquired over the years, and remembering the past. In fact, being able to weave past and present experiences was a strength that Kevin used to construct his current sense of identity. Although Kevin felt that being in charge was an important source of wellbeing, for him, having a sense of control did not entail close scrutiny, tight structure, and monitored progress. Instead, he took control of his days by following a loosely designed daily routine that allowed him the freedom of choice along the way. He felt that the more responsibility he had for making choices, the more he felt in charge. In addition, Kevin liked to work towards long-term goals. At the time of this study, the couple was in the process of planning a trip to South America. Financial security was also important to Kevin's sense of wellbeing. $\mathrm{He}$ photographed a dollar bill to symbolize the importance of material resources. 
Kevin also considered activities that kept his mind and body working an important source of wellbeing. To keep his mind active and engaged, he played Nintendo on a daily basis and from time to time, attended classes at a local community college. About a year ago, he had successfully completed a course in paranormal psychology and explained that he had managed to participate in class discussions and complete the course assignments with the help of elaborate note taking. In terms of physical exercise, Kevin considered basic yard tasks such as mowing the lawn, raking up leaves, and chopping wood the best way to get exercise.

This study provided Kevin with an opportunity to make a contribution to research on dementia, something that was very important to him. In addition, completing the photo-taking assignment facilitated a sense of accomplishment and pride for him.

Monica

Monica is a 59-year old, divorced Caucasian woman. She has a son who lives close-by but visits rarely and a step-daughter who she saw more frequently, touching base at least once a week. At the time of the study, she lived with her ex-husband's grandmother 'Nana' and later on moved to her own apartment. We had the opportunity to meet several times during this study. Our meetings took place at Nana's house, at Monica's new apartment, in restaurants, in my car, and for the dyadic interview, at Kathryn's house.

Monica was extremely likeable, intelligent, and inventive, a truly remarkable person. She enjoyed talking about her journey with dementia and I admired her for not allowing the many bumps in the road to derail her determination to cope with the illness. Our conversations brought to my awareness that regaining a sense of wellbeing while living with early-stage dementia requires hard work, persistence, and a positive attitude. Monica was diagnosed with 'early-onset Alzheimer's disease' while working as an accountant. During our first meeting, Monica discussed her journey with dementia and spoke of some of the difficult times she had experienced. Hard fortune seemed to have followed her unmercifully since the onset of dementia. As the illness started to unfold, Monica had difficulty attributing her growing forgetfulness and poor decisionmaking to illness. It was not until she was fired from her [second ???] job, that she sought medical help and was tested for dementia. By that time she had lost not only her job but also her savings, her retirement account, and her house as a result of the illness. However, Monica had an extraordinary network of family members and friends whose support ultimately prevented her from being homeless. Monica has used the time since her diagnosis of about 5 years ago to reconstruct her life. She successfully applied for Social Security disability benefits, established a trust fund, and secured a low-rent apartment, which she moved into between our second and third meeting. While recounting her moving 
story, Monica did not allow self-pity to override her positive perspective. She remained focused on how she had managed and what she had learned from her experiences. Although Monica clearly missed her professional life, there was a sense of contentment with and gratitude for the life that she was currently able to live.

Monica was immediately drawn to this study and created a portfolio of 27 photographs; she saw her participation in this project as a source of empowerment and a means through which to explore her options. A series of Monica's photographs expressed that setting, pursuing, and achieving goals is a strength that she draws heavily on when coping with the illness. When discussing her photographs, she indicated that working towards goals is, for her, a great way to take charge and to create the life she wants to live, given the reality of early-stage dementia. Her portfolio included images of various types of goals (long-term and short-term) and displayed different stages of goal achievement (those that she had accomplished and those that she was in the process of pursuing). Monica also talked about how achieving goals gave her a sense of accomplishment, which contributed to her wellbeing as it increased her self-respect. How instrumental goals were to Monica's sense of wellbeing becomes also apparent in the way she approaches daily life. Monica described that she started her days by asking herself what she can do today to get one step, however small, closer to achieving her goals. Monica's photographs clearly expressed how her sense of wellbeing was connected to the possibilities that lay ahead for her and her determination to continue to have dreams in life. At the time of this study, Monica focused much of her attention on her primary goal (moving into a new apartment to enjoy her independence 'one last time') but also pursued a number of secondary goals (such as working out daily, maintaining a healthy diet, and keeping a daily life log). Interestingly, although Monica was focused on goals, she considered being focused on the here and now an important strength as well. When discussing her photographs, she highlighted that she copes with the illness by making the best of each and every day for as long as she can. Monica also took a series of photographs to convey that she considers having a daily routine and staying organized important resources. For Monica, having a daily routine represented a commitment and a promise to herself. It set the tone for the day and got her started on the right track. Staying organized helped her maintain her routine. Part of Monica's daily routine is focused on managing her own medication. Monica considered access to and taking dementia drugs one of the resources necessary to cope with the illness. In addition, Monica found that prescribing to a healthy life style which included a mindful diet, daily exercise, and brain fitness workouts also was a valuable resource that helped her cope with the illness. Monica included a self-portrait in her portfolio. The self-portrait served as a reminder to her that self-love can be a powerful source of strength in coping with the illness. Monica took a photograph of her attorney's business card to symbolize the importance of material resources and the value she placed on having access to professionals who supported her in accessing needed benefits. Although 
accessing benefits had been difficult for Monica, she described the interactions with healthcare and social service professionals who helped her in the process as primarily positive.

Monica is the only participant who spoke to 'images not taken'. She explained that being involved with organizations such as the Alzheimer's Association is an important resource although she had not included an image. She explained the lack of this image by talking about the difficulties she encountered when trying to utilize this resource. Her difficulties included living in the countryside with limited funds, decreasing driving abilities, and no access to public transportation. At the time of this study, Monique participated in an early-stage memory loss support group, which required her to negotiate a 40-mile drive. Monica took photographs of photographs to symbolize her relationships with her father and son both of whom, unfortunately, struggled with accepting her illness and provided little support. Monica explained that talking to her father and son about her illness continues to be difficult as they both cope with the reality of her illness by ignoring it. In fact, Monica did not think she could burden her son with any of her problems or concerns and wondered who would take care of her when she would no longer be able to do so herself. In terms of decision-making, Monica felt that maintaining control over decisions pertaining her own life was essential to her sense of wellbeing. For important decisions she consulted with her stepdaughter whom she had designated as her durable power of attorney for health care. Monica did not include photographs of friends. Although she considered friends an important resource, her current circumstances (living in the countryside) were not conducive to maintaining and/or developing friendships. Monica spoke to out-of-town friends from time to time but noticed that, although she really wanted to keep the connection, phone conversations were increasingly difficult for her. From our conversations and her photographs, I felt a sense of loneliness and longing for more connection to other people. Monica explained that she considers time alone a resource that empowers the internal process necessary for coping with the illness but that participating in this study had brought to her awareness that she wanted to reach out and make more of an effort to connect with family, friends, and the community at large. In addition, Monica felt inspired by some of the new ideas that were sparked by our conversation and was looking forward to making these part of her goals and daily routine.

Monica was one of the four women chosen for the dyadic interviews. She looked forward to meeting other participants to exchange ideas. She was paired with Kathryn.

\section{Odette}

Odette is an 82-year-old, widowed Caucasian woman who had worked as a registered nurse for most of her working life. At the time of this study, she lived with her dog 'Sweet Pea' and her cat 'Nelli' in the same small house that she have 
lived in for more than 30 years. She has two sons. One of her sons lives close-by, the other in Portland but visits weekly. Odette was the first participant I interviewed for this study. She was incredibly friendly and easy-going and took great interest in me and this study. Our meetings took place in her home. Sitting in her small dinning room, I came to understand that everything about Odette's house and the feeling it invoked in me was a reflection of the contentment she had found in the everyday doings of her life. Odette was happy with her life, eager to have more of the same.

Odette was told that she had 'the early stages of senile dementia' during a routine check-up with her general care physician about three years ago. Odette seemed untouched by her diagnosis as if she had made a careful and deliberate decision not to let her life come unravelled by the illness. What she missed most was the strength to fix things around the house and maintain a full-blown vegetable garden like she used to.

Odette was fascinated with the prospect of taking photographs for this study. She immediately felt inspired by some of the ideas sparked by our initial conversation and was looking forward to recording her experiences visually. Odette took her first photograph even before I left after our first meeting. However, although eager to follow the study instructions to the dot, Odette only managed to take 11 images. Initially, Odette was somewhat disappointed with her performance but quickly found a creative way to compensate for the lack of images by talking about the photographs she had planned to take but never got around to do. Odette took two photographs of her pets to show the important role that 'Sweet Pea' and 'Nelli' play in her life. She explained that their companionship has been enormously comforting to her and that she draws strength from the sense of continuity they provide. Five of Odette's photographs portrayed family members. To symbolize the emotional support she received from her family, Odette took two photographs of her son who lives in Portland but spends every weekend with her. Odette considers her son's weekly visits a major source of support and stimulation. She enjoyed going to the movies with her son enormously and did so every Friday sitting through three of four movies at a time not because she liked every one but she thought being exposed to different images and new ideas contributed to her mental fitness. Odette dedicated the remaining three family pictures to represent the practical support she receives from her family. She took two photographs of her son mowing the lawn and one of her daughter-in-law delivering a home-cooked dinner. When discussing these images, Odette reiterated how much she treasured this support as it ultimately enabled her to remain in the comfort of her own home which she considered essential to her sense of wellbeing. In fact, at the time of this study, remaining in her house as long as she could was Odette's primary goal.

Odette's house was pleasantly cluttered with the papers, books, photographs, and mementos of life. She seemed too busy living life to waste energy on neatness. 
To symbolize that she was always able to find things she could be interested in, Odette included two photographs of her favorite activities in her portfolio, letterwriting and gardening. Pursuing these meaningful interests clearly contributed to her sense of wellbeing. Interestingly, Odette thought of household chores in much the same way as she did of other creative activities she pursued. Finding richness in her daily doings was certainly a strength that Odette truly valued. Odette followed a loosely designed daily routine and valued being in charge of her day. In terms of making important decisions, Odette viewed her sons as an important resource. She consulted with them, considered their advice, but ultimately, made her own decisions. Odette explained that she had planned to take a photograph of the local senior center where she had taken several courses and volunteered her time. For Odette, programs for senior citizens were a resource that she treasured and that enhanced her quality of life by providing social interactions as well as mental stimulation. Odette also wanted to take photographs to represent her belief in the value of a healthy diet and regular exercise. At the time of this study, Odette met three times a week with a neighbor maintaining a walking routine and was in the process of becoming a vegetarian. Spirituality was also a strength that Odette drew on. She explained that she prays often, largely in a non-formalized way that is hers alone.

For Odette this study stimulated three areas of her life. What she valued most was the mental stimulation it provided. Odette was so taken by photography and the camera that I had given her that she was planning on buying a disposable camera to continue the project by herself. To support her enthusiasm, I sent her two cameras by mail. Odette felt that this study provided her with an opportunity to share what she had learned in life and made her grateful and more aware of the beautiful life she was living.

\section{Romilda}

Romilda is a 76-year-old, widowed Caucasian woman who, at the time of this study, lived alone in one of two apartments in her own house; the other was rented to a long-term tenant with whom she maintained a loose friendship. Romilda considered being able to live in her own house a major resource as it allowed her to pursue two of her most valued activities, painting and gardening. Her house featured a garden cottage that Romilda had turned into studio space and where she spent large parts of her days. Romilda has a daughter who lives out-of-town but calls her several times a week and visits frequently. I knew Romilda because I had interviewed her as part of another study two years ago. However, Romilda did not remember me. Romilda was no longer able to recall the details of her diagnosis of "mild Alzheimer's" but remembered that she had noticed changes in her memory several years prior to undergoing medical testing.

For Romilda, being diagnosed with 'mild Alzheimer's' did not take away her sense of freedom, expression, and action. Romilda had worked most of her professional life in an office. After she retired, she picked up painting as a hobby. 
At the time of this study, she was a well-respected member of the local art community and contributed regularly to regional art exhibits. Romillda was fascinated with the idea of creating a portfolio of her experiences. She approached this study with a lot of passion and created a portfolio of 22 photographs. Some of her photographs portrayed the important role that her artwork played for her in coping with the illness. For Romilda, 'doing art' and being creative was clearly a strength that supported her sense of self-worth and facilitated a sense of meaning and purpose.

Retirement had brought a number of additional activities into Romilda's life that she considered important resources for coping with the illness and that provided her with much enjoyment. At the time of this study, Romilda's hobbies included reading, gardening, hiking, and making bead necklaces. Romilda portrayed that she viewed the close contact she maintains with her daughter a major strength by including a series of photographs of a recent day-hike. Although her daughter was not involved in her day-to-day life, Romilda drew strength from knowing that she could call her any time and consult with her on important decisions. Romilda took a few photographs to symbolize her extraordinary support system of friends to whom she was immensely grateful. Her well-developed support network of friends contributed to Romilda's active life and provided her with a sense of structure. Romilda met regularly with her friends for lunch and she had joined an exercise class at the local community. Being a member of this exercise class, she explained, addressed not only her desire for physical exercise but also fulfilled a number of her emotional and social needs. When talking about her friendships, Romilda reiterated that she considers being able to cover up her forgetfulness a major strength. To her, it was important not to tire her friends by repeating herself and asking the same question over and over again. Through a series of images, Romilda expressed the important role that different types of memory aids (such as journals, calendars, and memory aids around the house) play for her in coping with the illness. Romilda considered keeping a journal a great way to treasure her experiences and create a record of her life. To her, looking back on her life was something deeply satisfying. It reminded her of the everyday things that were meaningful to her, the good and the bad. To make sure journaling was as easy as possible, Romilda kept her notebook where she would not miss it. She put it where she had coffee in the morning and explained that when she sees the notebook as she sits down to drink coffee, she remembers to journal. At the time of this study, she tried to have an entry for just about every day and recorded a few bullet points for interesting or important things that happened each day. By keeping each day's entry short and simple, she felt, she made journaling so easy that there could be no excuses. A photograph of recent deck repairs in Romilda's portfolio symbolized the importance of material resources and the value she placed on keeping her house in good repair. Living in a house that was paid off, supplementing her income with monthly payments from her tenant, and being able to afford to pay for needed home repairs were resources that provided much comfort to Romilda. 
This study allowed Romilda to realize that she lives a truly meaningful and satisfying life while coping with her illness, something she had not been fully aware of.

\section{Rosabelle}

Rosabelle is a 82-year-old Caucasian woman who lives with 'Kolby', her husband of 52 years, on the Olympic peninsula. At the time of this study, the couple lived in the house where they had lived for most of their married life. The couple has two children (a daughter and a son), both of whom live out of town but visit frequently. Rosabelle worked as a nurse for most of her professional life and at the time of the study, still provided in-home pedicure to one of her old patients. Rosabelle received her diagnosis of 'mild senile dementia' from her primary care physician after expressing concerns about her growing forgetfulness during a routine check-up. Rosabelle coped with the diagnosis as she had with other challenges in her life; she approached it as yet another bump in the road and made the best of things. Due to distance, I met with Rosabelle only twice. Each time, we sat at her dinning room table facing the woods. Rosabelle was warm, amiable, and insightful, and there was an openness to her that made me like her from the moment we met. This feeling seemed to be mutual as she invited me to vacation at her house several times. Accustomed to the give and take of an engaged life, Rosabelle talked with charming frankness about her feelings and experiences.

Rosabelle was excited about the opportunity to take part in a study that would allow her to portray and talk about her life. She considered her participation as an opportunity for self-discovery and self-actualization and approached each activity of this study with a great sense of purpose. Several of our phone conversations during the photo-taking phase of this study centered around her concern to 'mess up' my research by not following the instructions correctly. Rosabelle took 27 photographs. Many of her images symbolized the strength that she drew from being in and observing nature. Coping by being in nature was a strategy that Rosabelle had developed as a child when she escaped difficult situations at home by seeking refuge outdoors. At the time of this study, Rosabelle drew much strength from being a member of a local hiking club, which provided her with an opportunity to go on long hikes and enjoy the outdoors in the company of fellow nature enthusiasts. For Rosabelle, the hiking club was also very much a social group that organized potlucks and regularly went to nearby restaurants after outings. When discussing the hiking club, Rosabelle considered herself lucky; she felt that she was better off physically than many others in the group. When Rosabelle was not hiking, she spent time watching the wildlife in her backyard or enjoyed walking her granddog 'Thor' in the woods close to her house. Rosabelle included a number of photographs of her home in her portfolio to convey the important role that being home played for her sense of wellbeing. Home was a place where Rosabelle could find peace and quiet, a place of rest where she could 
be herself without having to compensate for her memory problems and find richness in her daily life. Rosabelle did not take a photograph that would symbolize her belief that organizations providing services to people with dementia are an important resource. However, during our conversations, she mentioned numerous times that she would be interested in joining a support group for people living with the illness. Unfortunately, none was offered where she lives. There were also no photographs of medications or memory aids in Rosabelle's portfolio. However, she explained that she takes medications regularly and that she attributes much of her mental clarity to taking dementia drugs. In terms of memory aids, Rosabelle felt that memory aids were not much of a practical use in her life. She kept a calendar to remind her on her appointments but generally referred to her husband as being in charge of keeping track of things. The couple kept a loosely designed daily routine, which allowed Rosabelle to feel in control of her days. Having choices was more important to Rosabelle than the certainty that comes from a tightly structured routine.

Rosabelle photographed her husband and one of her children to illustrate that she considered being closely connected to her family an important strength. When discussing her photographs, Rosabelle explained that Kolby is her main source of support and at the time of this study, she had transferred some of her everyday duties such as cooking, shopping, and cleaning the house to him. Interestingly, Rosabelle did not perceive giving up some of her roles and responsibilities as a loss. Instead, she felt that freedom from these obligations provided her with more control over her life as it enabled her to spend more time pursuing activities that she liked. Contributing to a good cause was also important to Rosabelle's sense of wellbeing. She symbolized this by including a photograph of her Friday lunch group and explained that she and Kolby support an 'elderly gentlemen' by taking him to lunch every Friday.

For Rosabelle, participating in this study validated the realness of her experience. She felt valued, genuinely appreciated, and respected and thought that portraying her experiences visually and then talking about her images facilitated a growth experience.

Ruby

Ruby is a 89-year-old, widowed Caucasian woman. Ruby and her husband were involved in a car accident seven years ago, which killed her husband and left her a paraplegic. At the time of this study, Ruby lived with her daughter Beatrice who was her primary caregiver. I knew Ruby because I had interviewed her as part of another study two years ago. However, Ruby did not remember me. Ruby discovered that she had early-stage dementia after the accident. The medical care she received as part of her rehabilitation included extensive cognitive testing, the results of which were 'suggestive of early-stage Alzheimer's disease'. Overall, Ruby seemed to have adjusted well to her dual diagnosis and the resulting life circumstances but occasionally found it difficult to accept that she was dependent 
in all activities of daily living. Ruby appeared to be a living example of the notion that choice remains even in the face of adversity. She seemed to have understood that it is not life circumstances that determine wellbeing but the attitude we assume towards them. Ruby did not shrug away her problems but maintained a positive attitude.

Initially, Ruby was ambivalent toward her participation in this study. On one hand, she wanted to contribute to research on dementia; on the other hand, she doubted the value of her contribution and was afraid that her physical limitations would not allow her to successfully complete the photo-taking activity. However, after a problem-solving session during which with encouragement from me and Beatrice's offer to assist with photo-taking, Ruby was eventually convinced of the value that her participation would bring to the study. In the end, Ruby was wholeheartedly engaged in this study and created an interesting portfolio composed of 18 photographs. She would ask whomever was available at the time to assist her with operating the camera (her daughter, her grandchildren, her private caregiver, and adult day care staff members). I met with Ruby three times. Our meetings took place at her daughter's kitchen table, a place where Ruby, sitting in her wheelchair, spends most of her time. Her daughter Beatrice was present to provide assistance if needed but did not participate in the interview. During the interviews, Ruby was enthusiastic about discussing what was most familiar to her and what played a role in her everyday life.

Overall, Ruby's portfolio speaks to the importance of finding richness in the details of daily life and ordinary situations and how small, everyday interactions can provide a sense of connection. Ruby photographed a flower basket hanging on her daughter's deck to show that although she can no longer spend much time in nature, she enjoys watching the change of seasons and its attending colors and fragrances. Ruby took a photograph of her daughter to show the important role that her daughter plays in her life as her primary caregiver and daily companion. When talking about her photograph, Ruby shared that she feels tremendous gratitude towards her daughter for her willingness to care for her and the kindness and respect with which she treats her. Ruby's portfolio also included a photograph of her daughter's dog whom Ruby valued for the daily companionship he provided. Ruby spent much of her time at home - often in her bed due to danger of bedsores - also took a photograph of that. Ruby photographed the bus that takes her to and from the adult day care to show the important role that wheelchair accessible transportation resources play in her life. The center offers door-to-door transportation at an additional cost but for all her other transportation needs Ruby is dependent on her daughter. Ruby explained she is grateful to her daughter Beatrice who used her financial resources to purchase a wheelchair accessible minivan. The minivan was a vital link that provided transportation to and from medical appointments and enabled her to participate in other recreational activities such going to the movie theater and accompanying her daughter to performances of a small theater company where she worked. 
Ruby took several photographs to show that she considered having the opportunity to attend an adult day care program a major resource. She found interacting and chatting with friends at the adult day care refreshing but mourned the fact that her financial resources allowed her to attend adult day care only once a week. At the time of this study, one of Ruby's goals was to attend the adult day care at least twice a week. She and Beatrice were in the process of developing a more creative use of their finances to achieve this goal. That financial resources played a major role in Ruby's life became also apparent when she spoke about the photographs she had taken of her private caregiver. Ruby expressed concern about being a burden to her daughter and relieving her daughter from her caregiving responsibilities was very important to her. However, her financial resources allowed her to hire a private caregiver only two days a week. Ruby's portfolio also included a photograph of a Hoyer lift in front of her handmade quilt. One of Ruby's hobbies had been quilting, an activity she had enjoyed very much but is no longer able to do. Ruby explained that she had taken the photograph to capture the different times of her life, relating the past to the present. Although Ruby did not include a photograph in her portfolio that symbolized humor and the role it payed in her life, it became clear in our conversations that Ruby had a tremendous sense of humor and used laughter to cope with setbacks, not taking herself or her situation too seriously.

This study provided Ruby with an opportunity to share her story. Simply being listened to and having her experiences acknowledged had been immensely helpful to her.

Sam is a 80 -year-old Caucasian man who, at the time of this study, lived alone in his own apartment. He is retired sociology professor and a lifelong bachelor. I understood that the factor that has given the greatest meaning to his life was believing that all knowledge was connected and understanding relationships between the components was his passion. Sam's curiosity and interest in just about everything was amazing; it seemed to be the life force that kept him going. His way of coping with the illness could be described a forward momentum created by the very process of seeking knowledge, not so much about the illness but about the nature/complexity of life and the interrelationship of its aspects. Sam had been informed by his primary care physician six years ago that he has 'benign senility'. Initially, Sam felt overwhelmed by the diagnosis and reacted with a variety of emotions such as confusion, helplessness, and fear and coped with his diagnosis and the resulting feelings as he had approached everything else in his life. He read everything he could find and knew everything there was to know about the illness. In the process he found that most of the information on his illness was depressing and some of it even wrong. Other information, he felt, might apply years from now or never, so he stopped reading. Sam found that the most difficult aspect of the illness was the loss of control over his life and the 
uncertainty of his future. For him, it was important that he took control of his illness and not allow it to control him. Knowing that he could not change his illness, he eventually came to realize that he had control over his attitude and the perspective from which he viewed his life. He made a conscious decision to adopt a more positive attitude and to reinforce his internal effort by infusing his daily life with positive moments and encounters. Initially, trying to translate this theoretical understanding into a practical, everyday approach had been challenging for Sam. At times, he felt overwhelmed by the task of organizing his life in meaningful ways. Over time and with practice, adopting a positive attitude and striving to be happy made a big difference in the quality of Sam's life. Sam volunteered for this study because he hoped that his participation would provide him with some additional insight and/or practical advice about what else he could do. I met with Sam several times, in his apartment and in a local restaurant. Participating in this study was a challenge for Sam. He struggled to understand how his photographs and our conversations would contribute to science. His primary motivation for participation was that he wanted to help me complete this study. During the photo-taking activity and the interviews, he had a tendency to over-think the simplest of tasks, making them more complicated than they needed to be. Overly concerned about the scientific value of his contribution and how to approach the project in a theoretically logical way, he got stuck 'carefully considering' the smallest decisions. Several of our phone conversations were about logistics and focused on reframing the photo-taking activity into a simple task rather than an overwhelming process. For example, before taking his first photograph, he investigated the workings of the disposable camera because he wanted to take the best picture possible. He ended up having difficulties taking any photographs because rather than following the simple instruction sheet provided, he had to read the fine-print on the camera and was confused by it. Another example was that he put so much thought into the selection of his subject matter and how it would relate to my research question that he ended up taking only 14 photographs. During the interviews he kept asking me what I wanted to know and what he should tell me rather than discussing his photographs. Overall, Sam was a very private person and did not like to talk about himself. During our conversations, Sam enjoyed discussing ideas and theories related to aging, particularly the politics of aging and medicalization of aging. However, Sam considered talking about his daily activities unscientific and therefore not worth talking about.

Sam's portfolio predominantly spoke to the notion of challenges associated with aging in general and dementia in particular and the inner strength needed to take control. Sam's sense of wellbeing seemed to come from taking control over those aspects of his life that he could. He took a photograph of a collection of books, magazines, and journal articles, which focused_exclusively on the negative aspects of aging to show that he needs willpower and determination to combat stereotyped perceptions of aging. A few of his photographs showed that he achieved his positive attitude by maintaining a daily routine that provided a calm 
and yet engaging flow to the day. Sam carefully constructed his days to include a mixture of routine activities and small projects. He included a photograph of a pair of shoes that he had managed to purchase over the internet to express that he draws much strength from setting goals, working towards them, and achieving them. Setting goals inspired him to focus his acquisition of knowledge, striving towards them helped him to organize his time, and achieving them allowed him to celebrate small successes, which in turn raised his level of self-confidence. In other words, pursuing goals not only allowed him to keep his mind active and engaged but also celebrate his competence, both of which were very important to him. Sam also liked to infuse his day with pleasant events and uplifting visual images. He took a photograph of the flowers he had planted on his balcony not because he wanted to show that he likes gardening but to convey that he finds it important to look at something visually pleasing when he drinks his coffee in the morning. Sam photographs speak to the importance of and his ability to create a balance. Achieving a balance between being alone at home where he derived a sense of wellbeing primarily from thinking and reading and socializing with other people outside of his home was essential to Sam. Family relationships played a minor role in Sam's life. Being a devout bachelor, he had developed a different type of support system. He took several photographs to show that his support network consisted of long-term friends. When discussing his photographs, he explained that meeting with friends whom he could trust provided him with a sense of certainty and continuity. At the time of this study, Sam met regularly with his friends for lunch or to pursue other activities such as visiting community exhibits, local arts and craft shows and fairs, or Saturday's farmer's market. Sam's portfolio also included photographs that symbolized the importance of financial resources and access to adequate, affordable health care. Sam was generally outraged at the cost of healthcare for seniors and at the time of this study, was fighting with his periodontist about what he believed to be outrageous charges. Although Sam did not include a photograph that symbolized his belief in the importance of mental and physical fitness, he did consider maintaining a healthy life style an important resource. He exercised frequently, maintained a balanced diet, adhered to a medication regimen, and took over-the-counter supplements. Sam did not include a photograph of his car in his portfolio. However, being able to drive his own car was a resource that Sam truly valued. It enabled him to stay connected him with his friends and allowed him access to those activities that he considered essential to his wellbeing such as grocery shopping and/or making small outings. At the time of this study, Sam was able to operate his own car but limited himself to driving side streets and staying in familiar surroundings.

This study allowed Sam to, once again, take on the role of a professor by helping a student with a project. He was truly concerned about this study's completion and the contribution it would make to science. 
Sima is a 79-year-old, divorced Caucasian woman. At the time of this study, she lived with her cat 'Tina' in a house that she had owned for many years. She has a son who lives in town. For most of her adult life Sima had worked as a clerical assistant; she retired about ten years ago. I knew Sima from a 10-week educational support group for people with early-stage memory loss that I had conducted for the Alzheimer's Association three years ago. Sima recalled attending the support group but did not remember meeting me as one of two group facilitators. I met with Sima three times in her home. Sima was very talkative and had many stories to share about her life, both in Israel and in the United States, and her extensive travels in Asia. It soon became clear that it was important for Sima to tell her story and for me to listen with care and understanding. Sima was not interested in me or the particulars of this study. Rather, she was eager to understand the study instructions correctly and get started with taking photographs. During our first meeting, Sima talked at length about her mother who had lived with the illness for several years, first, with Sima as her primary caregiver and later, in a nursing home where she had died a few years ago. Given her first-hand experiences with dementia as a caregiver to her mother, Sima had tried to learn everything there is to learn about dementia and contribute to as many research studies as she could. She explained that she had not only participated in several studies investigating memory problems but had also attended numerous support groups and educational seminars, initially, as a caregiver and for the past three years, as a person living with the illness. Sima was diagnosed with 'suspected early-stage Alzheimer's disease' after undergoing comprehensive testing, which she had initiated because of the changes she had observed in her memory and overall functioning. She pointed out that she had never felt shame or embarrassment about her diagnosis. Instead, she looked at the diagnosis as a positive step, because she felt now that she knew what the problem was, she could start dealing with it. Sima coped with her diagnosis by identifying and utilizing a number of resources in the community; she reached out to organizations that provide services to people like her.

Sima approached the photo-taking activity with diligence and vigor, paying careful attention the research protocol and following the instructions to the dot. Her portfolio of 25 photographs included a variety of images, reflecting numerous aspects of her identity, her life at home and in the community as well as past and present experiences. Sima's illness did not dominate her life. Instead, Sima saw herself as a person who is living with a challenging but manageable condition, one that requires self-moitoring and self-care, as well as good medical attention. Sima considered being able to self-manage the illness an important strength because it contributed to her sense of control and independence. To portray the patterns she had designed to help her cope with the illness she included photographs of her calendar and the lists she kept to manage her forgetfulness. Having a plan for the day was a strength that Sima drew on daily. Her daily 
schedule included a balanced mixture of rest and activity, both at home and in the community.

Sima took a photograph of her cat to portray the important role that Tina played in her coping with the illness. She felt a sense of calm and peace when petting Tina and her recollection of times watching Tina elicited smiles and laughter. Sima took great pride in her home. For her, spending time at home and recalling memories of past travels were major contributors to wellbeing. She took a number of photographs of furniture and art objects that she had brought back from her travels and explained that decorating her walls with mementos gives her lots of pleasure and keeps her memory sharp. Sima included photographs of fellow support group members in her portfolio to symbolize that having access to programming that target older adults and/or people who experience memory problems is a major resource for her. When discussing these photographs, she explained that she draws strength from being with people who have similar experiences. At the time of this study, Sima attended three different support groups, a 'living alone' group, a 'relationships with adult children' group and an 'early-stage memory loss' group. Sima felt that participating in groups was a great way for her to give and receive both emotional and practical support as well as to exchange information. Sima's portfolio did not include a self-portrait or photographs of family members. She described her relationship with her son as strained. Lacking emotional and practical support from her family, Sima had put a lot of effort into developing a support network of friends. In addition to being an active member of the local senior center, she was involved in a Jewish Temple community. Sima expressed a strong sense of faith in her religion. For her religious practices fostered feelings of optimism and hope and provided a sense of connectedness, which contributed positively to her day-to-day coping. Sima found surrounding herself with inspirational quotes an important resource. As an example she took a photograph of a wall hanging that said "do one thing every day that makes you happy". Sima also photographed motivational refrigerator magnets to symbolize her belief that maintaining a positive attitude is an important strength. Having plans for the future was also an important strength for Sima. Although aware of the progressive nature of the illness, Sima looked at her future optimistically. To visually express how she is taking charge of her future, she photographed an old friend whom she had designated as her durable power of attorney and executer of her will. She also explained that she had reserved herself a spot in a local retirement community where she plans to live when she is no longer able to drive. The retirement community she had chosen offered not only extensive programming but also provided three levels of care (independent living, assisted living, and nursing home care). In talking about her future plans, Sima explained that feeling financially secure was important to her sense of wellbeing as it allowed her to plan effectively for the future. In planning for this move, Sima was in the process of simplifying her life and reducing her personal belongings. She took a photograph of her book case to symbolize that she was in the process of sorting out her books. Downsizing, so she felt, would allow her to remain flexible if her circumstances would change. 
Participating in this study provided Sima with an opportunity to tell her life story, recall and share cherished memories, and feel validated for the efforts she put into planning her future.

Tiffany

Tiffany is a 61-year old, divorced Caucasian woman. At the time of this study, she was living in a rented apartment with her dog 'Igor' and her cat 'Tatyana'. She has two sons both of whom live in town and visit frequently. Tiffany holds a Masters degree in nursing and has worked for over 30 years as an oncology nurse. Tiffany was 56 years old and working as a nurse when she received her diagnosis of 'early-onset dementia of the Alzheimer's type'. Tiffany was one of two participants who contacted me via e-mail the day after she had received the study announcement during a support group meeting. For Tiffany, it was important to share her story almost immediately after we met before she even signed the consent form. She could not wait to get started. She recounted that her first inkling that something was different was when she experienced difficulties learning the required computer skills when her employer switched from paper charts to computerized charting. Initially, Tiffany attributed her difficulties to not being from a generation that grew up using personal computers and approached the challenge as she had approached other challenges in her life; she was determined to overcome it. However, although she fully immersed herself in the task, she was unable to learn the necessary computer skills. Eventually, Tiffany left her job of 30 years because she was unable to perform the required computerized charting tasks and took on a teaching job.

In her new job, Tiffany experienced difficulties fulfilling the required teaching assignments and her memory problems became more noticeable, to her students and fellow co-workers at first and eventually, to her employer. After she was fired from her teaching job, Tiffany no longer interpreted her difficulties within the general context of being stressed and sought medical advice. However, congruent with her life-long coping style and because of financial needs, Tiffany did not give up immediately but tried to find a solution. While undergoing an extensive series of diagnostic testing, which eventually established her diagnosis, she applied for a number of different nursing jobs that required a lower technical skill level. However, each time, she was let go during the new employee orientation phase because she was unable to learn company policies and procedures and perform the required clinical documentation needs. Simultaneously, she applied for Social Security disability benefits, which was granted after her second appeal. Tiffany recalled that she had responded to the diagnosis and her inability to hold a nursing job with a sense of great loss and grief and likened her process to the five stages of grief (denial, anger, bargaining, depression, and acceptance) described by Elizabeth Kuebler Ross in her 1969 book "On Death And Dying". Her initial reaction was "wait a minute; this was not part of my plan'; the changes felt very much like betrayal. Eventually, 
Tiffany made the conscious decision to live her life more vibrantly and meaningfully.

Listening to Tiffany, I noticed that her words came easily as if they were the expression of a long-evolved understanding and it became obvious to me Tiffany had come to a quiet acceptance. Tiffany seemed to have integrated her experiences with the illness into a new philosophy for living. She was grateful for the life she was living and the opportunities it provided for her. Part of her determination to live purposefully was her decision to volunteer for research on dementia. At the time of this study, she had already participated in a number of drug trials and at least one other study investigating the dementia experience. Her decision to participate in so many research project was in part driven by her hope to be in the group that spearheaded a cure for the illness. I knew Tiffany from a 10-week educational support group that I had conducted for the Alzheimer's Association. However, Tiffany did not remember me or the group. I met with Tiffany four times. She preferred to meet at a location other than her home and suggested the offices of the local chapter of the Alzheimer's Association where, at the time of this study, Tiffany volunteered her time on a weekly basis. Tiffany was incredibly bright, lively, and out-going, and very enthusiastic about this study. She loved the idea that she would be able to contribute to another study on dementia and was excited about the opportunity to create and share a portfolio of her experiences.

Tiffany took 26 carefully composed photographs capturing a wide array of personal and social coping resources. The notion of 'self-development' and 'personal growth' figured prominently in Tiffany's portfolio. Her images spoke to the great effort she had put into designing a meaningful and satisfying pattern of living. Her home and familiar environments were important resources for Tiffany, which she portrayed by taking a series of photographs of her apartment and her immediate neighborhood. She explained that, following her diagnosis, she had sold her house, reduced her belongings, and moved into a rental apartment. She had made a careful decision to simplify her lifestyle and downsize. She felt that living with a small, carefully and deliberately chosen selection of objects increased her sense of freedom and control. It increased her selfsufficiency, decreased the stress of having too many responsibilities, and allowed her to remain flexible if and when her circumstances changed. Tiffany seemed somewhat of a loner. While working as a nurse, she had had a small group of work-related friends. However, after she received her diagnosis, she decided to end these relatively loose friendships because she wanted people to appreciate her the way she was now and not compare her to the way she had been prior to her illness. At the time of this study, Tiffany maintained casual contacts with some of her neighbors. Simply meeting her neighbors on her daily walks with her dog fulfilled her need for social interaction and provided her with a sense of security and connectedness. She had informed her neighbors of her diagnosis and felt they were looking out for her. In addition, Tiffany considered being connected to the 
Alzheimer's Association a major strength and at the time of this study, participated in the association's programming and advocacy work. She portrayed this important resource by taking photographs of fellow support group members and explained that she appreciates being with people with the illness because it provides an opportunity for her to be both a 'fellow traveler' and a 'guide.

Tiffany took several photographs of 'Igor' and 'Tatyana' to express the special bond she had with her pets. She often found it easier to confide her feelings to them than to other human beings. Spending time with 'Igor' and 'Tatyana' made her happy. She enjoyed their life affirming attitude and the way they brightened her day and made her laugh. Caring for her pets also enforced a routine that helped her get up in the morning and keep on moving through the day. For Tiffany, family support and planning for the future were interconnected. At the time of this study, Tiffany did not rely on family support but drew strength from knowing that her son Kyle will be there for her in the future. She visually expressed the intricate connection between these two resources by taking a photograph of her son Kyle whom she had designated as her future care partner and durable power of attorney. When discussing this photograph she explained that one of the hardest things she had had to do since her diagnosis was to inform her son about her illness and ask him to become her care partner. Staying fit, physically, mentally, and emotionally was also an important strength for Tiffany. She included photographs in her portfolio that symbolized physical and mental exercise and a healthy diet were part of her daily routine. Tiffany believed that physical exercise promotes general health but also enhances brain health and brain performance. To further improve her mental fitness, she also played brain fitness games on her computer. In terms of diet, Tiffany had lost her sense of taste as a result of the illness and had decided that she might as well eat what is healthy for her. She maintained a balanced diet, which included ample amounts of fresh vegetables and fruit. Nature played an important part in Tiffany's life. She took a series of photographs of distant mountain ranges and large bodies of waters to express how spending time outdoors and reflecting upon nature spurred her imagination, increased her sense of freedom, and helped her to keep things in perspective. Tiffany conveyed the importance of financial security by taking a photograph of an ATM machine. At the time of this study, Tiffany was still able to manage her own finances, something she was very proud of. She gave balancing her checkbook and keeping track of her finances much thought and had developed an elaborate system of checks and balances which protected her from making mistakes. Tiffany also dedicated two photographs to the crucial role that having access to adequate transportation played in her life. Although Tiffany felt that it was important to keep her car for emergencies, at the time of this study, the local transit system was her primary means of transportation. Lately, she had discovered the simple pleasure of going on bus rides with no particular destination in mind just enjoying the scenery as it passed by. Tiffany did not take photographs of some of the resources she considered important, perhaps, because she had talked about them at length during our initial meeting. For example, 
Tiffany considered dementia drugs a valuable resource. She said that it had taken a while for her doctor to find the right combination of medications for her.

Tiffany felt that her current medication regiment kept her symptoms well under control. Journaling was another strength that Tiffany commented on but did not bother to take a photograph of. She had journaled all her life and journaling had taken on an increasingly important role since her diagnosis as a means of studying and clarifying her own thinking. At the time of this study, she was reading old journal entries to see how her thinking had changed over the years and as a way of cross-checking whether what people told her about herself was indeed right.

For Tiffany, participating in this study had several benefits. It provided an opportunity for self-exploration, which she considered a major strength in her life. She was able to share her perspective on living with the illness to which she had quite obviously given a great deal of thought. Lastly, she realized her own resourcefulness in coping with the illness. Tiffany was one of the four women who took part in a dyadic interview; she was paired with Corinne.

$\underline{\text { Uma }}$

Uma is a 58-year-old, single Caucasian woman who, at the time of this study, was living in a rented apartment. She has a son who lives out of town and does not know of his mother's illness. Uma's boyfriend Theo has been a key person in her life for over twenty years. Staying legally single had been a conscious decision for Uma because she treasured solitude, wanted to avoid both the boredom and entrapment she associated with marriage, and to keep her independence while nurturing a relationship. Uma was proud of the fact that she had been able to provide for herself and her son all her life. She had a colorful work history that ranged from working as a commercial fisher woman to maintaining a dog walking business. At the time of the study, she had two clients. Her portfolio included two photographs of the five dogs that she continued to walk every morning. Walking these dogs was an important strength to her because it structured her day and centered her. At the time of this study, one of Uma's goals was to continue leading an independent life that included dog walking as she viewed this activity and the connection to the dogs as the most important, calming, and balancing event of her day.

Uma's mother died of Alzheimer's disease two months ago. Visiting her mother daily had been an important part of Uma's life. Uma took a photograph of a sign for the memory care center where her mother had lived for three and a half years to convey that she is still in the process of grieving. Witnessing her mother's decline had been emotionally challenging for Uma and at the time of this study, she focused much of her energy on saying her final goodbyes. Uma had received her own diagnosis of 'mild dementia' about a year ago when she participated in a research study investigating family members of individuals living with the illness. After her diagnosis, Uma found herself wondering about her own journey with the 
illness and her thoughts and feelings alternated between anxiety and acceptance. Eventually, she acknowledged that she had to face the disruption that the illness had brought to her life and coping for her seemed to be a constant negotiation of priorities.

Uma felt inspired by this study and put much thought in selecting her images and composing her 26 photographs. Her contribution to this study showed that she drew from a diverse set of coping resources. Her overall portfolio spoke to the important role that 'independence' and 'control' played in her coping and how she achieved a sense of continuity and stability by developing personally meaningful and emotionally satisfying rituals and routines. Doing things a certain way had a practical value in terms of helping her to remember but more importantly, expressed how she felt about herself and the priorities in her life. I met with Uma three times in her apartment, which felt like a private sanctuary where not many people are invited to.

Uma took a photograph of her front door to express that her home was an important resource for her. For Uma, home was a safe and comfortable place where she could face the disruption that the illness had brought to her life. Spending time at home allowed her to sort out her feelings and do the inner work needed to cope with the illness, its symptoms and their effects. A number of Uma's photographs captured other meaningful neighborhoods, places, and sites. Uma gave meaning to places by events that had occurred there; each place could have many events linked to it. She drew strength from visiting these sites and reconnecting to the feeling she had had at the time of these events. In other words, places for Uma were links to important memories and became symbols for feeling good, feeling loved, or being at peace. For example, she included three different photographs of the Leif Erikson statue in Seattle. She loved this spot and had walked there many times and expressed that feeling by having her photograph taken in front of the sculpture. The photograph of Theo in front of the sculpture shows that she considers him an important person in her life. Uma drew strength from her relationship with Theo, which she showed by taking a photograph of the couple in front of the sculpture. With another photograph she captured her favorite spot in a local park which signified peace for her. She visited the site weekly and drew strength from spending time in the tranquility of the park. Uma took a photograph of her pills to show that she considers access to and taking dementia drugs one of the resources necessary to cope with the illness. Being able to manage her daily medications meant independence to Uma and she put much effort in diligently taking her medications twice a day. Being in charge of her day and not having too many obligations was another resource that Uma considered important. She followed a loosely designed daily routine in which rituals played a more and more important role. Many of Uma's rituals centered around activities of daily living such as reading her mail once a week or having an afternoon cocktail to mark the end of her working day. Maintaining these and 
other rituals reassured her that she was still in control by giving a time-structure to her life on a daily and weekly level.

Uma took photographs of Theo's cats to show that spending time with pets is an important resource for her. Uma considered these cats her friends and spoke about how interacting with them brought her a lot of pleasure. It brightened her days and influenced positively how she felt about herself. Uma included a photograph of the local library in her portfolio to show that learning is an important resource for her. At the time of this study, she was reading books on Alzheimer's disease and stories about animals with startling abilities to predict seizures and death or detect various types of cancers. Uma also considered being connected to other people with early-stage dementia an important resource. She took a photograph of her support group facilitator to express that being part of a group of people who are in a similar situation was reassuring to her and increased her sense of self-efficacy. She liked the opportunity to share her story and have it validated as 'not crazy'. Uma did not include photographs of friends in her portfolio. However, she did include photographs of her son, her mailbox, and her cell phone to show that she considers being connected to other people an important strength. For Uma, a sense of connectedness was important because it decreased her sense of loneliness and helped her remember her past life. At the time of this study, the social ties she maintained were her membership in the support group and the relationships with her son, her boyfriend, and some out-of town friends. Uma also included a photograph in her portfolio to show the importance of knowing her family history. For Uma, being aware of her ancestral roots provided a link with previous generations and enabled her to stay connected with herself. Uma's portfolio also addressed the importance of access to transportation. She took a photograph of her car to show that, due to an inadequate public transport system, being able to drive was an essential resource for her. It allowed her to continue her current lifestyle of visiting meaningful places and neighborhood parks. Uma did not portray resources that helped her stay organized and manage her life and the illness. At the time of this study, she used a calendar but felt that it may be helpful to develop a chart system to monitor her personal activities of daily living more accurately.

For Uma, this study provided an opportunity to examine how she responded to the circumstances of her life, to recognize areas of improvement (such as nutrition and exercise), and to realize her commitment to areas in which she finds fulfillment.

\section{Vincent}

Vincent is a 74-year-old Caucasian man, who lives with his wife Sheila on their small farm. At the time of this study, Sheila was Vincent's primary source of support and the couple's three children lived near-by and visited often. Vincent's family is a close-knit social unit and although the couple's children are not 
involved in the day-to-day lives of their parents, they nevertheless maintain a strong presence. They look after their parents and Vincent and Sheila feel that they can depend on them. Generally speaking, Vincent was the dominant figure in the family and was treated as the much respected head of the household. Vincent learned about his illness after he suffered a stroke. The medical care he received as part of his post-stroke rehabilitation included comprehensive cognitive testing, the results of which were suggestive of the "early stages of vascular dementia'.

Vincent did not feel that the illness had changed him or influenced his attitude to life. Instead, he considered himself lucky that he had recovered from the stroke and went about his life as he had prior to the stroke. He enjoyed his family and did not think too much about his illness. Due to distance, I met with Vincent only twice. He chose to meet me at a local restaurant where we met for breakfast each time. His choice of our meeting place outside of his home was an extra incentive to participate in the study because food and meals were an important part of Vincent's life. He drew strength from sharing meals with people. Given that at the time of this study he no longer drove, he opted for Sheila to join us in the dialogue sessions. Much to Sheila's surprise, Vincent was immediately interested in participating in this study and was willing to listen to my instructions. Sheila shared that generally, Vincent does not like people to tell him what to do, a character trait that had created barriers to his post-stroke rehabilitation because he demonstrated low levels of therapeutic compliance.

Vincent approached the photo-taking activity with a sense of determination and purpose. He took 28 photographs. The notion of 'simple pleasures' and 'family life' figured prominently in his portfolio. Vincent enjoyed the small things in life, liked spending time at home, and was grateful for the simplicity of his quiet days. He liked his life the way it was and felt that there was no place he had to be (accept being at home with his family), there was nothing he had to do (accept attending to his animals), and there was nothing he was missing out on. For him, early-stage dementia was no reason to give up the small joys of life. For Vincent, spending time at home on the farm was his greatest strength. He took a series of photographs portraying different aspects of farm life. Vincent took photographs of their dogs, horses, and goats and referred to them as 'part of the family'. He enjoyed the sense of calmness and peace he experienced when he attended to the needs of these animals. It provided him with a reason to remain active and lifefocused, fostered feelings of competence and control, and ensured continuity and consistency. For Vincent, taking care of the animals not only provided an opportunity to fulfill a family responsibility but provided routine and structure for his daily life. Vincent had a healthy self-image; he approved of himself, was happy with who he was, and took pride in his family. He took a photograph of his wife Sheila to show that he draws much strength from their relationship. At the time of this study, Sheila had taken over some of his responsibilities in managing the couple's finances and the overall needs of the household. For Vincent, this 
role change was neither good not bad; he just accepted it. Vincent included photographs of family members in his portfolio to portray that he draws strength from his tight-knit family network because it created a sense of certainty and belonging. For Vincent, it was important to be treated with respect and was pleased that his family members treated each other with respect and consideration. He connected the value he placed on 'family' to his Italian heritage, something he was very proud of. Although Vincent did not include photographs that symbolized that his life followed a certain order, it became clear through our conversations that structure and routine played an important role in his life. Vincent deferred to Sheila to provide a basic structure for his days but generally liked to be in control of how he spent his time. Vincent liked to follow a loosely scheduled daily routine that included a balanced mix of activities and rest as well as time spent at home and outside. Simple pleasures also played an important role in Vincent's routine. For example, he included two photographs in his portfolio to symbolize his love for taking naps. Vincent usually took a nap every day after lunch; in the summer he loved to do it in the fresh air outside in the hammock. Vincent took a photograph of a grocery shop to show that his routine also included accompanying Sheila on her weekly shopping trips. When discussing his photograph, it became clear that he drew strength from 'being part of' certain activities, enjoying the activity without having the responsibility of attending to the details. Vincent's portfolio also included a photograph of a car. He was no longer able to drive but enjoyed riding in cars as a passenger. In fact, car rides without a particular destination had proven to be one of his favorite activities. He enjoyed trips around the city as much as he enjoyed more adventurous drives on scenic routes where he enjoyed water and mountain views. For Vincent, it was important to have an adventure now and then. He took photographs of a coin he had found, a lottery ticked he had purchased, and the outside of a casino that he used to gamble in to show that looking out for and being aware of opportunity and chance is a strength that he has drawn on all his life. Vincent's one regret was that he cannot gamble anymore but he continues to enjoy playing the lottery and never forgets to buy a ticket. When discussing his portfolio, Vincent complained that the camera did not have a sufficient number of exposures available. Unfortunately, Vincent was not able to recall all of the photographs he would have liked to take but remembered that he had not been able to photograph some of the couple's weekly activities such attending exercise classes or playing cards at the senior center.

This study provided an opportunity for Vincent to take stock of his life and realize how proud he was of his life and his family. To tell his own story seemed to have been an important experience for Vincent. His participation in this study also showed that there are activities that are interesting enough for him to put a considerable amount of thought, effort, and energy into. 


\section{Appendix U: Poster Boards}

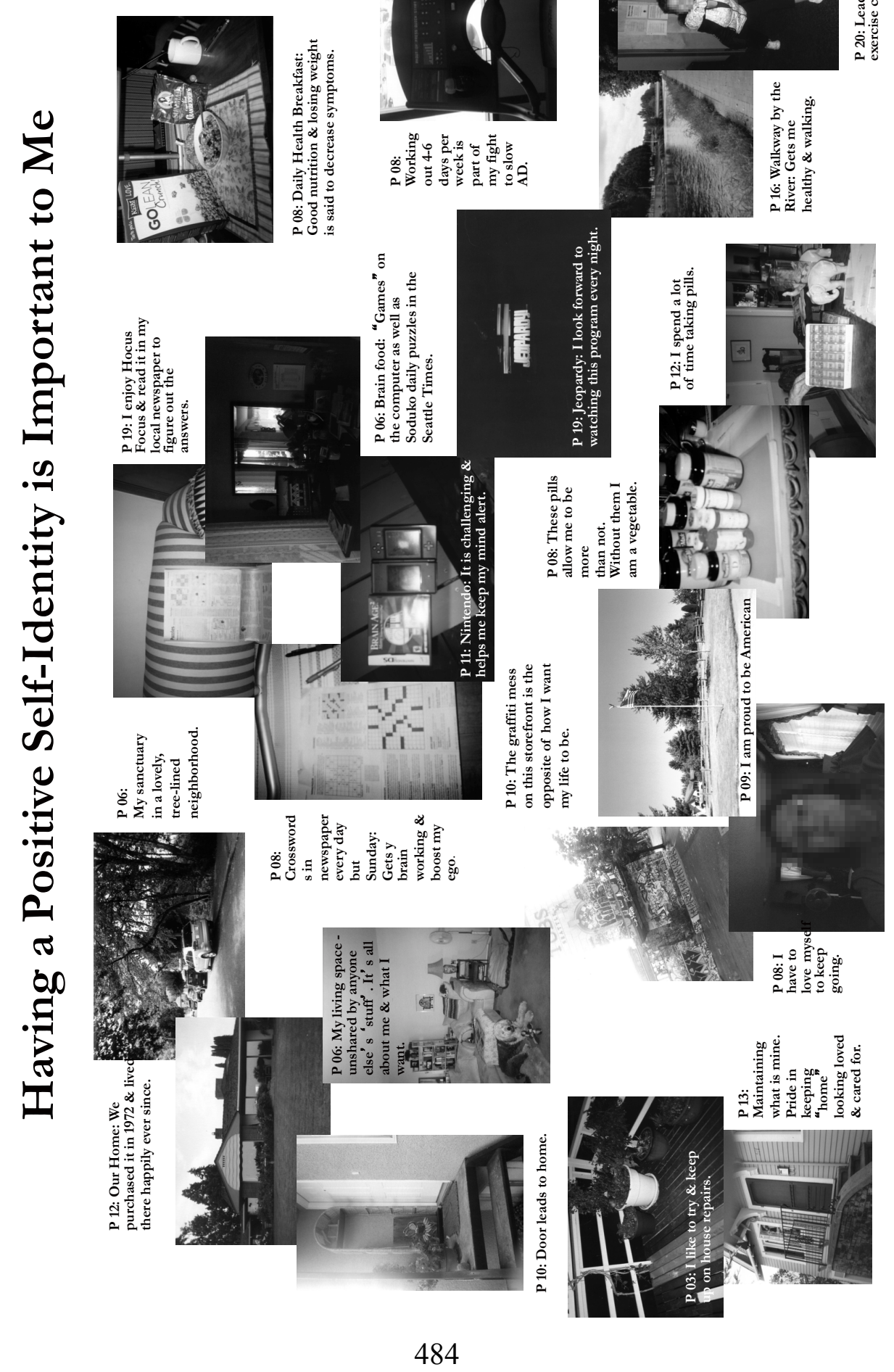




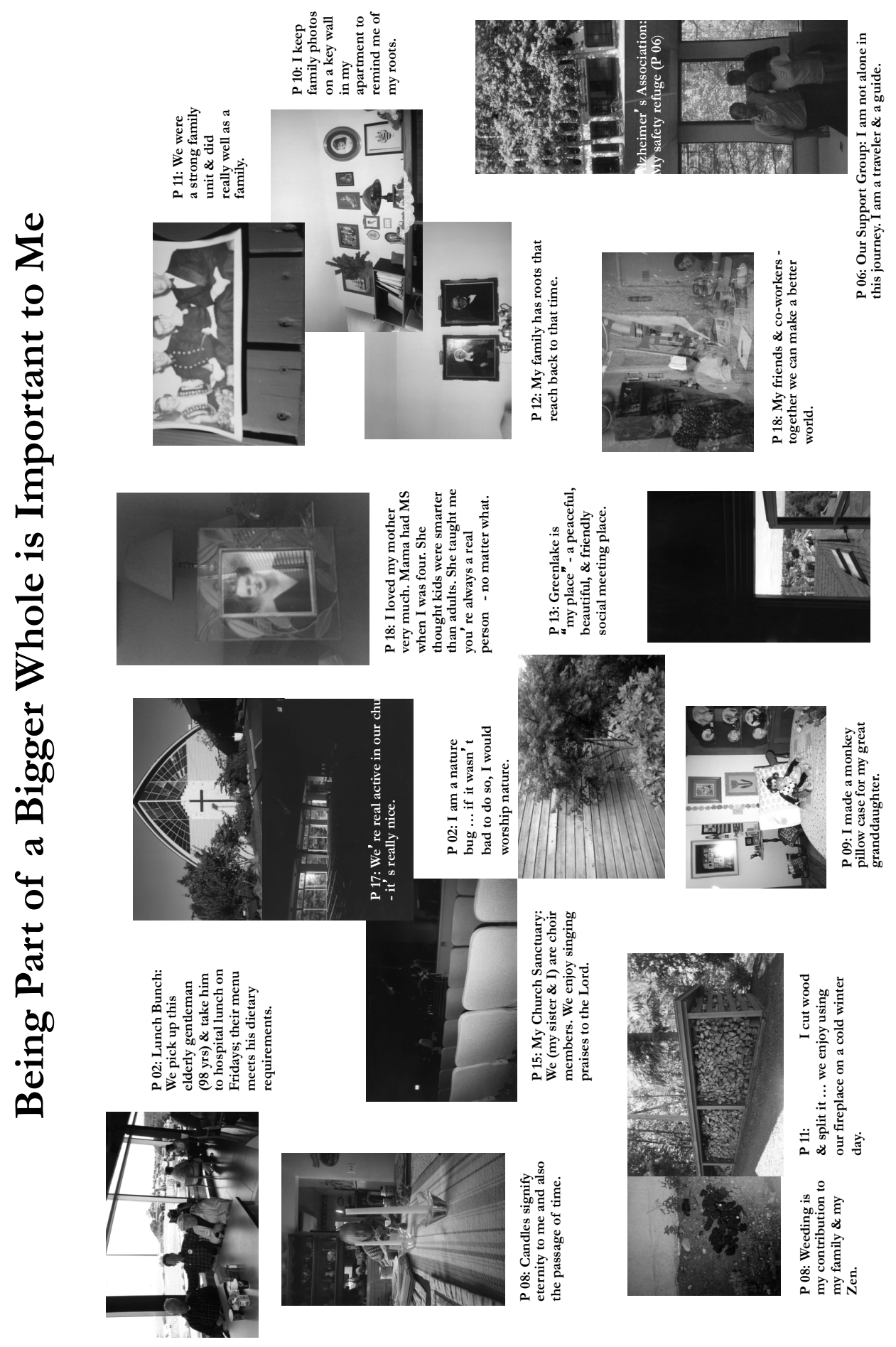




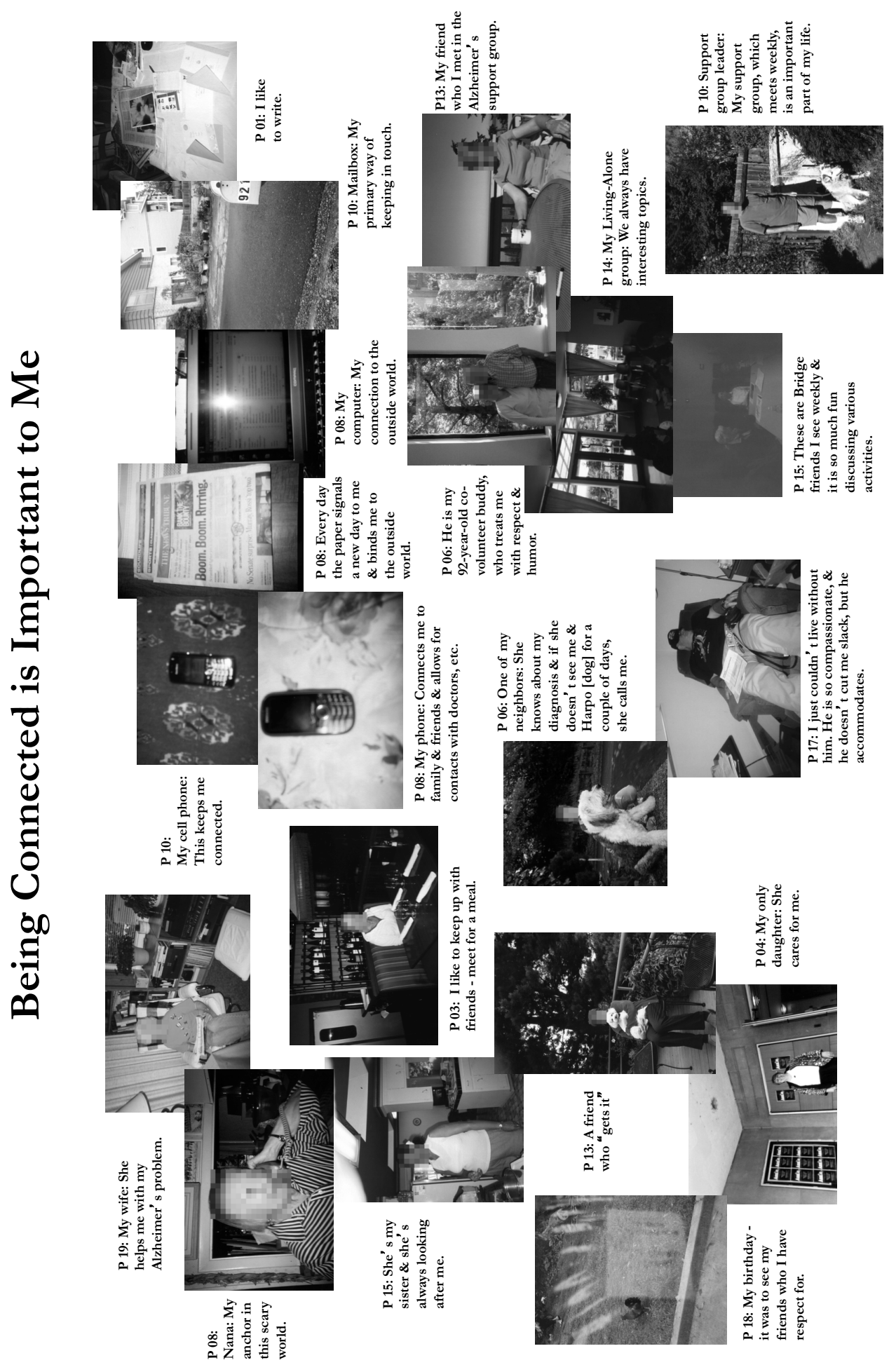




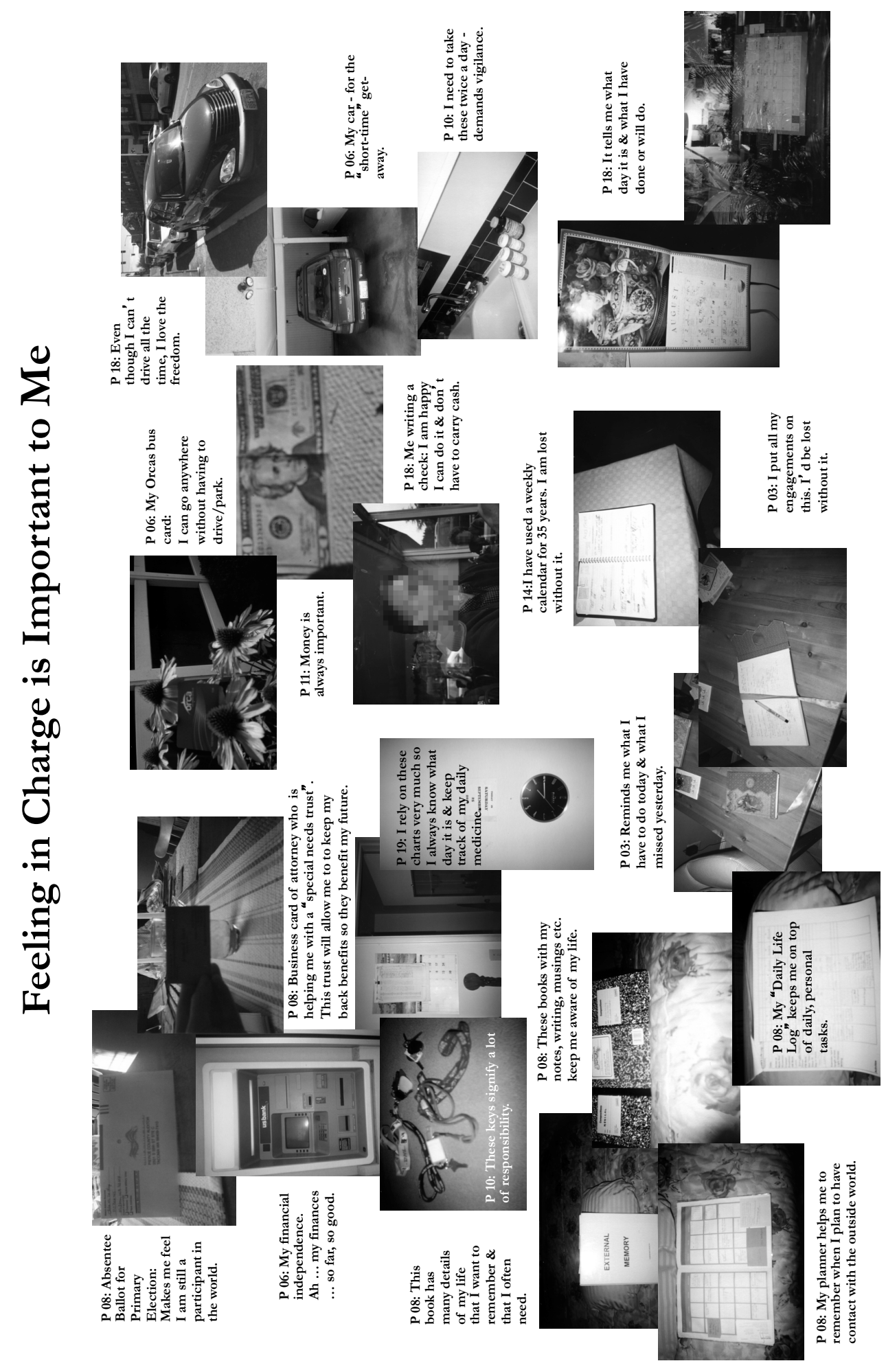




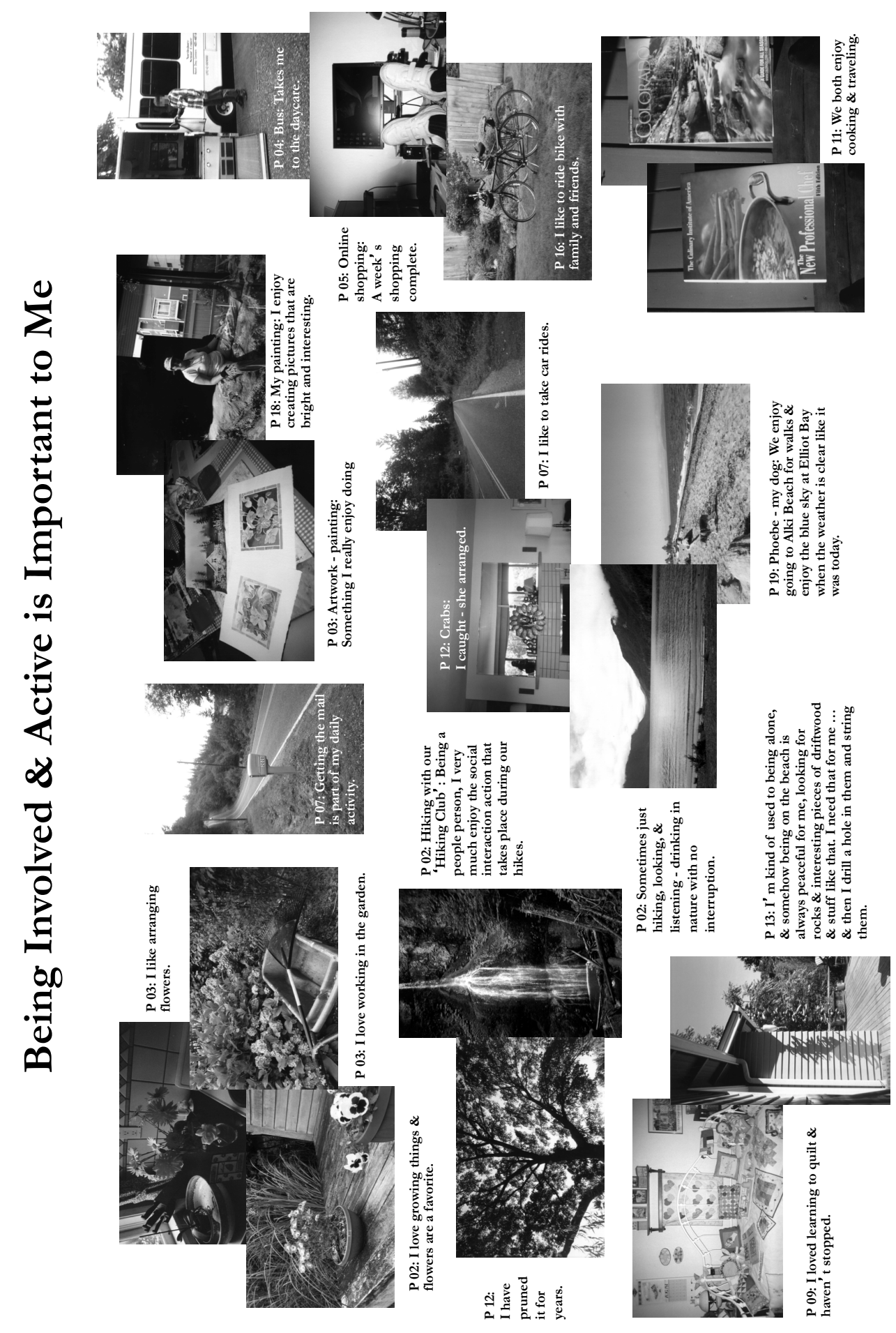




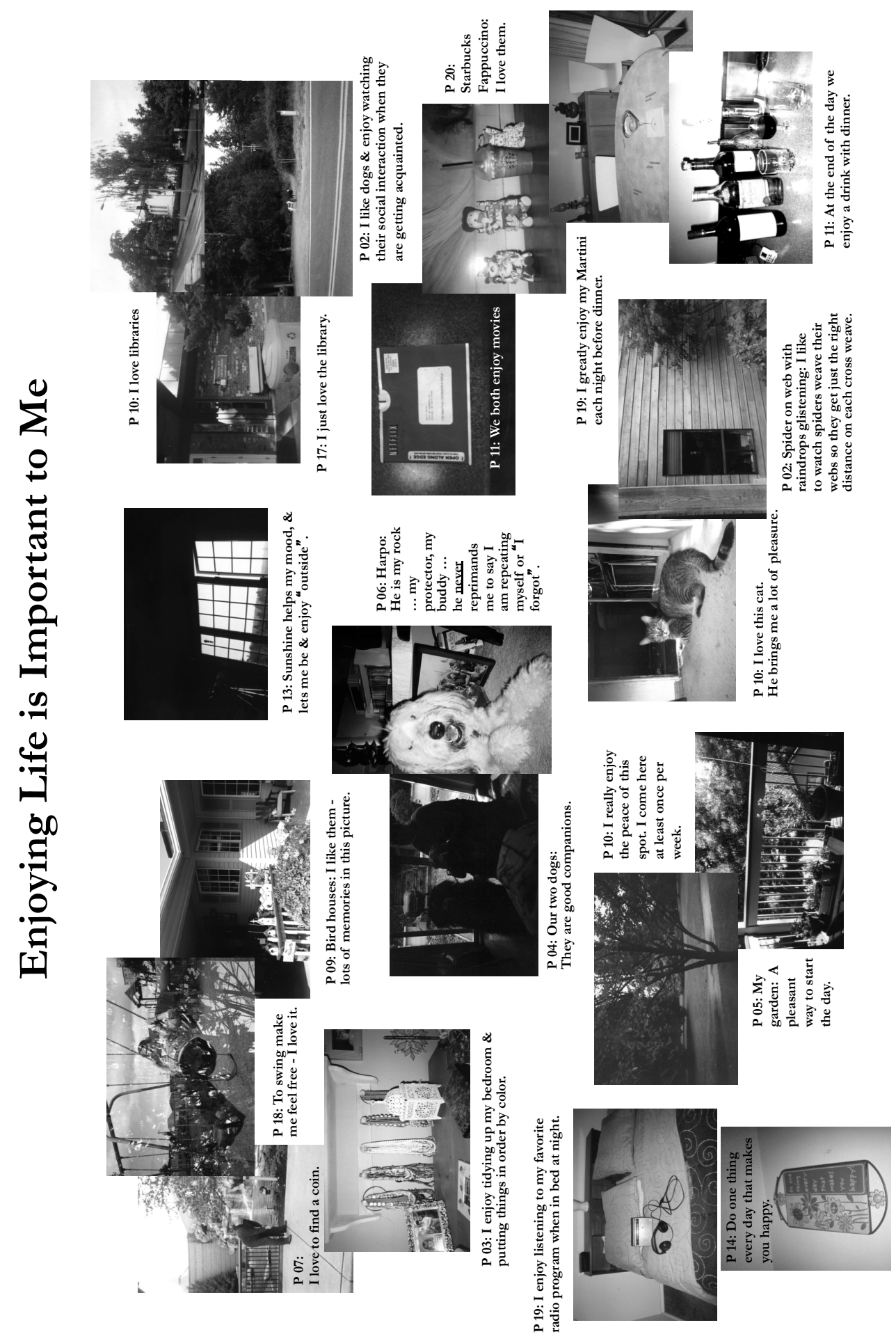


Appendix V: Participant Generated Photographs and Photo-Diary Entries

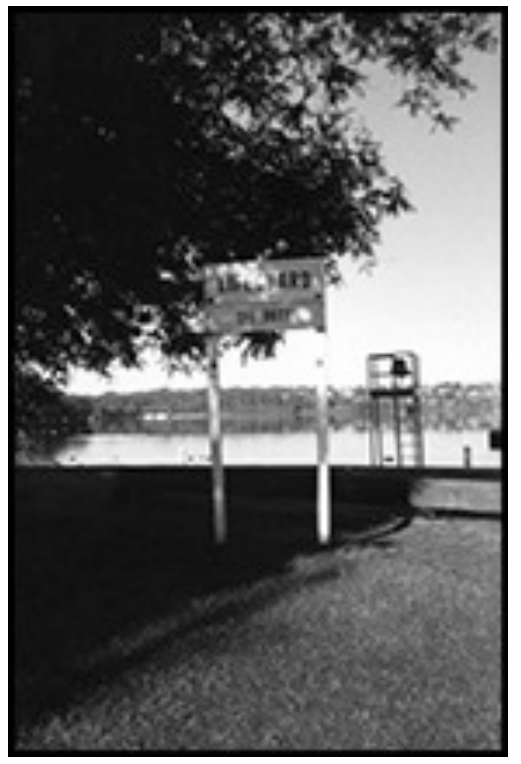

Figure 2: Lifeguard Off Duty: Sometimes I feel like my lifeguard is off duty.

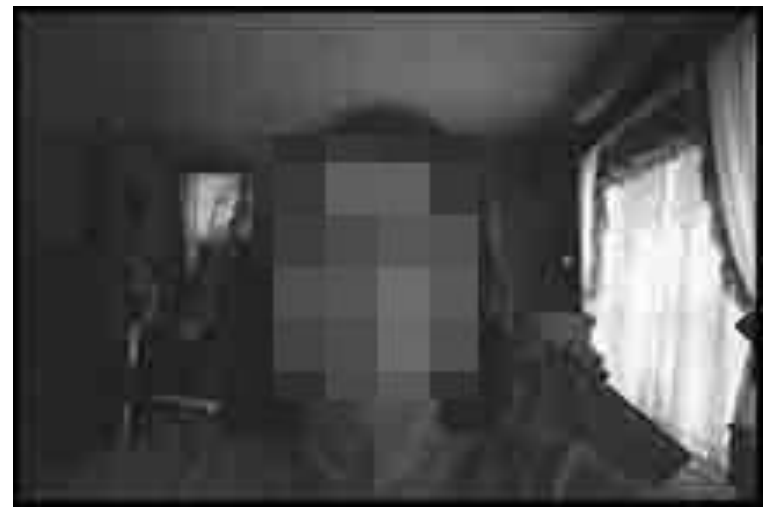

Figure 3: I Have to Love Myself to Keep Going. 


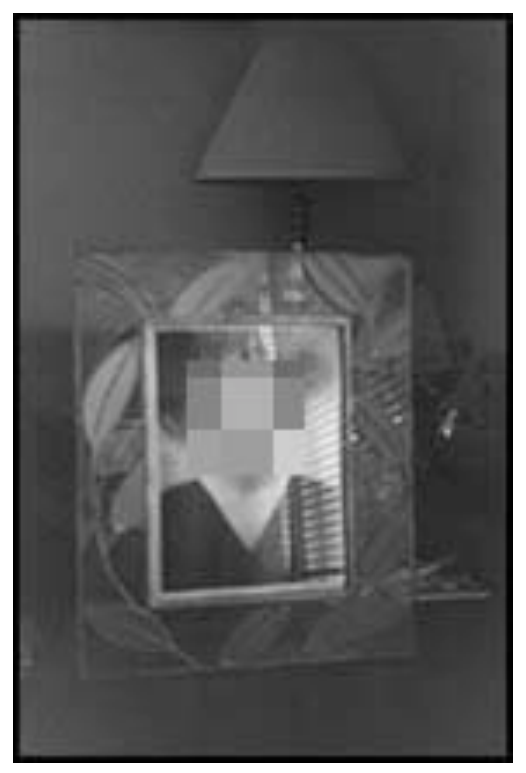

Figure 4: My Mother: She's my role model: You're always a real person - no matter what.

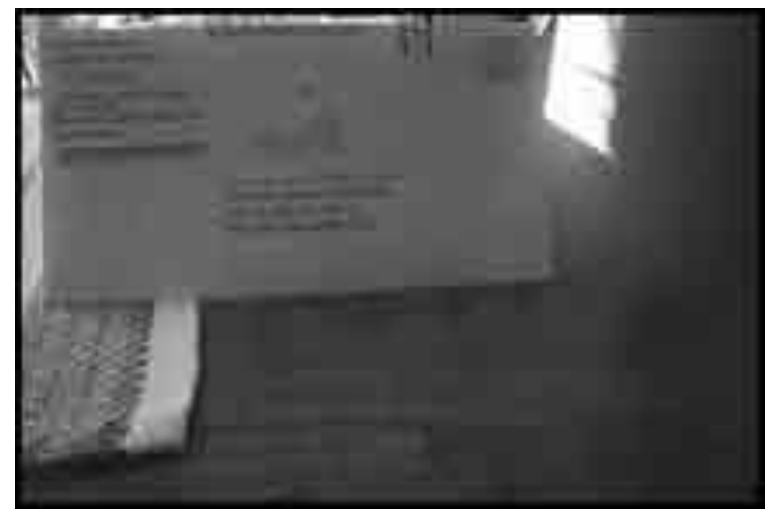

Figure 5: Absentee Ballot for Primary Election: Makes me feel like I am still a participant in the world. 


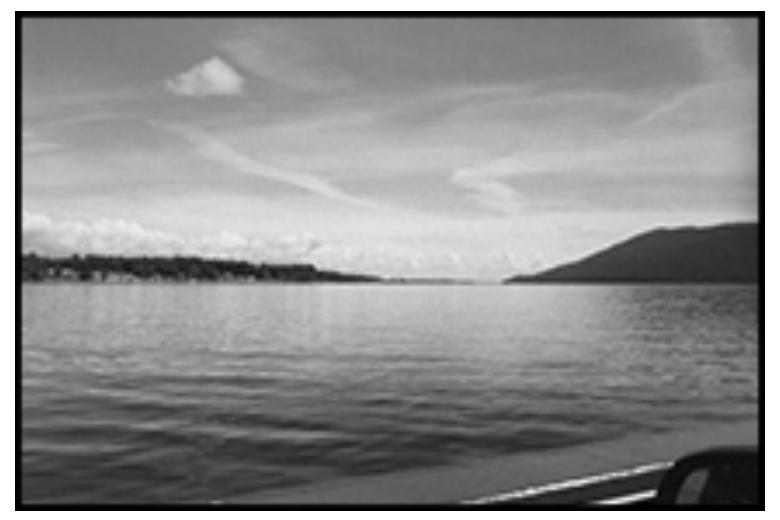

Figure 6: Ferry to Lummi.

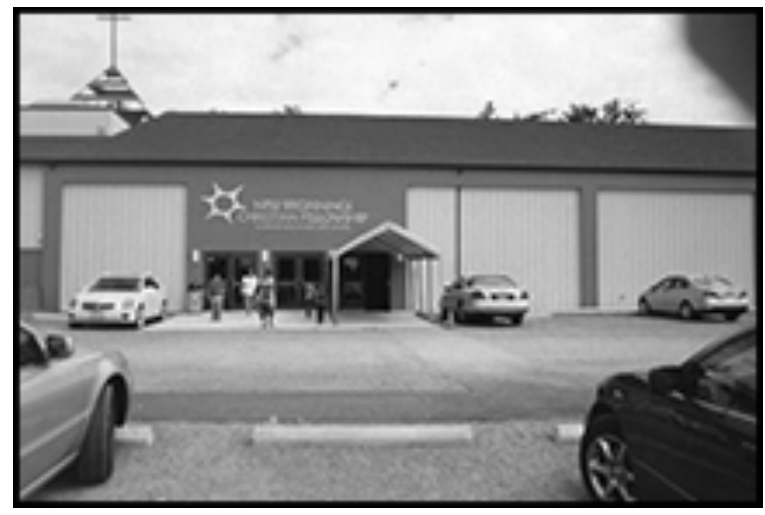

Figure 7: Entrance to My Church: Worshiping the Lord gives me strength to get through each and every day! Praise the Lord - Glory Hallelujah.

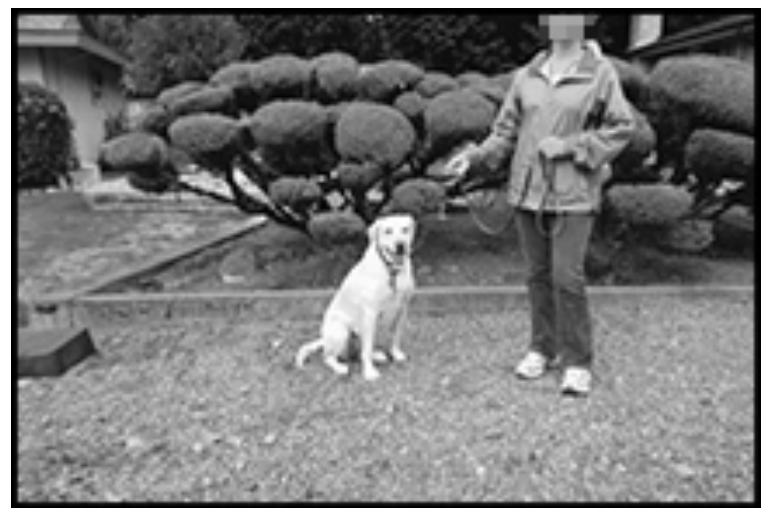

Figure 8: Nina: Took her for a walk. 


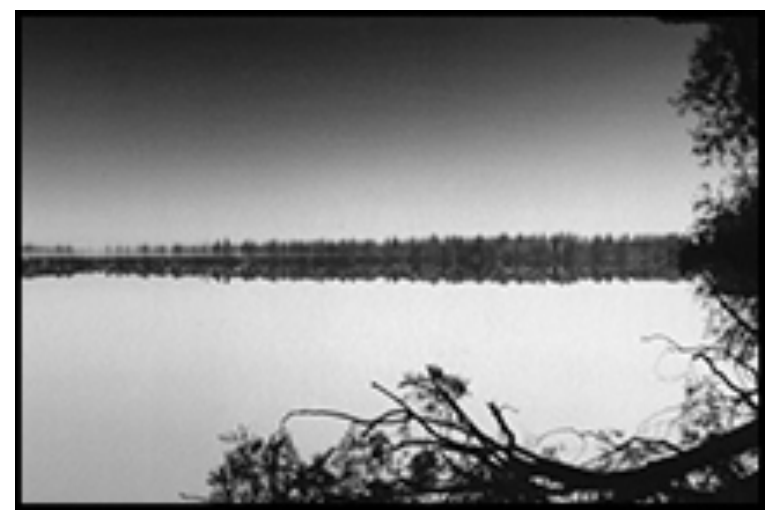

Figure 9: Off-the-Path View at Greenlake: I lost it for a while and then I found it - YES!

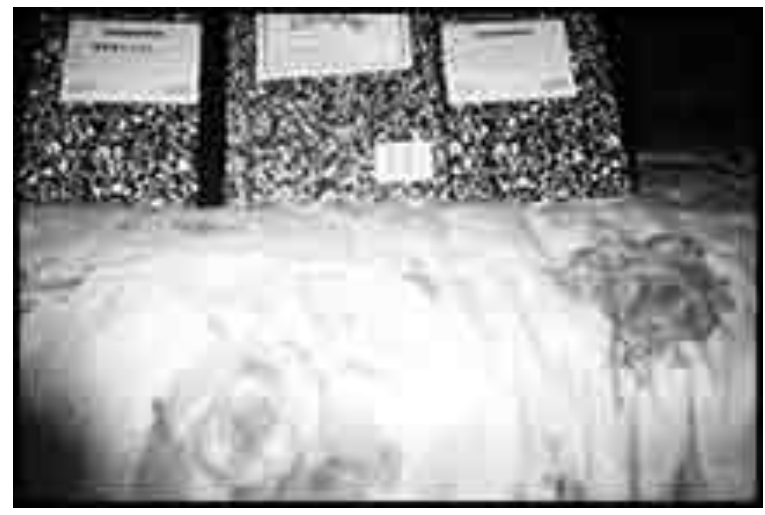

Figure 10: Composition Books: Medical, Diary, and Miscellaneous: These books with my notes, writings, musings etc. keep me aware of my life.

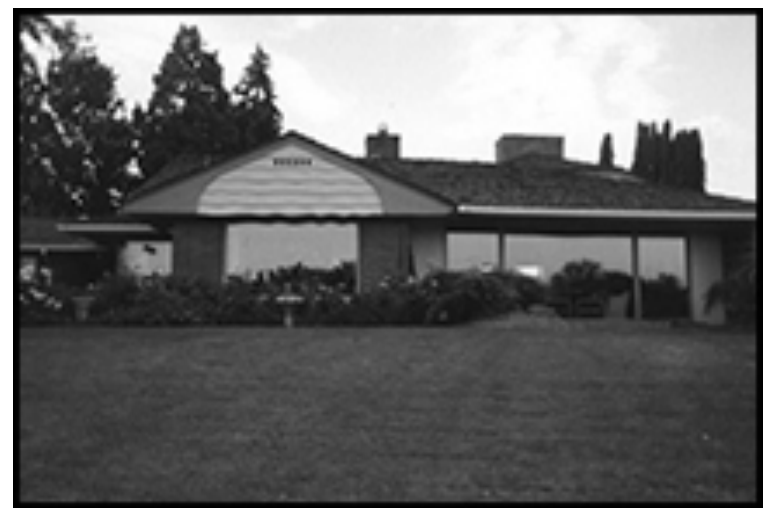

Figure 11: Our Home: The house we purchased in 1972 and lived there happily ever since. 


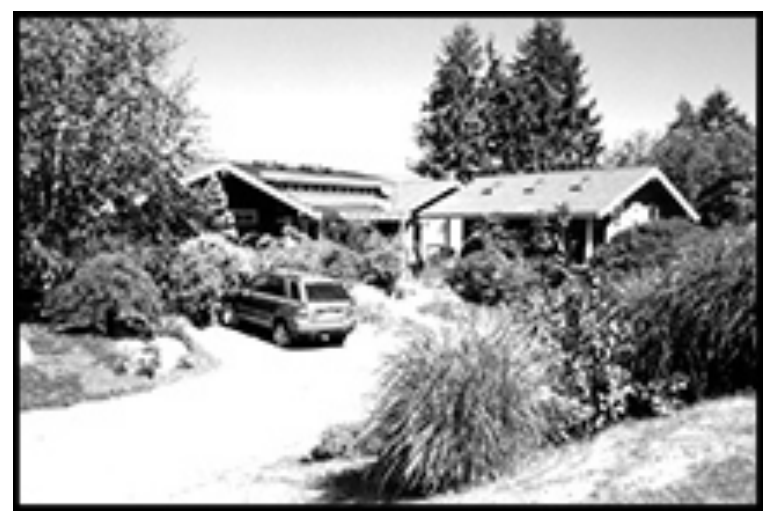

Figure 12: Our Home: It is gorgeous - we had it designed and built for us.

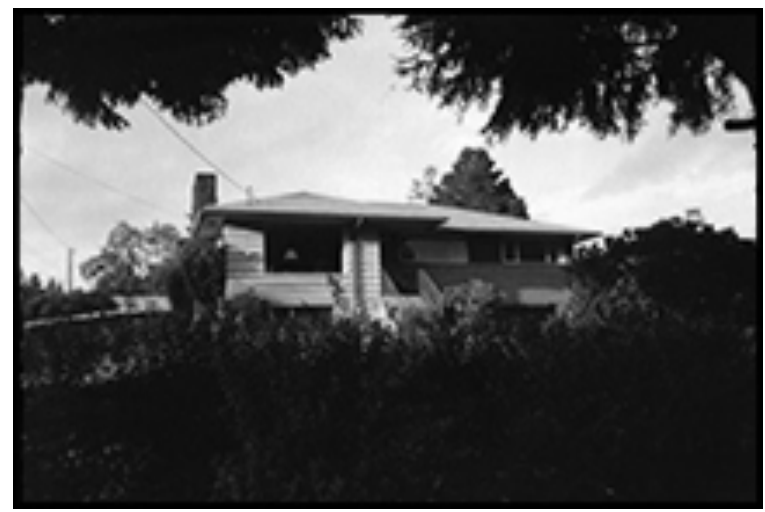

Figure 13: Our House: We have lived here for 52 years and love this area and our neighbors. We're the only ones that ever lived in this house. 


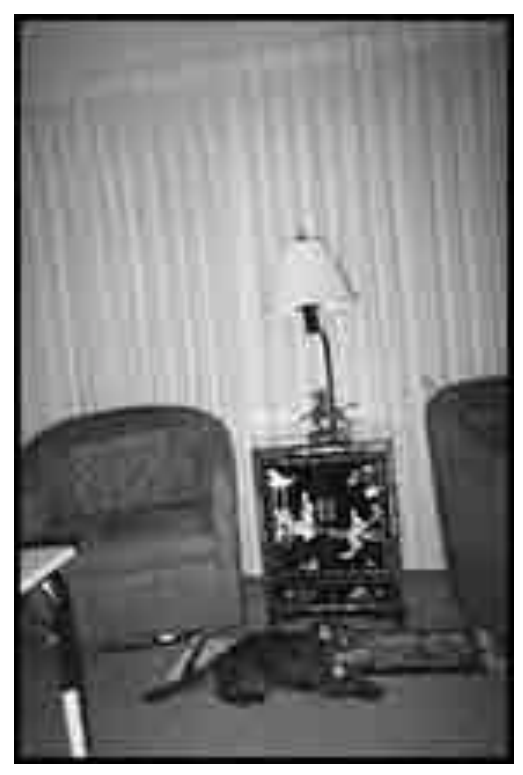

Figure 14: My Favorite Piece of Furniture and Sculpture of Tang Horse on Top: All inlaid and calms me when I look at it. I never get tired looking at it!! Also the Tang horse and the Chinese rug in front. My cat decided to pose in front so I took a picture of all of them. It also reminds what a good shopper I am - got it for less than 1/4 of its value.

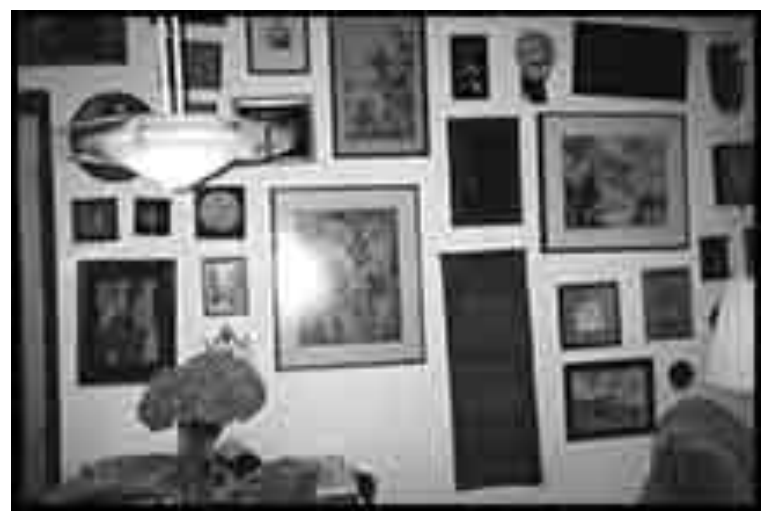

Figure 15: My Living Wall over My Sofa: It has art, pictures, etc. from all over the world. Some I bought, some are gifts. Every time I look at it I remember where I got it, the occasion. It helps to appreciate my life and keep my memory sharp. 


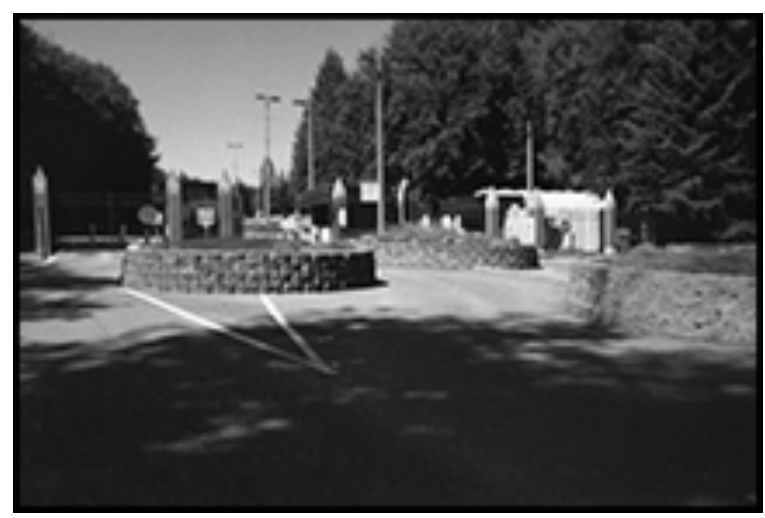

Figure 16: Navel Sub Base - Bangor: It reminds me of my career in the Navy.

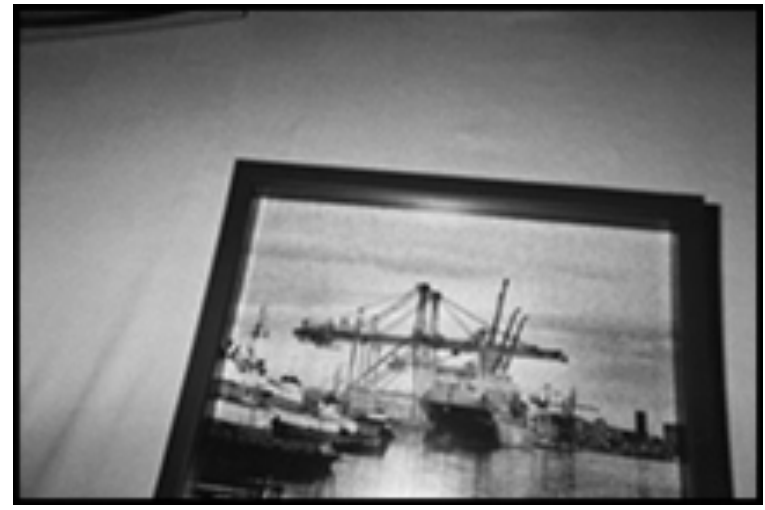

Figure 17: Crane: My job was a crane operator for 40 years on the Seattle waterfront.

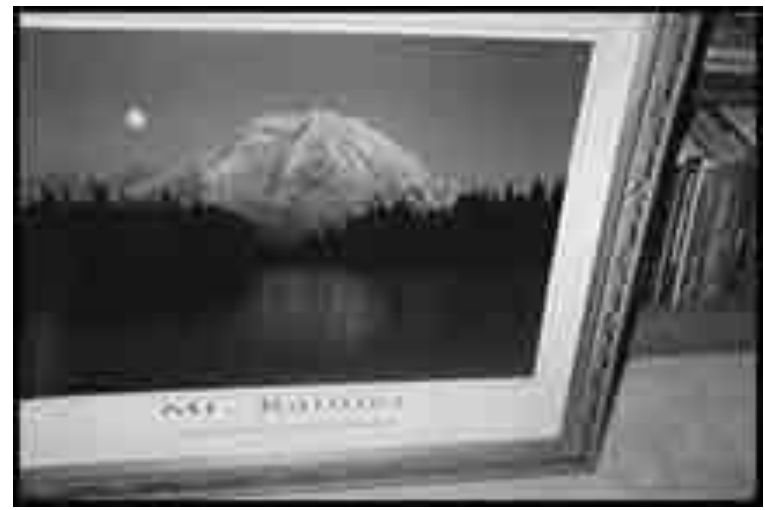

Figure 18: Picture of Mount Rainier: Climbed it three times - twice with my daughter. 


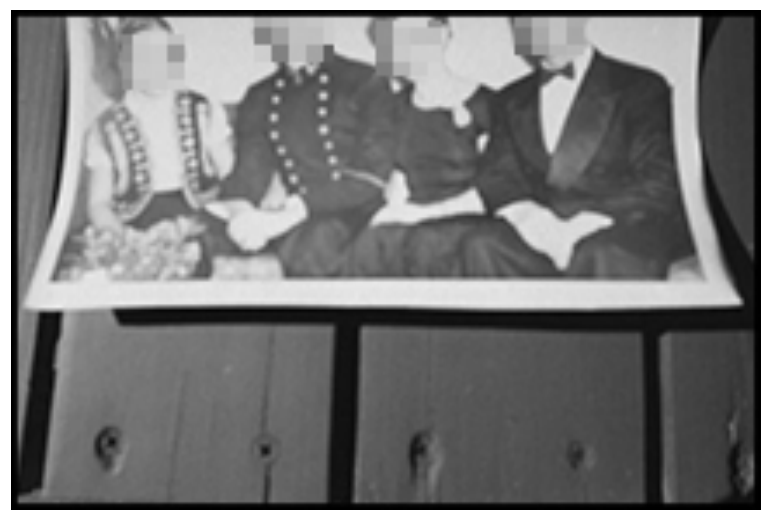

Figure 19: My Sister Mary, Me, and Mom and Dad in 1953: We were a strong family unit and did really well as a family.

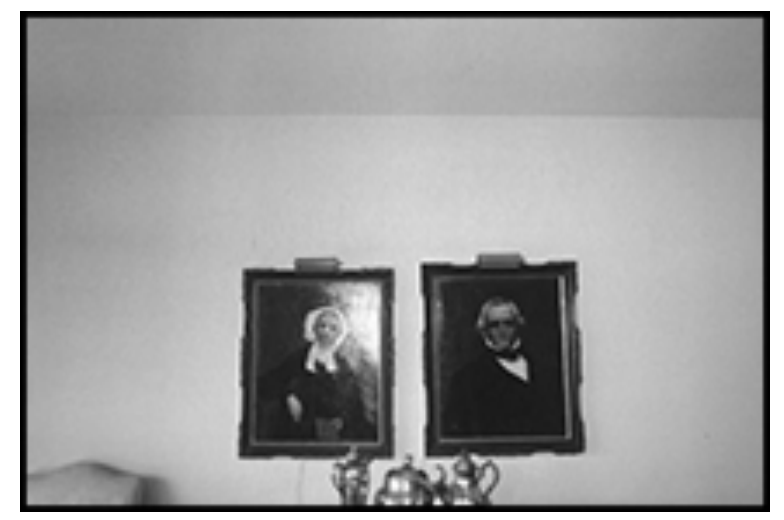

Figure 20: Paintings George and Johanna Klint: Bankers in Albany, NY mid 1800; had four sons, one daughter. Two were National Guard - came to California during Mexican War: Our family has inheritance back to those days. 


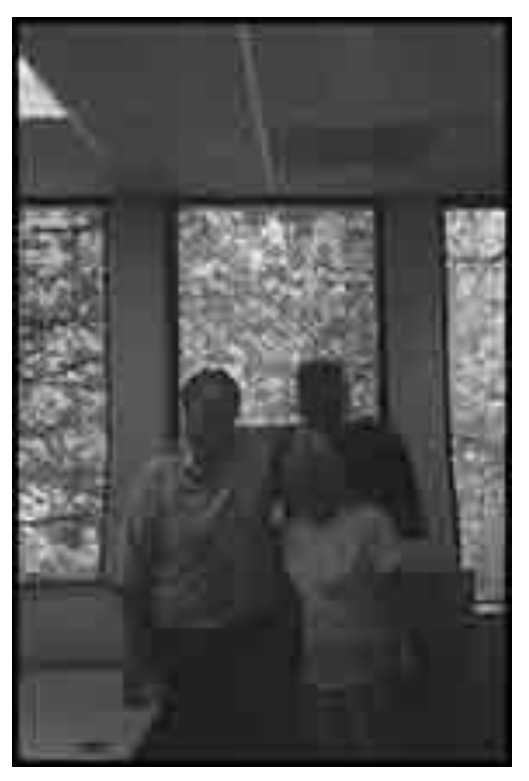

Figure 22: Our Support Group: I am not alone in this journey. I am a traveler and a guide.

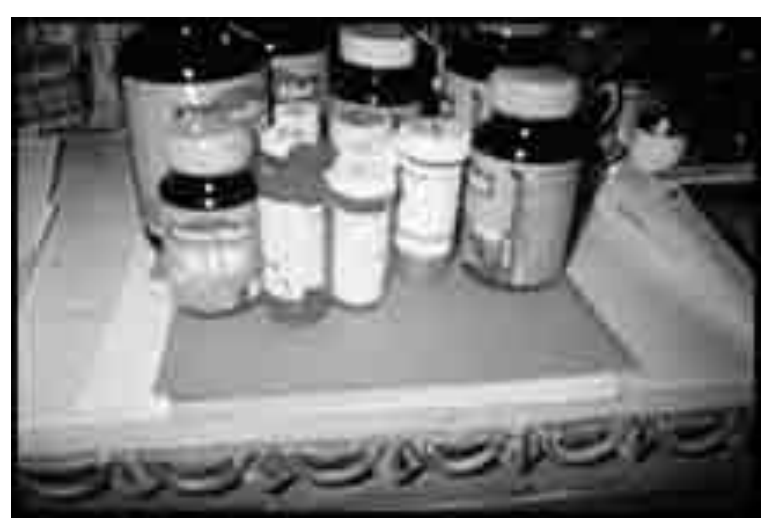

Figure 23: My Medications and Supplements: These pills allow me to be more me than not. Without them, I am a vegetable.

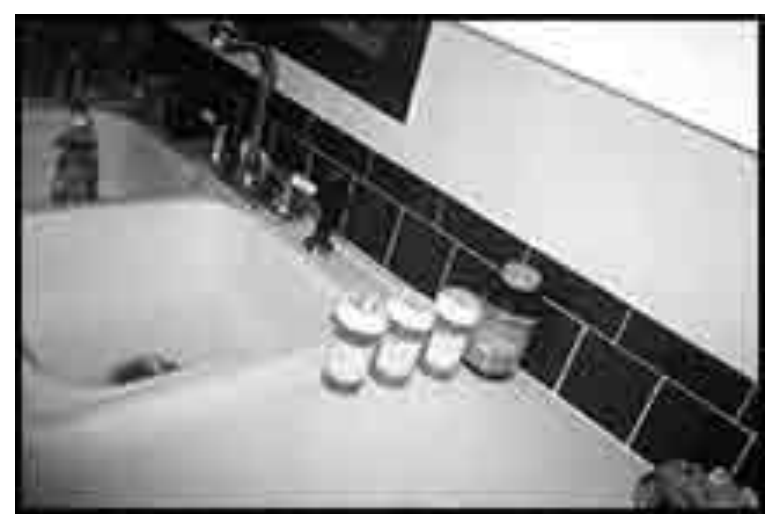

Figure 24: My Pills: I have to take these twice a day; demands vigilance. 


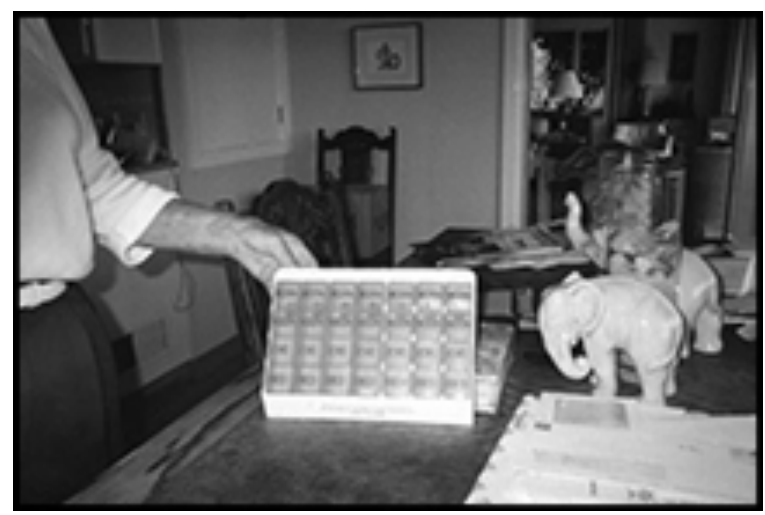

Figure 25: Daily Pill Box: I spend a lot of time taking pills.

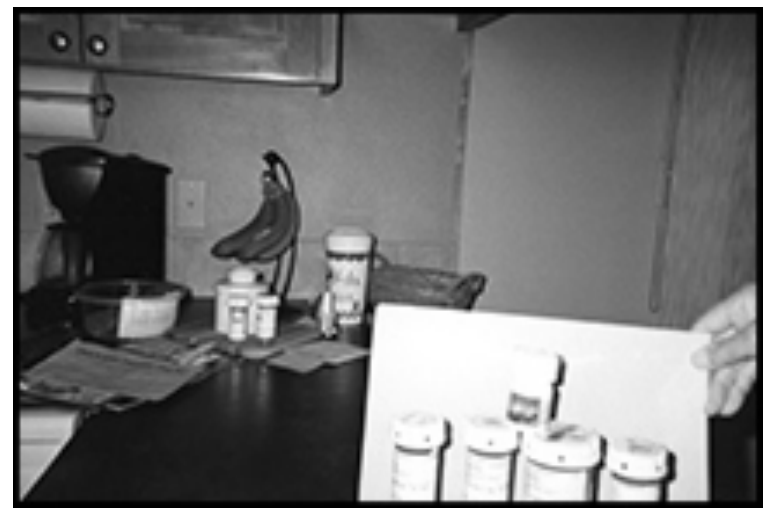

Figure 26: My Pills: For my health.

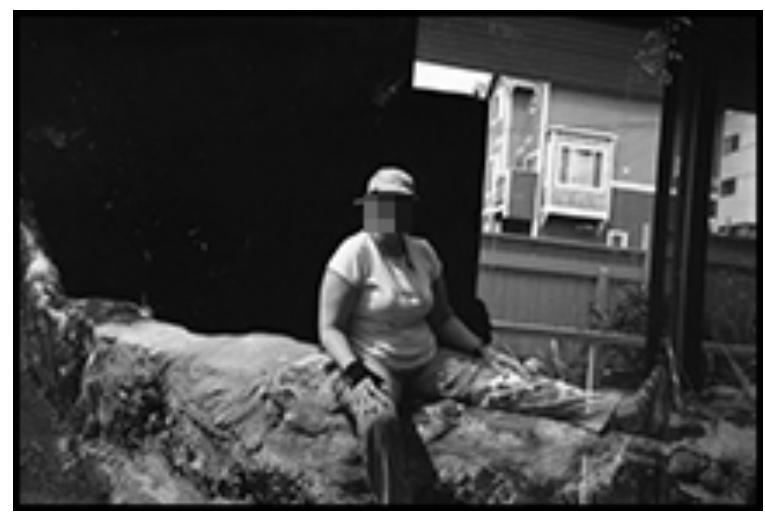

Figure 27: My Flowers on My Deck: They make me happy for the gorgeous colors. 


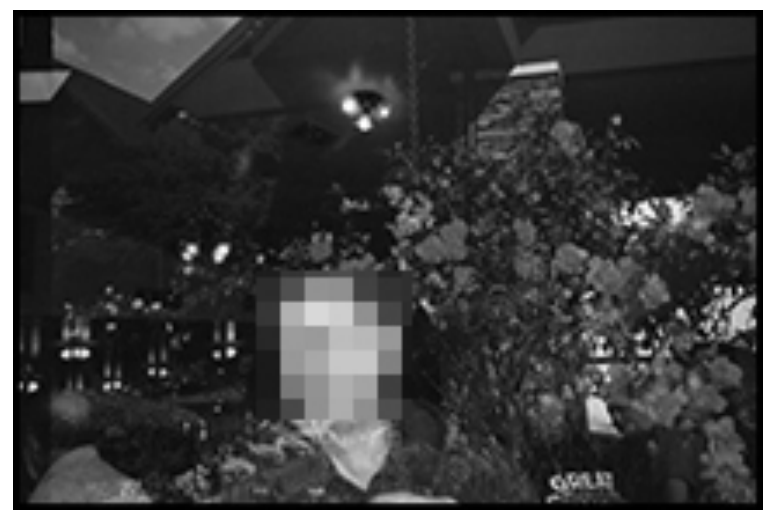

Figure 28: Roses: They are pretty; the colors are bright.

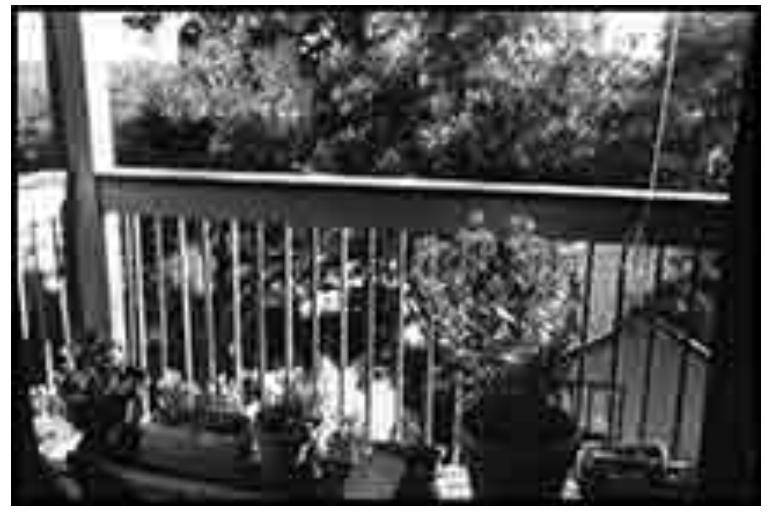

Figure 29: My Garden: Visual pleasure.

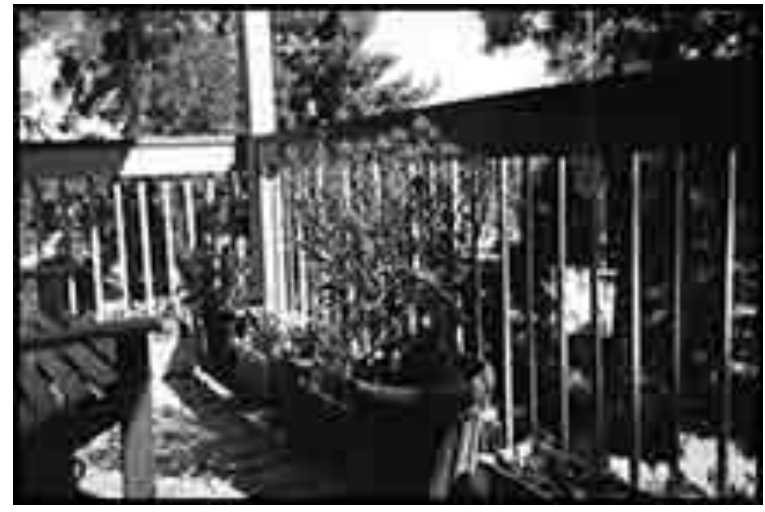

Figure 30: My Garden: Calm restored after battle. 


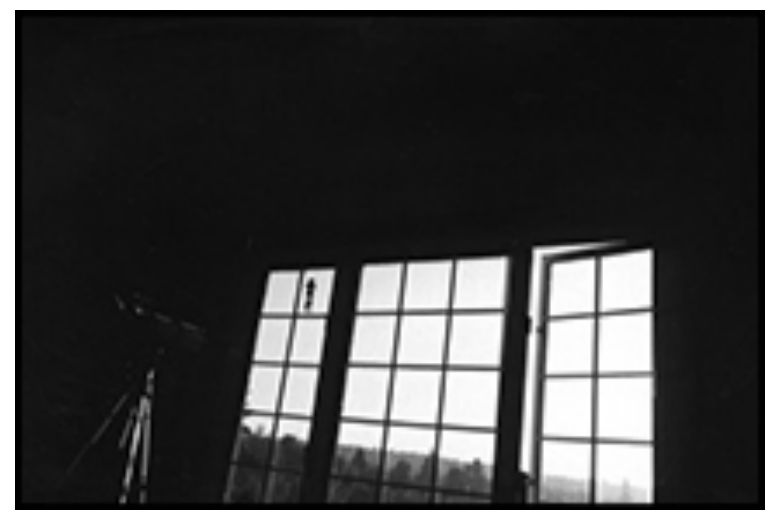

Figure 31: Sunshine in the Window in the Morning: Sunshine helps my mood and lets me be and enjoy 'outside'.

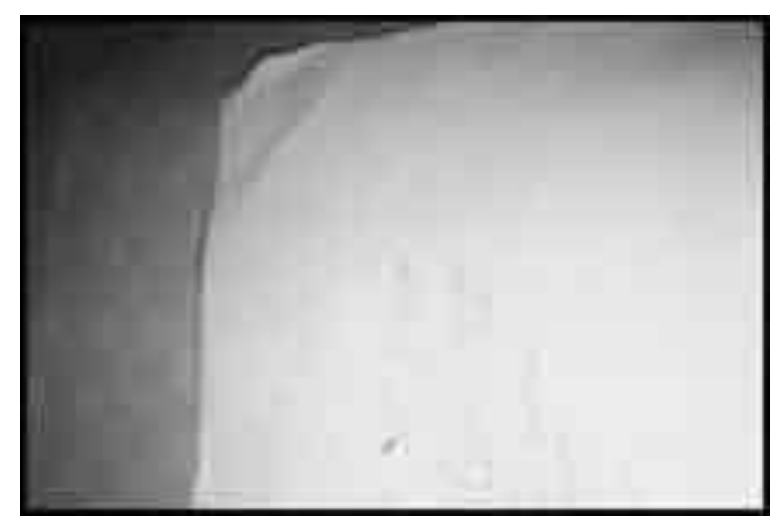

Figure 32: To-Do List: So I can remember where I need to go and what I need to do today.

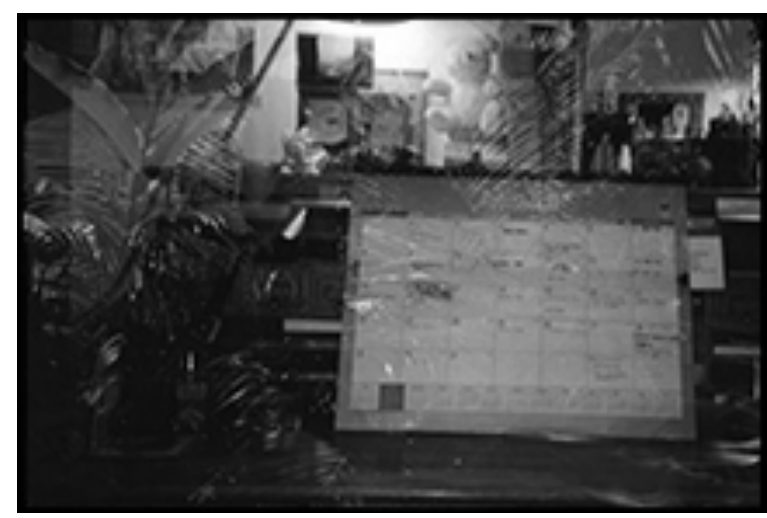

Figure 33: My Desk Calendar: So I get to places on time. 


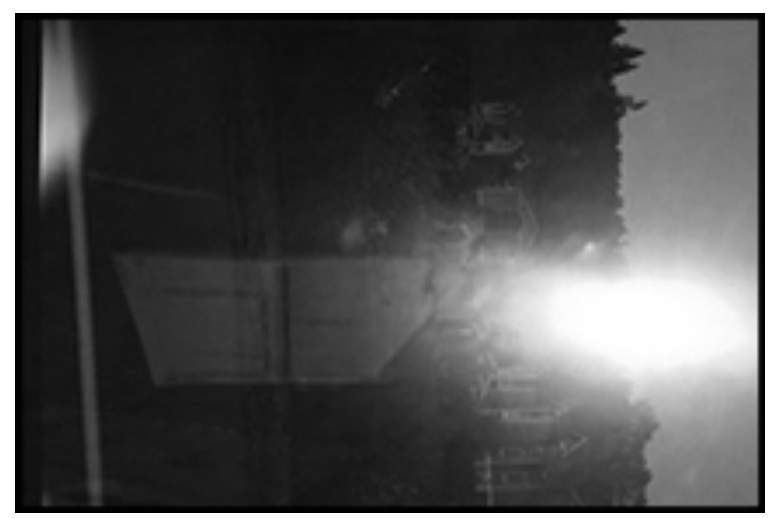

Figure 34: My Purse Calendar: It tells me what day it is and what I have done and what I will do.

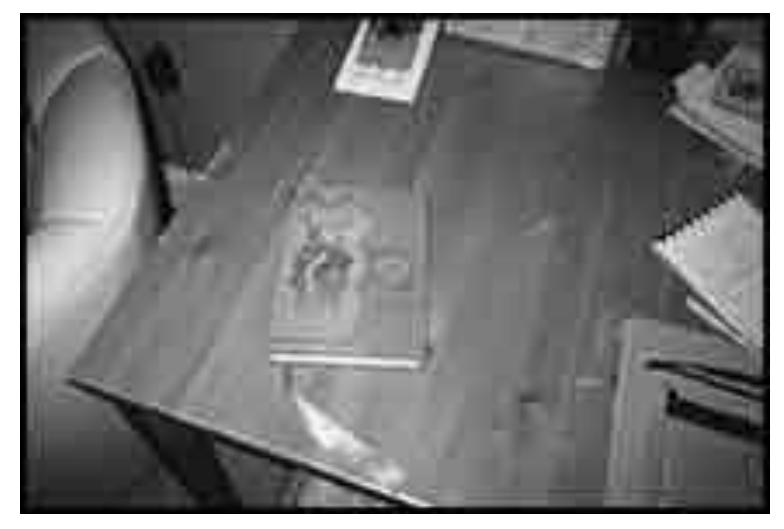

Figure 35: My Daily Notebook: Reminds me what I have to do today and what I missed yesterday. 


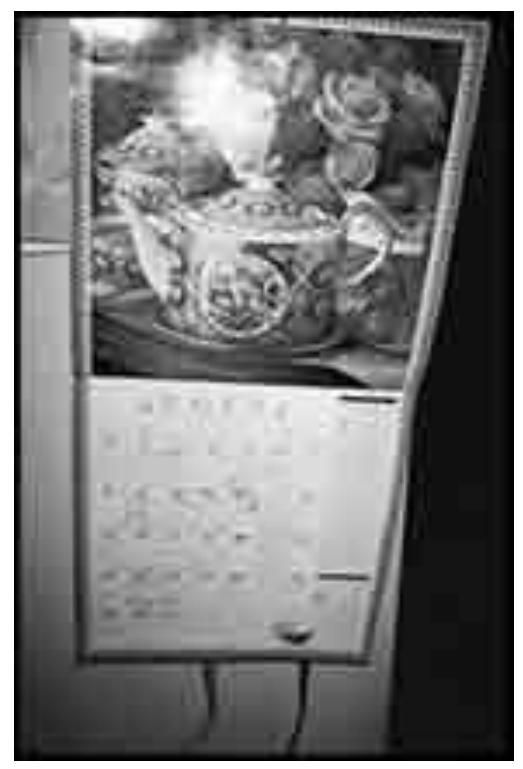

Figure 36: My Calendar: I put all my engagements on this. I'd be lost without this reminder.

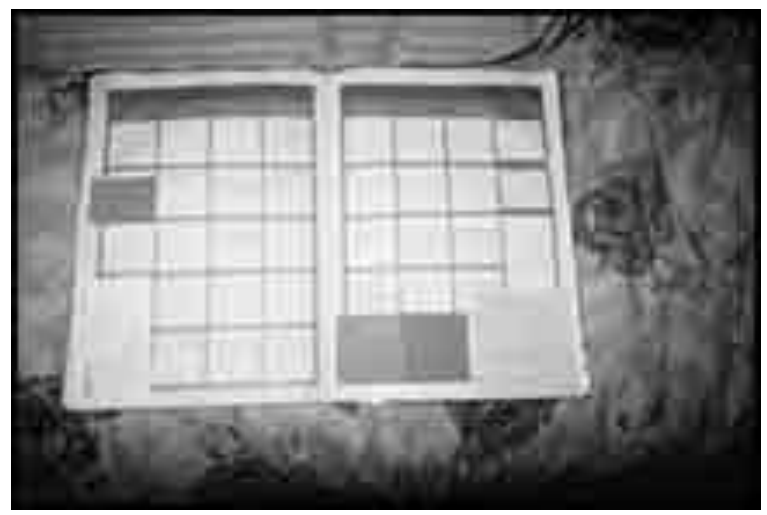

Figure 37: Daily Planner: My planner helps me to remember when I plan to have contact with the outside world. 


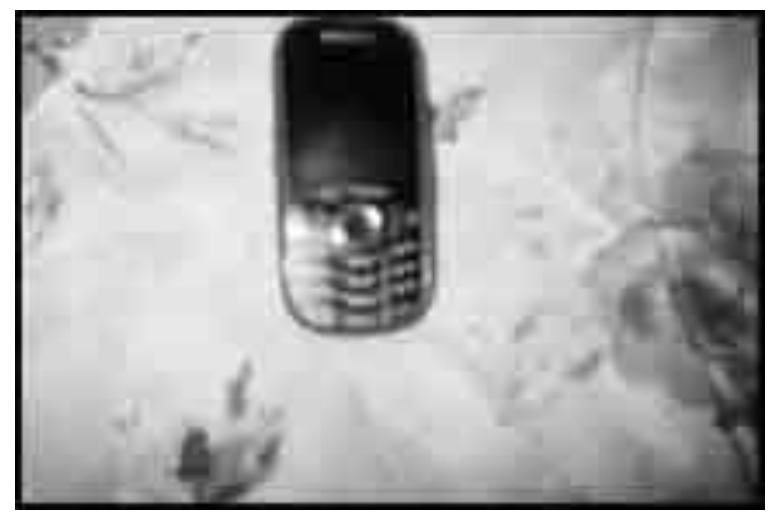

Figure 38: Cell Phone: Connects me to family and friends and allows for contacts with doctors.

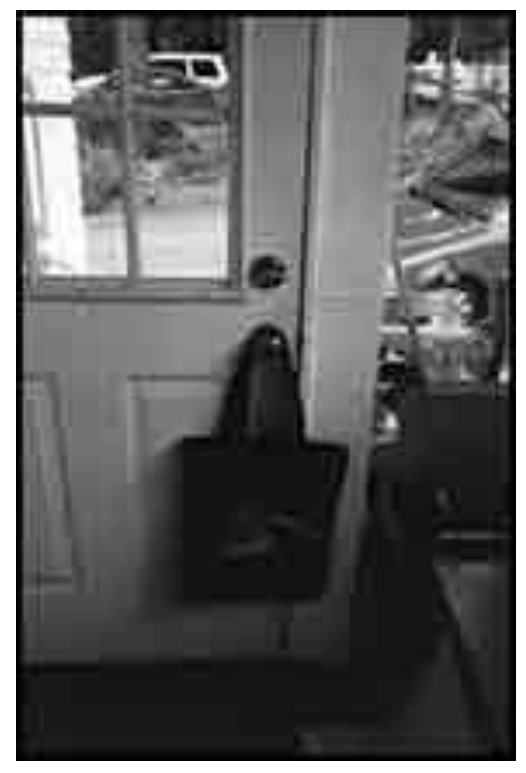

Figure 39: Shopping Bag on Door Handle: A reminder to take the bag with me when shopping. 


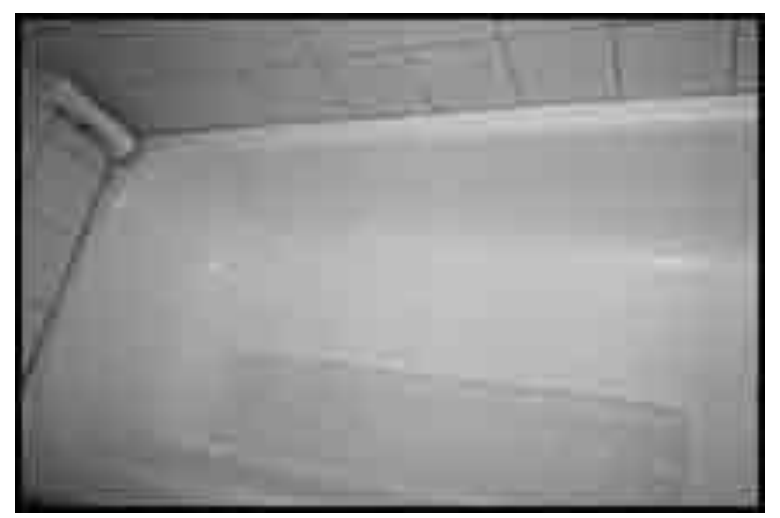

Figure 40: Reminder.

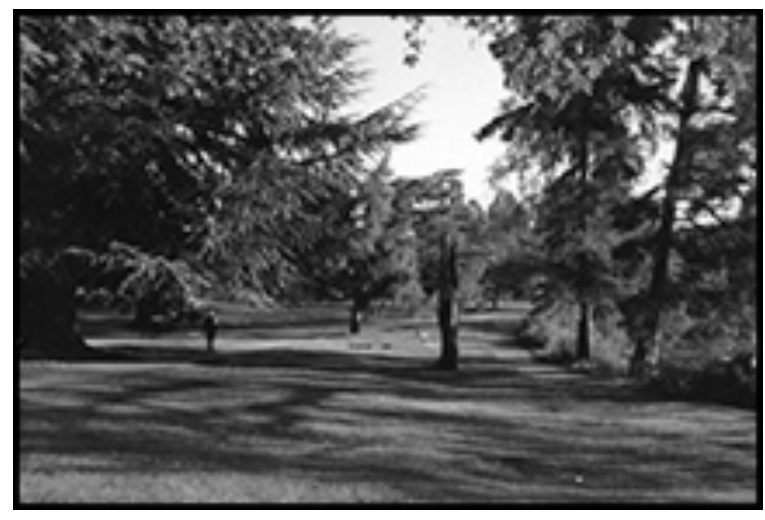

Figure 41: Off-the Path View at Greenlake.

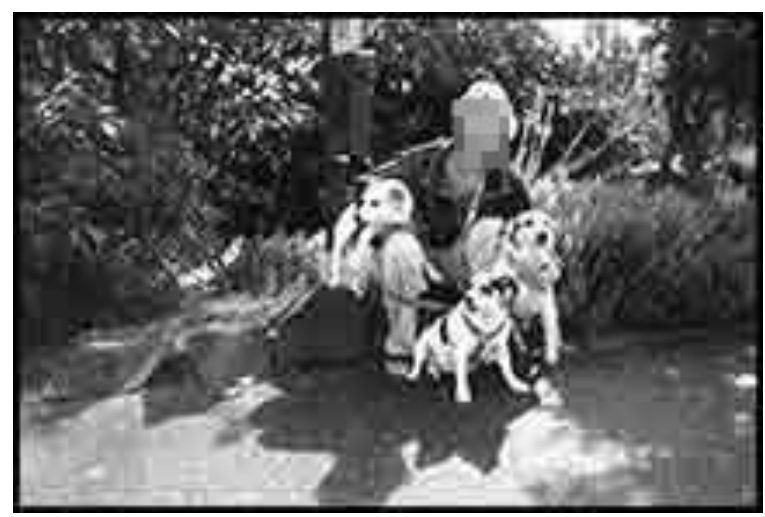

Figure 42:Three Dogs I Walk M-F: Walking these dogs, which I do first thing in the morning, centers me. We love each other. 


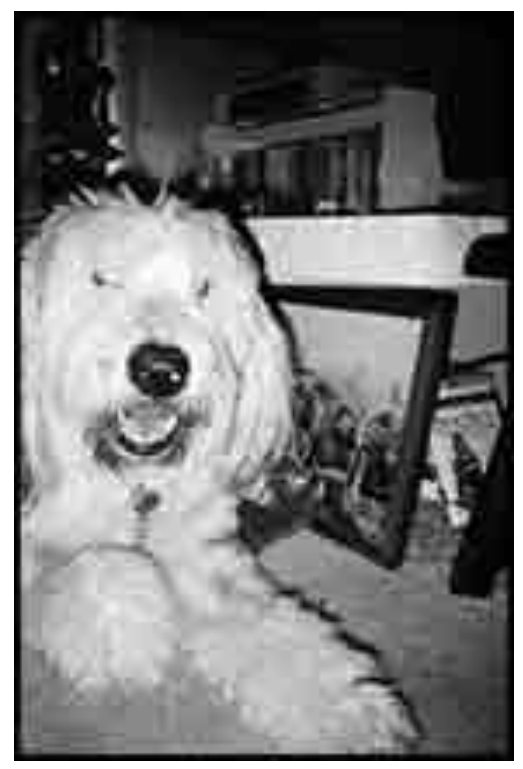

Figure 43: Knowing What Day it Is, Is Important to Me.

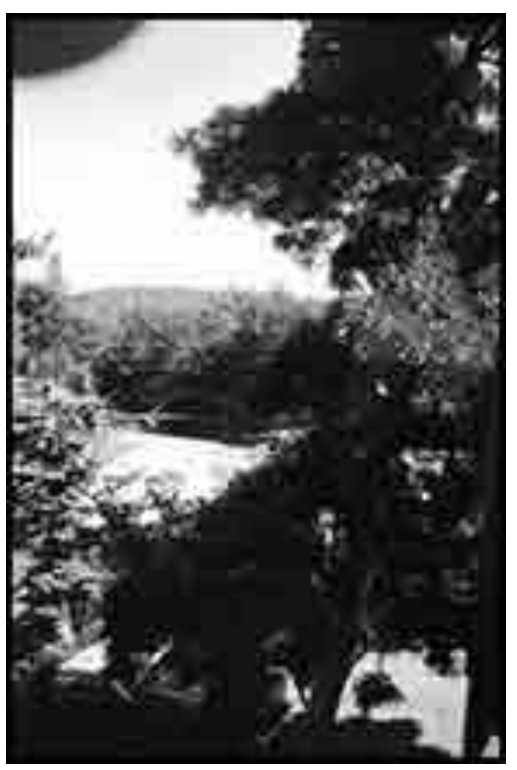

Figure 44: Start of the Day. 


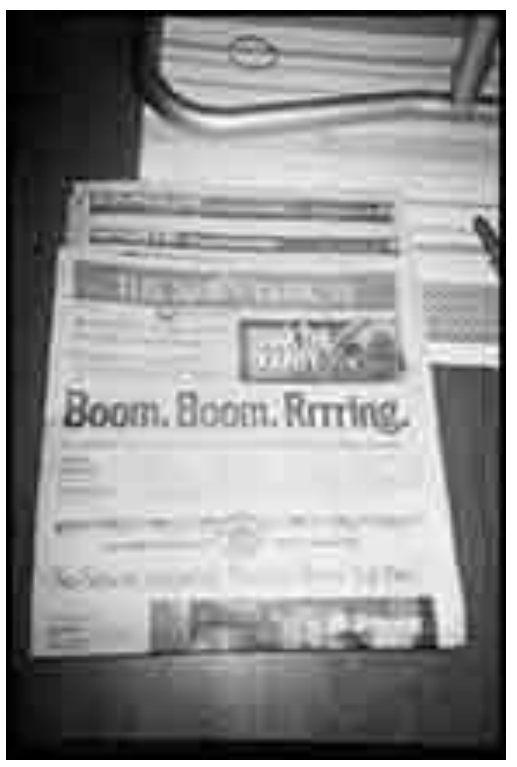

Figure 45: Newspaper Sections Every Day: The paper signals a new day to me each morning. And binds me to the outside world.

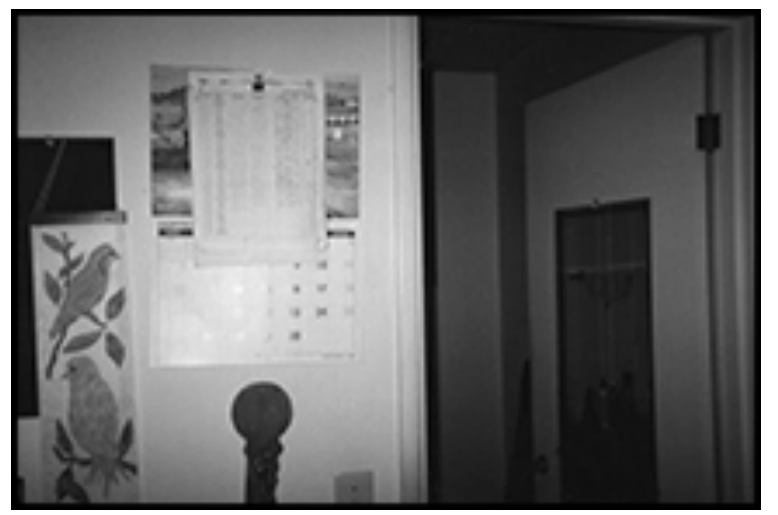

Figure 46: My Chart: To let me know what day it is and keep track of my daily medicines, etc. 


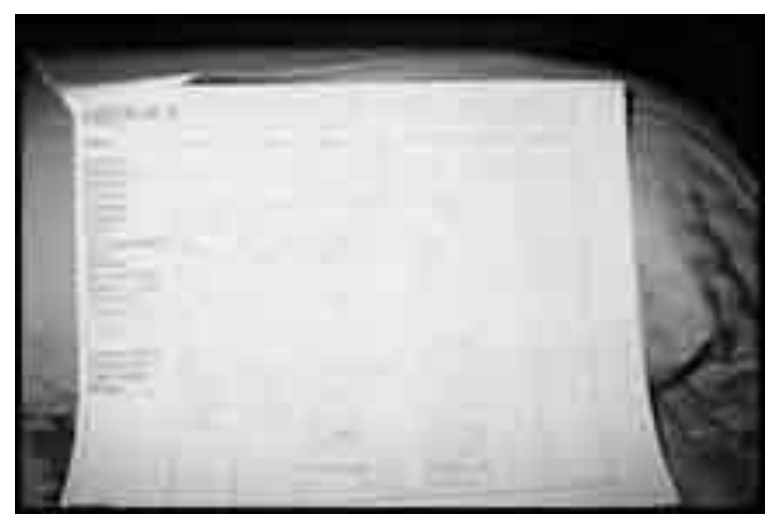

Figure 47: Daily Life Log: Keeps me on top of daily personal tasks - ensures I complete them or tracks failure to do so.

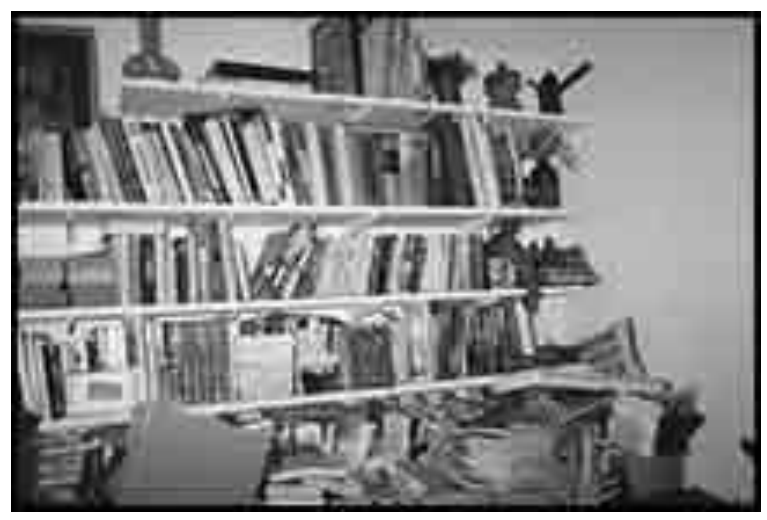

Figure 48: Wall in My Den: Shows some of my books that give me lots of pleasure. I have been a book reader and collector most of my life. I am now sorting to get rid of some old ones. 


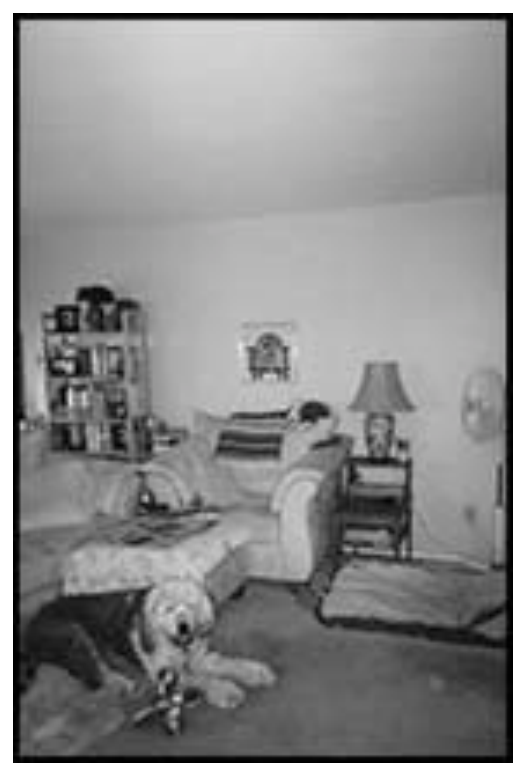

Figure 49: My Living Room - Books, Desks, Igor, and His Toy: My living space unshared by anyone else's stuff. It's all about me and what I want.

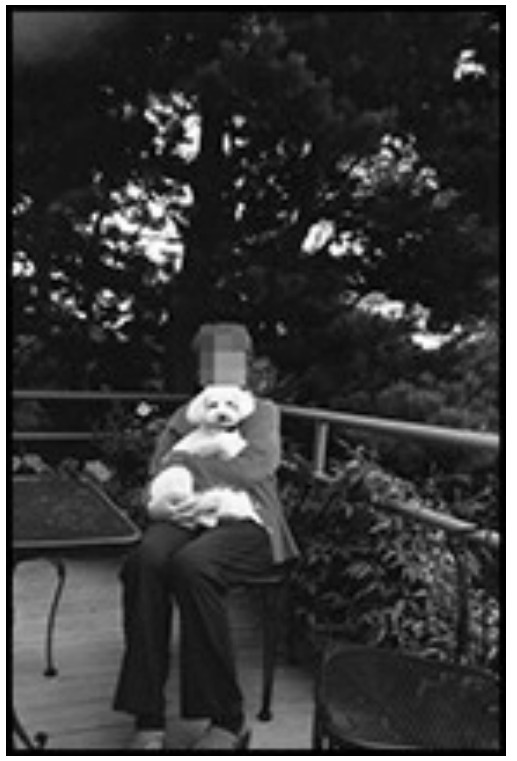

Figure 50: Gisela. 


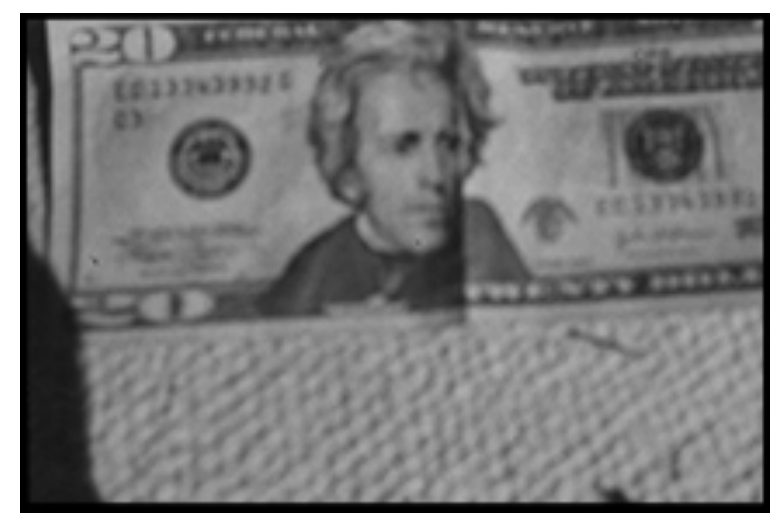

Figure 51: Money Is Always Important: For how we live, travel, and enjoy ourselves.

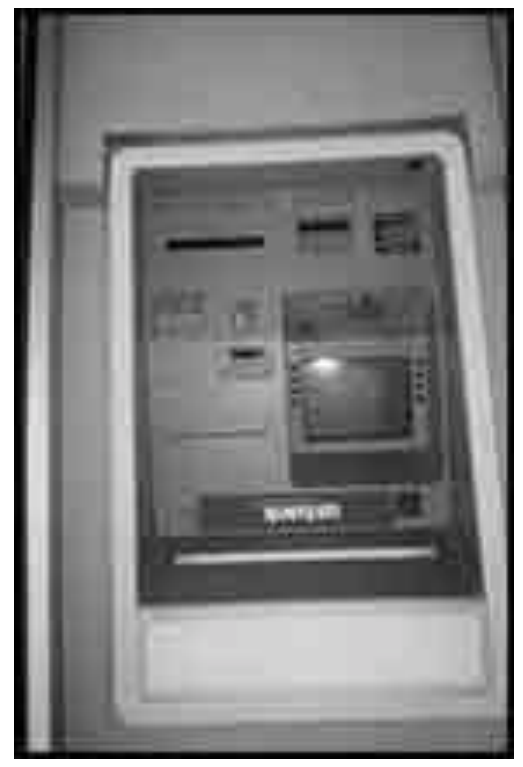

Figure 52: The US Bank Cash Machine: My financial independence. Ah ... my finances ... so far, so good.

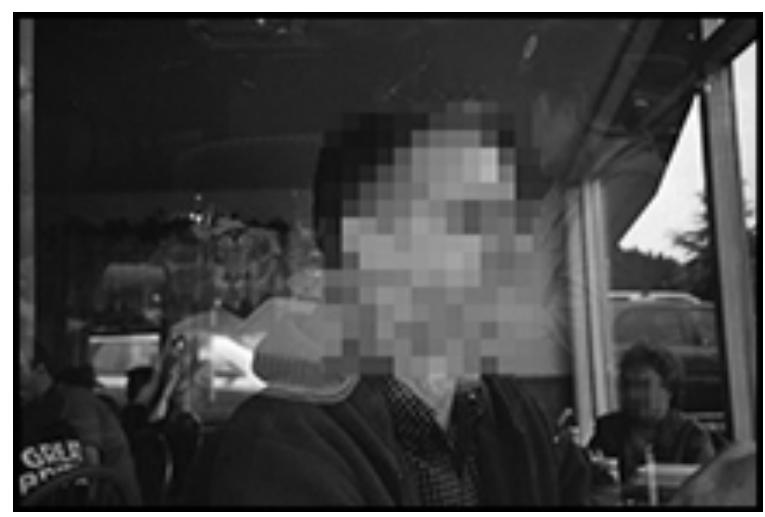

Figure 53: Me Writing a Check: I'm happy I can do it and don't carry cash. 510 


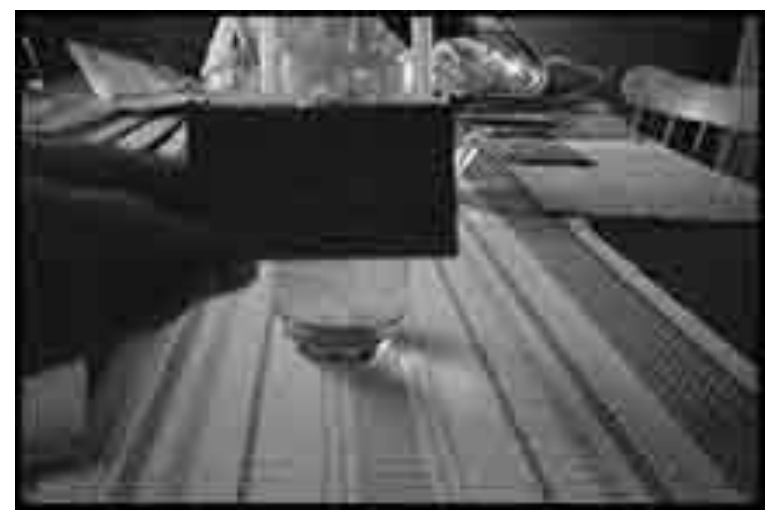

Figure 54: Business Card of Attorney: He's helping me with a special needs trust. The trust will allow me to keep my back benefits so they benefit my future.

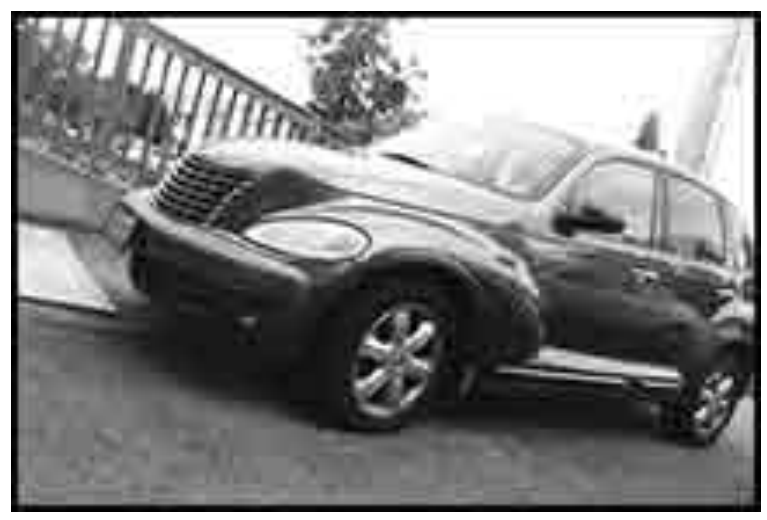

Figure 55: My Car: In Seattle, a car is essential.

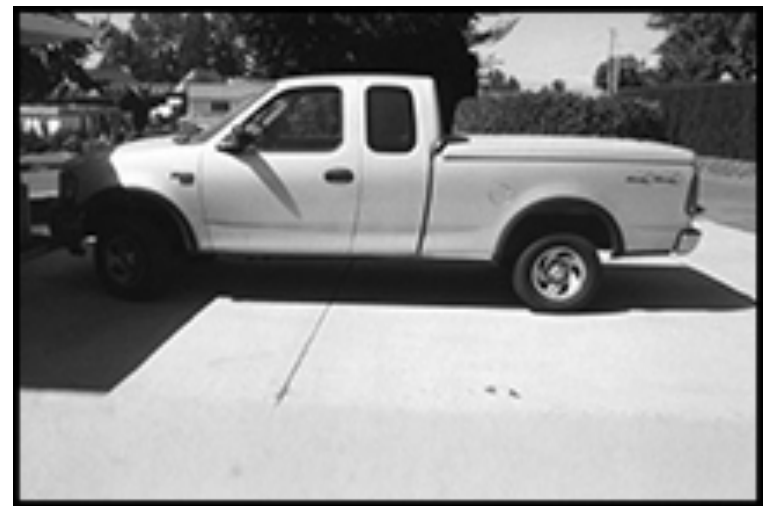

Figure 56: My Pick-up: Still works and looks good after 100,000 miles: I can still drive it to downtown. 


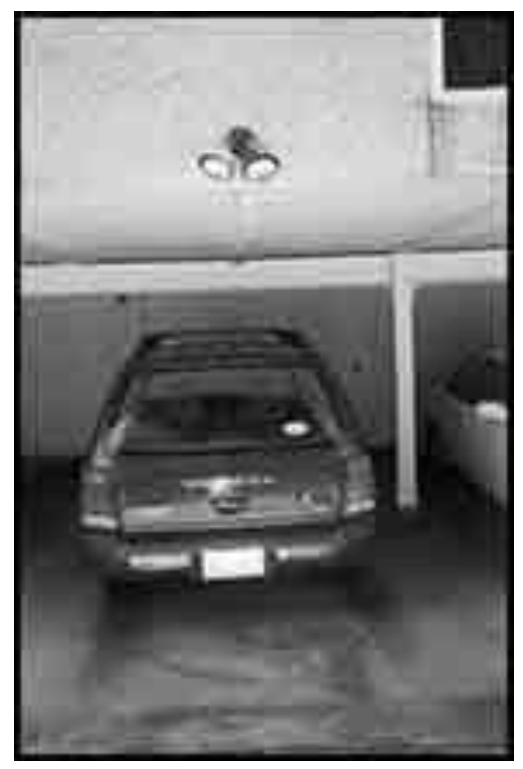

Figure 57: My Forester: My car (paid off). My politics (bumper stickers) for the 'short time' get-away.

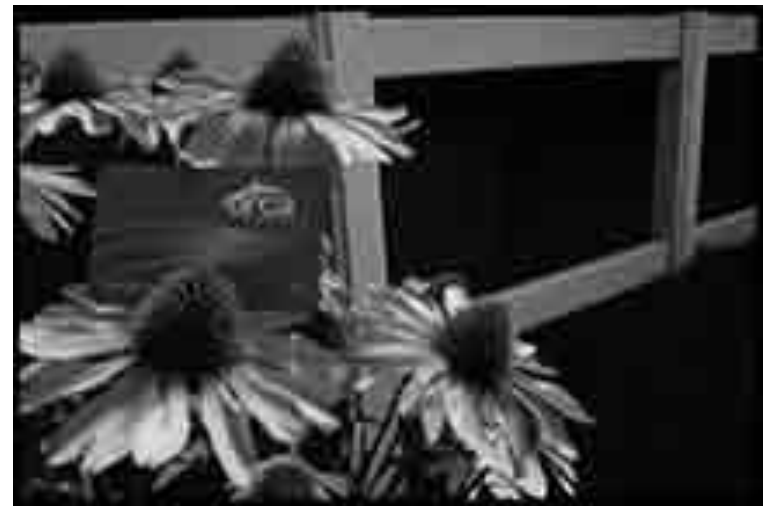

Figure 58: Freedom - My Orca Bus Card: I can go anywhere without having to drive/park etc. 


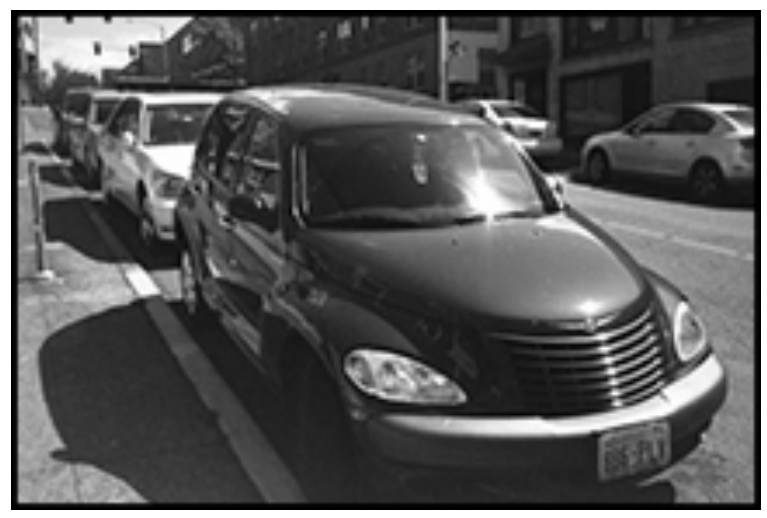

Figure 59: My PT Cruiser: My independence.

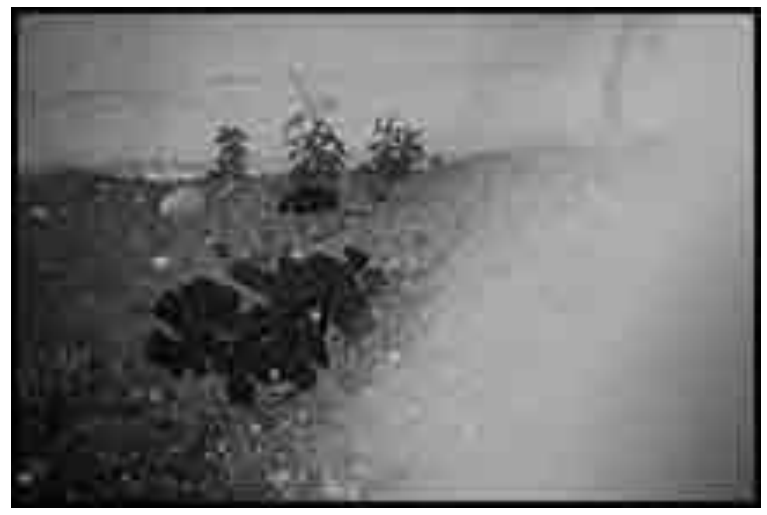

Figure 60: Weeds: Weeding is my contribution to my family and my Zen.

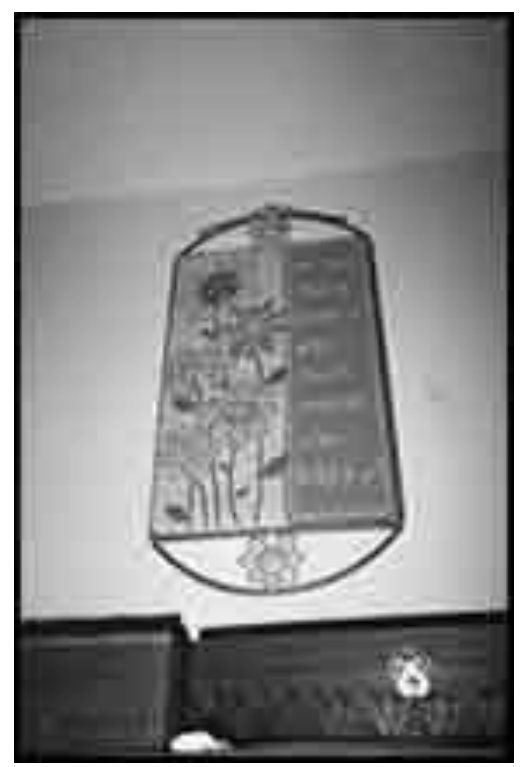

Figure 61: Do One Thing Every Day that Makes You Happy: Hanging on my bedroom wall. It makes me think about how I do and I try to follow its advice. 


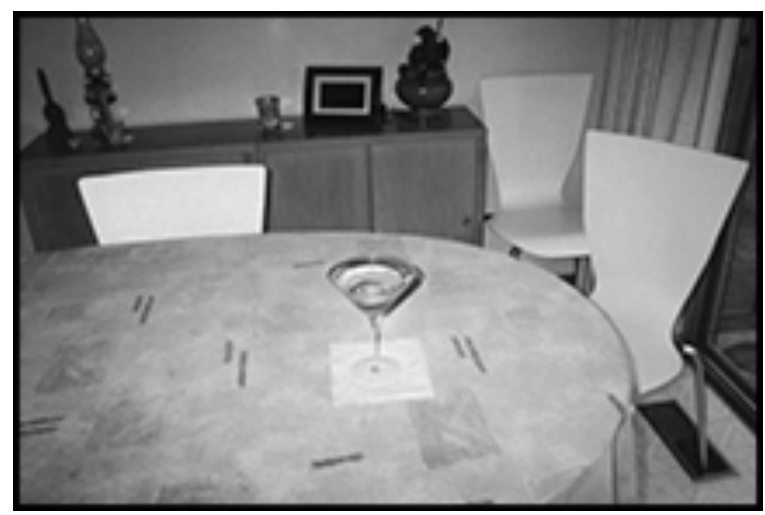

Figure 62: My Evening Martini: I greatly enjoy my Martini each night before dinner.

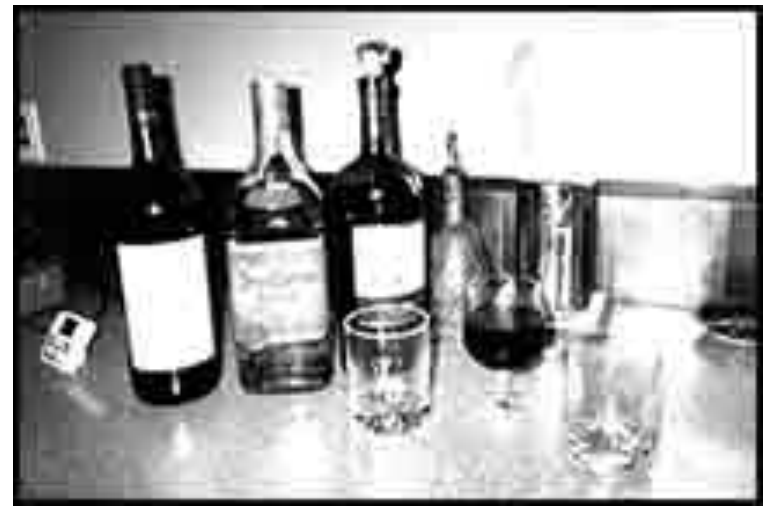

Figure 63: Wine and Tequila Bottles: At the end of the day we enjoy a drink with dinner.

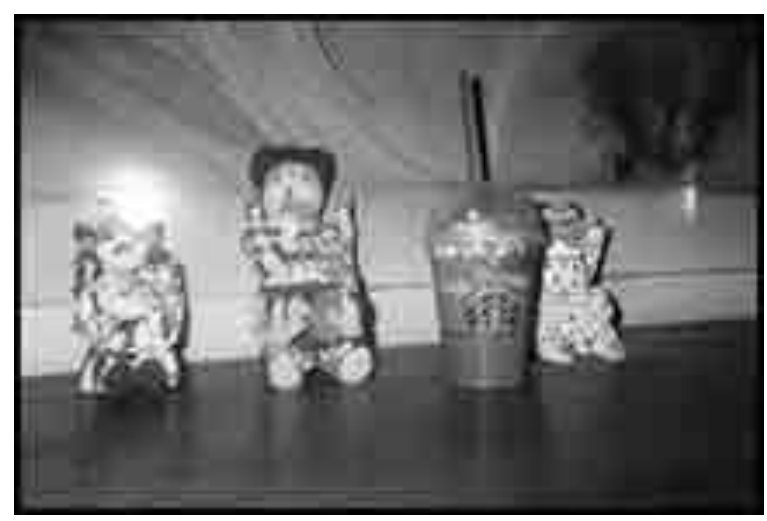

Figure 64: Starbucks Frappuchino: I love them. 


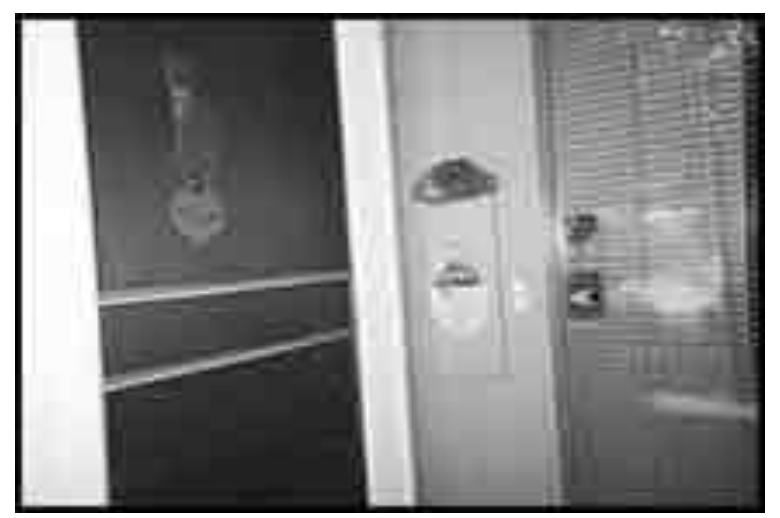

Figure 65: My Front Door With My two Welcoming Signs: Makes me and my guests feel good - that they know they're welcome.

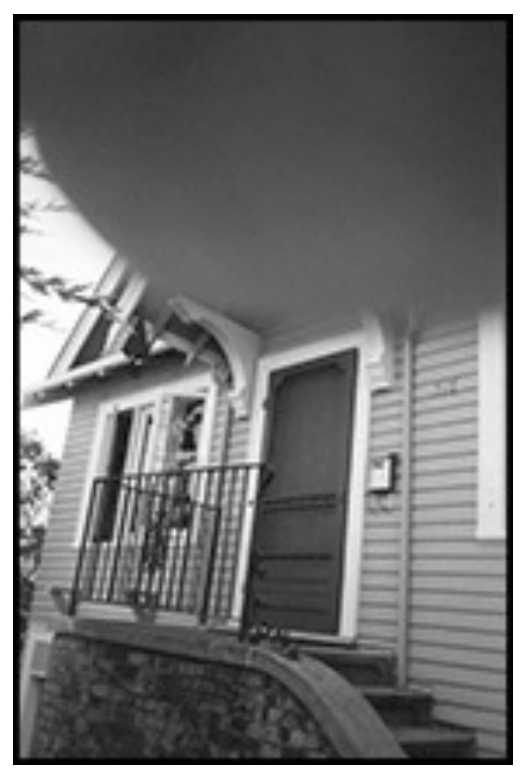

Figure 66: New Paint Job on House: Maintaining what is mine. Pride in 'home' looking good and care for. 


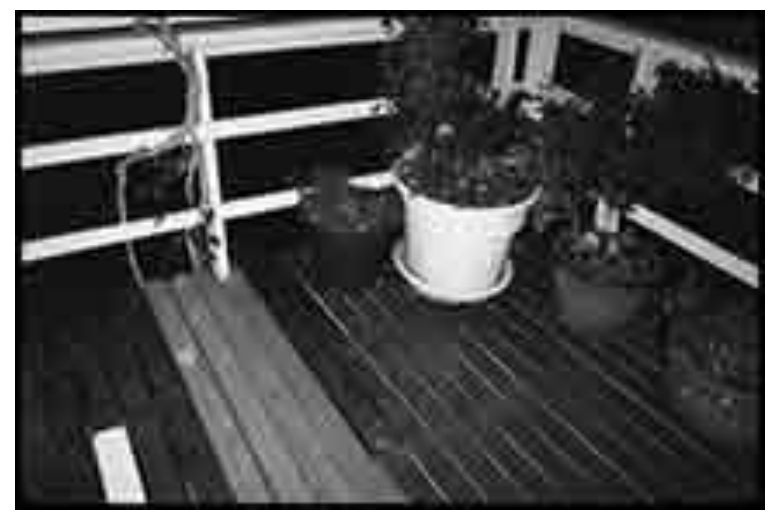

Figure 67: Repairs to My Deck: I like to try and keep up on house repairs.

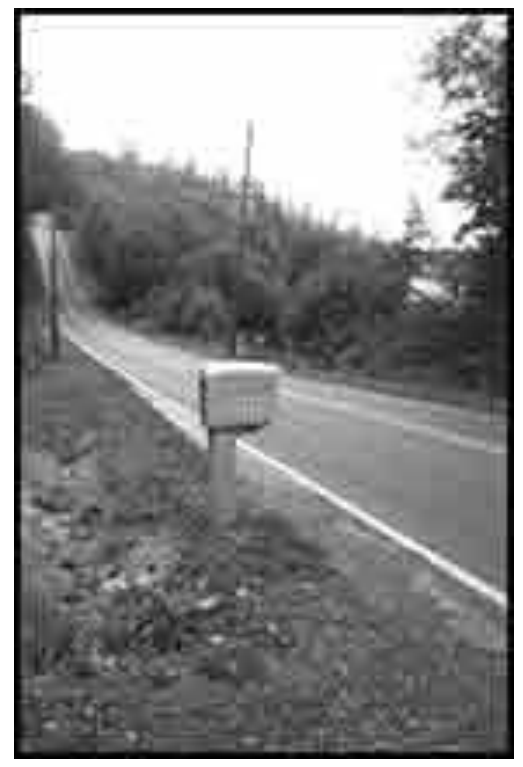

Figure 68: Mailbox: Getting the mail is part of my daily activity.

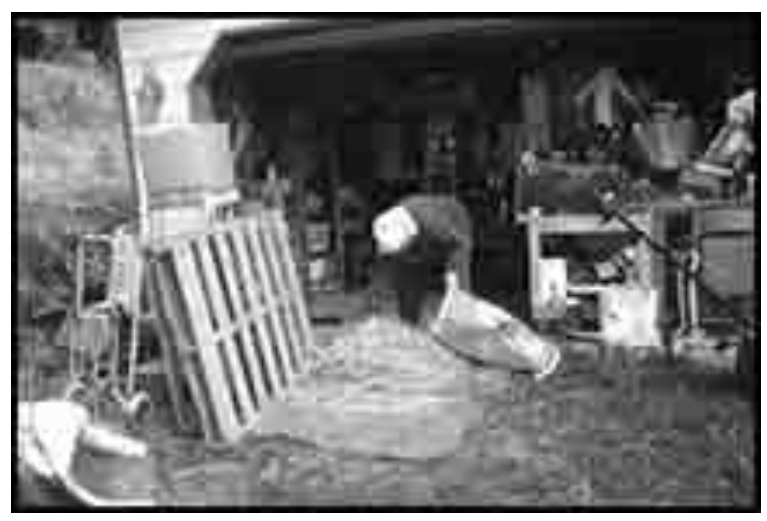

Figure 69: Hay for the Animals. 


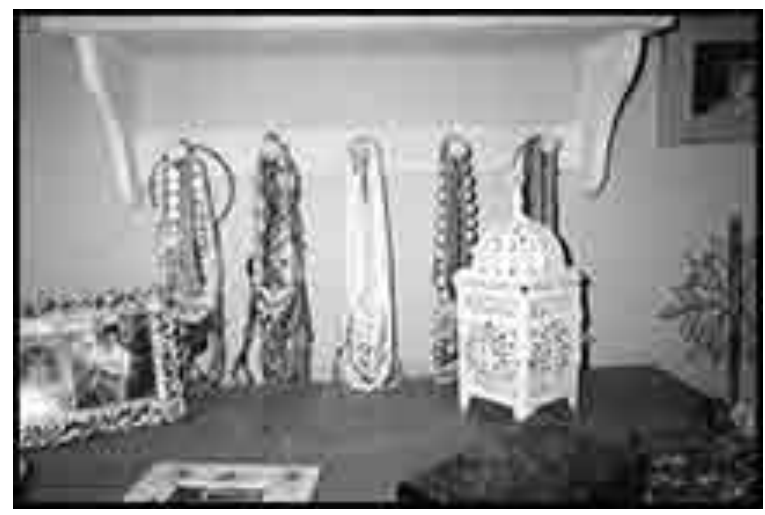

Figure 70: Scarves and Beads: I enjoy tidying up my bedroom and putting things in order by color.

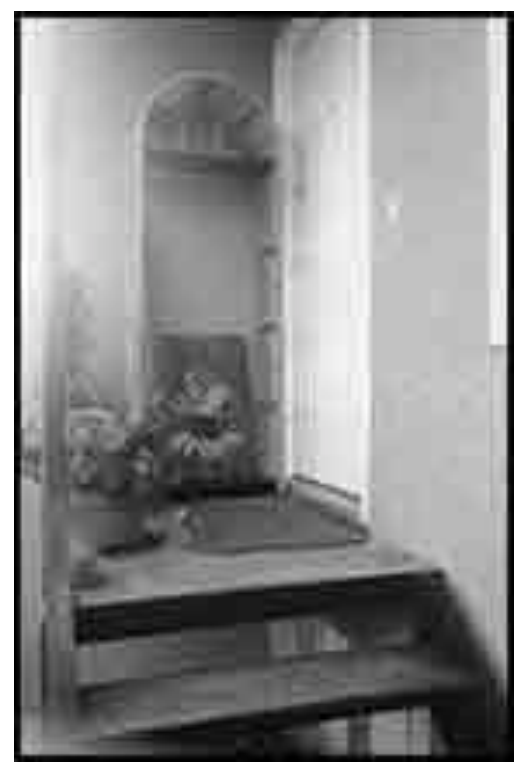

Figure 71: My Apartment Door: Door leads to home. 


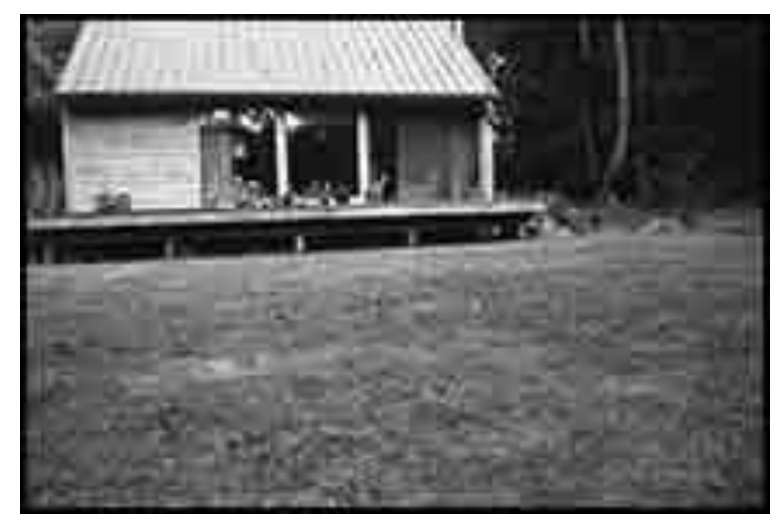

Figure 72: Our House: I absolutely love where we live. I just feel so fortunate to have this house on the hill.

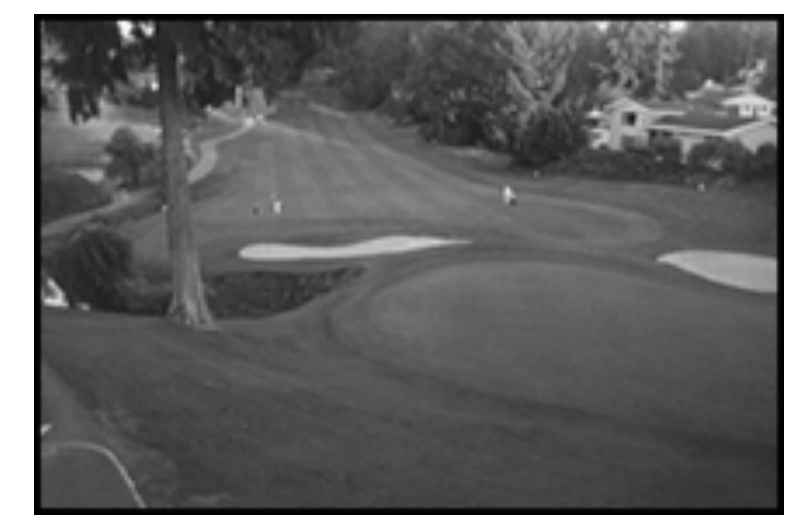

Figure 73: Our Home at the Golf Course: I just sit and watch them play. 


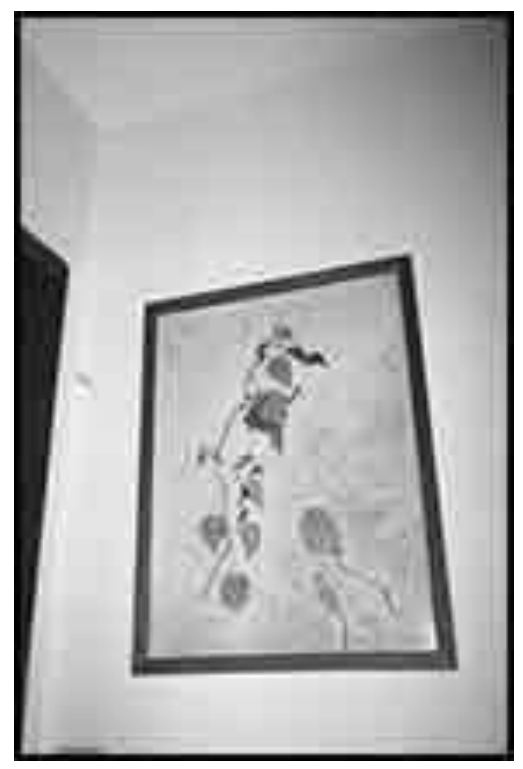

Figure 74: Painting on My Bedroom Wall: My Israeli friend painted it and gave it to me in 1970 in Israel. I love it. It reminds me of her. I miss her smile and our conversations.

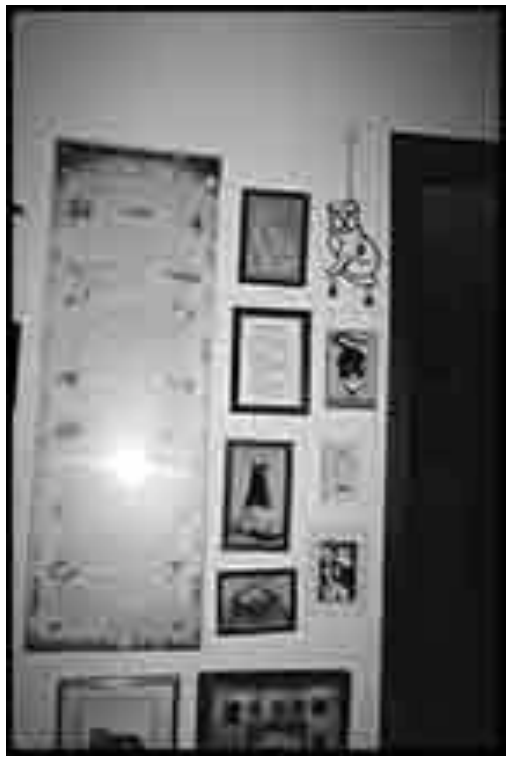

Figure 75: My 'Cat Room': My cat artwork that has been given to me or I collected it. I love looking at it - it reminds me on my favorite companions. 


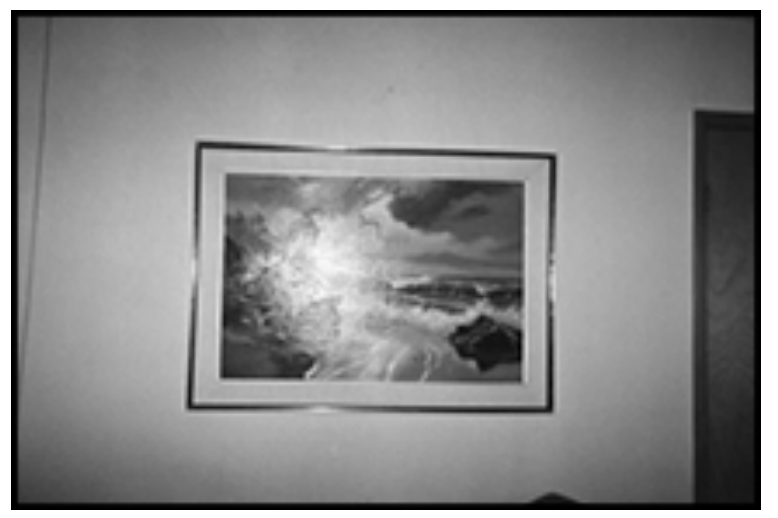

Figure 76: Picture of a Hulan Fleming Painting: We have had this painting in our living room for many years and has been my favorite one.

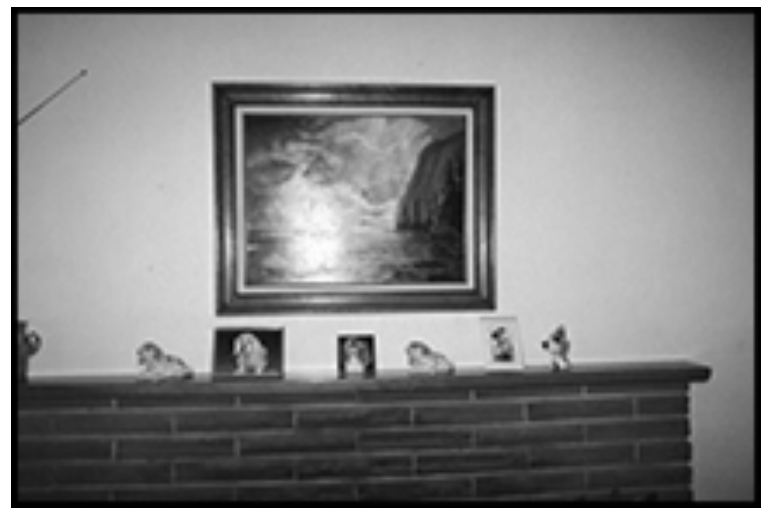

Figure 77: Picture of My Little Dog Figurines and One of My Favorite Pictures: I look at them every day and enjoy them every day.

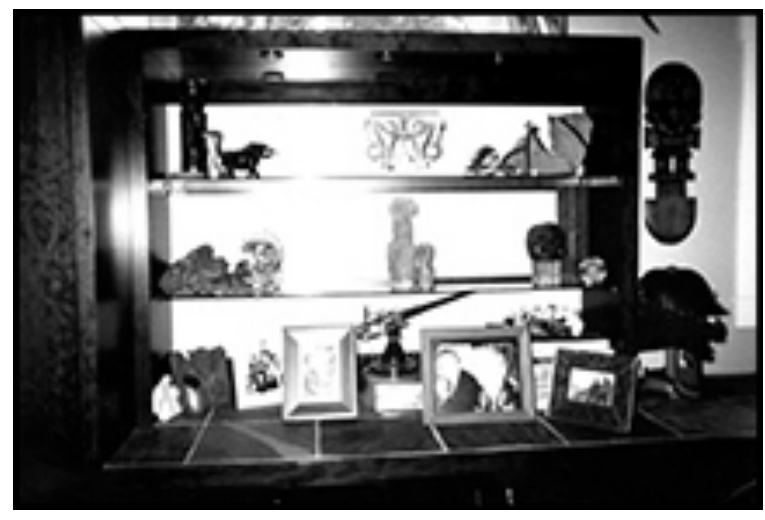

Figure 78: Mementos in Our House: Mementos from our worldwide travels and some family pictures. 


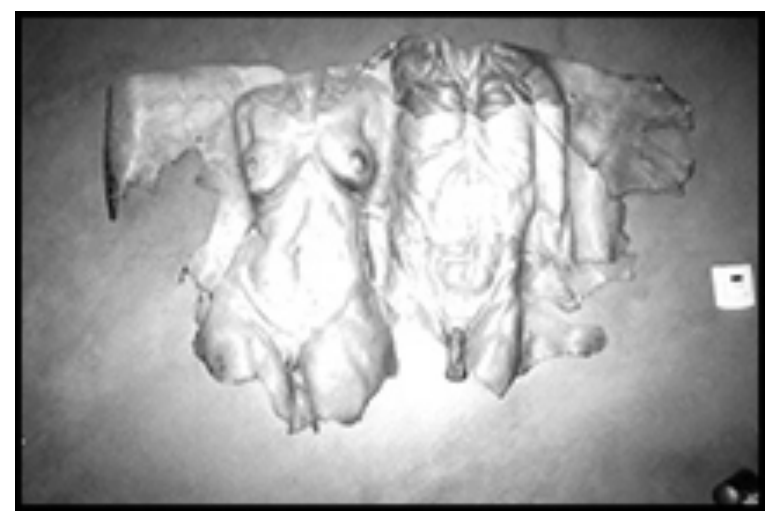

Figure 79: Leather Art Work: We commissioned this work and hung it in our bedroom.

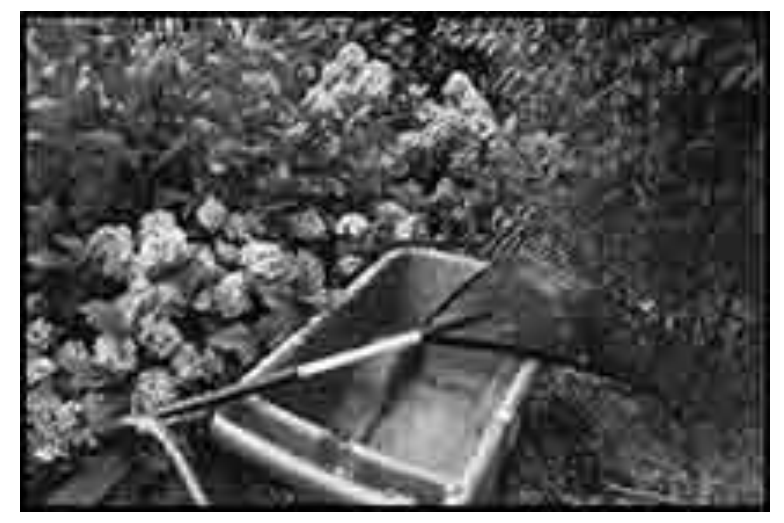

Figure 80: Garden Tools: I love working in the garden.

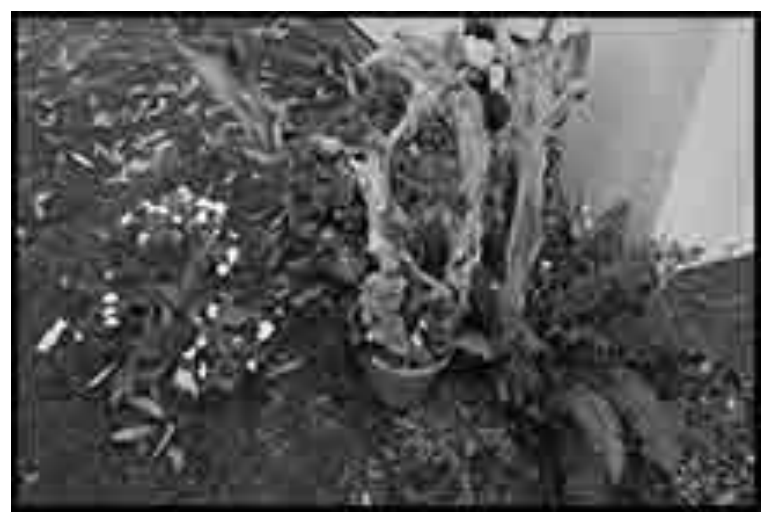

Figure 81: Decorative Roots with Flowers: I love to create interesting corners in my garden. 


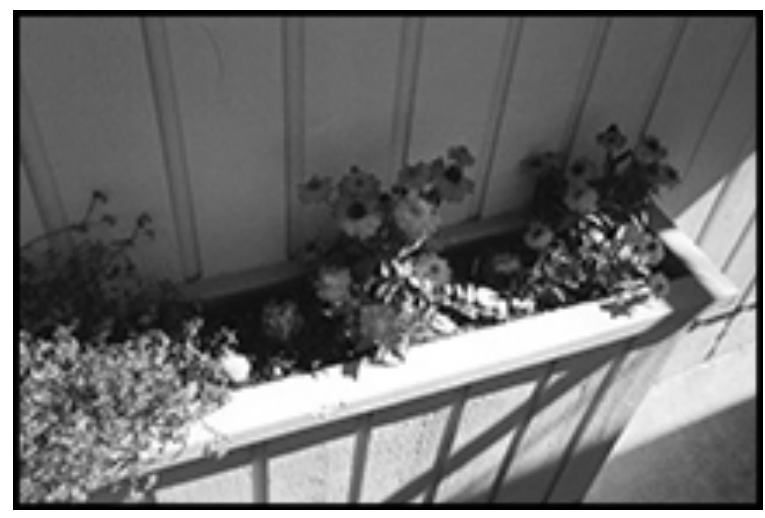

Figure 82: Bright Flowers at Lummi: Hope, beauty, color, joy.

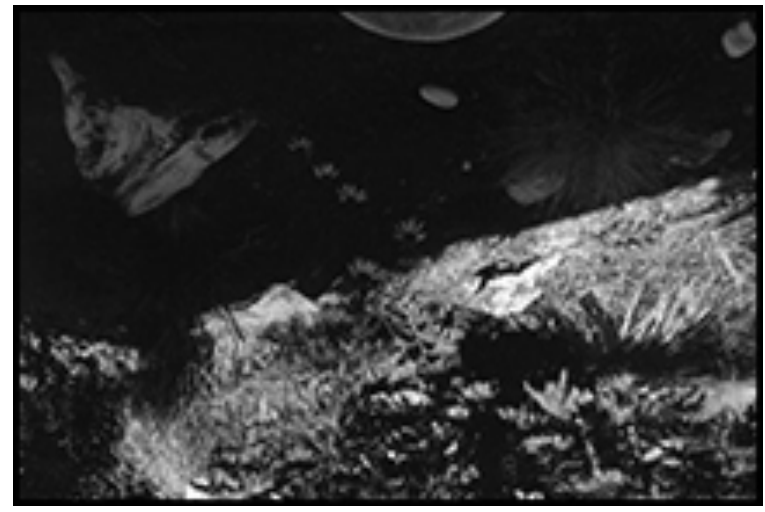

Figure 83: Flowers in Backyard: Contentment in gardening and seeing beauty in nature. 


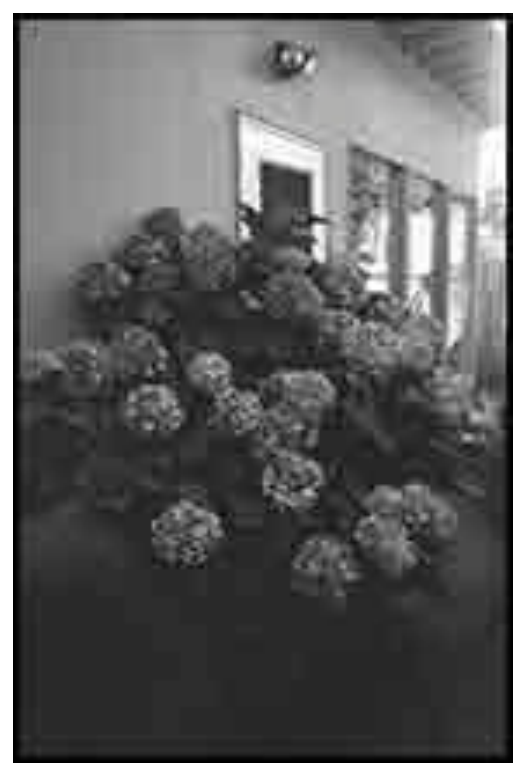

Figure 84: My Hydrangea Bush in Full Bloom: My friend/tenant planted it 15 years ago 18 " high at the time. I love the flowers and always think of her when I see it. I cut flowers for my tables when it is in season.

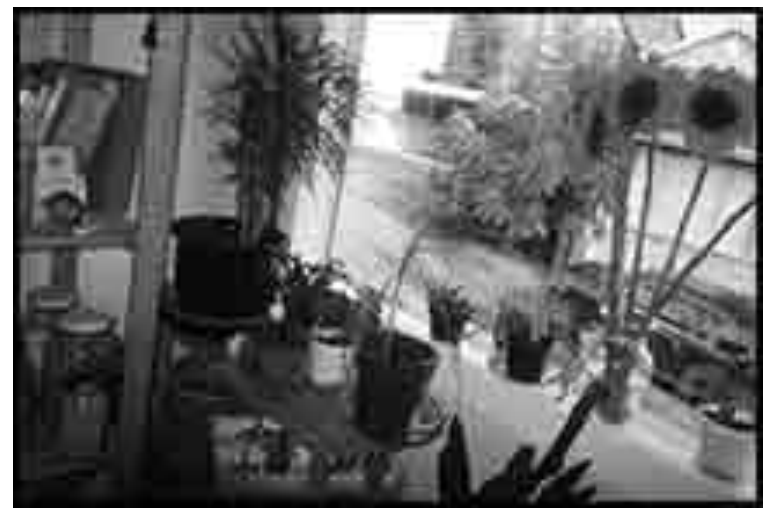

Figure 85: My Indoor Plants: "Water Us!", they say. These plants always remind me to stay in the present. 


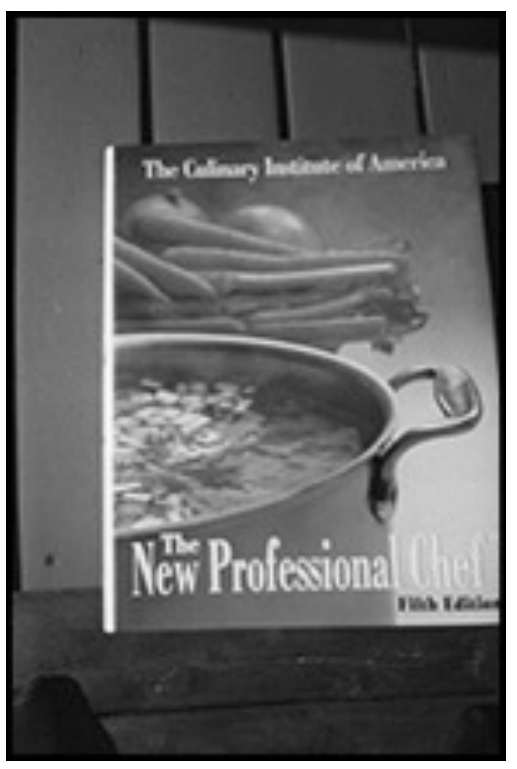

Figure 86: Cookbook: We both enjoy cooking and also eating the fruits of our labor.

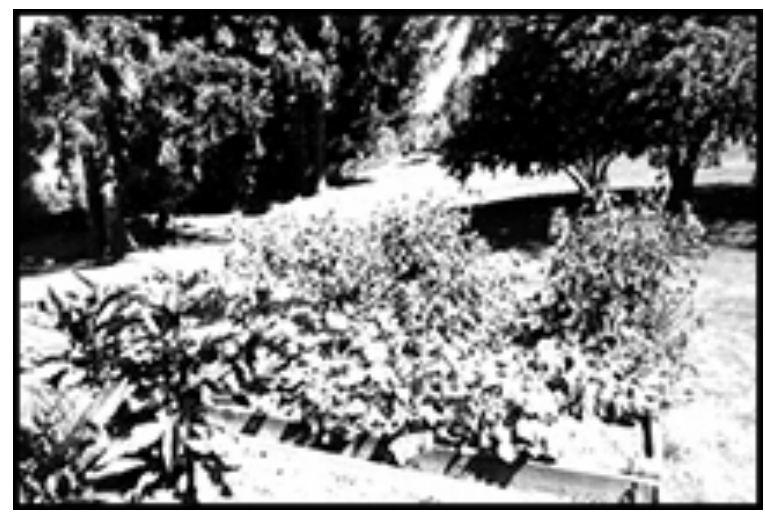

Figure 87: Vegetable Garden Next to Our House: We enjoy having really fresh vegetables, such as tomatoes and lettuce and eggplant.

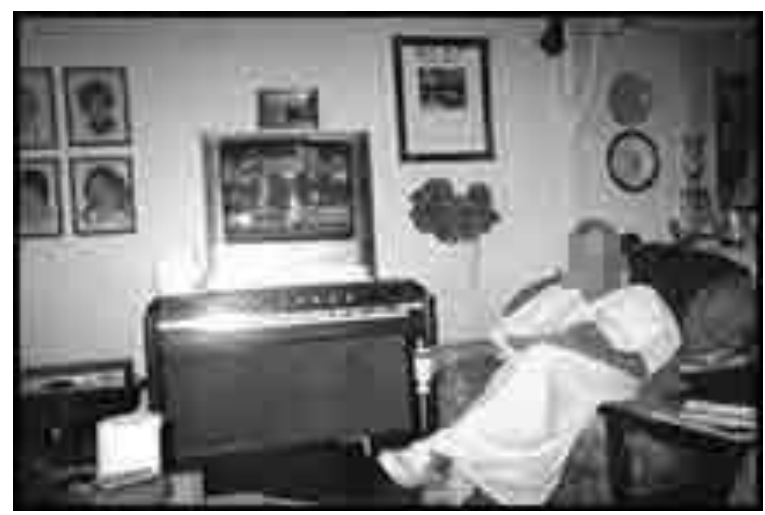

Figure 88: TV: I like watching TV. 


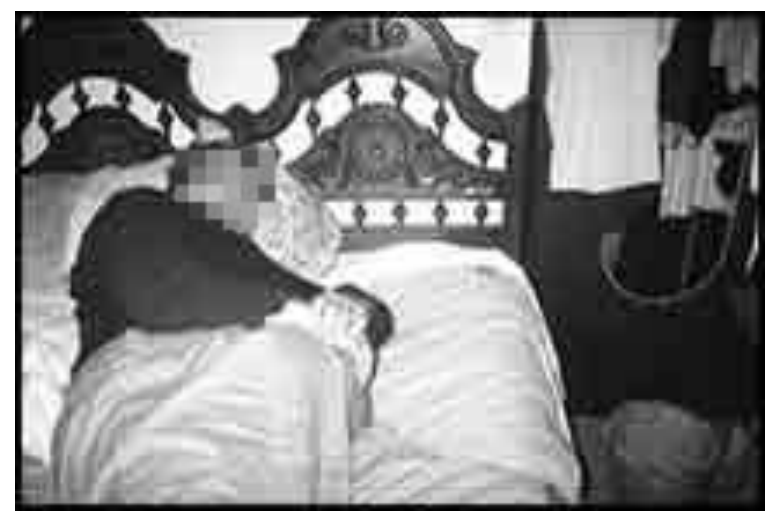

Figure 89: Napping: I like taking naps.

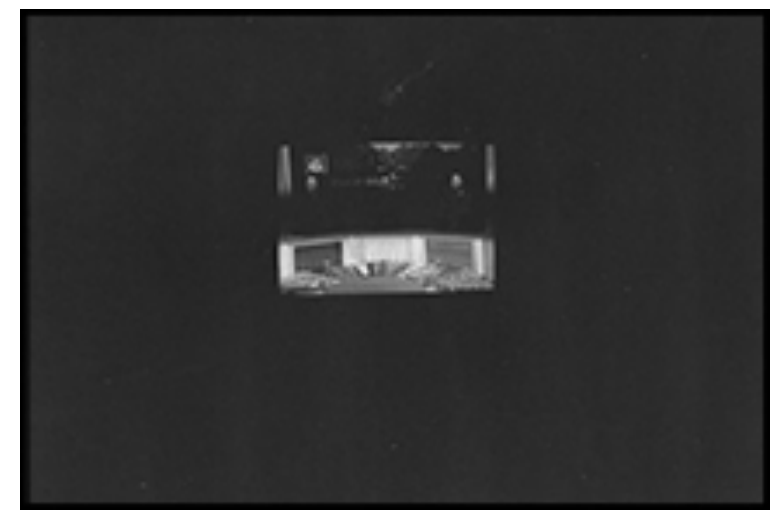

Figure 90: Wheel of Fortune Program: I look forward to watching this program every night.

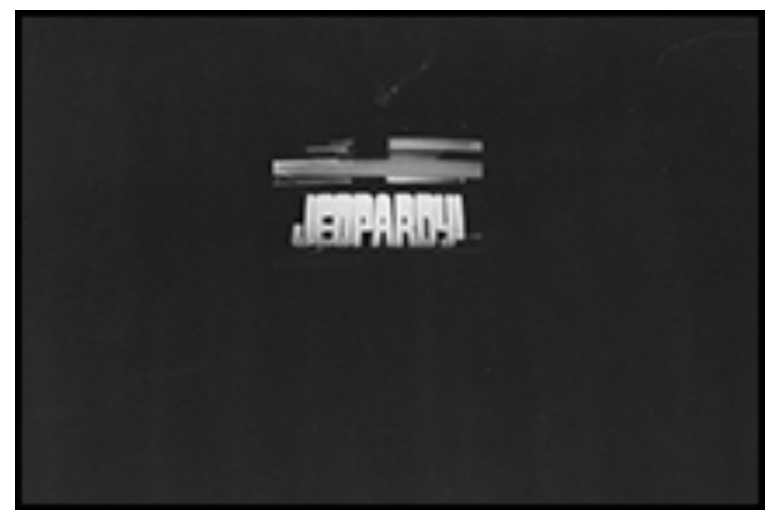

Figure 91: Jeopardy Program: I look forward to watching this program every night. 


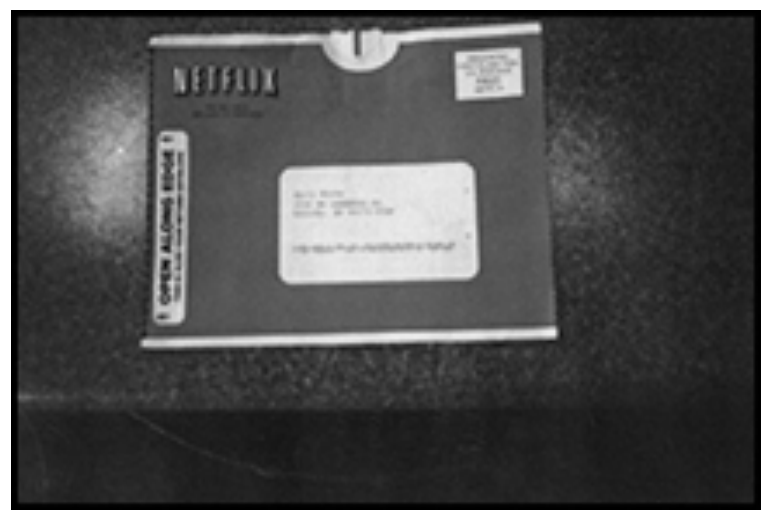

Figure 92: Netflix: We both enjoy movies of our choice in the evening.

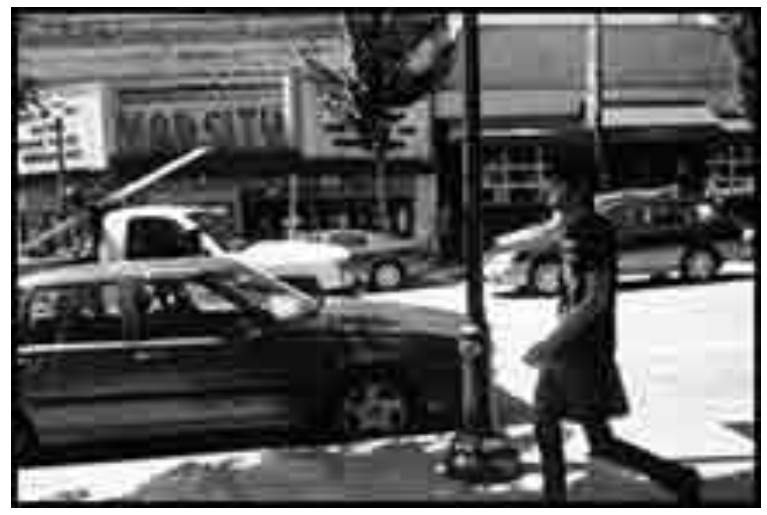

Figure 93: The Varsity Theater: My haunts of long ago starting in 1980. I still return often.

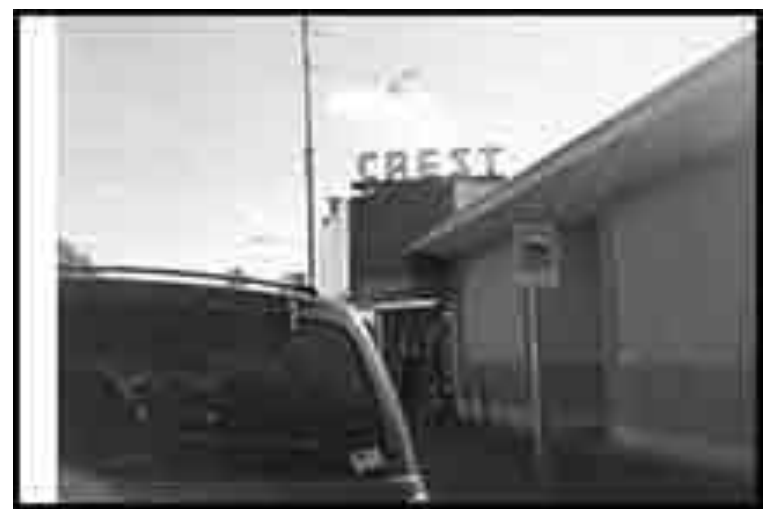

Figure 94: Crest Theater: When I feel down or want a change, I go to see a movie in my favorite theater, which is also very inexpensive (\$3). 


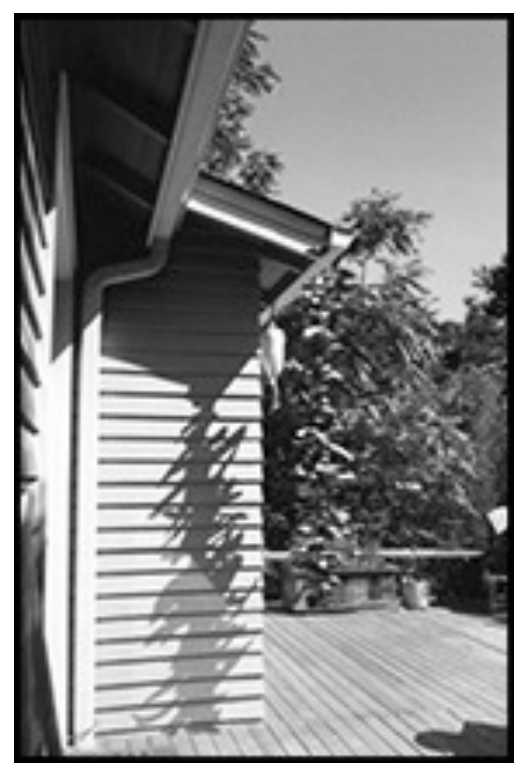

Figure 95: My Artwork.

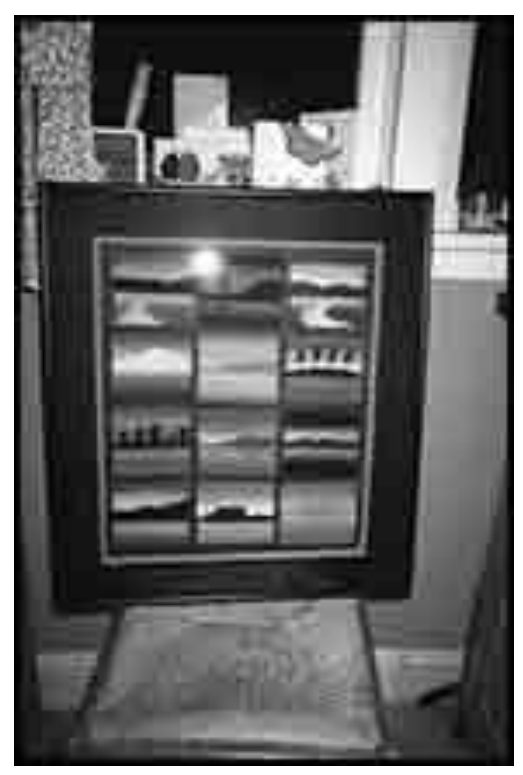

Figure 96: One of My Pastels: This painting I am entering at a juried art show. 


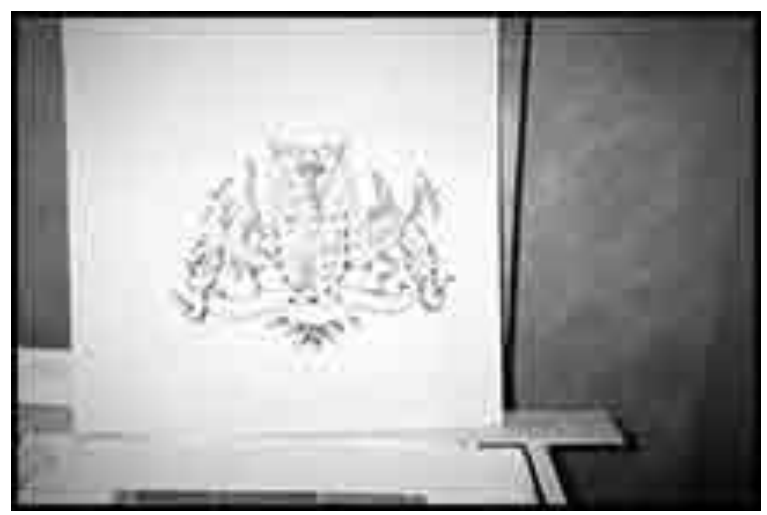

Figure 97: Black and White Drawing of a Crest: Part of my work - a commission.

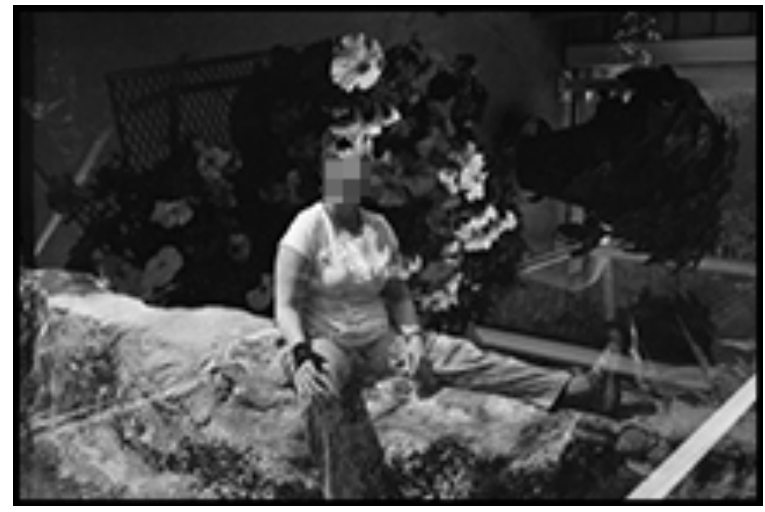

Figure 98: My Painting: I enjoy creating pictures that are bright and interesting.

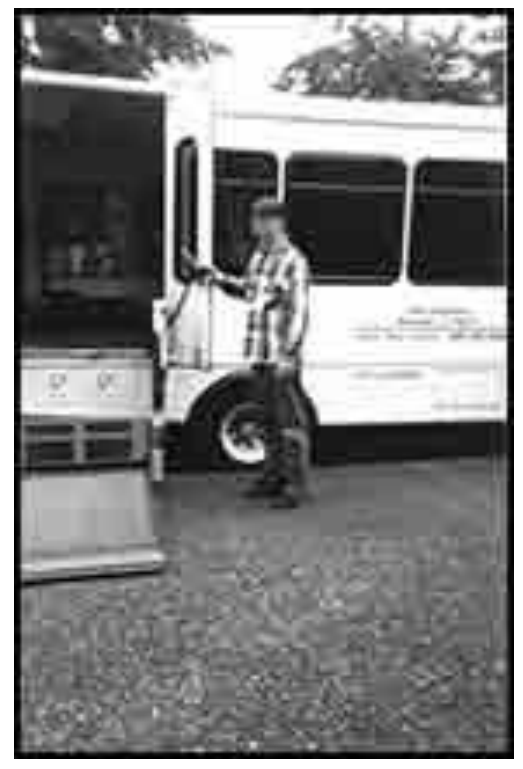

Figure 99: North Shore Bus. 


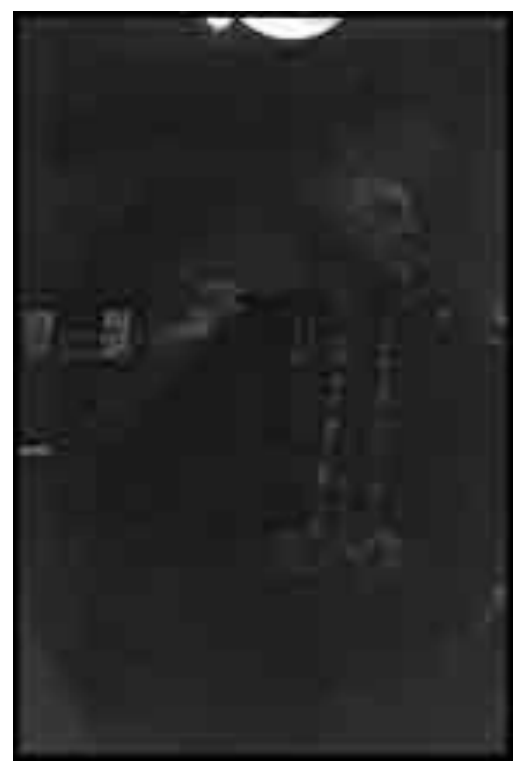

Figure 100: At the Day Care.

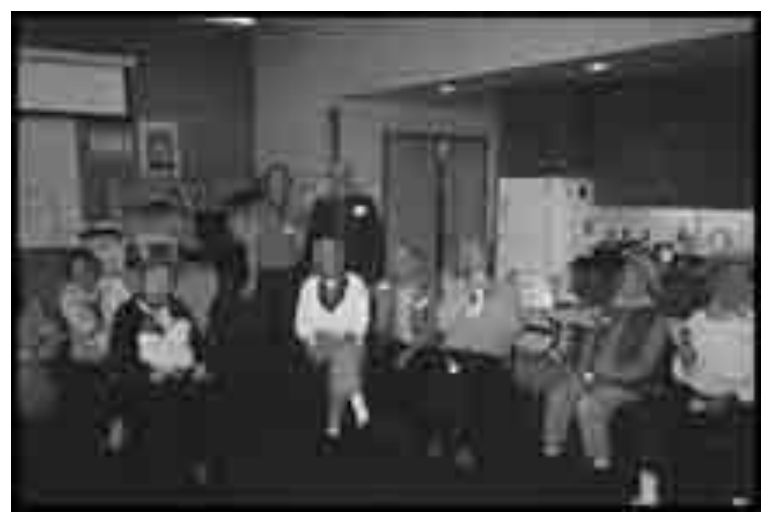

Figure 101: Senior Social: In enjoy that group.

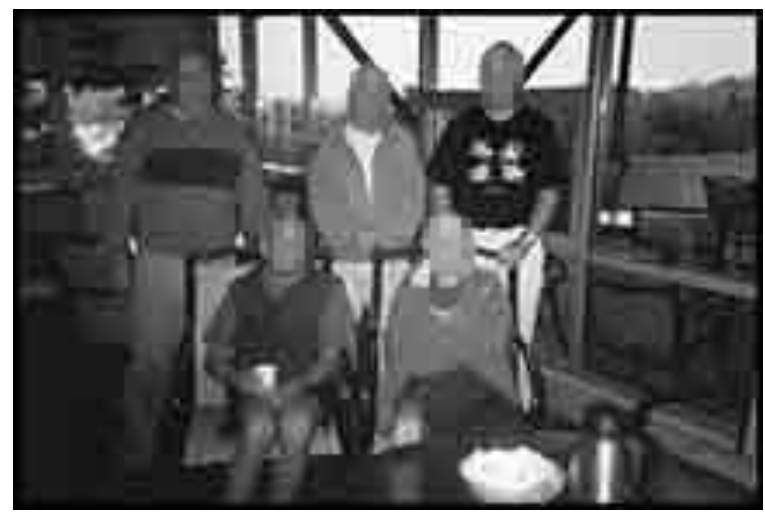

Figure 102: Coffee Group. 


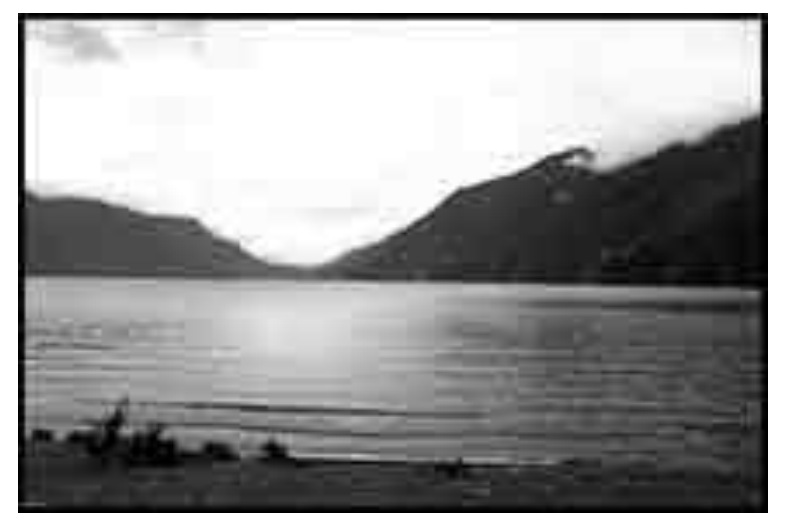

Figure 103: Lake Crescent and Mountains towards West of the Lake: We hike here in the mountains around the lake and especially enjoy the hikes here.

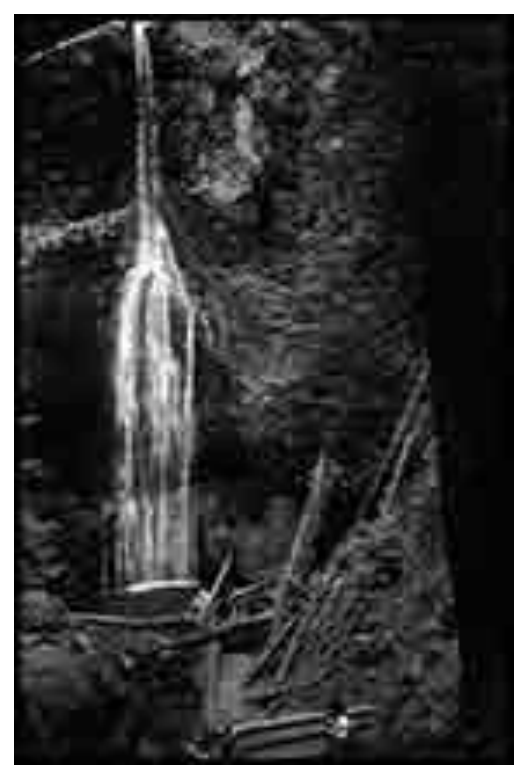

Figure 104: Falls in Olympic North Park: Hiking with a Group from Our Hiking Club: Being a people person, I very much enjoy the social interaction that takes place during our hikes and sometimes, just hiking, looking, and listening - drinking in nature with no interruption. 


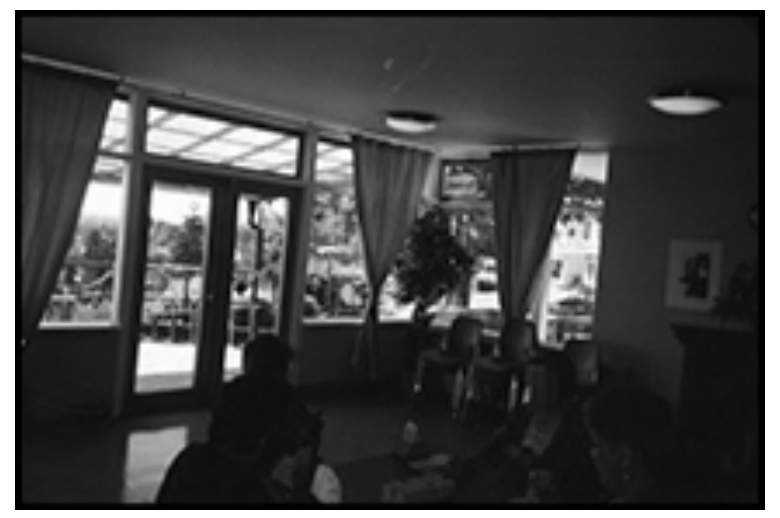

Figure 105: The Central Area Senior Center Bridge Group: Mon, Wed, Fri - 11:25 AM 3:00 PM: Sitting at the bridge table $3 \mathrm{x}$ a week is so important to me.

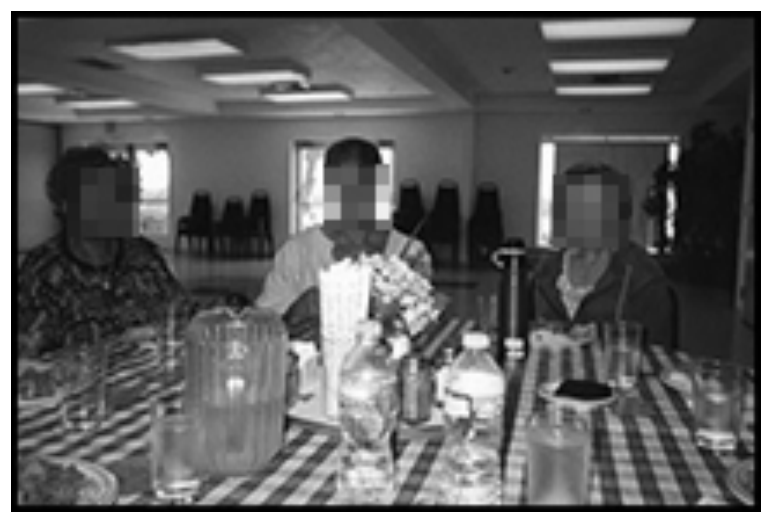

Figure 106: Bridge Group at the Central Area Senior Center: Lunchtime in the center's dining room. It's our lunch period where we can catch up on trips and other activities. It is so much fun discussing various topics. 


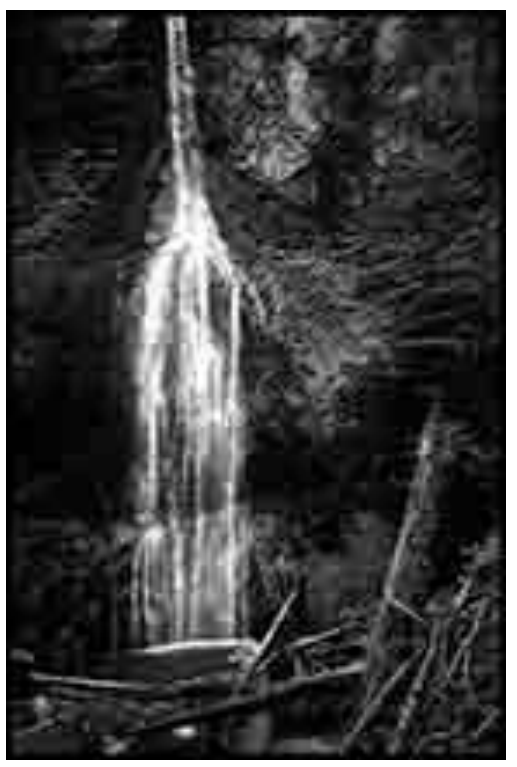

Figure 107: Falls in Olympic North Park: Hiking with a Group from Our Hiking Club: We all share a keen interest in nature and ecology. Four or five of our good friends were with us.

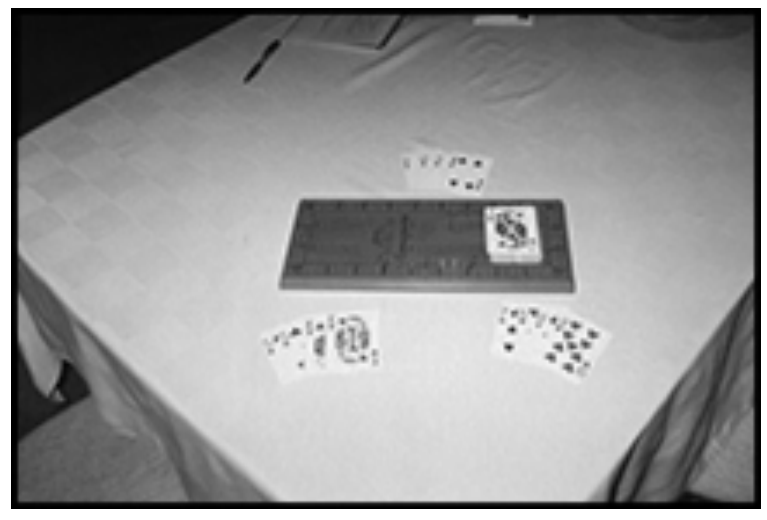

Figure 108: Cribbage Board: My favorite card game. 


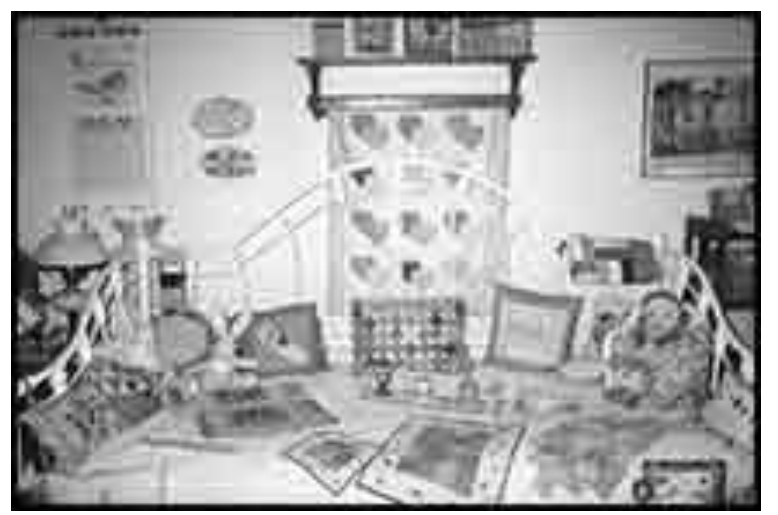

Figure 109: I Love to Quilt: My new white metal bed frame and some of my quilts I've made.

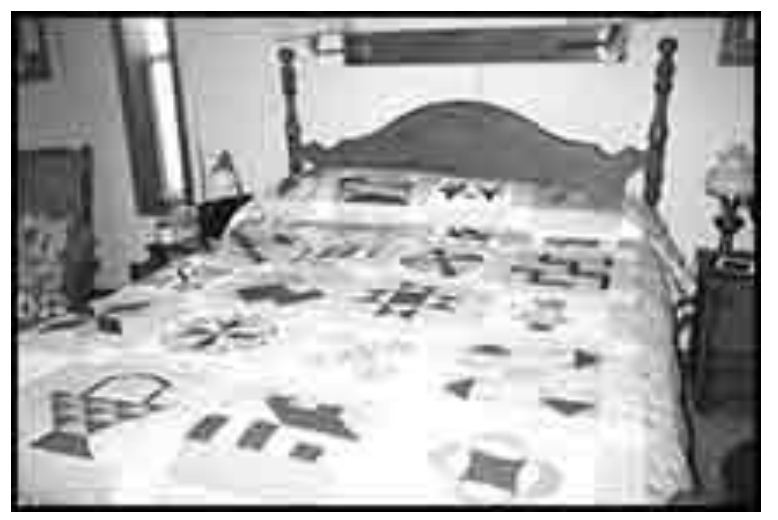

Figure 110: Quilt on Bed: Quilt I made in 1990. Loved learning to quilt and haven't stopped sewing.

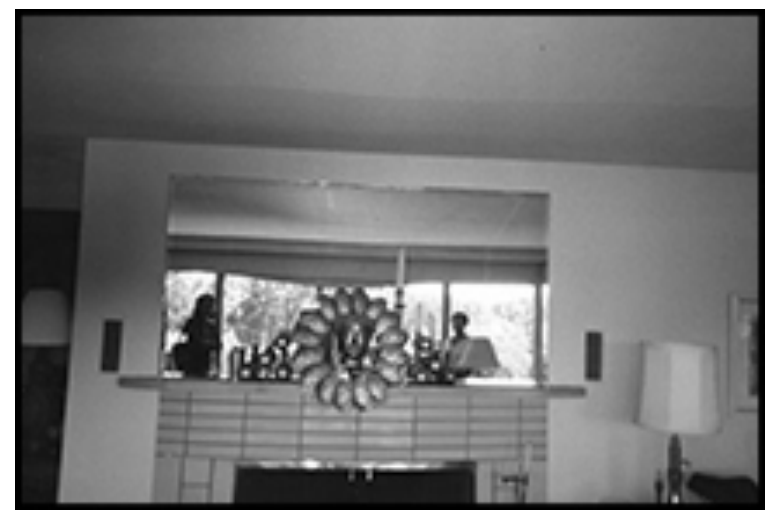

Figure 111: Crab Wreath: Crabs from Port Townsend. I caught and she arranged. 


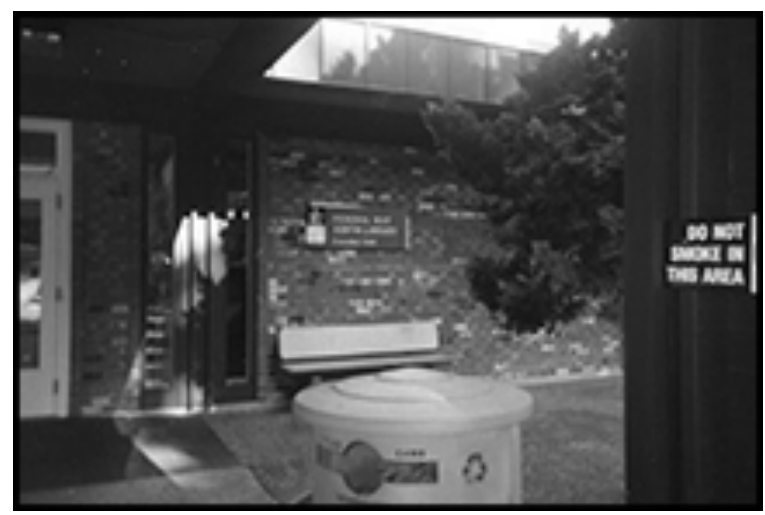

Figure 112: Untitled.

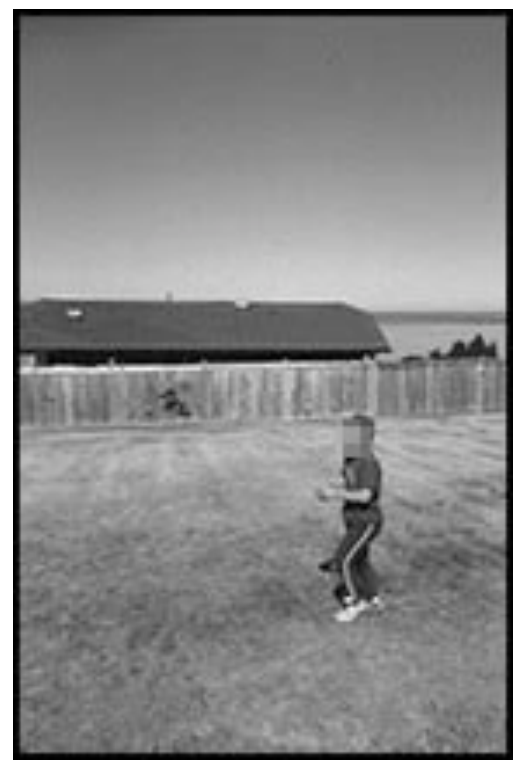

Figure 113: Eric and His Friend Playing Baseball: Children (our future, our hope) playing with them and taking enrichment in just play. 


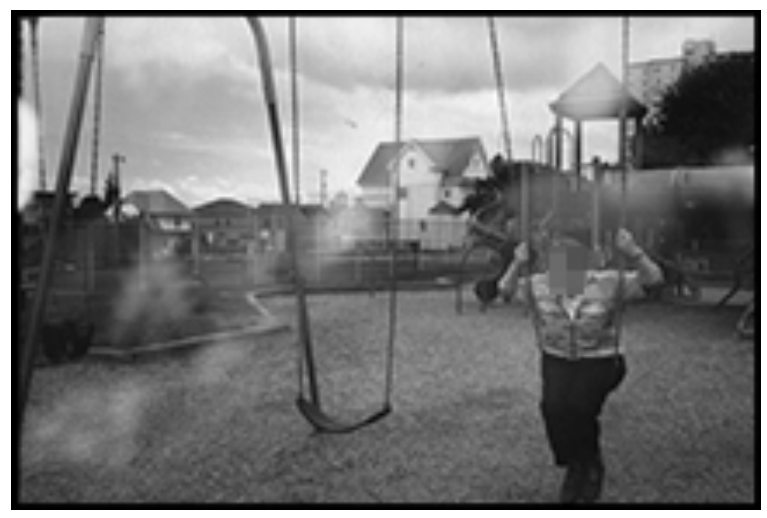

Figure 114: Me in a Swing Near My House: To swing makes me feel free - I love it.

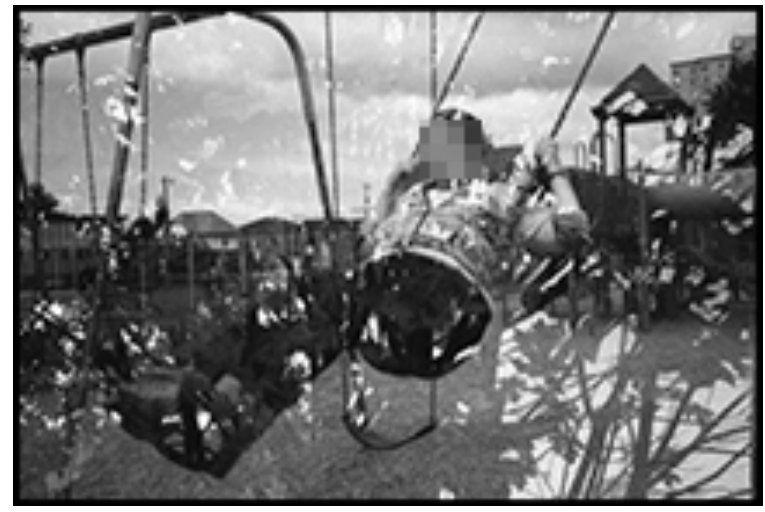

Figure 115: Another Photo of Me in a Swing: I love it! - So much, I wanted two photos.

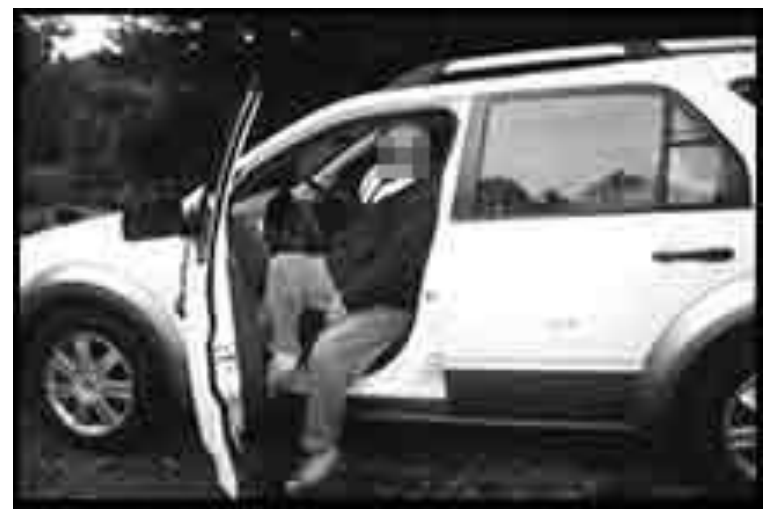

Figure 116: Car Rides: I like taking road trips. 


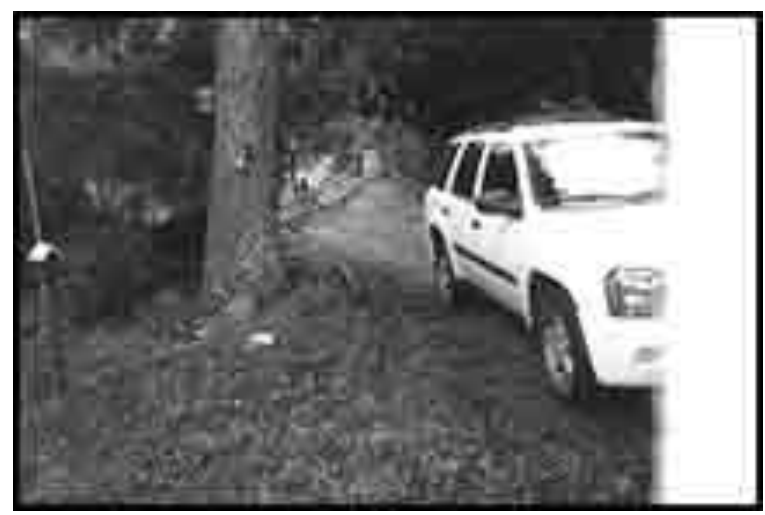

Figure 117: Car Rides: Me getting into the car.

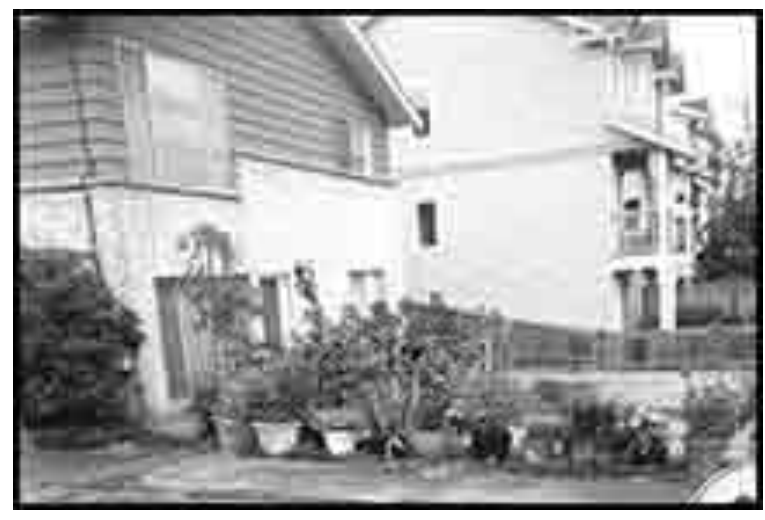

Figure 118: My Mailbox: Mailbox = primary way of keeping in touch.

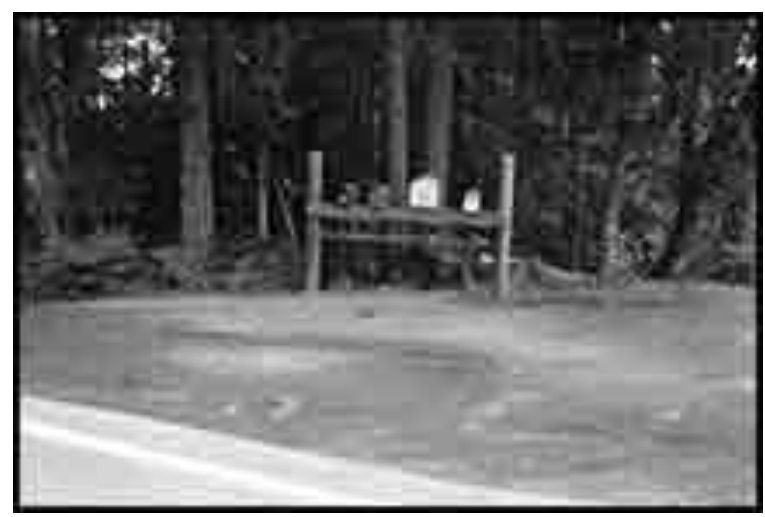

Figure 119: Country Mailbox Shared with Neighbors: I look forward to the mail every day. 


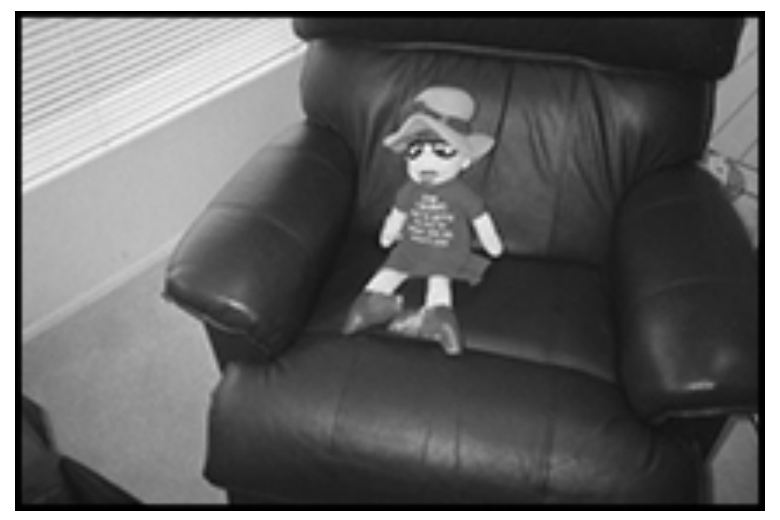

Figure 120: Singing and Dancing Doll: "Don't you wish your grandma was a freak like me?”. My grandsons gave me that. It's so much fun.

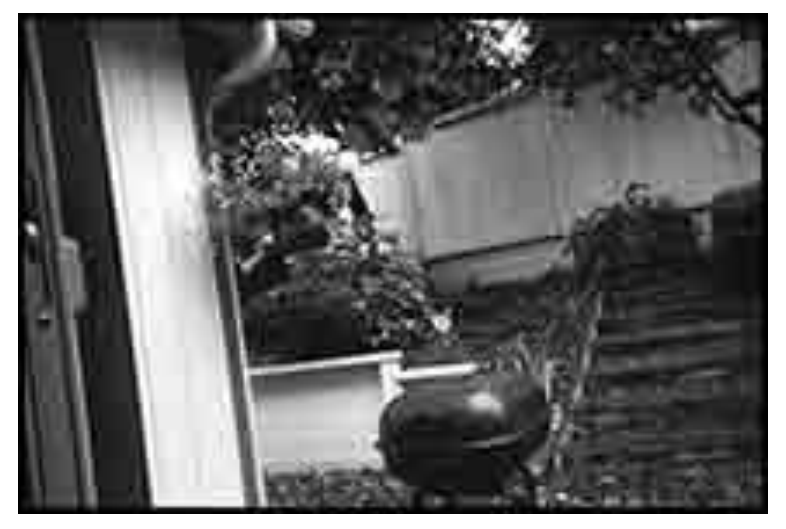

Figure 121: Hanging Flowers: It's the most flowers I can see. They're important to me.

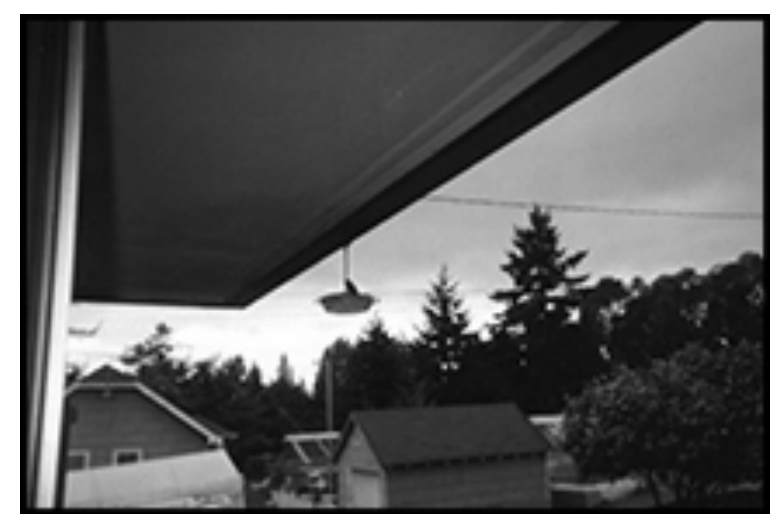

Figure 122: Picture of Hummingbird on Our Back Porch Bird Feeder: I enjoy watching these little birds flying in and out. 


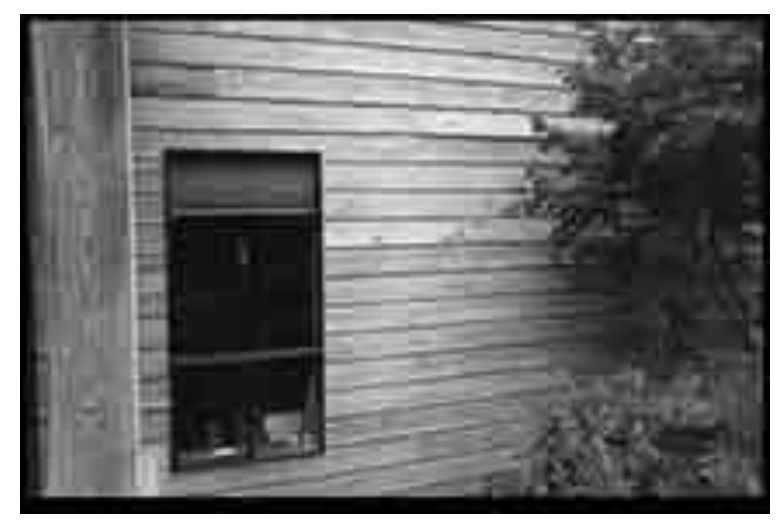

Figure 123: Spider on Web with Raindrops Glistening on the Web: I like to watch spiders weave their webs so they get just the right distance on each cross weave.

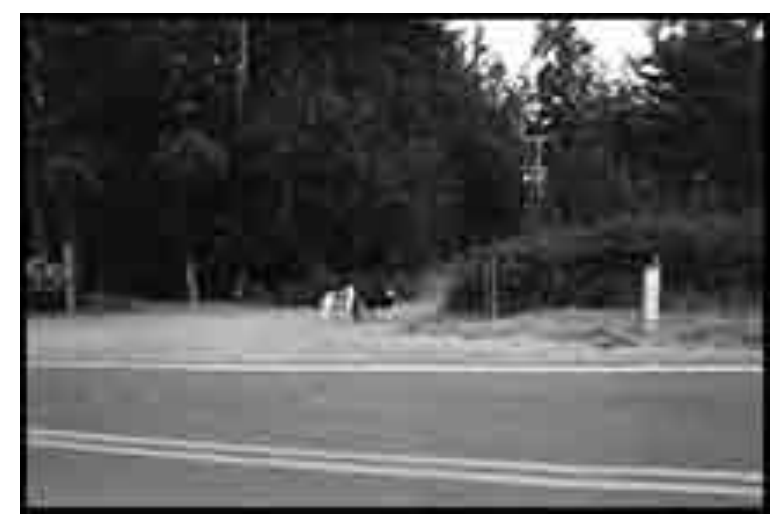

Figure 124: Neighborhood Dogs - Five of Them and Our Dog was with Them: I like dogs and enjoy watching their social interaction when they are getting acquainted. 


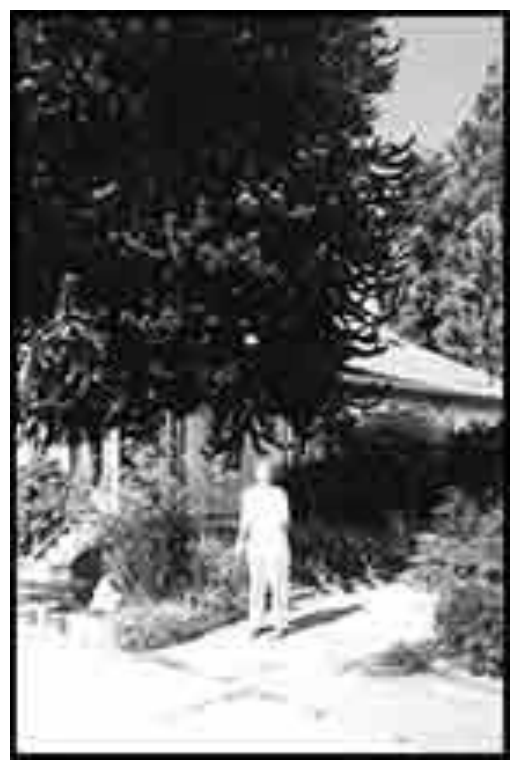

Figure 125: Me under a Monkey Tree at NW 61st and 11th NW: I love this kind of tree and have walked here many times.

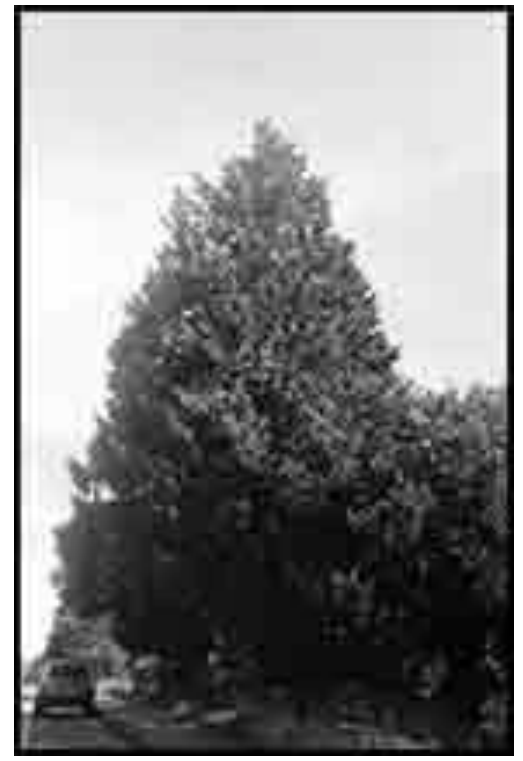

Figure 126: Cedar Tree: Cedar trees are a recurring theme in my life. 


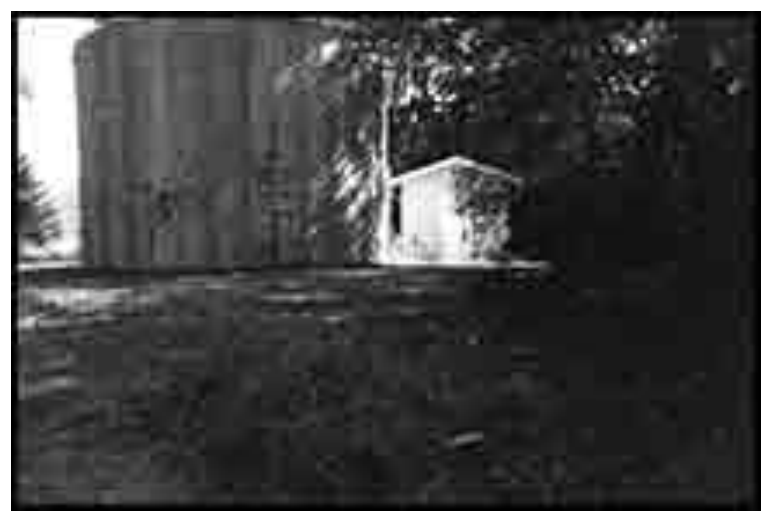

Figure 127: Water Tank and Pump House: Our water for home use is stored there. I often walk here.

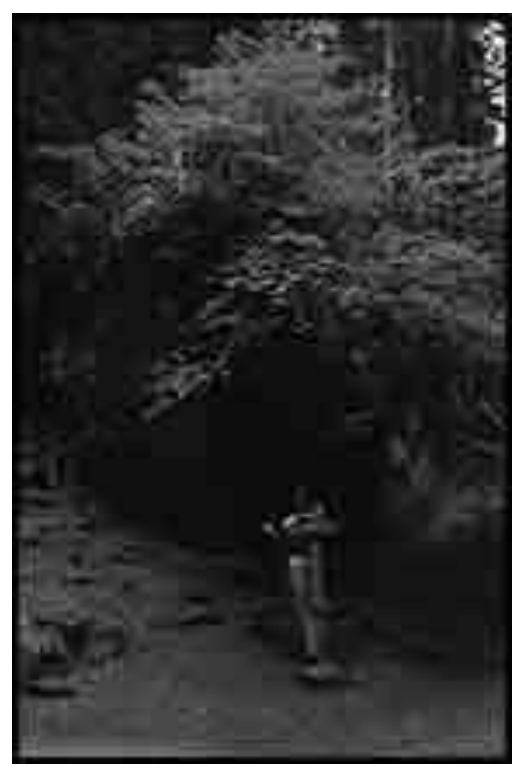

Figure 128: Sign to Storm King: Very large stone with a person draped over it. Another place where we like to hike. 


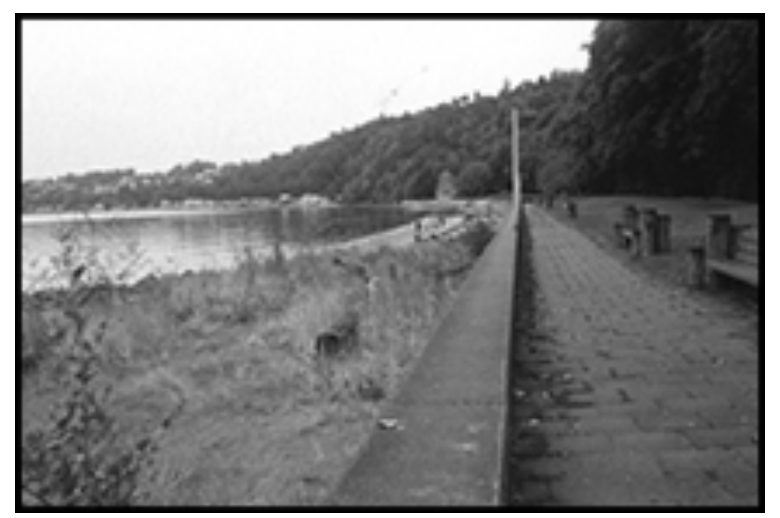

Figure 129: Took Picture of Seahurst Park Beach Area: We live close to this park and enjoy walking it about 4 or 5 times every week.

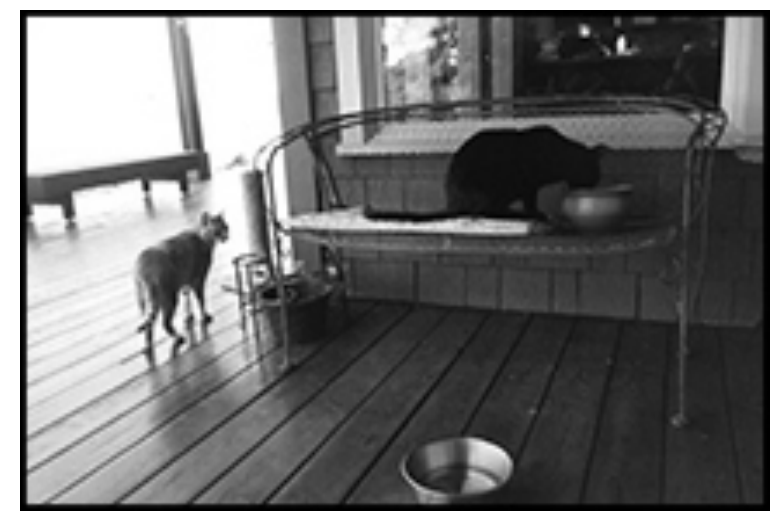

Figure 130: I like cats.

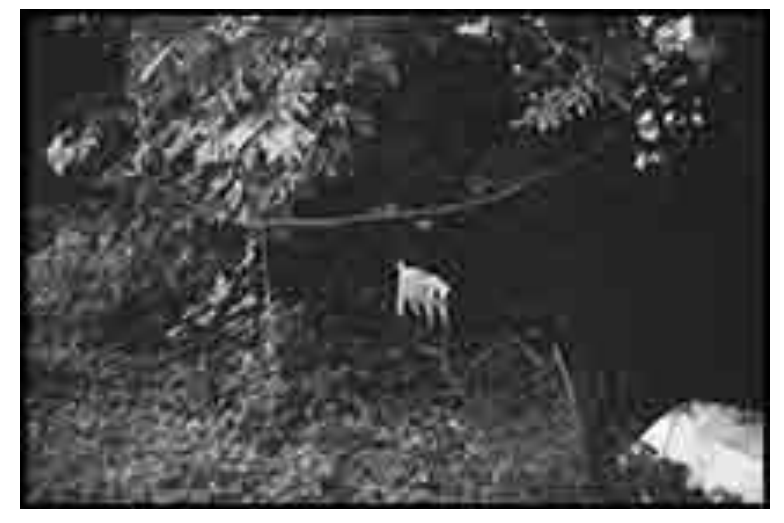

Figure 131: Billy Scruff, Our Goat: He is part of the family. 


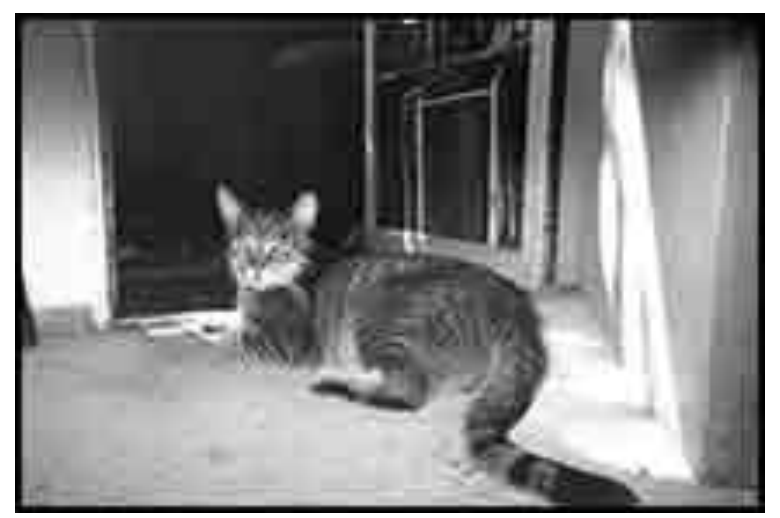

Figure 132: Boyfriend's Cat: I love this cat! He brings me a lot of pleasure.

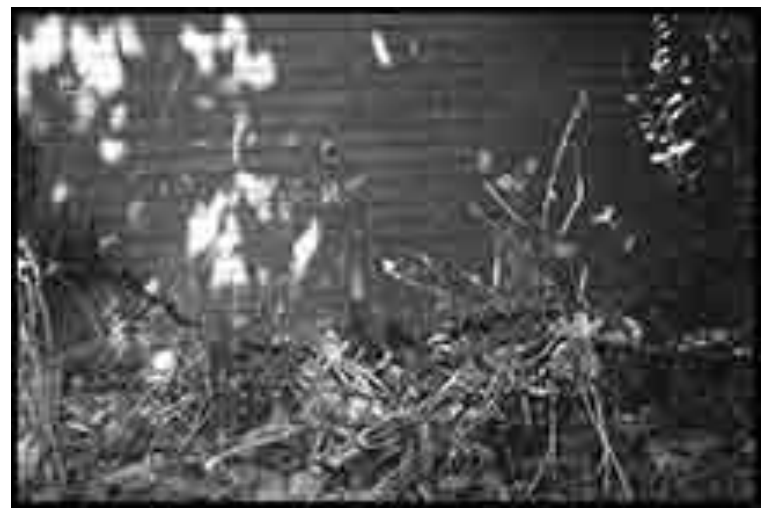

Figure 133: Another of Boyfriend's Cat: I've known this cat for years so he feels like an old friend.

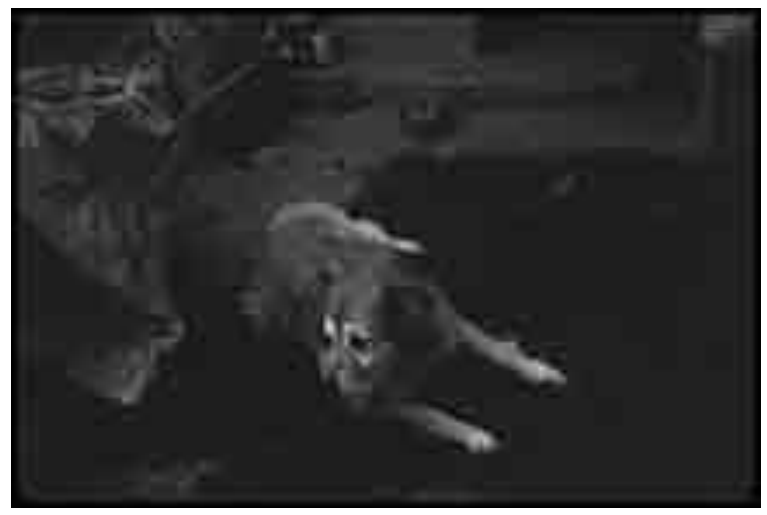

Figure 134: My Dog 'Sweet Pea'. 


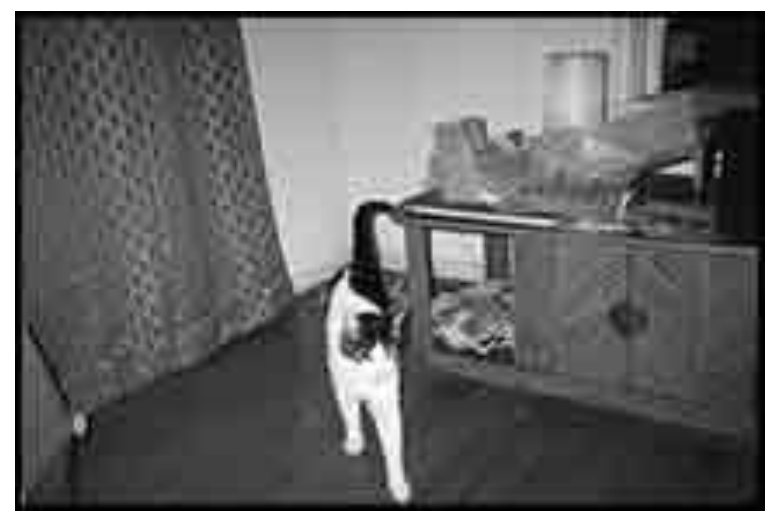

Figure 135: 'Nelli' - My Cat.

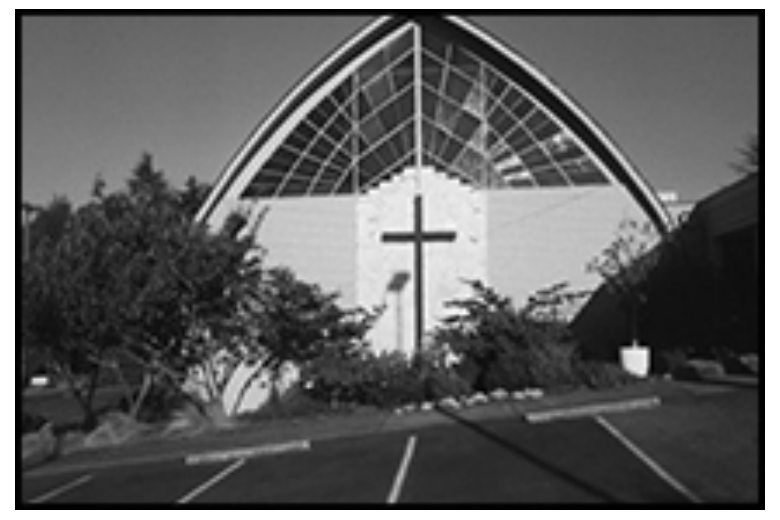

Figure 136: Our Church: My peace and salvation and friendships that go with it.

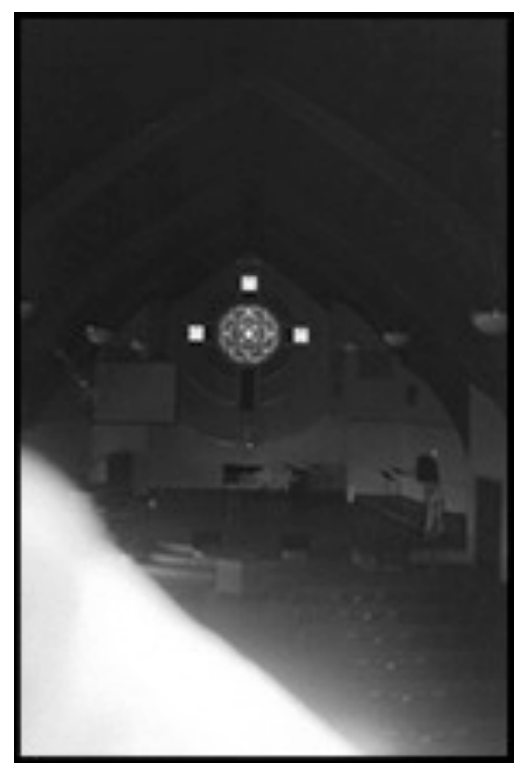

Figure 137: Our Church: We're real active in our church. It's really nice because we have small groups spread out from that. 


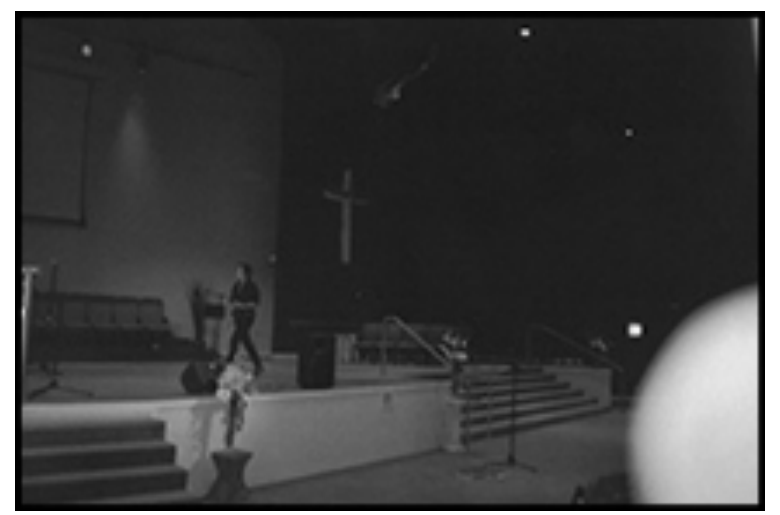

Figure 138 My Church Sanctuary: I was raised in church where we attended church six days a week (Saturday night was off night!).

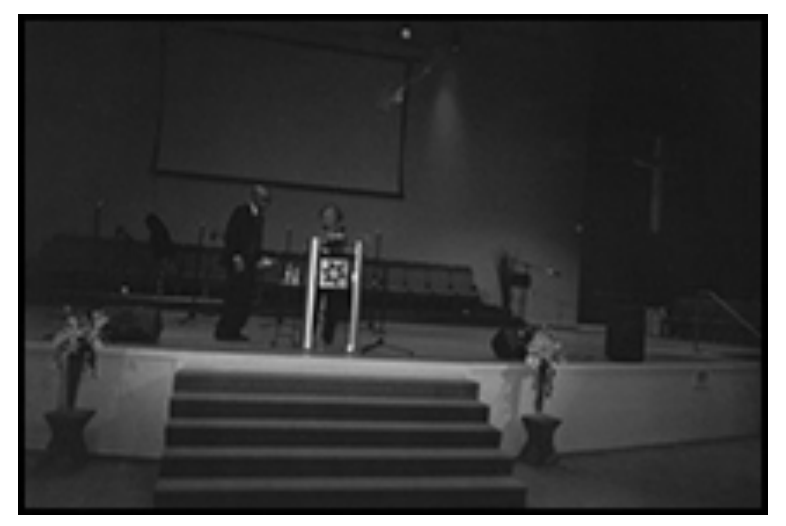

Figure 139: My Church Sanctuary: My Christian Upbringing keeps me attending church each and every Sunday: Worshiping the Lord Gives me strength to get through each and every day! Praise the Lord - Glory Hallelujah. 


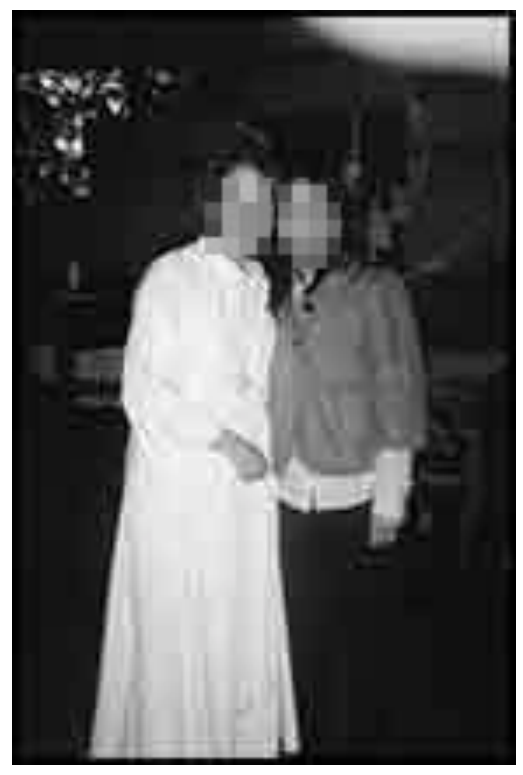

Figure 140: Our Choir Director and Organist.

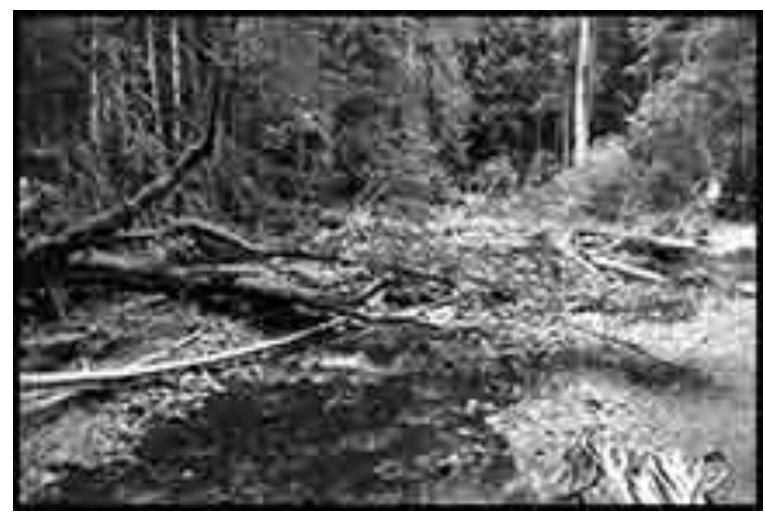

Figure 141: The Mountains: We hike here in the mountains and especially enjoy the hikes here.

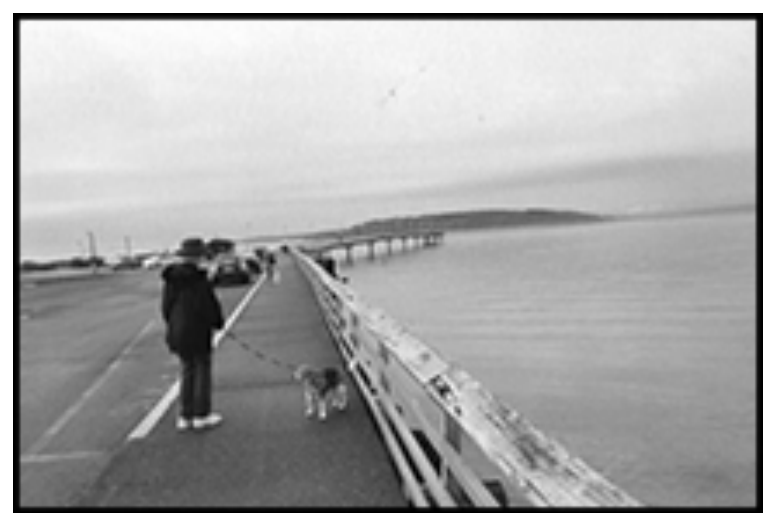

Figure142: Waterfront Picture at Des Moines: We frequently walk there. 


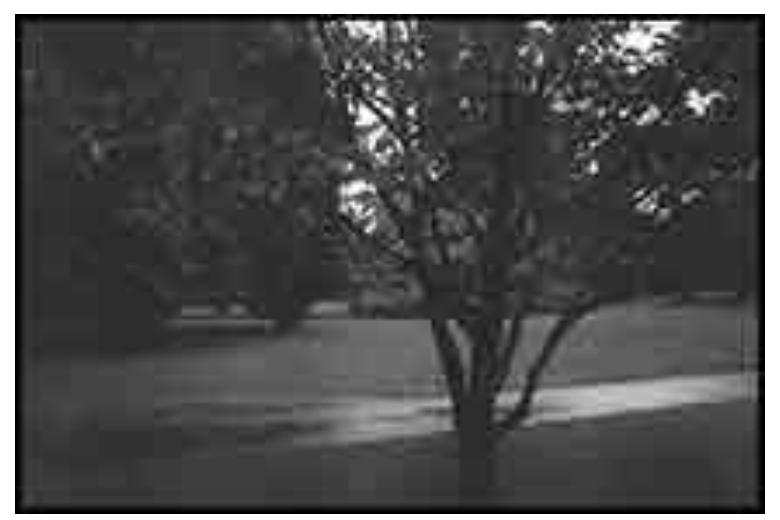

Figure 143: Volunteer Park: I really enjoy the peace of this spot. I come here at least once per week.

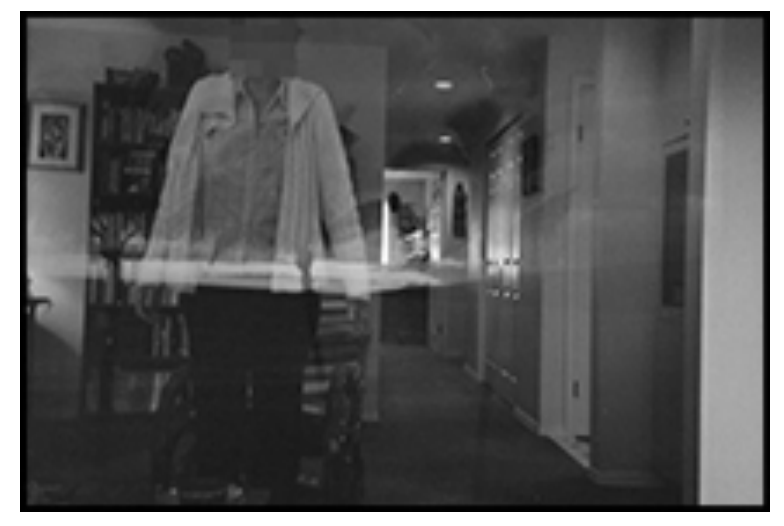

Figure 144: My Sister: Hetty is my friend and helps me a lot.

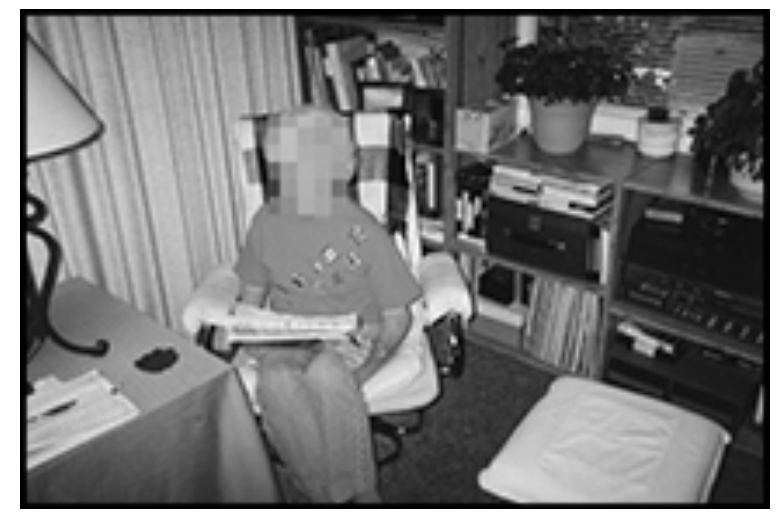

Figure 145: My Wife: We have had a lovely life together for 56 years. She helps me with my Alzheimer's problem. 


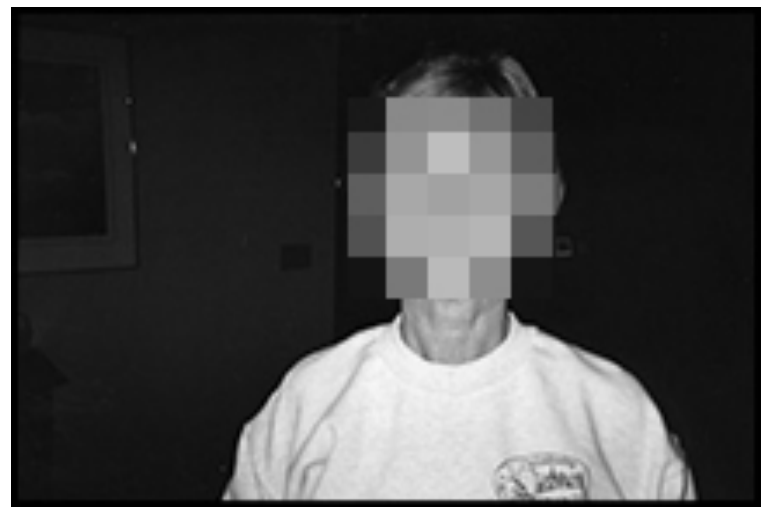

Figure 146: My Wife: Because she takes care of me. She is the love of my life.

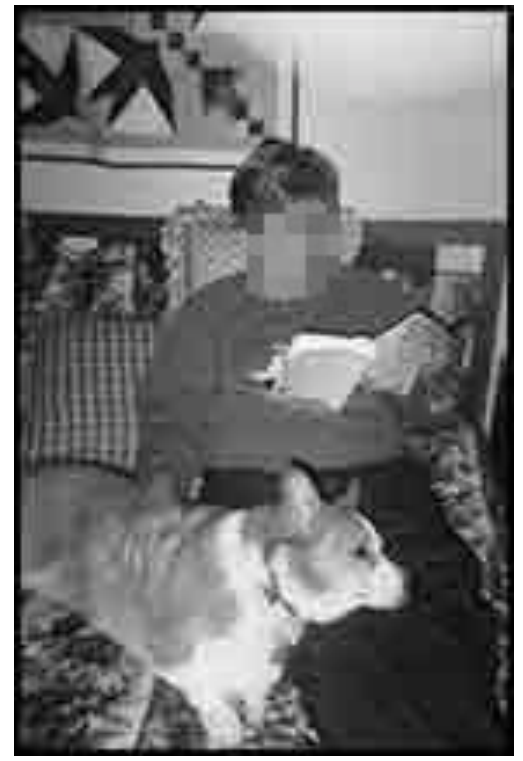

Figure 147: Sally and Nicolas: Sally is my wonderful wife and I can't imagine life without her.

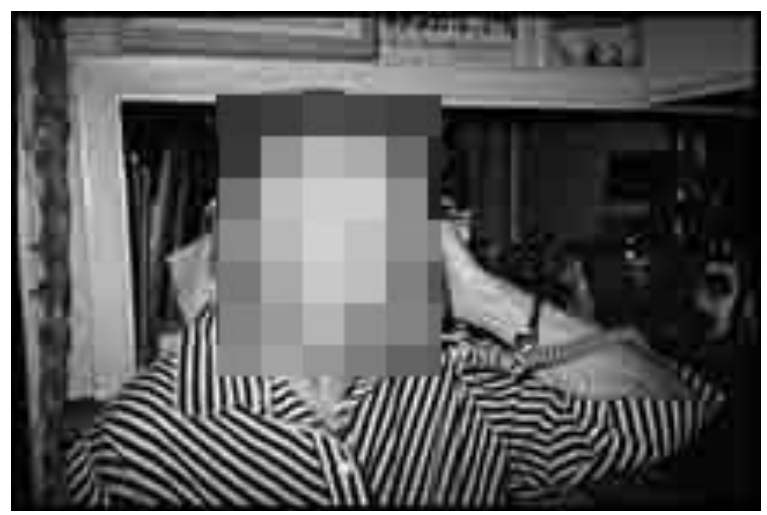

Figure 148: Nana: My anchor in this scary world. 


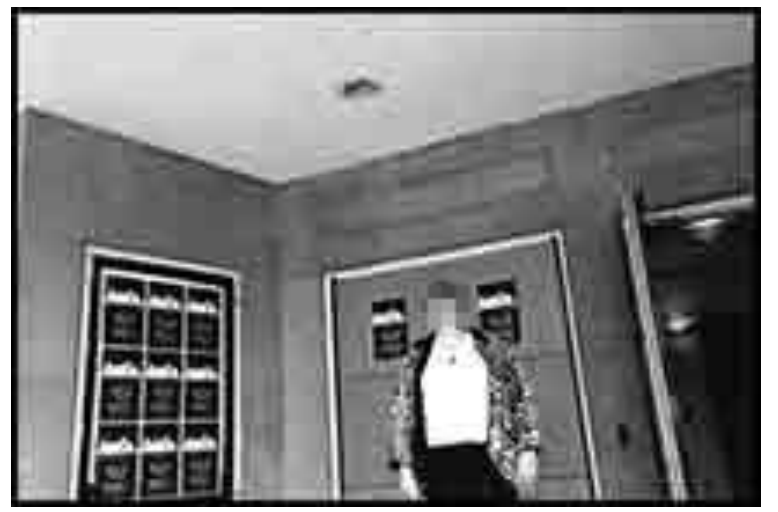

Figure 149: Daughter Beatrice: She cares for me. She is my only daughter.

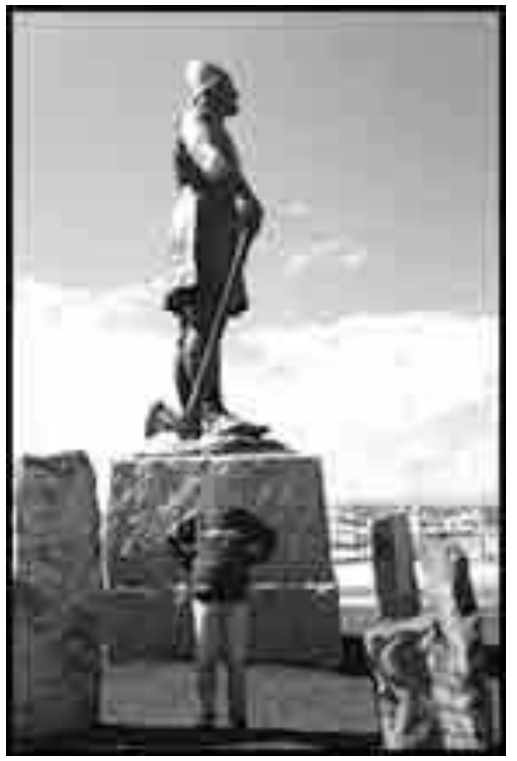

Figure 150: Boyfriend at Leif Erikson Statue: Boyfriend has been a key person in my life for 20 years. 


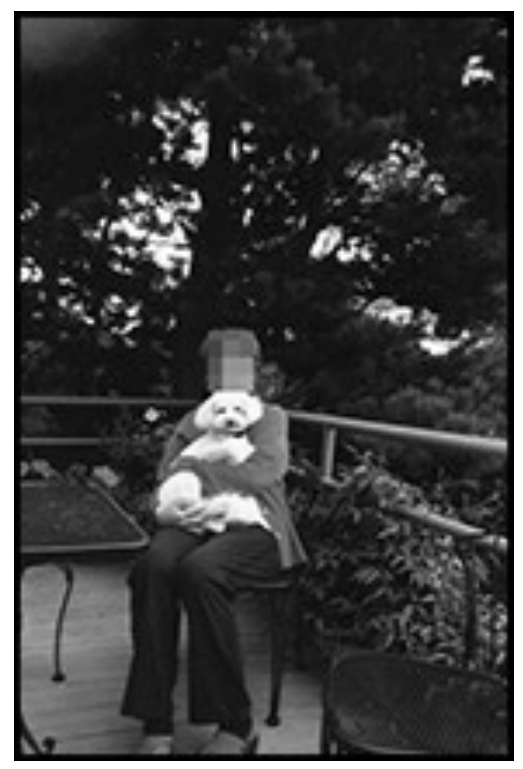

Figure 151: Kamil and Edda: A friend who 'gets it' (re: Alz) and who talks with me about my experiences and reactions to what is happening to me.

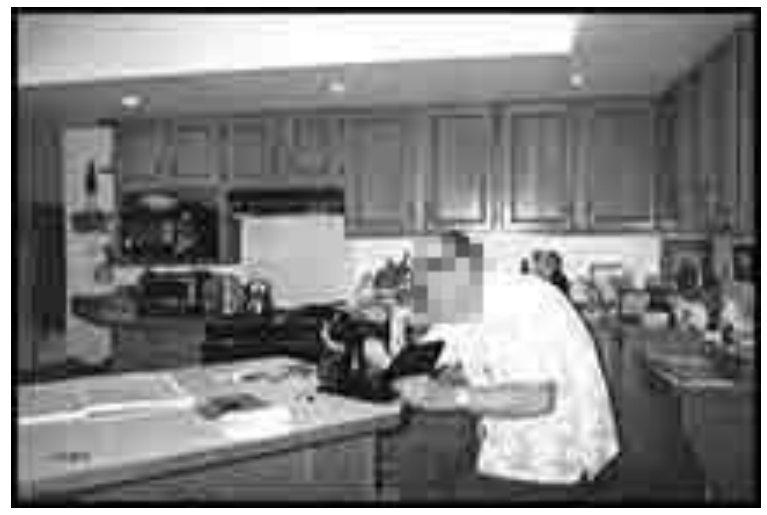

Figure 152: Ben: Husband.

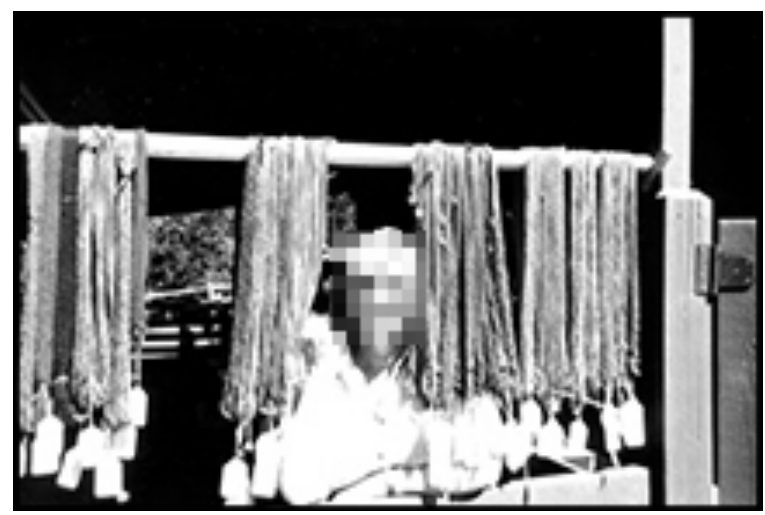

Figure 153: Beatrice: My wife and love of my life. 


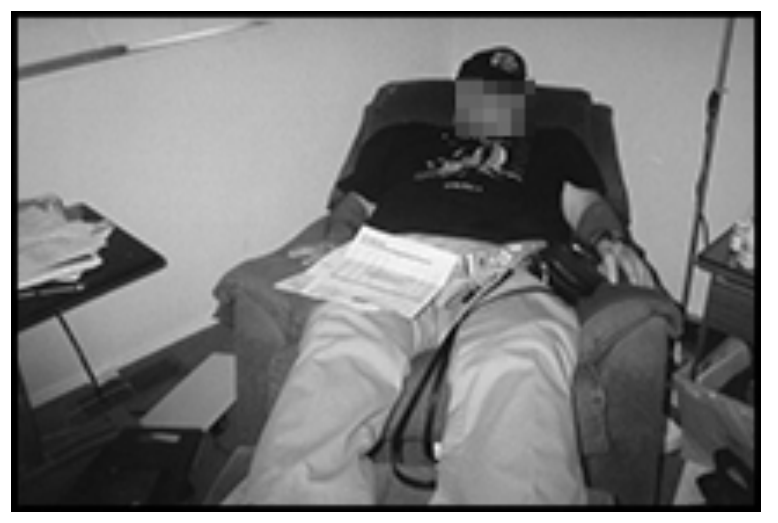

Figure 154: Ken doing his favorite thing.

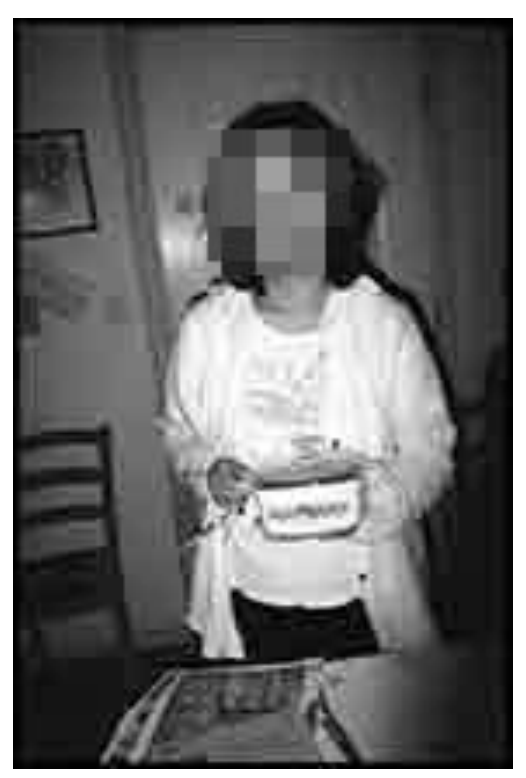

Figure 155: Young Tia with Dinner for Me. 


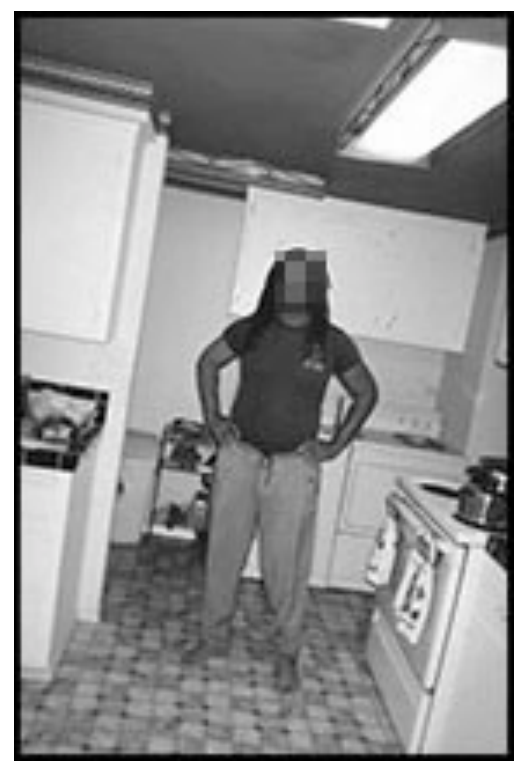

Figure 156: My Son Van: He is \# 1 protector of his aunt Kacie and his mother!

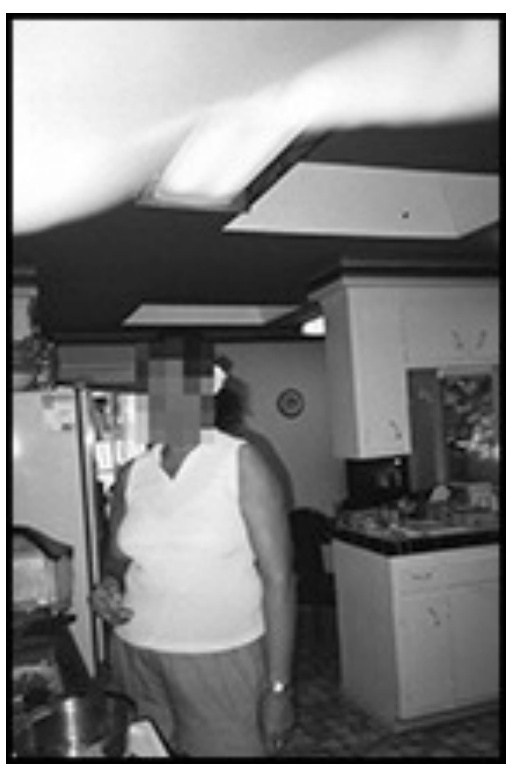

Figure 157: Kacie Fixing Vegetable: She's my sister and she's always looking after me. Each day is special because we do so many things together each day. 


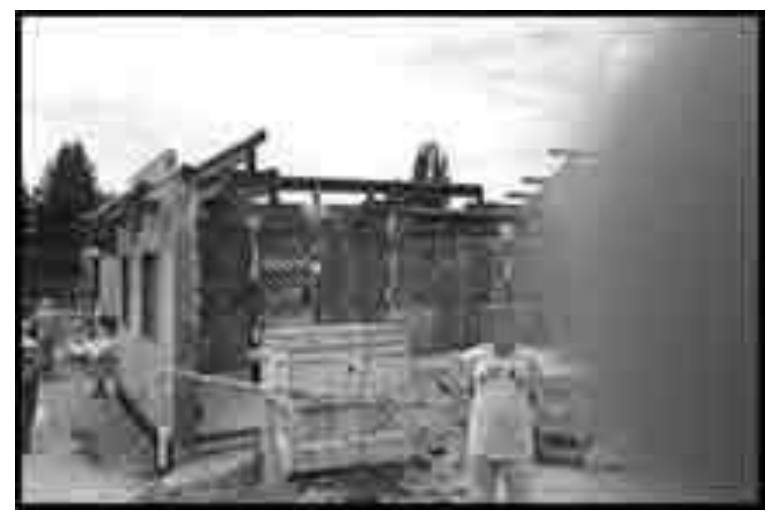

Figure 158: Paul: Seattle Chinese Garden: Paul is an old friend who I trust completely and is the executer of my will. He took me to see the Chinese Garden in West Seattle as he knew I wanted to see how it is progressing but couldn't drive there.

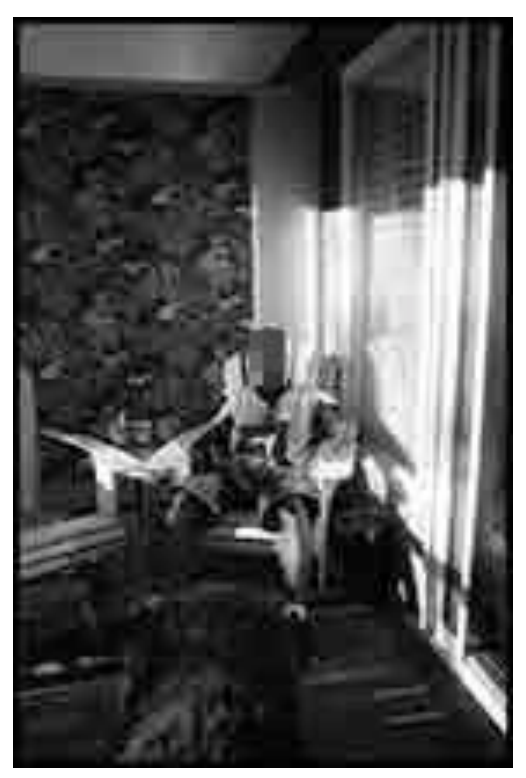

Figure 159: My Son Kristopher: The most important person in my life ... from the moment he. 


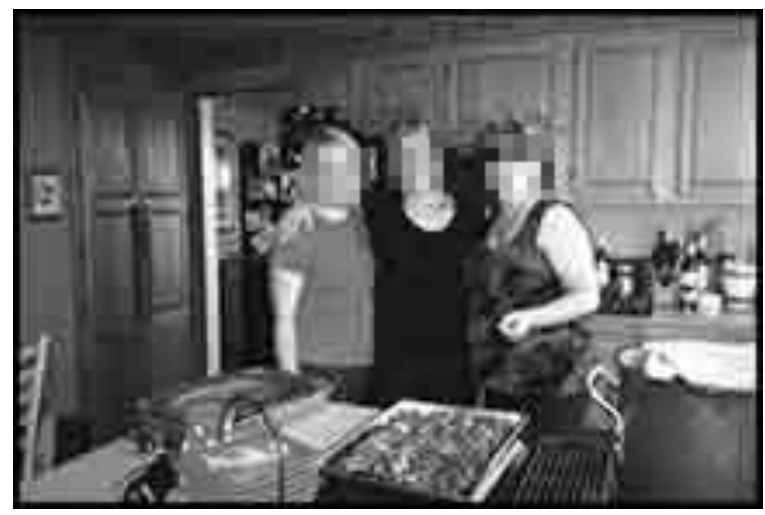

Figure 160: Family Dinner: I did not help.

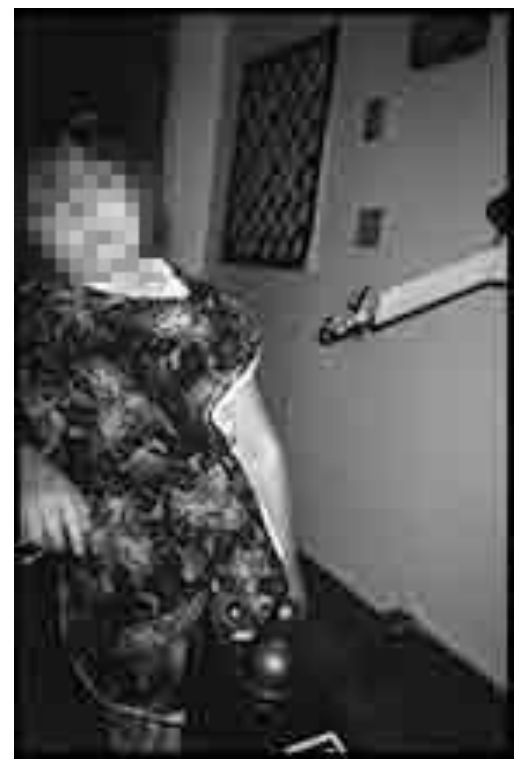

Figure 161: My Granddaughter: Cooking her dad's birthday cake. 


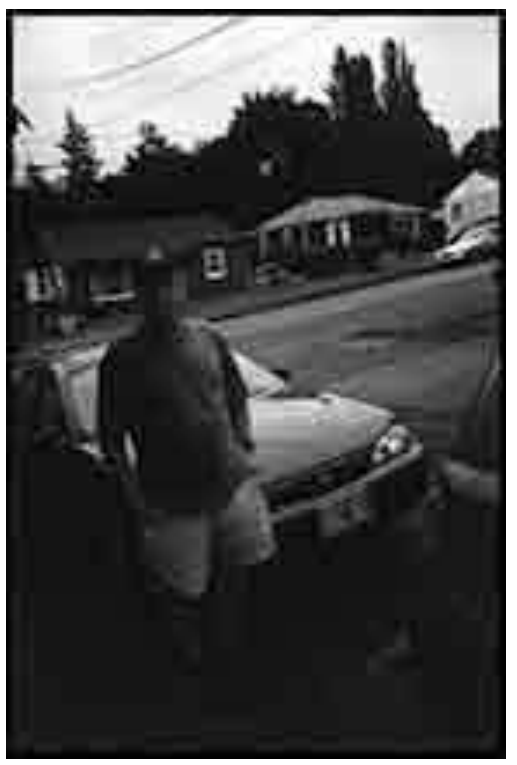

Figure 162: Son Kyle: He came from Oregon and visited.

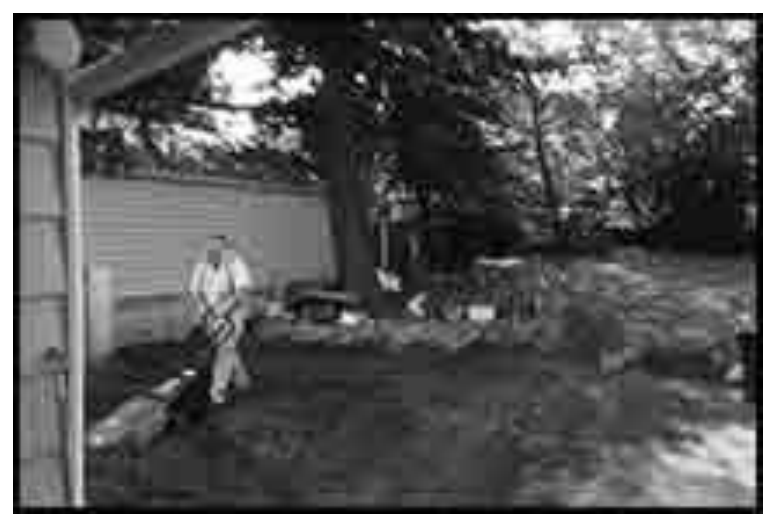

Figure 163: Son Don: He is very important to me.

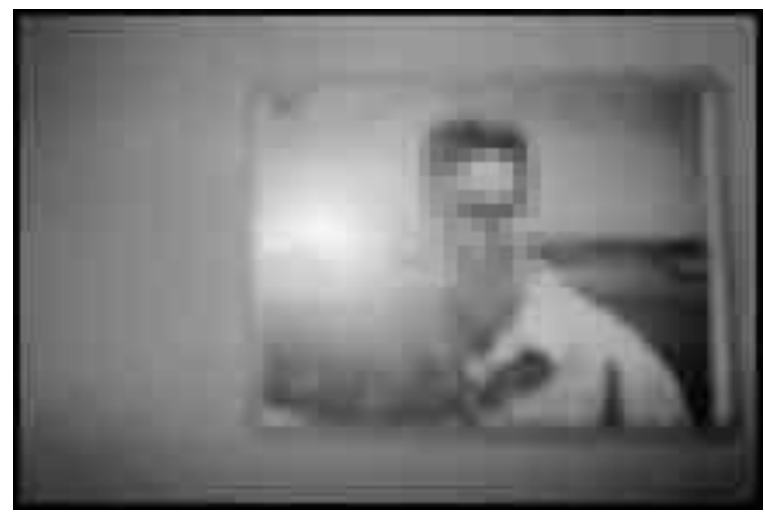

Figure 164: Picture of a Picture of My Son. 


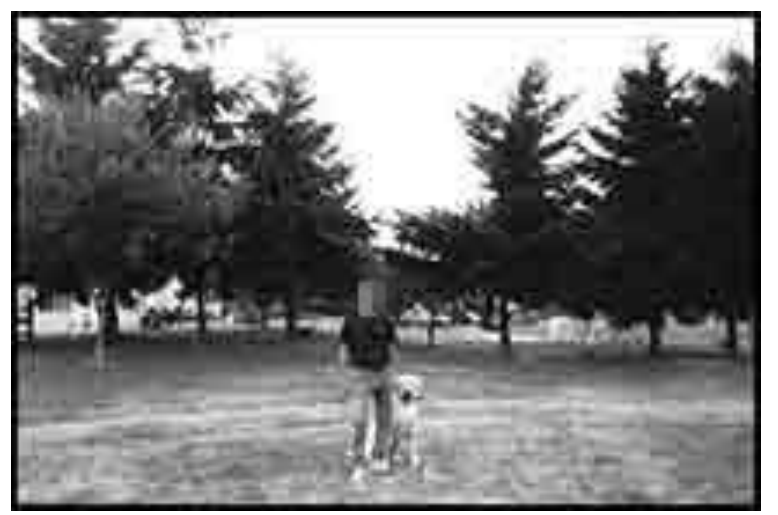

Figure 165: My Son: My son and his big dog, his 'buddy'.

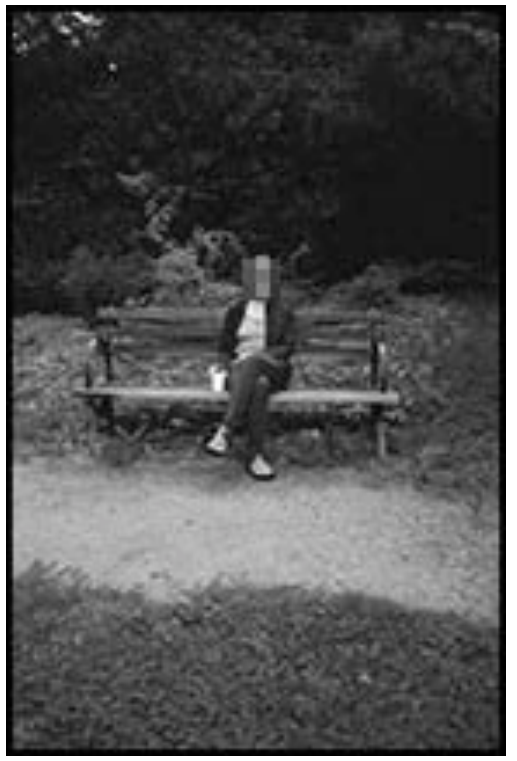

Figure 166: Gisela.

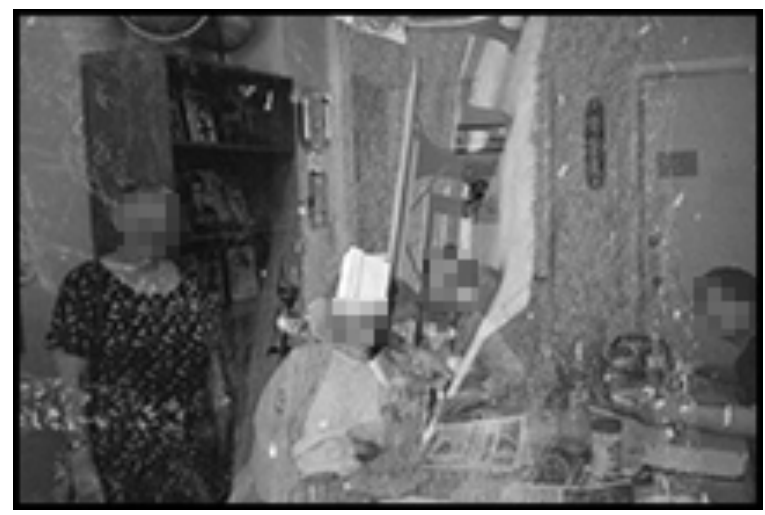

Figure 167: My Friends and Co-worker because together we can make a better world. 


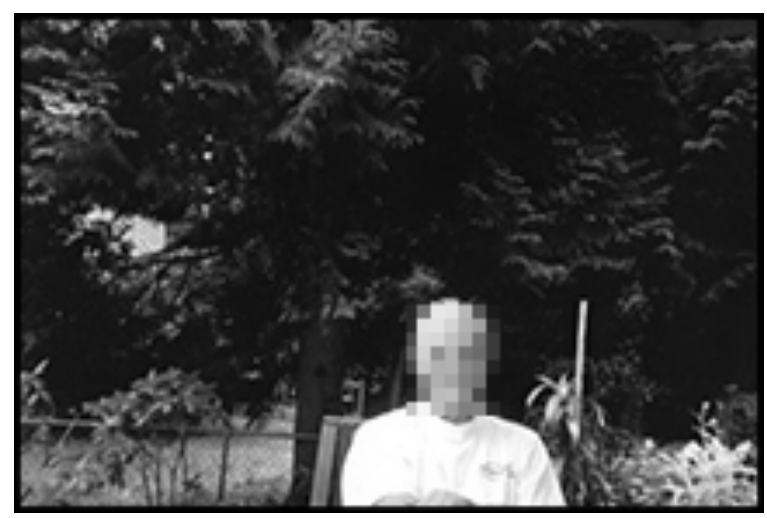

Figure 168: My best friend.

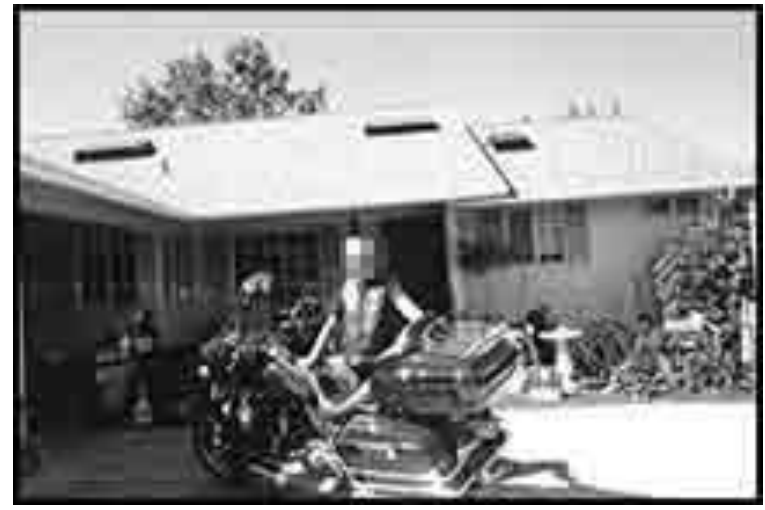

Figure 169: A Friend: He stopped by on his motorcycle to say hi.

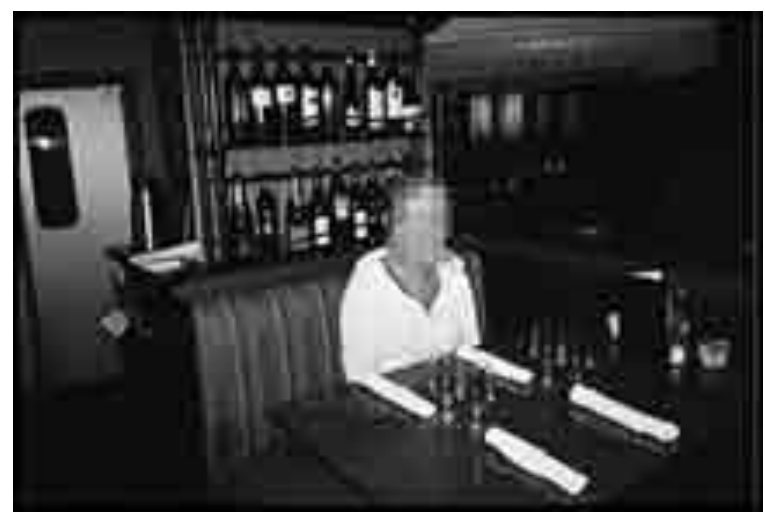

Figure 170: My Good Friend Edna at Lunch: I like to keep up with friends - meet for lunch. 


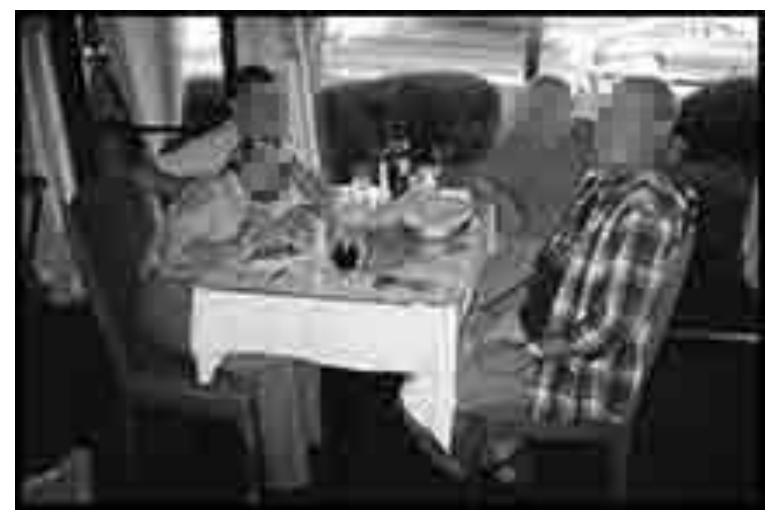

Figure 171: Friends: We met for lunch.

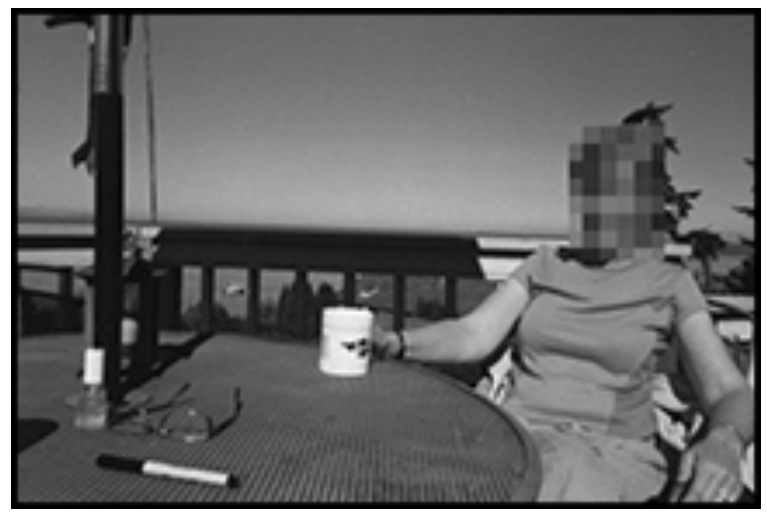

Figure 172: My Friend Who I Met in the Alzheimer's Support Group.

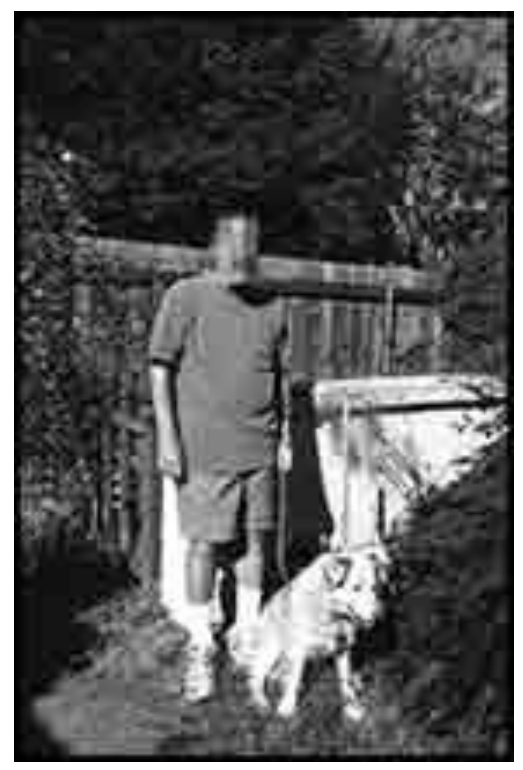

Figure 175: Psychologist Ed Who Leads the Support Group I'm in and His Dog: Support group, which meets weekly, is an important part of my life. 


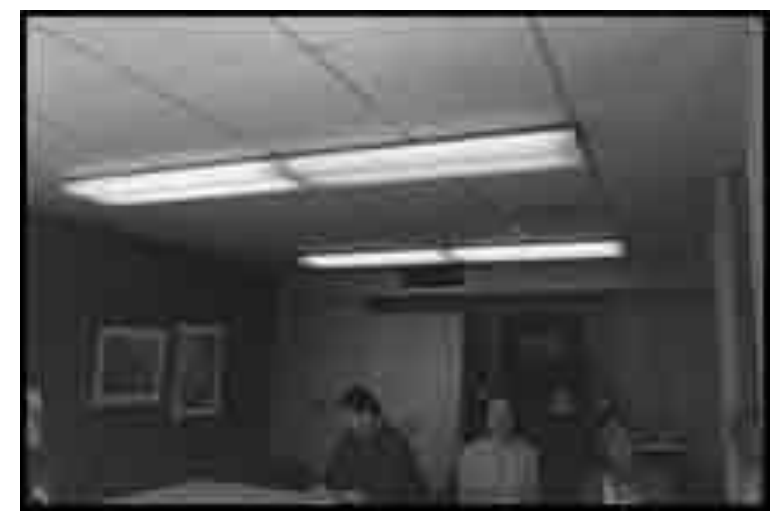

Figure 173: My "Family and Relationships" Group: Support group meeting once a month to discuss issues with family and relationships. Good and not so good. Gives us a chance to talk about things that we worry about - all is confidential.

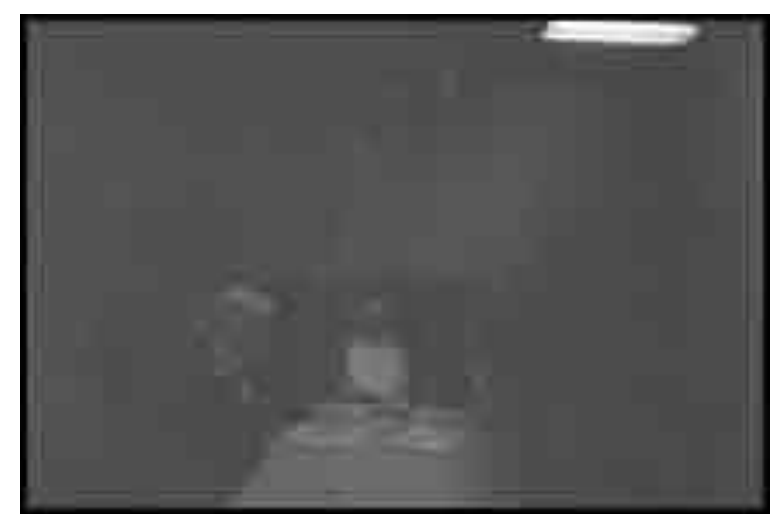

Figure 174: My "Living Alone" Group: We always have interesting topics. Today - we talked about what to do when we find a mouse/spider etc. we're afraid to touch. Will we call a neighbor, a friend, or plumbing emergency? 


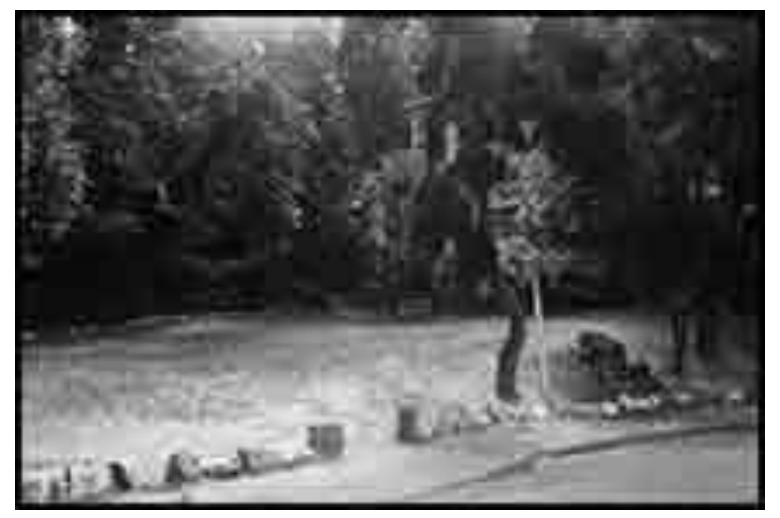

Figure 176: The Park: This is where many of us "dog owners" meet each other and last weekend had a band and potluck picnic.

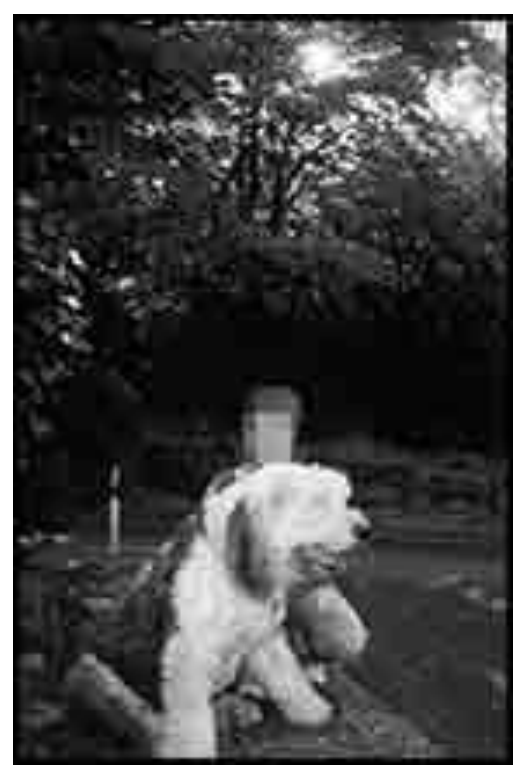

Figure 177: Bea - One of My Neighbors - Another Dog Owner: She knows about my diagnosis and if she doesn't see me and Igor for a couple of days ... she calls me. 


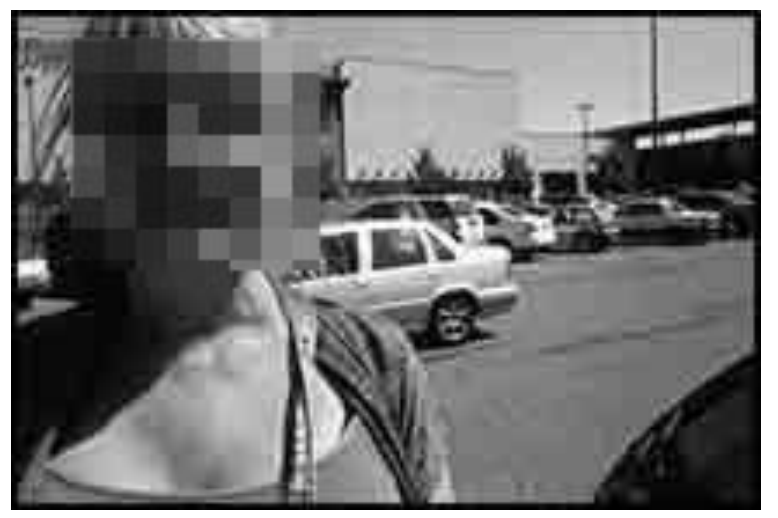

Figure 178: My Cousin: After an afternoon shopping.

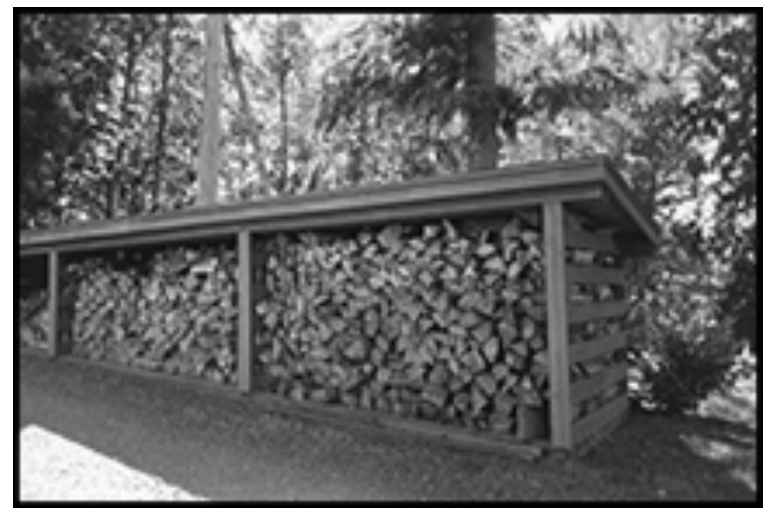

Figure 179: Wood Pile: I cut wood and split if. I enjoy the physical exercise and furthermore we enjoy using our fireplace on a cold winter day.

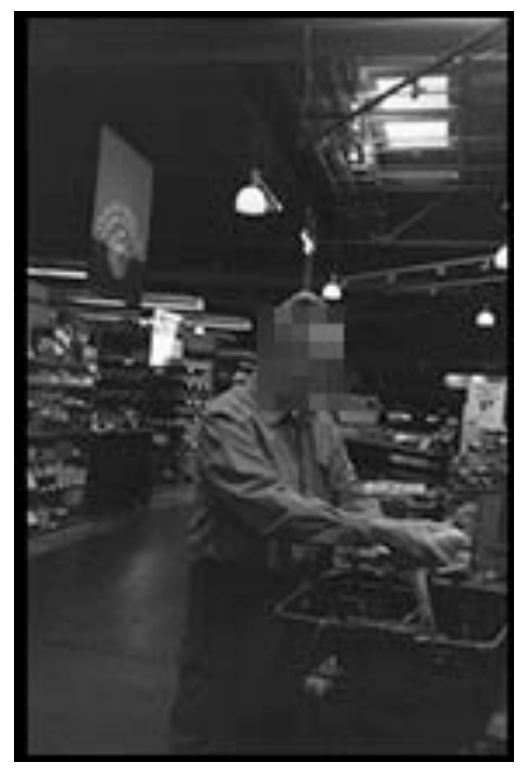

Figure 180: Me Shaving Corn at Central Market: I do most of our grocery shopping. 560 


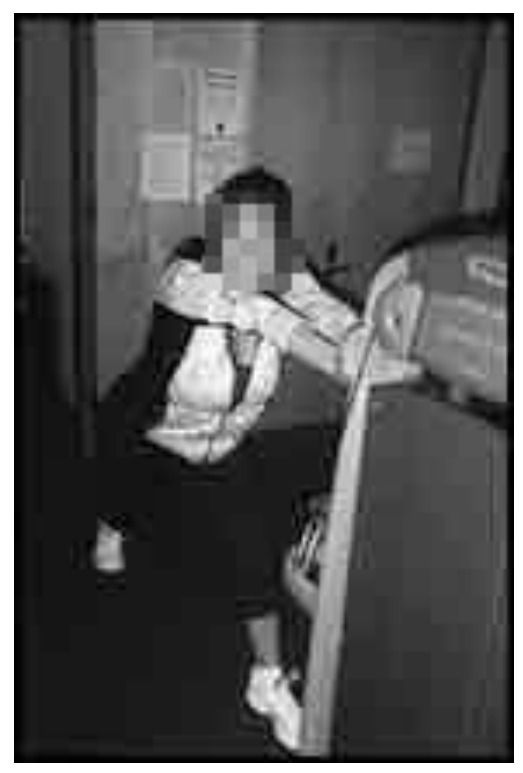

Figure 181:

Leader of the Exercise Class.

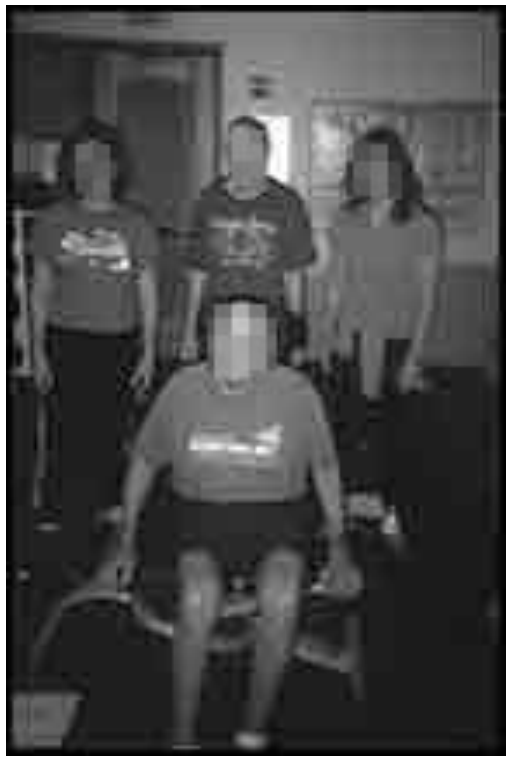

Figure 182:

Members of the Exercise Class. 


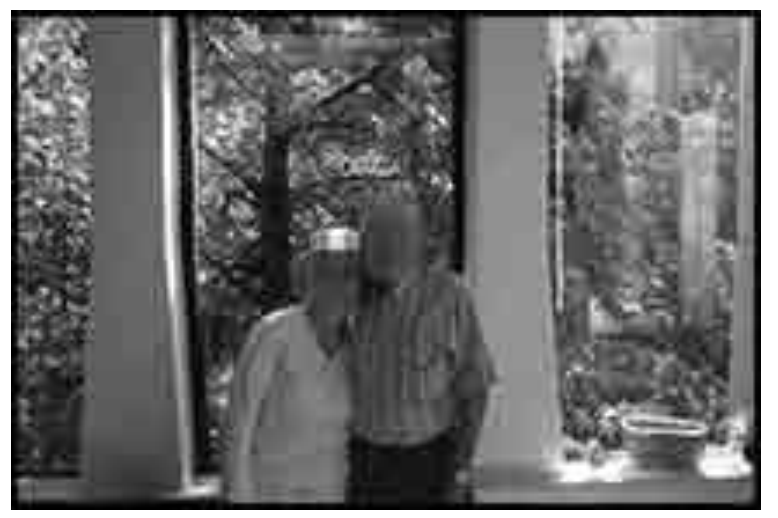

Figure 183: Me and Eric: He is my 92-year-old co-volunteer buddy, who treats me with respect and humor.

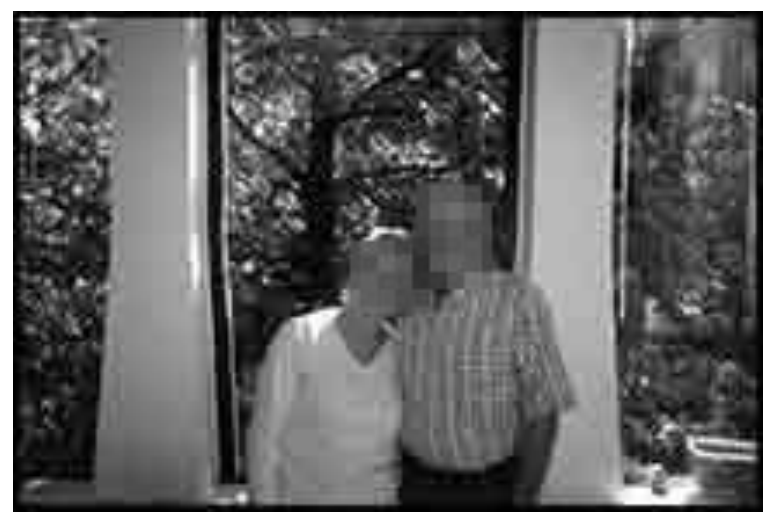

Figure 184: My volunteer buddy - his wife had expired from Alz Dx and was involved with this center. 


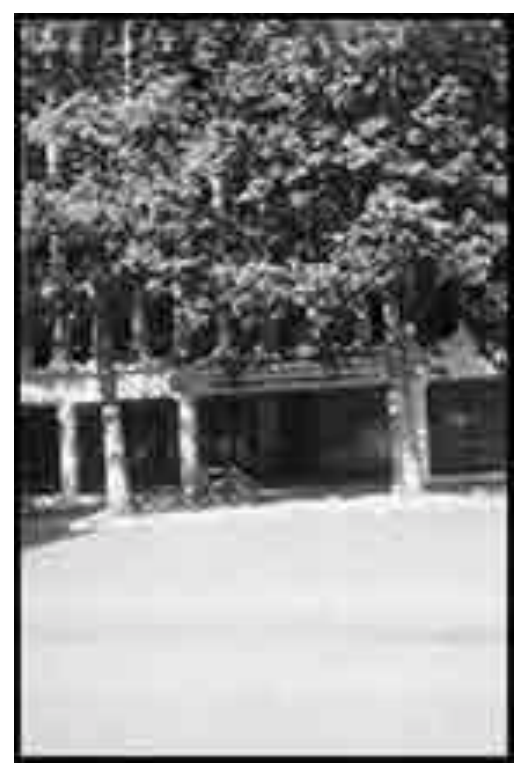

Figure 185: Alzheimer's Center Building: My safety refuge with fabulous support persons.

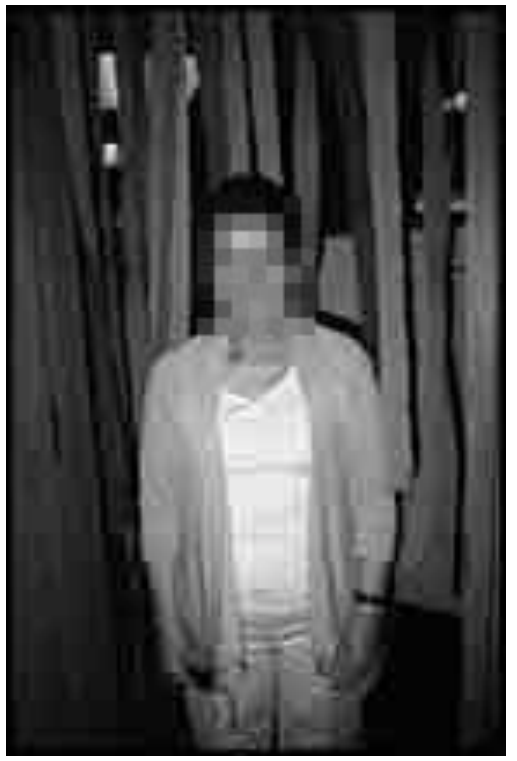

Figure 186: Melissa: She is a true angel of mercy who has guided me through the first stages of this journey. 


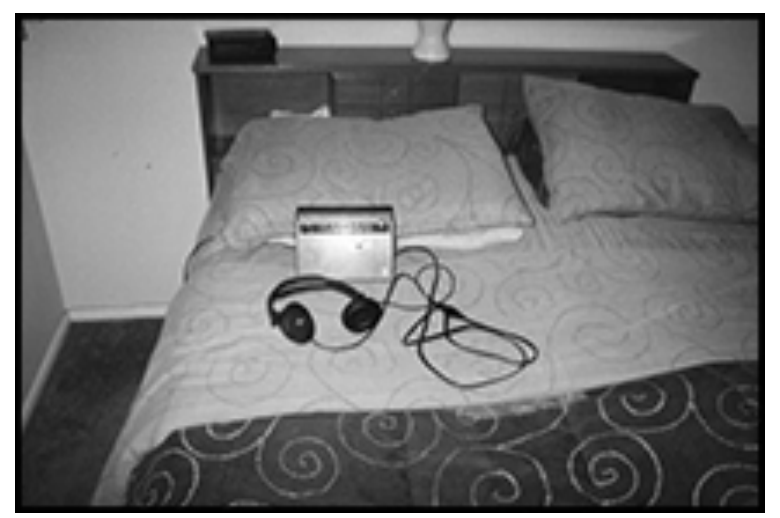

Figure 187: My Radio and Ear Phones: I enjoy listening to my favorite radio program when in bed at night.

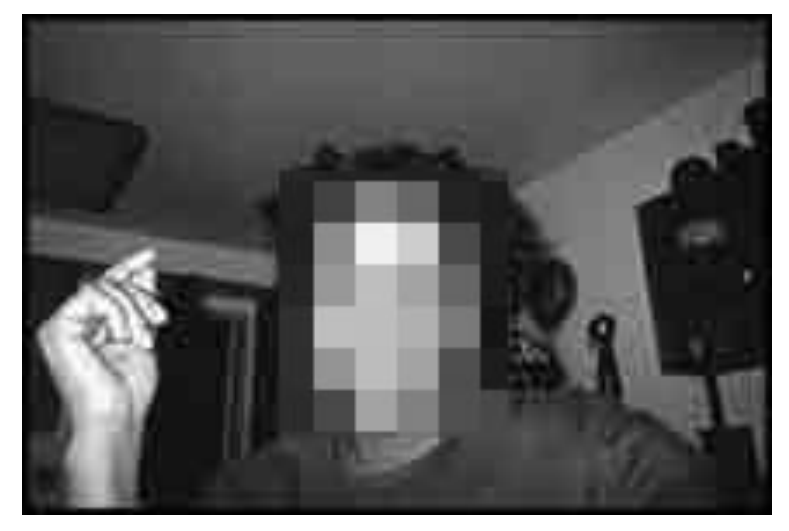

Figure 188: My Weekend Caregiver: I like Tamara.

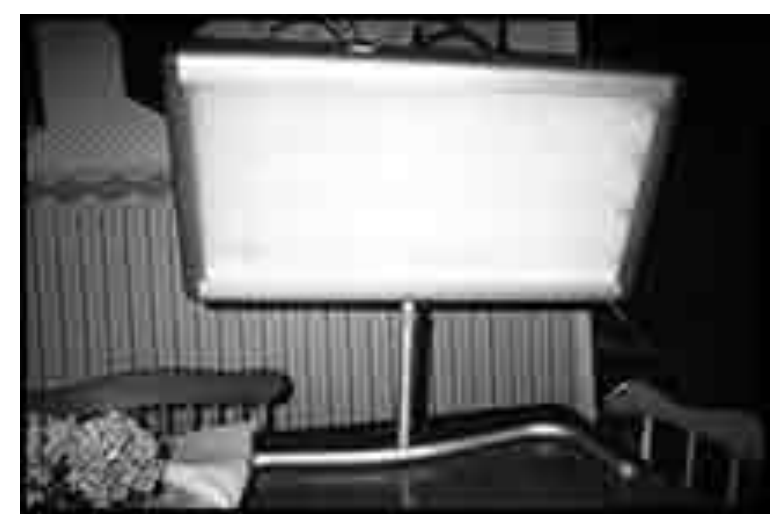

Figure 189: Sun-Replacement Lamp: It keeps me from getting very depressed. 


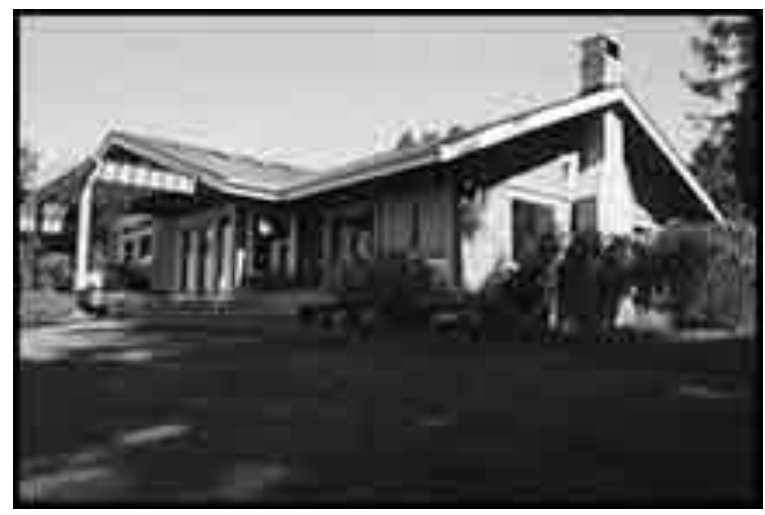

Figure 190: The Rear of Our House: Facing the Hood Canal.

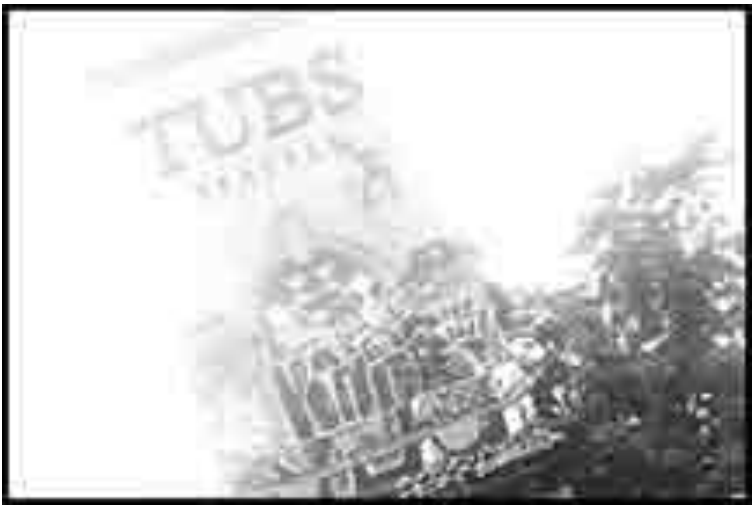

Figure 191: The Graffiti Menace of this Storefront Is the Opposite of How I Want my Life to Be.

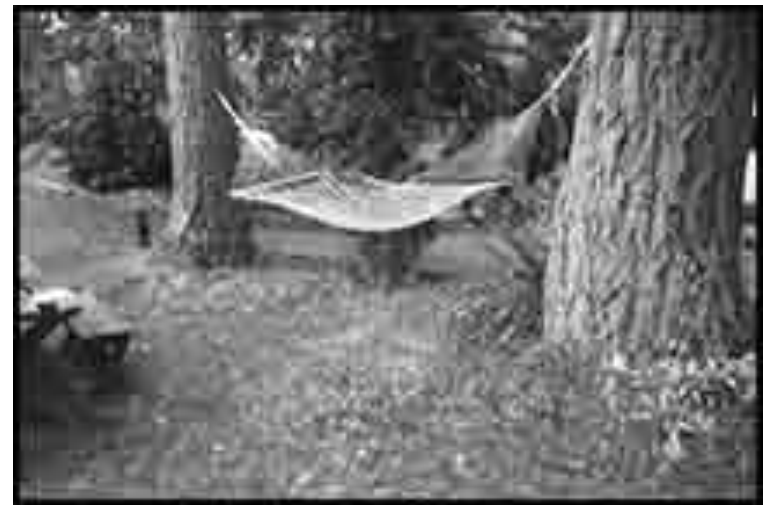

Figure 192: Hammock: For resting. 


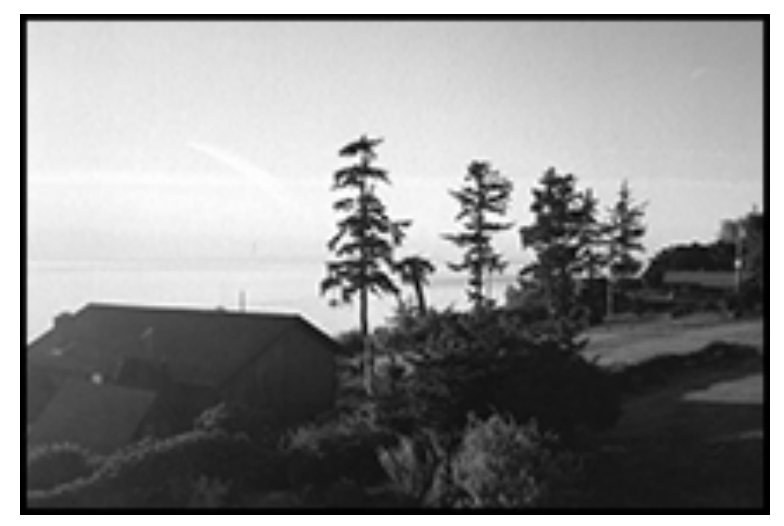

Figure 193: The Sunset and Evening Sky: Restful feelings and contentment.

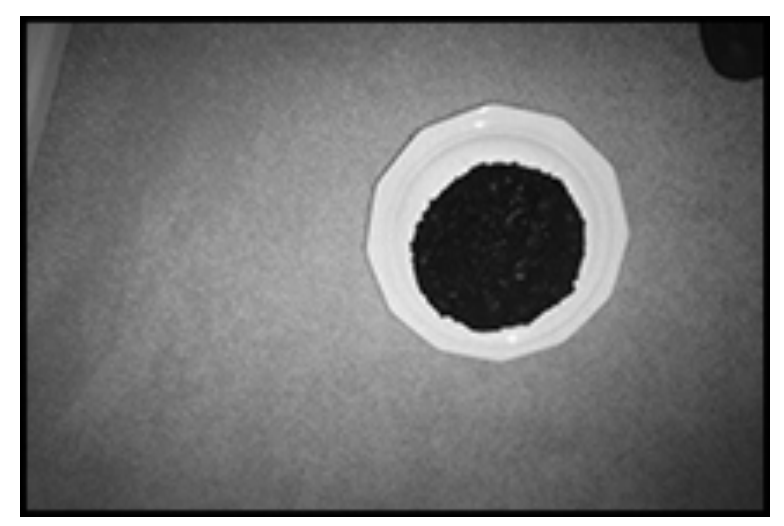

Figure 194: My Cooking Abilities: That's why we eat out almost every day.

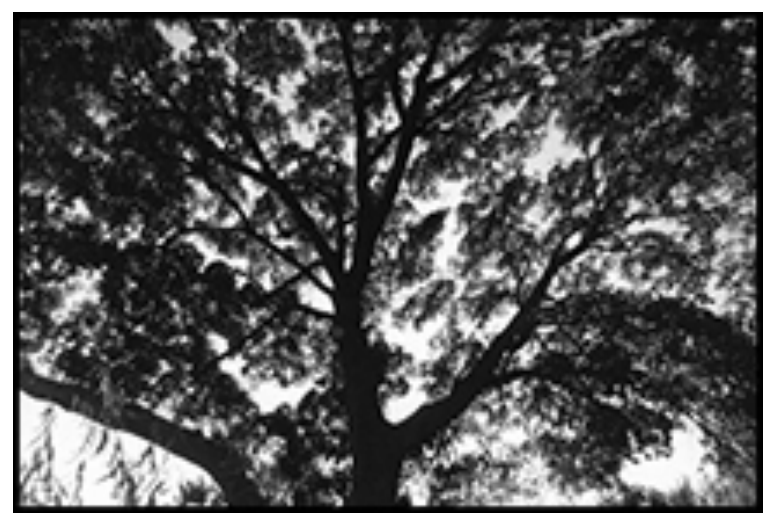

Figure 195: Maple Tree in Our Yard: I have pruned it for years. 


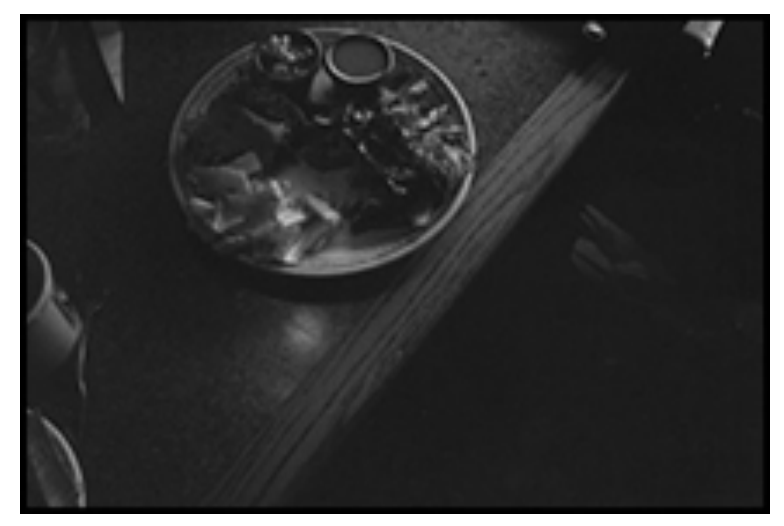

Figure 196: Dinner at the Golf Club: I love salads.

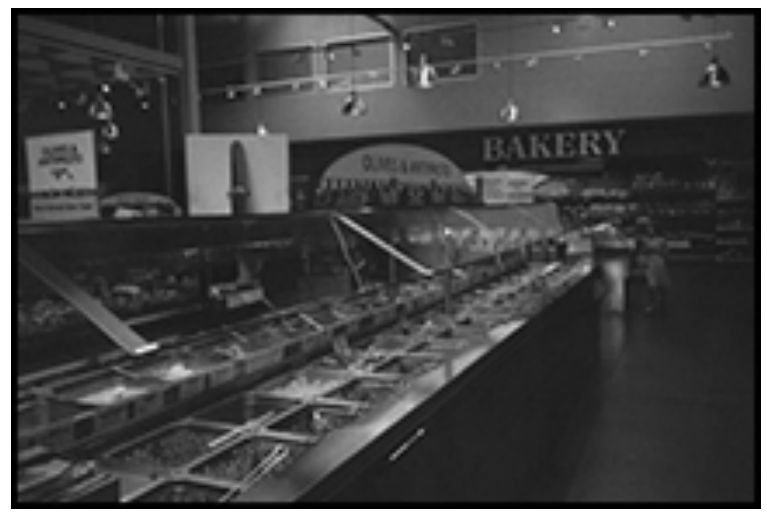

Figure 197: Display of Goodies: I shop a lot at Central Market for really healthy food.

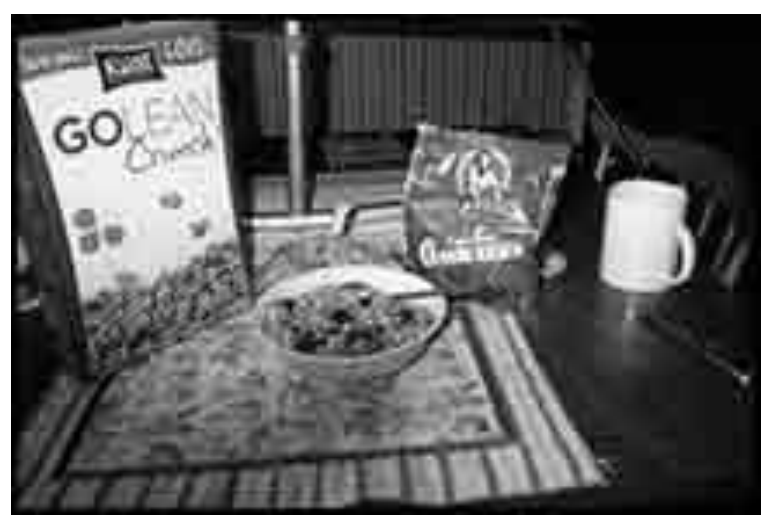

Figure 198: Daily Health Breakfast: Good nutrition and losing weight is said to decrease symptoms. 


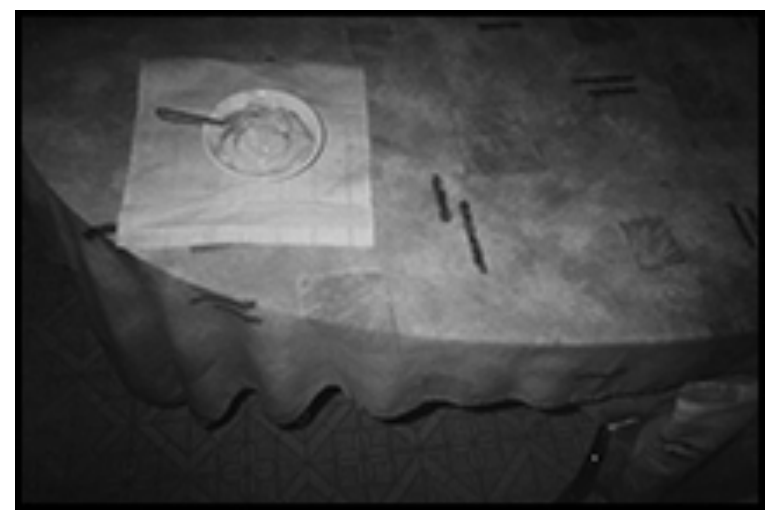

Figure 199:Dish of Strawberry Ice Cream: My favorite kind of ice cream that I eat for lunch daily.

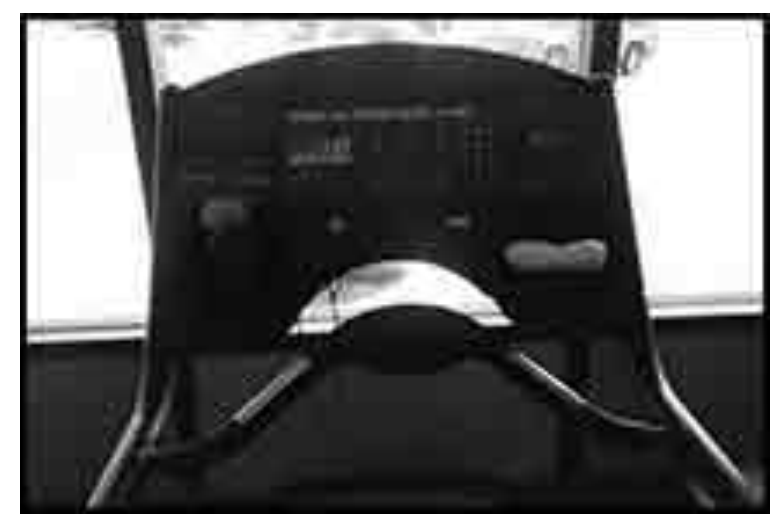

Figure 200: Working out 4-6 times a week is part of my fight to slow AD [Alzheimer's Disease].

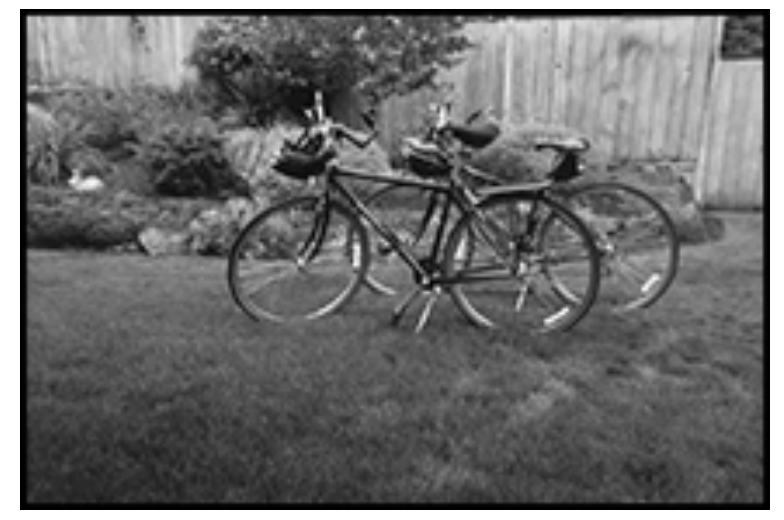

Figure 201: Bikes: I like to ride bikes with family and friends. 


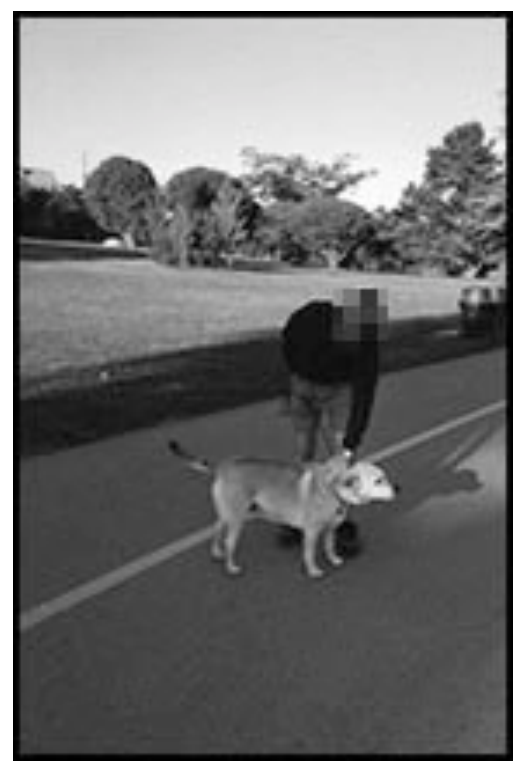

Figure 202: Dora at Greenlake: Just follow the yellow line and you can't get lost at Greenlake.

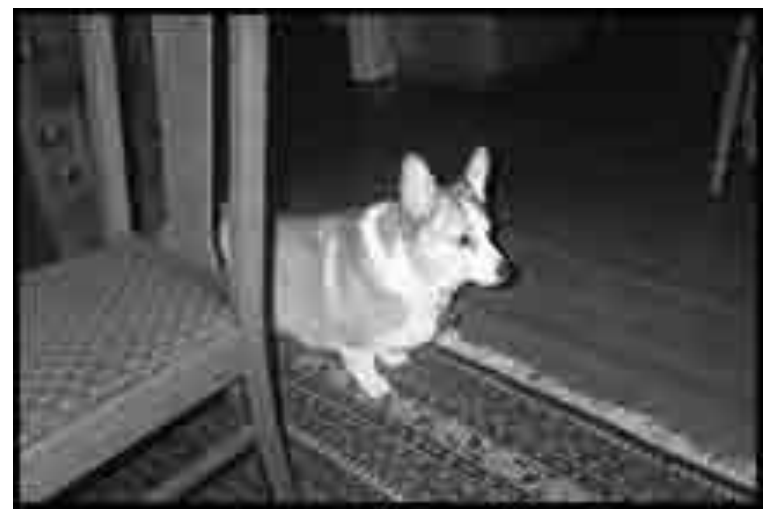

Figure 203: Nicolas: Great pet - I walk him every day. 


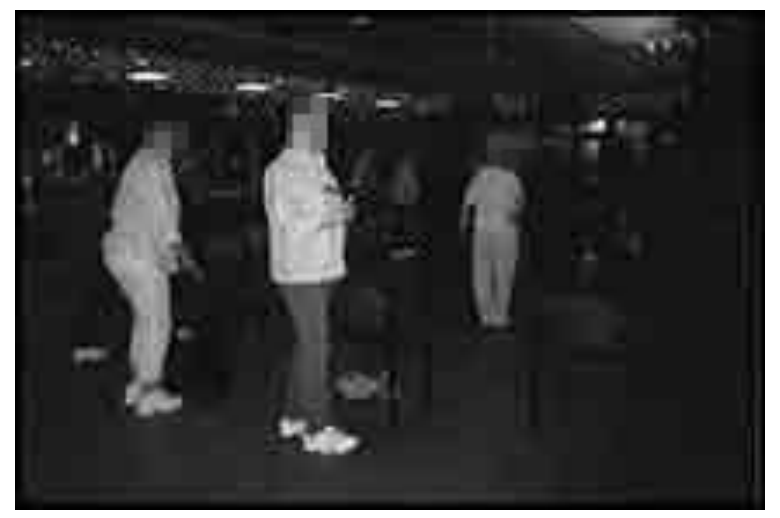

Figure 204: My Exercise Class: It is good - it makes me exercise and I meet lots of nice people.

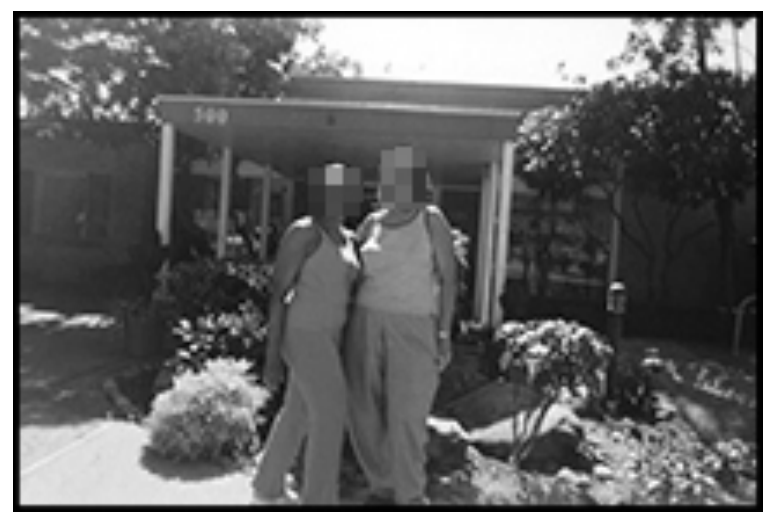

Figure 205: My Sound Step Walking Partners: I walked 2.7 miles. At least 3x week, 8:30 AM to 11:00 AM, after our exercise class, we walk 2.7 miles for our Sound Step Program.

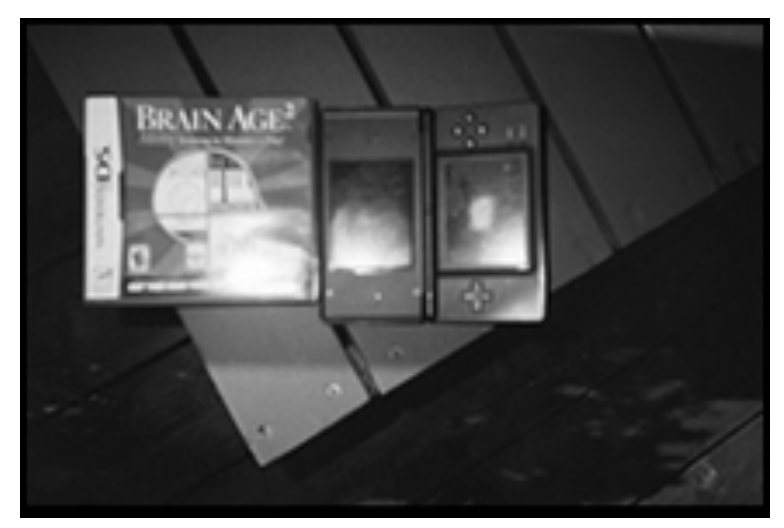

Figure 206: Nintendo: It is challenging and helps me keep my mind alert. 


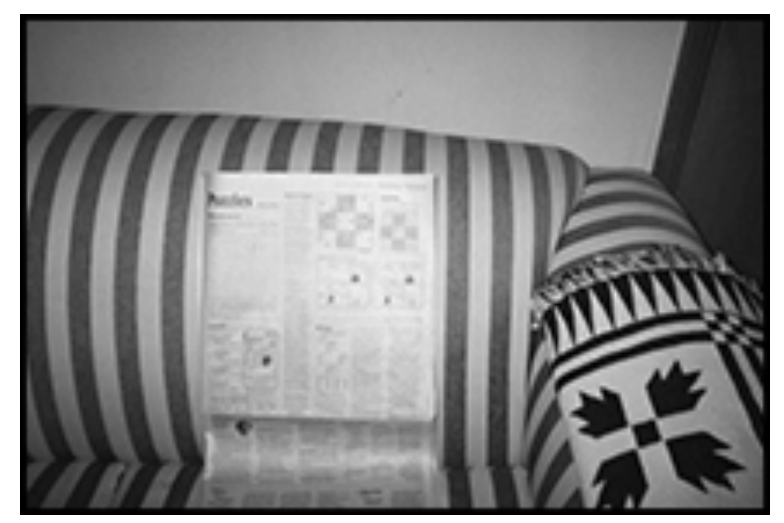

Figure 207: Hocus Focus: I enjoy Hocus Focus and read it in my local newspaper to figure out the answers.

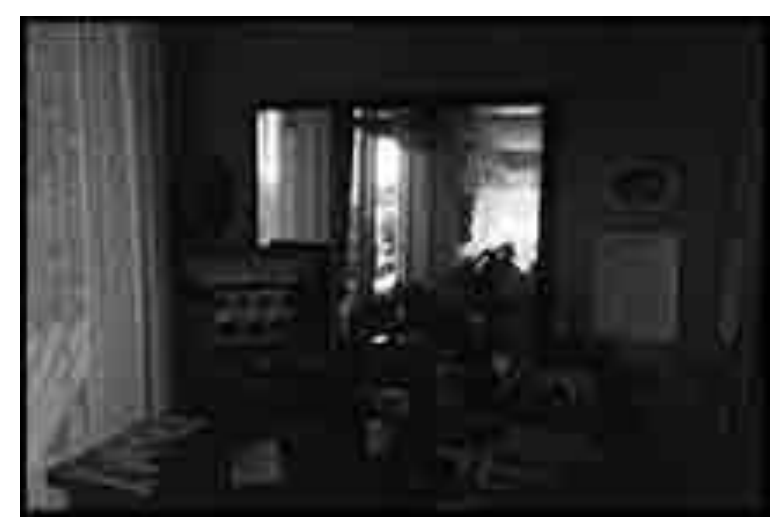

Figure 208: Brain Food: My computer 'games'... as well as Soduko daily puzzles in the Seattle Times. 


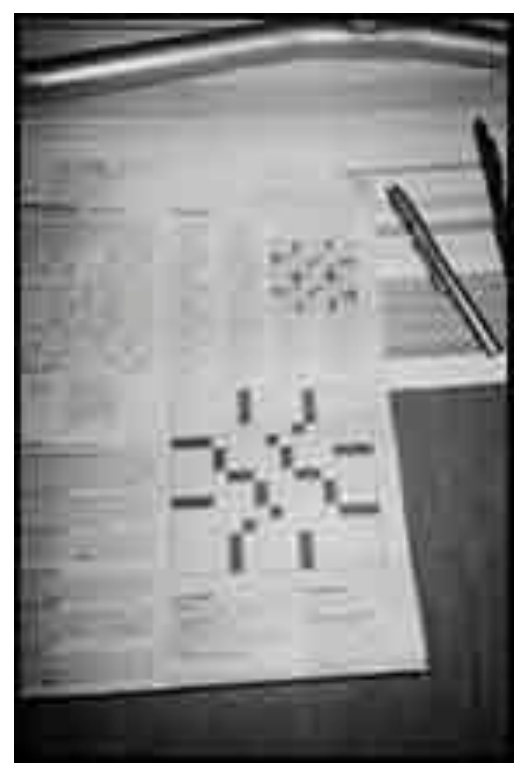

Figure 209: Crosswords in Newspaper Every Day but Sunday: Gets my brain working and boosts my ego.

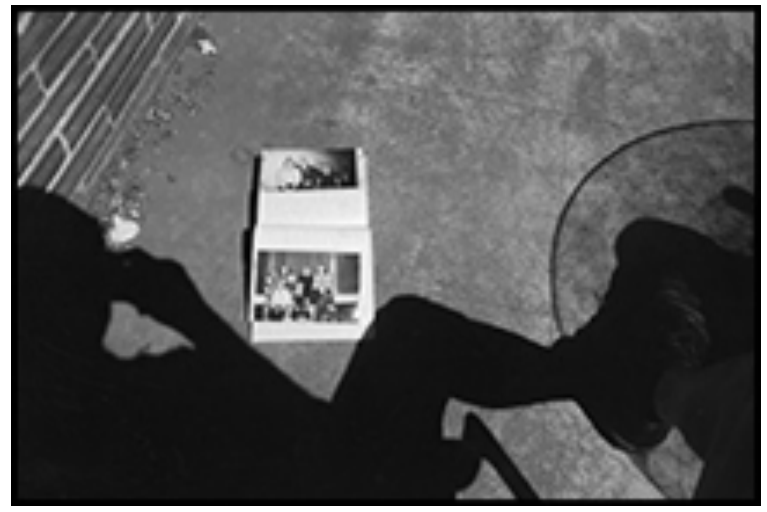

Figure 210: Our Family at Our Port Townsend Beach Cabin: Most everyone is doing great, as is the old cabin. 